Electronic Supporting Information

\title{
Enantioselective Hydrogenation toward Chiral 3-Aryloxy Tetrahydrofurans Enabled by Spiro Ir-PNN Catalysts Containing an Unusual 5-Substituted Chiral Oxazoline Unit
}

\author{
Xue-Song Gu, ${ }^{a}$ Ying Xiong, ${ }^{a}$ Fan Yang, ${ }^{a}$ Na Yu, ${ }^{a}$ Pu-Cha Yan,${ }^{b, *}$ Jian-Hua Xie,,${ }^{a, *}$ Qi-Lin Zhou ${ }^{a}$ \\ ${ }^{a}$ State Key Laboratory and Institute of Elemento-organic Chemistry, College of Chemistry, Nankai \\ University, Tianjin 300071, China \\ ${ }^{b}$ Raybow (Hangzhou) Pharmaceutical CO., Ltd. Hangzhou 310018, China \\ E-mail: jhxie@nankai.edu.cn \\ pucha.yan@raybowpharma.com
}

Table of Contents

General.

(A) Preparation of Spiro-5-OAP ligands and Their Iridium Catalysts.................................................S2

(B) Preparation of Racemic $\alpha$-Aryloxy Lactones..................................................................................S11

(C) Asymmetric Hydrogenation of Racemic $\alpha$-Aryloxy Lactones.............................S16

(D) Enantioselective Synthesis of 3-Aryloxy Substituted Tetrahydrofurans 6...................S26

(E) Enantioselective Synthesis of the Advanced Chiral Intermediate (S)-13.....................S29

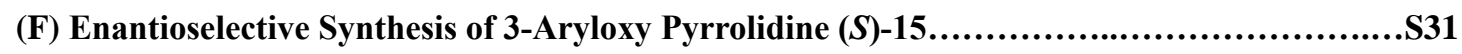

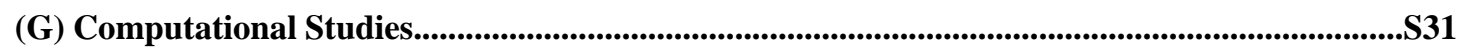

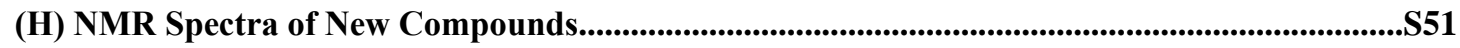

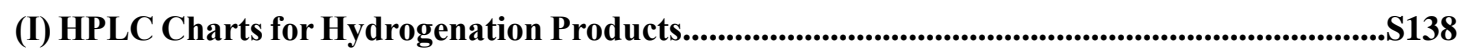

General: All reactions and manipulations which are sensitive to moisture or air were performed under inert atmosphere of nitrogen or argon. All chemicals were purchased from Adamas, Innochem and Aldrich and were used as received. Hydrogen gas (99.999\%) was purchased from Boc Gas Inc., Tianjin. Anhydrous THF was distilled from sodium benzophenone ketyl. Anhydrous $\mathrm{CH}_{2} \mathrm{Cl}_{2}, n \mathrm{PrOH}$ and $\mathrm{Et}_{3} \mathrm{~N}$ were freshly distilled from calcium hydride. Anhydrous EtOH was freshly distilled from magnesium. Melting points were measured on a RY-I apparatus and uncorrected. ${ }^{1} \mathrm{H}$ NMR spectra were recorded at $400 \mathrm{MHz}$ on Bruker AV 400 spectrometer. ${ }^{13} \mathrm{C}$ NMR spectra were recorded at $100 \mathrm{MHz}$ on Bruker AV 400 spectrometer. NMR spectra were recorded in deuterated chloroform $\left(\mathrm{CDCl}_{3}\right)$ as a solvent, with residual chloroform ( $\delta 7.26 \mathrm{ppm}$. for ${ }^{1} \mathrm{H}$ NMR and $\delta 77.00 \mathrm{ppm}$. for $\left.{ }^{13} \mathrm{C} \mathrm{NMR}\right)$ or tetramethylsilane (TMS, $\delta 0.00 \mathrm{ppm}$. for ${ }^{1} \mathrm{H}$ NMR) taken as the inert standard, and were reported in ppm. Abbreviations for signal coupling are as follows: s, singlet; $d$, doublet; $t$, triplet; $q$, quartet; m, multiplet. Coupling constants were taken from the spectra directly and are uncorrected. Optical rotations were determined using a Perkin Elmer 341 polarimeter. HRMS were recorded on APEXII and ZAB-HS spectrometer. HPLC analyses were performed using Hewlett Packard Model HP1100 instruments with Chiralcel OJ-3, OD-3, AD-3, IC-3, IF-3, IG-3, IH-3 column. 


\section{(A) Preparation of Spiro-5-OAP Ligands and Their Iridium Catalysts.}

The procedure for the synthesis of spiro-5-OAP ligands $\mathbf{S 6}$ was outlined as below. Among them, spiro amino acid $\mathbf{S} \mathbf{4}$ could be synthesized from spiro aminophosphine (S)-SpiroAP and ethyl 2-oxoacetate via two steps in around $85 \%$ yield according to our previously established procedure. ${ }^{1}$

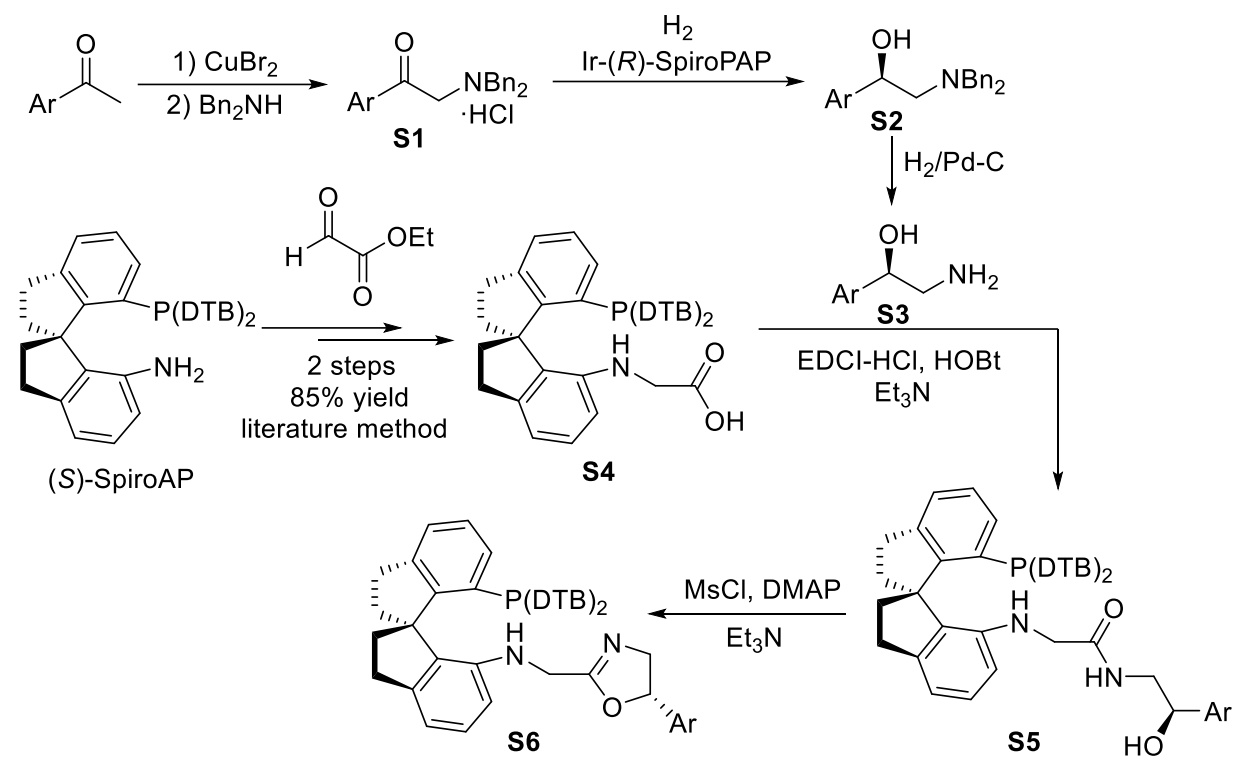

\section{Preparation of $\alpha$-dibenzylamino acetophenones S1.}

The $\alpha$-amino acetophenones $\mathbf{S 1}$ were prepared from the corresponding aryl ketones according to literature procedure. $^{2}$

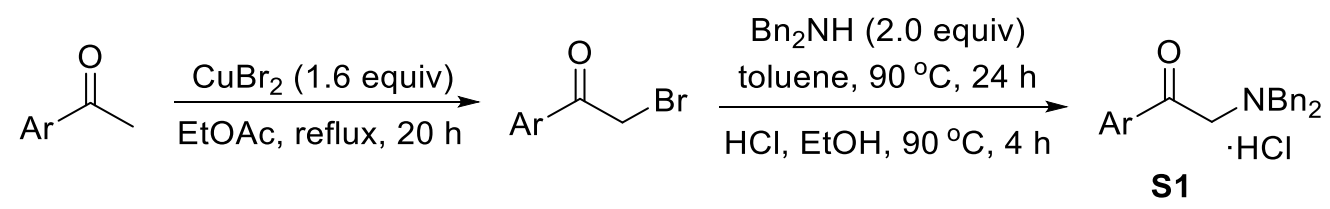

General procedure: To a solution of acetophenones $(50.0 \mathrm{mmol})$ in ethyl acetate $(50 \mathrm{~mL})$ was added $\mathrm{CuBr}_{2}(18.3 \mathrm{~g}, 82.0 \mathrm{mmol})$. The reaction mixture was heated at reflux in an oil bath for $6 \mathrm{~h}$. Then the solution was cooled, and the resulting solid was filtered off and washed with ethyl acetate $(20 \mathrm{~mL})$. The filtrate was concentrated in vacuo. The residue was chromatographed on silica gel column with ethyl acetate/petroleum ether (from 20:1 to 10:1) as an eluent to offer the corresponding product as white/yellow solid or red oil. The yielding solid or oil was resolved in toluene $(20 \mathrm{~mL})$, and then dibenzylamine $(19.7 \mathrm{~g}, 100.0 \mathrm{mmol})$ was added. The resulting mixture was heated to $90^{\circ} \mathrm{C}$ in an oil bath for $24 \mathrm{~h}$. The formed hydrobromide salt was then filtered and the filtrate was evaporated to give the corresponding $\alpha$-amino acetophenone. The yielded $\alpha$-amino acetophenone was then treated with hydrochloric acid and recrystallized in hot anhydrous $\mathrm{EtOH}$ to give the corresponding hydrochloride product as a white crystalline solid.

\section{2-(Dibenzylamino)-1-(3,5-dimethylphenyl)ethanone hydrochloride (S1a)}




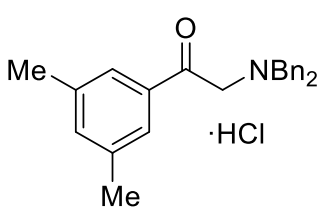

White solid, mp $102-104^{\circ} \mathrm{C}, 9.4 \mathrm{~g}, 50 \%$ yield. $\mathrm{R}_{f}=0.42$ (petroleum ether/ethyl acetate $=10: 1, \mathrm{v} / \mathrm{v}) .{ }^{1} \mathrm{H}$ NMR $\left(400 \mathrm{MHz}, \mathrm{CDCl}_{3}\right) \delta: 13.13(\mathrm{~s}, 1 \mathrm{H}), 7.85-7.69$ (m, 4H), 7.46-7.33 (m, 6H), $7.22(\mathrm{~s}, 3 \mathrm{H}), 4.80-4.55(\mathrm{~m}, 4 \mathrm{H}), 4.15$ (s, 2H), 2.29 (s, 6H). ${ }^{13} \mathrm{C}$ NMR $\left(101 \mathrm{MHz}, \mathrm{CDCl}_{3}\right) \delta: 192.8,138.8,136.6,133.9,131.6$, 344.2012 .

\section{2-(Dibenzylamino)-1-(4-methoxyphenyl)ethanone hydrochloride (S1b)}<smiles>COc1ccc(C(=O)CNC(=O)c2ccccc2)cc1</smiles>

White solid, mp $139-140{ }^{\circ} \mathrm{C}, 13.3 \mathrm{~g}, 70 \%$ yield. $\mathrm{R}_{f}=0.39$ (petroleum ether/ethyl acetate $=10: 1, \mathrm{v} / \mathrm{v}) .{ }^{1} \mathrm{H}$ NMR $\left(400 \mathrm{MHz}, \mathrm{CDCl}_{3}\right) \delta: 13.10(\mathrm{~s}$, 1H), 7.83-7.72 (m, 4H), 7.66-7.57 (m, 2H), 7.42-7.30 (m, 6H), 6.91$6.81(\mathrm{~m}, 2 \mathrm{H}), 4.81-4.63(\mathrm{~m}, 4 \mathrm{H}), 4.11(\mathrm{~d}, J=2.0 \mathrm{~Hz}, 2 \mathrm{H}), 3.93-3.81(\mathrm{~m}$, 3H). ${ }^{13} \mathrm{C} \mathrm{NMR}\left(101 \mathrm{MHz}, \mathrm{CDCl}_{3}\right) \delta: 190.6,164.9,131.6,130.1,130.0,129.3(2), 126.9,114.3,57.4$, 55.7, 50.6. HRMS (ESI) Calcd for $\mathrm{C}_{23} \mathrm{H}_{24} \mathrm{NO}_{2}\left([\mathrm{M}+\mathrm{H}]^{+}\right)$: 346.1802; Found: 346.1803.

\section{2-(Dibenzylamino)-1-(4-(trifluoromethyl)phenyl)ethanone hydrochloride (S1c)}<smiles>O=C(CNc1ccccc1)c1ccc(C(F)(F)F)cc1</smiles>

White solid, mp $155-156{ }^{\circ} \mathrm{C}, 13.4 \mathrm{~g}, 64 \%$ yield. $\mathrm{R}_{f}=0.40$ (petroleum ether/ethyl acetate $=10: 1, \mathrm{v} / \mathrm{v}) .{ }^{1} \mathrm{H} \mathrm{NMR}\left(400 \mathrm{MHz}, \mathrm{CDCl}_{3}\right) \delta: 13.35(\mathrm{~s}$, 1H), 7.80-7.71 (m, 6H), 7.66 (d, J=8.0 Hz, 2H), 7.40-7.30 (m, 6H), 4.86$4.60(\mathrm{~m}, 4 \mathrm{H}), 4.25(\mathrm{~s}, 2 \mathrm{H}) .{ }^{13} \mathrm{C} \mathrm{NMR}\left(101 \mathrm{MHz}, \mathrm{CDCl}_{3}\right) \delta: 192.0,136.4$, $136.0\left(\mathrm{q},{ }^{2} J_{\mathrm{C}-\mathrm{F}}=33.3 \mathrm{~Hz}\right), 131.5,130.2,129.4,129.1,128.1,126.1\left(\mathrm{q},{ }^{3} J_{\mathrm{C}-\mathrm{F}}=4.0 \mathrm{~Hz}\right), 123.1\left(\mathrm{q},{ }^{1} J_{\mathrm{C}-\mathrm{F}}=\right.$ $273.7 \mathrm{~Hz}), 57.5$, 51.4. HRMS (ESI) Calcd for $\mathrm{C}_{23} \mathrm{H}_{21} \mathrm{~F}_{3} \mathrm{NO}\left([\mathrm{M}+\mathrm{H}]^{+}\right)$: 384.1570; Found: 384.1581 .

\section{Preparation of $\alpha$-dibenzylamino arylethanols S2.}

The preparation of amino alcohols $\mathbf{S 2}$ from aminoketone $\mathbf{S 1}$ via catalytic asymmetric hydrogenation was according to our previously established procedure. ${ }^{3}$<smiles>[13CH][18O]CC(=O)Br</smiles>

s1

$$
\underset{t \mathrm{HuOK}, \mathrm{EtOH}, 30^{\circ} \mathrm{C}}{\stackrel{\mathrm{H}_{2}(10 \mathrm{~atm})}{0.1 \mathrm{~mol} \% \mathrm{Ir}-(R)-S p i r o P A P}}
$$

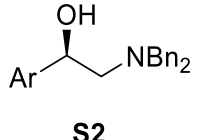

S2

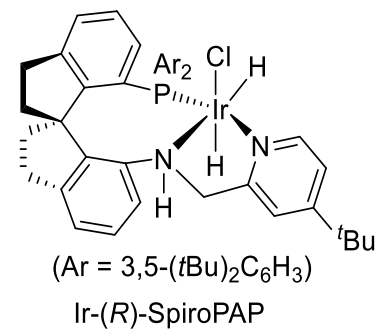

Ir-(R)-SpiroPAP

General procedure: To a suitable glass tube equipped with a magnetic stir bar was added $\alpha$ dibenzylamino acetophenones (as a hydrochloride, $7.0 \mathrm{mmol}), \mathrm{BuOK}(0.94 \mathrm{~g}, 8.4 \mathrm{mmol})$, iridium catalyst Ir- $(R)$-SpiroPAP $(7.0 \mathrm{mg}, 7.0 \mu \mathrm{mol})$ under argon atmosphere. Then the tube was placed in a stainless vessel and anhydrous EtOH $(40 \mathrm{~mL})$ was then added via an injection port. The vessel was then closed and quickly purged with $\mathrm{H}_{2}$ for three times. After the vessel was pressurized to 10 atm with $\mathrm{H}_{2}$, the reaction mixture was stirred at room temperature $\left(30^{\circ} \mathrm{C}\right)$ until no obvious hydrogen pressure drop 
was observed (12 h). After releasing the hydrogen pressure carefully, the solvent of the reaction mixture was removed under reduced pressure and the residue was purified by recrystallization of the crude solid from ethyl acetate/hexane to afford the desired chiral amino alcohol. The enantiomeric excess of the product was determined by chiral HPLC analysis of the chiral amino alcohol directly.

\section{(R)-2-(Dibenzylamino)-1-(3,5-dimethylphenyl)ethanol (S2a)}

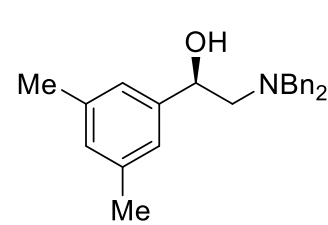

White solid, mp $80-81{ }^{\circ} \mathrm{C}, 1.5 \mathrm{~g}, 64 \%$ yield. $\mathrm{R}_{f}=0.41$ (petroleum ether/ethyl acetate $=5: 1, \mathrm{v} / \mathrm{v}) .>99.9 \%$ ee. $[\alpha]_{\mathrm{D}}^{25}-26.0(c$ 1.0, EtOH $) .{ }^{1} \mathrm{H}$ NMR $(400 \mathrm{MHz}$, $\left.\mathrm{CDCl}_{3}\right) \delta: 7.41-7.26(\mathrm{~m}, 10 \mathrm{H}), 6.91-6.79(\mathrm{~m}, 3 \mathrm{H}), 4.70-4.60(\mathrm{~m}, 1 \mathrm{H}), 3.91$

$(\mathrm{d}, J=13.6 \mathrm{~Hz}, 2 \mathrm{H}), 3.74(\mathrm{~s}, 1 \mathrm{H}), 3.48$ (d, $J=13.2 \mathrm{~Hz}, 2 \mathrm{H}), 2.71-2.59$ (m, 2H) 2.27 (s, 6H). ${ }^{13} \mathrm{C}$ NMR (101 MHz, $\left.\mathrm{CDCl}_{3}\right) \delta: 142.0,138.4,137.8,129.2,129.1,128.5,127.4,123.7$, 69.6, 62.0, 58.3, 21.4. HRMS (ESI) Calcd for $\mathrm{C}_{24} \mathrm{H}_{28} \mathrm{NO}\left([\mathrm{M}+\mathrm{H}]^{+}\right): 346.2165$; Found: 346.2173. HPLC conditions: Chiralcel IC-3 column ( $25 \mathrm{~cm} \times 0.46 \mathrm{~cm}$ ID); $n$-hexane/2-propanol = 95:5; temp, rt; flow rate $=1.0 \mathrm{~mL} / \mathrm{min} ; 220 \mathrm{~nm} \mathrm{UV}$ detector; $t_{\mathrm{R}}(R)=6.8 \mathrm{~min}$ (major); $t_{\mathrm{R}}(S)=9.6 \mathrm{~min}$ (minor).

\section{(R)-2-(Dibenzylamino)-1-(4-methoxyphenyl)ethanol (S2b)}<smiles>COc1ccc([C@@H](O)CNCc2ccccc2)cc1</smiles>

White solid, mp $81-82{ }^{\circ} \mathrm{C}, 1.5 \mathrm{~g}, 62 \%$ yield. $\mathrm{R}_{f}=0.37$ (petroleum ether/ethyl acetate $=5: 1, \mathrm{v} / \mathrm{v}) . \quad>99.9 \%$ ee. $[\alpha]_{\mathrm{D}}^{25}-32.2(c 1.0, \mathrm{EtOH}) .{ }^{1} \mathrm{H}$ NMR $(400$ $\left.\mathrm{MHz}, \mathrm{CDCl}_{3}\right) \delta: 7.41-7.24(\mathrm{~m}, 10 \mathrm{H}), 7.14(\mathrm{~d}, J=8.8 \mathrm{~Hz}, 2 \mathrm{H}), 6.82(\mathrm{~d}, J=$ $8.8 \mathrm{~Hz}, 2 \mathrm{H}), 4.66(\mathrm{dd}, J=10.0,4.0 \mathrm{~Hz}, 1 \mathrm{H}), 3.91$ (d, $J=13.6 \mathrm{~Hz}, 2 \mathrm{H}), 3.82-3.73$ (m, 4H), 3.46 (d, $J=$ $13.6 \mathrm{~Hz}, 2 \mathrm{H}), 2.70-2.52$ (m, 2H). ${ }^{13} \mathrm{C} \mathrm{NMR}\left(101 \mathrm{MHz}, \mathrm{CDCl}_{3}\right) \delta: 159.0,138.4,134.2,129.2,128.5$, 127.4, 127.2, 113.7, 69.2, 61.9, 58.3, 55.3. HRMS (ESI) Calcd for $\mathrm{C}_{23} \mathrm{H}_{26} \mathrm{NO}_{2}\left([\mathrm{M}+\mathrm{H}]^{+}\right): 348.1958$; Found: 348.1966. HPLC conditions: Chiralcel IC-3 column $(25 \mathrm{~cm} \times 0.46 \mathrm{~cm} \mathrm{ID)}$; $n$-hexane/2-propanol $=95: 5 ;$ temp, rt; flow rate $=1.0 \mathrm{~mL} / \mathrm{min} ; 210 \mathrm{~nm} \mathrm{UV} \mathrm{detector} ; t_{\mathrm{R}}(R)=19.1 \mathrm{~min}$ (major); $t_{\mathrm{R}}(S)=21.2$ $\min$ (minor).

\section{(R)-2-(Dibenzylamino)-1-(4-(trifluoromethyl)phenyl)ethanol (S2c)}<smiles>O[C@H](CNCCc1ccccc1)c1ccc(C(F)(F)F)cc1</smiles>

White solid, mp 104-105 ${ }^{\circ} \mathrm{C}, 1.7 \mathrm{~g}, 62 \%$ yield. $\mathrm{R}_{f}=0.39$ (petroleum ether/ethyl acetate $=5: 1, \mathrm{v} / \mathrm{v}) .>99.9 \%$ ee. $[\alpha]_{\mathrm{D}}^{25}-11.0(c 1.0, \mathrm{EtOH}) .{ }^{1} \mathrm{H}$ NMR (400 MHz, $\left.\mathrm{CDCl}_{3}\right) \delta: 7.53(\mathrm{~d}, J=8.0 \mathrm{~Hz}, 2 \mathrm{H}), 7.43-7.26(\mathrm{~m}, 12 \mathrm{H})$, $4.72(\mathrm{dd}, J=10.0,4.0 \mathrm{~Hz}, 1 \mathrm{H}), 3.92(\mathrm{~d}, J=13.6 \mathrm{~Hz}, 2 \mathrm{H}), 3.87$ (s, 1H), 3.50 $(\mathrm{d}, J=13.6 \mathrm{~Hz}, 2 \mathrm{H}), 2.70-2.55(\mathrm{~m}, 2 \mathrm{H}) .{ }^{13} \mathrm{C} \mathrm{NMR}\left(101 \mathrm{MHz}, \mathrm{CDCl}_{3}\right) \delta: 146.3,138.1,129.6\left(\mathrm{q},{ }^{2} J_{\mathrm{C}-\mathrm{F}}=\right.$ $32.3 \mathrm{~Hz}), 129.1,128.6,127.5,126.1,125.2\left(\mathrm{q},{ }^{3} J_{\mathrm{C}-\mathrm{F}}=3.0 \mathrm{~Hz}\right), 124.2\left(\mathrm{q},{ }^{1} J_{\mathrm{C}-\mathrm{F}}=272.7 \mathrm{~Hz}\right), 69.1,61.7$, 58.5. HRMS (ESI) Calcd for $\mathrm{C}_{23} \mathrm{H}_{23} \mathrm{~F}_{3} \mathrm{NO}\left([\mathrm{M}+\mathrm{H}]^{+}\right)$: 386.1726; Found: 386.1732. HPLC conditions: Chiralcel AD-H column $(25 \mathrm{~cm} \times 0.46 \mathrm{~cm} \mathrm{ID}) ; n$-hexane $/ 2$-propanol $=95: 5$; temp, rt; flow rate $=1.0$ $\mathrm{mL} / \mathrm{min} ; 210 \mathrm{~nm} \mathrm{UV}$ detector; $t_{\mathrm{R}}(R)=9.2 \min \left(\right.$ major); $t_{\mathrm{R}}(S)=8.6 \min$ (minor).

\section{Preparation of $\alpha$-amino alcohols S3.}




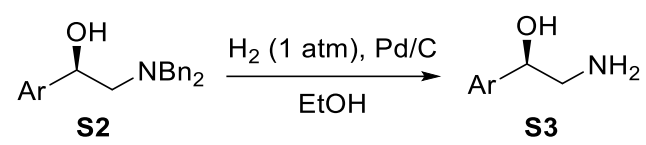

General procedure: A mixture of $\mathbf{S 2}(2.0 \mathrm{mmol}), 10 \% \mathrm{Pd} / \mathrm{C}(51-59 \mathrm{mg}, 10 \% \mathrm{wt})$, and EtOH $(10 \mathrm{~mL}) \mathrm{in}$ a $50 \mathrm{~mL}$ Schlenk tube was stirred under ambient $\mathrm{H}_{2}$ pressure at room temperature for $24 \mathrm{~h}$. The reaction mixture was then filtered through a Celite, and the filtrate was concentrated in vacuo to afford the desired $\alpha$-amino alcohol $\mathbf{S 3}$ as a yellow oil or solid.

\section{(R)-2-Amino-1-(3,5-dimethylphenyl)ethanol (S3a)}<smiles>Cc1cc(C)cc([C@@H](O)CN)c1</smiles>

Yellow oil, $277.0 \mathrm{mg}, 84 \%$ yield. $[\alpha]_{\mathrm{D}}^{25}-32.2\left(c\right.$ 1.0, EtOH). $\mathrm{R}_{f}=0.35$ (dichloromethane/methyl alcohol $=10: 1, \mathrm{v} / \mathrm{v}) .{ }^{1} \mathrm{H}$ NMR $\left(400 \mathrm{MHz}, \mathrm{CDCl}_{3}\right) \delta$ : 6.99-6.92 (m, 2H), $6.90(\mathrm{~s}, 1 \mathrm{H}), 4.55(\mathrm{dd}, J=7.6,4.0 \mathrm{~Hz}, 1 \mathrm{H}), 2.97-2.85$ (m,

1H), 2.85-2.75 (m, 1H), $2.58(\mathrm{br}, 3 \mathrm{H}), 2.30(\mathrm{~s}, 6 \mathrm{H}) .{ }^{13} \mathrm{C} \mathrm{NMR}\left(101 \mathrm{MHz}, \mathrm{CDCl}_{3}\right)$

$\delta: 142.5,138.0,129.2,123.7,74.3,49.2,21.4$. HRMS (ESI) Calcd for $\mathrm{C}_{10} \mathrm{H}_{16} \mathrm{NO}\left([\mathrm{M}+\mathrm{H}]^{+}\right): 166.1226$; Found: 166.1228 .

\section{(R)-2-Amino-1-(4-methoxyphenyl)ethanol (S3b)}<smiles>COc1ccc([C@@H](O)CN)cc1</smiles>

Yellow solid, mp $99-100{ }^{\circ} \mathrm{C}, 250.0 \mathrm{mg}, 75 \%$ yield. $[\alpha]_{\mathrm{D}}^{25}-36.2(c 1.0, \mathrm{EtOH})$. $\mathrm{R}_{f}=0.32$ (dichloromethane/methyl alcohol = 10:1, v/v). ${ }^{1} \mathrm{H} \mathrm{NMR}(400 \mathrm{MHz}$, $\left.\mathrm{CDCl}_{3}\right) \delta: 7.28(\mathrm{~d}, J=8.4 \mathrm{~Hz}, 2 \mathrm{H}), 6.89(\mathrm{~d}, J=8.4 \mathrm{~Hz}, 2 \mathrm{H}), 4.58(\mathrm{dd}, J=8.0$, $4.0 \mathrm{~Hz}, 1 \mathrm{H}), 3.80(\mathrm{~s}, 3 \mathrm{H}), 3.01-2.92(\mathrm{~m}, 1 \mathrm{H}), 2.84-2.76(\mathrm{~m}, 1 \mathrm{H}), 1.82(\mathrm{br}, 3 \mathrm{H}) .{ }^{13} \mathrm{C} \mathrm{NMR}(101 \mathrm{MHz}$, $\left.\mathrm{CDCl}_{3}\right) \quad \delta:$ 159.0, 134.7, 127.1, 113.8, 74.1, 55.3, 49.3. HRMS (ESI) Calcd for $\mathrm{C}_{9} \mathrm{H}_{14} \mathrm{NO}_{2}$ $\left([\mathrm{M}+\mathrm{H}]^{+}\right): 168.1019 ;$ Found: 168.1015 .

(R)-2-Amino-1-(4-(trifluoromethyl)phenyl)ethanol (S3c $)^{4}$<smiles>NC[C@@H](O)c1ccc(C(F)(F)F)cc1</smiles>

Yellow solid, mp $98-100{ }^{\circ} \mathrm{C}, 309.0 \mathrm{mg}, 75 \%$ yield. $[\alpha]_{\mathrm{D}}^{25}-14.2$ (c 1.0, EtOH). $\mathrm{R}_{f}=0.31$ (dichloromethane/methyl alcohol = 10:1, v/v). ${ }^{1} \mathrm{H}$ NMR (400 MHz, $\left.\mathrm{CDCl}_{3}\right) \delta: 7.61(\mathrm{~d}, J=8.0 \mathrm{~Hz}, 2 \mathrm{H}), 7.48(\mathrm{~d}, J=8.0 \mathrm{~Hz}, 2 \mathrm{H}), 4.69(\mathrm{dd}, J=7.8$, $3.8 \mathrm{~Hz}, 1 \mathrm{H}), 3.16-2.98$ (m, 1H), 2.82-2.68 (m, 1H), 2.53 (br, 3H).

\section{Preparation of spiro amides S5}

Spiro amides $\mathbf{S 5}$ were prepared from $\alpha$-amino alcohols $\mathbf{S 3}$ and spiro amino acid $\mathbf{S} \mathbf{4}$ according to our previously established procedure. ${ }^{1}$

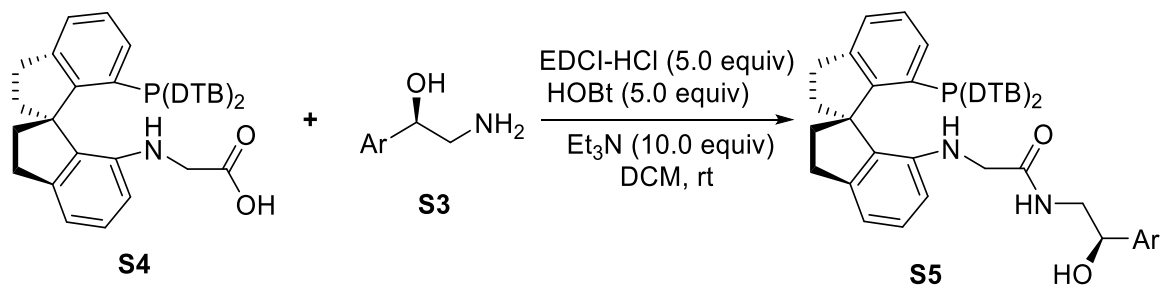


General procedure: To a solution of $\mathbf{S 4}(210.0 \mathrm{mg}, 0.3 \mathrm{mmol}), \alpha$-amino alcohol $\mathbf{S 3}(0.9 \mathrm{mmol}), \mathrm{HOBt}$ (203.0 mg, $1.5 \mathrm{mmol}$ ), and $\mathrm{Et}_{3} \mathrm{~N}$ (433 $\left.\mu \mathrm{L}, 3.0 \mathrm{mmol}\right)$ in $\mathrm{CH}_{2} \mathrm{Cl}_{2}(25 \mathrm{~mL}$ ) was added $\mathrm{EDCI} \cdot \mathrm{HCl}(288.0$ $\mathrm{mg}, 1.5 \mathrm{mmol}$ ) under argon atmosphere at room temperature. The resulting mixture was stirred at room temperature for $12 \mathrm{~h}$. The reaction mixture was then filtered through Celite and the filtrate was then concentrated in vacuo to yield a residue. The residue was purified by silica gel column chromatography with petroleum ether/ethyl acetate (from 5:1 to 3:1) as eluent to afford S5 as a white solid.

\section{Compound $\left(S_{\mathrm{a}}, R\right)$-S5a}

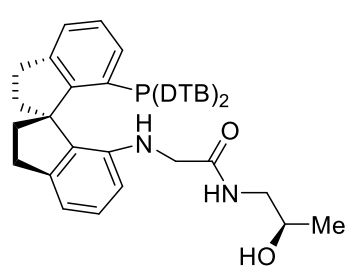

White solid, mp $101-102{ }^{\circ} \mathrm{C}, 186.0 \mathrm{mg}, 82 \%$ yield. $[\alpha]_{\mathrm{D}}^{25}-170.0(c) 0.1$, $\left.\mathrm{CHCl}_{3}\right) . \mathrm{R}_{f}=0.45$ (petroleum ether/ethyl acetate $\left.=2: 1, \mathrm{v} / \mathrm{v}\right) .{ }^{1} \mathrm{H}$ NMR $(400$ $\left.\mathrm{MHz}, \mathrm{CDCl}_{3}\right)$ 8: 7.60-7.51 (m, 1H), 7.35-7.29 (m, 2H), 7.22-7.08 (m, 3H), $6.88(\mathrm{dd}, J=8.0,1.8 \mathrm{~Hz}, 2 \mathrm{H}), 6.76(\mathrm{dd}, J=8.4,2.0 \mathrm{~Hz}, 2 \mathrm{H}), 6.70(\mathrm{~d}, J=7.2$

$\mathrm{Hz}, 1 \mathrm{H}), 6.11(\mathrm{~d}, J=8.0 \mathrm{~Hz}, 1 \mathrm{H}), 3.88-3.79(\mathrm{~m}, 1 \mathrm{H}), 3.77-3.68(\mathrm{~m}, 1 \mathrm{H})$, 3.45-3.32 (m, 2H), 3.20-3.11 (m, 1H), 3.09-2.94 (m, 2H), 2.88-2.70 (m, 2H), 2.60-2.42 (m, 2H), 2.34$2.21(\mathrm{~m}, 1 \mathrm{H}), 2.13-1.99(\mathrm{~m}, 2 \mathrm{H}), 1.89-1.77$ (m, 1H), 1.20 (s, 18H), 1.17 (s, 18H), 0.96 (d, J = 6.0 Hz, 3H). ${ }^{13} \mathrm{C}$ NMR (101 MHz, $\left.\mathrm{CDCl}_{3}\right) \delta: 171.9,151.7,151.5,150.4,150.4,150.2,150.1,145.1(2), 144.1$, 144.0, 143.1(2), 136.1, 136.0, 135.3, 135.2, 134.1, 133.9, 133.1, 131.6(2), 128.5, 128.4, 128.3, 127.7, 127.5, 127.3, 126.1, 122.4, 122.2, 115.6, 108.3, 67.3, 61.7, 61.6, 47.7, 46.67, 37.7(2), 36.2, 34.8, 34.7, 31.4, 31.3, 30.7, 30.5, 20.3. ${ }^{31} \mathrm{P}$ NMR (162 MHz, $\left.\mathrm{CDCl}_{3}\right) \delta$ : - 15.9. HRMS (ESI) Calcd for $\mathrm{C}_{50} \mathrm{H}_{68} \mathrm{~N}_{2} \mathrm{O}_{2} \mathrm{P}$ $\left([\mathrm{M}+\mathrm{H}]^{+}\right):$759.5013; Found: 759.5022 .

\section{Compound $\left(S_{\mathrm{a}}, \boldsymbol{R}\right)$-S5b}

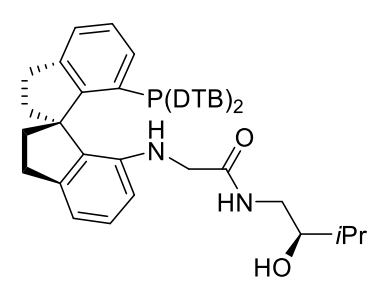

White solid, mp $94-96^{\circ} \mathrm{C}, 175.0 \mathrm{mg}, 74 \%$ yield. $[\alpha]_{\mathrm{D}}^{25}-136.0\left(c 0.1, \mathrm{CHCl}_{3}\right)$. $\mathrm{R}_{f}=0.47$ (petroleum ether/ethyl acetate $\left.=2: 1, \mathrm{v} / \mathrm{v}\right) .{ }^{1} \mathrm{H}$ NMR $(400 \mathrm{MHz}$, $\left.\mathrm{CDCl}_{3}\right)$ : $7.65-7.57(\mathrm{~m}, 1 \mathrm{H}), 7.34-7.28(\mathrm{~m}, 3 \mathrm{H}), 7.24-7.12(\mathrm{~m}, 3 \mathrm{H}), 6.86-$ $6.75(\mathrm{~m}, 4 \mathrm{H}), 6.70(\mathrm{~d}, J=7.4 \mathrm{~Hz}, 1 \mathrm{H}), 6.13(\mathrm{~d}, J=8.0 \mathrm{~Hz}, 1 \mathrm{H}), 3.91-3.84$ (m, 1H), $3.46(\mathrm{dd}, J=18.0,6.0 \mathrm{~Hz}, 1 \mathrm{H}), 3.42-3.35(\mathrm{~m}, 1 \mathrm{H}), 3.26(\mathrm{dd}, J=$ 18.0, $5.2 \mathrm{~Hz}, 1 \mathrm{H}), 3.19-3.14(\mathrm{~m}, 1 \mathrm{H}), 3.10-2.93(\mathrm{~m}, 2 \mathrm{H}), 2.85-2.73(\mathrm{~m}, 2 \mathrm{H}), 2.55-2.41(\mathrm{~m}, 2 \mathrm{H}), 2.31-$ $2.20(\mathrm{~m}, 1 \mathrm{H}), 2.11-1.94(\mathrm{~m}, 2 \mathrm{H}), 1.77-1.66(\mathrm{~m}, 1 \mathrm{H}), 1.56-1.47(\mathrm{~m}, 1 \mathrm{H}), 1.18(\mathrm{~d}, J=3.8 \mathrm{~Hz}, 36 \mathrm{H}), 0.82$ $(\mathrm{d}, J=6.8 \mathrm{~Hz}, 3 \mathrm{H}), 0.63(\mathrm{~d}, J=6.8 \mathrm{~Hz}, 3 \mathrm{H}) .{ }^{13} \mathrm{C} \mathrm{NMR}\left(101 \mathrm{MHz}, \mathrm{CDCl}_{3}\right) \delta: 172.2(2), 151.8,151.5$, 150.5, 150.4(2), 150.3, 145.3, 145.2, 144.2, 144.1, 143.3(2), 135.9, 135.8, 135.6, 135.5, 134.2, 134.0, 132.9, 131.3(2), 128.8, 128.6, 128.4, 127.6, 127.4(2), 126.3, 122.5, 122.3, 115.6, 108.4, 76.4, 61.8(2), 47.8, 43.4, 37.7, 37.6, 36.3, 34.9, 34.8, 31.6, 31.5, 31.4, 30.8, 30.6, 18.8, 18.0. ${ }^{31} \mathrm{P}$ NMR (162 MHz, $\mathrm{CDCl}_{3}$ ) $\delta$ : -15.07. HRMS (ESI) Calcd for $\mathrm{C}_{52} \mathrm{H}_{72} \mathrm{~N}_{2} \mathrm{O}_{2} \mathrm{P}\left([\mathrm{M}+\mathrm{H}]^{+}\right)$: 787.5326; Found: 787.5328.

\section{Compound $\left(S_{\mathrm{a}}, R\right)-\mathrm{S} 5 \mathrm{c}$}




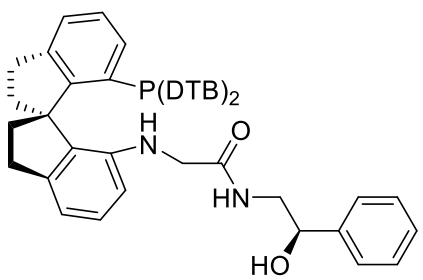

White solid, mp $96-98{ }^{\circ} \mathrm{C}, 216.0 \mathrm{mg}, 88 \%$ yield. $[\alpha]_{\mathrm{D}}^{25}-144.0$ (c 0.1 , $\mathrm{CHCl}_{3}$ ). $\mathrm{R}_{f}=0.50$ (petroleum ether/ethyl acetate $=2: 1, \mathrm{v} / \mathrm{v}$ ). ${ }^{1} \mathrm{H} \mathrm{NMR}$ $\left(400 \mathrm{MHz}, \mathrm{CDCl}_{3}\right) \delta: 7.58-7.51(\mathrm{~m}, 1 \mathrm{H}), 7.39-7.30(\mathrm{~m}, 3 \mathrm{H}), 7.30-7.24$ (m, 3H), 7.21-7.11 (m, 4H), $6.92(\mathrm{~d}, J=8.0 \mathrm{~Hz}, 2 \mathrm{H}), 6.81(\mathrm{~d}, J=8.0$ $\mathrm{Hz}, 2 \mathrm{H}), 6.76(\mathrm{~d}, J=7.6 \mathrm{~Hz}, 1 \mathrm{H}), 6.07(\mathrm{~d}, J=8.0 \mathrm{~Hz}, 1 \mathrm{H}), 4.72-4.65$ (m, 1H), 3.86-3.79 (m, 1H), 3.53-3.44 (m, 1H), $3.37(\mathrm{dd}, J=17.8,6.4$ $\mathrm{Hz}, 1 \mathrm{H}), 3.29-3.21(\mathrm{~m}, 1 \mathrm{H}), 3.16-3.13(\mathrm{~m}, 1 \mathrm{H}), 3.12-3.00$ (m, 2H), 2.94-2.83 (m, 2H), 2.68-2.58 (m, $1 \mathrm{H}), 2.30-2.18(\mathrm{~m}, 1 \mathrm{H}), 2.15-2.04(\mathrm{~m}, 2 \mathrm{H}), 1.97-1.85(\mathrm{~m}, 1 \mathrm{H}), 1.24(\mathrm{~s}, 18 \mathrm{H}), 1.21(\mathrm{~s}, 18 \mathrm{H}) .{ }^{13} \mathrm{C}$ NMR $\left(101 \mathrm{MHz}, \mathrm{CDCl}_{3}\right) \delta: 170.9,150.8,150.6,149.4(2), 149.2,149.1,144.1,143.1,143.0,142.1(2), 140.5$, 135.4, 135.3, 134.4, 134.3, 133.2, 133.0, 132.2, 130.9(2), 127.4(2), 127.2(2), 126.7, 126.5, 126.4, 126.3, 125.1, 124.7, 121.5, 121.0, 114.6, 107.2, 72.3, 60.7, 60.6, 46.7, 46.3, 36.9, 35.2, 33.8, 33.7, 30.4, 30.3, 29.8, 29.6. ${ }^{31} \mathrm{P}$ NMR (162 MHz, $\left.\mathrm{CDCl}_{3}\right) \delta$ : -16.35 . HRMS (ESI) Calcd for $\mathrm{C}_{50} \mathrm{H}_{70} \mathrm{~N}_{2} \mathrm{O}_{2} \mathrm{P}\left([\mathrm{M}+\mathrm{H}]^{+}\right)$: 821.5169; Found: 821.5174.

\section{Compound $\left(S_{\mathrm{a}}, S\right)$-S5c}

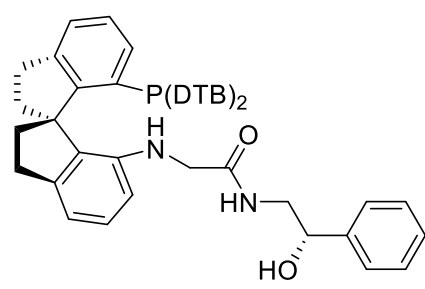

White solid, mp $90-92{ }^{\circ} \mathrm{C}, 197 \mathrm{mg}, 80 \%$ yield. $[\alpha]_{\mathrm{D}}^{25}-128.0(c) .1$, $\mathrm{CHCl}_{3}$ ). $\mathrm{R}_{f}=0.50$ (petroleum ether/ethyl acetate $=2: 1, \mathrm{v} / \mathrm{v}$ ). ${ }^{1} \mathrm{H} \mathrm{NMR}$

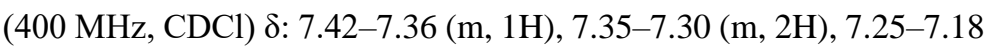
(m, 4H), 7.17-7.10 (m, 2H), 7.09-7.03 (m, 2H), $6.87(\mathrm{~d}, J=7.6 \mathrm{~Hz}$, $2 \mathrm{H}), 6.77-6.66(\mathrm{~m}, 3 \mathrm{H}), 6.04(\mathrm{~d}, J=8.0 \mathrm{~Hz}, 1 \mathrm{H}), 4.60-4.54(\mathrm{~m}, 1 \mathrm{H})$, $3.77-3.70(\mathrm{~m}, 1 \mathrm{H}), 3.42-3.25(\mathrm{~m}, 2 \mathrm{H}), 3.17(\mathrm{~d}, J=3.6 \mathrm{~Hz}, 1 \mathrm{H}), 3.13-2.96(\mathrm{~m}, 4 \mathrm{H}), 2.91-2.81(\mathrm{~m}, 1 \mathrm{H})$, 2.66-2.58 (m, 1H), 2.28-2.16 (m, 1H), 2.15-2.03 (m, 2H), 2.01-1.91 (m, 1H), 1.19 (s, 18H), $1.15(\mathrm{~s}$, 18H). ${ }^{13} \mathrm{C}$ NMR (101 MHz, $\left.\mathrm{CDCl}_{3}\right) \delta: 170.8,150.6,150.3,149.2,149.1,148.9(2), 143.8(2), 142.8,142.7$, 141.8(2), 140.1, 135.3, 135.2, 134.0, 133.9, 132.8, 132.6, 132.0, 130.8(2), 127.1, 126.9, 126.8, 126.6, 126.5, 126.3, 126.1, 126.0, 124.9, 124.3, 121.3, 120.7, 114.4, 107.1, 72.3, 60.3(2), 46.4, 46.2, 36.8, 36.7, 34.9, 33.5, 33.4, 30.1, 30.0, 29.5, 29.3. ${ }^{31} \mathrm{P}$ NMR (162 MHz, $\left.\mathrm{CDCl}_{3}\right)$ 8: -16.67. HRMS (ESI) Calcd for $\mathrm{C}_{50} \mathrm{H}_{70} \mathrm{~N}_{2} \mathrm{O}_{2} \mathrm{P}\left([\mathrm{M}+\mathrm{H}]^{+}\right)$: 821.5169; Found: 821.5174.

\section{Compound $\left(S_{\mathrm{a}}, \boldsymbol{R}\right)$-S5d}

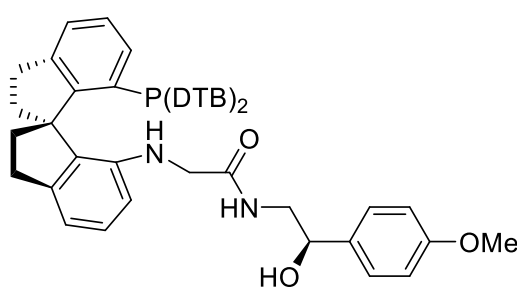

White solid, mp $100-102{ }^{\circ} \mathrm{C}, 199.0 \mathrm{mg}, 78 \%$ yield. $[\alpha]_{\mathrm{D}}^{25}-100.0$ (c $0.1, \mathrm{CHCl}_{3}$ ). $\mathrm{R}_{f}=0.42$ (petroleum ether/ethyl acetate $=2: 1$, v/v). ${ }^{1} \mathrm{H}$ NMR $\left(400 \mathrm{MHz}, \mathrm{CDCl}_{3}\right) \delta: 7.50-7.43(\mathrm{~m}, 1 \mathrm{H}), 7.36-$ $7.26(\mathrm{~m}, 3 \mathrm{H}), 7.24-7.19(\mathrm{~m}, 1 \mathrm{H}), 7.17-7.08(\mathrm{~m}, 2 \mathrm{H}), 7.07-7.02$ $(\mathrm{m}, 2 \mathrm{H}), 6.91-6.85(\mathrm{~m}, 2 \mathrm{H}), 6.79-6.70(\mathrm{~m}, 5 \mathrm{H}), 6.02(\mathrm{~d}, J=8.0$ $\mathrm{Hz}, 1 \mathrm{H}), 4.65-4.55(\mathrm{~m}, 1 \mathrm{H}), 3.81-3.71(\mathrm{~m}, 4 \mathrm{H}), 3.43-3.35$ (m, $1 \mathrm{H}), 3.30(\mathrm{dd}, J=17.8,6.8 \mathrm{~Hz}, 1 \mathrm{H}), 3.11-2.92(\mathrm{~m}, 4 \mathrm{H}), 2.91-2.79(\mathrm{~m}, 2 \mathrm{H}), 2.61(\mathrm{dd}, J=16.0,9.2 \mathrm{~Hz}$, $1 \mathrm{H}), 2.26-2.15(\mathrm{~m}, 1 \mathrm{H}), 2.11-2.00(\mathrm{~m}, 2 \mathrm{H}), 1.98-1.87(\mathrm{~m}, 1 \mathrm{H}), 1.20(\mathrm{~s}, 18 \mathrm{H}), 1.16(\mathrm{~s}, 18 \mathrm{H}) .{ }^{13} \mathrm{C}$ NMR $\left(101 \mathrm{MHz}, \mathrm{CDCl}_{3}\right)$ 8: 171.7, 158.9, 151.9, 151.6, 150.4, 150.6, 150.2, 150.1, 145.1, 145.1, 144.1, 144.0, 
$143.1,143.0,136.5,136.4,135.3,135.2,134.2,134.0,133.7,133.3,132.0(2), 128.4,128.3,128.1$, 127.6(2), 127.3, 127.0, 126.2, 122.5, 122.0, 115.6, 113.6, 108.2, 72.8, 61.6, 55.2, 47.7, 47.2, 38.0, 37.9, 36.1, 34.8, 34.7, 31.4, 31.3, 30.8, 30.6. ${ }^{31} \mathrm{P} \mathrm{NMR}\left(162 \mathrm{MHz}, \mathrm{CDCl}_{3}\right)$ \&: -16.71. HRMS (ESI) Calcd for $\mathrm{C}_{56} \mathrm{H}_{72} \mathrm{~N}_{2} \mathrm{O}_{3} \mathrm{P}\left([\mathrm{M}+\mathrm{H}]^{+}\right)$: 851.5275; Found: 851.5270.

\section{Compound $\left(S_{\mathrm{a}}, R\right)-\mathrm{S} 5 \mathrm{e}$}

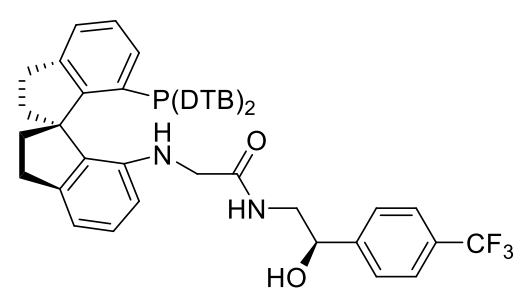

White solid, mp $110-111^{\circ} \mathrm{C}, 240.0 \mathrm{mg}, 90 \%$ yield. $[\alpha]_{\mathrm{D}}^{25}-110.0$ (c $0.1, \mathrm{CHCl}_{3}$ ). $\mathrm{R}_{f}=0.48$ (petroleum ether/ethyl acetate $=2: 1$, v/v). ${ }^{1} \mathrm{H}$ NMR $\left(400 \mathrm{MHz}, \mathrm{CDCl}_{3}\right) \delta: 7.72-7.60(\mathrm{~m}, 1 \mathrm{H}), 7.46(\mathrm{~d}$, $J=8.0 \mathrm{~Hz}, 2 \mathrm{H}), 7.35-7.28(\mathrm{~m}, 3 \mathrm{H}), 7.24-7.13(\mathrm{~m}, 3 \mathrm{H}), 7.11-$ $7.05(\mathrm{~m}, 1 \mathrm{H}), 6.87(\mathrm{~d}, J=7.6 \mathrm{~Hz}, 2 \mathrm{H}), 6.79(\mathrm{~d}, J=8.4 \mathrm{~Hz}, 2 \mathrm{H})$, $6.71(\mathrm{~d}, J=7.6 \mathrm{~Hz}, 1 \mathrm{H}), 6.01$ (d, $J=8.0 \mathrm{~Hz}, 1 \mathrm{H}), 4.70$ (d, $J=7.8 \mathrm{~Hz}, 1 \mathrm{H}), 3.96-3.81(\mathrm{~m}, 1 \mathrm{H}), 3.61$ (s, 1H), 3.48-3.33 (m, 2H), 3.19 (dd, $J=18.0,4.4 \mathrm{~Hz}, 1 \mathrm{H}), 3.08-2.92(\mathrm{~m}, 2 \mathrm{H}), 2.88-2.71(\mathrm{~m}, 2 \mathrm{H}), 2.58-$ $2.48(\mathrm{~m}, 1 \mathrm{H}), 2.23-2.11(\mathrm{~m}, 1 \mathrm{H}), 2.08-1.97(\mathrm{~m}, 2 \mathrm{H}), 1.84-1.75(\mathrm{~m}, 1 \mathrm{H}), 1.18(\mathrm{~s}, 36 \mathrm{H}) .{ }^{13} \mathrm{C} \mathrm{NMR}(101$ $\left.\mathrm{MHz}, \mathrm{CDCl}_{3}\right) \delta: 172.2,151.7,151.5,150.5$ (2), 150.4, 150.3, 145.6, 145.4, 144.1(2), 143.0(2), 135.9, 135.8, 135.5, 135.4, 134.0, 133.8, 133.0, 131.6 (2), 129.6 (q, $J=32.3 \mathrm{~Hz}), 128.6,128.4,127.6,127.4$, $126.2,126.1,125.1$ (q, $J=4.0 \mathrm{~Hz}), 124.1(\mathrm{q}, J=273.7 \mathrm{~Hz}), 122.4,122.3,115.8,107.9,72.7,61.7(2)$,

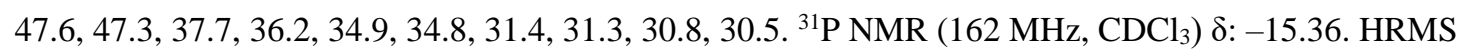
(ESI) Calcd for $\mathrm{C}_{58} \mathrm{H}_{69} \mathrm{~F}_{3} \mathrm{~N}_{2} \mathrm{O}_{2} \mathrm{P}\left([\mathrm{M}+\mathrm{H}]^{+}\right)$: 889.5043; Found: 889.5046.

\section{Compound $\left(S_{\mathrm{a}}, \boldsymbol{R}\right)$-S5f}

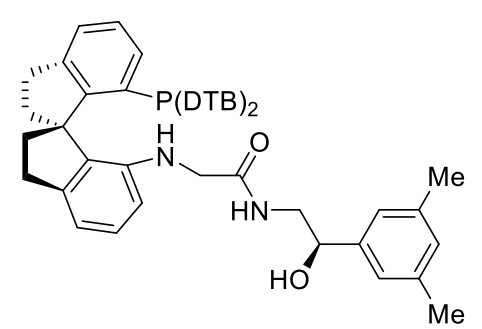

White solid, mp 99-100 ${ }^{\circ} \mathrm{C}, 200.0 \mathrm{mg}, 78 \%$ yield. $[\alpha]_{\mathrm{D}}^{25}-142.0(c 0.1$, $\left.\mathrm{CHCl}_{3}\right) \cdot \mathrm{R}_{f}=0.66$ (petroleum ether/ethyl acetate $\left.=2: 1, \mathrm{v} / \mathrm{v}\right) .{ }^{1} \mathrm{H}$ NMR $\left(400 \mathrm{MHz}, \mathrm{CDCl}_{3}\right) \delta: 7.45(\mathrm{~s}, 1 \mathrm{H}), 7.35-7.29(\mathrm{~m}, 2 \mathrm{H}), 7.24-7.18(\mathrm{~m}$, $1 \mathrm{H}), 7.15-7.08(\mathrm{~m}, 2 \mathrm{H}), 6.89(\mathrm{~d}, J=8.0 \mathrm{~Hz}, 2 \mathrm{H}), 6.86-6.79(\mathrm{~m}, 3 \mathrm{H})$, $6.75(\mathrm{dd}, J=14.4,7.6 \mathrm{~Hz}, 3 \mathrm{H}), 6.06(\mathrm{~d}, J=8.0 \mathrm{~Hz}, 1 \mathrm{H}), 4.60(\mathrm{~d}, J=$ $8.6 \mathrm{~Hz}, 1 \mathrm{H}), 3.81-3.74(\mathrm{~m}, 1 \mathrm{H}), 3.51-3.43(\mathrm{~m}, 1 \mathrm{H}), 3.31(\mathrm{dd}, J=18.0$, $6.8 \mathrm{~Hz}, 1 \mathrm{H}), 3.12-2.78(\mathrm{~m}, 6 \mathrm{H}), 2.68-2.50(\mathrm{~m}, 2 \mathrm{H}), 2.22(\mathrm{~s}, 6 \mathrm{H}), 2.12-2.04(\mathrm{~m}, 2 \mathrm{H}), 1.99-1.90(\mathrm{~m}, 1 \mathrm{H})$, 1.19 (s, 18H), 1.16 (s, 18H). ${ }^{13} \mathrm{C}$ NMR (101 MHz, $\left.\mathrm{CDCl}_{3}\right) \delta: 171.8,151.9,151.7,150.5,150.4,150.2(2)$, 145.2, 145.1, 144.1, 144.0, 143.2(2), 141.5, 137.8, 136.6(2), 135.3, 135.2, 134.4, 134.2, 133.4, 132.2(2), 129.1, 128.4, 128.3, 128.1, 127.9, 127.7, 127.4, 126.2, 123.5, 122.6, 122.0, 115.7, 108.3, 73.3, 61.7(2), $47.9,47.3,46.2,38.0(2), 36.2,34.9,34.8,31.4,31.3,30.9,30.6,21.3 .{ }^{31} \mathrm{P} \mathrm{NMR}\left(162 \mathrm{MHz}, \mathrm{CDCl}_{3}\right) \delta:-$ 17.02. HRMS (ESI) Calcd for $\mathrm{C}_{57} \mathrm{H}_{74} \mathrm{~N}_{2} \mathrm{O}_{2} \mathrm{P}\left([\mathrm{M}+\mathrm{H}]^{+}\right)$: 849.5482; Found: 849.5479.

\section{Preparation of SpiroOAP ligands S6.}

SpiroOAP ligands S6 were prepared from spiro amides S5 via dehydration according to our previously established procedure. ${ }^{1}$ 

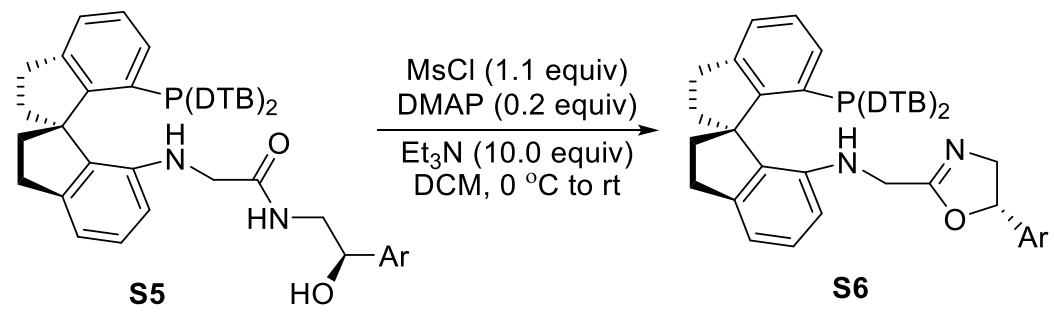

General procedure: To a solution of $\mathbf{S 5}(0.2 \mathrm{mmol}), \mathrm{Et}_{3} \mathrm{~N}(230.0 \mu \mathrm{L}, 1.6 \mathrm{mmol})$, and DMAP ( $4.8 \mathrm{mg}$, $0.04 \mathrm{mmol})$ in $\mathrm{CH}_{2} \mathrm{Cl}_{2}(10 \mathrm{~mL})$ was added $\mathrm{MsCl}(17.0 \mu \mathrm{L}, 0.22 \mathrm{mmol})$ at $0{ }^{\circ} \mathrm{C}$ (ice-water bath). The resulting mixture was then heated to $40{ }^{\circ} \mathrm{C}$ in an oil bath and maintained at the same temperature for 24 h. The reaction mixture was then filtered through Celite and the filtrate was then concentrated in vacuo to yield a residue. The residue was purified by chromatography on silica gel petroleum ether/ethyl acetate (10:1) as eluent to afford $\mathbf{S 6}$ as a white solid.

\section{Ligand $\left(S_{\mathrm{a}, S}\right)$-S6a}

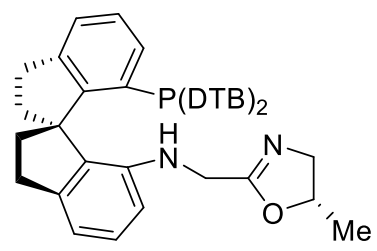

White solid, mp $83-84{ }^{\circ} \mathrm{C}, 107.0 \mathrm{mg}, 72 \%$ yield. $[\alpha]_{\mathrm{D}}^{25}-206.0$ (c 0.1 , $\left.\mathrm{CHCl}_{3}\right) . \mathrm{R}_{f}=0.40$ (petroleum ether/ethyl acetate $\left.=5: 1, \mathrm{v} / \mathrm{v}\right) .{ }^{1} \mathrm{H}$ NMR $(400$ $\left.\mathrm{MHz}, \mathrm{CDCl}_{3}\right)$ ): 7.35-7.26 (m, 2H), 7.20-7.05 (m, 4H), 6.95-6.87 (m, 2H), 6.75-6.64 (m, 3H), $6.07(\mathrm{~d}, J=8.0 \mathrm{~Hz}, 1 \mathrm{H}), 4.55-4.37(\mathrm{~m}, 1 \mathrm{H}), 3.80-3.69$ (m, 1H), 3.66-3.57 (m, 1H), 3.33-3.18 (m, 2H), 3.09-2.82 (m, 5H), 2.45$2.34(\mathrm{~m}, 1 \mathrm{H}), 2.29-2.17(\mathrm{~m}, 2 \mathrm{H}), 2.15-2.07(\mathrm{~m}, 1 \mathrm{H}), 1.23-1.10(\mathrm{~m}, 39 \mathrm{H}) .{ }^{13} \mathrm{C}$ NMR $\left(101 \mathrm{MHz}, \mathrm{CDCl}_{3}\right)$ $\delta: 163.8,151.6,151.3,148.9,148.8,148.7(2), 143.3(2), 143.0(2), 142.4(2), 137.6,137.5,134.9,134.8$, 133.6, 133.4, 133.0, 132.9, 131.7(2), 127.3, 127.2, 127.0, 126.7, 126.5, 125.8, 124.7, 121.3, 120.2, 113.1, 107.3, 75.1, 60.5, 60.4, 59.9, 39.9, 38.0(2), 34.9, 33.7, 33.6, 30.4, 30.3, 30.2, 29.8, 28.7, 19.9. ${ }^{31} \mathrm{P}$ NMR $\left(162 \mathrm{MHz}, \mathrm{CDCl}_{3}\right) \delta:-19.18$. HRMS (ESI) Calcd for $\mathrm{C}_{50} \mathrm{H}_{66} \mathrm{~N}_{2} \mathrm{OP}\left([\mathrm{M}+\mathrm{H}]^{+}\right)$: 741.4907; Found:741.4916.

\section{Ligand $\left(S_{\mathrm{a}}, S\right)-\mathrm{S} 6 \mathrm{~b}$}

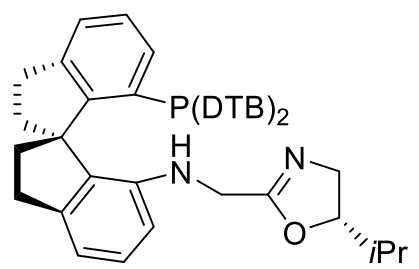

White solid, mp $78-79^{\circ} \mathrm{C}, 100.0 \mathrm{mg}, 65 \%$ yield. $[\alpha]_{\mathrm{D}}^{25}-158.0(c 0.1$, $\mathrm{CHCl}_{3}$ ). $\mathrm{R}_{f}=0.46$ (petroleum ether/ethyl acetate $=5: 1, \mathrm{v} / \mathrm{v}$ ). ${ }^{1} \mathrm{H} \mathrm{NMR}$ (400 MHz, $\left.\mathrm{CDCl}_{3}\right)$ 8: 7.33-7.24 (m, 2H), 7.20-7.13 (m, 2H), 7.12-7.04 $(\mathrm{m}, 2 \mathrm{H}), 6.93(\mathrm{~d}, J=8.1 \mathrm{~Hz}, 2 \mathrm{H}), 6.72-6.64(\mathrm{~m}, 3 \mathrm{H}), 6.07(\mathrm{~d}, J=8.0$ $\mathrm{Hz}, 1 \mathrm{H}), 4.13-4.03(\mathrm{~m}, 1 \mathrm{H}), 3.70-3.54(\mathrm{~m}, 2 \mathrm{H}), 3.43-3.31(\mathrm{~m}, 1 \mathrm{H})$, 3.24-3.16 (m, 1H), 3.10-2.82 (m, 5H), 2.47-2.34 (m, 1H), 2.30-2.18 (m, 2H), 2.15-2.06 (m, 1H), 1.70$1.59(\mathrm{~m}, 1 \mathrm{H}), 1.21(\mathrm{~s}, 18 \mathrm{H}), 1.14(\mathrm{~s}, 18 \mathrm{H}), 0.81$ (dd, $J=14.4,6.4 \mathrm{~Hz}, 6 \mathrm{H}) .{ }^{13} \mathrm{C} \mathrm{NMR}\left(101 \mathrm{MHz}, \mathrm{CDCl}_{3}\right)$ $\delta: 165.3,152.7,152.5,150.0,149.9,149.8,149.7,144.4(2), 144.0(2), 143.5(2), 138.7,138.6,136.0$, 135.9, 134.7, 134.4, 134.1, 134.0, 132.8(2), 128.3, 128.0, 127.7, 127.5, 126.9, 125.8, 122.3, 121.2, 114.2, 108.5, 85.0, 61.5(2), 57.1, 40.9, 39.2, 39.1, 35.9, 34.8, 34.7, 32.5, 31.4, 31.3, 31.2, 30.9, 17.9, 17.5. ${ }^{31} \mathrm{P}$ NMR (162 MHz, $\left.\mathrm{CDCl}_{3}\right) \delta$ : -19.34 . HRMS (ESI) Calcd for $\mathrm{C}_{52} \mathrm{H}_{70} \mathrm{~N}_{2} \mathrm{OP}\left([\mathrm{M}+\mathrm{H}]^{+}\right)$: 769.5220; Found: 769.5226 . 


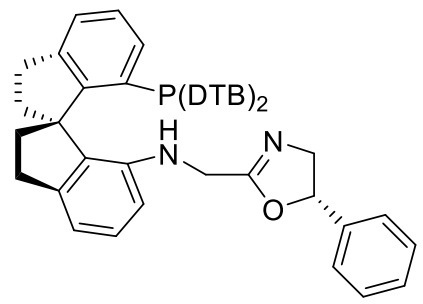

White solid, $\mathrm{mp} 77-78^{\circ} \mathrm{C}, 128.0 \mathrm{mg}, 80 \%$ yield. $[\alpha]_{\mathrm{D}}^{25}-144.0(c \quad 0.1$, $\mathrm{CHCl}_{3}$ ). $\mathrm{R}_{f}=0.55$ (petroleum ether/ethyl acetate $=5: 1, \mathrm{v} / \mathrm{v}$ ). ${ }^{1} \mathrm{H} \mathrm{NMR}$ $\left(400 \mathrm{MHz}, \mathrm{CDCl}_{3}\right)$ ס: 7.34-7.26 (m, 4H), 7.24-7.18 (m, 2H), 7.16-7.06 (m, 5H), $6.95(\mathrm{dd}, J=8.4,2.0 \mathrm{~Hz}, 2 \mathrm{H}), 6.77-6.64(\mathrm{~m}, 3 \mathrm{H}), 6.14(\mathrm{~d}, J=$ $8.0 \mathrm{~Hz}, 1 \mathrm{H}), 5.38-5.25(\mathrm{~m}, 1 \mathrm{H}), 4.15-4.02(\mathrm{~m}, 1 \mathrm{H}), 3.73-3.59(\mathrm{~m}, 2 \mathrm{H})$, $3.32(\mathrm{dd}, J=16.4,6.0 \mathrm{~Hz}, 1 \mathrm{H}), 3.15-2.85(\mathrm{~m}, 5 \mathrm{H}), 2.47-2.34(\mathrm{~m}, 1 \mathrm{H}), 2.29-2.19(\mathrm{~m}, 2 \mathrm{H}), 2.16-2.06$ (m, 1H), $1.22(\mathrm{~s}, 18 \mathrm{H}), 1.14(\mathrm{~s}, 18 \mathrm{H}) .{ }^{13} \mathrm{C} \mathrm{NMR}\left(101 \mathrm{MHz}, \mathrm{CDCl}_{3}\right) \delta: 165.2,152.8,152.5,150.1,150.0$, 149.9, 149.8, 144.5, 144.5, 144.1(2), 143.5(2), 140.8, 138.9 138.7, 136.1, 136.0, 134.7, 134.5, 134.2(2), 133.0, 133.0, 128.8, 128.4, 128.3, 128.2, 127.8, 127.6, 127.0, 125.9, 125.8, 122.5, 121.3, 114.4, 108.6, 81.1, 62.5, 61.6, 61.6, 41.0, 39.3(2), 36.1, 34.9, 34.8, 31.6, 31.4, 31.3, 30.9, 29.8, 29.8. ${ }^{31}$ P NMR (162 $\left.\mathrm{MHz}, \mathrm{CDCl}_{3}\right) \delta:-19.46$. HRMS (ESI) Calcd for $\mathrm{C}_{55} \mathrm{H}_{68} \mathrm{~N}_{2} \mathrm{OP}\left([\mathrm{M}+\mathrm{H}]^{+}\right)$: 803.5064; Found: 803.5068

\section{Ligand $\left(S_{\mathrm{a}}, R\right)$-S6c}

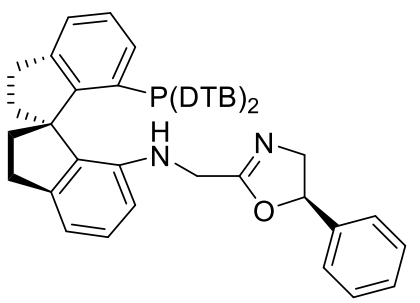

White solid, mp $77-78{ }^{\circ} \mathrm{C}, 128.0 \mathrm{mg}, 80 \%$ yield. $[\alpha]_{\mathrm{D}}^{25}-232.0(c 0.05$, $\mathrm{CHCl}_{3}$ ). $\mathrm{R}_{f}=0.55$ (petroleum ether/ethyl acetate $=5: 1, \mathrm{v} / \mathrm{v}$ ). ${ }^{1} \mathrm{H} \mathrm{NMR}$ (400 MHz, $\left.\mathrm{CDCl}_{3}\right)$ ס: 7.33-7.27 (m, 4H), 7.20-7.15 (m, 1H), 7.14-6.99 $(\mathrm{m}, 5 \mathrm{H}), 6.98-6.93(\mathrm{~m}, 2 \mathrm{H}), 6.89(\mathrm{~d}, J=7.2 \mathrm{~Hz}, 1 \mathrm{H}), 6.73(\mathrm{~d}, J=7.6 \mathrm{~Hz}$, $1 \mathrm{H}), 6.66(\mathrm{dd}, J=7.6,2.0 \mathrm{~Hz}, 2 \mathrm{H}), 6.12(\mathrm{~d}, J=8.0 \mathrm{~Hz}, 1 \mathrm{H}), 5.25(\mathrm{dd}, J$ $=10.2,7.2 \mathrm{~Hz}, 1 \mathrm{H}), 4.23-4.02(\mathrm{~m}, 1 \mathrm{H}), 3.75-3.58(\mathrm{~m}, 2 \mathrm{H}), 3.54-3.43(\mathrm{~m}, 1 \mathrm{H}), 3.04-2.74(\mathrm{~m}, 5 \mathrm{H}), 2.41-$ $2.14(\mathrm{~m}, 3 \mathrm{H}), 2.08-1.97(\mathrm{~m}, 1 \mathrm{H}), 1.21(\mathrm{~s}, 18 \mathrm{H}), 1.13(\mathrm{~s}, 18 \mathrm{H}) .{ }^{13} \mathrm{C} \mathrm{NMR}\left(101 \mathrm{MHz}, \mathrm{CDCl}_{3}\right) \delta: 165.0$, $152.8,152.5,150.1,150.0,149.9,149.8,144.5,144.4$, 144.0, 143.9, 143.4(2), 141.1, 138.9, 138.8, 136.0, 135.9, 134.6, 134.4, 134.2(2), 133.5(2), 128.7, 128.5, 128.4, 128.2(2), 127.7, 127.5, 126.9, 125.9, 125.6, 122.6, 121.3, 114.6, 108.9, 80.7, 62.7, 61.6(2), 41.0, 39.3(2), 35.8, 34.9, 34.8, 31.5, 31.4, 31.3, 30.9. ${ }^{31} \mathrm{P}$ NMR (162 MHz, $\left.\mathrm{CDCl}_{3}\right) \delta:-19.79$. HRMS (ESI) Calcd for $\mathrm{C}_{55} \mathrm{H}_{68} \mathrm{~N}_{2} \mathrm{OP}\left([\mathrm{M}+\mathrm{H}]^{+}\right)$: 803.5064; Found: 803.5053 .

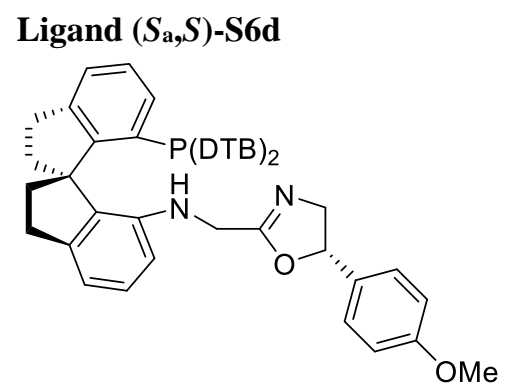

White solid, mp $78-79^{\circ} \mathrm{C}, 199.0 \mathrm{mg}, 20 \%$ yield. $[\alpha]_{\mathrm{D}}^{25}-148.0(c$ $0.1, \mathrm{CHCl}_{3}$ ). $\mathrm{R}_{f}=0.52$ (petroleum ether/ethyl acetate $=5: 1, \mathrm{v} / \mathrm{v}$ ). ${ }^{1} \mathrm{H}$ NMR (400 MHz, $\left.\mathrm{CDCl}_{3}\right)$ ): 7.33-7.28 (m, 1H), 7.25-7.17 (m, $2 \mathrm{H}), 7.15-7.03(\mathrm{~m}, 5 \mathrm{H}), 6.94(\mathrm{dd}, J=8.0,2.0 \mathrm{~Hz}, 2 \mathrm{H}), 6.88-6.81$ $(\mathrm{m}, 2 \mathrm{H}), 6.74-6.63(\mathrm{~m}, 3 \mathrm{H}), 6.12(\mathrm{~d}, J=8.0 \mathrm{~Hz}, 1 \mathrm{H}), 5.37-5.18$ $(\mathrm{m}, 1 \mathrm{H}), 4.04(\mathrm{dd}, J=14.4,10.0 \mathrm{~Hz}, 1 \mathrm{H}), 3.79(\mathrm{~s}, 3 \mathrm{H}), 3.68-3.59$ $(\mathrm{m}, 2 \mathrm{H}), 3.29(\mathrm{dd}, J=16.2,6.0 \mathrm{~Hz}, 1 \mathrm{H}), 3.13-2.84(\mathrm{~m}, 5 \mathrm{H}), 2.45-$ $2.34(\mathrm{~m}, 1 \mathrm{H}), 2.31-2.19(\mathrm{~m}, 2 \mathrm{H}), 2.15-2.08(\mathrm{~m}, 1 \mathrm{H}), 1.21(\mathrm{~s}, 18 \mathrm{H}), 1.13(\mathrm{~s}, 18 \mathrm{H}) .{ }^{13} \mathrm{C}$ NMR $(101 \mathrm{MHz}$, $\left.\mathrm{CDCl}_{3}\right)$ 8: 163.2, 157.7, 150.7, 150.5, 148.0(2), 147.9, 147.8, 142.5(2), 142.1, 142.0, 141.5(2), 136.8, 136.7, 134.1, 134.0, 132.7, 132.5, 132.1, 132.1, 131.0, 130.9, 130.7, 126.3, 126.3, 126.1, 125.8, 125.6(2), 
125.0, 123.9, 120.4, 119.3, 112.3, 112.1, 106.6, 79.1, 60.1, 59.6, 59.6, 53.4, 38.95, 38.0, 37.2, 34.0, 32.9, 32.8, 29.5, 29.4, 29.5, 28.9. ${ }^{31} \mathrm{P}$ NMR (162 MHz, $\mathrm{CDCl}_{3}$ ) $\delta:-19.45$. HRMS (ESI) Calcd for $\mathrm{C}_{56} \mathrm{H}_{70} \mathrm{~N}_{2} \mathrm{O}_{2} \mathrm{P}$ $\left([\mathrm{M}+\mathrm{H}]^{+}\right)$: 833.5169; Found: 833.5176.

\section{Ligand $\left(S_{\mathrm{a}}, S\right)-$ S6e}

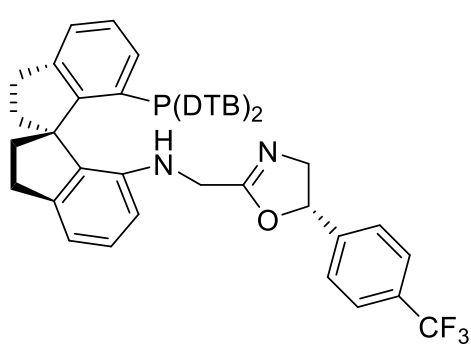

White solid, mp $90-91{ }^{\circ} \mathrm{C}, 110.0 \mathrm{mg}, 66 \%$ yield. $[\alpha]_{\mathrm{D}}^{25}-128.0(c 0.1$, $\mathrm{CHCl}_{3}$ ). $\mathrm{R}_{f}=0.53$ (petroleum ether/ethyl acetate $=5: 1, \mathrm{v} / \mathrm{v}$ ). ${ }^{1} \mathrm{H}$ NMR (400 MHz, $\left.\mathrm{CDCl}_{3}\right) \delta: 7.57(\mathrm{~d}, J=8.0 \mathrm{~Hz}, 2 \mathrm{H}), 7.38-7.32(\mathrm{~m}$, 1H), 7.26-7.21 (m, 3H), 7.19-7.08 (m, 3H), 6.99 (dd, $J=8.0,1.6$ $\mathrm{Hz}, 2 \mathrm{H}), 6.79-6.68(\mathrm{~m}, 3 \mathrm{H}), 6.18(\mathrm{~d}, J=8.0 \mathrm{~Hz}, 1 \mathrm{H}), 5.38(\mathrm{dd}, J=$ $J=16.4,6.4 \mathrm{~Hz}, 1 \mathrm{H}), 3.19$ (dd, $J=16.4,4.8 \mathrm{~Hz}, 1 \mathrm{H}), 3.11-2.85$ (m, 4H), 2.46-2.34 (m, 1H), 2.30-2.20 (m, 2H), 2.18-2.11 (m, 1H), 1.24 (s, 18H), 1.17 (s, 18H). $\left.{ }^{13} \mathrm{C} \mathrm{NMR} \mathrm{(101} \mathrm{MHz,} \mathrm{CDCl}_{3}\right)$ 8: 163.9, 151.4, 151.1, 148.8, 148.7, 148.6(2), 143.5, 143.3(2), 142.9, 142.8, 142.2, 142.1, 137.3, 137.2, 134.9, 134.8, 133.5, 133.2, 132.9, 132.8, $131.8(2), 129.1$ (q, $\left.{ }^{2} J_{C-F}=32.3 \mathrm{~Hz}\right), 127.1,126.9,126.8,126.5,126.3,125.7$, 124.6, $124.4\left(\mathrm{q},{ }^{3} J_{C-F}=4.0 \mathrm{~Hz}\right), 122.8\left(\mathrm{q},{ }^{1} J_{C-F}=273.7 \mathrm{~Hz}\right), 121.2,120.1,113.3,107.3,78.8,61.2,60.4$, 60.3, 39.7, 37.9, 37.8, 34.8, 33.6, 33.5, 30.2, 30.1, 30.0, 29.6. ${ }^{31} \mathrm{P}$ NMR $\left(162 \mathrm{MHz}, \mathrm{CDCl}_{3}\right) \delta:-19.52$. HRMS (ESI) Calcd for $\mathrm{C}_{58} \mathrm{H}_{67} \mathrm{~F}_{3} \mathrm{~N}_{2} \mathrm{OP}\left([\mathrm{M}+\mathrm{H}]^{+}\right)$: 871.4938; Found: 871.4944.

\section{Ligand $\left(S_{\mathrm{a}}, S\right)-$ S6f}

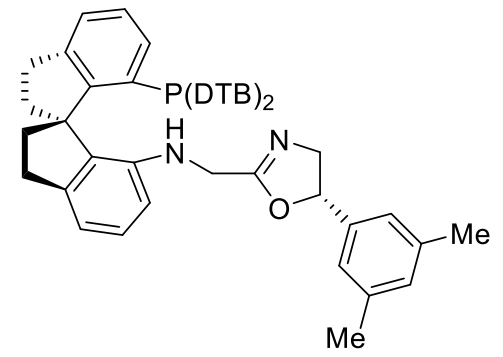

White solid, mp $179-180{ }^{\circ} \mathrm{C}, 91.0 \mathrm{mg}, 55 \%$ yield. $[\alpha]_{\mathrm{D}}^{25}-144.0(c$ $0.1, \mathrm{CHCl}_{3}$ ). $\mathrm{R}_{f}=0.64$ (petroleum ether/ethyl acetate $=5: 1, \mathrm{v} / \mathrm{v}$ ). ${ }^{1} \mathrm{H}$ NMR (400 MHz CDCl $)$ ) 7.31 (s, 1H), 7.26-7.17 (m, 2H), 7.15$7.06(\mathrm{~m}, 3 \mathrm{H}), 6.98-6.90(\mathrm{~m}, 3 \mathrm{H}), 6.78(\mathrm{~s}, 2 \mathrm{H}), 6.73-6.66(\mathrm{~m}, 3 \mathrm{H})$, $6.14(\mathrm{~d}, J=8.0 \mathrm{~Hz}, 1 \mathrm{H}), 5.24(\mathrm{t}, J=8.8 \mathrm{~Hz}, 1 \mathrm{H}), 4.08-3.97(\mathrm{~m}, 1 \mathrm{H})$, 3.69-3.59 (m, 2H), 3.28 (dd, $J=16.4,6.0 \mathrm{~Hz}, 1 \mathrm{H}), 3.13-2.89$ (m, 5H), 2.46-2.34 (m, 1H), 2.31-2.20 (m, 8H), 2.16-2.08 (m, 1H), 1.22 (s, 18H), 1.14 (s, 18H). ${ }^{13} \mathrm{C}$ NMR (101 MHz, $\mathrm{CDCl}_{3}$ ) $\delta: 164.1,151.7,151.4,148.9(2), 148.7(2), 143.4,143.3,143.0,142.9,142.3(2), 139.6$, 137.7, 137.6, 137.3, 135.0, 134.9, 133.6, 133.4, 133.1, 133.0, 131.9, 131.8, 128.8, 127.2, 127.0, 126.6, 126.4, 125.9, 124.8, 122.5, 121.3, 120.1, 113.2, 107.5, 80.2, 61.3, 60.5(2), 39.8, 38.2(2), 34.9, 33.8, 33.7, 30.4, 30.3, 30.2, 29.8, 25.9, 20.2. ${ }^{31} \mathrm{P}$ NMR $\left(162 \mathrm{MHz}, \mathrm{CDCl}_{3}\right) \delta$ : -19.62 . HRMS (ESI) Calcd for $\mathrm{C}_{57} \mathrm{H}_{72} \mathrm{~N}_{2} \mathrm{OP}\left([\mathrm{M}+\mathrm{H}]^{+}\right): 831.5377$; Found: 831.5381 .

\section{(B) Preparation of Racemic $\alpha$-Aryloxy Lactones.}

\section{Preparation of racemic $\alpha$-aryloxy $\gamma$-butyrolactones $4 a-m$.}

Racemic $\alpha$-aryloxy $\gamma$-butyrolactones $\mathbf{4 a - m}$ were prepared according to the literature method illustrated as below: ${ }^{5}$ 


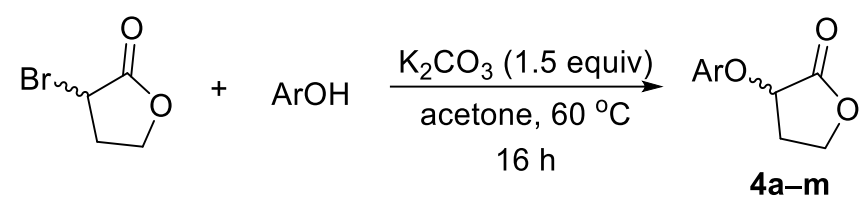

General procedure: A dry two neck flask charged with phenol (50.0 mmol), $\mathrm{K}_{2} \mathrm{CO}_{3}(10.4 \mathrm{~g}, 75.0 \mathrm{mmol})$ and anhydrous acetone $(100 \mathrm{~mL})$. The resulting mixture was heated to $60{ }^{\circ} \mathrm{C}$ in an oil bath, followed by the slow addition of $\alpha$-bromo- $\gamma$-butyrolactone $(12.4 \mathrm{~g}, 75.0 \mathrm{mmol})$ at the same temperature. The reaction mixture was continued to stir at $60{ }^{\circ} \mathrm{C}$ in an oil bath for $12 \mathrm{~h}$ and cooled to room temperature. The reaction mixture was filtered through a Celite and the filtrate was concentrated in vacuo to give a residue. The residue was chromatographed on silica gel column with petroleum ether/ethyl acetate (from 5:1 to 3:1) as eluent to offer the corresponding racemic $\alpha$-aryloxy $\gamma$-butyrolactone as white solid or colorless oil.

\section{3-Phenoxydihydrofuran-2-one (4a)}<smiles>O=C1OCC[C@@H]1Oc1ccccc1</smiles>

White solid, $\mathrm{mp} 81-82^{\circ} \mathrm{C}, 7.1 \mathrm{~g}, 80 \%$ yield. $\mathrm{R}_{f}=0.28$ (petroleum ether/ethyl acetate $=5: 1, \mathrm{v} / \mathrm{v}) .{ }^{1} \mathrm{H}$ NMR $\left(400 \mathrm{MHz}, \mathrm{CDCl}_{3}\right) \delta: 7.36-7.27(\mathrm{~m}, 2 \mathrm{H}), 7.09-6.96(\mathrm{~m}, 3 \mathrm{H})$, $4.95(\mathrm{t}, J=7.6 \mathrm{~Hz}, 1 \mathrm{H}), 4.58-4.48(\mathrm{~m}, 1 \mathrm{H}), 4.40-4.31(\mathrm{~m}, 1 \mathrm{H}), 2.76-2.67(\mathrm{~m}, 1 \mathrm{H})$, 2.52-2.41 (m, 1H). ${ }^{13} \mathrm{C}$ NMR (101 MHz, $\left.\mathrm{CDCl}_{3}\right) \delta: 173.6,157.3,129.7,122.4,115.9,72.4,65.4,29.8$. HRMS (ESI) Calcd for $\mathrm{C}_{10} \mathrm{H}_{11} \mathrm{O}_{3}\left([\mathrm{M}+\mathrm{H}]^{+}\right)$: 179.0703; Found: 179.0700.

\section{3-(p-Tolyloxy)dihydrofuran-2-one (4b)}<smiles>Cc1ccc(O[C@@H]2CCOC2=O)cc1</smiles>

White solid, mp $52-53{ }^{\circ} \mathrm{C}, 6.9 \mathrm{~g}, 72 \%$ yield. $\mathrm{R}_{f}=0.25$ (petroleum ether/ethyl acetate $=5: 1, \mathrm{v} / \mathrm{v}) .{ }^{1} \mathrm{H} \mathrm{NMR}\left(400 \mathrm{MHz}, \mathrm{CDCl}_{3}\right) \delta: 7.10(\mathrm{~d}, J=8.4 \mathrm{~Hz}, 2 \mathrm{H}), 6.98$ $6.90(\mathrm{~m}, 2 \mathrm{H}), 4.90(\mathrm{t}, J=8.0 \mathrm{~Hz}, 1 \mathrm{H}), 4.56-4.46(\mathrm{~m}, 1 \mathrm{H}), 4.38-4.29(\mathrm{~m}, 1 \mathrm{H})$, 2.75-2.64 (m, 1H), 2.53-2.38 (m, 1H), $2.30(\mathrm{~s}, 3 \mathrm{H}) .{ }^{13} \mathrm{C}$ NMR (101 MHz, $\left.\mathrm{CDCl}_{3}\right) \delta: 173.6,155.2,131.8$, 130.1, 115.9(2), 72.7, 65.3, 29.8, 20.5. HRMS (ESI) Calcd for $\mathrm{C}_{11} \mathrm{H}_{13} \mathrm{O}_{3}\left([\mathrm{M}+\mathrm{H}]^{+}\right)$: 193.0859; Found: 193.0855 .

\section{3-(4-Chlorophenoxy)dihydrofuran-2-one (4c)}<smiles>O=C1OCC[C@H]1Oc1ccc(Cl)cc1</smiles>
White solid, mp 78-79 ${ }^{\circ} \mathrm{C}, 9.1 \mathrm{~g}, 86 \%$ yield. $\mathrm{R}_{f}=0.23$ (petroleum ether/ethyl acetate $=5: 1, \mathrm{v} / \mathrm{v}) .{ }^{1} \mathrm{H}$ NMR $\left(400 \mathrm{MHz}, \mathrm{CDCl}_{3}\right) \delta: 7.31-7.18(\mathrm{~m}, 2 \mathrm{H}), 7.02-6.90$ $(\mathrm{m}, 2 \mathrm{H}), 4.91(\mathrm{t}, J=8.0 \mathrm{~Hz}, 1 \mathrm{H}), 4.55-4.44(\mathrm{~m}, 1 \mathrm{H}), 4.39-4.28(\mathrm{~m}, 1 \mathrm{H}), 2.76$ $2.65(\mathrm{~m}, 1 \mathrm{H}), 2.51-2.37(\mathrm{~m}, 1 \mathrm{H}) .{ }^{13} \mathrm{C} \mathrm{NMR}\left(101 \mathrm{MHz}, \mathrm{CDCl}_{3}\right) \delta: 173.4,155.9,129.5,127.3,117.3,72.7$, 65.4, 29.7. HRMS (ESI) Calcd for $\mathrm{C}_{10} \mathrm{H}_{10} \mathrm{O}_{3} \mathrm{Cl}\left([\mathrm{M}+\mathrm{H}]^{+}\right)$: 213.0313; Found: 213.0310.

\section{3-(4-(tert-Butyl)phenoxy)dihydrofuran-2-one (4d)}<smiles>CC(C)(C)c1ccc(O[C@H]2CCOC2=O)cc1</smiles>

White solid, mp $66-67^{\circ} \mathrm{C}, 10.0 \mathrm{~g}, 86 \%$ yield. $\mathrm{R}_{f}=0.31$ (petroleum ether/ethyl acetate $=5: 1, \mathrm{v} / \mathrm{v}) .{ }^{1} \mathrm{H} \mathrm{NMR}\left(400 \mathrm{MHz}, \mathrm{CDCl}_{3}\right) \delta: 7.32(\mathrm{~d}, J=8.8 \mathrm{~Hz}, 2 \mathrm{H}), 6.97$ $(\mathrm{d}, J=8.8 \mathrm{~Hz}, 2 \mathrm{H}), 4.93(\mathrm{t}, J=7.6 \mathrm{~Hz}, 1 \mathrm{H}), 4.55-4.47(\mathrm{~m}, 1 \mathrm{H}), 4.39-4.29(\mathrm{~m}$, 
1H), 2.75-2.66 (m, 1H), 2.50-2.39 (m, 1H), $1.30(\mathrm{~s}, 9 \mathrm{H}) .{ }^{13} \mathrm{C}$ NMR (101 MHz, $\left.\mathrm{CDCl}_{3}\right) \delta: 173.8,155.2$, 145.3, 126.6, 115.5, 72.6, 65.4, 34.3, 31.6, 30.0. HRMS (ESI) Calcd for $\mathrm{C}_{14} \mathrm{H}_{19} \mathrm{O}_{3} \mathrm{~N}$ $\left(\left[\mathrm{M}+\mathrm{NH}_{4}\right]^{+}\right): 252.1594 ;$ Found:252.1595.

\section{3-(3-Methoxyphenoxy)dihydrofuran-2-one (4e)}<smiles>COc1cccc(OC2CCOC2=O)c1</smiles>

Colorless oil, $8.9 \mathrm{~g}, 86 \%$ yield. $\mathrm{R}_{f}=0.24$ (petroleum ether/ethyl acetate $=5: 1$, v/v). ${ }^{1} \mathrm{H}$ NMR $\left(400 \mathrm{MHz}, \mathrm{CDCl}_{3}\right) \delta: 7.24-7.15(\mathrm{~m}, 1 \mathrm{H}), 6.65-6.55(\mathrm{~m}, 3 \mathrm{H})$, 4.98-4.90 (m, 1H), 4.54-4.46 (m, 1H), 4.38-4.29 (m, 1H), 3.78 (s, 3H), 2.77$2.67(\mathrm{~m}, 1 \mathrm{H}), 2.49-2.38(\mathrm{~m}, 1 \mathrm{H}) .{ }^{13} \mathrm{C} \mathrm{NMR}\left(101 \mathrm{MHz}, \mathrm{CDCl}_{3}\right) \delta: 173.5,160.9,158.5,130.1,108.0$, 107.7, 102.4, 72.4, 65.4, 55.4, 29.8. HRMS (ESI) Calcd for $\mathrm{C}_{11} \mathrm{H}_{13} \mathrm{O}_{4}\left([\mathrm{M}+\mathrm{H}]^{+}\right)$: 209.0808; Found: 209.0806.

\section{3-(3-Chlorophenoxy)dihydrofuran-2-one (4f)}<smiles>O=C1OCCC1Oc1cccc(Cl)c1</smiles>

Colorless oil, $8.5 \mathrm{~g}, 80 \%$ yield. $\mathrm{R}_{f}=0.22$ (petroleum ether/ethyl acetate $=5: 1$,

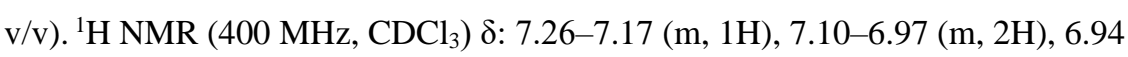
$(\mathrm{d}, J=8.4 \mathrm{~Hz}, 1 \mathrm{H}), 4.94(\mathrm{t}, J=8.0 \mathrm{~Hz}, 1 \mathrm{H}), 4.56-4.48(\mathrm{~m}, 1 \mathrm{H}), 4.40-4.32(\mathrm{~m}$, 1H), 2.78-2.68 (m, 1H), 2.52-2.40 (m, 1H). ${ }^{13} \mathrm{C}$ NMR (101 MHz, $\left.\mathrm{CDCl}_{3}\right)$ 8: 173.1, 158.0, 135.0, 130.4, 122.6, 116.4, 114.2, 72.6, 65.3, 29.7. HRMS (ESI) Calcd for $\mathrm{C}_{10} \mathrm{H}_{10} \mathrm{O}_{3} \mathrm{Cl}\left([\mathrm{M}+\mathrm{H}]^{+}\right)$:213.0313; Found:213.0316.

\section{3-(3-Bromophenoxy)dihydrofuran-2-one (4g)}<smiles>O=C1OCCC1Oc1cccc(Br)c1</smiles>

Colorless oil, $9.2 \mathrm{~g}, 72 \%$ yield. $\mathrm{R}_{f}=0.20$ (petroleum ether/ethyl acetate $=5: 1, \mathrm{v} / \mathrm{v}$ ). ${ }^{1} \mathrm{H}$ NMR $\left(400 \mathrm{MHz}, \mathrm{CDCl}_{3}\right) \delta: 7.25-7.10(\mathrm{~m}, 3 \mathrm{H}), 7.03-6.92(\mathrm{~m}, 1 \mathrm{H}), 4.94(\mathrm{t}, J$ $=8.0 \mathrm{~Hz}, 1 \mathrm{H}), 4.59-4.46(\mathrm{~m}, 1 \mathrm{H}), 4.40-4.28(\mathrm{~m}, 1 \mathrm{H}), 2.79-2.67(\mathrm{~m}, 1 \mathrm{H}), 2.49-$ $2.36(\mathrm{~m}, 1 \mathrm{H}) .{ }^{13} \mathrm{C} \mathrm{NMR}\left(101 \mathrm{MHz}, \mathrm{CDCl}_{3}\right) \delta: 173.3,158.0,130.8,125.5,122.8,119.2,114.7,72.6,65.4$, 29.7. HRMS (ESI) Calcd for $\mathrm{C}_{10} \mathrm{H}_{10} \mathrm{O}_{3} \mathrm{Br}\left([\mathrm{M}+\mathrm{H}]^{+}\right)$: 256.9808; Found: 256.9805.

\section{3-(2-Methylphenoxy)oxolan-2-one (4h)}<smiles>COc1ccccc1OC1CCOC1=O</smiles>

Colorless oil, $6.8 \mathrm{~g}, 71 \%$ yield. $\mathrm{R}_{f}=0.30$ (petroleum ether/ethyl acetate $=5: 1, \mathrm{v} / \mathrm{v}$ ). ${ }^{1} \mathrm{H}$ NMR (400 MHz, $\left.\mathrm{CDCl}_{3}\right)$ ): 7.21-7.12 (m, 2H), 7.02-6.91 (m, 2H), $4.92(\mathrm{t}, J=$ $7.6 \mathrm{~Hz}, 1 \mathrm{H}), 4.56-4.49(\mathrm{~m}, 1 \mathrm{H}), 4.39-4.32(\mathrm{~m}, 1 \mathrm{H}), 2.77-2.67(\mathrm{~m}, 1 \mathrm{H}), 2.53-2.43$ (m, 1H), 2.26 (s, 3H). ${ }^{13} \mathrm{C}$ NMR (101 MHz, $\left.\mathrm{CDCl}_{3}\right) \delta: 173.6,155.6,131.1,127.8,126.9,122.2,113.4$, 72.9, 65.3, 30.1, 16.2. HRMS (ESI) Calcd for $\mathrm{C}_{11} \mathrm{H}_{13} \mathrm{O}_{3}\left([\mathrm{M}+\mathrm{H}]^{+}\right)$: 193.0859; Found: 193.0862.

\section{3-(2-Methoxyphenoxy)dihydrofuran-2-one (4i)}<smiles>COc1ccccc1OC1CCOC1=O</smiles>

Colorless oil, $8.9 \mathrm{~g}, 86 \%$ yield. $\mathrm{R}_{f}=0.33$ (petroleum ether/ethyl acetate $=5: 1, \mathrm{v} / \mathrm{v}$ ) ${ }^{1} \mathrm{H}$ NMR $\left(400 \mathrm{MHz}, \mathrm{CDCl}_{3}\right) \delta:$ 7.17-7.09 (m, 1H), 7.08-7.01 (m, 1H), 6.96-6.86 (m, 2H), $4.94(\mathrm{t}, J=8.0 \mathrm{~Hz}, 1 \mathrm{H}), 4.56-4.46(\mathrm{~m}, 1 \mathrm{H}), 4.36-4.26(\mathrm{~m}, 1 \mathrm{H}), 3.86(\mathrm{~s}, 3 \mathrm{H})$, 
2.70-2.61 (m, 1H), 2.60-2.47 (m, 1H). $\left.{ }^{13} \mathrm{C} \mathrm{NMR} \mathrm{(101} \mathrm{MHz,} \mathrm{CDCl}_{3}\right)$ 8: 173.9, 150.5, 146.4, 123.8, 120.9, 118.3, 112.4, 74.0, 65.4, 55.9, 29.7. HRMS (ESI) Calcd for $\mathrm{C}_{11} \mathrm{H}_{13} \mathrm{O}_{4}\left([\mathrm{M}+\mathrm{H}]^{+}\right)$: 209.0808; Found: 209.0808.

\section{3-(2-Chlorophenoxy)dihydrofuran-2-one $(4 \mathbf{j})$}

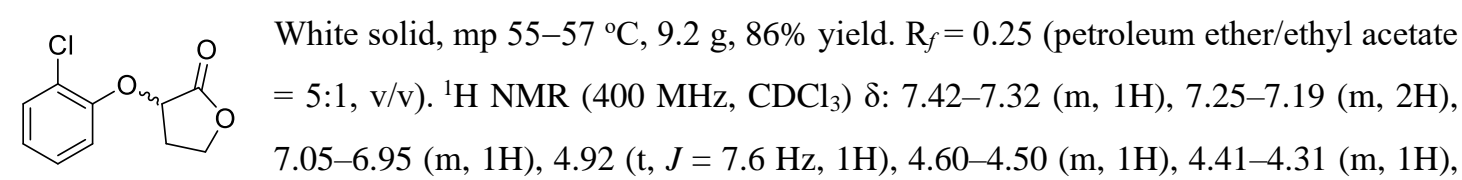

2.76-2.67 (m, 1H), 2.63-2.52 (m, 1H). ${ }^{13} \mathrm{C}$ NMR (101 MHz, $\left.\mathrm{CDCl}_{3}\right)$ o: 173.2, 153.1, 130.5, 127.9, 124.3, 123.7, 117.5, 117.4, 74.1, 65.5, 29.8. HRMS (ESI) Calcd for $\mathrm{C}_{10} \mathrm{H}_{10} \mathrm{O}_{3} \mathrm{Cl}\left([\mathrm{M}+\mathrm{H}]^{+}\right)$:213.0313; Found:213.0317.

\section{3-(2-Fluorophenoxy)dihydrofuran-2-one (4k)}<smiles>O=C1OCCC1Oc1ccccc1F</smiles>

White solid, mp 59-60 ${ }^{\circ} \mathrm{C}, 4.9 \mathrm{~g}, 50 \%$ yield. $\mathrm{R}_{f}=0.29$ (petroleum ether/ethyl acetate $=5: 1, \mathrm{v} / \mathrm{v}) .{ }^{1} \mathrm{H}$ NMR $\left(400 \mathrm{MHz}, \mathrm{CDCl}_{3}\right) \delta: 7.24-7.16(\mathrm{~m}, 1 \mathrm{H}), 7.15-6.95(\mathrm{~m}, 3 \mathrm{H})$, $4.93(\mathrm{t}, J=8.0 \mathrm{~Hz}, 1 \mathrm{H}), 4.57-4.48(\mathrm{~m}, 1 \mathrm{H}), 4.38-4.29(\mathrm{~m}, 1 \mathrm{H}), 2.75-2.65(\mathrm{~m}, 1 \mathrm{H})$, $2.60-2.47(\mathrm{~m}, 1 \mathrm{H}) .{ }^{13} \mathrm{C}$ NMR $\left(101 \mathrm{MHz}, \mathrm{CDCl}_{3}\right) \delta: 173.2,153.3\left(\mathrm{~d},{ }^{1} J_{\mathrm{C}-\mathrm{F}}=247.5 \mathrm{~Hz}\right), 145.1\left(\mathrm{~d},{ }^{2} J_{\mathrm{C}-\mathrm{F}}=\right.$ $11.1 \mathrm{~Hz}), 124.5\left(\mathrm{~d},{ }^{3} J_{\mathrm{C}-\mathrm{F}}=4.0 \mathrm{~Hz}\right), 123.7\left(\mathrm{~d},{ }^{3} J_{\mathrm{C}-\mathrm{F}}=7.1 \mathrm{~Hz}\right), 118.9,116.6\left(\mathrm{~d},{ }^{2} J_{\mathrm{C}-\mathrm{F}}=18.2 \mathrm{~Hz}\right), 74.4,65.4$, 29.8. HRMS (ESI) Calcd for $\mathrm{C}_{10} \mathrm{H}_{10} \mathrm{O}_{3} \mathrm{~F}\left([\mathrm{M}+\mathrm{H}]^{+}\right)$: 197.0608; Found: 197.0606.

\section{3-(2,5-Dimethylphenoxy)dihydrofuran-2-one (4I)}<smiles>O=C1OCCC1Oc1cc([N+](=O)[O-])ccc1[N+](=O)[O-]</smiles>

White solid, $\mathrm{mp} 53-54^{\circ} \mathrm{C}, 7.2 \mathrm{~g}, 70 \%$ yield. $\mathrm{R}_{f}=0.35$ (petroleum ether/ethyl acetate $\left.=5: 1, \mathrm{v} / \mathrm{v}) .{ }^{1} \mathrm{H} \operatorname{NMR}\left(400 \mathrm{MHz}, \mathrm{CDCl}_{3}\right)\right) \delta: 7.03(\mathrm{~d}, J=7.5 \mathrm{~Hz}, 1 \mathrm{H}), 6.81(\mathrm{~s}, 1 \mathrm{H})$, $6.75(\mathrm{~d}, J=7.6 \mathrm{~Hz}, 1 \mathrm{H}), 4.90(\mathrm{t}, J=8.0 \mathrm{~Hz}, 1 \mathrm{H}), 4.54-4.46(\mathrm{~m}, 1 \mathrm{H}), 4.38-4.29(\mathrm{~m}$, $1 \mathrm{H}), 2.75-2.65(\mathrm{~m}, 1 \mathrm{H}), 2.49-2.41(\mathrm{~m}, 1 \mathrm{H}), 2.31(\mathrm{~s}, 3 \mathrm{H}), 2.20(\mathrm{~s}, 3 \mathrm{H}) .{ }^{13} \mathrm{C} \mathrm{NMR}(101$ $\left.\mathrm{MHz}, \mathrm{CDCl}_{3}\right) \delta: 173.9,155.5,136.8,130.8,124.6,122.8,114.4,72.9,65.3,30.1,21.4,15.8$. HRMS (ESI) Calcd for $\mathrm{C}_{12} \mathrm{H}_{15} \mathrm{O}_{3}\left([\mathrm{M}+\mathrm{H}]^{+}\right)$: 207.1016; Found: 207.1015.

\section{3-(Naphthalen-2-yloxy)dihydrofuran-2-one (4m)}<smiles>O=C1OCCC1Oc1ccc2ccccc2c1</smiles>

White solid, mp $133-135^{\circ} \mathrm{C}, 7.9 \mathrm{~g}, 70 \%$ yield. $\mathrm{R}_{f}=0.34$ (petroleum ether/ethyl acetate $=5: 1, \mathrm{v} / \mathrm{v}) .{ }^{1} \mathrm{H}$ NMR $\left(400 \mathrm{MHz}, \mathrm{CDCl}_{3}\right) \delta: 7.85-7.71(\mathrm{~m}, 3 \mathrm{H}), 7.51-7.43$ (m, 1H), 7.42-7.35 (m, 1H), 7.34-7.29 (m, 1H), 7.28-7.20 (m, 1H), 5.09 (t, $J=$ $7.8 \mathrm{~Hz}, 1 \mathrm{H}), 4.62-4.49(\mathrm{~m}, 1 \mathrm{H}), 4.45-4.30(\mathrm{~m}, 1 \mathrm{H}), 2.85-2.74(\mathrm{~m}, 1 \mathrm{H}), 2.56-2.43(\mathrm{~m}, 1 \mathrm{H}) .{ }^{13} \mathrm{C} \mathrm{NMR}$ (101 MHz, $\left.\mathrm{CDCl}_{3}\right) \delta: 173.4,155.1,134.2,129.8,129.7,127.7,127.0,126.6,124.4,118.8,109.1,72.4$, 65.4, 29.8. HRMS (ESI) Calcd for $\mathrm{C}_{14} \mathrm{H}_{12} \mathrm{O}_{3}\left([\mathrm{M}+\mathrm{H}]^{+}\right)$: 229.0859; Found: 229.0858.

\section{Preparation of racemic $\alpha$-aryloxy $\delta$-valerolactones 7a-g.}

Racemic $\alpha$-aryloxy $\delta$-valerolactones 7a-g were synthesized from 3-bromotetrahydro-2 $H$-pyran-2-one 
and phenols. Among them, 3-bromotetrahydro-2H-pyran-2-one was prepared according to the literature method. ${ }^{6}$

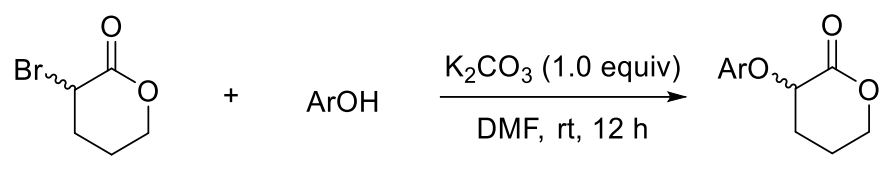

$7 \mathrm{a}-\mathrm{g}$

General procedure: To a dry two neck flask was added phenol (20.0 mmol), $\mathrm{K}_{2} \mathrm{CO}_{3}(2.8 \mathrm{~g}, 20.0 \mathrm{mmol})$, and anhydrous DMF $(33.0 \mathrm{~mL}$ ). The resulting mixture was stirred at room temperature for $5 \mathrm{~min}$ and $\alpha$ bromo- $\delta$-valerolactone $(5.3 \mathrm{~g}, 30.0 \mathrm{mmol})$ was added slowly also at room temperature. The reaction mixture was continued to stir at room temperature for $12 \mathrm{~h}$, filtered through a Celite pad, and the filtrate was concentrated in vacuo to give a residue. The residue was chromatographed on silica gel column with petroleum ether/ethyl acetate (from 5:1 to 3:1) as eluent to offer the corresponding racemic $\alpha$-aryloxy $\delta$ valerolactone as a white solid.

\section{3-Phenoxytetrahydro-2H-pyran-2-one (7a)}<smiles>O=C1OCCCC1Oc1ccccc1</smiles>

White solid, $\mathrm{mp} 68-71^{\circ} \mathrm{C}, 1.8 \mathrm{~g}, 47 \%$ yield. $\mathrm{R}_{f}=0.32$ (petroleum ether/ethyl acetate $=3: 1, \mathrm{v} / \mathrm{v}) .{ }^{1} \mathrm{H}$ NMR $\left(400 \mathrm{MHz}, \mathrm{CDCl}_{3}\right) \delta: 7.37-7.26(\mathrm{~m}, 2 \mathrm{H}), 7.07-6.94(\mathrm{~m}, 3 \mathrm{H})$, $4.90-4.77(\mathrm{~m}, 1 \mathrm{H}), 4.53-4.34(\mathrm{~m}, 2 \mathrm{H}), 2.50-2.37(\mathrm{~m}, 1 \mathrm{H}), 2.22-1.98(\mathrm{~m}, 3 \mathrm{H}) .{ }^{13} \mathrm{C}$ NMR (101 MHz, $\left.\mathrm{CDCl}_{3}\right) \delta: 170.0,157.5,129.6,122.2,116.0,72.5,68.1,25.8,20.8$. HRMS (ESI) Calcd for $\mathrm{C}_{11} \mathrm{H}_{13} \mathrm{O}_{3}\left([\mathrm{M}+\mathrm{H}]^{+}\right)$: 193.0859; Found:193.0862.

\section{3-(p-Tolyloxy)tetrahydro-2H-pyran-2-one (7b)}<smiles>Cc1ccc(OC2CCCOC2=O)cc1</smiles>

White solid, $\mathrm{mp} 74-75^{\circ} \mathrm{C}, 2.3 \mathrm{~g}, 56 \%$ yield. $\mathrm{R}_{f}=0.35$ (petroleum ether/ethyl acetate $=3: 1, \mathrm{v} / \mathrm{v}) .{ }^{1} \mathrm{H} \mathrm{NMR}\left(400 \mathrm{MHz}, \mathrm{CDCl}_{3}\right) \delta: 7.08(\mathrm{~d}, J=8.0 \mathrm{~Hz}, 2 \mathrm{H}), 6.89$ $(\mathrm{d}, J=8.0 \mathrm{~Hz}, 2 \mathrm{H}), 4.77(\mathrm{t}, J=7.2 \mathrm{~Hz}, 1 \mathrm{H}), 4.50-4.41(\mathrm{~m}, 1 \mathrm{H}), 4.40-4.32(\mathrm{~m}$, $1 \mathrm{H}), 2.47-2.36(\mathrm{~m}, 1 \mathrm{H}), 2.29(\mathrm{~s}, 3 \mathrm{H}), 2.18-1.97$ (m, 3H). ${ }^{13} \mathrm{C} \mathrm{NMR}\left(101 \mathrm{MHz}, \mathrm{CDCl}_{3}\right) \delta: 170.1,155.5$, 131.6, 130.0, 116.1, 72.8, 68.0, 25.8, 20.8, 20.5. HRMS (ESI) Calcd for $\mathrm{C}_{12} \mathrm{H}_{15} \mathrm{O}_{3}\left([\mathrm{M}+\mathrm{H}]^{+}\right)$: 207.1016; Found: 207.1019 .

\section{3-(m-Tolyloxy)tetrahydro-2H-pyran-2-one (7c)}<smiles>Cc1cccc(OC2CCCOC2=O)c1</smiles>

Colorless oil, $2.3 \mathrm{~g}, 56 \%$ yield. $\mathrm{R}_{f}=0.48$ (petroleum ether/ethyl acetate $=3: 1$, v/v). ${ }^{1} \mathrm{H}$ NMR $\left(400 \mathrm{MHz}, \mathrm{CDCl}_{3}\right) \delta: 7.15(\mathrm{t}, J=8.0 \mathrm{~Hz}, 1 \mathrm{H}), 6.85-6.73(\mathrm{~m}, 3 \mathrm{H})$, $4.80(\mathrm{t}, J=8.0 \mathrm{~Hz}, 1 \mathrm{H}), 4.47-4.30(\mathrm{~m}, 2 \mathrm{H}), 2.45-2.36(\mathrm{~m}, 1 \mathrm{H}), 2.31(\mathrm{~s}, 3 \mathrm{H})$, 2.16-1.95 (m, 3H). $\left.{ }^{13} \mathrm{C} \mathrm{NMR} \mathrm{(101} \mathrm{MHz,} \mathrm{CDCl}_{3}\right) \delta: 170.1,157.6,139.7,129.3,123.0,116.8,112.9,72.4$, 68.0, 25.8, 21.5, 20.8. HRMS (ESI) Calcd for $\mathrm{C}_{12} \mathrm{H}_{14} \mathrm{O}_{3} \mathrm{Na}\left([\mathrm{M}+\mathrm{Na}]^{+}\right)$: 229.0837; Found: 229.0841 .

\section{3-(o-Tolyloxy)tetrahydro-2H-pyran-2-one (7d)}


Colorless oil, $2.8 \mathrm{~g}, 69 \%$ yield. $\mathrm{R}_{f}=0.46$ (petroleum ether/ethyl acetate $=3: 1, \mathrm{v} / \mathrm{v}$ ). ${ }^{1} \mathrm{H}$ NMR (400 MHz, $\mathrm{CDCl}_{3}$ ) $\delta: ~ 7.24-7.10$ (m, 2H), 7.01-6.87 (m, 2H), 4.80 (t, $J=7.6$ $\mathrm{Hz}, 1 \mathrm{H}), 4.53-4.45(\mathrm{~m}, 1 \mathrm{H}), 4.43-4.33(\mathrm{~m}, 1 \mathrm{H}), 2.50-2.39(\mathrm{~m}, 1 \mathrm{H}), 2.27(\mathrm{~s}, 3 \mathrm{H})$, 2.23-1.97 (m, 3H). ${ }^{13} \mathrm{C}$ NMR (101 MHz, $\left.\mathrm{CDCl}_{3}\right) \delta: 170.1,155.9,131.0,127.7,126.8,121.9,113.5,73.0$, 68.1, 26.1, 20.8, 16.4. HRMS (ESI) Calcd for $\mathrm{C}_{12} \mathrm{H}_{15} \mathrm{O}_{3}\left([\mathrm{M}+\mathrm{H}]^{+}\right)$: 207.1016; Found: 207.1013.

\section{3-(2-Bromophenoxy)tetrahydro-2H-pyran-2-one (7e)}

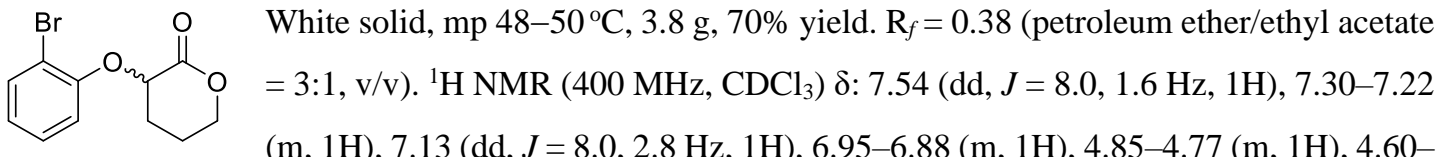
$(\mathrm{m}, 1 \mathrm{H}), 7.13(\mathrm{dd}, J=8.0,2.8 \mathrm{~Hz}, 1 \mathrm{H}), 6.95-6.88(\mathrm{~m}, 1 \mathrm{H}), 4.85-4.77(\mathrm{~m}, 1 \mathrm{H}), 4.60$ $4.51(\mathrm{~m}, 1 \mathrm{H}), 4.42-4.34(\mathrm{~m}, 1 \mathrm{H}), 2.51-2.40$ (m, 1H), 2.37-2.25 (m, 1H), 2.23-2.11 (m, 1H), 2.09-1.98

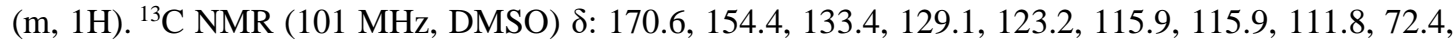
67.8, 25.2, 20.8. HRMS (ESI) Calcd for $\mathrm{C}_{11} \mathrm{H}_{11} \mathrm{O}_{3} \mathrm{BrNa}\left([\mathrm{M}+\mathrm{Na}]^{+}\right)$: 292.9784; Found: 292.9789

\section{3-(2-Chlorophenoxy)tetrahydro-2H-pyran-2-one (7f)}

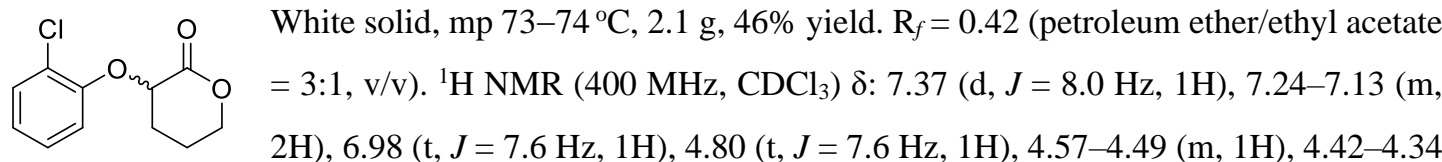
(m, 1H), 2.51-2.40 (m, 1H), 2.35-2.23 (m, 1H), 2.21-2.10 (m, 1H), 2.09-1.98 (m, 1H). ${ }^{13} \mathrm{C}$ NMR (101 $\left.\mathrm{MHz}, \mathrm{CDCl}_{3}\right) \delta: 169.3,153.4,130.4,127.8,124.3,123.5,117.6,74.3,68.3,26.0,20.6$. HRMS (ESI) Calcd for $\mathrm{C}_{11} \mathrm{H}_{12} \mathrm{ClO}_{3}\left([\mathrm{M}+\mathrm{H}]^{+}\right)$: 227.0469; Found: 227.0471 .

\section{3-(2,4-Dimethylphenoxy)tetrahydro-2H-pyran-2-one (7g)}<smiles>Cc1ccc(O[C@@H]2CCCOC2=O)c(C)c1</smiles>

White solid, mp $57-58{ }^{\circ} \mathrm{C}, 2.3 \mathrm{~g}, 52 \%$ yield. $\mathrm{R}_{f}=0.62$ (petroleum ether/ethyl acetate $=3: 1, \mathrm{v} / \mathrm{v}) .{ }^{1} \mathrm{H}$ NMR $\left(400 \mathrm{MHz}, \mathrm{CDCl}_{3}\right) \delta: 7.00-6.89(\mathrm{~m}, 2 \mathrm{H}), 6.82(\mathrm{~d}$, $J=8.0 \mathrm{~Hz}, 1 \mathrm{H}), 4.80-4.69(\mathrm{~m}, 1 \mathrm{H}), 4.52-4.44(\mathrm{~m}, 1 \mathrm{H}), 4.40-4.33(\mathrm{~m}, 1 \mathrm{H})$, 2.48-2.36 (m, 1H), $2.25(\mathrm{~s}, 3 \mathrm{H}), 2.24(\mathrm{~s}, 3 \mathrm{H}), 2.21-2.08(\mathrm{~m}, 2 \mathrm{H}), 2.05-1.97$ (m, 1H). ${ }^{13} \mathrm{C}$ NMR (101 MHz, $\left.\mathrm{CDCl}_{3}\right) \delta: 170.2,153.8,131.8,131.3,127.6,127.1,113.9,73.4,68.1,26.1$, 20.8, 20.5, 16.3. HRMS (ESI) Calcd for $\mathrm{C}_{13} \mathrm{H}_{17} \mathrm{O}_{3}\left([\mathrm{M}+\mathrm{H}]^{+}\right)$: 221.1172; Found: 221.1175.

\section{(C) Asymmetric Hydrogenation of Racemic $\alpha$-Aryloxy Lactones.}

\section{The preparation of chiral spiro iridium catalysts.}

The chiral spiro iridium catalysts $(R)-\mathbf{1 a} \mathbf{a}-\mathbf{c},\left(S_{\mathrm{a}}, R\right)-\mathbf{2} \mathbf{a}-\mathbf{b},\left(S_{\mathrm{a}}, S\right)-\mathbf{3 a} \mathbf{a}-\mathbf{f}$ etc. were prepared in situ from $[\operatorname{Ir}(\mathrm{COD}) \mathrm{Cl}]_{2}$ and the corresponding chiral spiro ligands according to our previous procedure. ${ }^{7}$

General procedure: ${ }^{7}$ To a suitable glass tube equipped with a magnetic stir bar was added $[\operatorname{Ir}(\mathrm{COD}) \mathrm{Cl}]_{2}$ $(1.0 \mathrm{mg}, 1.5 \mu \mathrm{mol})$, chiral spiro ligand $(3.2 \mu \mathrm{mol})$, and anhydrous $n \mathrm{PrOH}(3 \mathrm{~mL})$ under argon atmosphere at room temperature. After this, the glass tube was placed in a stainless vessel and the reaction mixture was stirred at room temperature for 20 min under argon atmosphere. The argon atmosphere was replaced 
with $\mathrm{H}_{2}$ and the reaction mixture was continued to stir at room temperature under 10 atm of $\mathrm{H}_{2}$ pressure for another $10 \mathrm{~min}$. After releasing the pressure, the yielding solution was direct used for the asymmetric hydrogenations $(2.0 \mathrm{~mL}, 2.0 \mu \mathrm{mol}$ of catalyst $)$.

\section{Asymmetric hydrogenation of $\alpha$-aryloxy $\gamma$-butyrolactones.}<smiles>O=C1OCC[C@@H]1O</smiles>

4a-m

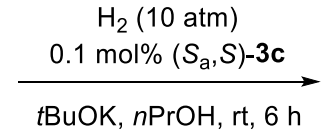

tBuOK, $n \mathrm{PrOH}, \mathrm{rt}, 6 \mathrm{~h}$<smiles>OCC[C+](CO)O[Na]</smiles>

5a-m

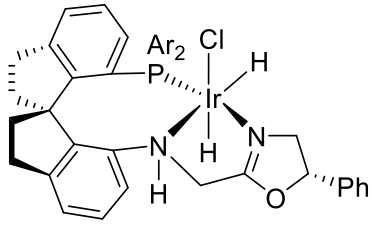

$\left(\mathrm{Ar}=3,5-\mathrm{BBu}_{2} \mathrm{C}_{6} \mathrm{H}_{3}\right)$

$\left(S_{a}, S\right)-3 c$

General procedure: To a suitable glass tube equipped with a magnetic stir bar was added racemic $\alpha$ aryloxy $\gamma$-butyrolactone 4a-m (1.0 mmol), $t$ BuOK $(22.4 \mathrm{mg}, 0.2 \mathrm{mmol})$, and $n \mathrm{PrOH}(2.0 \mathrm{~mL})$ under argon atmosphere. Then the tube was placed in a stainless vessel and the in situ generated iridium catalyst $\left(S_{\mathrm{a}}, S\right)$-3c $(2.0 \mu \mathrm{mol}$ in $2.0 \mathrm{~mL} n \mathrm{PrOH})$ was added via an injection port. The vessel was then closed and quickly purged with $\mathrm{H}_{2}$ for three times. After the vessel was pressurized to $10 \mathrm{~atm}$ with $\mathrm{H}_{2}$, the reaction mixture was stirred at room temperature for $6 \mathrm{~h}$ to complete the reaction. The hydrogen gas was then released slowly and carefully, the solvent was removed in vacuo, and the residue was purified by column chromatography on silica gel with petroleum ether/ethyl acetate (from 1:1 to 1:2) as eluent to afford the corresponding product. The enantiomeric excess of the product was determined by HPLC on a chiral stationary phase.

(+)-2-Phenoxybutane-1,4-diol ((+)-5a)<smiles>OCCC(CO)Oc1ccccc1</smiles>

Colorless oil, $171.0 \mathrm{mg}, 94 \%$ yield, $94 \%$ ee. $[\alpha]_{\mathrm{D}}^{25}+37.6(c 0.5, \mathrm{EtOH}) . \mathrm{R}_{f}=0.42$ (petroleum ether/ethyl acetate $=1: 5, \mathrm{v} / \mathrm{v}) .{ }^{1} \mathrm{H}$ NMR $\left(400 \mathrm{MHz}, \mathrm{CDCl}_{3}\right) \delta: 7.32-7.25$ $(\mathrm{m}, 2 \mathrm{H}), 7.04-6.86(\mathrm{~m}, 3 \mathrm{H}), 4.62-4.50(\mathrm{~m}, 1 \mathrm{H}), 3.89-3.71(\mathrm{~m}, 4 \mathrm{H}), 2.56(\mathrm{br}, 2 \mathrm{H})$, 2.06-1.91 (m, 2H). ${ }^{13} \mathrm{C}$ NMR (101 MHz, $\left.\mathrm{CDCl}_{3}\right) \delta: 157.8,129.7,121.5,116.1,76.3,64.2,58.9,33.8$. HRMS (ESI) Calcd for $\mathrm{C}_{10} \mathrm{H}_{14} \mathrm{O}_{3} \mathrm{Na}\left([\mathrm{M}+\mathrm{Na}]^{+}\right)$: 205.0835; Found: 205.0834. HPLC conditions: Chiralcel AD-3 column $(25 \mathrm{~cm} \times 0.46 \mathrm{~cm} \mathrm{ID}) ; n$-hexane/2-propanol $=90: 10$; temp, rt; flow rate $=1.0$ $\mathrm{mL} / \mathrm{min} ; 210 \mathrm{~nm}$ UV detector; $t_{\mathrm{R}}(S)=9.0 \mathrm{~min}$ (minor); $t_{\mathrm{R}}(R)=9.6 \mathrm{~min}$ (major).

(+)-2-(p-Tolyloxy)butane-1,4-diol ((+)-5b)<smiles>Cc1ccc(OC(CO)CCO)cc1</smiles>

Colorless oil, $176.4 \mathrm{mg}, 90 \%$ yield, $95 \%$ ee. $[\alpha]_{\mathrm{D}}^{25}+21.6(c 0.3, \mathrm{EtOH}) . \mathrm{R}_{f}=0.46$ (petroleum ether/ethyl acetate $=1: 5, \mathrm{v} / \mathrm{v}) .{ }^{1} \mathrm{H} \mathrm{NMR}\left(400 \mathrm{MHz}, \mathrm{CDCl}_{3}\right) \delta: 7.07$ $(\mathrm{d}, J=8.4 \mathrm{~Hz}, 2 \mathrm{H}), 6.86(\mathrm{~d}, J=8.4 \mathrm{~Hz}, 2 \mathrm{H}), 4.54-4.46(\mathrm{~m}, 1 \mathrm{H}), 3.87-3.71(\mathrm{~m}$, 4H), $2.62(\mathrm{br}, 1 \mathrm{H}), 2.39(\mathrm{br}, 1 \mathrm{H}), 2.28(\mathrm{~s}, 3 \mathrm{H}), 2.04-1.91(\mathrm{~m}, 2 \mathrm{H}) .{ }^{13} \mathrm{C} \mathrm{NMR}\left(101 \mathrm{MHz}, \mathrm{CDCl}_{3}\right) \delta: 155.6$, 130.9, 130.1, 116.2, 76.7, 64.2, 59.0, 33.8, 20.5. HRMS (ESI) Calcd for $\mathrm{C}_{11} \mathrm{H}_{16} \mathrm{O}_{3} \mathrm{Na}\left([\mathrm{M}+\mathrm{Na}]^{+}\right)$: 219.0992; Found: 219.0991. HPLC conditions: Chiralcel IG-3 column $(25 \mathrm{~cm} \times 0.46 \mathrm{~cm}$ ID); $n$ - 
hexane/2-propanol = 92:8; temp, rt; flow rate $=1.0 \mathrm{~mL} / \mathrm{min} ; 210 \mathrm{~nm} \mathrm{UV}$ detector; $t_{\mathrm{R}}(S)=14.1 \mathrm{~min}$ (minor); $t_{\mathrm{R}}(R)=14.8 \min$ (major).

(+)-2-(4-Chlorophenoxy)butane-1,4-diol ((+)-5c)

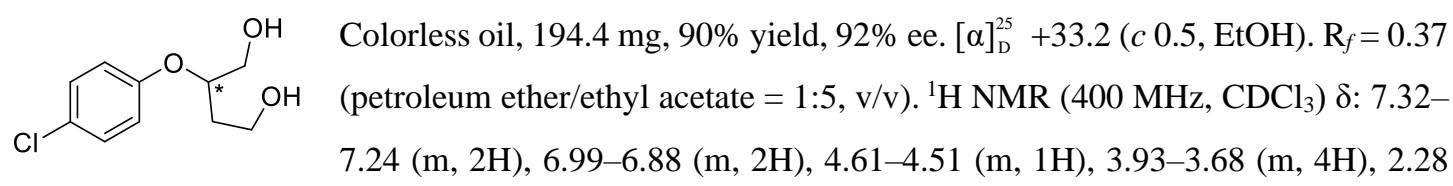
(br, 1H), 2.12-1.83 (m, 3H). $\left.{ }^{13} \mathrm{C} \mathrm{NMR} \mathrm{(101} \mathrm{MHz,} \mathrm{CDCl}_{3}\right) \delta: 156.5,129.5,126.2,117.5,76.7,63.9(2)$, 58.6(2), 33.8. HRMS (ESI) Calcd for $\mathrm{C}_{10} \mathrm{H}_{13} \mathrm{O}_{3} \mathrm{ClNa}\left([\mathrm{M}+\mathrm{Na}]^{+}\right)$: 239.0445; Found: 239.0441. HPLC conditions: Chiralcel IC-3 column $(25 \mathrm{~cm} \times 0.46 \mathrm{~cm} \mathrm{ID);} n$-hexane/2-propanol = 90:10; temp, rt; flow rate $=1.0 \mathrm{~mL} / \mathrm{min} ; 220 \mathrm{~nm} \mathrm{UV}$ detector; $t_{\mathrm{R}}(R)=9.1 \mathrm{~min}$ (major); $t_{\mathrm{R}}(S)=14.7 \mathrm{~min}$ (minor).

(+)-2-(4-(tert-Butyl)phenoxy)butane-1,4-diol ((+)-5d)

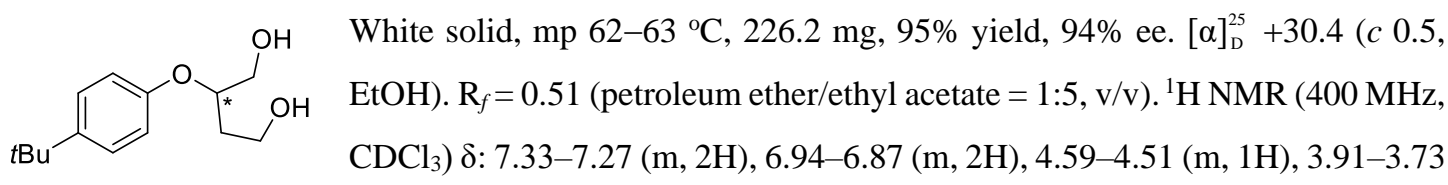
(m, 4H), 2.20-1.90 (m, 4H), 1.30 (s, 9H). $\left.{ }^{13} \mathrm{C} \mathrm{NMR} \mathrm{(101} \mathrm{MHz,} \mathrm{CDCl}_{3}\right)$ 8: 155.5, 144.3, 126.5, 115.6, 76.5, 64.3, 59.2, 34.1, 33.8, 31.5. HRMS (ESI) Calcd for $\mathrm{C}_{14} \mathrm{H}_{22} \mathrm{O}_{3} \mathrm{Na}\left([\mathrm{M}+\mathrm{Na}]^{+}\right)$: 261.1461; Found: 261.1458. HPLC conditions: Chiralcel AD-3 column $(25 \mathrm{~cm} \times 0.46 \mathrm{~cm} \mathrm{ID}) ; n$-hexane/2-propanol = 92:8; temp, rt; flow rate $=1.0 \mathrm{~mL} / \mathrm{min} ; 210 \mathrm{~nm} \mathrm{UV}$ detector; $t_{\mathrm{R}}(S)=24.5 \mathrm{~min}($ minor $) ; t_{\mathrm{R}}(R)=26.8 \mathrm{~min}$ (major).

\section{(+)-2-(3-Methoxyphenoxy)butane-1,4-diol ((+)-5e)}<smiles>COc1cccc(O[C@@H](CO)CCO)c1</smiles>

Colorless oil, $199.3 \mathrm{mg}, 94 \%$ yield, $91 \%$ ee. $[\alpha]_{\mathrm{D}}^{25}+33.6(c 0.5, \mathrm{EtOH}) . \mathrm{R}_{f}=$ 0.46 (petroleum ether/ethyl acetate $=1: 5, \mathrm{v} / \mathrm{v}) .{ }^{1} \mathrm{H}$ NMR $\left(400 \mathrm{MHz}, \mathrm{CDCl}_{3}\right) \delta$ : $7.17(\mathrm{t}, J=8.0 \mathrm{~Hz}, 1 \mathrm{H}), 6.67-6.46(\mathrm{~m}, 3 \mathrm{H}), 4.63-4.47(\mathrm{~m}, 1 \mathrm{H}), 3.94-3.70(\mathrm{~m}$, 7H), 2.63-2.33 (m, 1H), 2.30-2.06 (m, 1H), 2.04-1.79 (m, 2H). ${ }^{13} \mathrm{C} \mathrm{NMR} \mathrm{(101} \mathrm{MHz,} \mathrm{CDCl} 3$ ) $\delta: 160.9$, 159.1, 130.1, 108.2, 106.9, 102.6, 76.3, 64.1, 58.9, 55.3, 33.8. HRMS (ESI) Calcd for $\mathrm{C}_{11} \mathrm{H}_{16} \mathrm{O}_{4} \mathrm{Na}$ $\left([\mathrm{M}+\mathrm{Na}]^{+}\right): \quad 235.0941$; Found: 235.0938. HPLC conditions: Chiralcel OJ-3 column $(25 \mathrm{~cm} \times 0.46 \mathrm{~cm}$ ID); $n$-hexane/2-propanol = 90:10; temp, rt; flow rate $=1.0 \mathrm{~mL} / \mathrm{min} ; 210 \mathrm{~nm} \mathrm{UV}$ detector; $t_{\mathrm{R}}(S)=15.4$ $\min \left(\right.$ minor); $t_{\mathrm{R}}(R)=16.2 \min$ (major).

\section{(+)-2-(3-Chlorophenoxy)butane-1,4-diol ((+)-5f)}

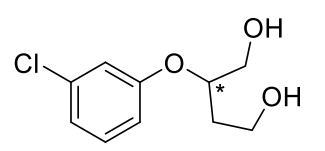

Colorless oil, $203.0 \mathrm{mg}, 94 \%$ yield, $93 \%$ ee. $[\alpha]_{\mathrm{D}}^{25}+38.0(c 0.5, \mathrm{EtOH}) . \mathrm{R}_{f}=0.39$ (petroleum ether/ethyl acetate $=1: 5, \mathrm{v} / \mathrm{v}) .{ }^{1} \mathrm{H} \mathrm{NMR}\left(400 \mathrm{MHz}, \mathrm{CDCl}_{3}\right) \delta: 7.19$ $(\mathrm{t}, J=8.0 \mathrm{~Hz}, 1 \mathrm{H}), 7.01-6.91(\mathrm{~m}, 2 \mathrm{H}), 6.88-6.81(\mathrm{~m}, 1 \mathrm{H}), 4.60-4.47(\mathrm{~m}, 1 \mathrm{H})$, 3.92-3.72 (m, 4H), 2.96-2.33 (m, 2H), 2.03-1.91 (m, 2H). $\left.{ }^{13} \mathrm{C} \mathrm{NMR} \mathrm{(101} \mathrm{MHz,} \mathrm{CDCl}_{3}\right) \delta: 158.7,135.0$, 130.4, 121.6, 116.5, 114.3, 76.6, 64.0, 58.7, 33.7. HRMS (ESI) Calcd for $\mathrm{C}_{10} \mathrm{H}_{13} \mathrm{O}_{3} \mathrm{ClNa}\left([\mathrm{M}+\mathrm{Na}]^{+}\right)$: 
239.0445; Found: 239.0440. HPLC conditions: Chiralcel OJ-3 column (25 cm $\times 0.46 \mathrm{~cm} \mathrm{ID);} n$ hexane $/ 2$-propanol $=95: 5$; temp, rt; flow rate $=1.0 \mathrm{~mL} / \mathrm{min} ; 220 \mathrm{~nm} \mathrm{UV}$ detector; $t_{\mathrm{R}}(S)=15.8 \mathrm{~min}$ (minor); $t_{\mathrm{R}}(R)=16.8 \min$ (major).

\section{(+)-2-(3-Bromophenoxy)butane-1,4-diol ((+)-5g)}

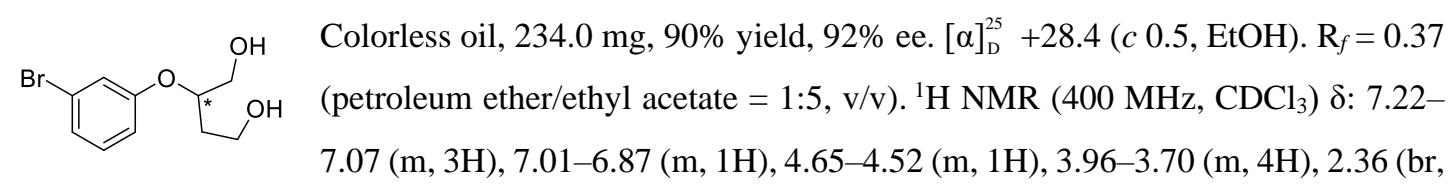
1H), 2.12-1.90 (m, 3H). $\left.{ }^{13} \mathrm{C} \mathrm{NMR} \mathrm{(101} \mathrm{MHz,} \mathrm{CDCl}_{3}\right) \delta: 158.7,130.8,124.4,122.9,119.4,114.8,76.6$, 63.9, 58.6, 33.7. HRMS (ESI) Calcd for $\mathrm{C}_{10} \mathrm{H}_{13} \mathrm{O}_{3} \mathrm{BrNa}\left([\mathrm{M}+\mathrm{Na}]^{+}\right)$: 282.9940; Found: 282.9937. HPLC conditions: Chiralcel AS-3 column $(25 \mathrm{~cm} \times 0.46 \mathrm{~cm} \mathrm{ID)}$; $n$-hexane/2-propanol = 93:7; temp, rt; flow rate $=1.0 \mathrm{~mL} / \mathrm{min} ; 210 \mathrm{~nm} \mathrm{UV}$ detector; $t_{\mathrm{R}}(S)=14.7 \mathrm{~min}($ minor $) ; t_{\mathrm{R}}(R)=16.1 \mathrm{~min}$ (major).

\section{(+)-2-(o-Tolyloxy)butane-1,4-diol ((+)-5h)}<smiles>Cc1ccccc1OC(CO)CCO</smiles>

Colorless oil, $188.2 \mathrm{mg}, 96 \%$ yield, $93 \%$ ee. $[\alpha]_{\mathrm{D}}^{25}+44.0(c 1.0, \mathrm{EtOH}) . \mathrm{R}_{f}=0.46$ (petroleum ether/ethyl acetate $=1: 5, \mathrm{v} / \mathrm{v}) .{ }^{1} \mathrm{H} \mathrm{NMR}\left(400 \mathrm{MHz}, \mathrm{CDCl}_{3}\right) \delta: 7.17-7.09$ $(\mathrm{m}, 2 \mathrm{H}), 6.96-6.84(\mathrm{~m}, 2 \mathrm{H}), 4.63-4.50(\mathrm{~m}, 1 \mathrm{H}), 3.89-3.69(\mathrm{~m}, 4 \mathrm{H}), 2.60(\mathrm{br}, 2 \mathrm{H})$,

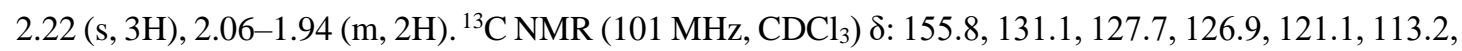
76.3, 64.2(2), 59.0(2), 34.1(2), 16.4. HRMS (ESI) Calcd for $\mathrm{C}_{11} \mathrm{H}_{16} \mathrm{O}_{3} \mathrm{Na}\left([\mathrm{M}+\mathrm{Na}]^{+}\right)$: 219.0992; Found: 219.0991. HPLC conditions: Chiralcel OD-3 column $(25 \mathrm{~cm} \times 0.46 \mathrm{~cm} \mathrm{ID})$; $n$-hexane/2-propanol = 95:5; temp, rt; flow rate $=1.0 \mathrm{~mL} / \mathrm{min} ; 220 \mathrm{~nm} \mathrm{UV} \mathrm{detector;} t_{\mathrm{R}}(R)=18.4 \mathrm{~min}($ major $) ; t_{\mathrm{R}}(S)=20.0 \mathrm{~min}$ (minor).

\section{(+)-2-(2-Methoxyphenoxy)butane-1,4-diol ((+)-5i)}<smiles>COc1ccccc1O[C@@H](CO)CCO</smiles>

Colorless oil, $199.2 \mathrm{mg}, 94 \%$ yield, $96 \%$ ee. $[\alpha]_{\mathrm{D}}^{25}+33.0(c 0.2, \mathrm{EtOH}) . \mathrm{R}_{f}=0.43$ (petroleum ether/ethyl acetate $=1: 5, \mathrm{v} / \mathrm{v}) .{ }^{1} \mathrm{H} \mathrm{NMR}\left(400 \mathrm{MHz}, \mathrm{CDCl}_{3}\right) \delta: 7.12-6.98$ $(\mathrm{m}, 2 \mathrm{H}), 6.97-6.88(\mathrm{~m}, 2 \mathrm{H}), 4.42-4.32(\mathrm{~m}, 1 \mathrm{H}), 3.87(\mathrm{~m}, 5 \mathrm{H}), 3.84-3.74(\mathrm{~m}, 1 \mathrm{H})$, 3.70-3.61 (m, 1H), 3.11 (br, 1H), 2.75 (br, 1H), 2.16-2.06 (m, 1H), 1.99-1.91 (m, 1H). ${ }^{13} \mathrm{C}$ NMR (101 $\mathrm{MHz}_{\mathrm{CDCl}}$ ) $\delta:$ 151.0, 147.0, 123.4, 121.4, 119.5, 112.1, 80.9, 63.7, 59.4, 55.8, 34.1. HRMS (ESI) Calcd for $\mathrm{C}_{11} \mathrm{H}_{16} \mathrm{O}_{4} \mathrm{Na}\left([\mathrm{M}+\mathrm{Na}]^{+}\right)$: 235.0941; Found: 235.0940. HPLC conditions: Chiralcel IC-3 column (25 $\mathrm{cm} \times 0.46 \mathrm{~cm} \mathrm{ID)} ; n$-hexane/2-propanol = 80:20; temp, rt; flow rate $=1.0 \mathrm{~mL} / \mathrm{min} ; 210 \mathrm{~nm} \mathrm{UV}$ detector; $t_{\mathrm{R}}(R)=12.8 \min ($ major $) ; t_{\mathrm{R}}(S)=19.7 \min ($ minor $)$.

\section{(+)-2-(2-Chlorophenoxy)butane-1,4-diol ((+)-5j)}

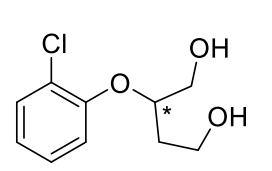

Colorless oil, $196.6 \mathrm{mg}, 91 \%$ yield, $95 \%$ ee. $[\alpha]_{\mathrm{D}}^{25}+38.0(c 0.5, \mathrm{EtOH}) . \mathrm{R}_{f}=0.39$ (petroleum ether/ethyl acetate $=1: 5, \mathrm{v} / \mathrm{v}) .{ }^{1} \mathrm{H}$ NMR $\left(400 \mathrm{MHz}, \mathrm{CDCl}_{3}\right) \delta: 7.39-7.30$ $(\mathrm{m}, 1 \mathrm{H}), 7.23-7.14(\mathrm{~m}, 1 \mathrm{H}), 7.11-7.02(\mathrm{~m}, 1 \mathrm{H}), 6.96-6.86(\mathrm{~m}, 1 \mathrm{H}), 4.60-4.48(\mathrm{~m}$, 1H), 3.90-3.69 (m, 4H), 3.39-2.60 (m, 2H), 2.10-1.87 (m, 2H). $\left.{ }^{13} \mathrm{C} \mathrm{NMR} \mathrm{(101} \mathrm{MHz,} \mathrm{CDCl}_{3}\right) \delta: 153.6$, 
130.4, 127.9, 124.1, 122.4, 116.6, 78.7, 63.9, 58.7, 33.8. HRMS (ESI) Calcd for $\mathrm{C}_{10} \mathrm{H}_{14} \mathrm{O}_{3} \mathrm{Cl}\left([\mathrm{M}+\mathrm{H}]^{+}\right)$: 217.0626; Found: 217.0624. HPLC conditions: Chiralcel IC-3 column $(25 \mathrm{~cm} \times 0.46 \mathrm{~cm}$ ID); $n$-hexane/2propanol $=90: 10$; temp, rt; flow rate $=1.0 \mathrm{~mL} / \mathrm{min} ; 210 \mathrm{~nm} \mathrm{UV} \mathrm{detector;} t_{\mathrm{R}}(R)=11.0$ min (major); $t_{\mathrm{R}}$ $(S)=18.1 \min ($ minor $)$.

\section{(+)-2-(2-Fluorophenoxy)butane-1,4-diol ((+)-5k)}<smiles>OCC[C+](CCO)Oc1ccccc1F</smiles>

Colorless oil, $182.0 \mathrm{mg}, 91 \%$ yield, $95 \%$ ee. $[\alpha]_{\mathrm{D}}^{25}+36.4(c 0.5, \mathrm{EtOH}) . \mathrm{R}_{f}=0.43$ (petroleum ether/ethyl acetate $=1: 5, \mathrm{v} / \mathrm{v}) .{ }^{1} \mathrm{H} \mathrm{NMR}\left(400 \mathrm{MHz}, \mathrm{CDCl}_{3}\right) \delta: 7.16-7.00$ $(\mathrm{m}, 3 \mathrm{H}), 6.98-6.88(\mathrm{~m}, 1 \mathrm{H}), 4.53-4.45(\mathrm{~m}, 1 \mathrm{H}), 3.91-3.72(\mathrm{~m}, 4 \mathrm{H}), 2.87-2.18(\mathrm{~m}$, 2H), 2.10-1.91 (m, 2H). ${ }^{13} \mathrm{C} \mathrm{NMR}\left(101 \mathrm{MHz}, \mathrm{CDCl}_{3}\right) \delta: 153.8\left(\mathrm{~d},{ }^{1} J_{\mathrm{C}-\mathrm{F}}=246.4 \mathrm{~Hz}\right), 145.7\left(\mathrm{~d},{ }^{2} J_{\mathrm{C}-\mathrm{F}}=\right.$ $11.1 \mathrm{~Hz}), 124.5\left(\mathrm{~d},{ }^{3} J_{\mathrm{C}-\mathrm{F}}=4.0 \mathrm{~Hz}\right), 122.5\left(\mathrm{~d},{ }^{3} J_{\mathrm{C}-\mathrm{F}}=7.1 \mathrm{~Hz}\right), 118.7,116.6\left(\mathrm{~d},{ }^{2} J_{\mathrm{C}-\mathrm{F}}=18.2 \mathrm{~Hz}\right), 79.3,64.0$, 58.9, 33.9. HRMS (ESI) Calcd for $\mathrm{C}_{10} \mathrm{H}_{13} \mathrm{O}_{3} \mathrm{FNa}\left([\mathrm{M}+\mathrm{Na}]^{+}\right)$: 223.0741; Found: 223.0739. HPLC conditions: Chiralcel IF-3 column $(25 \mathrm{~cm} \times 0.46 \mathrm{~cm} \mathrm{ID})$; $n$-hexane/2-propanol = 97:3; temp, rt; flow rate $=1.0 \mathrm{~mL} / \mathrm{min} ; 220 \mathrm{~nm} \mathrm{UV}$ detector; $t_{\mathrm{R}}(R)=59.2 \min$ (major); $t_{\mathrm{R}}(S)=67.8 \mathrm{~min}$ (minor).

\section{(R)-2-(2,5-Dimethylphenoxy)butane-1,4-diol ((R)-5l)}<smiles>Cc1ccc(C)c(O[C@H](CO)CCO)c1</smiles>

White solid, mp $54-55^{\circ} \mathrm{C}, 197.4 \mathrm{mg}, 94 \%$ yield, $91 \%$ ee. $[\alpha]_{\mathrm{D}}^{25}+34.0(c 0.5$, EtOH). $\mathrm{R}_{f}=0.49$ (petroleum ether/ethyl acetate $\left.=1: 5, \mathrm{v} / \mathrm{v}\right) .{ }^{1} \mathrm{H}$ NMR $\left(400 \mathrm{MHz}, \mathrm{CDCl}_{3}\right) \delta$ : $7.02(\mathrm{~d}, J=7.6 \mathrm{~Hz}, 1 \mathrm{H}), 6.76(\mathrm{~s}, 1 \mathrm{H}), 6.70(\mathrm{~d}, J=7.6 \mathrm{~Hz}, 1 \mathrm{H}), 4.62-4.54(\mathrm{~m}, 1 \mathrm{H})$, 3.93-3.72 (m, 4H), $2.30(\mathrm{~m}, 4 \mathrm{H}), 2.18(\mathrm{~s}, 3 \mathrm{H}), 2.09-1.93(\mathrm{~m}, 3 \mathrm{H}) .{ }^{13} \mathrm{C}$ NMR $(101$ $\mathrm{MHz}_{\mathrm{CDCl}}$ ) $\delta:$ 155.6, 136.7, 130.8, 124.4, 121.8, 114.2, 76.3, 64.3, 59.2, 34.1, 21.1, 16.0. HRMS (ESI) Calcd for $\mathrm{C}_{12} \mathrm{H}_{18} \mathrm{O}_{3} \mathrm{Na}\left([\mathrm{M}+\mathrm{Na}]^{+}\right)$: 233.1148; Found: 233.1150. HPLC conditions: Chiralcel AD-3 column $(25 \mathrm{~cm} \times 0.46 \mathrm{~cm} \mathrm{ID}) ; n$-hexane/2-propanol $=95: 5$; temp, $\mathrm{rt}$; flow rate $=1.0 \mathrm{~mL} / \mathrm{min} ; 210 \mathrm{~nm}$ UV detector; $t_{\mathrm{R}}(R)=11.7 \mathrm{~min}$ (major); $t_{\mathrm{R}}(S)=12.7 \mathrm{~min}$ (minor).

The product $(R)-5 \mathbf{l}(20.0 \mathrm{mg})$ was dissolved in mixture solvents of ethyl acetate $(1.0 \mathrm{~mL})$ and hexane $(1.0 \mathrm{~mL})$. After slowly evaporation of solvents at ambient temperautre, the fine crystals which were suitable for the X-ray diffraction analyses were obtained. The data of X-ray diffraction analysis of $(R)$ $\mathbf{5 l}$ are summarized in Table S1.

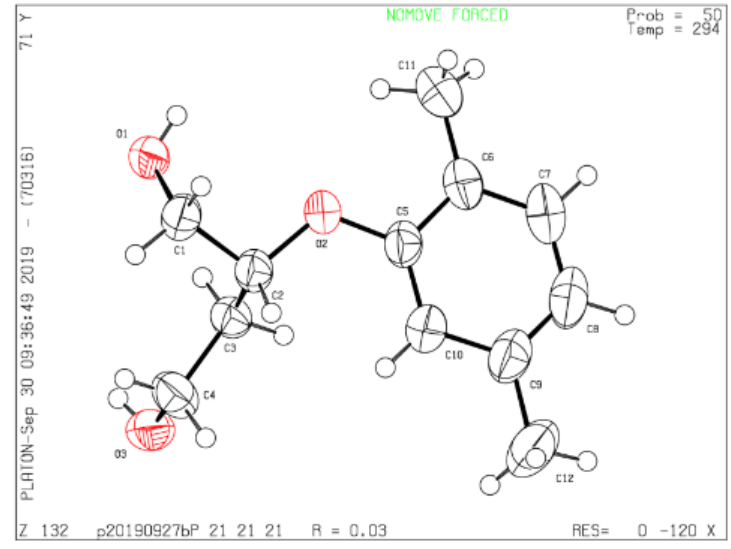


Table S1. Crystal data and structure refinement for $(R)-5 \mathbf{l}(C C D C: 1973935)$

\begin{tabular}{|c|c|}
\hline Identification code & P20190927b \\
\hline Empirical formula & $\mathrm{C}_{12} \mathrm{H}_{18} \mathrm{NO}_{3}$ \\
\hline Formula weight & 210.26 \\
\hline Temperature & $294 \mathrm{~K}$ \\
\hline Crystal system & orthorhombic \\
\hline Space group & $\mathrm{P} 2{ }_{1}{ }_{1} 2_{1}$ \\
\hline $\mathrm{a} / \AA$ & $4.62040(10)$ \\
\hline $\mathrm{b} / \AA$ & $7.01440(10)$ \\
\hline $\mathrm{c} / \AA$ & $36.1467(5)$ \\
\hline$\alpha /^{\circ}$ & 90 \\
\hline$\beta /{ }^{\circ}$ & 90 \\
\hline$\gamma /{ }^{\circ}$ & 90 \\
\hline Volume/ $/ \AA^{3}$ & $1171.49(3)$ \\
\hline $\mathrm{Z}$ & 4 \\
\hline$\rho_{\text {calc }} \mathrm{g} / \mathrm{cm}^{3}$ & 1.192 \\
\hline$\mu / \mathrm{mm}^{-1}$ & 0.684 \\
\hline $\mathrm{F}(000)$ & 456.0 \\
\hline Crystal size $/ \mathrm{mm}^{3}$ & $0.28 \times 0.24 \times 0.12$ \\
\hline Radiation & $\mathrm{CuK} \alpha(\lambda=1.54184)$ \\
\hline $2 \Theta$ range for data collection ${ }^{\circ}$ & 9.788 to 158.51 \\
\hline Index ranges & $-2 \leq \mathrm{h} \leq 5,-8 \leq \mathrm{k} \leq 8,-45 \leq 1 \leq 44$ \\
\hline Reflections collected & 9238 \\
\hline Independent reflections & $2444\left[R_{\text {int }}=0.0156, R_{\text {sigma }}=0.0135\right]$ \\
\hline Data/restraints/parameters & $2444 / 0 / 147$ \\
\hline Goodness-of-fit on $\mathrm{F}^{2}$ & 1.031 \\
\hline Final $R$ indices $[I>2 \sigma(I)]$ & $\mathrm{R}_{1}=0.0318, \mathrm{wR}_{2}=0.0960$ \\
\hline Final R indices (all data) & $\mathrm{R}_{1}=0.0325, \mathrm{wR}_{2}=0.0966$ \\
\hline Largest diff. peak and hole e $\AA^{-3}$ & $0.13 /-0.10$ \\
\hline Flack parameter & $0.02(6)$ \\
\hline
\end{tabular}

(+)-2-(Naphthalen-2-yloxy)butane-1,4-diol ((+)-5m)

Colorless oil, $213.4 \mathrm{mg}, 92 \%$ yield, 91\% ee. $[\alpha]_{\mathrm{D}}^{25}+26.8\left(c\right.$ 0.5, EtOH). $\mathrm{R}_{f}=$
0.44 (petroleum ether/ethyl acetate $=1: 5, \mathrm{v} / \mathrm{v}) .{ }^{1} \mathrm{H}$ NMR $\left(400 \mathrm{MHz}, \mathrm{CDCl}_{3}\right) \delta$ : $7.11(\mathrm{~m}, 1 \mathrm{H}), 4.77-4.62(\mathrm{~m}, 1 \mathrm{H}), 3.97-3.70(\mathrm{~m}, 4 \mathrm{H}), 3.06-2.26(\mathrm{~m}, 2 \mathrm{H}), 2.15-1.74(\mathrm{~m}, 3 \mathrm{H}) .{ }^{13} \mathrm{C}$ NMR $\left(101 \mathrm{MHz}, \mathrm{CDCl}_{3}\right) \delta: 155.6,134.4,129.8,129.2,127.6,126.8,126.5,124.0,119.3,109.2,76.3,64.1$, 
59.0, 33.8. HRMS (ESI) Calcd for $\mathrm{C}_{12} \mathrm{H}_{18} \mathrm{O}_{3} \mathrm{Na}\left([\mathrm{M}+\mathrm{Na}]^{+}\right)$: 233.1148; Found: 233.1150. HRMS (ESI) Calcd for $\mathrm{C}_{14} \mathrm{H}_{16} \mathrm{O}_{3} \mathrm{Na}\left([\mathrm{M}+\mathrm{H}]^{+}\right)$: 255.0992; Found: 255.0992. HPLC conditions: Chiralcel OD-3 column $(25 \mathrm{~cm} \times 0.46 \mathrm{~cm}$ ID); $n$-hexane/2-propanol = 90:10; temp, rt; flow rate $=1.0 \mathrm{~mL} / \mathrm{min} ; 210 \mathrm{~nm}$ UV detector; $t_{\mathrm{R}}(R)=14.5 \min \left(\right.$ major); $t_{\mathrm{R}}(S)=20.5 \min$ (minor).

The results of evaluation of chiral spiro iridium catalysts and optimization of the reaction conditions with $\left(S_{a}, S\right)-3 \mathbf{c}$ were summarized in Table S2 and S3, respectively.

Table S2. The results of the evaluation of chiral spiro iridium catalysts.<smiles>O=C1OCC[C@@H]1Oc1ccccc1</smiles>

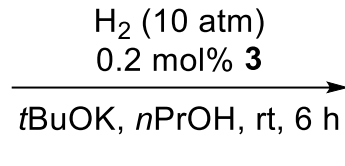<smiles>OCC[C@H](CO)Oc1ccccc1</smiles>

\begin{tabular}{|c|c|c|c|c|}
\hline Entry & Cat. & $R$-group & Yield of $\mathbf{5 a}(\%)$ & ee of $\mathbf{5 a}(\%)$ \\
\hline 1 & $(R)-\mathbf{1 a}$ & 3-Me & 94 & 67 \\
\hline 2 & $(R)-\mathbf{1 b}$ & $4-\mathrm{C}(\mathrm{OH}) \mathrm{Cy}_{2}$ & 93 & 77 \\
\hline 3 & $(R)-\mathbf{1 c}$ & 4- $t \mathrm{Bu}$ & 94 & 72 \\
\hline 4 & $\left(S_{\mathrm{a}}, R\right)-\mathbf{2 a}$ & 4-Me & 94 & 70 \\
\hline 5 & $\left(S_{\mathrm{a}}, S\right)-\mathbf{2} \mathbf{a}$ & 4-Me & 75 & 67 \\
\hline 6 & $\left(S_{\mathrm{a}}, R\right)-\mathbf{2 b}$ & $4-\mathrm{C}_{6} \mathrm{H}_{5}$ & 77 & 77 \\
\hline 7 & $\left(S_{\mathrm{a}}, R\right)-\mathbf{2 b}$ & $4-\mathrm{C}_{6} \mathrm{H}_{5}$ & 90 & 43 \\
\hline 8 & $\left(S_{\mathrm{a}}, S\right)-\mathbf{3 a}$ & $5-\mathrm{Me}$ & 66 & 90 \\
\hline 9 & $\left(S_{\mathrm{a}}, S\right)-\mathbf{3 b}$ & 5-iPr & 66 & 90 \\
\hline 10 & $\left(S_{\mathrm{a}}, S\right)-\mathbf{3 c}$ & $5-\mathrm{C}_{6} \mathrm{H}_{5}$ & 94 & 91 \\
\hline 11 & $\left(S_{\mathrm{a}}, R\right)-\mathbf{3 c}$ & $5-\mathrm{C}_{6} \mathrm{H}_{5}$ & 43 & 68 \\
\hline 12 & $\left(S_{\mathrm{a}}, S\right)-\mathbf{3 d}$ & $5-\left(4-\mathrm{MeOC}_{6} \mathrm{H}_{4}\right)$ & 93 & 86 \\
\hline 13 & $\left(S_{\mathrm{a}}, S\right)-\mathbf{3 e}$ & $5-\left(4-\mathrm{CF}_{3} \mathrm{C}_{6} \mathrm{H}_{4}\right)$ & 93 & 86 \\
\hline 14 & $\left(S_{\mathrm{a}}, S\right)-\mathbf{3 f}$ & $5-\left(3,5-(\mathrm{Me})_{2} \mathrm{C}_{6} \mathrm{H}_{3}\right)$ & 92 & 90 \\
\hline
\end{tabular}


Table S3. The results of the optimization of the reaction conditions with $\left(S_{a}, S\right)-3 \mathrm{c}$.

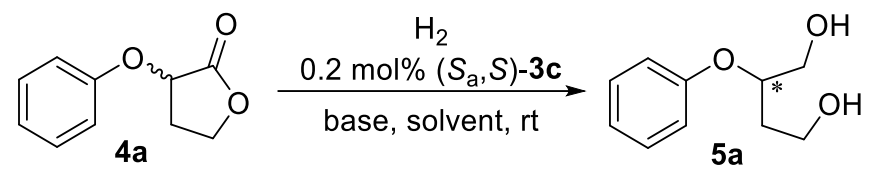

\begin{tabular}{|c|c|c|c|c|c|c|c|}
\hline Entry & base & $\mathrm{S} / \mathrm{B}$ & solvent & $P \mathrm{H}_{2}$ (atm) & Time (h) & Yield $(\%)^{[\mathrm{b}]}$ & $\operatorname{Ee}(\%)^{[\mathrm{c}]}$ \\
\hline 1 & $t \mathrm{BuOK}$ & 10 & $n \mathrm{PrOH}$ & 10 & 6 & 94 & 91 \\
\hline 2 & $t \mathrm{BuOK}$ & 10 & $\mathrm{MeOH}$ & 10 & 20 & 84 & 64 \\
\hline 3 & $t \mathrm{BuOK}$ & 10 & $\mathrm{EtOH}$ & 10 & 12 & 90 & 86 \\
\hline 4 & $t \mathrm{BuOK}$ & 10 & $i \mathrm{PrOH}$ & 10 & 12 & 90 & 88 \\
\hline 5 & $t \mathrm{BuONa}$ & 10 & $n \mathrm{PrOH}$ & 10 & 20 & 93 & 86 \\
\hline 6 & $\mathrm{KOH}$ & 10 & $n \mathrm{PrOH}$ & 10 & 24 & NR & - \\
\hline 7 & $\mathrm{~K}_{2} \mathrm{CO}_{3}$ & 10 & $n \mathrm{PrOH}$ & 10 & 24 & NR & - \\
\hline 8 & $t \mathrm{BuOK}$ & 5 & $n \mathrm{PrOH}$ & 10 & 6 & 94 & 94 \\
\hline 9 & $t \mathrm{BuOK}$ & 1 & $n \mathrm{PrOH}$ & 10 & 6 & 90 & 94 \\
\hline 10 & $t \mathrm{BuOK}$ & 20 & $n \mathrm{PrOH}$ & 10 & 24 & NR & - \\
\hline 11 & $t \mathrm{BuOK}$ & 5 & $n \mathrm{PrOH}$ & 5 & 8 & 93 & 93 \\
\hline 12 & $t \mathrm{BuOK}$ & 5 & $n \mathrm{PrOH}$ & 30 & 3 & 92 & 86 \\
\hline
\end{tabular}

[a] Reaction conditions: $1.0 \mathrm{mmol}$ 4a, $0.2 \mathrm{~mol} \%\left(S_{\mathrm{a}}, S\right)-3 \mathbf{c}, 5-100 \mathrm{~mol} \% \mathrm{t} \mathrm{BuOK}, n \mathrm{PrOH}(4.0 \mathrm{~mL})$, room temperature $\left(25-30{ }^{\circ} \mathrm{C}\right)$. [b] Isolated yield. [c] Determined by HPLC using chiral column.

3. Asymmetric hydrogenation of $\alpha$-aryloxy $\delta$-valerolactones 7 .<smiles>O=C1OCCC[C@@H]1OC1CCCCC1</smiles>

7a-g

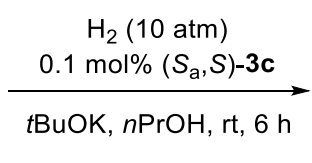

$t \mathrm{BuOK}, n \mathrm{PrOH}, \mathrm{rt}, 6 \mathrm{~h}$<smiles>OCCC[C+](CO)CO</smiles>

8a-g

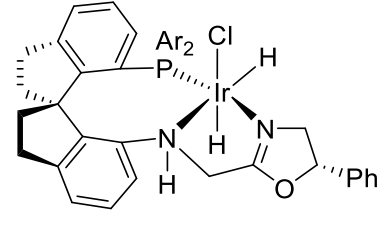

$\left(\mathrm{Ar}=3,5-\mathrm{BBu}_{2} \mathrm{C}_{6} \mathrm{H}_{3}\right)$

$\left(S_{a}, S\right)-3 c$

General procedure: To a suitable glass tube equipped with a magnetic stir bar was added $\alpha$-aryloxy $\delta$ valerolactones 7a-g $(1.0 \mathrm{mmol}), t \mathrm{BuOK}(112.0 \mathrm{mg}, 1.0 \mathrm{mmol})$, and $n \mathrm{PrOH}(2.0 \mathrm{~mL})$ under argon atmosphere. Then the tube was placed in a stainless vessel and the in situ generated iridium catalyst $\left(S_{\mathrm{a}}, S\right)-3 \mathbf{c}(2.0 \mu \mathrm{mol}$ in $2.0 \mathrm{~mL} n \mathrm{PrOH})$ was added via an injection port. The vessel was then closed and quickly purged with $\mathrm{H}_{2}$ for three times. After the vessel was pressurized to $10 \mathrm{~atm}$ with $\mathrm{H}_{2}$, the reaction mixture was stirred at room temperature for $6 \mathrm{~h}$ to complete the reaction. The hydrogen gas was then released slowly and carefully, the solvent was removed in vacuo, and the residue was purified by column chromatography on silica gel with petroleum ether/ethyl acetate (from 1:1 to 1:2) as eluent to afford the corresponding product. The enantiomeric excess of the product was determined by HPLC on a chiral stationary phase. 
(+)-2-Phenoxypentane-1,5-diol ((+)-8a)<smiles>OCCCC(CO)Oc1ccccc1</smiles>

Colorless oil, $176.4 \mathrm{mg}, 90 \%$ yield, $93 \%$ ee. $[\alpha]_{\mathrm{D}}^{25}+11.6\left(c 0.5\right.$, EtOH). $\mathrm{R}_{f}=0.45$ (petroleum ether/ethyl acetate $=1: 5, \mathrm{v} / \mathrm{v}) .{ }^{1} \mathrm{H}$ NMR $\left(400 \mathrm{MHz}, \mathrm{CDCl}_{3}\right) \delta: 7.38-7.27$ (m, 2H), 7.07-6.90 (m, 3H), 4.48-4.37 (m, 1H), 3.90-3.72 (m, 2H), 3.70-3.61 (m, $2 \mathrm{H}), 2.66-2.41(\mathrm{~m}, 1 \mathrm{H}), 2.19-2.00(\mathrm{~m}, 1 \mathrm{H}), 1.87-1.64(\mathrm{~m}, 4 \mathrm{H}) .{ }^{13} \mathrm{C} \mathrm{NMR}\left(101 \mathrm{MHz}, \mathrm{CDCl}_{3}\right) \delta: 158.0$, 129.7, 121.3, 116.1, 78.5, 64.1(2), 62.6(2), 28.2, 27.1. HRMS (ESI) Calcd for $\mathrm{C}_{11} \mathrm{H}_{16} \mathrm{O}_{3} \mathrm{Na}\left([\mathrm{M}+\mathrm{Na}]^{+}\right)$: 219.0992; Found: 219.0989. HPLC conditions: Chiralcel AD-3 column $(25 \mathrm{~cm} \times 0.46 \mathrm{~cm} \mathrm{ID}) ; n$ hexane $/ 2$-propanol $=90: 10$; temp, rt; flow rate $=1.0 \mathrm{~mL} / \mathrm{min} ; 210 \mathrm{~nm} \mathrm{UV}$ detector; $t_{\mathrm{R}}(S)=10.4 \mathrm{~min}$ $\left(\right.$ minor); $t_{\mathrm{R}}(R)=12.9 \min$ (major).

\section{(+)-2-(p-Tolyloxy)pentane-1,5-diol ((+)-8b)}<smiles>Cc1ccc(OC(CO)CCCO)cc1</smiles>

Colorless oil, $189.0 \mathrm{mg}, 90 \%$ yield, $93 \%$ ee, $[\alpha]_{\mathrm{D}}^{25}+5.6\left(c 0.5\right.$, EtOH). $\mathrm{R}_{f}=0.47$ (petroleum ether/ethyl acetate $=1: 5, \mathrm{v} / \mathrm{v}) .{ }^{1} \mathrm{H}$ NMR $\left(400 \mathrm{MHz}, \mathrm{CDCl}_{3}\right) \delta: 7.06$ (d, $J=8.4 \mathrm{~Hz}, 2 \mathrm{H}), 6.87-6.80(\mathrm{~m}, 2 \mathrm{H}), 4.34-4.26(\mathrm{~m}, 1 \mathrm{H}), 3.81-3.68(\mathrm{~m}, 2 \mathrm{H})$, $3.61(\mathrm{t}, J=6.0 \mathrm{~Hz}, 2 \mathrm{H}), 2.47(\mathrm{br}, 2 \mathrm{H}), 2.28(\mathrm{~s}, 3 \mathrm{H}), 1.81-1.56(\mathrm{~m}, 4 \mathrm{H}) .{ }^{13} \mathrm{C} \mathrm{NMR}\left(101 \mathrm{MHz}, \mathrm{CDCl}_{3}\right) \delta$ : 155.9, 130.7, 130.1, 116.2, 78.8, 64.0, 62.5, 28.2, 27.1, 20.1 HRMS (ESI) Calcd for $\mathrm{C}_{12} \mathrm{H}_{18} \mathrm{O}_{3} \mathrm{Na}$ $\left([\mathrm{M}+\mathrm{Na}]^{+}\right)$: 233.1148; Found: 233.1151. HPLC conditions: Chiralcel AD-3 column $(25 \mathrm{~cm} \times 0.46 \mathrm{~cm}$ ID); $n$-hexane $/ 2$-propanol $=90: 10$; temp, rt; flow rate $=1.0 \mathrm{~mL} / \mathrm{min} ; 210 \mathrm{~nm} \mathrm{UV}$ detector; $t_{\mathrm{R}}(S)=10.2$ $\min (\operatorname{minor}) ; t_{\mathrm{R}}(R)=14.1 \mathrm{~min}$ (major).

\section{(+)-2-(m-Tolyloxy)pentane-1,5-diol ((+)-8c)}<smiles>Cc1cccc(OC(CO)CCCO)c1</smiles>

Colorless oil, $189.0 \mathrm{mg}$, 90\%yield, $92 \%$ ee, $[\alpha]_{\mathrm{D}}^{25}+12.0(c 0.5$, EtOH $) . \mathrm{R}_{f}=0.46$ (petroleum ether/ethyl acetate $=1: 5, \mathrm{v} / \mathrm{v}) .{ }^{1} \mathrm{H}$ NMR $\left(400 \mathrm{MHz}, \mathrm{CDCl}_{3}\right) \delta: 7.15$ (t, $J=7.6 \mathrm{~Hz}, 1 \mathrm{H}), 6.80-6.71(\mathrm{~m}, 3 \mathrm{H}), 4.40-4.32(\mathrm{~m}, 1 \mathrm{H}), 3.82-3.69(\mathrm{~m}, 2 \mathrm{H})$, 3.66-3.59 (m, 2H), 2.89-2.39 (m, 2H), 2.31 (s, 3H), 1.82-1.73 (m, 2H), 1.71-1.59 (m, 2H). ${ }^{13} \mathrm{C}$ NMR $\left(101 \mathrm{MHz}, \mathrm{CDCl}_{3}\right) \delta: 158.0,139.7,129.4,122.2,117.0,112.9,78.3,63.9,62.5,28.2,27.1,21.5$. HRMS (ESI) Calcd for $\mathrm{C}_{12} \mathrm{H}_{18} \mathrm{O}_{3} \mathrm{Na}\left([\mathrm{M}+\mathrm{Na}]^{+}\right)$: 233.1148; Found: 233.1152. HPLC conditions: Chiralcel IC-3 column $(25 \mathrm{~cm} \times 0.46 \mathrm{~cm} \mathrm{ID}) ; n$-hexane $/ 2$-propanol = 90:10; temp, rt; flow rate $=1.0 \mathrm{~mL} / \mathrm{min} ; 210 \mathrm{~nm}$ $\mathrm{UV}$ detector; $t_{\mathrm{R}}(R)=12.9 \mathrm{~min}$ (major); $t_{\mathrm{R}}(S)=23.0 \mathrm{~min}$ (minor).

\section{(+)-2-(o-Tolyloxy)pentane-1,5-diol ((+)-8d)}

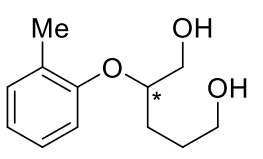

Colorless oil, $197.4 \mathrm{mg}, 94 \%$ yield, $93 \%$ ee, $[\alpha]_{\mathrm{D}}^{25}+19.2(c 0.5, \mathrm{EtOH}) . \mathrm{R}_{f}=0.45$ (petroleum ether/ethyl acetate $=1: 5, \mathrm{v} / \mathrm{v}) .{ }^{1} \mathrm{H}$ NMR $\left(400 \mathrm{MHz}, \mathrm{CDCl}_{3}\right) \delta: 7.17-7.10$ (m, 2H), 6.92-6.83 (m, 2H), 4.46-4.37 (m, 1H), 3.85-3.73 (m, 2H), 3.68-3.61 (m, 2H), 2.23 (s, 3H), 1.85-1.76 (m, 2H), 1.74-1.59 (m, 2H). $\left.{ }^{13} \mathrm{C} \mathrm{NMR} \mathrm{(101} \mathrm{MHz,} \mathrm{CDCl}_{3}\right)$ 8: 156.0, 131.1, 127.6, 126.8, 120.9, 113.0, 78.3, 64.2, 62.7, 28.2, 27.2, 16.5. HRMS (ESI) Calcd for $\mathrm{C}_{12} \mathrm{H}_{18} \mathrm{O}_{3} \mathrm{Na}$ $\left([\mathrm{M}+\mathrm{Na}]^{+}\right)$: 233.1148; Found: 233.1149. HPLC conditions: Chiralcel IC-3 column $(25 \mathrm{~cm} \times 0.46 \mathrm{~cm}$ ID); 
$n$-hexane/2-propanol $=90: 10$; temp, rt; flow rate $=1.0 \mathrm{~mL} / \mathrm{min} ; 210 \mathrm{~nm} \mathrm{UV}$ detector; $t_{\mathrm{R}}(R)=10.4 \mathrm{~min}$ (major); $t_{\mathrm{R}}(S)=17.1 \mathrm{~min}$ (minor).

\section{(+)-2-(2-Bromophenoxy)pentane-1,5-diol ((+)-8e)}<smiles>OCCCC(CO)Oc1ccccc1Br</smiles>

Colorless oil, $254.8 \mathrm{mg}, 93 \%$ yield, $92 \%$ ee. $[\alpha]_{\mathrm{D}}^{25}+32.8(c 0.5, \mathrm{EtOH}) . \mathrm{R}_{f}=0.37$ (petroleum ether/ethyl acetate $=1: 5, \mathrm{v} / \mathrm{v}) .{ }^{1} \mathrm{H} \mathrm{NMR}\left(400 \mathrm{MHz}, \mathrm{CDCl}_{3}\right) \delta: 7.53$ $(\mathrm{dd}, J=8.0,1.6 \mathrm{~Hz}, 1 \mathrm{H}), 7.28-7.22(\mathrm{~m}, 1 \mathrm{H}), 7.01(\mathrm{~d}, J=8.4 \mathrm{~Hz}, 1 \mathrm{H}), 6.89-6.82$ $(\mathrm{m}, 1 \mathrm{H}), 4.46-4.37(\mathrm{~m}, 1 \mathrm{H}), 3.84-3.73(\mathrm{~m}, 2 \mathrm{H}), 3.70-3.62(\mathrm{~m}, 2 \mathrm{H}), 2.49(\mathrm{br}, 1 \mathrm{H})$,

1.99-1.77 (m, 3H), 1.75-1.60 (m, 2H). $\left.{ }^{13} \mathrm{C} \mathrm{NMR} \mathrm{(101} \mathrm{MHz,} \mathrm{CDCl}_{3}\right) \delta: 154.6,133.5,128.6,122.7,116.1$, 113.6, 81.0, 64.0, 62.5, 28.2, 27.1. HRMS (ESI) Calcd for $\mathrm{C}_{11} \mathrm{H}_{15} \mathrm{BrO}_{3} \mathrm{Na}\left([\mathrm{M}+\mathrm{Na}]^{+}\right)$: 297.0097; Found: 297.011. HPLC conditions: Chiralcel IC-3 column ( $25 \mathrm{~cm} \times 0.46 \mathrm{~cm}$ ID); $n$-hexane/2-propanol = 90:10; temp, $\mathrm{rt}$; flow rate $=1.0 \mathrm{~mL} / \mathrm{min} ; 210 \mathrm{~nm} \mathrm{UV} \mathrm{detector;} t_{\mathrm{R}}(R)=12.3 \mathrm{~min}($ major$) ; t_{\mathrm{R}}(S)=18.0 \mathrm{~min}$ (minor).

\section{(+)-2-(2-Chlorophenoxy)pentane-1,5-diol ((+)-8f)}<smiles>OCCC[C](CO)Oc1ccccc1Cl</smiles>
Colorless oil, $212.0 \mathrm{mg}, 92 \%$ yield, $92 \%$ ee. $[\alpha]_{\mathrm{D}}^{26}+23.6(c 0.5, \mathrm{EtOH}) . \mathrm{R}_{f}=0.40$ (petroleum ether/ethyl acetate $=1: 5, \mathrm{v} / \mathrm{v}) .{ }^{1} \mathrm{H}$ NMR $\left(400 \mathrm{MHz}, \mathrm{CDCl}_{3}\right) \delta: 7.36(\mathrm{dd}$, $J=8.0,1.6 \mathrm{~Hz}, 1 \mathrm{H}), 7.20(\mathrm{td}, J=8.0,7.6,1.6 \mathrm{~Hz}, 1 \mathrm{H}), 7.04(\mathrm{dd}, J=8.0,1.6 \mathrm{~Hz}$, $1 \mathrm{H}), 6.93(\mathrm{td}, J=7.6,1.6 \mathrm{~Hz}, 1 \mathrm{H}), 4.46-4.37(\mathrm{~m}, 1 \mathrm{H}), 3.86-3.74(\mathrm{~m}, 2 \mathrm{H}), 3.71-3.62(\mathrm{~m}, 2 \mathrm{H}), 2.03$ (br, 1H), 1.94-1.66 (m, 5H). $\left.{ }^{13} \mathrm{C} \mathrm{NMR} \mathrm{(101} \mathrm{MHz,} \mathrm{CDCl}_{3}\right) \delta: 153.8,130.5,127.9,124.2,122.3,116.5,80.9$, 63.9, 62.5, 28.1, 27.1. HRMS (ESI) Calcd for $\mathrm{C}_{11} \mathrm{H}_{15} \mathrm{ClO}_{3} \mathrm{Na}\left([\mathrm{M}+\mathrm{Na}]^{+}\right)$: 253.0602; Found: 253.0602 . HPLC conditions: Chiralcel OB-H column (25 cm $\times 0.46 \mathrm{~cm} \mathrm{ID);} n$-hexane/2-propanol = 90:10; temp, $\mathrm{rt}$; flow rate $=1.0 \mathrm{~mL} / \mathrm{min} ; 220 \mathrm{~nm} \mathrm{UV}$ detector; $t_{\mathrm{R}}(S)=7.6 \mathrm{~min}$ (minor); $t_{\mathrm{R}}(R)=10.1$ min (major).

\section{(+)-2-(2,4-Dimethylphenoxy)pentane-1,5-diol ((+)-8g)}<smiles>Cc1ccc(O[C](CO)CCCO)c(C)c1</smiles>

Colorless oil, $217.3 \mathrm{mg}, 97 \%$ yield, $92 \%$ ee. $[\alpha]_{\mathrm{D}}^{25}+16.4(c 0.5, \mathrm{EtOH}) . \mathrm{R}_{f}=$ 0.61 (petroleum ether/ethyl acetate $=1: 5, \mathrm{v} / \mathrm{v}) .{ }^{1} \mathrm{H} \mathrm{NMR}\left(400 \mathrm{MHz}, \mathrm{CDCl}_{3}\right)$ $\delta: 7.01-6.88(\mathrm{~m}, 2 \mathrm{H}), 6.77(\mathrm{~d}, J=8.0 \mathrm{~Hz}, 1 \mathrm{H}), 4.36-4.28(\mathrm{~m}, 1 \mathrm{H}), 3.83-3.68$ (m, 2H), 3.65-3.56 (m, 2H), 2.54 (br, 1H), 2.24 (s, 3H), 2.18 (s, 3H), 1.82-1.71 (m, 2H), 1.69-1.54 (m, 2H). ${ }^{13} \mathrm{C}$ NMR $\left(101 \mathrm{MHz}, \mathrm{CDCl}_{3}\right) \delta: 153.9,131.8,130.2,127.5,127.1,113.4,78.7,64.1,62.6,28.2$, 27.2, 20.5, 16.4. HRMS (ESI) Calcd for $\mathrm{C}_{13} \mathrm{H}_{20} \mathrm{O}_{3} \mathrm{Na}\left([\mathrm{M}+\mathrm{Na}]^{+}\right)$: 247.1305; Found: 247.1307. HPLC conditions: Chiralcel IC-3 column $(25 \mathrm{~cm} \times 0.46 \mathrm{~cm} \mathrm{ID);} n$-hexane/2-propanol = 90:10; temp, rt; flow rate $=1.0 \mathrm{~mL} / \mathrm{min} ; 210 \mathrm{~nm} \mathrm{UV}$ detector; $t_{\mathrm{R}}(R)=11.1 \mathrm{~min}$ (major); $t_{\mathrm{R}}(S)=19.2 \mathrm{~min}$ (minor). 
(D) Enantioselective Synthesis of 3-Aryloxy Substituted Tetrahydrofurans 6

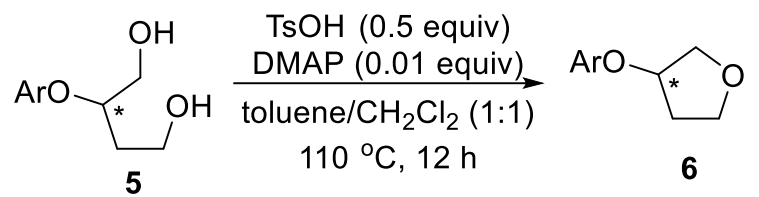

To a Schlenk tube was added diol 5 (1.0 mmol), p-toluenesulfonic acid (95 mg, 0.5 mmol), DMAP (1.2 $\mathrm{mg}, 0.01 \mathrm{mmol})$, toluene $(1 \mathrm{~mL}), \mathrm{CH}_{2} \mathrm{Cl}_{2}(1 \mathrm{~mL})$, and the resulting mixture was stirred in an oil-bath at $110{ }^{\circ} \mathrm{C}$ for $12 \mathrm{~h}$. The reaction mixture was then cooled to room temperature, diluted with water $(2 \mathrm{~mL})$, and extracted with ethyl acetate $(2 \mathrm{~mL} \times 3)$. The combined extracts were washed with brine, dried over $\mathrm{Mg}_{2} \mathrm{SO}_{4}$, and concentrated in vacuo to give a residue. The residue was chromatographed on silica gel column with petroleum ether/ethyl acetate/ (20:1) as eluent to give $\mathbf{6}$ as a colorless liquid.

\section{(-)-3-Phenoxytetrahydrofuran ((-)-6a) ${ }^{8}$}<smiles>c1ccc(OC2CCOC2)cc1</smiles>

Colorless liquid, $157 \mathrm{mg}, 96 \%$ yield, $93 \%$ ee. $[\alpha]_{\mathrm{D}}^{25}-14.4\left(\mathrm{c} 0.5, \mathrm{CHCl}_{3}\right) \cdot \mathrm{R}_{f}=0.33$ (petroleum ether/ethyl acetate $=10: 1, \mathrm{v} / \mathrm{v}) .{ }^{1} \mathrm{H}$ NMR $\left(400 \mathrm{MHz}, \mathrm{CDCl}_{3}\right) \delta: 7.33-7.24$ (m, 2H), 6.98-6.92 (m, 1H), 6.88-6.82 (m, 2H), 4.96-4.84 (m, 1H), 4.03-3.93 (m, 3H), 3.92-3.85 (m, 1H), 2.23-2.09 (m, 2H). $\left.{ }^{13} \mathrm{C} \mathrm{NMR} \mathrm{(101} \mathrm{MHz,} \mathrm{CDCl}_{3}\right) \delta: 157.4,129.6,121.0,115.4$, 77.2, 73.2, 67.2, 33.1. HPLC conditions: Chiralcel OD-H column $(25 \mathrm{~cm} \times 0.46 \mathrm{~cm} \mathrm{ID)}$; $n$-hexane/2propanol $=90: 10 ;$ temp, $\mathrm{rt}$; flow rate $=1.0 \mathrm{~mL} / \mathrm{min} ; 210 \mathrm{~nm} \mathrm{UV} \mathrm{detector;} t_{\mathrm{R}}(R)=7.1 \mathrm{~min}(\operatorname{minor}) ; t_{\mathrm{R}}(S)$ $=7.7$ min (major) (Note: analysis of the the corrsponding (+)-6a derived from (-)-5a)).

\section{(-)-3-(p-Tolyloxy)tetrahydrofuran ((-)-6b)}<smiles>Cc1ccc(OC2CCOC2)cc1</smiles>

Using Colorless liquid, $160 \mathrm{mg}, 90 \%$ yield, 93\% ee. $[\alpha]_{\mathrm{D}}^{25}-14.0\left(\mathrm{c} 0.3, \mathrm{CHCl}_{3}\right)$. $\mathrm{R}_{f}=0.45$ (petroleum ether/ethyl acetate $\left.=8: 1, \mathrm{v} / \mathrm{v}\right) .{ }^{1} \mathrm{H} \mathrm{NMR}\left(400 \mathrm{MHz}, \mathrm{CDCl}_{3}\right)$ $\delta: 7.06(\mathrm{~d}, J=8.0 \mathrm{~Hz}, 2 \mathrm{H}), 6.75(\mathrm{~d}, J=8.0 \mathrm{~Hz}, 2 \mathrm{H}), 4.90-4.82(\mathrm{~m}, 1 \mathrm{H}), 4.01-$ $3.91(\mathrm{~m}, 3 \mathrm{H}), 3.90-3.83(\mathrm{~m}, 1 \mathrm{H}), 2.27(\mathrm{~s}, 3 \mathrm{H}), 2.18-2.08(\mathrm{~m}, 2 \mathrm{H}) .{ }^{13} \mathrm{C} \mathrm{NMR}\left(101 \mathrm{MHz}, \mathrm{CDCl}_{3}\right) \delta: 155.3$, 130.2, 130.1, 115.3, 73.2, 67.2, 33.0, 20.5. HRMS (ESI) Calcd for $\mathrm{C}_{11} \mathrm{H}_{14} \mathrm{O}_{2} \mathrm{Na}\left([\mathrm{M}+\mathrm{Na}]^{+}\right)$: 201.0886; Found: 201.0880. HPLC conditions: Chiralcel OD-H column $(25 \mathrm{~cm} \times 0.46 \mathrm{~cm} \mathrm{ID)}$; $n$-hexane/2-propanol $=90: 10 ;$ temp, rt; flow rate $=1.0 \mathrm{~mL} / \mathrm{min} ; 210 \mathrm{~nm} \mathrm{UV} \mathrm{detector;} t_{\mathrm{R}}(R)=5.5 \mathrm{~min}$ (major); $t_{\mathrm{R}}(S)=6.0$ $\min$ (minor).

\section{(-)-3-(4-Chlorophenoxy)tetrahydrofuran ((-)-6c)}<smiles>Clc1ccc(OC2CCOC2)cc1</smiles>

Colorless liquid, $178 \mathrm{mg}, 90 \%$ yield, $[\alpha]_{\mathrm{D}}^{25}-11.3\left(\mathrm{c} 0.3, \mathrm{CHCl}_{3}\right) . \mathrm{R}_{f}=0.45$ (petroleum ether/ethyl acetate $=8: 1, \mathrm{v} / \mathrm{v}) .{ }^{1} \mathrm{H} \mathrm{NMR}\left(400 \mathrm{MHz}, \mathrm{CDCl}_{3}\right) \delta: 7.25-$ 7.19 (m, 2H), 6.82-6.75 (m, 2H), 4.91-4.82 (m, 1H), 4.02-3.93 (m, 3H), 3.92$3.85(\mathrm{~m}, 1 \mathrm{H}), 2.27-2.06(\mathrm{~m}, 2 \mathrm{H}) .{ }^{13} \mathrm{C} \mathrm{NMR}\left(101 \mathrm{MHz}, \mathrm{CDCl}_{3}\right) \delta: 156.0,129.5,125.9,116.6,77.7,73.0$, 67.2, 32.9. HRMS (EI) Calcd for $\mathrm{C}_{10} \mathrm{H}_{11} \mathrm{ClO}_{2}\left([\mathrm{M}]^{+}\right)$: 198.0442; Found: 198.0440. 
(-)-3-(4-(tert-Butyl)phenoxy)tetrahydrofuran ((-)-6d)<smiles>Brc1ccc(OC2CCOC2)cc1</smiles>

Colorless liquid, $200 \mathrm{mg}, 91 \%$ yield, $[\alpha]_{\mathrm{D}}^{25}-14.0\left(\right.$ c $\left.0.3, \mathrm{CHCl}_{3}\right) . \mathrm{R}_{f}=0.50$ (petroleum ether/ethyl acetate $=8: 1, \mathrm{v} / \mathrm{v}) .{ }^{1} \mathrm{H}$ NMR $\left(400 \mathrm{MHz}, \mathrm{CDCl}_{3}\right) \delta: 7.34-$ $7.28(\mathrm{~m}, 2 \mathrm{H}), 6.84-6.77(\mathrm{~m}, 2 \mathrm{H}), 4.93-4.88(\mathrm{~m}, 1 \mathrm{H}), 4.04-3.95(\mathrm{~m}, 3 \mathrm{H}), 3.94-$ $3.85(\mathrm{~m}, 1 \mathrm{H}), 2.23-2.14(\mathrm{~m}, 2 \mathrm{H}), 1.31$ (s, 9H). ${ }^{13} \mathrm{C}$ NMR $\left(101 \mathrm{MHz}, \mathrm{CDCl}_{3}\right) \delta: 155.1,143.7,126.4,114.8$, 77.2, 73.2, 67.2, 34.1, 33.1, 31.5. HRMS (ESI) Calcd for $\mathrm{C}_{14} \mathrm{H}_{24} \mathrm{NO}_{2}\left(\left[\mathrm{M}+\mathrm{NH}_{4}\right]^{+}\right)$: 238.1802; Found: 238.1800 .

\section{(-)-3-(3-Methoxyphenoxy)tetrahydrofuran ((-)-6e)}<smiles>COc1cccc(OC2CCOC2)c1</smiles>

Colorless liquid, $182 \mathrm{mg}, 94 \%$ yield, $[\alpha]_{\mathrm{D}}^{25}-6.67\left(c \quad 0.3, \mathrm{CHCl}_{3}\right) . \mathrm{R}_{f}=0.28$ (petroleum ether/ethyl acetate $=10: 1, \mathrm{v} / \mathrm{v}) .{ }^{1} \mathrm{H}$ NMR $\left(400 \mathrm{MHz}, \mathrm{CDCl}_{3}\right) \delta: 7.20$ (t, $J=8.0 \mathrm{~Hz}, 1 \mathrm{H}), 6.54(\mathrm{~m}, 1 \mathrm{H}), 6.50-6.43(\mathrm{~m}, 2 \mathrm{H}), 4.96-4.90(\mathrm{~m}, 1 \mathrm{H}), 4.04-3.97(\mathrm{~m}, 3 \mathrm{H}), 3.95-3.88$ (m, 1H), 3.80 (s, 3H), 2.25-2.14 (m, 2H). ${ }^{13} \mathrm{C}$ NMR (101 MHz, $\left.\mathrm{CDCl}_{3}\right) \delta: 160.9,158.7,130.0,107.4$, 106.5, 101.9, 77.3, 73.1, 67.2, 55.3, 33.0. HRMS (ESI) Calcd for $\mathrm{C}_{11} \mathrm{H}_{15} \mathrm{O}_{3}\left([\mathrm{M}+\mathrm{H}]^{+}\right)$: 195.1016; Found: 195.1018.

\section{(-)-3-(3-Chlorophenoxy)tetrahydrofuran ((-)-6f)}

Cl Colorless liquid, $182 \mathrm{mg}, 92 \%$ yield, $[\alpha]_{\mathrm{D}}^{25}-16.67\left(c \quad 0.3, \mathrm{CHCl}_{3}\right) . \mathrm{R}_{f}=0.28$ (petroleum ether/ethyl acetate $=10: 1, \mathrm{v} / \mathrm{v}){ }^{1} \mathrm{H}$ NMR $\left(400 \mathrm{MHz}, \mathrm{CDCl}_{3}\right) \delta: 7.19(\mathrm{t}$, $J=8.0 \mathrm{~Hz}, 1 \mathrm{H}), 6.98-6.89(\mathrm{~m}, 1 \mathrm{H}), 6.86(\mathrm{t}, J=2.0 \mathrm{~Hz}, 1 \mathrm{H}), 6.77-6.72(\mathrm{~m}, 1 \mathrm{H}), 4.93-4.85(\mathrm{~m}, 1 \mathrm{H})$, 4.02-3.86 (m, 4H), 2.25-2.09 (m, 2H). ${ }^{13} \mathrm{C}$ NMR (101 MHz, $\left.\mathrm{CDCl}_{3}\right) \delta$ 158.2, 135.0, 130.4, 121.2, 115.7, 113.8, 77.6, 73.0, 67.2, 33.0. HRMS (EI) Calcd for $\mathrm{C}_{10} \mathrm{H}_{11} \mathrm{ClO}_{2}\left([\mathrm{M}]^{+}\right)$: 198.0442; Found: 198.0442 .

\section{(-)-3-(3-Bromophenoxy)tetrahydrofuran ((-)-6g)}

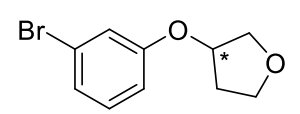

Colorless liquid, $225 \mathrm{mg}, 93 \%$ yield, $[\alpha]_{\mathrm{D}}^{25}-12.0\left(c \quad 0.3, \mathrm{CHCl}_{3}\right) . \mathrm{R}_{f}=0.43$ (petroleum ether/ethyl acetate $=10: 1, \mathrm{v} / \mathrm{v}){ }^{1} \mathrm{H}$ NMR $\left(400 \mathrm{MHz}, \mathrm{CDCl}_{3}\right) \delta: 7.16-$ 7.06 (m, 2H), 7.03-6.99 (m, 1H), 6.81-6.76 (m, 1H), 4.93-4.84 (m, 1H), 4.02$3.93(\mathrm{~m}, 3 \mathrm{H}), 3.92-3.85(\mathrm{~m}, 1 \mathrm{H}), 2.25-2.17(\mathrm{~m}, 1 \mathrm{H}), 2.16-2.08(\mathrm{~m}, 1 \mathrm{H}){ }^{13} \mathrm{C} \mathrm{NMR}\left(101 \mathrm{MHz}, \mathrm{CDCl}_{3}\right) \delta$ : 158.2, 130.7, 124.1, 122. 9, 118.6, 114.3, 77.6, 73.0, 67.2, 32.9. HRMS (ESI) Calcd for $\mathrm{C}_{10} \mathrm{H}_{11} \mathrm{BrO}_{2}$ $\left([\mathrm{M}]^{+}\right): 241.9937$; Found: 241.9937.

\section{(-)-3-(o-Tolyloxy)tetrahydrofuran ((-)-6h)}<smiles>O=[N+]([O-])c1ccccc1OC1CCOC1</smiles>

Colorless liquid, $171 \mathrm{mg}, 96 \%$ yield, 94\% ee. $[\alpha]_{\mathrm{D}}^{25}-8.67\left(c 0.3, \mathrm{CHCl}_{3}\right) . \mathrm{R}_{f}=0.55$ (petroleum ether/ethyl acetate $=8: 1, \mathrm{v} / \mathrm{v}){ }^{1} \mathrm{H}$ NMR $\left(400 \mathrm{MHz}, \mathrm{CDCl}_{3}\right) \delta: 7.16(\mathrm{t}, J=$ $8.0 \mathrm{~Hz}, 2 \mathrm{H}), 6.89(\mathrm{t}, J=7.6 \mathrm{~Hz}, 1 \mathrm{H}), 6.76(\mathrm{~d}, J=8.0 \mathrm{~Hz}, 1 \mathrm{H}), 4.98-4.91(\mathrm{~m}, 1 \mathrm{H})$, 4.07-3.91 (m, 4H), 2.23 (s, 3H), 2.22-2.13 (m, 2H). $\left.{ }^{13} \mathrm{C} \mathrm{NMR} \mathrm{(101} \mathrm{MHz,} \mathrm{CDCl}_{3}\right)$ o: 155.6, 131.0, 127.6, 126.6, 120.6, 112.0, 77.3, 73.3, 67.3, 33.3, 16.4. $\mathrm{C}_{11} \mathrm{H}_{14} \mathrm{O}_{2} \mathrm{Na}\left([\mathrm{M}+\mathrm{Na}]^{+}\right)$: 201.0886; Found: 201.0884. 
HPLC conditions: Chiralcel AD-H column (25 cm $\times 0.46 \mathrm{~cm}$ ID); $n$-hexane/2-propanol = 99:1; temp, rt; flow rate $=0.8 \mathrm{~mL} / \mathrm{min} ; 210 \mathrm{~nm} \mathrm{UV} \mathrm{detector;} t_{\mathrm{R}}(R)=5.6 \min$ (major); $t_{\mathrm{R}}(S)=5.9 \mathrm{~min}$ (minor).

\section{(-)-3-(2-Methoxyphenoxy)tetrahydrofuran ((-)-6i)}<smiles>COc1ccccc1OC1CCOC1</smiles>

Colorless liquid $175 \mathrm{mg}, 90 \%$ yield, $[\alpha]_{\mathrm{D}}^{25}-7.33\left(c 0.3, \mathrm{CHCl}_{3}\right) . \mathrm{R}_{f}=0.30$ (petroleum ether/ethyl acetate $=8: 1, \mathrm{v} / \mathrm{v}) .{ }^{1} \mathrm{H}$ NMR $\left(400 \mathrm{MHz}, \mathrm{CDCl}_{3}\right) \delta: 7.00-6.81(\mathrm{~m}, 4 \mathrm{H})$, 4.98-4.91 (m, 1H), 4.07-3.97 (m, 3H), 3.93-3.87 (m, 1H), 3.85 (s, 3H), 2.26-2.14 (m, 2H). ${ }^{13} \mathrm{C} \mathrm{NMR}\left(101 \mathrm{MHz}, \mathrm{CDCl}_{3}\right) \delta: 150.3,146.9,122.0,120.8,115.9,112.3,78.8,73.1,67.2,55.9$, 33.1. HRMS (ESI) Calcd for $\mathrm{C}_{11} \mathrm{H}_{15} \mathrm{O}_{3}\left([\mathrm{M}+\mathrm{H}]^{+}\right)$: 195.1016; Found: 195.1019 .

\section{(-)-3-(2-Chlorophenoxy)tetrahydrofuran ((-)-6j)}<smiles>Clc1ccccc1OC1CCOC1</smiles>

Colorless liquid, $182 \mathrm{mg}, 92 \%$ yield, $[\alpha]_{\mathrm{D}}^{25}-11.33\left(c 0.3, \mathrm{CHCl}_{3}\right) \cdot \mathrm{R}_{f}=0.40$ (petroleum ether/ethyl acetate $=8: 1, \mathrm{v} / \mathrm{v}) .{ }^{1} \mathrm{H}$ NMR $\left(400 \mathrm{MHz}, \mathrm{CDCl}_{3}\right) \delta: 7.36(\mathrm{dd}, J=8.0,1.6$ $\mathrm{Hz}, 1 \mathrm{H}), 7.23-7.14(\mathrm{~m}, 1 \mathrm{H}), 6.94-6.84(\mathrm{~m}, 2 \mathrm{H}), 4.99-4.91(\mathrm{~m}, 1 \mathrm{H}), 4.05-3.98$ (m,

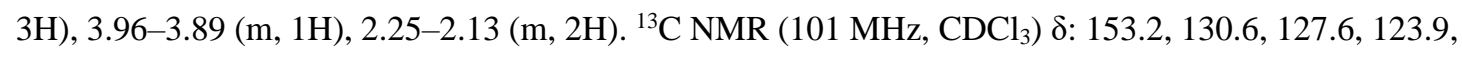
121.9, 114.9, 78.9, 73.0, 67.3, 33.1. HRMS (ESI) Calcd for $\mathrm{C}_{10} \mathrm{H}_{12} \mathrm{ClO}_{2}\left([\mathrm{M}+\mathrm{H}]^{+}\right)$: 199.0520; Found: 199.0520.

\section{(-)-3-(2-Fluorophenoxy)tetrahydrofuran ((-)-6k)}

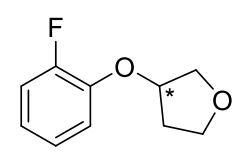

Colorless liquid, $175 \mathrm{mg}, 96 \%$ yield, $94 \%$ ee. $[\alpha]_{\mathrm{D}}^{25}-8.0\left(c \quad 0.3, \mathrm{CHCl}_{3}\right) . \mathrm{R}_{f}=0.40$ (petroleum ether/ethyl acetate $=8: 1, \mathrm{v} / \mathrm{v}){ }^{1} \mathrm{H} \mathrm{NMR}\left(400 \mathrm{MHz}, \mathrm{CDCl}_{3}\right) \delta: 7.15-7.03$ (m, 2H), 6.98-6.90 (m, 2H), 5.02-4.95 (m, 1H), 4.09-3.99 (m, 3H), 3.98-3.89 (m, 1H), 2.27-2.14 (m, 2H). ${ }^{13} \mathrm{C}$ NMR $\left(101 \mathrm{MHz}, \mathrm{CDCl}_{3}\right) \delta: 153.3\left(\mathrm{~d},{ }^{1} J_{\mathrm{C}-\mathrm{F}}=246.4 \mathrm{~Hz}\right), 145.4\left(\mathrm{~d},{ }^{2} J_{\mathrm{C}-\mathrm{F}}=\right.$ $10.1 \mathrm{~Hz}), 124.3\left(\mathrm{~d},{ }^{3} J_{\mathrm{C}-\mathrm{F}}=3.7 \mathrm{~Hz}\right), 121.9\left(\mathrm{~d},{ }^{3} J_{\mathrm{C}-\mathrm{F}}=7.1 \mathrm{~Hz}\right), 117.0,116.6\left(\mathrm{~d},{ }^{2} J_{\mathrm{C}-\mathrm{F}}=18.2 \mathrm{~Hz}\right), 79.3,73.0$, 67.2, 33.1. HRMS (ESI) Calcd for $\mathrm{C}_{10} \mathrm{H}_{15} \mathrm{FO}_{2}\left(\left[\mathrm{M}+\mathrm{N}_{4} \mathrm{H}\right]^{+}\right)$: 200.1081; Found: 200.1081. HPLC conditions: Chiralcel AD-3 column $(25 \mathrm{~cm} \times 0.46 \mathrm{~cm}$ ID); $n$-hexane/2-propanol = 99:1; temp, rt; flow rate $=0.9 \mathrm{~mL} / \mathrm{min} ; 210 \mathrm{~nm} \mathrm{UV} \mathrm{detector} ; t_{\mathrm{R}}(R)=6.4 \mathrm{~min}($ major $) ; t_{\mathrm{R}}(S)=6.8 \mathrm{~min}$ (minor).

\section{(R)-3-(2,5-Dimethylphenoxy)tetrahydrofuran ((R)-61)}

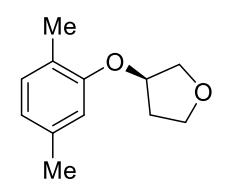

Colorless liquid, $200 \mathrm{mg}, 91 \%$ yield, $91 \%$ ee. $[\alpha]_{\mathrm{D}}^{25}-9.33\left(c 0.3, \mathrm{CHCl}_{3}\right) \cdot \mathrm{R}_{f}=0.50$ (petroleum ether/ethyl acetate $=8: 1, \mathrm{v} / \mathrm{v}){ }^{1} \mathrm{H}$ NMR $\left(400 \mathrm{MHz}, \mathrm{CDCl}_{3}\right) \delta: 7.05(\mathrm{~d}, J=$ $7.6 \mathrm{~Hz}, 1 \mathrm{H}), 6.71(\mathrm{~d}, J=7.6 \mathrm{~Hz}, 1 \mathrm{H}), 6.59(\mathrm{~s}, 1 \mathrm{H}), 4.97-4.91(\mathrm{~m}, 1 \mathrm{H}), 4.07-3.91(\mathrm{~m}$, $4 \mathrm{H}), 2.34(\mathrm{~s}, 3 \mathrm{H}), 2.22-2.16(\mathrm{~m}, 5 \mathrm{H}) .{ }^{13} \mathrm{C} \mathrm{NMR}\left(101 \mathrm{MHz}, \mathrm{CDCl}_{3}\right) \delta: 155.5,136.4$, 130.7, 124.4, 121.2, 113.1, 76.8, 73.3, 67.3, 33.3, 21.5, 16.0. HRMS (ESI) Calcd for $\mathrm{C}_{12} \mathrm{H}_{17} \mathrm{O}_{2}\left([\mathrm{M}+\mathrm{H}]^{+}\right)$: 193.1223; Found: 193.1224. HPLC conditions: Chiralcel AD-H column $(25 \mathrm{~cm} \times 0.46 \mathrm{~cm} \mathrm{ID})$; $n$ hexane/2-propanol $=99: 1$; temp, rt; flow rate $=0.9 \mathrm{~mL} / \mathrm{min} ; 210 \mathrm{~nm} \mathrm{UV}$ detector; $t_{\mathrm{R}}(R)=4.7 \mathrm{~min}$ (major); $t_{\mathrm{R}}(S)=5.1 \mathrm{~min}$ (minor). 
(-)-3-(Naphthalen-2-yloxy)tetrahydrofuran ((-)-6m)

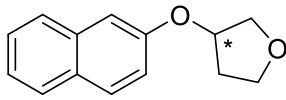

Colorless liquid $90 \mathrm{mg}, 42 \%$ yield, $90 \%$ ee. $[\alpha]_{\mathrm{D}}^{25}-16.0\left(c 0.3, \mathrm{CHCl}_{3}\right) . \mathrm{R}_{f}=0.45$

(petroleum ether/ethyl acetate $=3: 1, \mathrm{v} / \mathrm{v}){ }^{1} \mathrm{H} \mathrm{NMR}\left(400 \mathrm{MHz}, \mathrm{CDCl}_{3}\right) \delta: 7.83$

$7.68(\mathrm{~m}, 3 \mathrm{H}), 7.45(\mathrm{t}, J=7.6 \mathrm{~Hz}, 1 \mathrm{H}), 7.35(\mathrm{t}, J=7.6 \mathrm{~Hz}, 1 \mathrm{H}), 7.14(\mathrm{dd}, J=8.8$,

$2.0 \mathrm{~Hz}, 1 \mathrm{H}), 7.07$ (s, 1H), 5.12-5.02 (m, 1H), 4.13-3.99 (m, 3H), 3.98-3.87 (m, 1H), 2.35-2.18 (m, 2H).

${ }^{13} \mathrm{C}$ NMR $\left(101 \mathrm{MHz} \mathrm{CDCl}_{3}\right) \delta: 155.3,134.3,129.6,128.9,127.6,126.7,126.4,123.7,119.3,107.6$,

77.2, 73.1, 67.2, 33.0. HRMS (ESI) Calcd for $\mathrm{C}_{14} \mathrm{H}_{15} \mathrm{O}_{2}\left([\mathrm{M}+\mathrm{H}]^{+}\right)$: 215.1067; Found: 215.1065. HPLC conditions: Chiralcel OD-H column (25 cm $\times 0.46 \mathrm{~cm} \mathrm{ID);} n$-hexane/2-propanol = 90:10; temp, rt; flow rate $=1.0 \mathrm{~mL} / \mathrm{min} ; 210 \mathrm{~nm} \mathrm{UV} \mathrm{detector} ; t_{\mathrm{R}}(R)=7.3 \mathrm{~min}$ (major); $t_{\mathrm{R}}(S)=9.4 \mathrm{~min}$ (minor)

(E) Enantioselective Synthesis of the Advanced Chiral Intermediate (S)-13 Synthesis of racemic lactone $10^{8}$
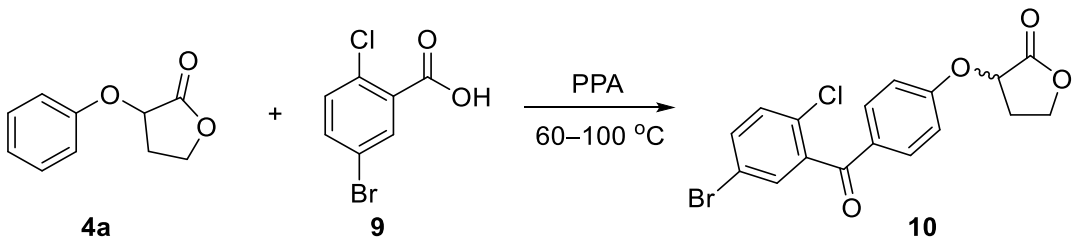

A $250 \mathrm{~mL}$ round-bottom flask was charged with PPA $(37.6 \mathrm{~g})$ and heated in an oil bath to $60{ }^{\circ} \mathrm{C}$. Then 5-bromo-2-chlorobenzoic acid $9(4.7 \mathrm{~g}, 20.0 \mathrm{mmol})$ and $4 \mathbf{a}(3.6 \mathrm{~g}, 20.0 \mathrm{mmol})$ were added to the flask. The mixture was stirred at $100{ }^{\circ} \mathrm{C}$ for $4 \mathrm{~h}$ to complete the reaction, and then cooled to $50{ }^{\circ} \mathrm{C}$ and quenched with water $(100.0 \mathrm{~mL})$. The resulting reaction mixture was allowed to stir at room temperature for $1 \mathrm{~h}$, then diluted with $\mathrm{CH}_{2} \mathrm{Cl}_{2}(50.0 \mathrm{~mL})$ and continued to stir for $1 \mathrm{~h}$. The organic phase was separated and aqueous was extracted with $\mathrm{CH}_{2} \mathrm{Cl}_{2}(30.0 \mathrm{~mL} \times 3)$. The combined organic layers were washed with brine, dried over $\mathrm{Mg}_{2} \mathrm{SO}_{4}$, and concentrated in vacuo to yield a residue. The residue was chromatographed on silica gel column with petroleum ether/ethyl acetate (4:1 to $2: 1)$ as an eluent to give lactone $\mathbf{1 0}$ (4.5 g, $58 \%$ yield) as a yellow solid. mp. $102-105^{\circ} \mathrm{C} .{ }^{1} \mathrm{H}$ NMR $\left(400 \mathrm{MHz}, \mathrm{CDCl}_{3}\right) \delta: 7.86-7.74(\mathrm{~m}, 2 \mathrm{H}), 7.55$ $(\mathrm{dd}, J=8.6,2.4 \mathrm{~Hz}, 1 \mathrm{H}), 7.48(\mathrm{~d}, J=2.4 \mathrm{~Hz}, 1 \mathrm{H}), 7.33$ (d, $J=8.4 \mathrm{~Hz}, 1 \mathrm{H}), 7.16-7.01$ (m, 2H), 5.08 (t, $J=8.0 \mathrm{~Hz}, 1 \mathrm{H}), 4.61-4.50(\mathrm{~m}, 1 \mathrm{H}), 4.45-4.35(\mathrm{~m}, 1 \mathrm{H}), 2.84-2.71(\mathrm{~m}, 1 \mathrm{H}), 2.58-2.44(\mathrm{~m}, 1 \mathrm{H}) .{ }^{13} \mathrm{C} \mathrm{NMR}$ $\left(101 \mathrm{MHz}, \mathrm{CDCl}_{3}\right) \delta:$ 192.0, 172.6, 161.7, 140.3, 133.9, 132.5, 131.6, 131.5, 130.2, 130.1, 120.6, 115.6, 72.1, 65.4, 29.7. HRMS (ESI) Calcd for $\mathrm{C}_{17} \mathrm{H}_{13} \mathrm{BrClO}_{4}\left([\mathrm{M}+\mathrm{H}]^{+}\right)$: 394.9680; Found: 394.9681 .

\section{Synthesis of lactone $11^{8}$}
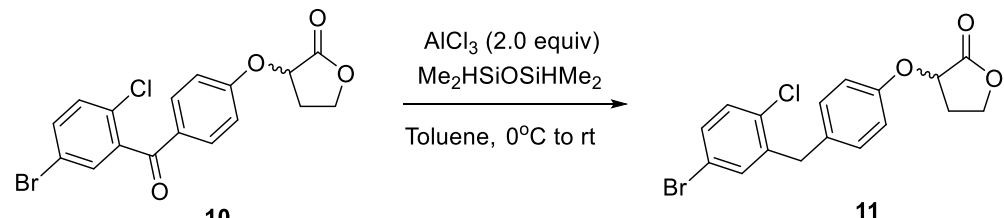

To an ice water cooled mixture of $\mathrm{AlCl}_{3}(2.4 \mathrm{~g}, 18.2 \mathrm{mmol})$ and lactone $\mathbf{1 0}$ (3.6 g, $\left.9.1 \mathrm{mmol}\right)$ in toluene (100.0 mL) was added 1,1,3,3-tetramethyldisiloxane $(2.4 \mathrm{~g}, 18.2 \mathrm{mmol})$ slowly at $0{ }^{\circ} \mathrm{C}$ under argon atmosphere. The reaction mixture was then allowed to stir at room temperature for $1 \mathrm{~h}$, then cooled to 0 
${ }^{\circ} \mathrm{C}$ and quenched with water $(30.0 \mathrm{~mL})$. The reaction mixture was extracted with ethyl acetate $(20.0 \mathrm{~mL}$ $\times 3$ ). The combined extracts were washed with brine, dried over $\mathrm{Mg}_{2} \mathrm{SO}_{4}$, and concentrated in vacuo to give a residue. The residue was chromatographed on silica gel column with petroleum ether/ethyl acetate (4:1 to $1: 1)$ as an eluent to give lactone 11 (2.5 g, 73\% yield) as a white solid. mp. 93-94 ${ }^{\circ} \mathrm{C} .{ }^{1} \mathrm{H}$ NMR $\left(400 \mathrm{MHz}, \mathrm{CDCl}_{3}\right) \delta: 7.29(\mathrm{dd}, J=8.4,2.4 \mathrm{~Hz}, 1 \mathrm{H}), 7.26-7.21(\mathrm{~m}, 2 \mathrm{H}), 7.15-7.08(\mathrm{~m}, 2 \mathrm{H}), 7.01-6.95$ $(\mathrm{m}, 2 \mathrm{H}), 4.93(\mathrm{t}, J=7.8 \mathrm{~Hz}, 1 \mathrm{H}), 4.56-4.49(\mathrm{~m}, 1 \mathrm{H}), 4.40-4.32(\mathrm{~m}, 1 \mathrm{H}), 4.00(\mathrm{~s}, 2 \mathrm{H}), 2.75-2.67(\mathrm{~m}, 1 \mathrm{H})$, 2.52-2.41 (m, 1H). ${ }^{13} \mathrm{C}$ NMR (101 MHz, $\left.\mathrm{CDCl}_{3}\right) \delta: 173.5,156.0,140.9,133.6,133.1,132.5,130.9$, 130.7, 130.1, 120.5, 116.1, 72.6, 65.3, 38.2, 29.9. HRMS (ESI) Calcd for $\mathrm{C}_{17} \mathrm{H}_{19} \mathrm{BrClO}_{3} \mathrm{Na}\left([\mathrm{M}+\mathrm{Na}]^{+}\right)$: 402.9707; Found: 402.9710.

\section{Asymmetric hydrogenation of lactone $11(\mathrm{~S} / \mathrm{C}=1000)$.}

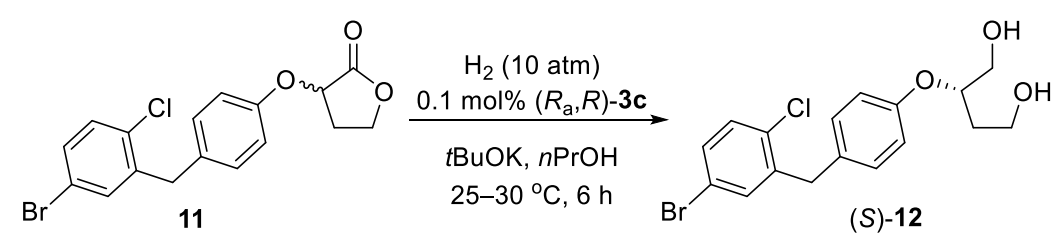

To a suitable glass tube equipped with a magnetic stirring bar was added $[\operatorname{Ir}(\mathrm{COD}) \mathrm{Cl}]_{2}(1.0 \mathrm{mg}, 1.5 \mu \mathrm{mol})$, $\left(R_{\mathrm{a}}, R\right)$-S6c $(2.6 \mathrm{mg}, 3.2 \mu \mathrm{mol})$, and anhydrous $n \mathrm{PrOH}(3 \mathrm{~mL})$ under argon atmosphere at room temperature. After this, the glass tube was placed in a stainless vessel and the reaction mixture was stirred at room temperature for $20 \mathrm{~min}$ under argon atmosphere. The argon atmosphere was replaced with $\mathrm{H}_{2}$ and the reaction mixture was continued to stir at room temperature under $10 \mathrm{~atm}$ of $\mathrm{H}_{2}$ pressure for another $10 \mathrm{~min}$. After releasing the pressure, the prepared iridium catalyst $\left(R_{\mathrm{a}}, R\right)-\mathbf{3 c}$ was then transferred into to a glass tube containing a mixture of lactone $11(1.14 \mathrm{~g}, 3.0 \mathrm{mmol}), \mathrm{BuOK}(0.34 \mathrm{~g}, 3.0 \mathrm{mmol})$ and $n \operatorname{PrOH}(20.0 \mathrm{~mL})$, which was previously placed in a $250 \mathrm{~mL}$ stainless vessel, through the injection port. The vessel was then pressurized to $10 \mathrm{~atm}$ of $\mathrm{H}_{2}$ pressure and the reaction mixture was allowed to stir at room temperature until no obvious hydrogen pressure drop was observed. After releasing the hydrogen pressure, the solvent was removed in vacuo, and the residue was chromatographed on silica gel column with petroleum ether / ethyl acetate (1:1 to 1:2) as an eluent to afford product $(S)-\mathbf{1 2}(0.93 \mathrm{~g}, 80 \%$ yield) as a colorless oil. The enantiomeric excess of the product was determined by HPLC on a chiral stationary phase. $93 \%$ ee. $[\alpha]_{\mathrm{D}}^{25}-18.8(c 0.5, \mathrm{EtOH}) .{ }^{1} \mathrm{H} \mathrm{NMR}\left(400 \mathrm{MHz}, \mathrm{CDCl}_{3}\right) \delta: 7.31-7.25(\mathrm{~m}, 2 \mathrm{H}), 7.23(\mathrm{~d}, J$ $=8.4 \mathrm{~Hz}, 1 \mathrm{H}), 7.09(\mathrm{~d}, J=8.8 \mathrm{~Hz}, 2 \mathrm{H}), 6.91(\mathrm{~d}, J=8.4 \mathrm{~Hz}, 2 \mathrm{H}), 4.59-4.52(\mathrm{~m}, 1 \mathrm{H}), 3.99(\mathrm{~s}, 2 \mathrm{H}), 3.89-$ $3.73(\mathrm{~m}, 4 \mathrm{H}), 2.38-1.89(\mathrm{~m}, 4 \mathrm{H}) .{ }^{13} \mathrm{C} \mathrm{NMR}\left(101 \mathrm{MHz}, \mathrm{CDCl}_{3}\right) \delta: 156.5,141.1,133.6,133.1,131.5$, 130.9, 130.6, 130.1, 120.5, 116.3, 76.4, 64.2, 59.0, 38.2, 33.8. HRMS (ESI) Calcd for $\mathrm{C}_{17} \mathrm{H}_{19} \mathrm{BrClO}_{3}$ $\left([\mathrm{M}+\mathrm{H}]^{+}\right)$: 385.0201; Found: 385.0199. HPLC conditions: Chiralcel IC-3 column $(25 \mathrm{~cm} \times 0.46 \mathrm{~cm}$ ID); $n$-hexane/2-propanol $=90: 10$; temp, rt; flow rate $=1.0 \mathrm{~mL} / \mathrm{min} ; 210 \mathrm{~nm} \mathrm{UV}$ detector; $t_{\mathrm{R}}(R)=12.0 \mathrm{~min}$ (minor); $t_{\mathrm{R}}(S)=13.7$ min (major).

\section{Synthesis of the advanced chiral intermediate $(S)$-13}




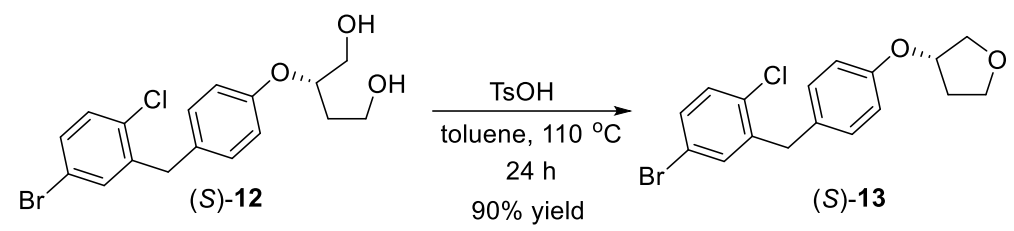

According to the procedure for the synthesis of 6, $(S)$-13 was synthesized from $(S)$-12 (384.0 mg, 1.0 mmol) as a colorless oil: $329.4 \mathrm{mg}, 90 \%$ yield, $92 \%$ ee. $[\alpha]_{\mathrm{D}}^{26}+6.8\left(c 0.5, \mathrm{CHCl}_{3}\right) .{ }^{1} \mathrm{H} \mathrm{NMR}(400 \mathrm{MHz}$, $\left.\mathrm{CDCl}_{3}\right) \delta: 7.31-7.20(\mathrm{~m}, 3 \mathrm{H}), 7.09$ (d, $\left.J=8.6 \mathrm{~Hz}, 2 \mathrm{H}\right), 6.80(\mathrm{~d}, J=8.8 \mathrm{~Hz}, 2 \mathrm{H}), 4.93-4.86(\mathrm{~m}, 1 \mathrm{H})$, 4.02-3.94 (m, 5H), 3.93-3.86 (m, 1H), 2.25-2.10 (m, 2H). ${ }^{13} \mathrm{C}$ NMR (101 MHz, $\left.\mathrm{CDCl}_{3}\right) \delta: 156.1,141.2$, 133.6, 133.1, 130.9, 130.6, 130.1, 120.5, 115.5, 77.3, 73.2, 67.2, 38.2, 33.0. HRMS (ESI) Calcd for $\mathrm{C}_{17} \mathrm{H}_{20} \mathrm{BrClNO}_{2}\left(\left[\mathrm{M}+\mathrm{NH}_{4}\right]^{+}\right)$: 384.0380; Found:384.0368. HPLC conditions: Chiralcel OJ-3 column (25 $\mathrm{cm} \times 0.46 \mathrm{~cm} \mathrm{ID}$ ); $n$-hexane $/ 2$-propanol $=85: 15$; temp, rt; flow rate $=1.0 \mathrm{~mL} / \mathrm{min} ; 210 \mathrm{~nm} \mathrm{UV}$ detector; $t_{\mathrm{R}}(S)=9.8 \mathrm{~min}$ (major); $t_{\mathrm{R}}(R)=11.0 \mathrm{~min}$ (minor).

\section{(F) Enantioselective Synthesis of 3-Aryloxy Pyrrolidine (S)-15}

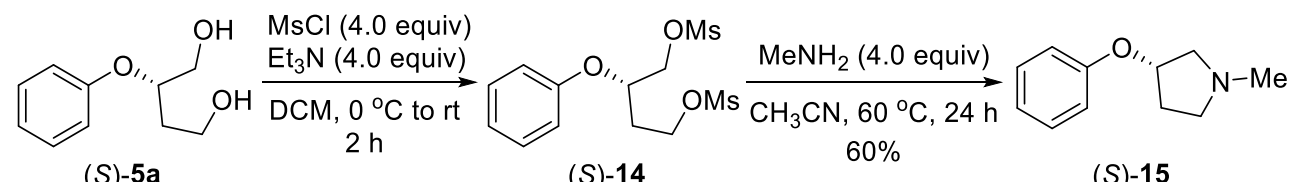

To an ice-cooled solution of (S)-5a (236.6 mg, $1.3 \mathrm{mmol})$ and $\mathrm{Et}_{3} \mathrm{~N}$ (525.2 mg, $\left.5.2 \mathrm{mmol}\right)$ in $\mathrm{CH}_{2} \mathrm{Cl}_{2}(2.0$ $\mathrm{mL}$ ) was added $\mathrm{MsCl}(592.8 \mathrm{mg}, 5.2 \mathrm{mmol}$ ) slowly under argon atmosphere. The reaction mixture was then stirred at $0{ }^{\circ} \mathrm{C}$ for $2 \mathrm{~h}$. The reaction mixture was concentrated in vacuo, diluted with $\mathrm{CH}_{3} \mathrm{CN}(2.0$ $\mathrm{mL})$, added a solution of $\mathrm{MeNH}_{2}(8.0 \mathrm{mmol}, 2 \mathrm{M}, 4.0 \mathrm{~mL})$ in methanol, and heated to $60{ }^{\circ} \mathrm{C}$ in an oil bath for $24 \mathrm{~h}$. The reaction mixture was concentrated again in vacuo, and the residue was chromatographed on silica gel column with petroleum ether/ethyl acetate (5:1) as eluent to give $(S)$-15 ( $138.0 \mathrm{mg}, 60 \%$ yield, $93 \%$ ee) as a yellow liquid. $\mathrm{R}_{f}=0.33$ (dichloromethane/methyl alcohol $=5: 1, \mathrm{v} / \mathrm{v}$ ). $[\alpha]_{\mathrm{D}}^{26}+14.0\left(c\right.$ 0.5, EtOH). ${ }^{13} \mathrm{C} \mathrm{NMR}\left(101 \mathrm{MHz}, \mathrm{CDCl}_{3}\right) \delta: 157.6,129.5,120.6,115.3,76.8,62.4,55.1$, 42.2, 32.9. HRMS (ESI) Calcd for $\mathrm{C}_{11} \mathrm{H}_{16} \mathrm{NO}\left([\mathrm{M}+\mathrm{H}]^{+}\right)$: 178.1226; Found: 178.1226. HPLC conditions: Chiralcel OD-3 column $(25 \mathrm{~cm} \times 0.46 \mathrm{~cm} \mathrm{ID}) ; n$-hexane/2-propanol $=85: 15$; temp, rt; flow rate $=1.0$ $\mathrm{mL} / \mathrm{min} ; 210 \mathrm{~nm}$ UV detector; $t_{\mathrm{R}}(S)=5.1 \mathrm{~min}$ (major); $t_{\mathrm{R}}(R)=10.3 \mathrm{~min}$ (minor).

\section{(G) Computational Studies}

\section{Density functional theory (DFT) Details.}

All DFT calculations have been carried out using the Gaussian 16 program package. ${ }^{9}$ The B3LYP ${ }^{10}$ method with def2svp basis set has been selected for geometry optimizations and calculation of Gibbs energy corrections at $298 \mathrm{~K}$. Final energies were retrieved from single-point calculations at the B3LYP/6$311+\mathrm{G}^{* *}$ level, including the GD3BJ dispersion correction scheme developed by Grimme. ${ }^{11}$ All structures have been optimized considering solvent effects using the SMD Model for $i$ PrOH. Reaction paths were traced by the intrinsic reaction coordinate method for all transition states. All energetics 
reported throughout the text are in $\mathrm{kcal} / \mathrm{mol}$. Structures were generated using CYLview. ${ }^{12}$

\section{DFT Structures}

The proposed mechanism for the iridium catalyzed asymmetric hydrogenation (AH) of exocyclic $\gamma, \delta$ unsaturated $\beta$-ketoesters was shown in as below:

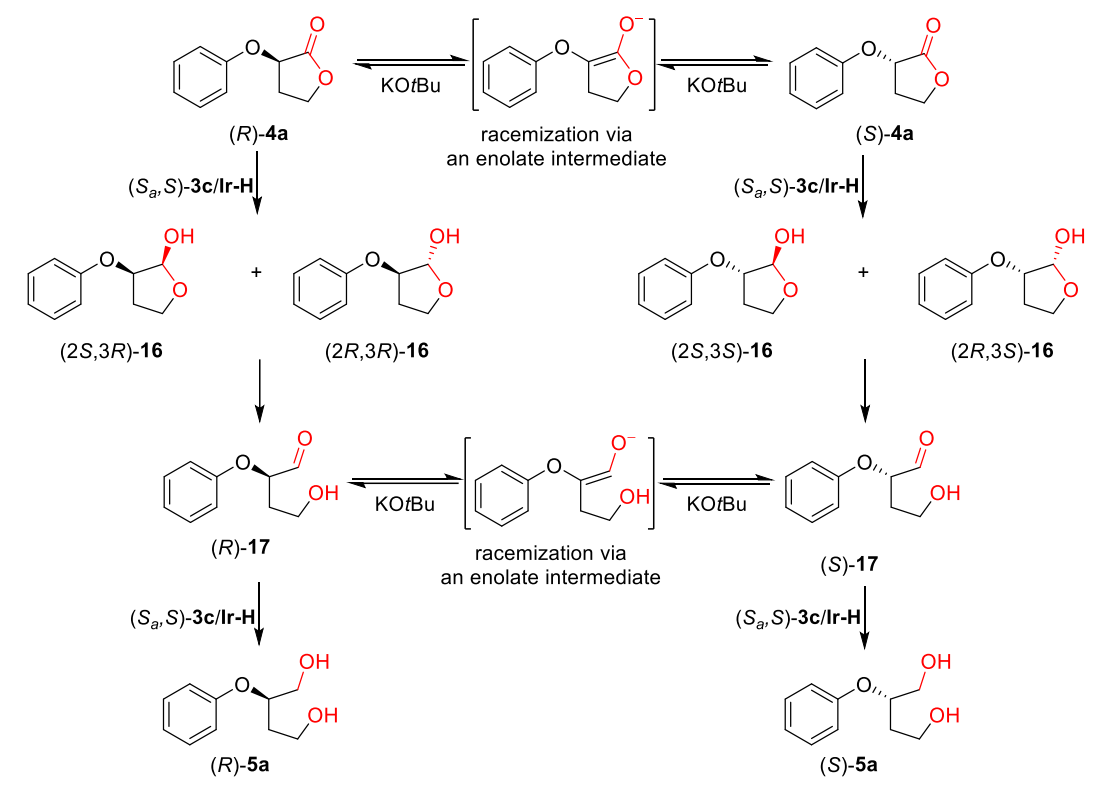

Owing to the effective chiral recognition of the active iridium species (generally $\operatorname{Ir}(\mathrm{H})_{3}$-species) generated in situ from $\left(S_{\mathrm{a}}, S\right)-3 \mathbf{c}$, one enantiomer of the racemic lactone [e.g., $(R)-\mathbf{4 a}$ ] would undergo a preferential reduction, thus leading to a faster hydrogenation than the other under the reaction conditions. Meanwhile, the residual enantioenriched lactone [e.g., (S)-4a] undergoes rapid racemization via basepromoted enol-keto tautomerization, eventually generating the corresponding hemiacetals [e.g., $(2 S, 3 R)$ 16] with two contiguous chiral centers by asymmetric hydrogenation via a dynamic kinetic resolution (DKR) process. Next, the resulting hemiacetals could be converted into the corresponding aldehyde [e.g., $(R)-17]$ via a base-promoted acetal cleavage. Finally, via a same process for the hydrogenation of racemic lactone [e.g., $(R)-\mathbf{4 a}$ ] to hemiacetals [e.g., $(2 S, 3 R)$-16], the resulting aldehyde $(R)-\mathbf{1 7}$ could be reduced to chiral diol $(R)-5 \mathbf{a}$. Enantioselectivity were rationalized by DFT calculations on the stereo-determining hydride/proton transfer step, assuming a bifunctional mechanism in which the keto group of the substrate forms a hydrogen bond to the $\mathrm{N}-\mathrm{H}$ group of the ligand, via a six-membered cyclic transition state. ${ }^{13}$ For the $(S a, S)$-3c catalyzed asymmetric hydrogenation, the chiral Ir-H intermediate can be generated in situ from the reaction mixture. Since Ir-H may act as an effective reductant for the $S i$ - or Re-attack on either of the $\alpha$-aryloxy lactones [(S)-4a and $(R)-\mathbf{4 a}$ ], four initial guessed structures of transition states need to be considered for the stereochemistry of the hydride/proton transfer step (Figure S1). 


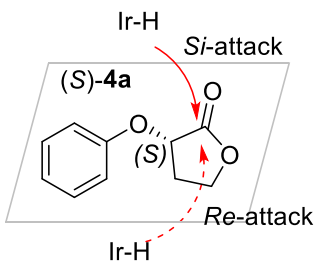

schematic representation of the transition state for $\mathrm{H}-/ \mathrm{H}+$ transfer

bifunctional mechanism

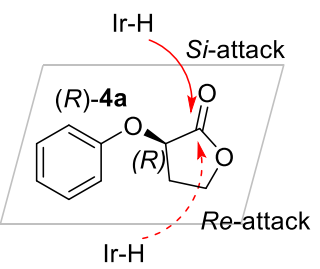

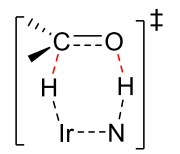

Figure S1 $S i$ - or $R e$-attack of the Ir-H species onto the keto carbonyl group of $(S)$ - or $(R)$ - $\alpha$-aryloxy

\section{lactones $\mathbf{4 a}$}

According to the bifunctional mechanism depicted in Figure S1, different spatial arrangements can exist for the reaction of Ir-H and the $\alpha$-aryloxy lactones [two conformers each for $(S)-\mathbf{4 a}$ or $(R)-\mathbf{4 a}, R e$ - or $S i$ attack] for the $\mathrm{H}^{-} / \mathrm{H}^{+}$transfer step. Although this can in principle lead to a number of distinct initially guessed transition state (TS) structures, some of them can safely be excluded due to the severe steric clashes between the substrate and the catalyst. For the reaction of Ir-H with 4a, four TS structures [TS1, TS2, TS3, and TS4] were found to be energetically viable, which would lead to stereoisomeric products $(2 S, 3 S)-,(2 S, 3 R)-,(2 R, 3 R)-$, and $(2 R, 3 S)-16$, respectively. These identified TS structures were and collectively shown in Figure S2. Remarkably, a comparison of the relative heights in free energy barriers for these TSs indicated that the hydride/proton transfer step was dominated by the reactivity of Ir-H.

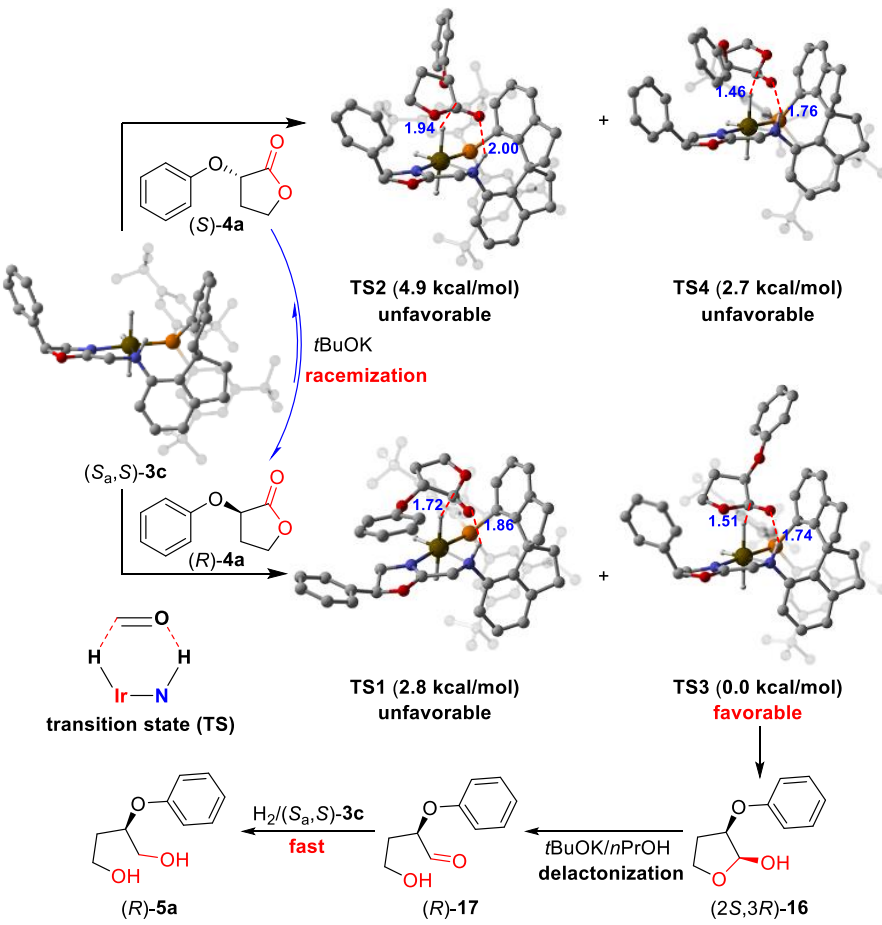

Figure S2 The transition state structures for the $\mathrm{H}^{-} / \mathrm{H}^{+}$transfer to the racemic $\alpha$-aryloxy lactones $4 \mathbf{a}$

For this multiple transition state reaction, the Boltzmann analysis of the four competing TS structures were summarized in Table S3. A calculation of the ee based on the Boltzmann distribution for each TS structure, indicated that TS3 is the most important single TS, leading to formation of $(2 S, 3 R)-\mathbf{1 6}$ as the 
major product with a calculated $97.9 \%$ ee, which are in agreement with the experimental results $(94.0 \%$ ee).

Table S4 DFT-calculated Boltzmann distribution of the TS structures for $(S a, S)$-3c catalyzed hydride/proton transfer to $\mathbf{4} \mathbf{a}^{a}$

\begin{tabular}{cccc}
\hline Transition States & Free energy in solution (a.u.) & relative G (kcal/mol) & Boltzmann weights \\
\hline TS1 & -3382.93488474 & 2.79862490 & 0.008691097 \\
TS2 & -3382.93073578 & 4.92896230 & 0.000238094 \\
TS3 & -3382.93780270 & 0.00000000 & 0.980625148 \\
TS4 & -3382.93216228 & 2.68972855 & 0.010445662 \\
\hline & & Total & 1.0000000 \\
\cline { 2 - 4 } & & ee $(\%)^{b}$ & 97.9 \\
\hline
\end{tabular}

${ }^{a}$ DFT-calculated transition states for the stereoselectivity-determining hydride/proton transfer to $\mathbf{4 a} .{ }^{b}$ Enantiomeric excess $(\%)$ of the $(2 S, 3 R)-\mathbf{1 6}=[\mathbf{T S 3}-\mathbf{T S 4}] /[\mathbf{T S 3}+\mathbf{T S 4}]$.

\section{Coordinates of All Stationary Points}

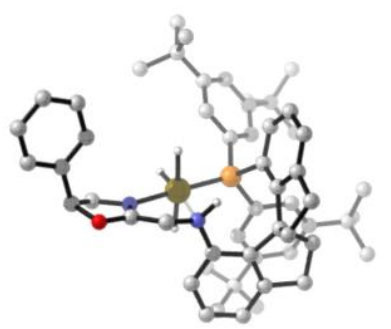

$\left(S_{a}, S\right)-3 c$

$\begin{array}{llll}\mathrm{C} & 0.84617 & -2.60066 & 2.28975 \\ \mathrm{C} & 2.39436 & -2.81346 & 2.29106 \\ \mathrm{C} & 2.6776 & -3.9747 & 1.31987 \\ \mathrm{C} & 1.44253 & -4.03457 & 0.45359 \\ \mathrm{C} & 0.44062 & -3.18797 & 0.94643 \\ \mathrm{C} & 1.21758 & -4.85134 & -0.65357 \\ \mathrm{C} & -0.03648 & -4.81635 & -1.27057 \\ \mathrm{C} & -1.0415 & -3.97362 & -0.79576 \\ \mathrm{C} & -0.8098 & -3.15411 & 0.31731 \\ \mathrm{C} & 0.50579 & -1.16821 & 2.75895 \\ \mathrm{C} & 0.2881 & -1.21408 & 4.15541 \\ \mathrm{C} & 0.32645 & -2.6265 & 4.68666 \\ \mathrm{C} & 0.19592 & -3.46571 & 3.41382 \\ \mathrm{C} & 0.50733 & 0.09034 & 2.10264 \\ \mathrm{C} & 0.33095 & 1.23957 & 2.89832 \\ \mathrm{C} & 0.13698 & 1.17474 & 4.27779 \\ \mathrm{C} & 0.09942 & -0.06307 & 4.91657 \\ \mathrm{~N} & -1.79998 & -2.23637 & 0.76866 \\ \mathrm{C} & -3.89458 & -2.01989 & -0.48244\end{array}$

$\begin{array}{cccc}\mathrm{H} & 0.00029 & 2.0977 & 4.84651 \\ \mathrm{H} & -0.0683 & -0.13609 & 5.99437 \\ \mathrm{H} & 2.89462 & 2.06502 & -0.23193 \\ \mathrm{H} & 1.18813 & 5.9995 & -0.36877 \\ \mathrm{H} & -1.32372 & 2.55613 & 0.2716 \\ \mathrm{H} & 3.15792 & 0.28733 & 1.39842 \\ \mathrm{H} & 5.44512 & -1.59551 & -1.71274 \\ \mathrm{H} & 1.17919 & -1.07649 & -2.13519 \\ \mathrm{H} & 4.77455 & 3.15297 & 0.36539 \\ \mathrm{H} & 5.67066 & 4.16983 & -0.78377 \\ \mathrm{H} & 4.55103 & 2.92676 & -1.38456 \\ \mathrm{H} & 4.88362 & 6.22097 & 0.35022 \\ \mathrm{H} & 3.91317 & 5.27524 & 1.5116 \\ \mathrm{H} & 3.15171 & 6.56792 & 0.55666 \\ \mathrm{H} & 2.80057 & 6.23611 & -1.98898 \\ \mathrm{H} & 4.53939 & 5.90007 & -2.16817 \\ \mathrm{H} & 3.33254 & 4.7248 & -2.7599 \\ \mathrm{H} & 5.15114 & 1.23659 & 1.92865 \\ \mathrm{H} & 6.48019 & 0.29412 & 2.63974\end{array}$




\begin{tabular}{|c|c|c|c|}
\hline & 0.46171 & 0.35497 & 0.25037 \\
\hline & 2.03534 & 2.72404 & -0.16318 \\
\hline & 2.20314 & 4.10349 & -0.31991 \\
\hline & 1.06384 & 4.92462 & -0.24424 \\
\hline & -0.21664 & 4.40398 & -0.02709 \\
\hline & -0.34666 & 3.01503 & 0.11842 \\
\hline & 3.22713 & -0.19596 & 0.4251 \\
\hline & 4.4576 & -0.64817 & -0.05366 \\
\hline & 4.48759 & -1.24697 & -1.32885 \\
\hline & 3.34139 & -1.40026 & -2.1147 \\
\hline & 2.11566 & -0.95704 & -1.5861 \\
\hline & 4.70178 & 3.68099 & -0.59751 \\
\hline & 3.58046 & 4.73248 & -0.58402 \\
\hline & 3.89747 & 5.75966 & 0.52264 \\
\hline & 3.56024 & 5.44066 & -1.95483 \\
\hline & 5.52957 & 0.21569 & 2.09002 \\
\hline & 5.75442 & -0.52257 & 0.7612 \\
\hline & 6.80496 & 0.25873 & -0.05496 \\
\hline & 6.29005 & -1.93572 & 1.0743 \\
\hline 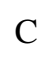 & 4.78555 & -2.42389 & -3.95195 \\
\hline & 3.37186 & -1.99904 & -3.52881 \\
\hline & 2.86081 & -0.9354 & -4.52447 \\
\hline & 2.451 & -3.23398 & -3.58322 \\
\hline r & -1.17637 & 6.76522 & -0.15057 \\
\hline$C$ & -1.48038 & 5.27333 & 0.04944 \\
\hline & -2.13325 & 5.08669 & 1.43548 \\
\hline 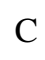 & -2.46877 & 4.82068 & -1.04657 \\
\hline 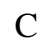 & -3.21441 & -2.61998 & 0.70802 \\
\hline 0 & -5.15595 & -2.36226 & -0.77167 \\
\hline & -5.64705 & -1.30751 & -1.68648 \\
\hline$\gamma$ & -4.32977 & -0.69118 & -2.21032 \\
\hline N & -3.36519 & -1.08987 & -1.18573 \\
\hline 1 & 2.77376 & -2.99306 & 3.30746 \\
\hline & 2.87296 & -1.90375 & 1.92061 \\
\hline $\mathrm{H}$ & 3.58904 & -3.80061 & 0.72625 \\
\hline & 2.81863 & -4.93348 & 1.84915 \\
\hline & 2.00329 & -5.51243 & -1.02565 \\
\hline & -0.23223 & -5.43902 & -2.14662 \\
\hline & -1.99358 & -3.91503 & -1.32398 \\
\hline $\mathrm{H}$ & -0.4657 & -2.825 & 5.42572 \\
\hline & 1.28841 & -2.81589 & 5.19522 \\
\hline & 0.66215 & -4.45968 & 3.47456 \\
\hline & -0.86957 & -3.62209 & 3.17726 \\
\hline & 0.32586 & 2.21757 & 2.42027 \\
\hline
\end{tabular}

\begin{tabular}{|c|c|c|c|}
\hline & 4.81384 & -0.31395 & 2.73692 \\
\hline & 7.04906 & -0.24859 & -0.99995 \\
\hline & 6.49124 & -2.50562 & 0.15472 \\
\hline & 7.22988 & -1.87634 & 1.64714 \\
\hline & 5.56148 & -2.50614 & 1.67107 \\
\hline & 5.48351 & -1.57227 & -3.96267 \\
\hline & 4.75975 & -2.84418 & -4.9693 \\
\hline & 5.19702 & -3.19667 & -3.28391 \\
\hline & 2.87115 & -1.335 & -5.5518 \\
\hline & 3.49685 & -0.03643 & -4.49882 \\
\hline & 1.83125 & -0.627 & -4.2 \\
\hline & 1.41992 & -2.98887 & -3.29202 \\
\hline & 2.43061 & -3.65158 & -4.60327 \\
\hline & 2.80821 & -4.0158 & -2.89694 \\
\hline & -0.49353 & 7.15096 & 0.62245 \\
\hline & -2.1092 & 7.34731 & -0.09042 \\
\hline & -0.72515 & 6.9609 & -1.13564 \\
\hline & -3.05044 & 5.69328 & 1.51454 \\
\hline & -1.44524 & 5.39943 & 2.23711 \\
\hline & -2.40489 & 4.03585 & 1.61441 \\
\hline & -2.74141 & 3.76095 & -0.93421 \\
\hline & -3.39383 & 5.41873 & -1.0005 \\
\hline & -2.02644 & 4.94755 & -2.04716 \\
\hline & -3.72631 & -2.21447 & 1.59665 \\
\hline & -6.22793 & -1.82231 & -2.46118 \\
\hline & -1.49296 & -0.33287 & -0.59879 \\
\hline & -2.30941 & 0.58136 & 0.60442 \\
\hline & -0.78462 & -1.2146 & -1.82913 \\
\hline & -3.35384 & -3.71379 & 0.71824 \\
\hline & -1.46279 & 0.89494 & -1.57646 \\
\hline & -1.56779 & -1.8513 & 1.68012 \\
\hline & -6.51749 & -0.3579 & -0.90203 \\
\hline & -7.91074 & -0.41854 & -1.02354 \\
\hline & -5.94337 & 0.57876 & -0.02384 \\
\hline & -8.72729 & 0.44071 & -0.28308 \\
\hline & -8.36129 & -1.14493 & -1.70556 \\
\hline & -6.7609 & 1.43041 & 0.71995 \\
\hline & -4.85663 & 0.65081 & 0.08582 \\
\hline & -8.1526 & 1.36559 & 0.59144 \\
\hline & -9.81327 & 0.38713 & -0.39031 \\
\hline & -6.30525 & 2.15393 & 1.39998 \\
\hline & -8.78835 & 2.03912 & 1.17122 \\
\hline & -4.01505 & -1.09935 & -3.18404 \\
\hline & -4.37207 & 0.40181 & -2.28991 \\
\hline
\end{tabular}




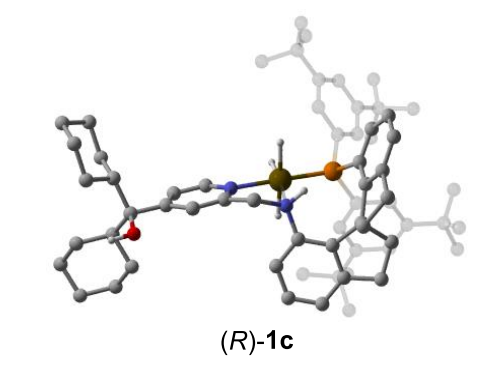
$\begin{array}{llll}\text { Ir } & -0.27721 & 0.36817 & 0.17400\end{array}$
$\begin{array}{llll}\text { P } & 1.97899 & 0.45408 & 0.37502\end{array}$
$\begin{array}{llll}\text { C } & 1.93640 & -2.29047 & 2.82402\end{array}$
$\begin{array}{llll}\text { C } & 1.47163 & -2.71291 & 4.25633\end{array}$
$\begin{array}{llll}\text { C } & 2.32055 & -1.88495 & 5.22024\end{array}$
$\begin{array}{llll}\text { C } & 2.59886 & -0.63394 & 4.42347\end{array}$
$\begin{array}{llll}\text { C } & 2.30646 & -0.79254 & 3.04586\end{array}$
$\begin{array}{llll}\text { C } & 2.53617 & 0.31220 & 2.17213\end{array}$
$\begin{array}{llll}\text { C } & 3.08489 & 1.48269 & 2.73548\end{array}$
$\begin{array}{llll}\text { C } & 3.38109 & 1.60697 & 4.09334\end{array}$
$\begin{array}{llll}\text { C } & 3.12202 & 0.54459 & 4.95318\end{array}$
$\begin{array}{llll}\text { C } & 3.21688 & -3.09249 & 2.42220\end{array}$
$\begin{array}{llll}\text { C } & 2.71893 & -4.43259 & 1.85458\end{array}$
$\begin{array}{llll}\text { C } & 1.32771 & -4.10676 & 1.36412\end{array}$
$\begin{array}{llll}\text { C } & 0.91014 & -2.85070 & 1.83219\end{array}$
$\begin{array}{llll}\text { C } & -0.38914 & -2.40430 & 1.53765\end{array}$
$\begin{array}{llll}\text { C } & -1.24936 & -3.23410 & 0.80217\end{array}$
$\begin{array}{llll}\text { C } & -0.81591 & -4.48011 & 0.34512\end{array}$
$\begin{array}{llll}\text { C } & 0.48151 & -4.92487 & 0.61228\end{array}$
$\begin{array}{llll}\mathrm{N} & -0.78240 & -1.08996 & 1.92224\end{array}$
$\begin{array}{llll}\text { C } & -2.18748 & -0.80672 & 2.25211\end{array}$
$\begin{array}{llll}\text { C } & -3.02828 & -0.39675 & 1.06293\end{array}$
C $3.36264 \quad-2.35985 \quad-2.32769$
$\begin{array}{llll}\text { C } & 4.70380 & -2.51402 & -1.92849\end{array}$
C $\quad 5.25891 \quad-1.81942 \quad-0.84433$
$\begin{array}{llll}\text { C } & 4.41828 & -0.93385 & -0.14332\end{array}$
C $\quad 2.82275 \quad-3.17095 \quad-3.52353$
C $1.35412-2.83065 \quad-3.83506$
$\begin{array}{llll}\text { C } & 2.91134 & -4.68032 & -3.19774\end{array}$
$\begin{array}{llll}\text { C } & 3.66712 & -2.86636 & -4.78239\end{array}$
$\begin{array}{llll}\text { C } & 6.72899 & -2.00351 & -0.40527\end{array}$

$\begin{array}{llll}\text { C } & -4.40632 & -0.61623 & 1.04014\end{array}$

$\begin{array}{llll}\text { C } & -5.18783 & -0.14379 & -0.02176\end{array}$

$\begin{array}{llll}\text { C } & -4.50397 & 0.53127 & -1.04567\end{array}$

$\begin{array}{llll}\text { C } & -3.12612 & 0.70082 & -0.97037\end{array}$

$\begin{array}{llll}\mathrm{N} & -2.39170 & 0.25224 & 0.06389\end{array}$

$\begin{array}{llll}\text { C } & 2.65964 & 2.10085 & -0.20127\end{array}$

$\begin{array}{llll}\text { C } & 3.80728 & 2.23485 & -0.99333\end{array}$

$\begin{array}{llll}\text { C } & 4.21648 & 3.48921 & -1.48260\end{array}$

C $3.45008 \quad 4.61238-1.13876$

$\begin{array}{llll}\text { C } & 2.29668 & 4.51796 & -0.34191\end{array}$

$\begin{array}{llll}\text { C } & 1.91167 & 3.24845 & 0.10663\end{array}$

$\begin{array}{llll}\text { C } & 5.48022 & 3.59284 & -2.36356\end{array}$

$\begin{array}{llll}\text { C } & 6.71813 & 3.21152 & -1.51983\end{array}$

$\begin{array}{llll}\text { C } & 5.36108 & 2.62900 & -3.56661\end{array}$

$\begin{array}{llll}\text { C } & 5.69634 & 5.01410 & -2.91564\end{array}$

C $\quad 1.43334 \quad 5.74219 \quad 0.02744$

$\begin{array}{llll}\text { C } & 1.35255 & 5.86778 & 1.56644\end{array}$

$\begin{array}{llll}\text { C } & 2.00717 & 7.05638 & -0.53216\end{array}$

$\begin{array}{llll}\text { C } & 0.00943 & 5.54997 & -0.54494\end{array}$

$\begin{array}{llll}\text { C } & 3.07708 & -0.75804 & -0.51085\end{array}$

$\begin{array}{llll}\text { C } & 2.55900 & -1.47321 & -1.59971\end{array}$

$\begin{array}{llll}\mathrm{H} & 6.63850 & 2.18908 & -1.12331\end{array}$

H $\quad 4.48364 \quad 2.87712 \quad-4.18489$

$\begin{array}{llll}\mathrm{H} & 6.25809 & 2.69823 & -4.20430\end{array}$

H $\quad \begin{array}{llll}5.25942 & 1.58043 & -3.24977\end{array}$

H $\quad 4.84612 \quad 5.34958 \quad-3.53012$

H $\quad 5.85022 \quad 5.75171 \quad-2.11269$

$\begin{array}{llll}\mathrm{H} & 6.59468 & 5.03229 & -3.55303\end{array}$

H $\quad 2.35416 \quad 6.00965 \quad 2.00410$

$\begin{array}{lllll}\mathrm{H} & 0.73222 & 6.73436 & 1.85048\end{array}$

$\begin{array}{llll}\mathrm{H} & 0.90719 & 4.97283 & 2.02594\end{array}$ 


\begin{tabular}{|c|c|c|c|c|c|c|c|}
\hline $\mathrm{C}$ & 6.77550 & -2.55135 & 1.03972 & $\mathrm{H}$ & 3.01777 & 7.26313 & -0.14541 \\
\hline $\mathrm{C}$ & 7.49184 & -2.98643 & -1.31243 & $\mathrm{H}$ & 2.05710 & 7.04931 & -1.63238 \\
\hline $\mathrm{C}$ & 7.46123 & -0.64374 & -0.45353 & $\mathrm{H}$ & 1.36218 & 7.89964 & -0.23785 \\
\hline $\mathrm{H}$ & -0.23498 & -0.83769 & -0.98517 & $\mathrm{H}$ & -0.63183 & 6.40690 & -0.27774 \\
\hline $\mathrm{H}$ & -0.13727 & 1.39785 & -1.00868 & $\mathrm{H}$ & 0.03556 & 5.47342 & -1.64384 \\
\hline $\mathrm{H}$ & 1.55289 & -3.79985 & 4.40469 & $\mathrm{H}$ & -0.46602 & 4.63621 & -0.15826 \\
\hline $\mathrm{H}$ & 0.40710 & -2.45191 & 4.37943 & $\mathrm{H}$ & 1.50524 & -1.32647 & -1.83904 \\
\hline $\mathrm{H}$ & 3.26637 & -2.39883 & 5.47020 & $\mathrm{H}$ & 5.33137 & -3.20772 & -2.48671 \\
\hline $\mathrm{H}$ & 1.81660 & -1.67475 & 6.17761 & $\mathrm{H}$ & 4.81314 & -0.37389 & 0.70767 \\
\hline $\mathrm{H}$ & 3.27416 & 2.34000 & 2.09163 & $\mathrm{H}$ & 1.00984 & -3.42632 & -4.69585 \\
\hline $\mathrm{H}$ & 3.80121 & 2.54260 & 4.47145 & $\mathrm{H}$ & 0.69015 & -3.05190 & -2.98618 \\
\hline 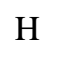 & 3.32974 & 0.62159 & 6.02420 & $\mathrm{H}$ & 1.22582 & -1.76779 & -4.09117 \\
\hline $\mathrm{H}$ & 3.91359 & -3.20456 & 3.26671 & $\mathrm{H}$ & 2.53040 & -5.28000 & -4.04132 \\
\hline $\mathrm{H}$ & 3.74145 & -2.53763 & 1.63587 & $\mathrm{H}$ & 3.94728 & -4.99964 & -3.00363 \\
\hline $\mathrm{H}$ & 2.67484 & -5.21837 & 2.63077 & $\mathrm{H}$ & 3.62099 & -1.79572 & -5.03805 \\
\hline $\mathrm{H}$ & 3.37047 & -4.81933 & 1.05444 & $\mathrm{H}$ & 4.72651 & -3.13372 & -4.64689 \\
\hline $\mathrm{H}$ & -2.25242 & -2.88987 & 0.54848 & $\mathrm{H}$ & 3.28907 & -3.43838 & -5.64597 \\
\hline $\mathrm{H}$ & -1.50114 & -5.10270 & -0.23600 & $\mathrm{H}$ & 6.27343 & -3.52936 & 1.10893 \\
\hline $\mathrm{H}$ & 0.82198 & -5.90083 & 0.25659 & $\mathrm{H}$ & 7.82016 & -2.68216 & 1.36778 \\
\hline $\mathrm{H}$ & -0.17650 & -0.72490 & 2.65266 & $\mathrm{H}$ & 6.28574 & -1.87137 & 1.75285 \\
\hline 4 & -2.66428 & -1.65199 & 2.77778 & $\mathrm{H}$ & 7.52019 & -2.64383 & -2.35862 \\
\hline $\mathrm{H}$ & -2.16849 & 0.05464 & 2.94126 & $\mathrm{H}$ & 8.53367 & -3.07911 & -0.96693 \\
\hline $\mathrm{H}$ & -4.88074 & -1.16365 & 1.85375 & $\mathrm{H}$ & 7.04850 & -3.99427 & -1.29437 \\
\hline 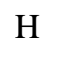 & 4.38437 & 1.34820 & -1.25245 & $\mathrm{H}$ & 6.99762 & 0.09665 & 0.21544 \\
\hline 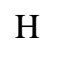 & 3.75337 & 5.58827 & -1.50900 & $\mathrm{H}$ & 8.51205 & -0.76048 & -0.14047 \\
\hline $\mathrm{H}$ & 0.99832 & 3.11875 & 0.68885 & $\mathrm{H}$ & 7.45577 & -0.22761 & -1.47322 \\
\hline 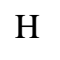 & 7.63640 & 3.26595 & -2.12864 & $\mathrm{H}$ & 2.30999 & -4.92359 & -2.30743 \\
\hline 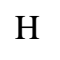 & 6.83649 & 3.89495 & -0.66368 & $\mathrm{H}$ & -8.78061 & -3.59204 & -2.64796 \\
\hline $\mathrm{H}$ & -0.39444 & 1.62464 & 1.32931 & $\mathrm{O}$ & -6.94564 & -1.36764 & 0.95139 \\
\hline $\mathrm{H}$ & -5.01923 & 0.91180 & -1.92743 & $\mathrm{H}$ & -7.89521 & -1.53160 & 0.99394 \\
\hline U & -2.56916 & 1.20195 & -1.76251 & $\mathrm{H}$ & -10.01611 & 3.10803 & 0.27630 \\
\hline & -6.71237 & -0.33363 & -0.00468 & $\mathrm{C}$ & -7.22247 & -0.80311 & -1.40994 \\
\hline $\mathrm{C}$ & -7.33055 & 1.02373 & 0.54586 & $\mathrm{C}$ & -6.51320 & -2.10156 & -1.85734 \\
\hline$r$ & -7.46015 & 2.15620 & -0.49060 & $\mathrm{C}$ & -8.75127 & -0.99979 & -1.49841 \\
\hline & -8.64863 & 0.87217 & 1.34002 & $\mathrm{H}$ & -6.96491 & -0.01070 & -2.13359 \\
\hline & -6.59262 & 1.36629 & 1.29351 & $\mathrm{C}$ & -6.96335 & -2.57772 & -3.24385 \\
\hline C & -7.88542 & 3.48051 & 0.16229 & $\mathrm{H}$ & -6.72273 & -2.88479 & -1.10906 \\
\hline & -8.20685 & 1.88175 & -1.25621 & $\mathrm{H}$ & -5.42206 & -1.96107 & -1.85302 \\
\hline
\end{tabular}




$\begin{array}{lllllllll}\mathrm{H} & -6.50789 & 2.30666 & -1.02094 & \mathrm{C} & -9.20242 & -1.48242 & -2.88561 \\ \mathrm{C} & -9.06092 & 2.19973 & 1.99433 & \mathrm{H} & -9.07326 & -1.74543 & -0.74608 \\ \mathrm{H} & -9.46845 & 0.53368 & 0.68469 & \mathrm{H} & -9.27980 & -0.06656 & -1.25917 \\ \mathrm{H} & -8.52470 & 0.10977 & 2.12475 & \mathrm{C} & -8.48167 & -2.76262 & -3.31561 \\ \mathrm{C} & -9.18076 & 3.32985 & 0.96659 & \mathrm{H} & -6.44710 & -3.51816 & -3.49959 \\ \mathrm{H} & -7.99972 & 4.25818 & -0.61135 & \mathrm{H} & -6.65109 & -1.83855 & -4.00501 \\ \mathrm{H} & -7.07848 & 3.82759 & 0.83327 & \mathrm{H} & -10.29535 & -1.63174 & -2.88789 \\ \mathrm{H} & -10.01232 & 2.06756 & 2.53658 & \mathrm{H} & -8.99865 & -0.68563 & -3.62480 \\ \mathrm{H} & -8.30713 & 2.47761 & 2.75373 & \mathrm{H} & -8.79126 & -3.05518 & -4.33304 \\ \mathrm{H} & -9.43615 & 4.27915 & 1.46667 & & & & \end{array}$

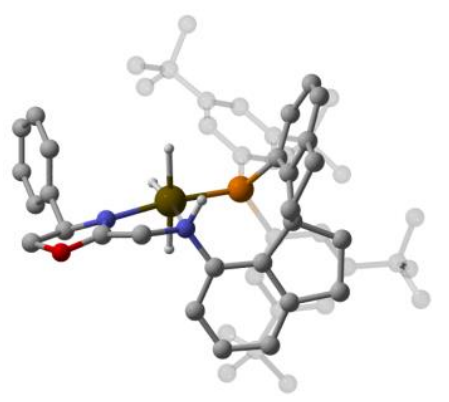

$\left(S_{a}, R\right)-\mathbf{2 b}$

\begin{tabular}{|c|c|c|c|}
\hline & -0.85423 & -3.11844 & \\
\hline & -2.34072 & -3.59009 & -1.93073 \\
\hline & -2.65468 & -4.55738 & -0.7841 \\
\hline & -1.64523 & -4.19514 & 0.272675 \\
\hline & -0.63575 & & \\
\hline & -1.61385 & -4.66 & 1.583956 \\
\hline & -0.53164 & -4.31661 & 2.393768 \\
\hline & 0.497579 & -3.525 & 1.889282 \\
\hline & 0.468623 & -3.0 & \\
\hline & -0.73965 & -1.76128 & -2.47112 \\
\hline & -0.44587 & -2.03913 & -3.81995 \\
\hline & -0.02098 & -3.47393 & -4.0203 \\
\hline & 0.099301 & -4.01092 & -2.5858 \\
\hline & -1.06203 & -0.4356 & -2.09966 \\
\hline & -1.21237 & 0.521189 & -3.12107 \\
\hline & -1.00784 & 0.204263 & -4.46382 \\
\hline & -0.58176 & -1.0746 & -4.81553 \\
\hline & 1.528096 & -2.25469 & 0.074486 \\
\hline & 3.360671 & -1.54521 & 1.533887 \\
\hline & -1.01927 & 0.166808 & -0.35223 \\
\hline & -1.59718 & 1.926616 & -0.47805 \\
\hline & -2.48924 & -0.63713 & 0.41693 \\
\hline & -2.95311 & 2.272416 & -0.4800 \\
\hline
\end{tabular}

$\begin{array}{rrrr}\mathrm{H} & -1.1407 & 0.974411 & -5.22772 \\ \mathrm{H} & -0.35781 & -1.32368 & -5.85622 \\ \mathrm{H} & -3.69907 & 1.487651 & -0.40593 \\ \mathrm{H} & -2.66591 & 5.644832 & -0.66494 \\ \mathrm{H} & 0.414805 & 2.644942 & -0.50935 \\ \mathrm{H} & -3.58281 & -0.69201 & -1.43214 \\ \mathrm{H} & -5.72771 & -1.95493 & 2.067902 \\ \mathrm{H} & -1.62995 & -0.64746 & 2.369346 \\ \mathrm{H} & -5.63875 & 2.12838 & -1.32609 \\ \mathrm{H} & -6.8213 & 3.128753 & -0.45619 \\ \mathrm{H} & -5.59645 & 2.212539 & 0.450301 \\ \mathrm{H} & -6.22537 & 5.096584 & -1.85253 \\ \mathrm{H} & -4.97643 & 4.168325 & -2.7262 \\ \mathrm{H} & -4.5553 & 5.703992 & -1.93457 \\ \mathrm{H} & -4.50345 & 5.834642 & 0.655293 \\ \mathrm{H} & -6.17284 & 5.2217 & 0.706561 \\ \mathrm{H} & -4.88272 & 4.385474 & 1.613508 \\ \mathrm{H} & -5.66777 & -0.21644 & -2.21458 \\ \mathrm{H} & -6.8267 & -1.49526 & -2.63859 \\ \mathrm{H} & -5.09132 & -1.8648 & -2.56119 \\ \mathrm{H} & -8.16198 & -1.00387 & -0.62529 \\ \mathrm{H} & -7.01811 & 0.249054 & -0.07566 \\ \mathrm{H} & -7.46468 & -1.08803 & 1.007454\end{array}$




\begin{tabular}{|c|c|c|c|c|c|c|c|}
\hline $\mathrm{C}$ & -3.35863 & 3.61169 & -0.55504 & $\mathrm{H}$ & -6.56457 & -3.48086 & 0.523071 \\
\hline & -2.36413 & 4.596884 & -0.62261 & $\mathrm{H}$ & -7.36053 & -3.36591 & -1.06528 \\
\hline & -0.99593 & 4.288175 & -0.61604 & $\mathrm{H}$ & -5.62909 & -3.77119 & -0.96176 \\
\hline & -0.63046 & 2.938667 & -0.54816 & $\mathrm{H}$ & -5.91818 & -1.33156 & 4.192189 \\
\hline & -3.62908 & -0.89501 & -0.3641 & $\mathrm{H}$ & -5.0564 & -2.08534 & 5.549547 \\
\hline & -4.80459 & -1.3777 & 0.21427 & $\mathrm{H}$ & -5.29743 & -2.99703 & 4.04317 \\
\hline & -4.81277 & -1.58696 & 1.606658 & $\mathrm{H}$ & -3.53926 & -0.11055 & 5.659813 \\
\hline & -3.70219 & -1.32128 & 2.413686 & $\mathrm{H}$ & -4.28948 & 0.647596 & 4.227976 \\
\hline & -2.53043 & -0.85709 & 1.790912 & $\mathrm{H}$ & -2.53522 & 0.358962 & 4.264937 \\
\hline & -5.77076 & 2.799402 & -0.4639 & $\mathrm{H}$ & -1.62932 & -2.07206 & 4.077691 \\
\hline & -4.84038 & 4.019432 & -0.54526 & $\mathrm{H}$ & -2.60782 & -2.46639 & 5.51477 \\
\hline & -5.1657 & 4.793444 & -1.83946 & $\mathrm{H}$ & -2.77878 & -3.4184 & 4.013378 \\
\hline & -5.11329 & 4.919084 & 0.678301 & $\mathrm{H}$ & -1.11167 & 6.687218 & 0.749865 \\
\hline & -5.89739 & -1.28524 & -2.08702 & $\mathrm{H}$ & 0.624811 & 7.075502 & 0.676864 \\
\hline & -6.07537 & -1.65631 & -0.60627 & $\mathrm{H}$ & 0.068876 & 5.620713 & 1.548132 \\
\hline & -7.24623 & -0.8263 & -0.03842 & $\mathrm{H}$ & 0.555911 & 7.137632 & -1.88245 \\
\hline & -6.42658 & -3.1558 & -0.51912 & $\mathrm{H}$ & -1.18563 & 6.784495 & -1.84898 \\
\hline & -5.08117 & -1.99782 & 4.452255 & $\mathrm{H}$ & -0.09227 & 5.746471 & -2.79224 \\
\hline & -3.73706 & -1.45514 & 3.944211 & $\mathrm{H}$ & 1.652999 & 4.294117 & -1.60477 \\
\hline & -3.51017 & -0.05681 & 4.559056 & $\mathrm{H}$ & 2.194858 & 5.718008 & -0.69182 \\
\hline & -2.62063 & -2.40694 & 4.414077 & $\mathrm{H}$ & 1.721127 & 4.242812 & 0.173145 \\
\hline & -0.1056 & 6.249819 & 0.661635 & $\mathrm{H}$ & 3.559488 & -2.48682 & -0.34388 \\
\hline & 0.044454 & 5.419527 & -0.6309 & $\mathrm{H}$ & 4.83783 & -0.84488 & 4.130266 \\
\hline & -0.18665 & 6.323505 & -1.85872 & $\mathrm{Ir}$ & 1.003834 & -0.05648 & 0.634621 \\
\hline & 1.481017 & 4.879462 & -0.68999 & $\mathrm{H}$ & 1.896006 & 0.23417 & -0.88511 \\
\hline & 2.896482 & -2.54954 & 0.530973 & $\mathrm{H}$ & 0.274203 & -0.35837 & 2.057911 \\
\hline & 4.388732 & -1.81272 & 2.34692 & $\mathrm{H}$ & 2.992192 & -3.56257 & 0.951269 \\
\hline & 4.639966 & -0.58548 & 3.083337 & $\mathrm{H}$ & 0.888477 & 1.441567 & 1.085344 \\
\hline & 3.355483 & 0.253391 & 2.867253 & $\mathrm{H}$ & 1.56267 & -2.17806 & -0.95247 \\
\hline & 2.783425 & -0.41134 & 1.68516 & $\mathrm{H}$ & 2.641333 & 0.093857 & 3.693038 \\
\hline & -2.4958 & -4.02416 & -2.9284 & $\mathrm{H}$ & 5.524149 & -0.10333 & 2.641919 \\
\hline & -2.99315 & -2.71495 & -1.8538 & $\mathrm{C}$ & 3.565222 & 1.737541 & 2.702734 \\
\hline & -3.68943 & -4.46226 & -0.4218 & $\mathrm{C}$ & 3.218193 & 2.614556 & 3.73588 \\
\hline & -2.51659 & -5.61148 & -1.08386 & $\mathrm{C}$ & 4.102361 & 2.254289 & 1.515957 \\
\hline & -2.41626 & -5.30293 & 1.962795 & $\mathrm{C}$ & 3.410624 & 3.992064 & 3.592949 \\
\hline & -0.48545 & -4.65814 & 3.430187 & $\mathrm{H}$ & 2.776449 & 2.219236 & 4.654595 \\
\hline & 1.323592 & -3.23876 & 2.541082 & $\mathrm{C}$ & 4.292043 & 3.629126 & 1.372231 \\
\hline & 0.929272 & -3.53703 & -4.57299 & $\mathrm{H}$ & 4.335608 & 1.580142 & 0.692473 \\
\hline & -0.77323 & -4.02999 & -4.60679 & $\mathrm{C}$ & 3.948073 & 4.502665 & 2.409306 \\
\hline & -0.15662 & -5.07601 & -2.48483 & $\mathrm{H}$ & 3.129218 & 4.667311 & 4.404519 \\
\hline & 1.131139 & -3.87773 & -2.23112 & $\mathrm{H}$ & 4.696606 & 4.022471 & 0.436461 \\
\hline & -1.47493 & 1.544891 & -2.85531 & $\mathrm{H}$ & 4.08909 & 5.579656 & 2.290531 \\
\hline
\end{tabular}




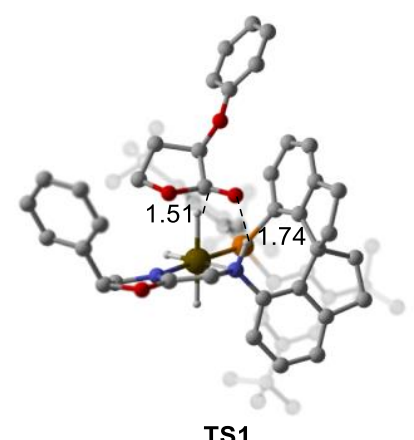

$\begin{array}{llll}\mathrm{C} & 1.06097 & -1.4119 & 3.04958 \\ \mathrm{C} & 2.52608 & -1.23007 & 3.58188 \\ \mathrm{C} & 3.24958 & -2.56527 & 3.35614 \\ \mathrm{C} & 2.44385 & -3.22984 & 2.27017 \\ \mathrm{C} & 1.22201 & -2.56764 & 2.06955 \\ \mathrm{C} & 2.76665 & -4.39768 & 1.58242 \\ \mathrm{C} & 1.83652 & -4.92296 & 0.68189 \\ \mathrm{C} & 0.60957 & -4.29239 & 0.48546 \\ \mathrm{C} & 0.28172 & -3.12421 & 1.19183 \\ \mathrm{C} & 0.52379 & -0.01029 & 2.69503 \\ \mathrm{C} & -0.06835 & 0.52318 & 3.85685 \\ \mathrm{C} & -0.22854 & -0.51776 & 4.93675 \\ \mathrm{C} & 0.11319 & -1.82748 & 4.21311 \\ \mathrm{C} & 0.66145 & 0.81267 & 1.55352 \\ \mathrm{C} & 0.3104 & 2.17314 & 1.67703 \\ \mathrm{C} & -0.20791 & 2.70029 & 2.86004 \\ \mathrm{C} & -0.43799 & 1.86143 & 3.94827 \\ \mathrm{~N} & -0.9706 & -2.48787 & 0.97825 \\ \mathrm{C} & -2.73842 & -3.23709 & -0.53863 \\ \mathrm{P} & 0.97042 & 0.16561 & -0.1568 \\ \mathrm{C} & 1.21019 & 1.69738 & -1.16915 \\ \mathrm{C} & 2.67897 & -0.50706 & -0.10237 \\ \mathrm{C} & 2.47457 & 2.23071 & -1.45462 \\ \mathrm{C} & 2.6113 & 3.37861 & -2.24201 \\ \mathrm{C} & 1.445 & 3.98623 & -2.73838 \\ \mathrm{C} & 0.16828 & 3.48031 & -2.47042 \\ \mathrm{C} & 0.07205 & 2.32484 & -1.68126 \\ \mathrm{C} & 3.64554 & 0.12027 & 0.70173 \\ \mathrm{C} & 4.98504 & -0.2692 & 0.64914 \\ \mathrm{C} & 5.33358 & -1.29877 & -0.24633\end{array}$

\begin{tabular}{llll}
$\mathrm{H}$ & 5.02689 & 5.88198 & -2.3647 \\
$\mathrm{H}$ & 3.88926 & 5.51783 & -1.0391 \\
$\mathrm{H}$ & 3.27635 & 6.05742 & -2.61899 \\
$\mathrm{H}$ & 3.42527 & 4.46347 & -4.65846 \\
$\mathrm{H}$ & 5.17904 & 4.32006 & -4.38973 \\
$\mathrm{H}$ & 4.16059 & 2.85586 & -4.46429 \\
$\mathrm{H}$ & 5.10069 & 2.36268 & 1.66272 \\
$\mathrm{H}$ & 6.32995 & 2.0401 & 2.90592 \\
$\mathrm{H}$ & 4.73576 & 1.26667 & 3.01772 \\
$\mathrm{H}$ & 7.9747 & 1.43187 & 1.18588 \\
$\mathrm{H}$ & 6.80308 & 1.65256 & -0.14055 \\
$\mathrm{H}$ & 7.68548 & 0.11839 & 0.02519 \\
$\mathrm{H}$ & 7.06912 & -1.50937 & 1.95652 \\
$\mathrm{H}$ & 7.47912 & -0.18576 & 3.07509 \\
$\mathrm{H}$ & 5.89374 & -0.99017 & 3.18685 \\
$\mathrm{H}$ & 6.92711 & -2.42632 & -2.27833 \\
$\mathrm{H}$ & 6.53119 & -4.05909 & -2.85202 \\
$\mathrm{H}$ & 6.58002 & -3.72594 & -1.10692 \\
$\mathrm{H}$ & 4.7683 & -3.08375 & -4.32437 \\
$\mathrm{H}$ & 5.03778 & -1.435 & -3.69474 \\
$\mathrm{H}$ & 3.41127 & -2.15248 & -3.64233 \\
$\mathrm{H}$ & 2.91109 & -4.07883 & -1.96695 \\
$\mathrm{H}$ & 4.24009 & -4.98506 & -2.73359 \\
$\mathrm{H}$ & 4.22861 & -4.71689 & -0.96835 \\
$\mathrm{H}$ & -0.30406 & 6.13971 & -3.34219 \\
$\mathrm{H}$ & -1.78644 & 5.77536 & -4.25022 \\
$\mathrm{H}$ & -0.23741 & 5.07847 & -4.77373 \\
$\mathrm{H}$ & -2.92127 & 5.03071 & -2.13787 \\
\hline & -2.21363 & 3.72964 & -1.15064
\end{tabular}




$$
\begin{array}{llll}
\mathrm{C} & 4.39936 & -1.92367 & -1.07845 \\
\mathrm{C} & 3.05531 & -1.52289 & -0.97705 \\
\mathrm{C} & 5.12964 & 3.20782 & -1.9016 \\
\mathrm{C} & 3.98629 & 3.97147 & -2.58791 \\
\mathrm{C} & 4.04453 & 5.44261 & -2.12679 \\
\mathrm{C} & 4.19797 & 3.89934 & -4.11467 \\
\mathrm{C} & 5.52156 & 1.58239 & 2.31528 \\
\mathrm{C} & 6.07438 & 0.40021 & 1.50363 \\
\mathrm{C} & 7.19831 & 0.92868 & 0.58729 \\
\mathrm{C} & 6.66297 & -0.63389 & 2.48541 \\
\mathrm{C} & 6.29468 & -3.30629 & -2.08421 \\
\mathrm{C} & 4.79882 & -2.96162 & -2.13916 \\
\mathrm{C} & 4.4833 & -2.37264 & -3.53169 \\
\mathrm{C} & 3.99468 & -4.26001 & -1.94049 \\
\mathrm{C} & -0.83733 & 5.34821 & -3.89093 \\
\mathrm{C} & -1.11899 & 4.12858 & -3.00177 \\
\mathrm{C} & -1.97292 & 4.58161 & -1.80063 \\
\mathrm{H} & -0.47633 & 3.75661 & 2.91069 \\
\mathrm{C} & -1.90867 & 3.09027 & -3.82673 \\
\mathrm{C} & -2.16603 & -3.33415 & 0.83379 \\
\mathrm{O} & -3.75882 & -4.02882 & -0.89106 \\
\mathrm{C} & -4.25725 & -3.52064 & -2.16104 \\
\mathrm{C} & -3.10317 & -2.59448 & -2.63891 \\
\mathrm{~N} & -2.32587 & -2.40147 & -1.41744 \\
\mathrm{H} & 2.5314 & -0.89928 & 4.63011 \\
\mathrm{H} & 3.01518 & -0.44754 & 2.99304 \\
\mathrm{H} & 4.30324 & -2.43076 & 3.06858 \\
\mathrm{H} & 3.24386 & -3.19522 & 4.26328 \\
\mathrm{H} & 3.72454 & -4.89466 & 1.75215 \\
\mathrm{H} & 2.06854 & -5.8287 & 0.11747 \\
\mathrm{H} & -0.09261 & -4.69133 & -0.2474 \\
\mathrm{H} & -1.24962 & -0.51573 & 5.34788 \\
\mathrm{H} & 0.46053 & -0.32044 & 5.77677 \\
\mathrm{H} & -2.58826 & 4.85652 \\
\mathrm{H} & -2.26244 & 3.79336 \\
\mathrm{H} & -1.06776
\end{array}
$$

$$
\begin{aligned}
& \begin{array}{llll}
\mathrm{H} & -2.16939 & 2.20555 & -3.22695
\end{array} \\
& \begin{array}{llll}
\mathrm{H} & -2.84447 & 3.5322 & -4.20626
\end{array} \\
& \begin{array}{llll}
\mathrm{H} & -1.31682 & 2.74389 & -4.68821
\end{array} \\
& \begin{array}{llll}
\mathrm{H} & -2.9304 & -2.94858 & 1.52778
\end{array} \\
& \mathrm{H} \quad-4.38744 \quad-4.39285 \quad-2.81793 \\
& \text { Ir } \quad-0.74268 \quad-1.12255 \quad-0.87829 \\
& \begin{array}{llll}
\mathrm{H} & -1.89862 & -0.11422 & 0.03728
\end{array} \\
& \text { H } \quad 0.26664 \quad-2.08264 \quad-1.71203 \\
& \begin{array}{llll}
\mathrm{H} & -1.96709 & -4.38694 & 1.08619
\end{array} \\
& \begin{array}{llll}
\mathrm{H} & -0.7775 & -0.26706 & -2.20104
\end{array} \\
& \begin{array}{llll}
\mathrm{H} & -1.21217 & -1.7761 & 1.68724
\end{array} \\
& \begin{array}{llll}
\text { C } & -5.57914 & -2.80849 & -1.9621
\end{array} \\
& \begin{array}{lllll}
\text { C } & -6.19959 & -2.74202 & -0.71083
\end{array} \\
& \begin{array}{llll}
\text { C } & -6.18473 & -2.1834 & -3.06197
\end{array} \\
& \begin{array}{llll}
\text { C } & -7.40143 & -2.0452 & -0.55843
\end{array} \\
& \begin{array}{llll}
\mathrm{H} & -5.72816 & -3.21572 & 0.14859
\end{array} \\
& \begin{array}{llll}
\text { C } & -7.38536 & -1.4889 & -2.90963
\end{array}
\end{aligned}
$$

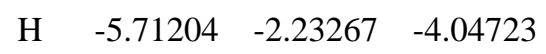

$$
\begin{aligned}
& \begin{array}{llll}
\text { C } & -7.99624 & -1.41497 & -1.65331
\end{array} \\
& \mathrm{H} \quad-7.86781 \quad-1.98738 \quad 0.42762 \\
& \begin{array}{llll}
\mathrm{H} & -7.8447 & -1.00222 & -3.77321
\end{array} \\
& \begin{array}{llll}
\mathrm{H} & -8.93322 & -0.86687 & -1.53045
\end{array}
\end{aligned}
$$

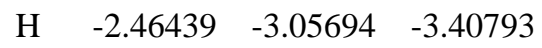

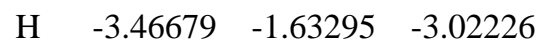

$$
\begin{aligned}
& \begin{array}{llll}
\text { C } & -3.12951 & 1.43904 & 1.11441
\end{array} \\
& \begin{array}{llll}
\text { C } & -4.18398 & 1.62556 & 0.00958
\end{array} \\
& \begin{array}{llll}
\text { C } & -4.37595 & 0.2109 & -0.55948
\end{array} \\
& \begin{array}{llll}
\text { O } & -4.01739 & -0.67387 & 0.49162
\end{array} \\
& \begin{array}{llll}
\text { C } & -2.96069 & -0.10156 & 1.2095
\end{array} \\
& \begin{array}{llll}
\mathrm{O} & -3.5719 & 1.87587 & 2.39156
\end{array} \\
& \begin{array}{llll}
\text { O } & -2.52649 & -0.68925 & 2.20435
\end{array} \\
& \begin{array}{llll}
\mathrm{H} & -2.16009 & 1.89952 & 0.88879
\end{array} \\
& \begin{array}{llll}
\mathrm{H} & -3.88726 & 2.34412 & -0.76605
\end{array} \\
& \mathrm{H} \quad-5.11185 \quad 1.98516 \quad 0.47721 \\
& \begin{array}{llll}
\mathrm{H} & -5.4069 & -0.01154 & -0.85914
\end{array} \\
& \begin{array}{llll}
\mathrm{H} & -3.69871 & 0.0435 & -1.41564
\end{array} \\
& \begin{array}{llll}
\text { C } & -3.45597 & 3.18003 & 2.72897
\end{array} \\
& \begin{array}{llll}
\text { C } & -3.65446 & 3.48895 & 4.08574
\end{array}
\end{aligned}
$$




$\begin{array}{llcllllll}\mathrm{H} & 1.5452 & 4.87609 & -3.35784 & \mathrm{C} & -3.14805 & 4.20818 & 1.82619 \\ \mathrm{H} & -0.89995 & 1.88095 & -1.46735 & \mathrm{C} & -3.52493 & 4.80129 & 4.53132 \\ \mathrm{H} & 3.33082 & 0.93569 & 1.35028 & \mathrm{H} & -3.89207 & 2.67088 & 4.76801 \\ \mathrm{H} & 6.37776 & -1.60127 & -0.30214 & \mathrm{C} & -3.01754 & 5.52217 & 2.28823 \\ \mathrm{H} & 2.2807 & -1.98394 & -1.59121 & \mathrm{H} & -2.99841 & 3.99469 & 0.77095 \\ \mathrm{H} & 5.03281 & 3.23478 & -0.80574 & \mathrm{C} & -3.19927 & 5.83014 & 3.63693 \\ \mathrm{H} & 6.09606 & 3.66576 & -2.16327 & \mathrm{H} & -3.67381 & 5.02532 & 5.59081 \\ \mathrm{H} & 5.16535 & 2.15256 & -2.21124 & \mathrm{H} & -2.77043 & 6.31179 & 1.57375 \\ & & & & \mathrm{H} & -3.09407 & 6.85805 & 3.99013\end{array}$

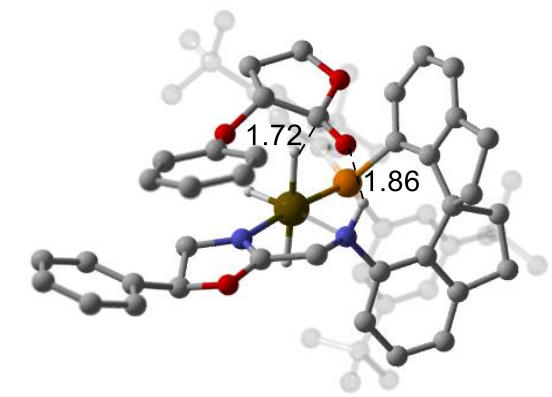

TS2

$\begin{array}{llllllll}\mathrm{C} & 1.02919 & -2.56139 & 2.32557 & \mathrm{H} & 7.15569 & 4.73543 & 0.08213 \\ \mathrm{C} & 2.48017 & -3.12398 & 2.5471 & \mathrm{H} & 5.95879 & 4.14367 & 1.26606 \\ \mathrm{C} & 2.62455 & -4.34595 & 1.63654 & \mathrm{H} & 5.58553 & 5.55353 & 0.24837 \\ \mathrm{C} & 1.60342 & -4.10203 & 0.55789 & \mathrm{H} & 5.16509 & 5.22148 & -2.28942 \\ \mathrm{C} & 0.69315 & -3.09793 & 0.93661 & \mathrm{H} & 6.74439 & 4.41267 & -2.42703 \\ \mathrm{C} & 1.47569 & -4.81147 & -0.63318 & \mathrm{H} & 5.26338 & 3.58925 & -2.98816 \\ \mathrm{C} & 0.39459 & -4.52088 & -1.46724 & \mathrm{H} & 6.09131 & -0.23399 & 1.71336 \\ \mathrm{C} & -0.53744 & -3.55615 & -1.0968 & \mathrm{H} & 7.1791 & -1.52221 & 2.27573 \\ \mathrm{C} & -0.42238 & -2.85777 & 0.11895 & \mathrm{H} & 5.4209 & -1.72061 & 2.42702 \\ \mathrm{C} & 1.09223 & -1.07306 & 2.72562 & \mathrm{H} & 8.32751 & -1.55637 & 0.0977 \\ \mathrm{C} & 0.89261 & -1.01012 & 4.11785 & \mathrm{H} & 7.24221 & -0.31956 & -0.58905 \\ \mathrm{C} & 0.34954 & -2.3083 & 4.66633 & \mathrm{H} & 7.45877 & -1.8761 & -1.41914 \\ \mathrm{C} & 0.06944 & -3.13945 & 3.40319 & \mathrm{H} & 6.41549 & -4.02549 & -0.40185 \\ \mathrm{C} & 1.4944 & 0.09167 & 2.03335 & \mathrm{H} & 7.38771 & -3.69823 & 1.05353 \\ \mathrm{C} & 1.823 & 1.22984 & 2.79514 & \mathrm{H} & 5.62861 & -3.93931 & 1.19064 \\ \mathrm{C} & 1.71234 & 1.24429 & 4.1856 & \mathrm{H} & 5.59722 & -2.46989 & -4.34646 \\ \mathrm{C} & 1.20339 & 0.13129 & 4.85208 & \mathrm{H} & 4.55721 & -3.36168 & -5.47648 \\ \mathrm{~N} & -1.41475 & -1.90244 & 0.46665 & \mathrm{H} & 4.87366 & -4.0255 & -3.8587 \\ \mathrm{C} & -3.35452 & -1.30765 & -0.90631 & \mathrm{H} & 3.1843 & -1.3121 & -5.80969 \\ \mathrm{P} & 1.32859 & 0.29597 & 0.19954 & \mathrm{H} & 4.12028 & -0.38996 & -4.60094\end{array}$




$$
\begin{aligned}
& \begin{array}{llll}
\text { C } & 2.08292 & 1.96453 & -0.1064
\end{array} \\
& \begin{array}{llll}
\text { C } & 2.62678 & -0.8105 & -0.49765
\end{array} \\
& \begin{array}{llll}
\text { C } & 3.45289 & 2.13537 & -0.33328
\end{array} \\
& \begin{array}{llll}
\text { C } & 3.99917 & 3.40924 & -0.54225
\end{array} \\
& \begin{array}{llll}
\text { C } & 3.13124 & 4.50845 & -0.51701
\end{array} \\
& \begin{array}{llll}
\text { C } & 1.75157 & 4.37459 & -0.30173
\end{array} \\
& \begin{array}{llll}
\text { C } & 1.243 & 3.08663 & -0.09805
\end{array} \\
& \begin{array}{llll}
\text { C } & 3.81414 & -1.03639 & 0.22009
\end{array} \\
& \begin{array}{llll}
\text { C } & 4.87964 & -1.73389 & -0.35307
\end{array} \\
& \begin{array}{llll}
\text { C } & 4.72868 & -2.18912 & -1.67691
\end{array} \\
& \begin{array}{llll}
\text { C } & 3.56954 & -1.96069 & -2.4245
\end{array} \\
& \begin{array}{llll}
\text { C } & 2.50792 & -1.27967 & -1.80331
\end{array} \\
& \begin{array}{llll}
\text { C } & 6.28184 & 2.30439 & -0.76691
\end{array} \\
& \begin{array}{llll}
\text { C } & 5.4969 & 3.62403 & -0.81105
\end{array} \\
& \begin{array}{llll}
\text { C } & 6.08019 & 4.57076 & 0.25821
\end{array} \\
& \begin{array}{llll}
\text { C } & 5.67596 & 4.25008 & -2.20997
\end{array} \\
& \begin{array}{llll}
\text { C } & 6.21683 & -1.32558 & 1.77836
\end{array} \\
& \begin{array}{llll}
\text { C } & 6.20372 & -1.98325 & 0.38956
\end{array} \\
& \begin{array}{llll}
\text { C } & 7.37354 & -1.3997 & -0.43136
\end{array} \\
& \begin{array}{llll}
\text { C } & 6.41978 & -3.5003 & 0.56511
\end{array} \\
& \begin{array}{llll}
\text { C } & 4.69293 & -3.09385 & -4.41717
\end{array} \\
& \begin{array}{llll}
\text { C } & 3.4467 & -2.35677 & -3.90448
\end{array} \\
& \begin{array}{llll}
\text { C } & 3.26391 & -1.06907 & -4.73733
\end{array} \\
& \begin{array}{llll}
\text { C } & 2.2248 & -3.27229 & -4.10571
\end{array} \\
& \begin{array}{llll}
\text { C } & 0.97225 & 6.31427 & -1.69234
\end{array} \\
& \begin{array}{llll}
\text { C } & 0.85445 & 5.62236 & -0.31811
\end{array} \\
& \begin{array}{llll}
\text { C } & 1.30958 & 6.59337 & 0.79096
\end{array} \\
& \begin{array}{llll}
\text { C } & -0.62235 & 5.2735 & -0.07942
\end{array} \\
& \begin{array}{llll}
\text { C } & -2.80831 & -2.23528 & 0.12557
\end{array} \\
& \begin{array}{llll}
\text { O } & -4.54141 & -1.54525 & -1.47621
\end{array} \\
& \begin{array}{llll}
\text { C } & -4.696 & -0.52989 & -2.52139
\end{array} \\
& \begin{array}{llll}
\text { C } & -3.62739 & 0.52015 & -2.14693
\end{array} \\
& \begin{array}{llll}
\mathrm{N} & -2.75137 & -0.23328 & -1.25479
\end{array} \\
& \text { H } \quad 2.66782 \quad-3.33841 \quad 3.60843 \\
& \begin{array}{llll}
\mathrm{H} & 3.19907 & -2.35764 & 2.24012
\end{array} \\
& \begin{array}{llll}
\mathrm{H} & 3.64167 & -4.45929 & 1.23319
\end{array} \\
& \begin{array}{llll}
\mathrm{H} & 2.38505 & -5.28457 & 2.16763
\end{array} \\
& \text { H } \quad 2.20299 \quad-5.58116 \quad-0.90119
\end{aligned}
$$

$\begin{array}{cccc}\mathrm{H} & 2.35406 & -0.52493 & -4.44423 \\ \mathrm{H} & 1.29822 & -2.7999 & -3.75051 \\ \mathrm{H} & 2.09988 & -3.51319 & -5.17413 \\ \mathrm{H} & 2.35051 & -4.21289 & -3.55056 \\ \mathrm{H} & 2.00514 & 6.62837 & -1.90462 \\ \mathrm{H} & 0.33422 & 7.21243 & -1.72776 \\ \mathrm{H} & 0.65539 & 5.63468 & -2.49866 \\ \mathrm{H} & 0.67929 & 7.49764 & 0.79594 \\ \mathrm{H} & 2.35322 & 6.91288 & 0.6513 \\ \mathrm{H} & 1.2344 & 6.11726 & 1.78134 \\ \mathrm{H} & -0.7739 & 4.81262 & 0.90691 \\ \mathrm{H} & -1.23306 & 6.18893 & -0.11648 \\ \mathrm{H} & -1.00794 & 4.57871 & -0.84049 \\ \mathrm{H} & -3.42652 & -2.10741 & 1.02747 \\ \mathrm{H} & -4.40478 & -1.03595 & -3.45989 \\ \mathrm{Ir} & -0.8104 & 0.1045 & -0.52929 \\ \mathrm{C} & -2.64086 & 2.76909 & 2.01152 \\ \mathrm{H} & -1.45149 & 0.76247 & 0.92154 \\ \mathrm{H} & -0.28409 & -0.5166 & -1.94763 \\ \mathrm{H} & -2.91962 & -3.27807 & -0.20474 \\ \mathrm{H} & -0.63862 & 1.49494 & -1.24734 \\ \mathrm{H} & -1.43301 & -1.58383 & 1.43173 \\ \mathrm{C} & -6.12872 & -0.08379 & -2.62343 \\ \mathrm{C} & -6.44564 & 1.22402 & -3.01137 \\ \mathrm{C} & -7.16501 & -0.99313 & -2.37378 \\ \mathrm{C} & -7.77974 & 1.62006 & -3.14146 \\ \mathrm{H} & -5.651 & 1.94627 & -3.21093 \\ \mathrm{C} & -8.49619 & -0.59786 & -2.50512 \\ \mathrm{H} & -6.92114 & -2.00527 & -2.04874 \\ \mathrm{C} & -8.8088 & 0.71028 & -2.88741 \\ \mathrm{H} & -8.01394 & 2.64459 & -3.43978 \\ \mathrm{H} & -9.29546 & -1.31308 & -2.29692 \\ \mathrm{H} & -9.85181 & 1.02004 & -2.98609 \\ \mathrm{H} & -3.06988 & 0.89747 & -3.0133 \\ \mathrm{H} & 1.24483 & 3.13863\end{array}$




$\begin{array}{cccc}\mathrm{H} & 0.27291 & -5.04464 & -2.4175 \\ \mathrm{H} & -1.35606 & -3.31591 & -1.77536 \\ \mathrm{H} & -0.55874 & -2.13912 & 5.26553 \\ \mathrm{H} & 1.08584 & -2.79722 & 5.32802 \\ \mathrm{H} & 0.22504 & -4.22009 & 3.53854 \\ \mathrm{H} & -0.9699 & -2.98228 & 3.08519 \\ \mathrm{H} & 2.15472 & 2.13391 & 2.2859 \\ \mathrm{H} & 1.98553 & 2.14578 & 4.73978 \\ \mathrm{H} & 1.05018 & 0.14847 & 5.93446 \\ \mathrm{H} & 4.09755 & 1.26258 & -0.35725 \\ \mathrm{H} & 3.54231 & 5.50544 & -0.6844 \\ \mathrm{H} & 0.17881 & 2.92274 & 0.04747 \\ \mathrm{H} & 3.89659 & -0.62977 & 1.2261 \\ \mathrm{H} & 5.55914 & -2.72196 & -2.13682 \\ \mathrm{H} & 1.57495 & -1.08449 & -2.33371 \\ \mathrm{H} & 6.20393 & 1.81904 & 0.21758 \\ \mathrm{H} & 7.34858 & 2.49535 & -0.9613 \\ \mathrm{H} & 5.92607 & 1.58998 & -1.52417\end{array}$

$\begin{array}{llll}\text { C } & -2.06569 & 0.48384 & 2.41626\end{array}$

$\begin{array}{llll}\text { O } & -4.16598 & 1.08334 & 1.15012\end{array}$

$\begin{array}{llll}\text { O } & -2.11015 & -0.74289 & 2.6104\end{array}$

$\begin{array}{llll}\mathrm{H} & -3.84241 & 1.3674 & 3.16178\end{array}$

$\begin{array}{llll}\mathrm{H} & -3.27447 & 3.59822 & 2.35509\end{array}$

$\begin{array}{llll}\mathrm{H} & -2.43317 & 2.89898 & 0.94134\end{array}$

H $\quad-0.45126 \quad 2.95646 \quad 2.25346$

$\begin{array}{llll}\mathrm{H} & -1.3599 & 3.18422 & 3.7725\end{array}$

$\begin{array}{llll}\text { C } & -5.26959 & 0.3222 & 1.42125\end{array}$

$\begin{array}{llll}\text { C } & -5.26729 & -0.72082 & 2.35977\end{array}$

$\begin{array}{llll}\text { C } & -6.43085 & 0.61222 & 0.69511\end{array}$

$\begin{array}{llll}\text { C } & -6.43454 & -1.46398 & 2.55863\end{array}$

H $\quad-4.34967 \quad-0.9563 \quad 2.90053$

$\begin{array}{llll}\text { C } & -7.58643 & -0.14024 & 0.90241\end{array}$

H $\quad-6.41334 \quad 1.43065 \quad-0.02498$

$\begin{array}{llll}\text { C } & -7.59602 & -1.18239 & 1.83454\end{array}$

H $\quad-6.42818 \quad-2.27821 \quad 3.28779$

H $\quad-8.48436 \quad 0.09516 \quad 0.32853$

H $\quad-8.50294 \quad-1.7691 \quad 1.99672$

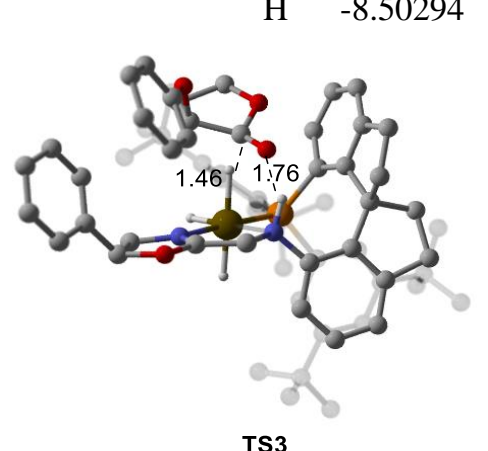
$\begin{array}{llll}\text { C } & 0.82177 & -2.49361 & 2.40023\end{array}$
$\begin{array}{llll}\text { C } & 2.23428 & -2.95349 & 2.92199\end{array}$
$\begin{array}{llll}\text { C } & 2.67422 & -4.13511 & 2.05324\end{array}$
$\begin{array}{llll}\text { C } & 1.85776 & -3.97744 & 0.79883\end{array}$
$\begin{array}{llll}\text { C } & 0.79868 & -3.06975 & 0.99015\end{array}$
$\begin{array}{llll}\text { C } & 2.03548 & -4.67053 & -0.39455\end{array}$
$\begin{array}{llll}\text { C } & 1.11943 & -4.46021 & -1.42686\end{array}$
$\begin{array}{llll}\text { C } & 0.04757 & -3.5912 & -1.24795\end{array}$
$\begin{array}{llll}\text { C } & -0.15079 & -2.91422 & -0.03065\end{array}$
$\begin{array}{llll}\text { C } & 0.73554 & -0.99244 & 2.73054\end{array}$
$\begin{array}{llll}\text { C } & 0.29764 & -0.88865 & 4.0634\end{array}$
$\begin{array}{llll}\text { C } & -0.28904 & -2.18913 & 4.55995\end{array}$

$\mathrm{H} \quad 7.0104 \quad 5.01057-1.14607$

$\mathrm{H} \quad 5.6543 \quad 5.68418 \quad-0.21886$

$\mathrm{H} \quad 5.45402 \quad 5.31251 \quad-1.95113$

H $\quad 5.45273 \quad 2.85418 \quad-2.74275$

H $\quad 7.03826 \quad 2.67836 \quad-1.94223$

$\mathrm{H} \quad 5.72101 \quad 1.54306 \quad-1.5732$

$\begin{array}{llll}\mathrm{H} & 5.70789 & -0.15557 & 2.50587\end{array}$

$\begin{array}{llll}\mathrm{H} & 6.86427 & -1.3676 & 3.09504\end{array}$

$\begin{array}{llll}\mathrm{H} & 5.14103 & -1.77965 & 2.96487\end{array}$

$\begin{array}{llll}\mathrm{H} & 8.27972 & -0.98499 & 1.09173\end{array}$

$\begin{array}{llll}\mathrm{H} & 7.11413 & 0.16889 & 0.39192\end{array}$

$\begin{array}{llll}\mathrm{H} & 7.65002 & -1.23735 & -0.55307\end{array}$ 


\begin{tabular}{|c|c|c|c|}
\hline & & & \\
\hline & 1.1983 & 0.16078 & \\
\hline & 1.35262 & 1.34026 & 2.8126 \\
\hline & 1.01421 & 1.39887 & 41652 \\
\hline & .43975 & 84 & \\
\hline & -1.27356 & -2.04894 & \\
\hline & -3.13726 & -1.54322 & -1.40153 \\
\hline & 1.30474 & 0.29089 & \\
\hline & 2.04994 & .96419 & \\
\hline & 2.71547 & -0.77913 & -0.27957 \\
\hline & 3.42373 & 2.14461 & -0.2371 \\
\hline & 3.96889 & 3.41334 & -0. \\
\hline & 3.09663 & 4.50864 & -0.5291 \\
\hline & & & \\
\hline & 1.20549 & 3.07959 & \\
\hline & 3.80016 & -0.96241 & 0.5942 \\
\hline & & -1.55 & \\
\hline & 5.05319 & -1.9564 & \\
\hline & 3.99679 & -1.77205 & -2.09408 \\
\hline & 2.81195 & -1.19518 & -1.60494 \\
\hline & 5.18189 & & \\
\hline & 5.48939 & 3.53 & -0.65 \\
\hline & 5.91814 & 4.96947 & 101348 \\
\hline & 5.94923 & & \\
\hline & 5.96052 & 1.2 & 2.48248 \\
\hline & 6.21897 & -1.720 & 10510 \\
\hline & & & \\
\hline & 6.62789 & -3.2 & \\
\hline & 5.46994 & -2.7203 & -395040 \\
\hline & & & \\
\hline & 3.98094 & -0.757 & 4.5 \\
\hline & 2.9989 & -3.05051 & -4.02657 \\
\hline & -0.30398 & 5.28798 & -1.52902 \\
\hline & & 5.55096 & -0.4189 \\
\hline & 44935 & 6.876 & -0.7257 \\
\hline & 0.01255 & 5.68988 & 00367 \\
\hline & -2.54705 & -2.53055 & -0.40019 \\
\hline & -4.27792 & -1.82823 & -2.02338 \\
\hline
\end{tabular}

\begin{tabular}{|c|c|c|c|}
\hline & & & \\
\hline & 7.5252 & -3.33394 & \\
\hline & 5.82141 & -3.8183 & 1.5445 \\
\hline & 6.31217 & -2.05004 & 371964 \\
\hline & 5.50597 & -2.93692 & -5.02937 \\
\hline & 5.62842 & -3.66896 & -3.4143 \\
\hline & 4.08482 & -0.93383 & -5.45567 \\
\hline & 4.75826 & -0.04123 & -4.06302 \\
\hline & 3.00281 & -0.28618 & -4.19712 \\
\hline & 2.00208 & -2.65636 & -3.78303 \\
\hline & 3.04271 & -3.2221 & -5.11442 \\
\hline & 3.10736 & -4.02064 & -3.5201 \\
\hline & 0.18811 & 5.18265 & -2.50839 \\
\hline & -1.0208 & 6.12309 & -1.59077 \\
\hline & -0.87332 & 4.36521 & -1.34343 \\
\hline & 0.7135 & 7.69384 & -0.77015 \\
\hline & 1.97046 & 6.84397 & -1.69489 \\
\hline & 2.18544 & 7.13377 & 0.0513 \\
\hline & 0.73306 & 5.86978 & 1.7500 \\
\hline & -0.69726 & 6.53272 & 0.913 \\
\hline & -0.55359 & 4.78134 & 1.18527 \\
\hline & -3.26589 & -2.70371 & 0.36561 \\
\hline & -4.55117 & -1.11209 & -3.93216 \\
\hline & -0.71198 & 0.00583 & -0.78299 \\
\hline & -1.48293 & 0.59466 & 0.61153 \\
\hline & -0.00887 & -0.54517 & -2.15061 \\
\hline & -2.42675 & -3.48908 & -0.97387 \\
\hline & -0.54527 & 1.42935 & -1.44037 \\
\hline & -1.50602 & -1.71032 & 1.04139 \\
\hline & -5.97735 & -0.1915 & -2.63359 \\
\hline & -7.05427 & -0.78935 & -3.30351 \\
\hline & -6.24097 & 0.8018 & -1.68137 \\
\hline & -8.36775 & -0.40707 & -3.02609 \\
\hline & -6.85886 & -1.56643 & -4.04749 \\
\hline & -7.55199 & 1.18744 & -1.40269 \\
\hline & -5.42612 & 1.2753 & -1.13511 \\
\hline 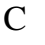 & -8.6179 & 0.58334 & -2.07286 \\
\hline & -9.19675 & -0.88252 & -3.55544 \\
\hline
\end{tabular}




$\begin{array}{cccc}\mathrm{C} & -4.58441 & -0.68898 & -2.91842 \\ \mathrm{C} & -3.39293 & 0.27559 & -2.67194 \\ \mathrm{~N} & -2.58232 & -0.4177 & -1.67319 \\ \mathrm{H} & 2.20488 & -3.18517 & 3.99564 \\ \mathrm{H} & 2.93808 & -2.12404 & 2.7955 \\ \mathrm{H} & 3.7561 & -4.13125 & 1.85131 \\ \mathrm{H} & 2.44175 & -5.10476 & 2.52817 \\ \mathrm{H} & 2.87242 & -5.36163 & -0.5163 \\ \mathrm{H} & 1.23857 & -4.97096 & -2.384 \\ \mathrm{H} & -0.63154 & -3.40452 & -2.07985 \\ \mathrm{H} & -1.30083 & -2.03614 & 4.96626 \\ \mathrm{H} & 0.32478 & -2.61887 & 5.37066 \\ \mathrm{H} & -0.12362 & -4.14664 & 3.51511 \\ \mathrm{H} & -1.26561 & -2.98291 & 2.79753 \\ \mathrm{H} & 1.72915 & 2.23935 & 2.32602 \\ \mathrm{H} & 1.15894 & 2.33114 & 4.7168 \\ \mathrm{H} & 0.10394 & 0.34571 & 5.82639 \\ \mathrm{H} & 4.08413 & 1.28218 & -0.20139 \\ \mathrm{H} & 3.50328 & 5.49767 & -0.72094 \\ \mathrm{H} & 0.13472 & 2.92159 & -0.00194 \\ \mathrm{H} & 3.71584 & -0.58488 & 1.61139 \\ \mathrm{H} & 5.98027 & -2.40019 & -1.55608 \\ \mathrm{H} & 1.95618 & -1.02408 & -2.25916 \\ \mathrm{H} & 5.88392 & 3.79635 & 1.48085 \\ \mathrm{H} & 7.27814 & 3.17747 & 0.5531 \\ \mathrm{H} & 5.91544 & 2.10159 & 0.94871\end{array}$

$\begin{array}{llll}\mathrm{H} & -7.73851 & 1.94877 & -0.64417\end{array}$

$\mathrm{H} \quad-9.64449 \quad 0.88237 \quad-1.84896$

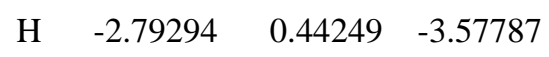

$\begin{array}{llll}\mathrm{H} & -3.69892 & 1.2608 & -2.29417\end{array}$

$\begin{array}{llll}\text { C } & -3.50008 & 1.26343 & 1.22848\end{array}$

$\begin{array}{llll}\text { C } & -2.86275 & 2.64306 & 1.32829\end{array}$

$\begin{array}{llll}\text { C } & -1.88635 & 2.49324 & 2.51578\end{array}$

$\begin{array}{llll}\text { O } & -1.74763 & 1.10023 & 2.76308\end{array}$

$\begin{array}{llll}\text { C } & -2.38346 & 0.3462 & 1.79813\end{array}$

$\begin{array}{llll}\text { O } & -2.48186 & -0.88704 & 1.98452\end{array}$

$\begin{array}{llll}\mathrm{H} & -3.62533 & 3.41481 & 1.49429\end{array}$

$\begin{array}{llll}\mathrm{H} & -2.32174 & 2.86715 & 0.40039\end{array}$

$\begin{array}{llll}\mathrm{H} & -0.89427 & 2.91597 & 2.29592\end{array}$

$\begin{array}{llll}\mathrm{H} & -2.2667 & 2.96356 & 3.43577\end{array}$

$\begin{array}{llll}\text { O } & -4.66364 & 1.25845 & 2.05686\end{array}$

$\begin{array}{llll}\mathrm{H} & -3.76264 & 0.96626 & 0.20611\end{array}$

$\begin{array}{llll}\text { C } & -5.68628 & 0.40938 & 1.76641\end{array}$

$\begin{array}{llll}\text { C } & -6.97913 & 0.82716 & 2.11636\end{array}$

$\begin{array}{llll}\text { C } & -5.5025 & -0.82489 & 1.13167\end{array}$

$\begin{array}{llll}\text { C } & -8.07553 & 0.02259 & 1.80644\end{array}$

$\begin{array}{llll}\mathrm{H} & -7.09886 & 1.789 & 2.61881\end{array}$

$\begin{array}{lllll}\text { C } & -6.60972 & -1.60993 & 0.80488\end{array}$

$\mathrm{H} \quad-4.48723 \quad-1.16706 \quad 0.95336$

$\begin{array}{llll}\text { C } & -7.90055 & -1.19258 & 1.13439\end{array}$

$\begin{array}{llll}\mathrm{H} & -9.08018 & 0.35746 & 2.07736\end{array}$

$\begin{array}{llll}\mathrm{H} & -6.45366 & -2.558 & 0.28478\end{array}$

H $\quad-8.76396 \quad-1.80772 \quad 0.8731$

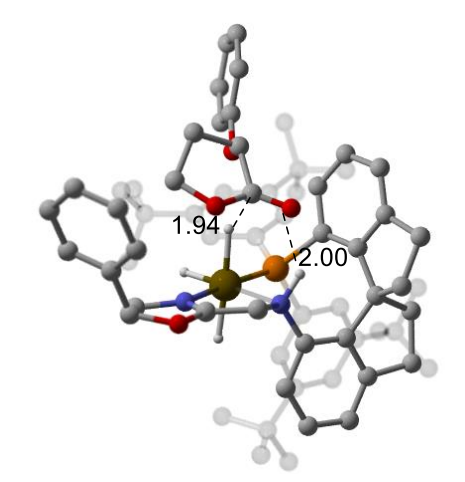

TS4
$\begin{array}{llll}\text { C } & -0.91764 & -2.23277 & -2.84289\end{array}$
$\begin{array}{llll}\text { C } & -2.42699 & -2.54643 & -3.13012\end{array}$

$\begin{array}{llll}\text { H } & -4.79365 & 6.038 & -0.6961\end{array}$

$\mathrm{H} \quad-3.12872 \quad 5.45191 \quad-0.97043$ 


$$
\begin{aligned}
& \text { C } \quad-2.73019 \quad-3.90731 \quad-2.48693 \\
& \text { C } \quad-1.62952-4.08235-1.47258 \\
& \text { C } \quad-0.62011 \quad-3.11952 \quad-1.64473 \\
& \begin{array}{llll}
\text { C } & -1.52819 & -5.09047 & -0.51815
\end{array} \\
& \begin{array}{llll}
\text { C } & -0.38025 & -5.14197 & 0.27664
\end{array} \\
& \text { C } \quad 0.63501 \quad-4.20375 \quad 0.11184 \\
& \text { C } \quad 0.53988 \quad-3.19274 \quad-0.86101 \\
& \begin{array}{llll}
\text { C } & -0.73677 & -0.70615 & -2.84717
\end{array} \\
& \text { C } \quad-0.45183 \quad-0.31602 \quad-4.1721 \\
& \text { C } \quad-0.20022 \quad-1.5068 \quad-5.06513 \\
& \text { C } \quad-0.04662-2.65655 \quad-4.06072 \\
& \begin{array}{llll}
\text { C } & -0.89456 & 0.28221 & -1.8503
\end{array} \\
& \begin{array}{llll}
\text { C } & -0.8187 & 1.62989 & -2.2557
\end{array} \\
& \begin{array}{llll}
\text { C } & -0.59843 & 2.00191 & -3.57974
\end{array} \\
& \begin{array}{llll}
\text { C } & -0.39103 & 1.0207 & -4.5494
\end{array} \\
& \begin{array}{llll}
\mathrm{N} & 1.59329 & -2.24403 & -0.98727
\end{array} \\
& \begin{array}{llll}
\text { C } & 3.66678 & -2.24783 & 0.32058
\end{array} \\
& \mathrm{P} \quad-0.83827 \quad-0.02871 \quad-0.02041 \\
& \begin{array}{llll}
\text { C } & -1.55148 & 1.51333 & 0.72475
\end{array} \\
& \begin{array}{llll}
\text { C } & -2.25784 & -1.14917 & 0.35169
\end{array} \\
& \begin{array}{llll}
\text { C } & -2.67845 & 2.14797 & 0.17361
\end{array} \\
& \begin{array}{llll}
\text { C } & -3.20886 & 3.30242 & 0.74753
\end{array} \\
& \begin{array}{llll}
\text { C } & -2.60644 & 3.79803 & 1.92116
\end{array} \\
& \begin{array}{llll}
\text { C } & -1.50951 & 3.17193 & 2.51494
\end{array} \\
& \begin{array}{llll}
\text { C } & -0.99071 & 2.02222 & 1.89226
\end{array} \\
& \begin{array}{llll}
\text { C } & -3.46525 & -1.01915 & -0.35377
\end{array} \\
& \begin{array}{llll}
\text { C } & -4.61193 & -1.70238 & 0.05241
\end{array} \\
& \begin{array}{llll}
\text { C } & -4.52373 & -2.5098 & 1.20316
\end{array} \\
& \begin{array}{llll}
\text { C } & -3.34174 & -2.64613 & 1.93625
\end{array} \\
& \begin{array}{llll}
\text { C } & -2.20033 & -1.96536 & 1.4784
\end{array} \\
& \begin{array}{llll}
\text { C } & -4.92631 & 3.35905 & -1.13406
\end{array} \\
& \begin{array}{llll}
\text { C } & -4.4011 & 4.05132 & 0.13288
\end{array} \\
& \begin{array}{llll}
\text { C } & -3.95667 & 5.48008 & -0.24548
\end{array} \\
& \text { C } \quad-5.55063 \quad 4.11706 \quad 1.15949 \\
& \text { C } \quad-5.84525 \quad-0.73423 \quad-1.9625 \\
& \begin{array}{llll}
\text { C } & -5.96525 & -1.54074 & -0.65984
\end{array} \\
& \begin{array}{llll}
\text { C } & -6.92894 & -0.79221 & 0.2865
\end{array} \\
& \text { C } \quad-6.55366 \quad-2.92365 \quad-1.00486
\end{aligned}
$$

\begin{tabular}{|c|c|c|c|}
\hline & -3.61285 & 6.0453 & \\
\hline & -5.24815 & 4.63858 & ค \\
\hline & -6.41484 & 4.65537 & 073725 \\
\hline & -5.88027 & 3.10432 & דוטד 1 \\
\hline & -5.49198 & 0.29111 & -1.7808 \\
\hline & -6.83077 & -0.66075 & -2.4476 \\
\hline & -5.1558 & -1.21014 & -2.67546 \\
\hline & -7.91275 & -0.65287 & -0.19111 \\
\hline & -6.52677 & 0.20033 & 0.54403 \\
\hline & -7.08263 & -1.34439 & 1.22584 \\
\hline & -6.71071 & -3.54133 & -0.10859 \\
\hline & -7.52805 & -2.81164 & -1.50695 \\
\hline & -5.88409 & -3.47673 & -1.68168 \\
\hline & -5.41707 & -3.42139 & 3.71884 \\
\hline & -4.48917 & -4.70106 & 4.5274 \\
\hline & -4.88273 & -4.85399 & 2.8006 \\
\hline & -2.88976 & -2.97349 & 5.35877 \\
\hline & -3.71105 & -1.66352 & 4.46514 \\
\hline & -1.97247 & -1.9486 & 4.22603 \\
\hline & & & \\
\hline & -2.07001 & -5.0339 & 4.13149 \\
\hline & -2.39015 & -5.24067 & 2.3872 \\
\hline & -1.48837 & 5.78185 & 3.62104 \\
\hline & -1.00984 & 5.28541 & 5.25776 \\
\hline & -2.57513 & 4.76094 & 4.59953 \\
\hline & 1.14963 & & \\
\hline & 0.70638 & 4.82745 & 2.71976 \\
\hline & 1.18924 & 3.16149 & 3.10448 \\
\hline & -0.37017 & 1.67765 & 4.54602 \\
\hline & -0.40714 & 2.93947 & 5.80138 \\
\hline & -1.93045 & 2.32192 & 5.10485 \\
\hline & 3.53077 & -2.37554 & -1.76171 \\
\hline & 5.53868 & -2.68781 & 2.56101 \\
\hline & 1.24844 & -0.63763 & 0.6457 \\
\hline & 1.94601 & 0.46021 & -0.47545 \\
\hline & 0.64925 & -1.61992 & 1.80066 \\
\hline & 3.00802 & -3.85658 & -0.91988 \\
\hline & 1.26292 & 0.47129 & $1.76^{\prime}$ \\
\hline
\end{tabular}




\begin{tabular}{|c|c|c|c|c|c|c|c|}
\hline $\mathrm{C}$ & -4.59276 & -4.13896 & 3.58629 & $\mathrm{H}$ & 1.59324 & -1.62167 & -1.79093 \\
\hline C & -3.26843 & -3.43595 & 3.25274 & $\mathrm{C}$ & 6.64717 & -1.21568 & 1.45985 \\
\hline $\mathrm{C}$ & -2.93949 & -2.44601 & 4.39191 & $\mathrm{C}$ & 6.99733 & -0.88158 & 0.14616 \\
\hline $\mathrm{C}$ & -2.16092 & -4.50297 & 3.16972 & $\mathrm{C}$ & 7.48205 & -0.82393 & 2.51517 \\
\hline $\mathrm{C}$ & -1.52151 & 4.95045 & 4.34221 & $\mathrm{C}$ & 8.16782 & -0.16038 & -0.10454 \\
\hline $\mathrm{C}$ & -0.83406 & 3.69215 & 3.79233 & $\mathrm{H}$ & 6.34844 & -1.18395 & -0.67625 \\
\hline $\mathrm{C}$ & 0.63872 & 4.03584 & 3.48204 & $\mathrm{C}$ & 8.64653 & -0.09598 & 2.26354 \\
\hline $\mathrm{C}$ & -0.88814 & 2.5914 & 4.87263 & $\mathrm{H}$ & 7.22097 & -1.09243 & 3.54296 \\
\hline $\mathrm{C}$ & 2.969 & -2.75744 & -0.89369 & $\mathrm{C}$ & 8.99279 & 0.23746 & 0.95076 \\
\hline $\mathrm{O}$ & 4.89929 & -2.68125 & 0.60209 & $\mathrm{H}$ & 8.43557 & 0.09198 & -1.13348 \\
\hline $\mathrm{C}$ & 5.36421 & -1.94458 & 1.76846 & $\mathrm{H}$ & 9.28955 & 0.20481 & 3.094 \\
\hline $\mathrm{C}$ & 4.15423 & -1.02069 & 2.11768 & $\mathrm{H}$ & 9.90687 & 0.80148 & 0.75121 \\
\hline $\mathrm{N}$ & 3.16853 & -1.36086 & 1.09847 & $\mathrm{H}$ & 3.74457 & -1.20945 & 3.12052 \\
\hline $\mathrm{H}$ & -2.64369 & -2.51607 & -4.20729 & $\mathrm{H}$ & 4.41112 & 0.04606 & 2.04571 \\
\hline $\mathrm{H}$ & -3.03951 & -1.77372 & -2.65806 & $\mathrm{C}$ & 2.6442 & 1.95818 & -2.12857 \\
\hline $\mathrm{H}$ & -3.72774 & -3.93652 & -2.02167 & $\mathrm{C}$ & 4.0857 & 2.42921 & -1.90559 \\
\hline $\mathrm{H}$ & -2.69652 & -4.72912 & -3.22379 & $\mathrm{C}$ & 4.58436 & 1.39531 & -0.89759 \\
\hline $\mathrm{H}$ & -2.32731 & -5.82557 & -0.39878 & $\mathrm{O}$ & 4.04692 & 0.17258 & -1.38639 \\
\hline $\mathrm{H}$ & -0.27615 & -5.91189 & 1.04398 & $\mathrm{C}$ & 2.74432 & 0.41155 & -1.87184 \\
\hline $\mathrm{H}$ & 1.5015 & -4.2293 & 0.77312 & $\mathrm{O}$ & 2.25301 & -0.44388 & -2.62651 \\
\hline $\mathrm{H}$ & 0.69334 & -1.36499 & -5.69243 & $\mathrm{H}$ & 4.13894 & 3.4667 & -1.54824 \\
\hline $\mathrm{H}$ & -1.0507 & -1.67455 & -5.7495 & $\mathrm{H}$ & 4.66445 & 2.34493 & -2.83896 \\
\hline $\mathrm{H}$ & -0.35051 & -3.63968 & -4.44965 & $\mathrm{H}$ & 5.67554 & 1.29972 & -0.84298 \\
\hline $\mathrm{H}$ & 1.00443 & -2.72848 & -3.74405 & $\mathrm{H}$ & 4.18798 & 1.60488 & 0.11346 \\
\hline $\mathrm{H}$ & -0.90828 & 2.41718 & -1.51412 & $\mathrm{C}$ & 1.34156 & 3.8413 & -1.40785 \\
\hline $\mathrm{H}$ & -0.54787 & 3.06265 & -3.83446 & $\mathrm{C}$ & 1.66346 & 4.62909 & -2.52284 \\
\hline $\mathrm{H}$ & -0.16928 & 1.29251 & -5.585 & $\mathrm{C}$ & 0.48235 & 4.35311 & -0.42039 \\
\hline $\mathrm{H}$ & -3.1227 & 1.74538 & -0.73154 & $\mathrm{C}$ & 1.11402 & 5.91218 & -2.64194 \\
\hline $\mathrm{H}$ & -3.01499 & 4.70036 & 2.3733 & $\mathrm{H}$ & 2.32926 & 4.25436 & -3.29947 \\
\hline $\mathrm{H}$ & -0.11983 & 1.51582 & 2.3043 & $\mathrm{C}$ & -0.05742 & 5.62811 & -0.5544 \\
\hline $\mathrm{H}$ & -3.49873 & -0.35883 & -1.21727 & $\mathrm{H}$ & 0.23822 & 3.72016 & 0.43086 \\
\hline $\mathrm{H}$ & -5.4203 & -3.02574 & 1.54201 & $\mathrm{C}$ & 0.2526 & 6.41956 & -1.66888 \\
\hline $\mathrm{H}$ & -1.25014 & -2.03902 & 2.00847 & $\mathrm{H}$ & 1.36894 & 6.51799 & -3.51536 \\
\hline $\mathrm{H}$ & -4.15522 & 3.29517 & -1.91677 & $\mathrm{H}$ & -0.73388 & 6.00085 & 0.21804 \\
\hline $\mathrm{H}$ & -5.77458 & 3.92745 & -1.54548 & $\mathrm{H}$ & -0.17261 & 7.41988 & -1.77411 \\
\hline \multirow[t]{2}{*}{$\mathrm{H}$} & -5.28226 & 2.34003 & -0.91996 & $\mathrm{H}$ & 2.26692 & 2.08456 & -3.15363 \\
\hline & & & & $\mathrm{O}$ & 1.76902 & 2.57079 & -1.20341 \\
\hline
\end{tabular}




\section{Reference}

(1) Zhang, F.-H.; Wang, C.; Xie, J.-H.; Zhou, Q.-L. Synthesis of Tridentate Chiral Spiro Aminophosphine-oxazoline Ligands and Application to Asymmetric Hydrogenation of $\alpha$-Keto Amides. Adv. Synth. Catal. 2019, 361, 2832-2835.

(2) Lee, Y.-S. E.; Chuang, S.-H.; Huang, L. Y. L.; Lai, C.-L.; Lin, Y.-H.; Yang, J.-Y.; Liu, C.-W.; Yang, S.-C.; Lin, H.-S.; Chang, C.-C.; Lai, J.-Y.; Jian, P.-S.; Lam, K.; Chang, J.-M.; Lau, J. Y. N.; Huang, J.-J. Discovery of 4-Aryl-N-arylcarbonyl-2-aminothiazoles as Hec1/Nek2 Inhibitors. Part I: Optimization of in Vitro Potencies and Pharmacokinetic Properties. J. Med. Chem. 2014, 57, 40984110.

(3) Yuan, M.-L.; Xie, J.-H.; Yang, X.-H.; Zhou, Q.-L. Enantioselective Synthesis of Chiral 1,2-Amino Alcohols via Asymmetric Hydrogenation of $\alpha$-Amino Ketones with Chiral Spiro Iridium Catalysts. Synthesis 2014, 46, 2910-2916.

(4) (a) Malkov, A. V.; Stewart-Liddon, A. J. P.; McGeoch, G. D.; Ramírez-López, P.; Kočovský, P. Catalyst development for organocatalytic hydrosilylation of aromatic ketones and ketimines. Org. Biomol. Chem. 2012, 10, 4864-4877. (b) Taban, I. M.; Zhu, J.; Deluca, H. F.; Simons, C. Synthesis, molecular modelling and CYP24A1 inhibitory activity of novel of (E)-N-(2-(1H-imidazol-1-yl)-2(phenylethyl)-3/4-styrylbenzamides. Bioorg. Med. Chem. 2017, 25, 4076-4087.

(5) Zhang, Y. R.; Shao, M. B.; Zhang, H. Z.; Li, Y. Q.; Liu, D. Y.; Cheng, Y.; Liu, G. Y.; Wang, J. H. Synthesis and reactivity of oxygen chelated ruthenium carbene metathesis catalysts. J. Organomet. Chem. 2014, 756, 1-9.

(6) Baskar, B.; Dakas, P.-Y.; Kumar, K. Natural Product Biosynthesis Inspired Concise and Stereoselective Synthesis of Benzopyrones and Related Scaffolds. Org. Lett. 2011, 13, 1988-1991.

(7) Yang, X.-H.; Xie, J.-H.; Zhou, Q.-L. Chiral spiro iridium catalysts with SpiroPAP ligands: highly efficient for asymmetric hydrogenation of ketones and ketoesters. Org. Chem. Front. 2014, 1, 190193.

(8) (a) Hrapchak, M.; Latli, B.; Wang, X.-j.; Lee, H.; Campbell, S.; Song, J. J.; Senanayake, C. H. Synthesis of empagliflozin, a novel and selective sodium-glucose co-transporter-2 inhibitor, labeled with carbon-14 and carbon-13 J. Label Compd. Radiopharm. 2014, 57, 687-694. (b) Wang, X. J.; Zhang, L.; Byrne, D.; Nummy, L.; Weber, D.; Krishnamurthy, D.; Yee, N.; Senanayake, C. H. Efficient synthesis of Empagliflozin, an inhibitor of SGLT-2, utilizing an $\mathrm{AlCl}_{3}$-promoted silane reduction of a, $\beta$-glycopyranoside. Org. Lett. 2014, 16, 4090-4093.

(9) Frisch, M. J.; Trucks, G. W.; Schlegel, H. B.; Scuseria, G. E.; Robb, M. A.; Cheeseman, J. R.; Scalmani, G.; Barone, V.; Petersson, G. A.; Nakatsuji, H.; Li, X.; Caricato, M.; Marenich, A. V.; Bloino, J.; Janesko, B. G.; Gomperts, R.; Mennucci, B.; Hratchian, H. P.; Ortiz, J. V.; Izmaylov, A. F.; Sonnenberg, J. L.; Williams-Young, F.; Ding, F.; Lipparini, F.; Egidi, F.; Goings, J.; Peng, B.; Petrone, A.; Henderson, T.; Ranasinghe, D.; Zakrzewski, V. G.; Gao, J.; Rega, N.; Zheng, G.; Liang, W.; Hada, M.; Ehara, M.; Toyota, K.; Fukuda, R.; Hasegawa, J.; Ishida, M.; Nakajima, T.; Honda, Y.; Kitao, O.; Nakai, H.; Vreven, T.; Throssell, K.; Montgomery Jr., J. A.; Peralta, J. E.; Ogliaro, F.; 
Bearpark, M. J.; Heyd, J. J.; Brothers, E. N.; Kudin, K. N.; Staroverov, V. N.; Keith, T. A.; Kobayashi, R.; Normand, J.; Raghavachari, K.; Rendell, A. P.; Burant, J. C.; Iyengar, S. S.; Tomasi, J.; Cossi, M.; Millam, J. M.; Klene, M.; Adamo, C.; Cammi, R.; Ochterski, J. W.; Martin, R. L.; Morokuma, K.; Farkas, O.; Foresman, J. B.; Fox, D. J. Gaussian 16, Revision A.03; Wallingford, CT, 2016.

(10) (a) Lee, C.; Yang, W.; Parr, R. G. Development of the Colle-Salvetti Correlation-Energy Formula into a Functional of the Electron density. Phys. Rev. B. 1988, 37, 785. (b) Becke, A. D. A New Mixing of Hartree-Fock and Local Density-functional theories. J. Chem. Phys. 1993, 98, 1372. (c) Becke, A. D. Density Functional Thermochemistry. III. The Role of Exact Exchange. J. Chem. Phys. 1993, $98,5648$.

(11) Grimme, S.; Antony, J.; Ehrlich, S.; Krieg, H. A Consistent and Accurate ab Initio Parametrization of Density Functional Dispersion Correction (DFT-D) for the 94 Elements H-Pu. J. Chem. Phys. 2010, 132, 154104.

(12) Legault, C. Y., CYLview, 1.0b (Université de Sherbrooke, 2009).

(13) Sandoval, C. A.; Ohkuma, T.; Muñiz, K.; Noyori, R. Mechanism of Asymmetric Hydrogenation of Ketones Catalyzed by BINAP/1,2-Diamine-Ruthenium(II) Complexes. J. Am. Chem. Soc. 2003, $125,13490-13503$. 
(H) NMR Spectra of New Compounds.

2-(Dibenzylamino)-1-(3,5-dimethylphenyl)ethanone hydrochloride (S1a)<smiles>C1=CCCCC1</smiles>

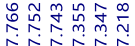

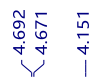<smiles>Cc1cc(C)cc(C(=O)CNc2ccccc2)c1</smiles>

${ }^{1} \mathrm{H}$ NMR $\left(400 \mathrm{MHz}, \mathrm{CDCl}_{3}\right)$
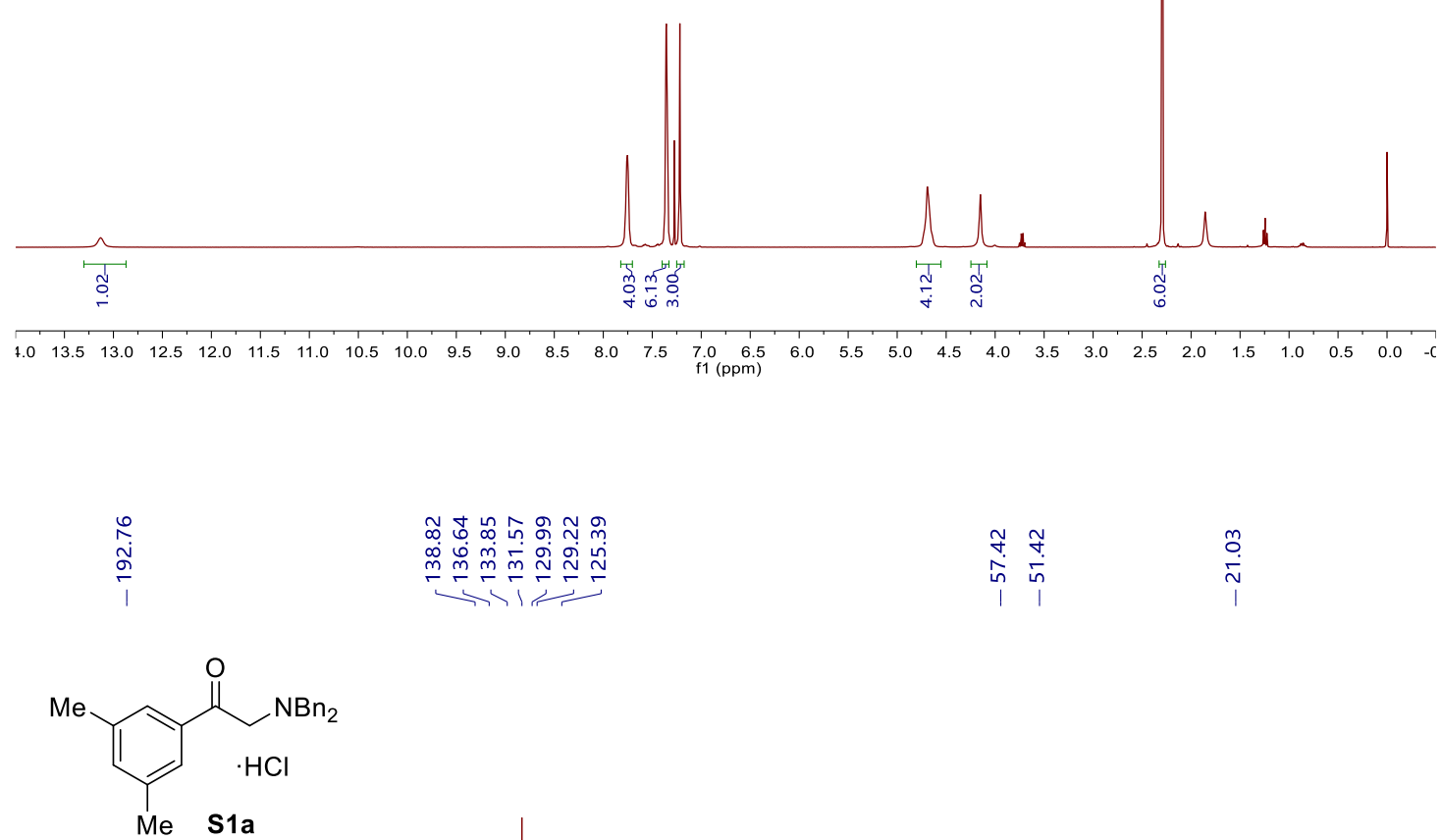

${ }^{13} \mathrm{C}$ NMR $\left(101 \mathrm{MHz}, \mathrm{CDCl}_{3}\right)$

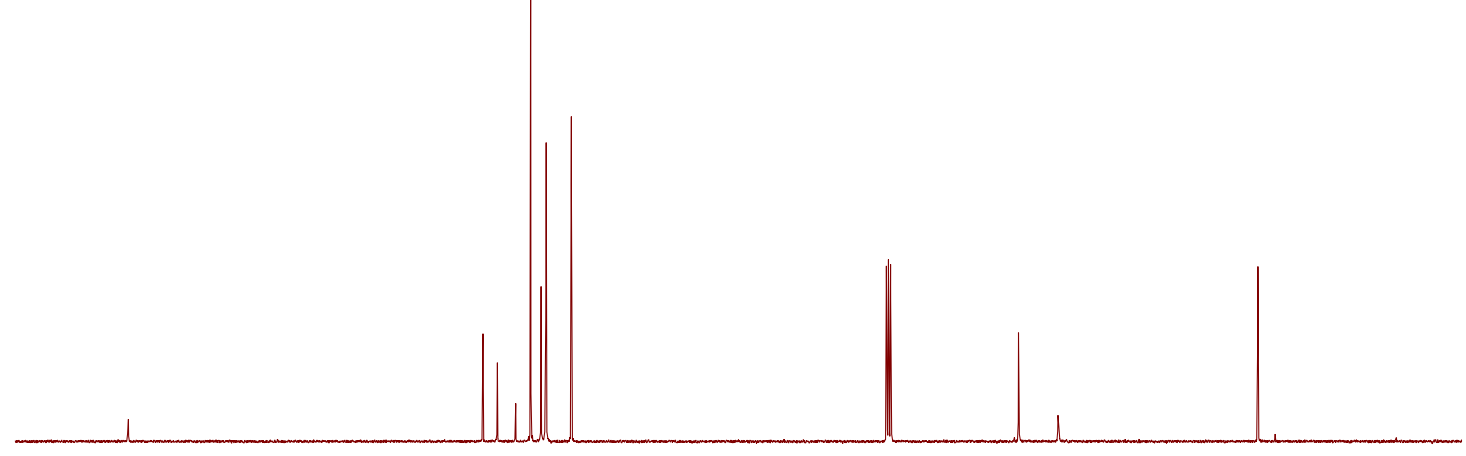

$\begin{array}{llllllllllllllllllllllll}10 & 200 & 190 & 180 & 170 & 160 & 150 & 140 & 130 & 120 & 110 & 100 & 90 & 80 & 70 & 60 & 50 & 40 & 30 & 20 & 10 & 0 & -\end{array}$ 
2-(Dibenzylamino)-1-(4-methoxyphenyl)ethanone hydrochloride (S1b)

$\stackrel{2}{3}$

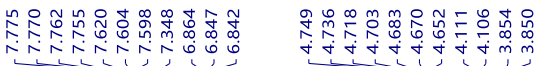

$\overbrace{\mathbf{S} 1 \mathbf{b}}^{\mathrm{HCl}}$

${ }^{1} \mathrm{H}$ NMR $\left(400 \mathrm{MHz}, \mathrm{CDCl}_{3}\right)$

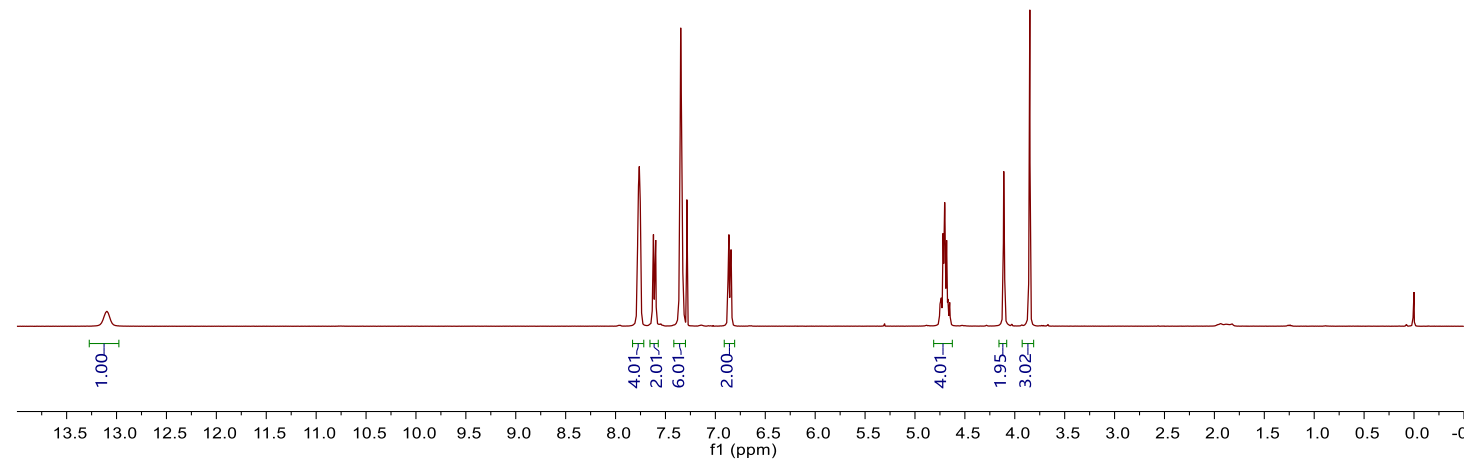<smiles></smiles>

${ }^{13} \mathrm{C}$ NMR $\left(101 \mathrm{MHz}, \mathrm{CDCl}_{3}\right)$

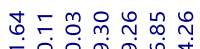

m.

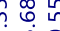

in

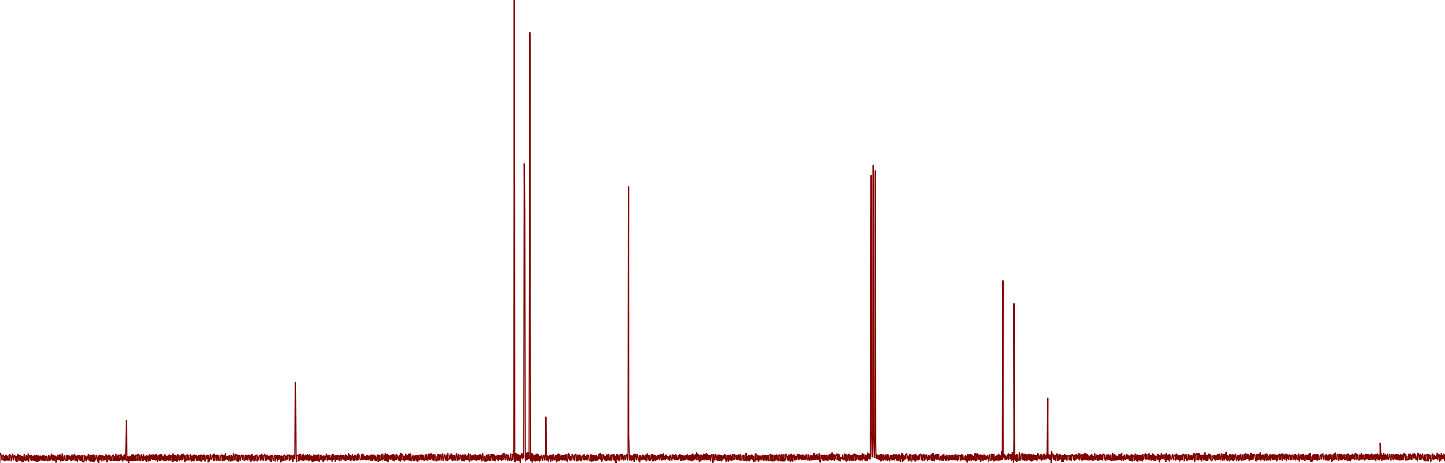

$\begin{array}{llllllllllllllllllllll}10 & 200 & 190 & 180 & 170 & 160 & 150 & 140 & 130 & 120 & 110 \underset{\mathrm{f} 1}{100}(\mathrm{ppm}) & 90 & 80 & 70 & 60 & 50 & 40 & 30 & 20 & 10 & 0 & -\end{array}$ 
2-(Dibenzylamino)-1-(4-(trifluoromethyl)phenyl)ethanone hydrochloride (S1c)

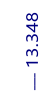

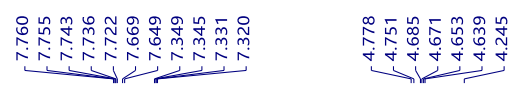<smiles>O=C(CNc1ccccc1)c1ccc(C(F)(F)F)cc1</smiles>

${ }^{1} \mathrm{H}$ NMR (400 MHz, $\mathrm{CDCl}_{3}$ )

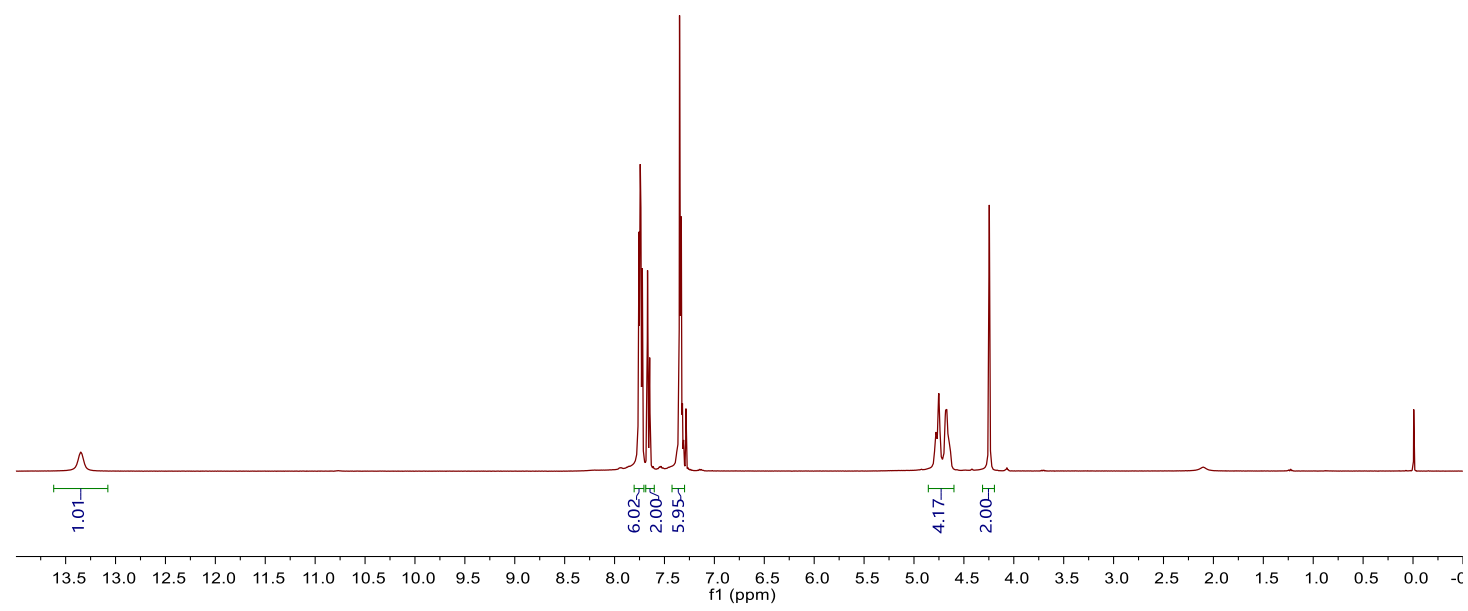

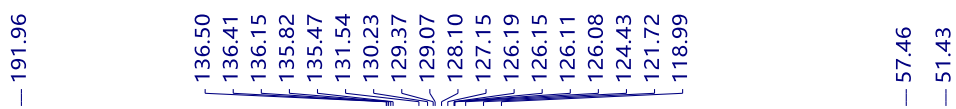

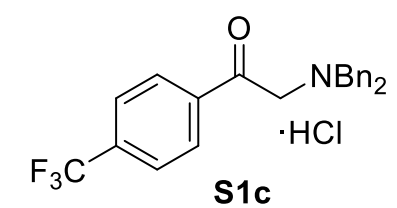

${ }^{13} \mathrm{C}$ NMR $\left(101 \mathrm{MHz}, \mathrm{CDCl}_{3}\right)$

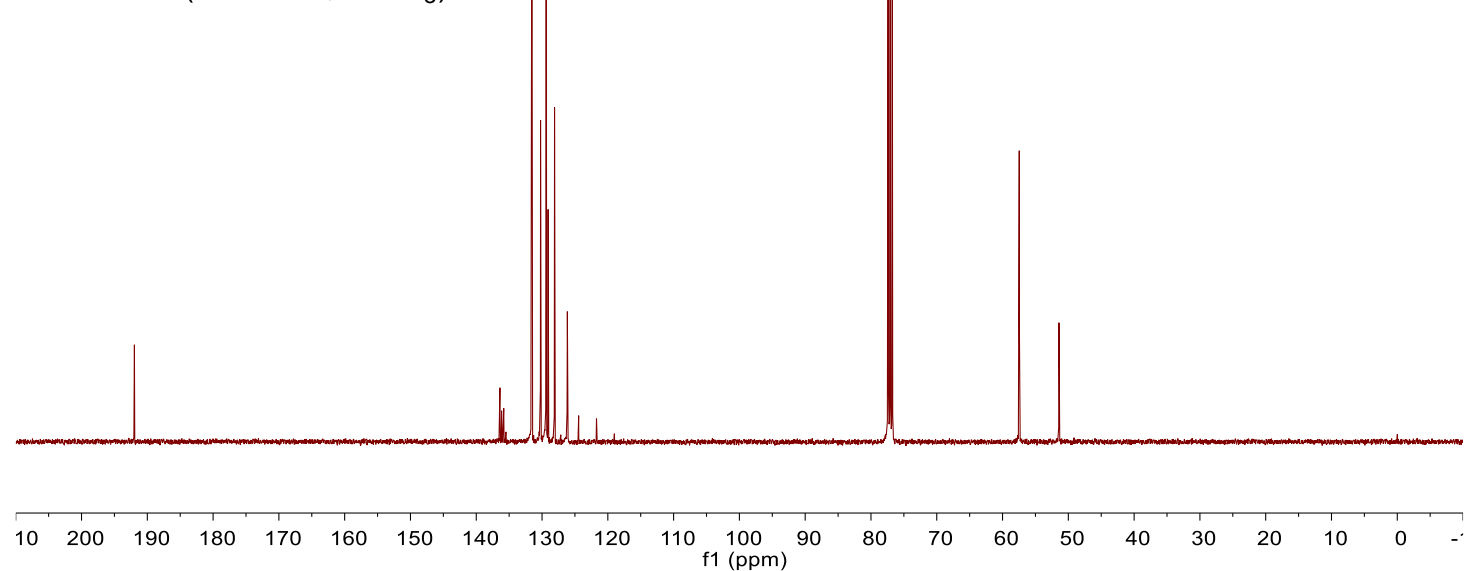


(R)-2-(Dibenzylamino)-1-(3,5-dimethylphenyl)ethanol (S2a)

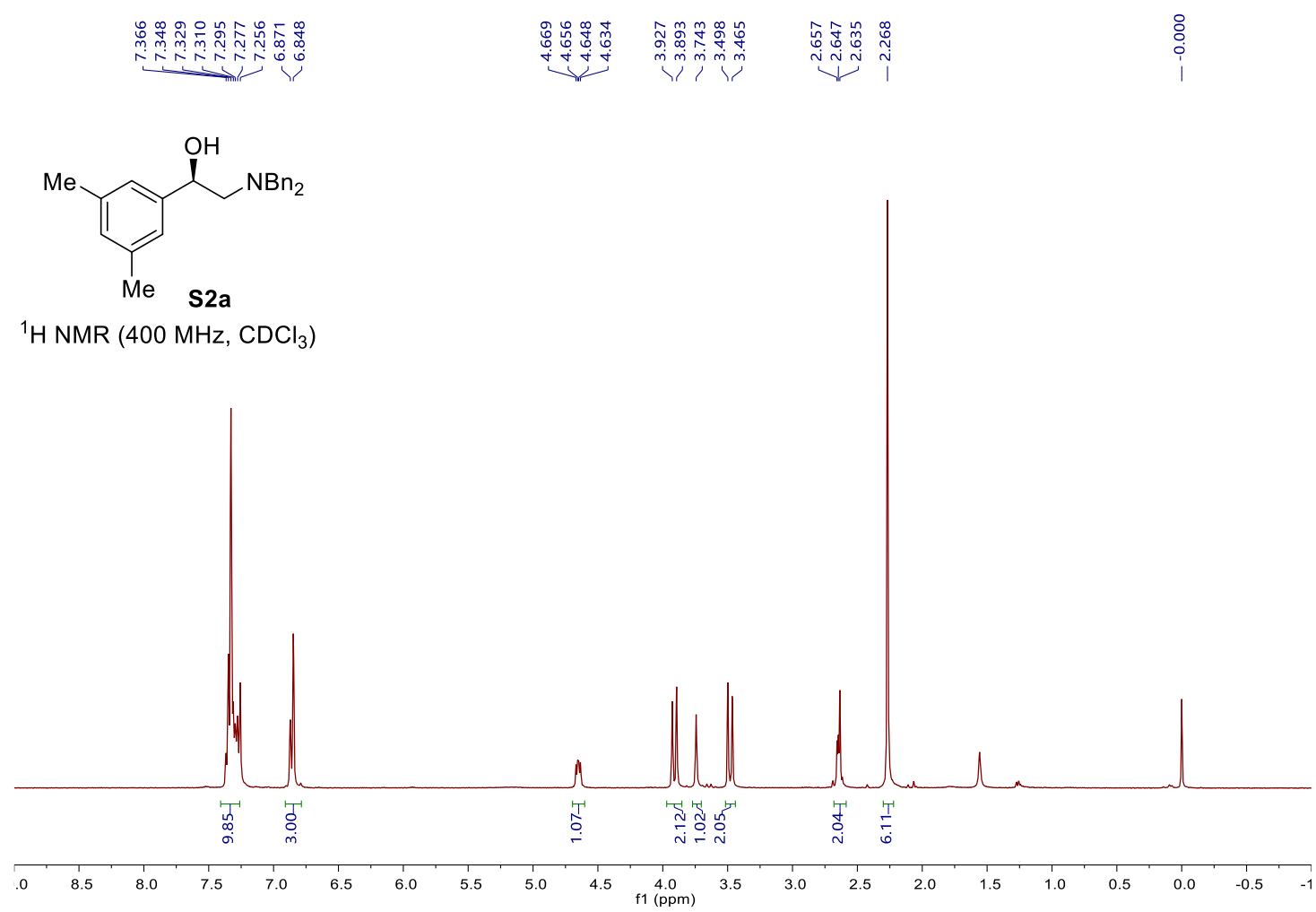

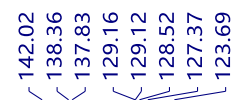

กิ

જู

$\stackrel{\substack{m \\ \sim}}{\sim}$

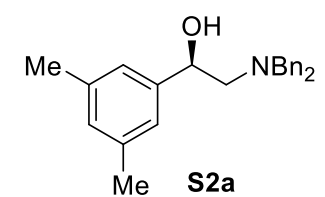

${ }^{13} \mathrm{C}$ NMR $\left(101 \mathrm{MHz}, \mathrm{CDCl}_{3}\right)$

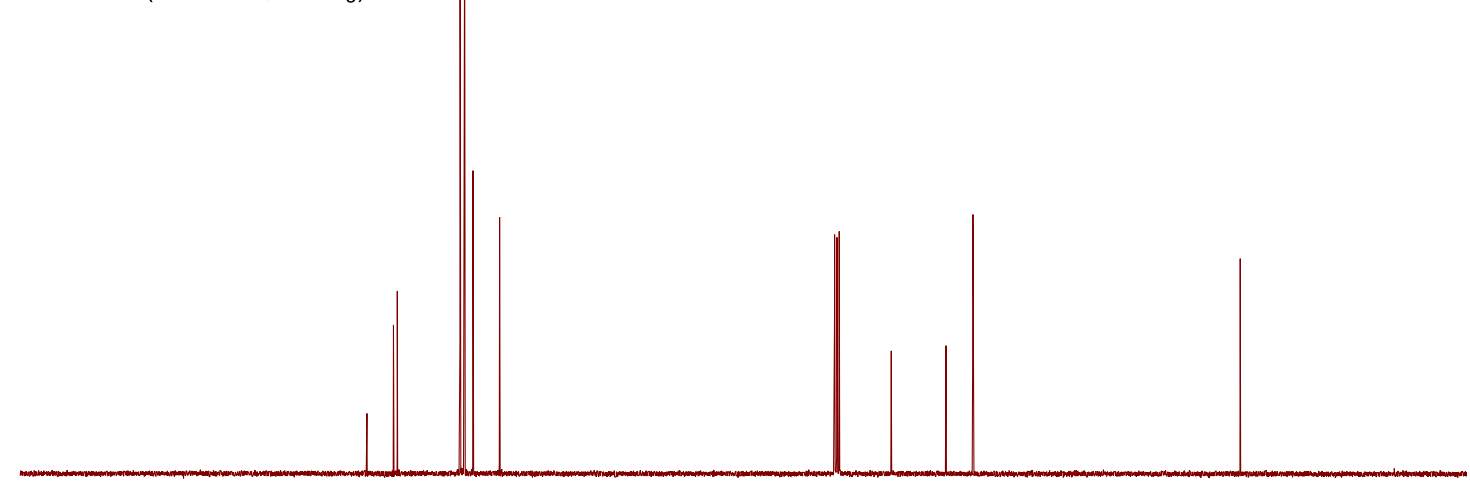

$\begin{array}{llllllllllllllllllllll}90 & 180 & 170 & 160 & 150 & 140 & 130 & 120 & 110 & 100 & 90 & 80 & 70 & 60 & 50 & 40 & 30 & 20 & 10 & 0 & -\end{array}$ 
(R)-2-(Dibenzylamino)-1-(4-methoxyphenyl)ethanol (S2b)

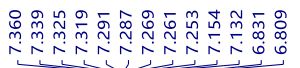

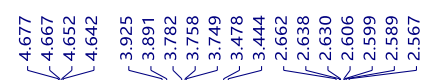

S2b

${ }^{1} \mathrm{H} \mathrm{NMR}\left(400 \mathrm{MHz}, \mathrm{CDCl}_{3}\right)$
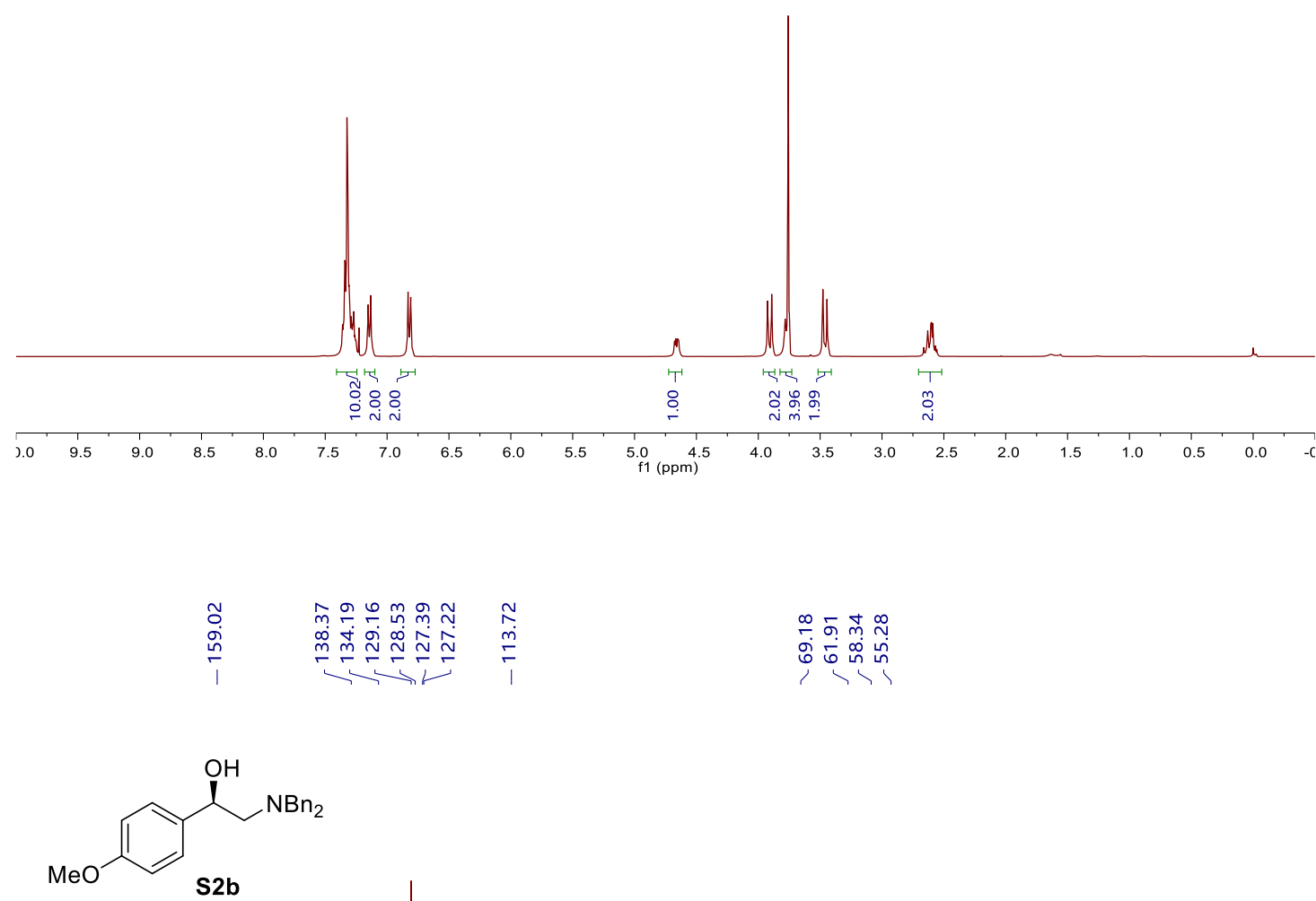

${ }^{13} \mathrm{C}$ NMR $\left(101 \mathrm{MHz}, \mathrm{CDCl}_{3}\right)$

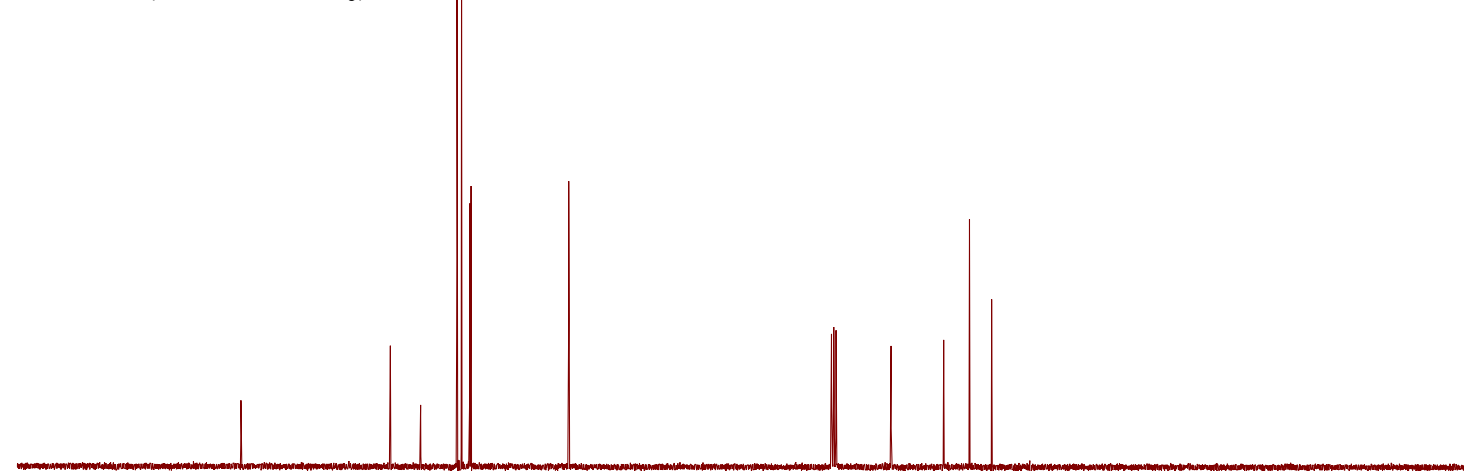

$\begin{array}{llllllllllllllllllllll}90 & 180 & 170 & 160 & 150 & 140 & 130 & 120 & 110 & 100 & 90 & 80 & 70 & 60 & 50 & 40 & 30 & 20 & 10 & 0 & -1\end{array}$ 


\section{(R)-2-(Dibenzylamino)-1-(4-(trifluoromethyl)phenyl)ethanol (S2c)}
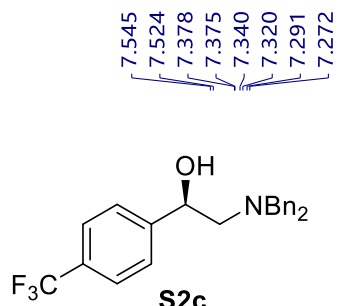

${ }^{1} \mathrm{H} \mathrm{NMR}\left(400 \mathrm{MHz}, \mathrm{CDCl}_{3}\right)$
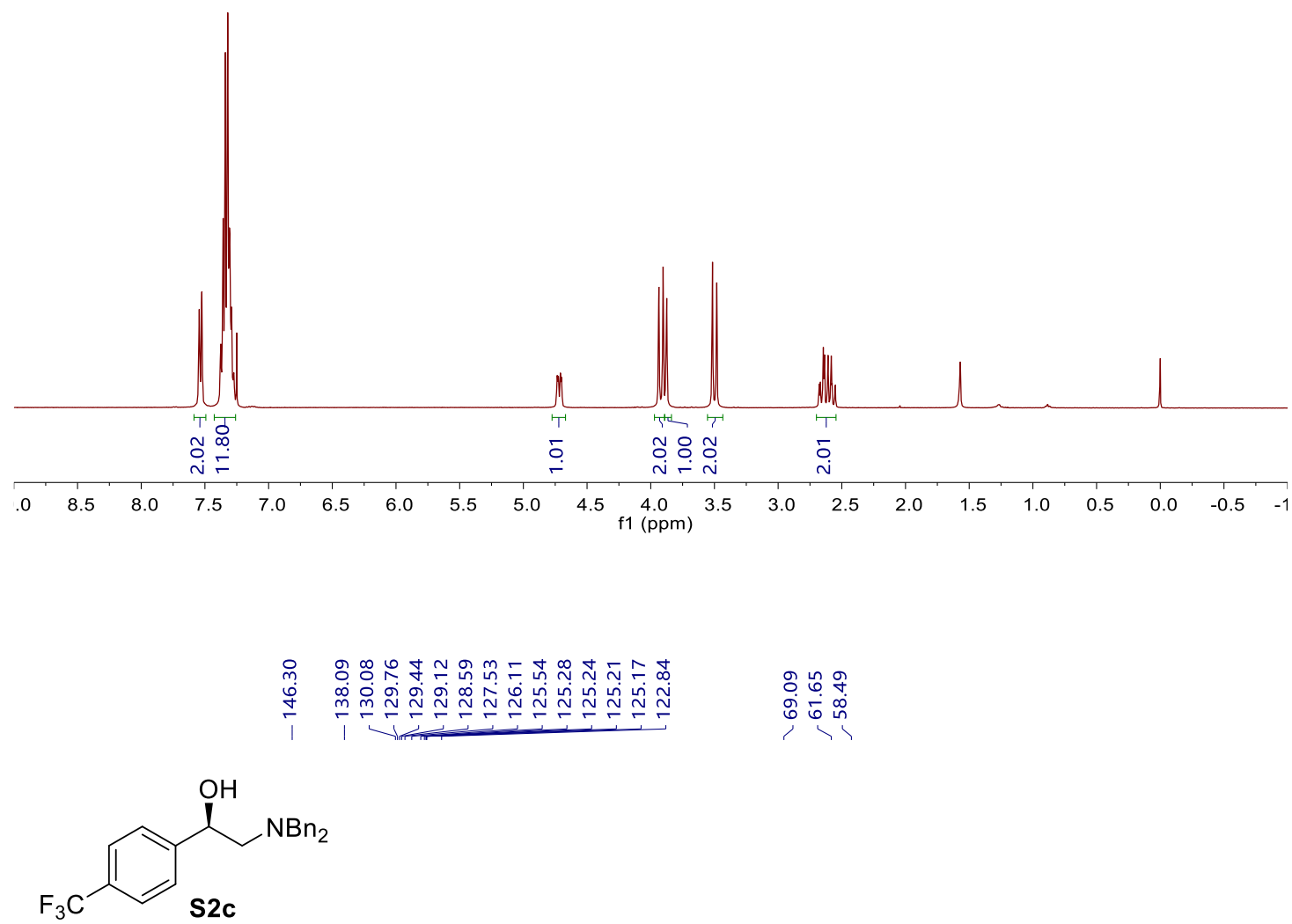

${ }^{13} \mathrm{C}$ NMR $\left(101 \mathrm{MHz}, \mathrm{CDCl}_{3}\right)$

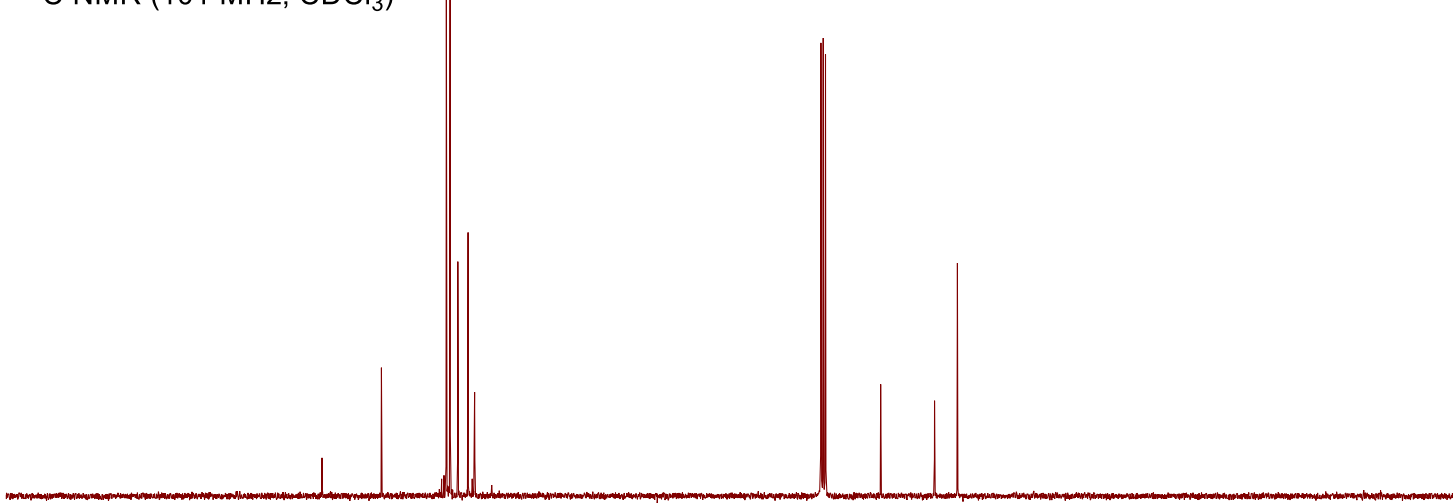

$\begin{array}{lllllllllllllllllll}180 & 170 & 160 & 150 & 140 & 130 & 120 & 110 & 100 & 90 & 80 & 70 & 60 & 50 & 40 & 30 & 20 & 10 & 0\end{array}$ 
(1R)-2-Amino-1-(3,5-dimethylphenyl)ethanol (S3a)

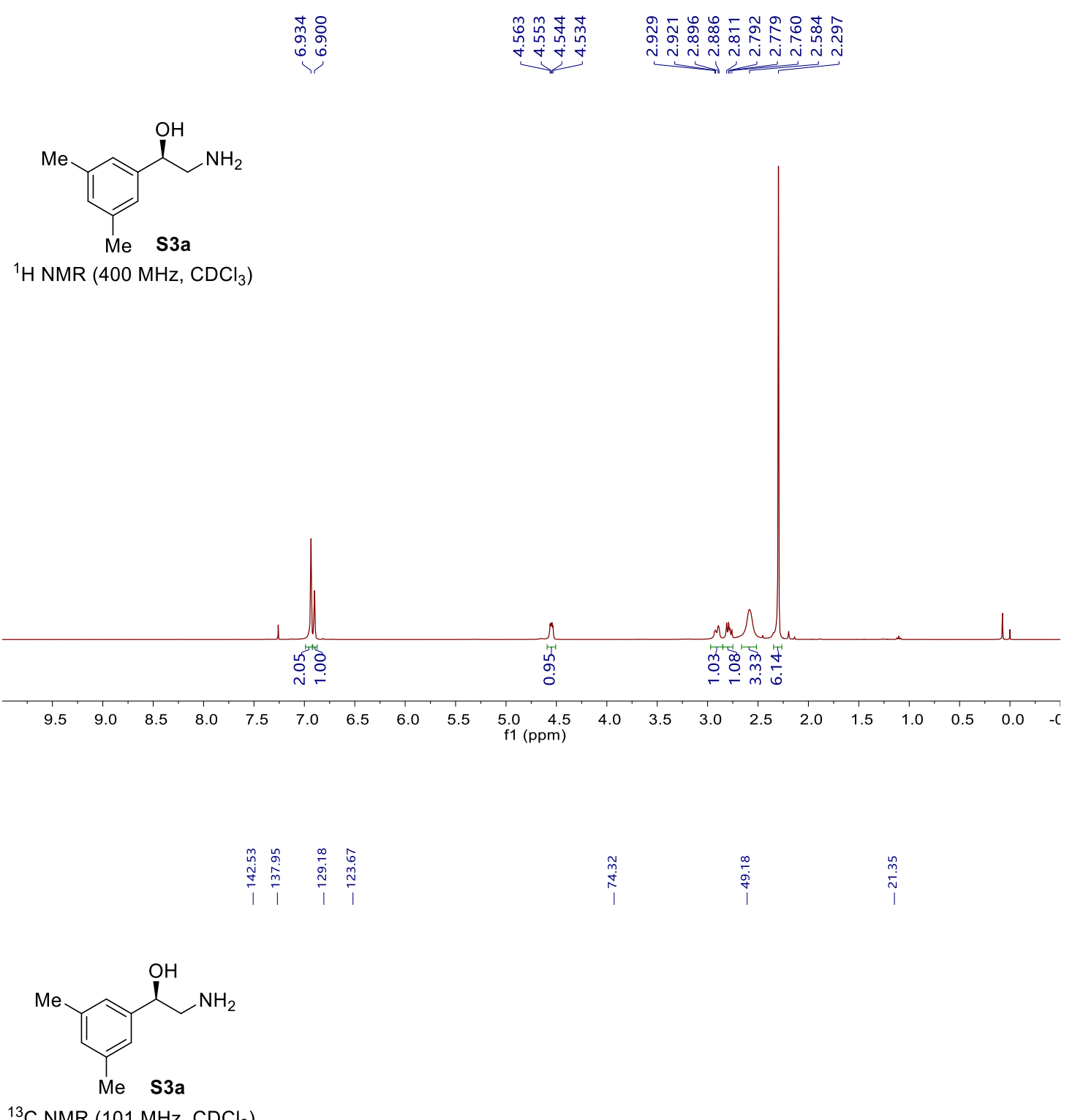

${ }^{13} \mathrm{C} \mathrm{NMR}\left(101 \mathrm{MHz}, \mathrm{CDCl}_{3}\right)$

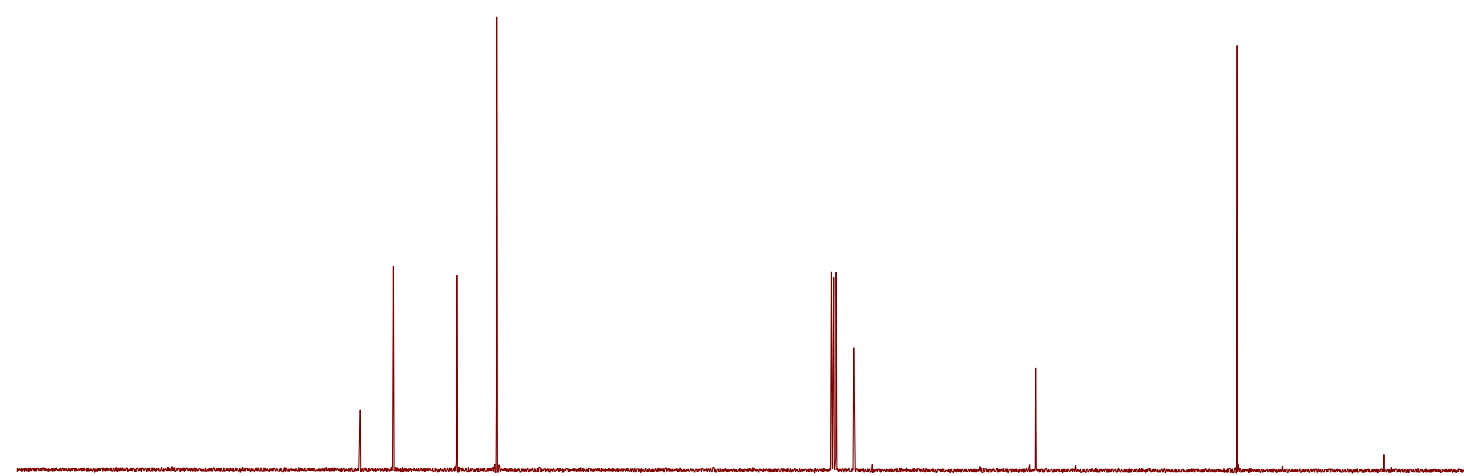

\begin{tabular}{lllllllllllllllllllll}
\hline 90 & 180 & 170 & 160 & 150 & 140 & 130 & 120 & 110 & 100 & $\underset{f}{\mathrm{f}}(\mathrm{ppm})$ & 80 & 70 & 60 & 50 & 40 & 30 & 20 & 10 & 0 & -
\end{tabular} 
(R)-2-Amino-1-(4-methoxyphenyl)ethanol (S3b)

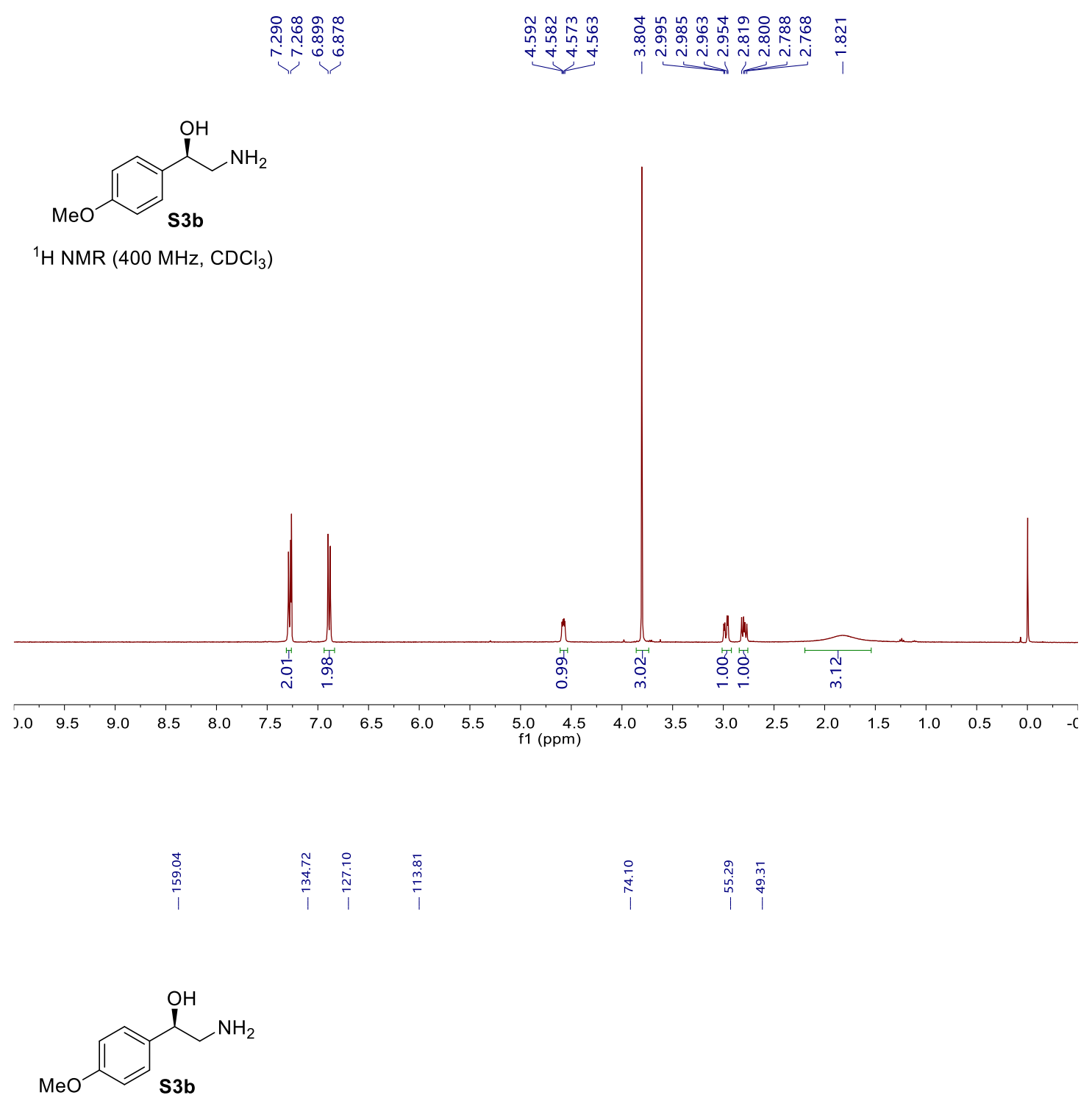

${ }^{13} \mathrm{C}$ NMR $\left(101 \mathrm{MHz}, \mathrm{CDCl}_{3}\right)$

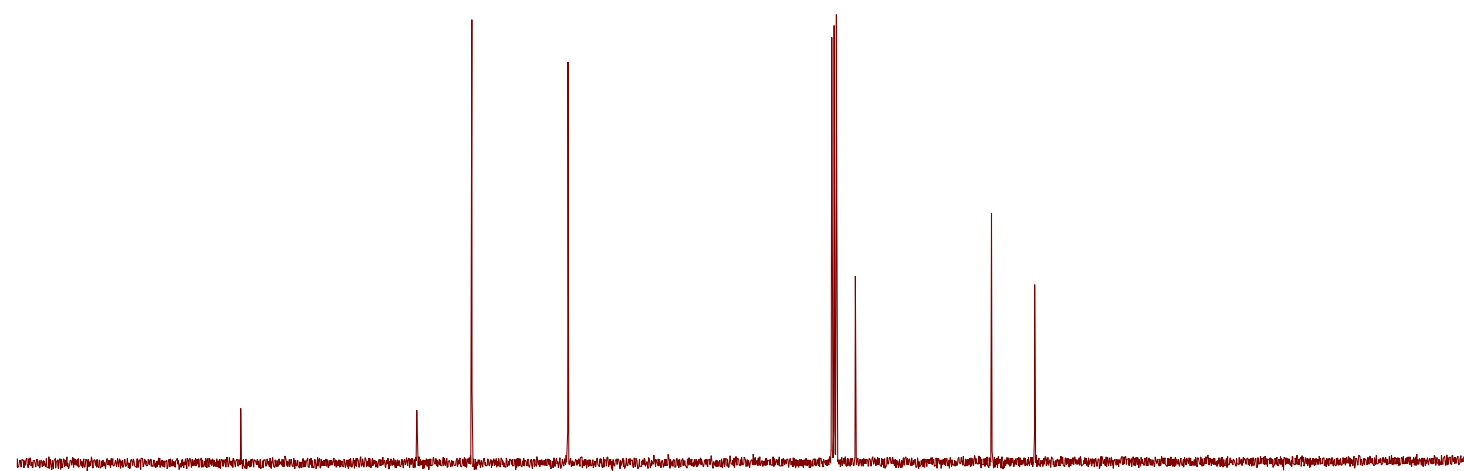

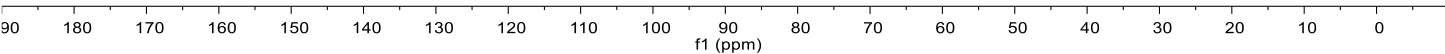




\section{Compound $\left(S_{\mathrm{a}}, R\right)-\mathrm{S} 5 \mathrm{a}$}

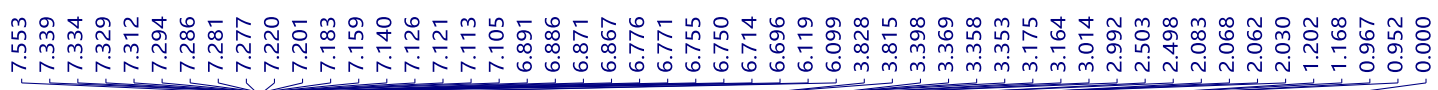

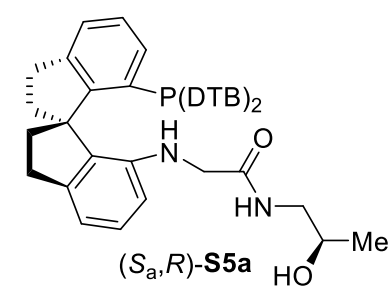

${ }^{1} \mathrm{H}$ NMR $\left(400 \mathrm{MHz}, \mathrm{CDCl}_{3}\right)$
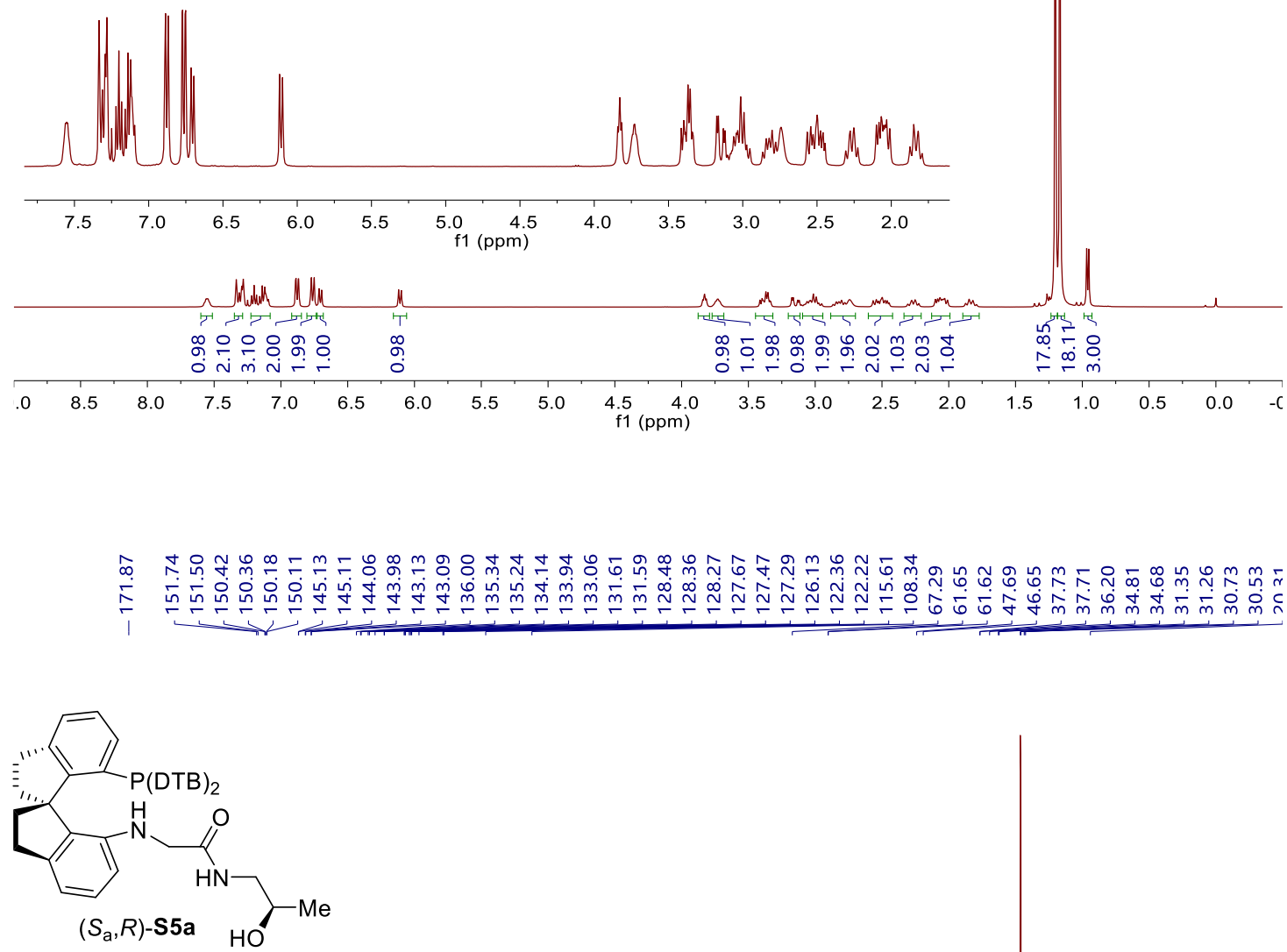

${ }^{13} \mathrm{C}$ NMR $\left(101 \mathrm{MHz}, \mathrm{CDCl}_{3}\right)$

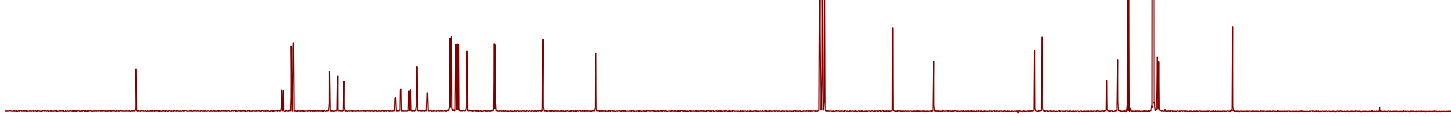

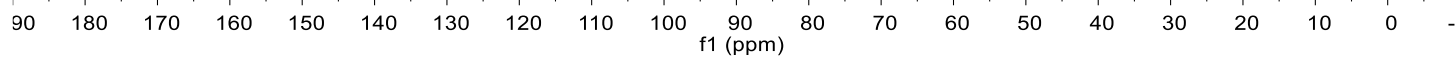




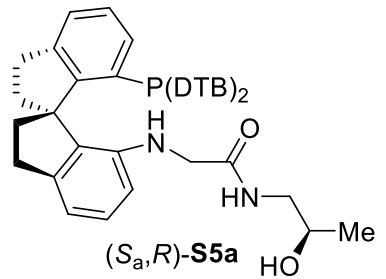

${ }^{31} \mathrm{P}$ NMR $\left(162 \mathrm{MHz}, \mathrm{CDCl}_{3}\right)$

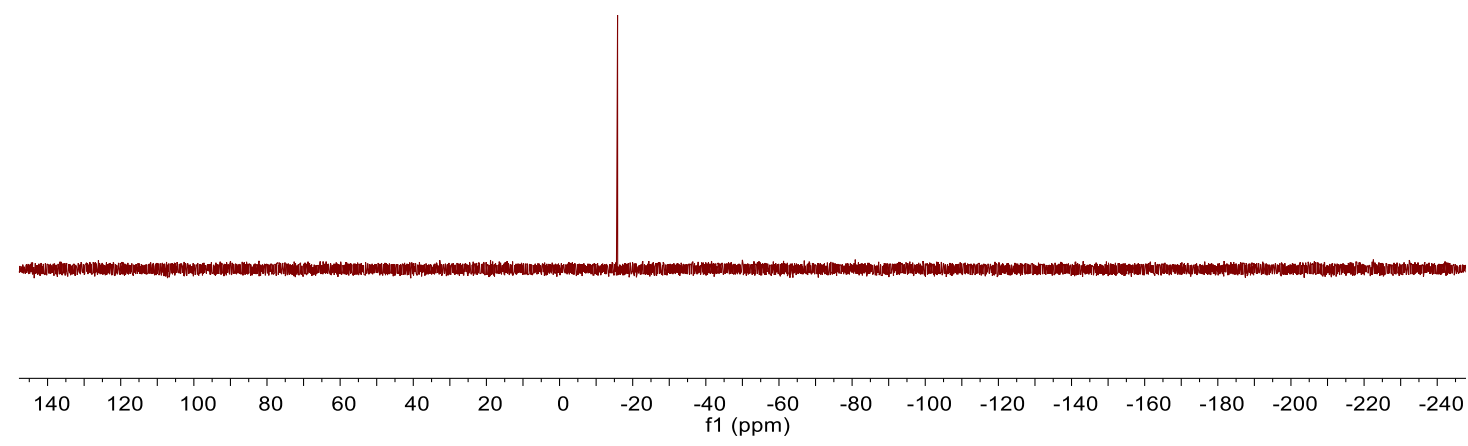

\section{Compound $\left(S_{\mathrm{a}}, \boldsymbol{R}\right)$-S5b}

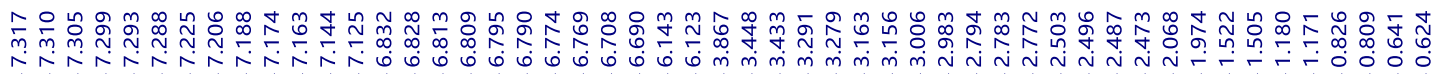

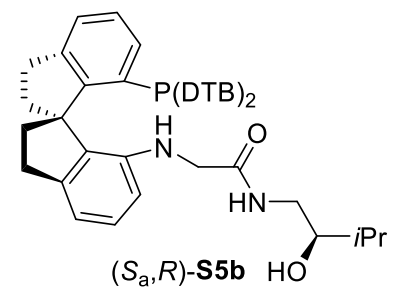

${ }^{1} \mathrm{H} \mathrm{NMR}\left(400 \mathrm{MHz}, \mathrm{CDCl}_{3}\right)$

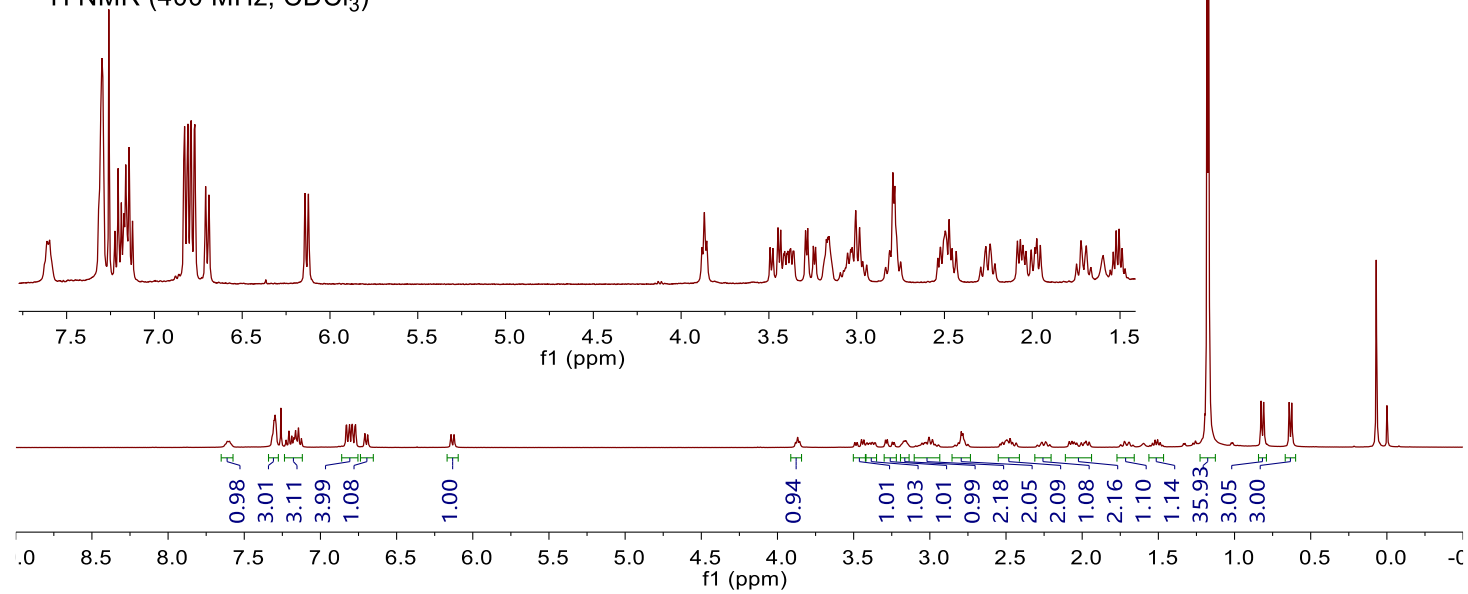




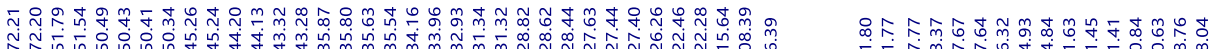

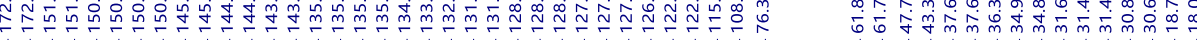

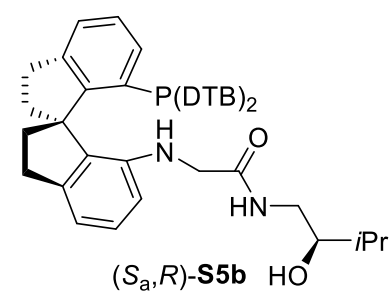

${ }^{13} \mathrm{C} \mathrm{NMR}\left(101 \mathrm{MHz}, \mathrm{CDCl}_{3}\right)$
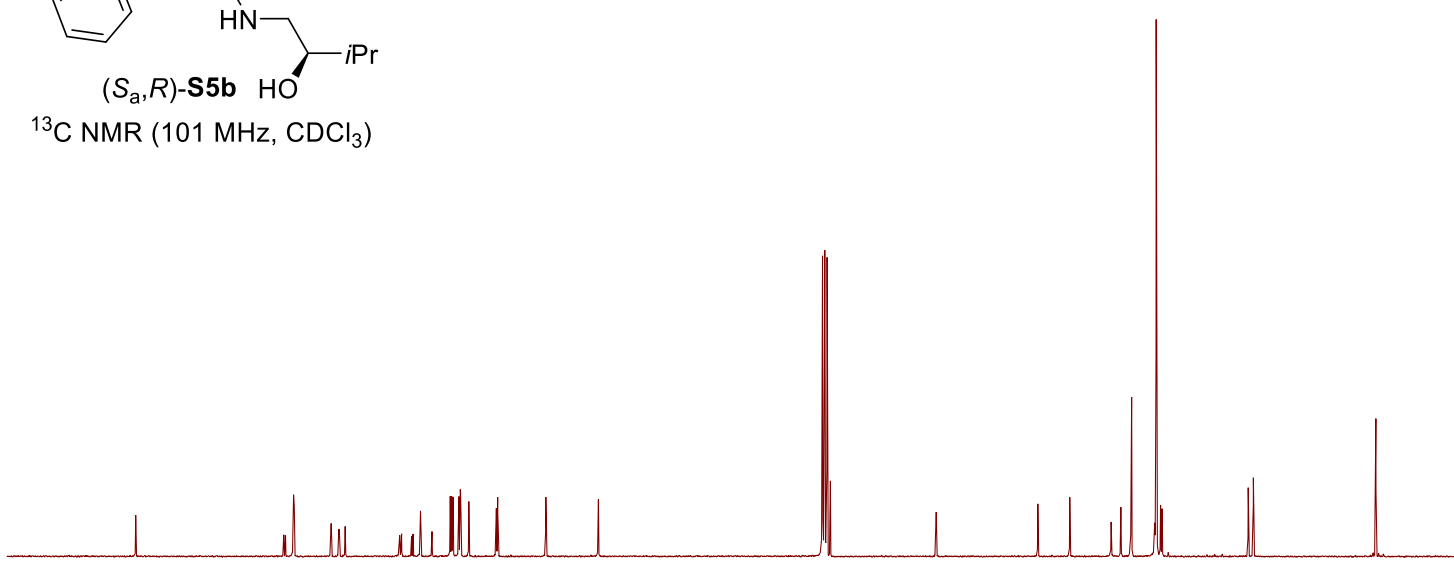

$\begin{array}{llllllllllllllllllllll}1 & 180 & 170 & 160 & 150 & 140 & 130 & 120 & 110 & 100 & 90 & 90 & 70 & 60 & 50 & 40 & 30 & 20 & 10 & 0 & -\end{array}$

$$
\begin{aligned}
& \hat{0} \\
& \text { in } \\
& i \\
& i
\end{aligned}
$$

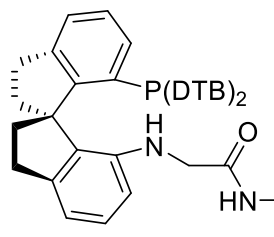

$\left(S_{\mathrm{a}}, R\right)-\mathbf{S 5 b} \mathrm{HO}$

${ }^{31} \mathrm{P}$ NMR $\left(162 \mathrm{MHz}, \mathrm{CDCl}_{3}\right)$

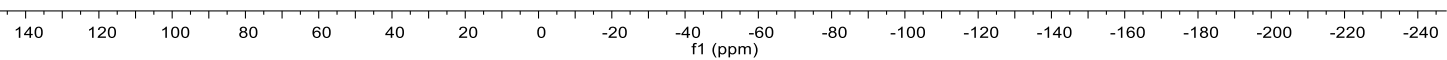




\section{Compound $\left(S_{\mathrm{a}}, \boldsymbol{R}\right)-\mathrm{S5c}$}
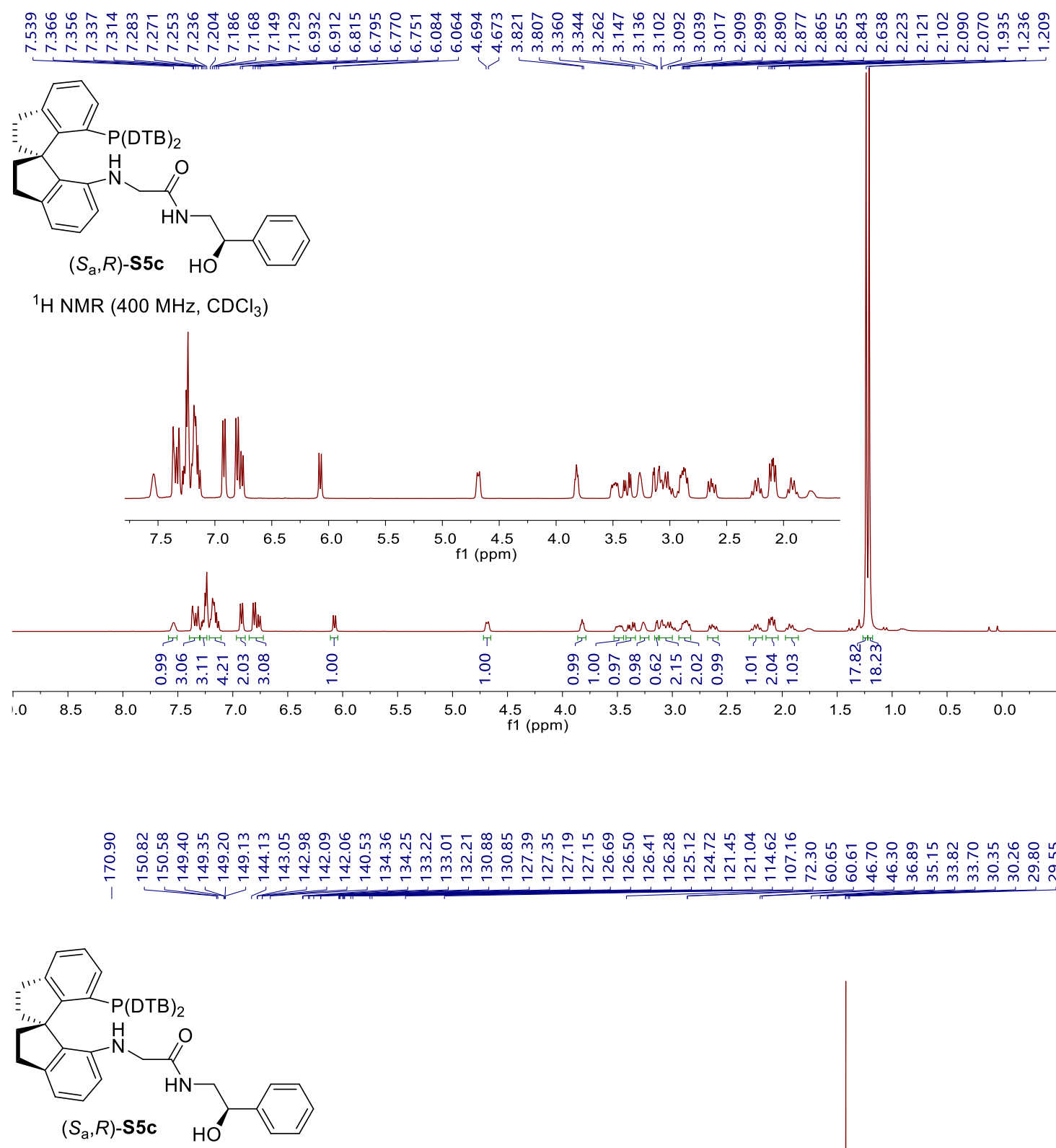

${ }^{13} \mathrm{C} \mathrm{NMR}\left(101 \mathrm{MHz}, \mathrm{CDCl}_{3}\right)$

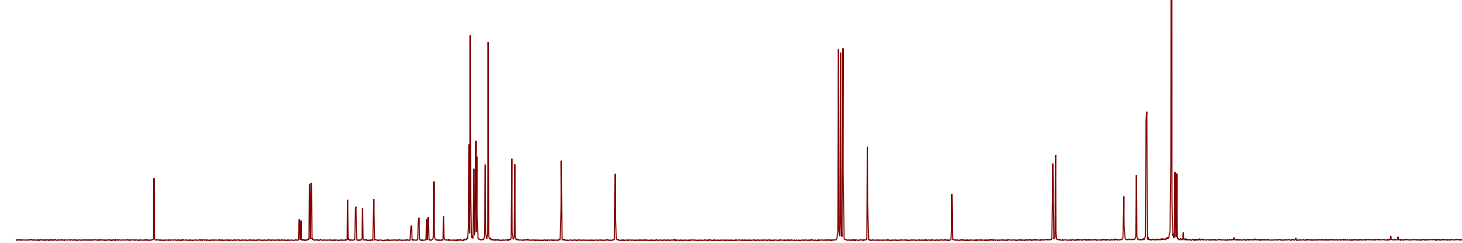

$\begin{array}{lllllllllllllllllllllllll}90 & 180 & 170 & 160 & 150 & 140 & 130 & 120 & 110 & 100 & 90 & 90 & 70 & 60 & 50 & 40 & 30 & 20 & 10 & 0 & -\end{array}$ 


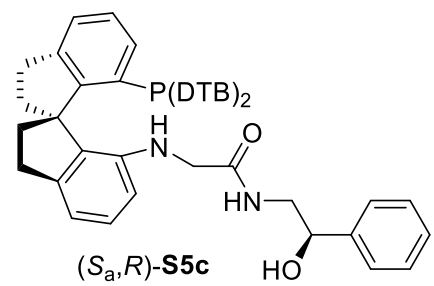

${ }^{31} \mathrm{P}$ NMR $\left(162 \mathrm{MHz}, \mathrm{CDCl}_{3}\right)$

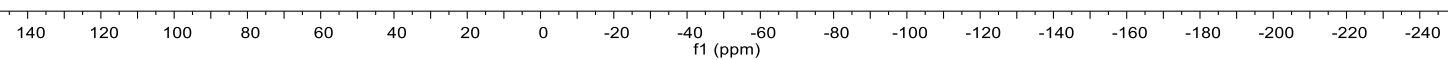

\section{Compound $\left(S_{\mathrm{a}}, S\right)$-S5c}

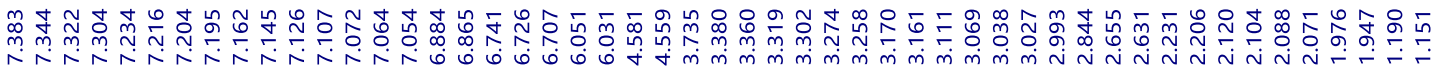

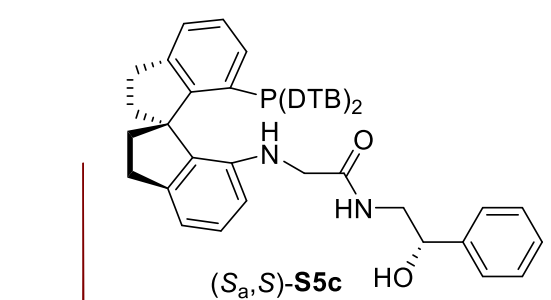

${ }^{1} \mathrm{H}$ NMR $\left(400 \mathrm{MHz}, \mathrm{CDCl}_{3}\right)$
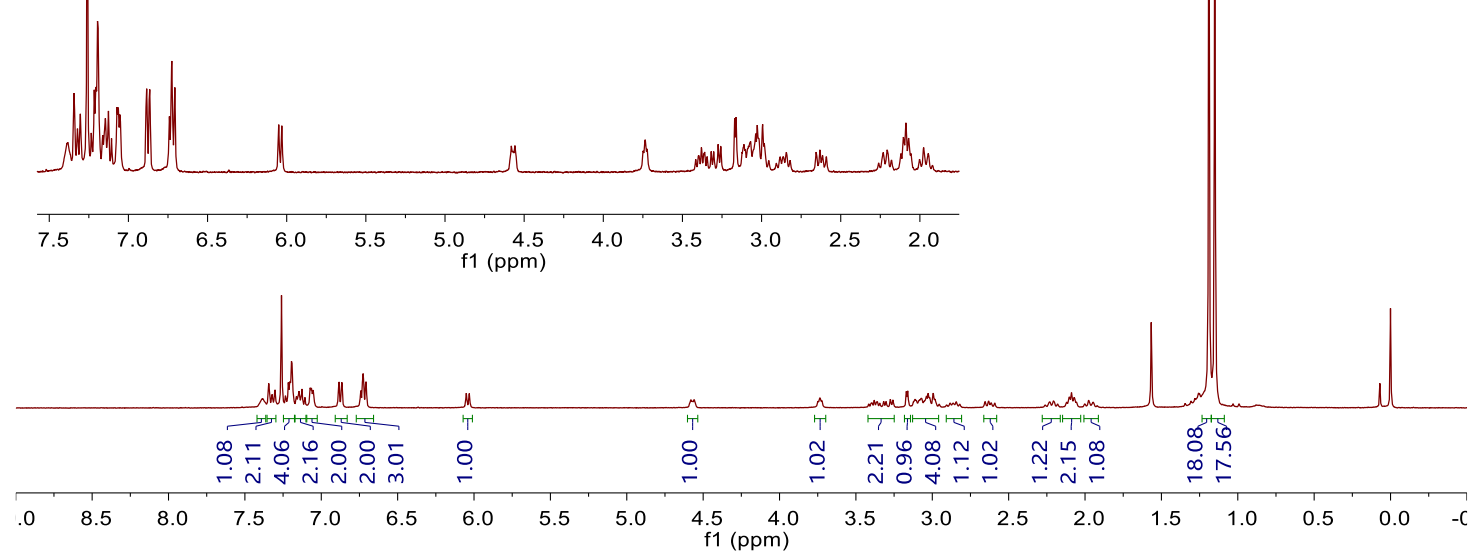


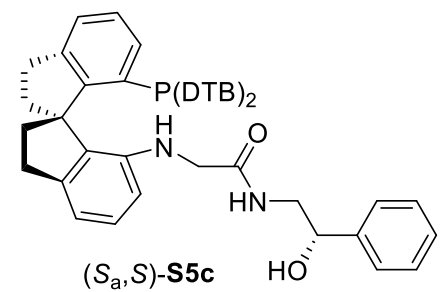

${ }^{13} \mathrm{C} \mathrm{NMR}\left(101 \mathrm{MHz}, \mathrm{CDCl}_{3}\right)$
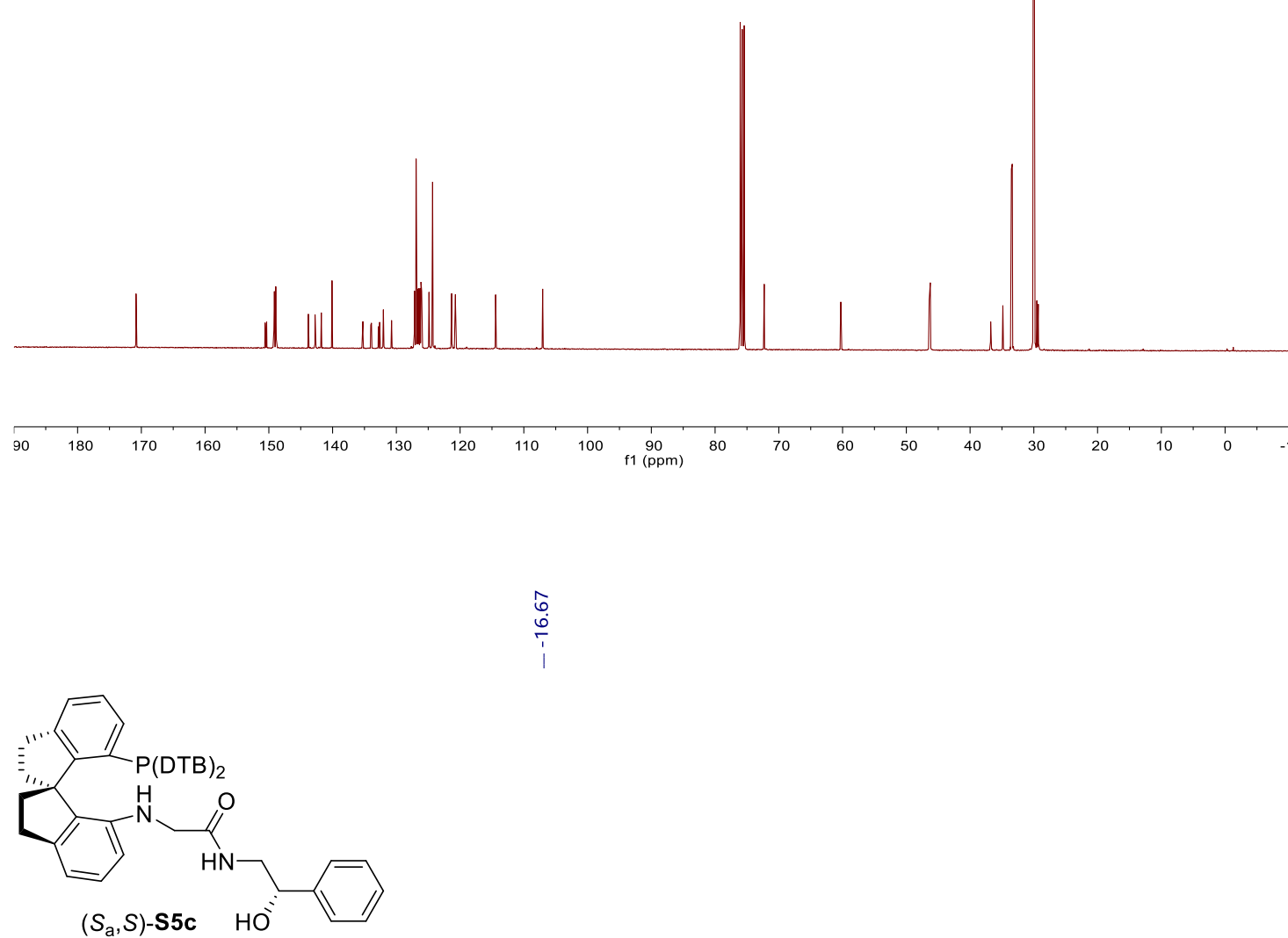

${ }^{31} \mathrm{P}$ NMR $\left(162 \mathrm{MHz}, \mathrm{CDCl}_{3}\right)$

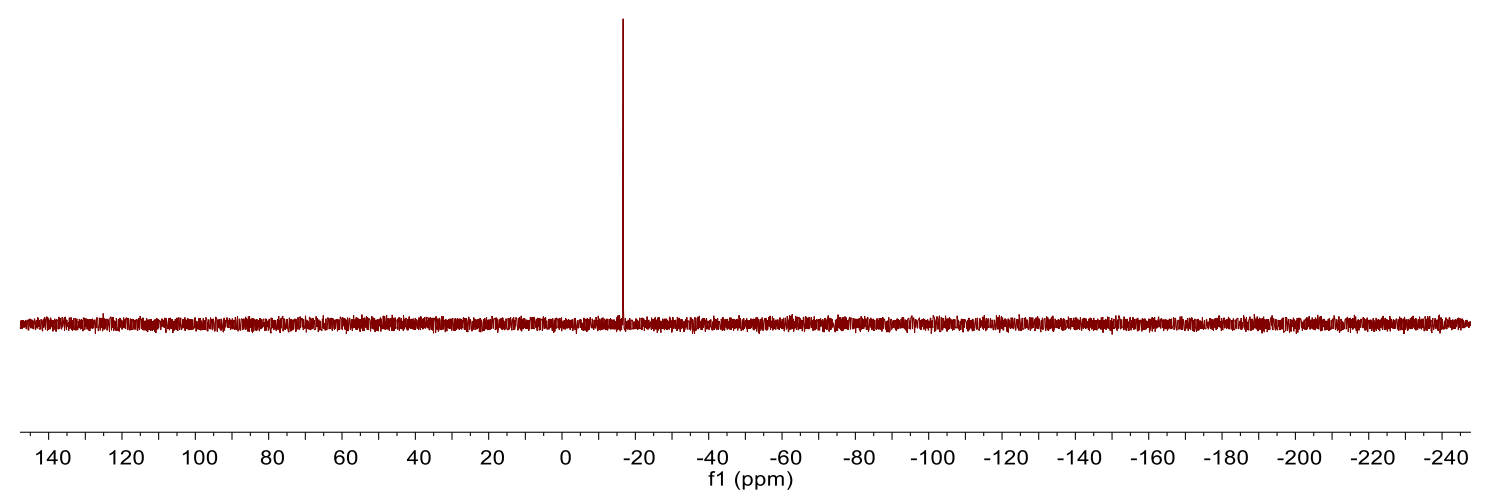




\section{Compound $\left(S_{\mathrm{a}}, R\right)-S 5 d$}

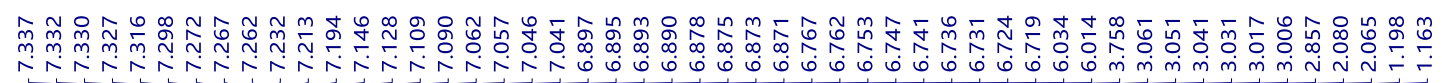

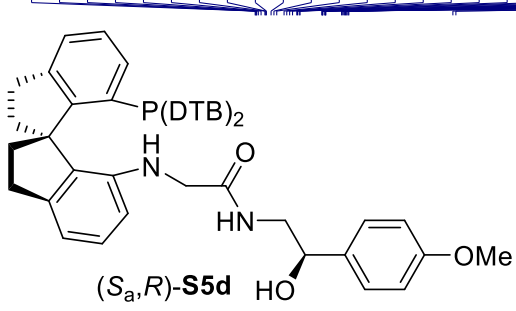

${ }^{1} \mathrm{H}$ NMR $\left(400 \mathrm{MHz}, \mathrm{CDCl}_{3}\right)$
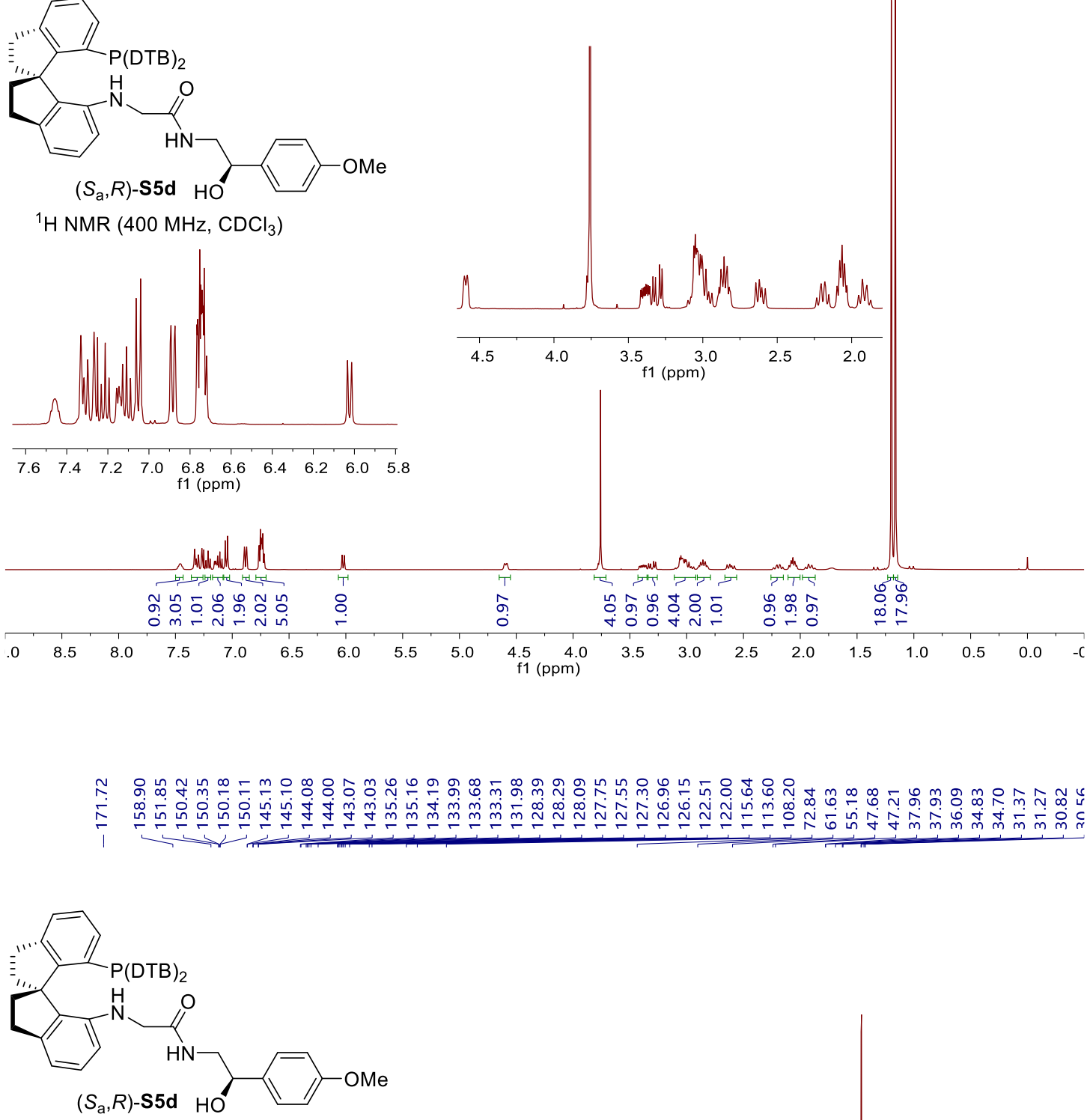

${ }^{13} \mathrm{C}$ NMR $\left(101 \mathrm{MHz}, \mathrm{CDCl}_{3}\right)$

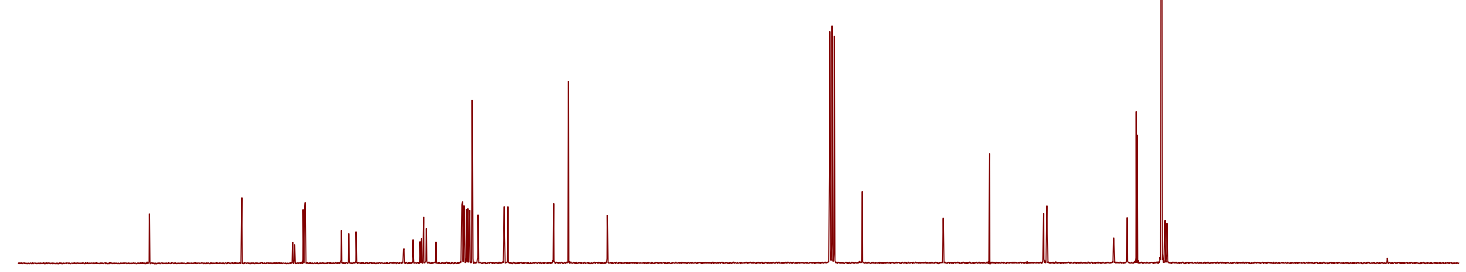

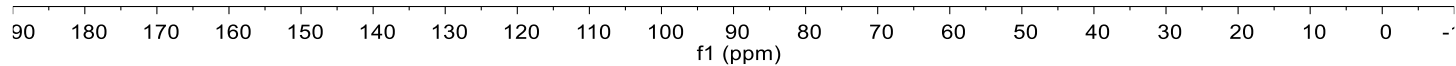




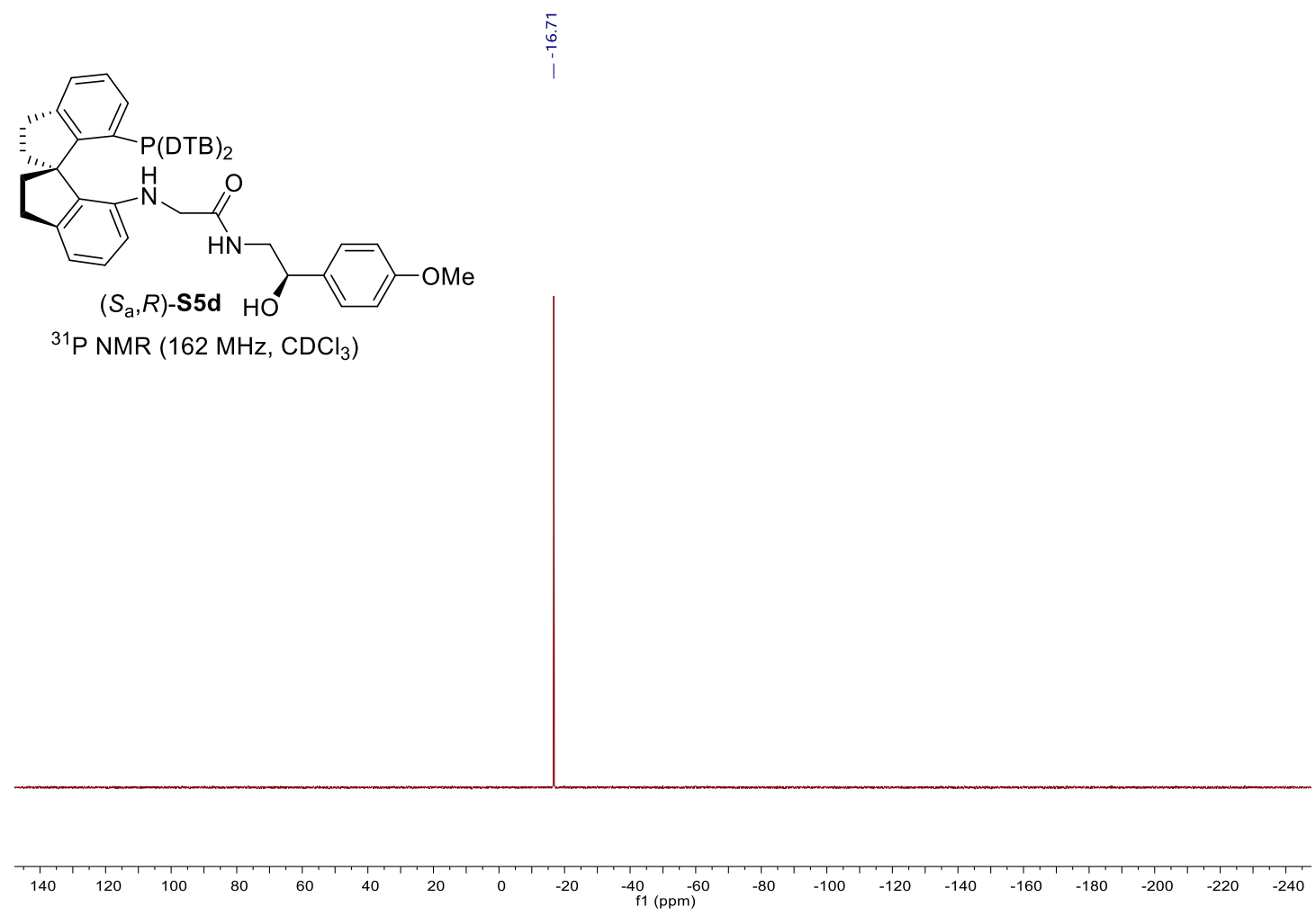

\section{Compound $\left(S_{\mathrm{a}}, R\right)-\mathrm{S} 5 \mathrm{e}$}

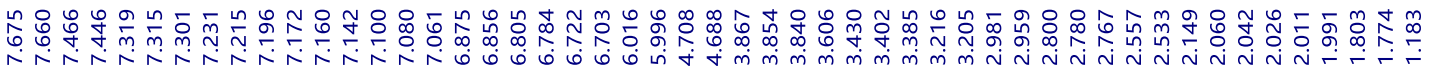

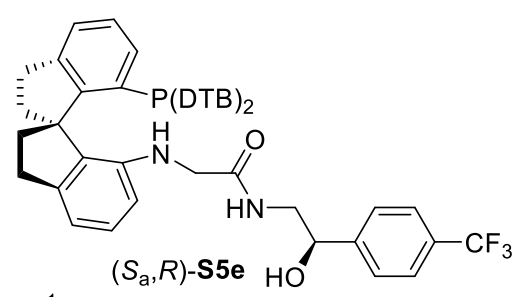

${ }^{1} \mathrm{H} \mathrm{NMR}\left(400 \mathrm{MHz}, \mathrm{CDCl}_{3}\right)$

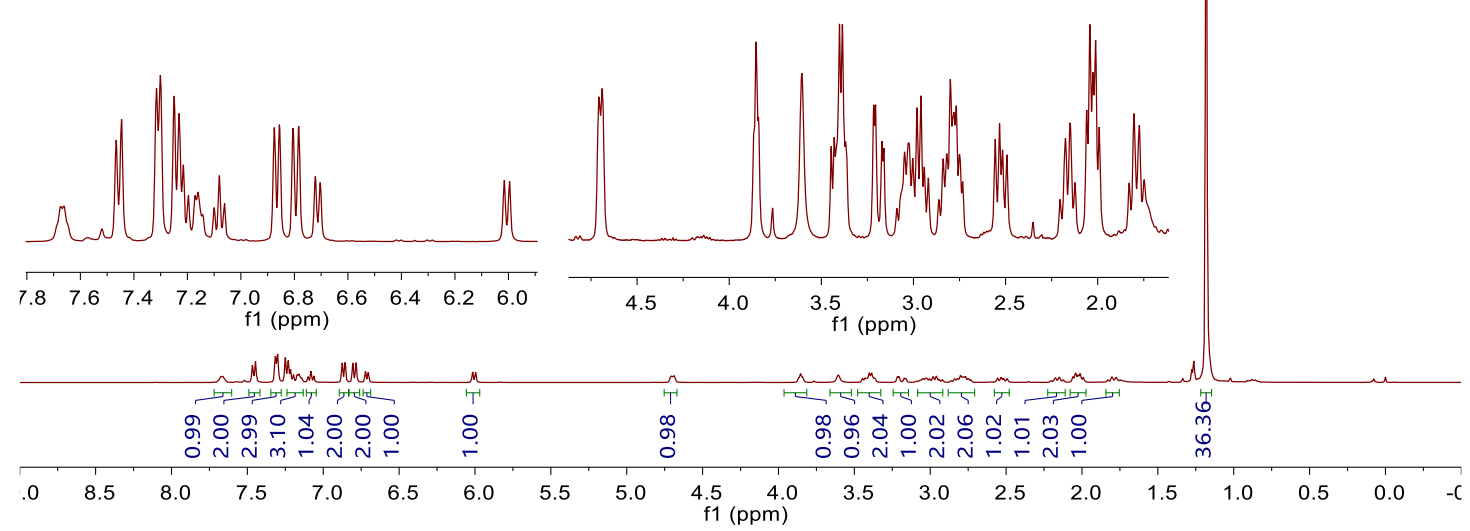




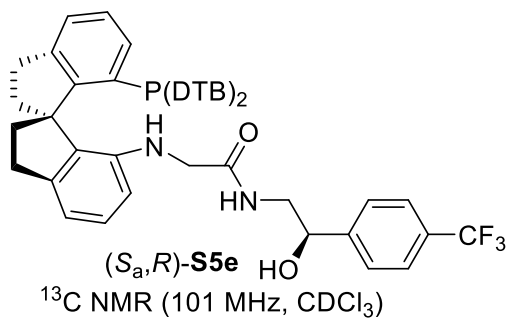

${ }^{3} \mathrm{C}$ NMR $\left(101 \mathrm{MHz}, \mathrm{CDCl}_{3}\right)$

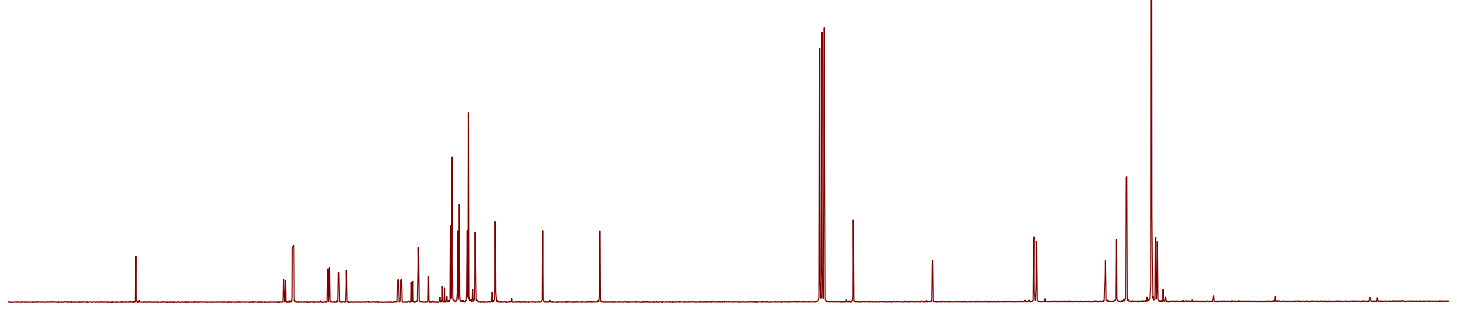

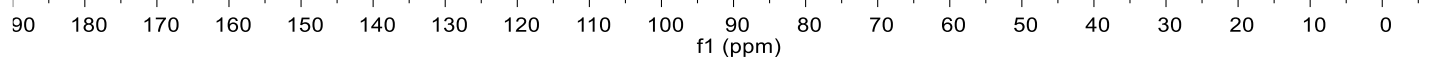<smiles>NC(=O)NCc1cccc2c1C1c3c(P)cccc3CCC21</smiles>

$\left(S_{\mathrm{a}}, R\right)$-S5e $\mathrm{HO}$

${ }^{31} \mathrm{P}$ NMR $\left(162 \mathrm{MHz}, \mathrm{CDCl}_{3}\right)$

$\begin{array}{llllllllllllllllllllll}140 & 120 & 100 & 80 & 60 & 40 & 20 & 0 & -20 & -40 & -60 & -80 & -100 & -120 & -140 & -160 & -180 & -200 & -220 & -240\end{array}$ 


\section{Compound $\left(S_{\mathrm{a}}, R\right)-\mathrm{S5f}$}

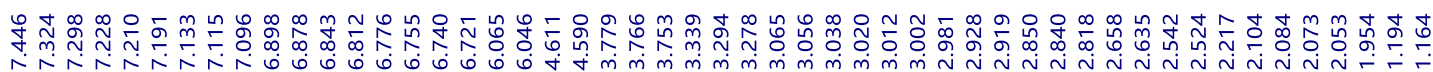

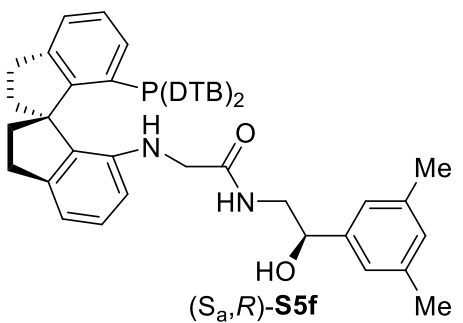

${ }^{1} \mathrm{H}$ NMR $\left(400 \mathrm{MHz}, \mathrm{CDCl}_{3}\right)$

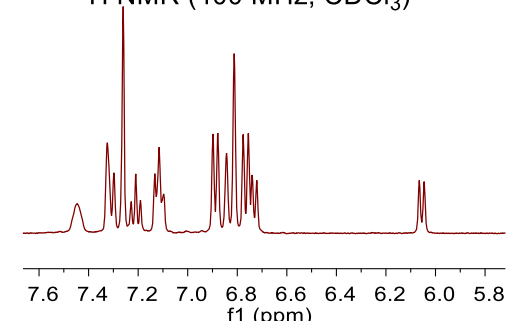
6.86 .6
$\mathrm{f} 1(\mathrm{ppm})$
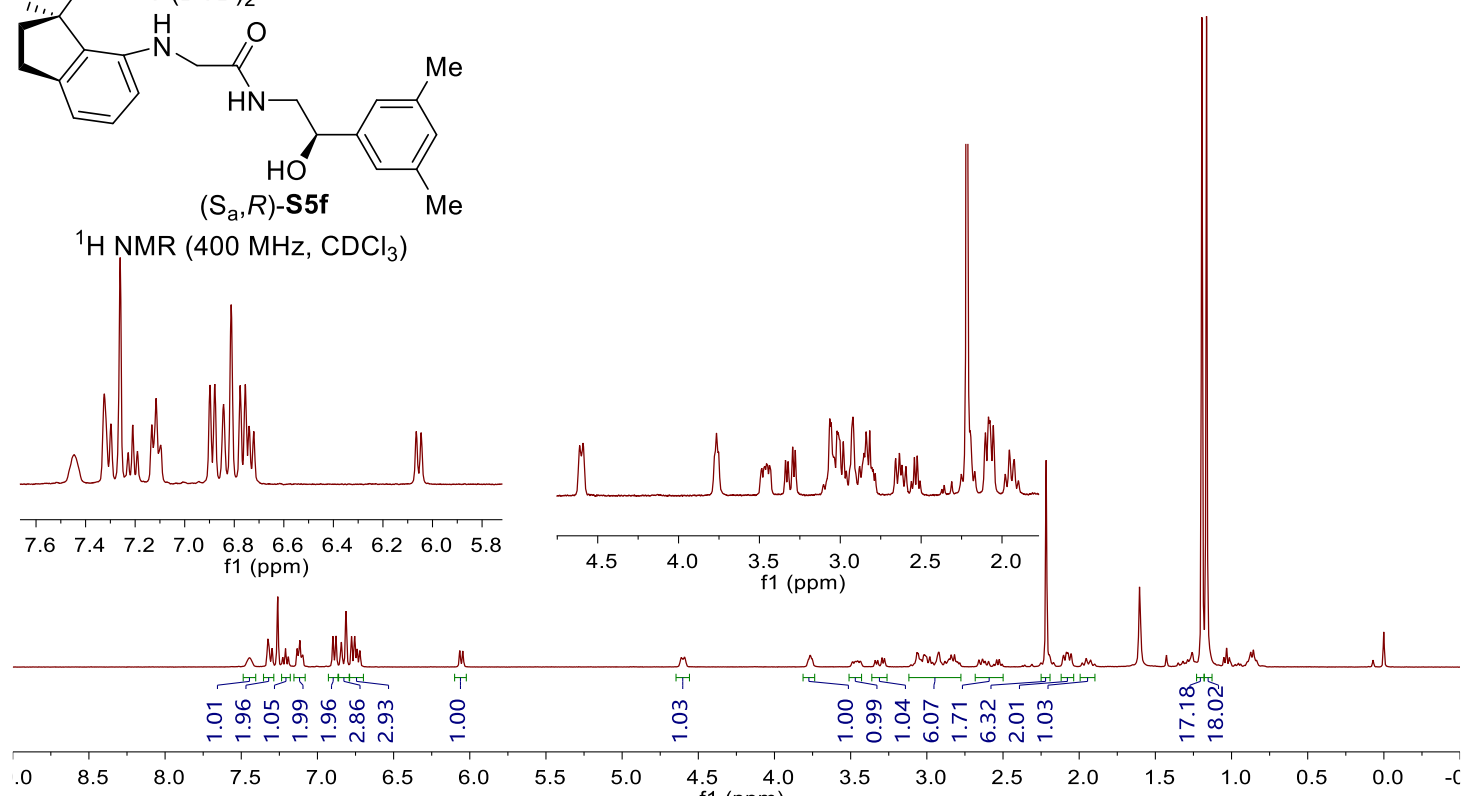

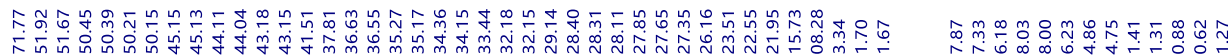

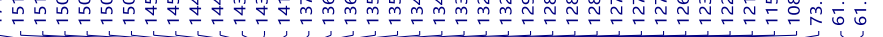

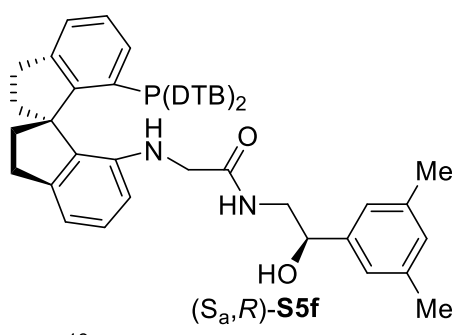

${ }^{13} \mathrm{C}$ NMR $\left(101 \mathrm{MHz}, \mathrm{CDCl}_{3}\right)$

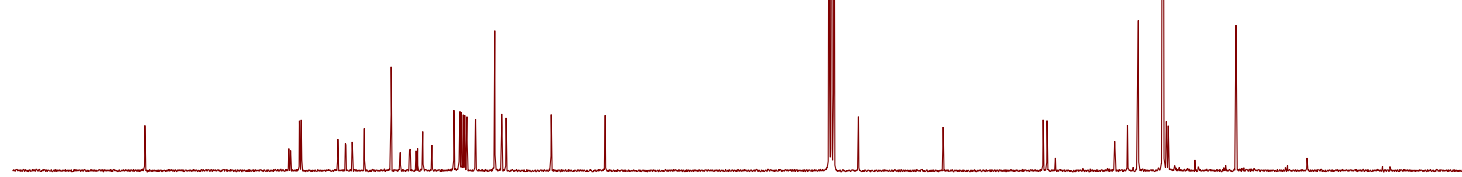

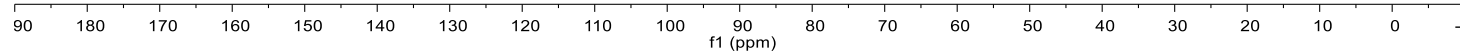



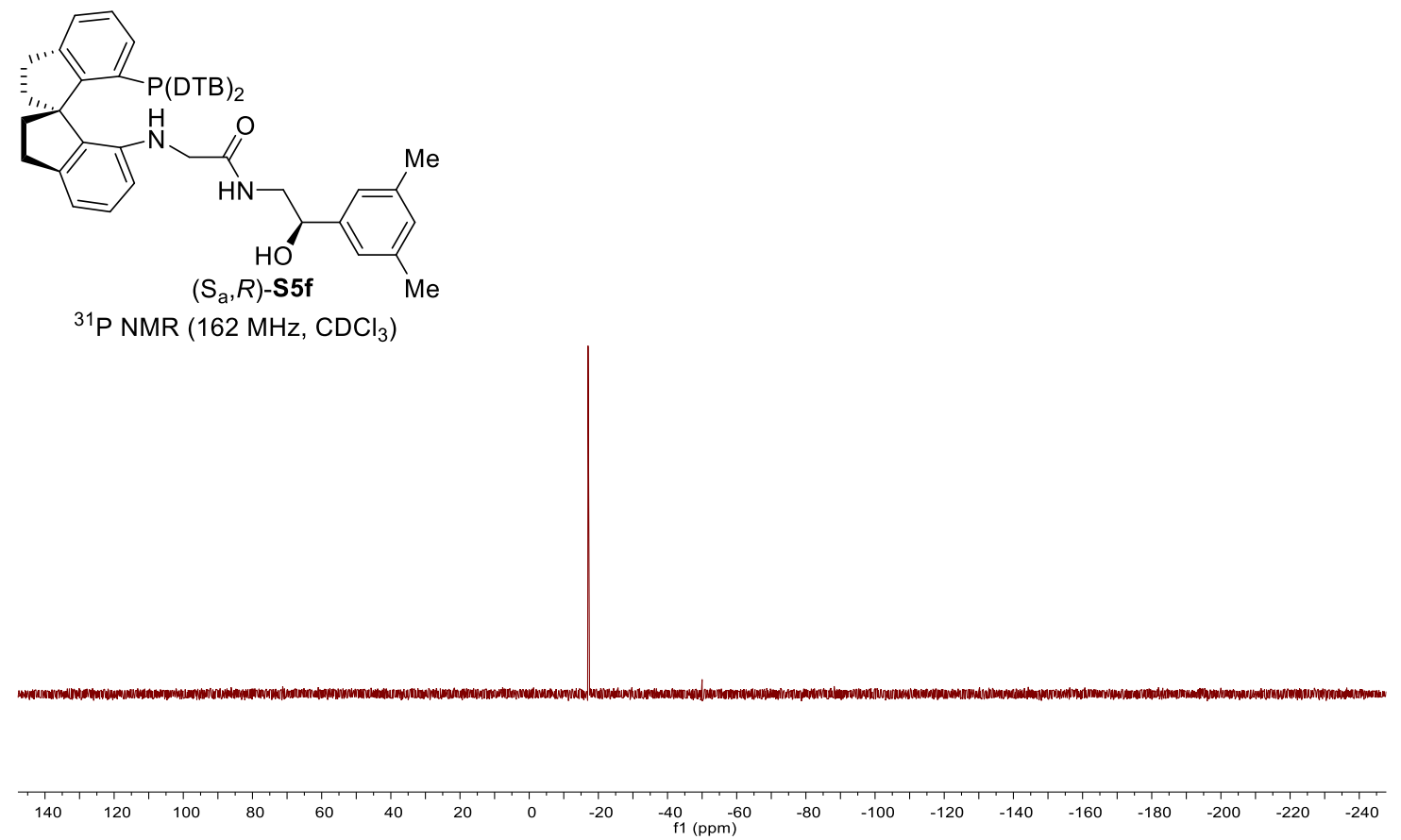

\section{Ligand $\left(S_{\mathrm{a}}, S\right)$-S6a}

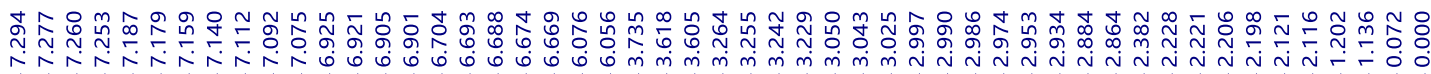

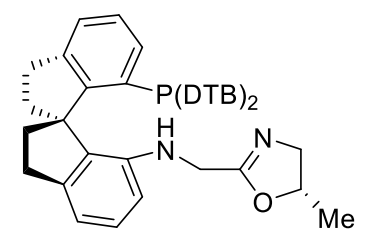

$\left(S_{a}, S\right)-\mathbf{S 6 a}$

${ }^{1} \mathrm{H}$ NMR $\left(400 \mathrm{MHz}, \mathrm{CDCl}_{3}\right)$

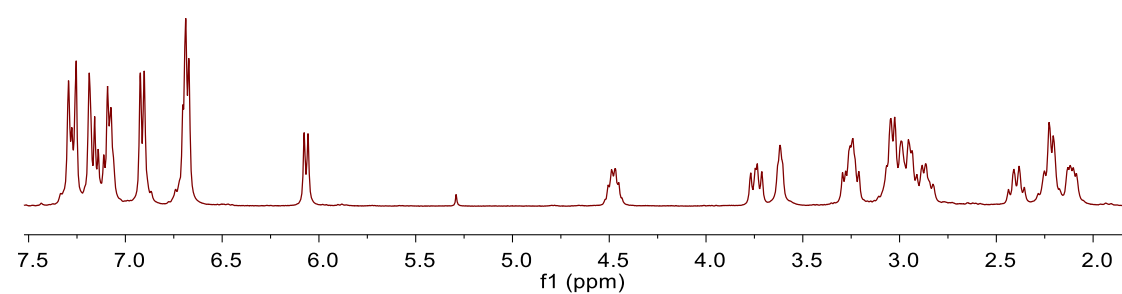

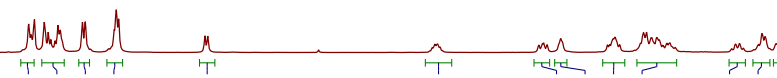

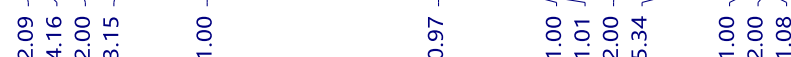
它

\begin{tabular}{llllllllllllllllllllll}
\hline .0 & 8.5 & 8.0 & 7.5 & 7.0 & 6.5 & 6.0 & 5.5 & 5.0 & 4.5 & $\underset{\mathrm{f} 1}{4}(\mathrm{ppm})$ & 3.5 & 3.0 & 2.5 & 2.0 & 1.5 & 1.0 & 0.5 & 0.0 & -0.5 & -1
\end{tabular} 


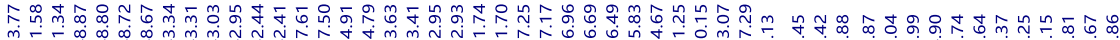

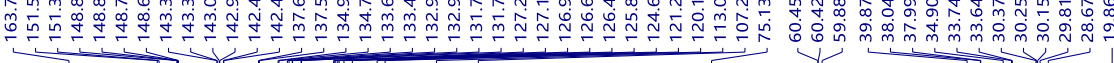

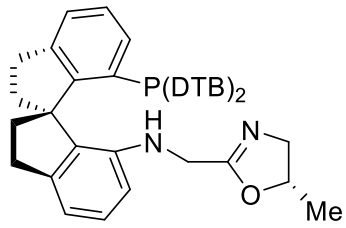

$\left(S_{\mathrm{a}}, S\right)-\mathbf{S 6 a}$

${ }^{13} \mathrm{C}$ NMR $\left(101 \mathrm{MHz}, \mathrm{CDCl}_{3}\right)$

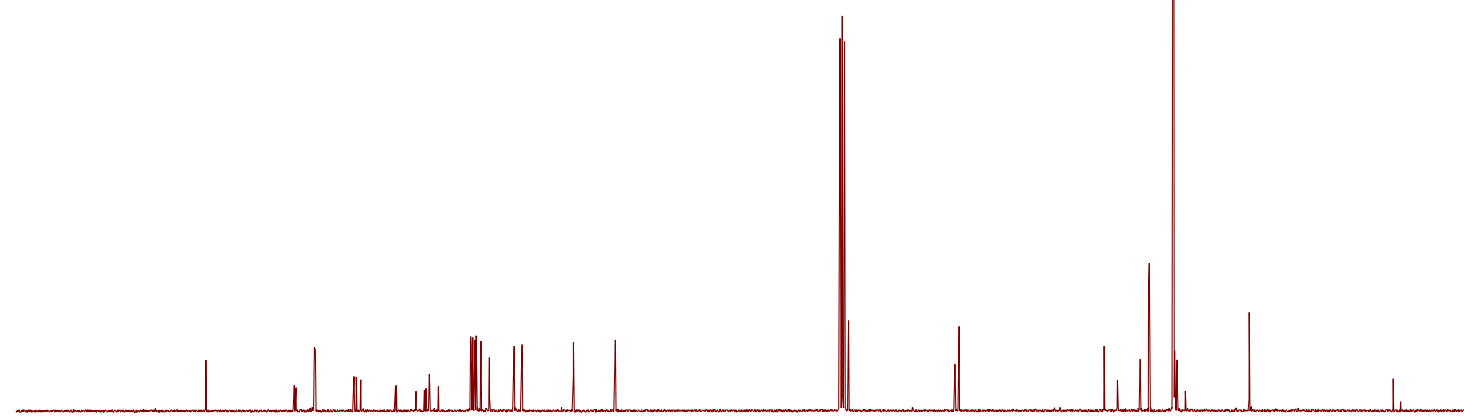

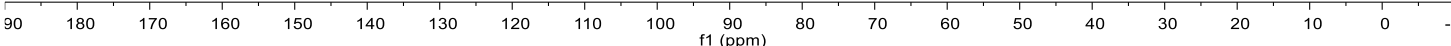

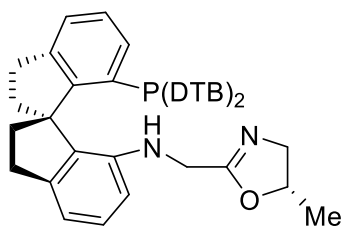

$\frac{\infty}{\frac{\pi}{1}}$

$\left(S_{a}, S\right)-\mathbf{S 6 a}$

${ }^{31} \mathrm{P}$ NMR $\left(162 \mathrm{MHz}, \mathrm{CDCl}_{3}\right)$

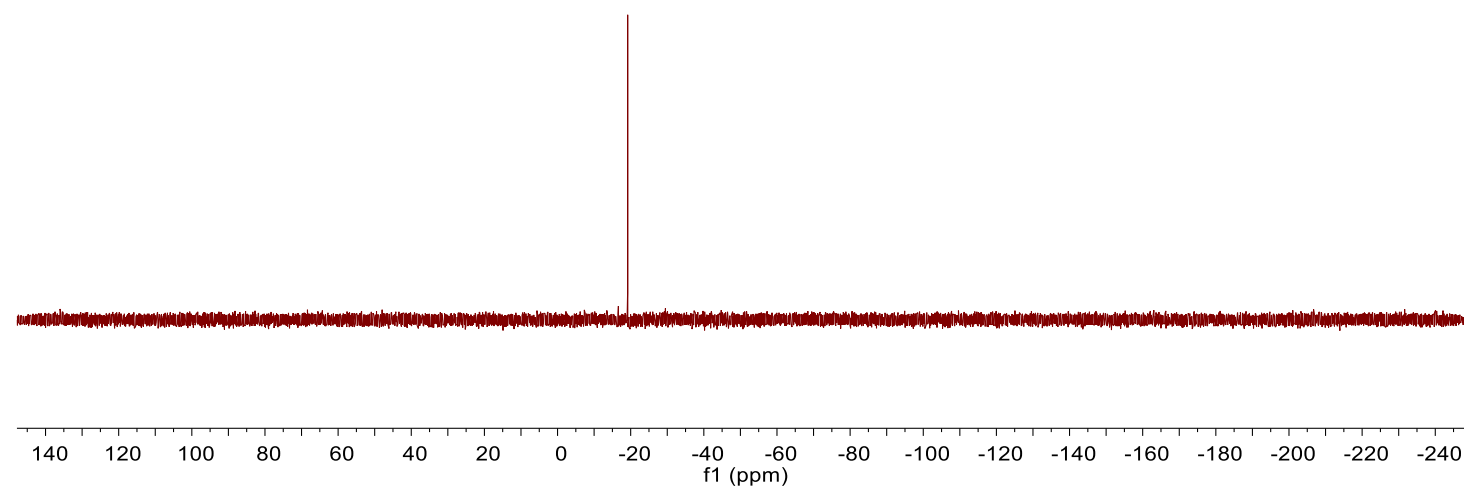




\section{Ligand $\left(S_{\mathrm{a}}, S\right)$-S6b}

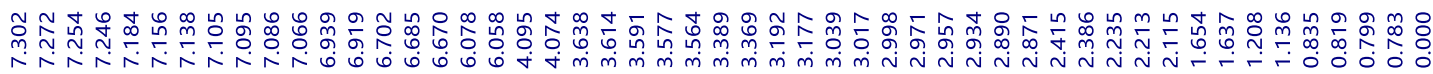

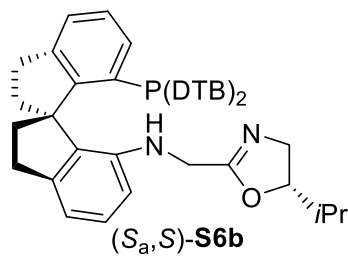

${ }^{1} \mathrm{H} \mathrm{NMR}\left(400 \mathrm{MHz}, \mathrm{CDCl}_{3}\right)$
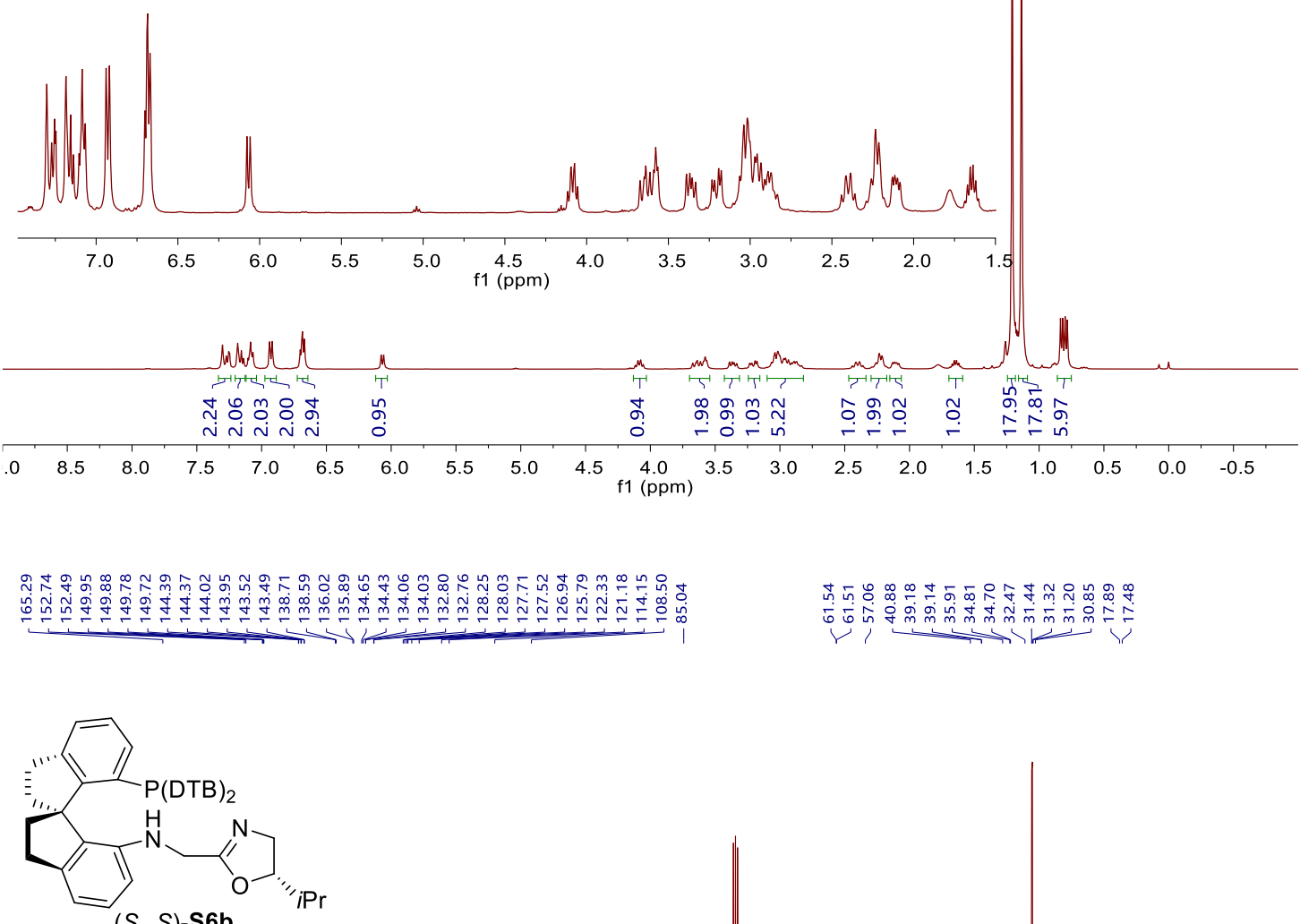

$\left(S_{a}, S\right)-\mathbf{S 6 b}$

${ }^{13} \mathrm{C}$ NMR $\left(101 \mathrm{MHz}, \mathrm{CDCl}_{3}\right)$

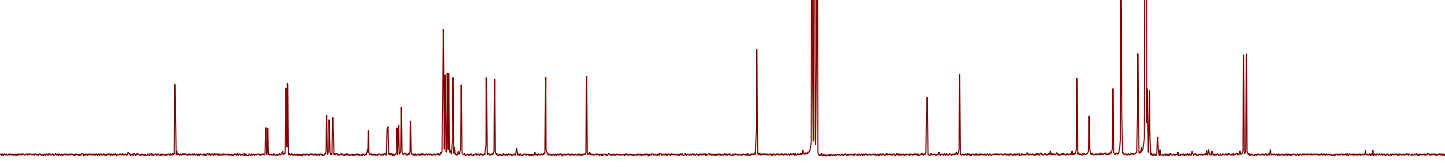

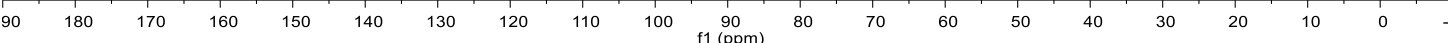




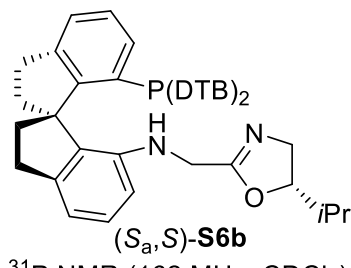

${ }^{31} \mathrm{P}$ NMR $\left(162 \mathrm{MHz} \mathrm{CDCl}_{3}\right)$

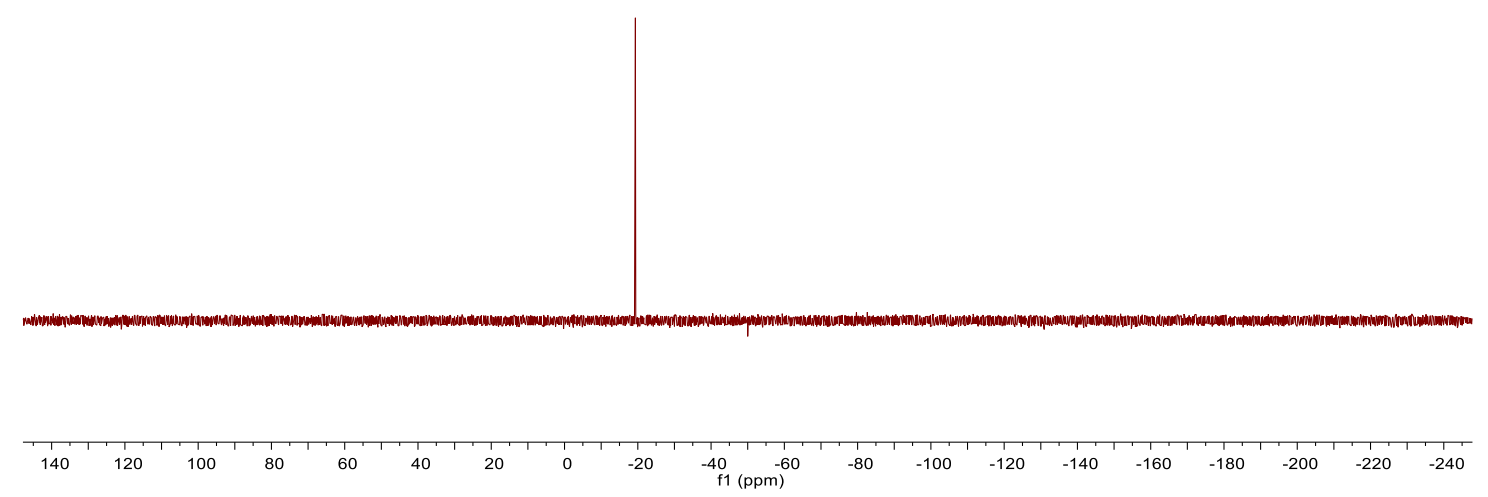

\section{Ligand $\left(S_{\mathrm{a}}, S\right)$-S6c}

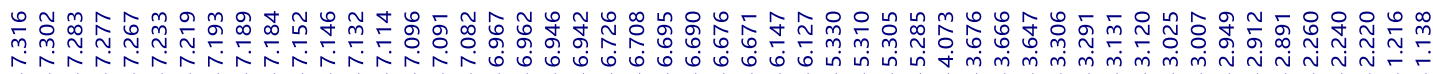

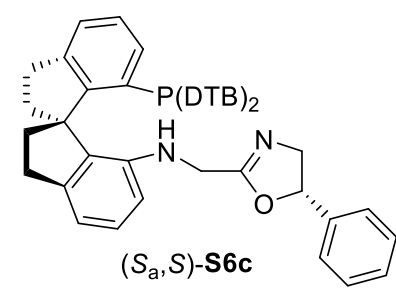

${ }^{1} \mathrm{H}$ NMR $\left(400 \mathrm{MHz}, \mathrm{CDCl}_{3}\right)$

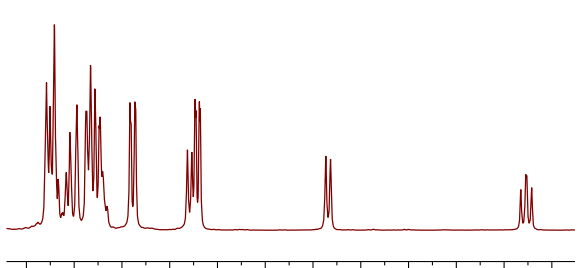
$\begin{array}{lllllllllllllll}7.4 & 7.2 & 7.0 & 6.8 & 6.6 & 6.4 & 6.2 & 6.0 & 5.8 & 5.6 & 5.4 & 5.2 \\ & & & & & & f(p p m)\end{array}$

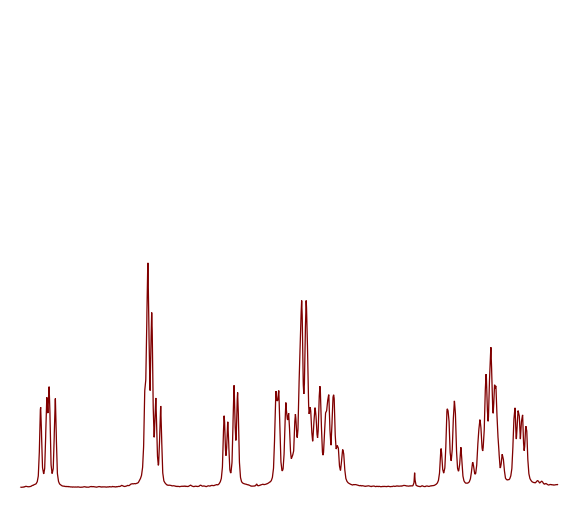

$\begin{array}{lllllllllll}4.0 & 3.8 & 3.6 & 3.4 & 3.2 & 3.0 & 2.8 & 2.6 & 2.4 & 2.2 & 2.0\end{array}$

whe

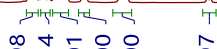

o̊

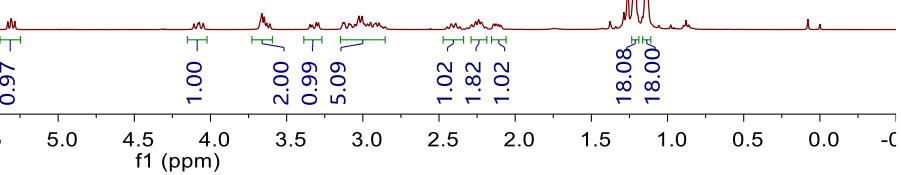




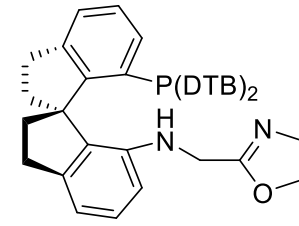

$\left(S_{\mathrm{a}}, S\right)-\mathbf{S 6 c}$<smiles>[c]1ccccc1</smiles>

${ }^{13} \mathrm{C} \mathrm{NMR}\left(101 \mathrm{MHz}, \mathrm{CDCl}_{3}\right)$

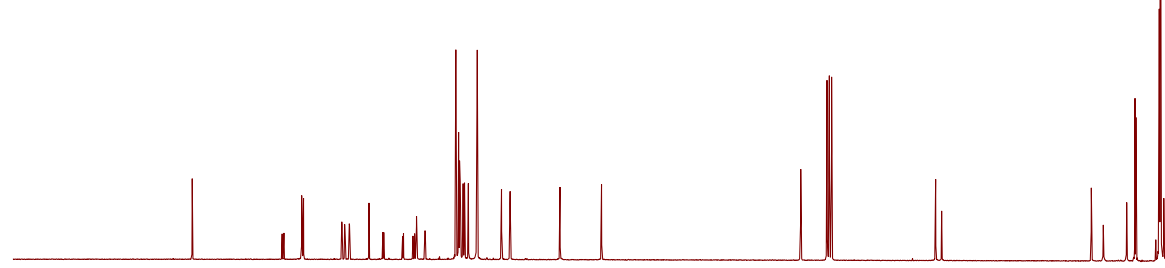

$\begin{array}{lllllllllllllllllllll}90 & 180 & 170 & 160 & 150 & 140 & 130 & 120 & 110 & 100 & \begin{array}{c}90 \\ \mathrm{f} 1(\mathrm{ppm})\end{array} & 80 & 70 & 60 & 50 & 40 & 30 & 20 & 10 & 0 & -\end{array}$

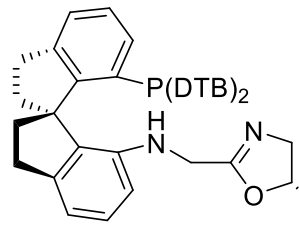

$\left(S_{\mathrm{a}}, S\right)-\mathrm{S} 6 \mathrm{c}$<smiles>c1ccccc1</smiles>

${ }^{31} \mathrm{P}$ NMR $\left(162 \mathrm{MHz}, \mathrm{CDCl}_{3}\right)$

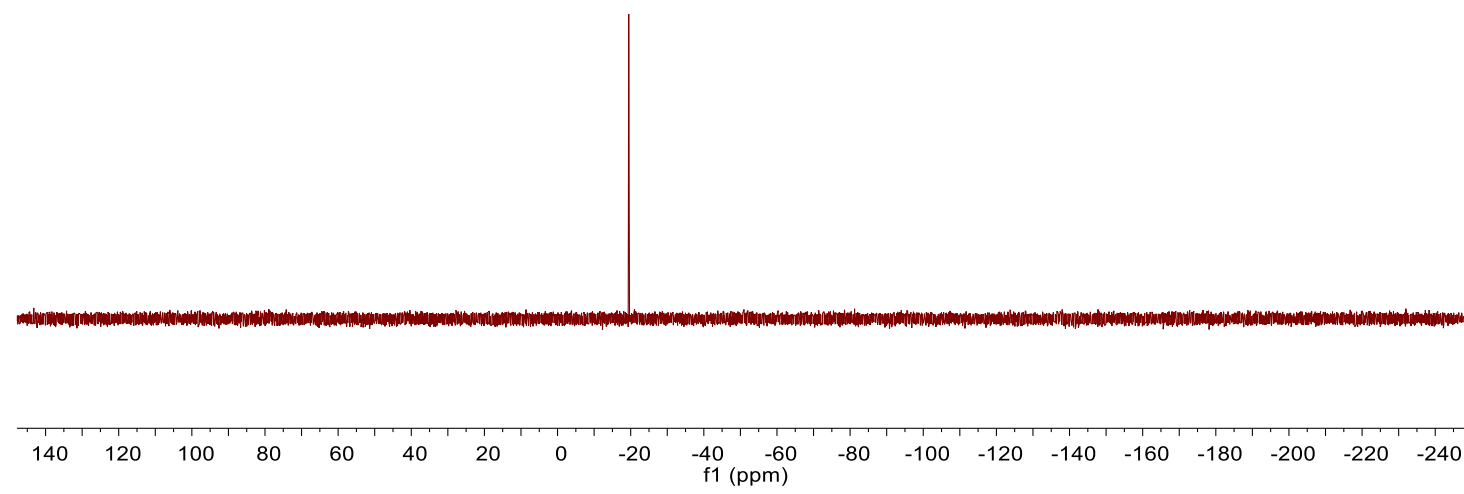




\section{Ligand $\left(S_{\mathrm{a},}, R\right)$-S6c}

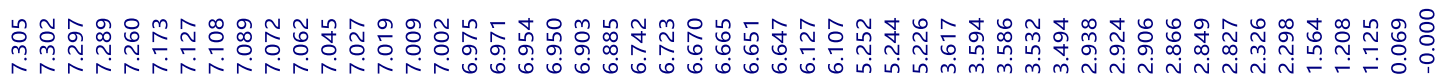

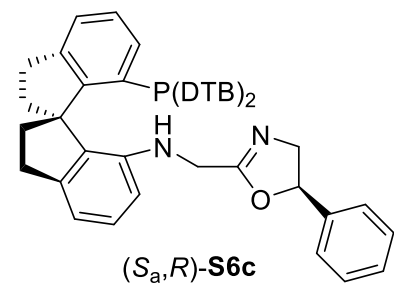

${ }^{1} \mathrm{H} \mathrm{NMR}\left(400 \mathrm{MHz}, \mathrm{CDCl}_{3}\right)$
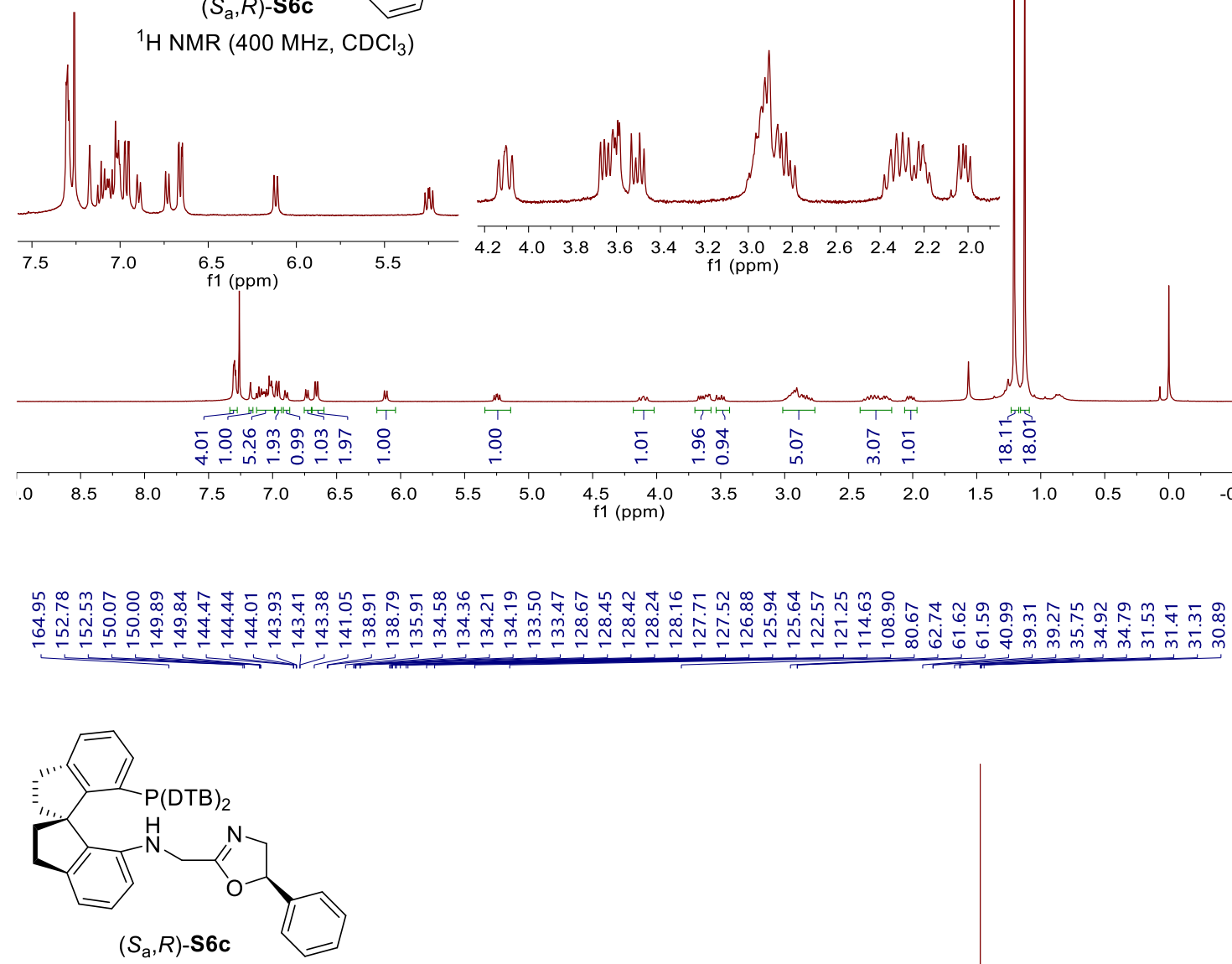

${ }^{13} \mathrm{C}$ NMR $\left(101 \mathrm{MHz}, \mathrm{CDCl}_{3}\right)$

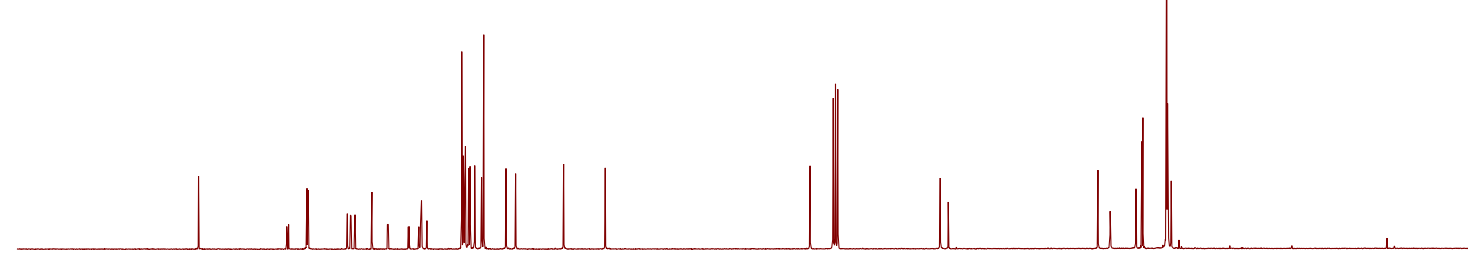

$\begin{array}{llllllllllllllllllllllll}90 & 180 & 170 & 160 & 150 & 140 & 130 & 120 & 110 & 100 & 90 & 80 & 70 & 60 & 50 & 40 & 30 & 20 & 10 & 0 & -\end{array}$ 


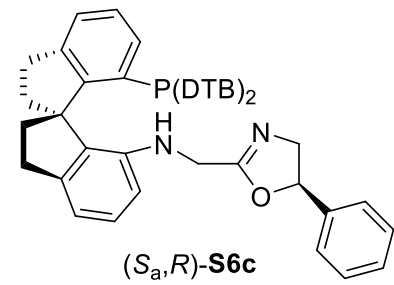

${ }^{31} \mathrm{P}$ NMR $\left(162 \mathrm{MHz}, \mathrm{CDCl}_{3}\right)$

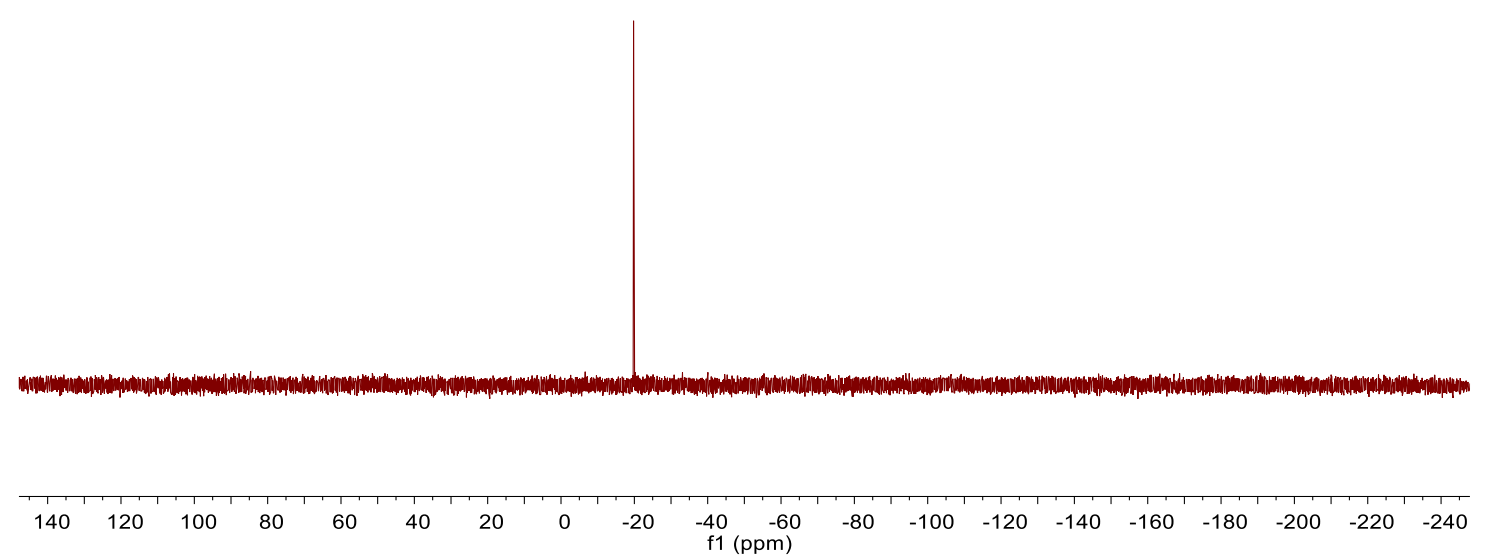

\section{Ligand $\left(S_{\mathrm{a}}, S\right)$-S6d}

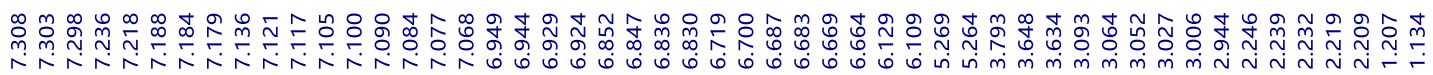

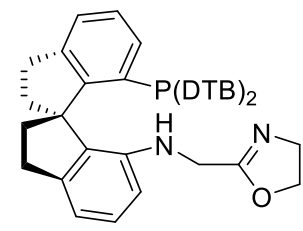

$\left(S_{\mathrm{a}}, S\right)-\mathbf{S 6 d}$

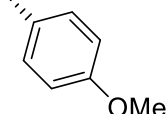

${ }^{1} \mathrm{H}$ NMR $\left(400 \mathrm{MHz}, \mathrm{CDCl}_{3}\right)$

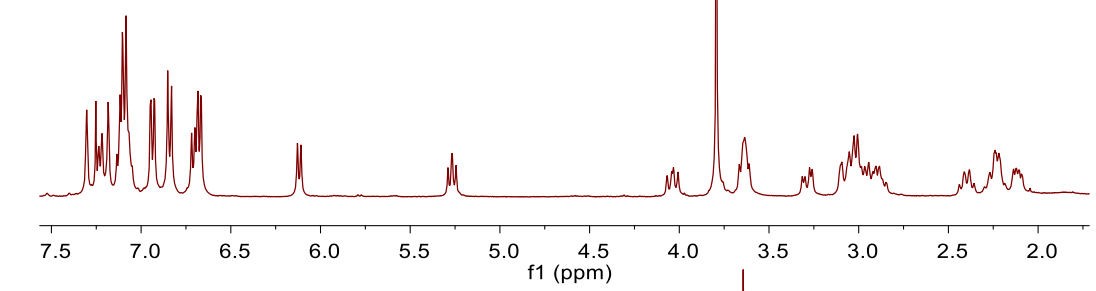

molld

0.1500

ดे نं نं

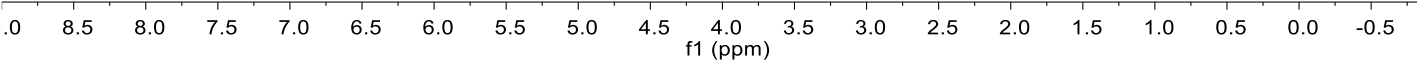




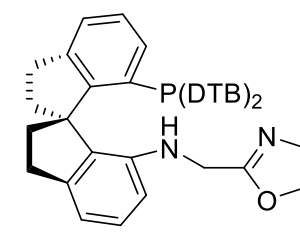

$\left(S_{a}, S\right)-\mathbf{S 6 d}$ ${ }^{13} \mathrm{C}$ NMR $\left(101 \mathrm{MHz}, \mathrm{CDCl}_{3}\right)$<smiles>COc1ccccc1</smiles> 


\section{Ligand $\left(S_{\mathrm{a}, S}\right)-\mathrm{S} 6 \mathrm{e}$}

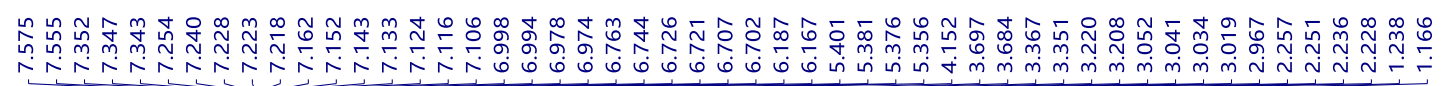

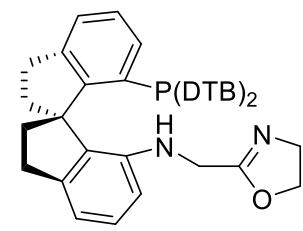

$\left(S_{\mathrm{a}}, S\right)-\mathrm{S6e}$

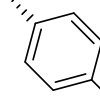

${ }^{1} \mathrm{H}$ NMR $\left(400 \mathrm{MHz}, \mathrm{CDCl}_{3}\right) \quad \mathrm{CF}_{3}$
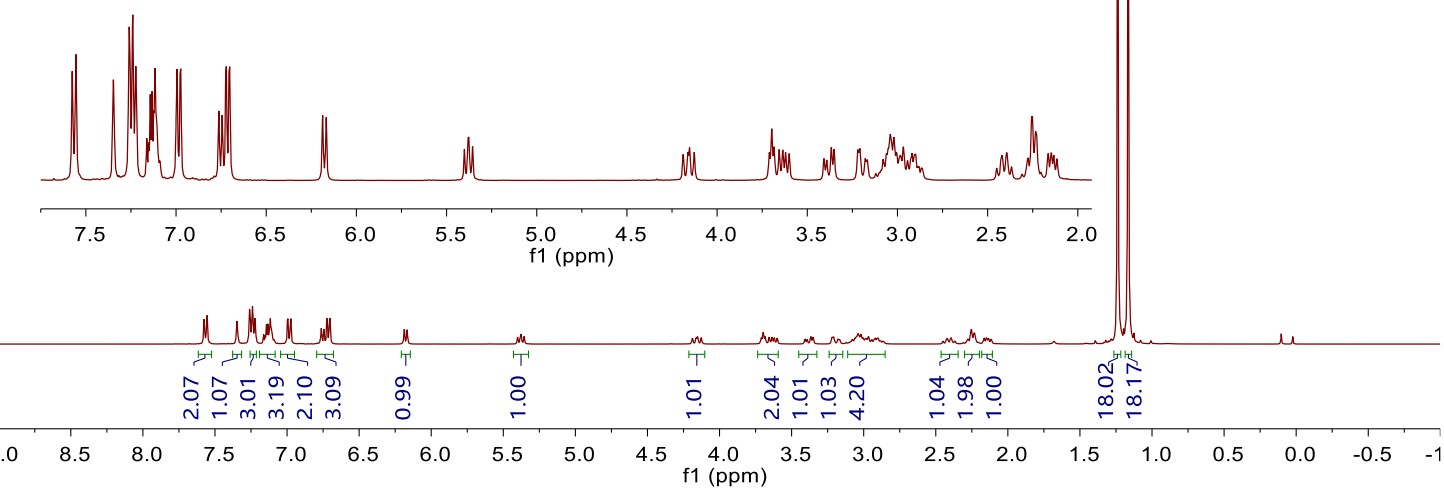

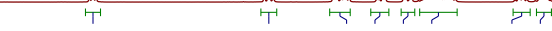

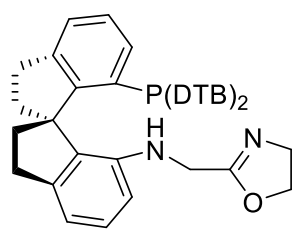

$\left(S_{a}, S\right)-\mathbf{S 6 e}$

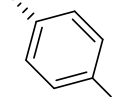

${ }^{13} \mathrm{C}$ NMR $\left(101 \mathrm{MHz}, \mathrm{CDCl}_{3}\right) \mathrm{CF}_{3}$

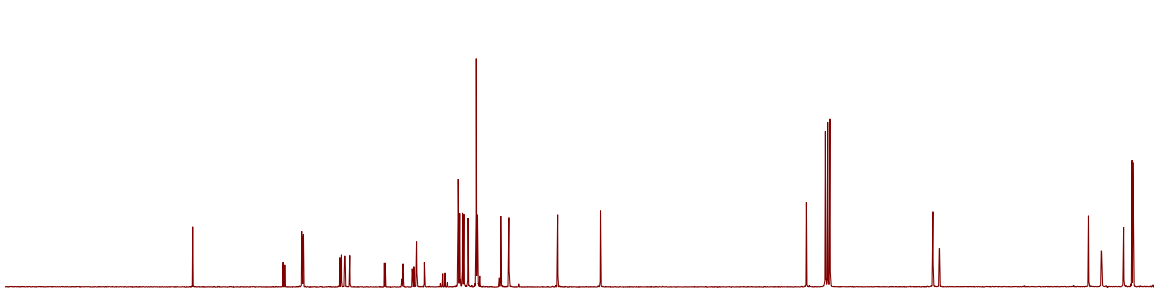

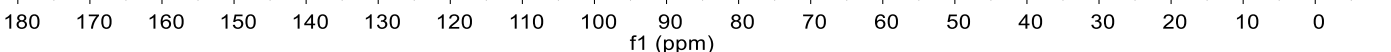




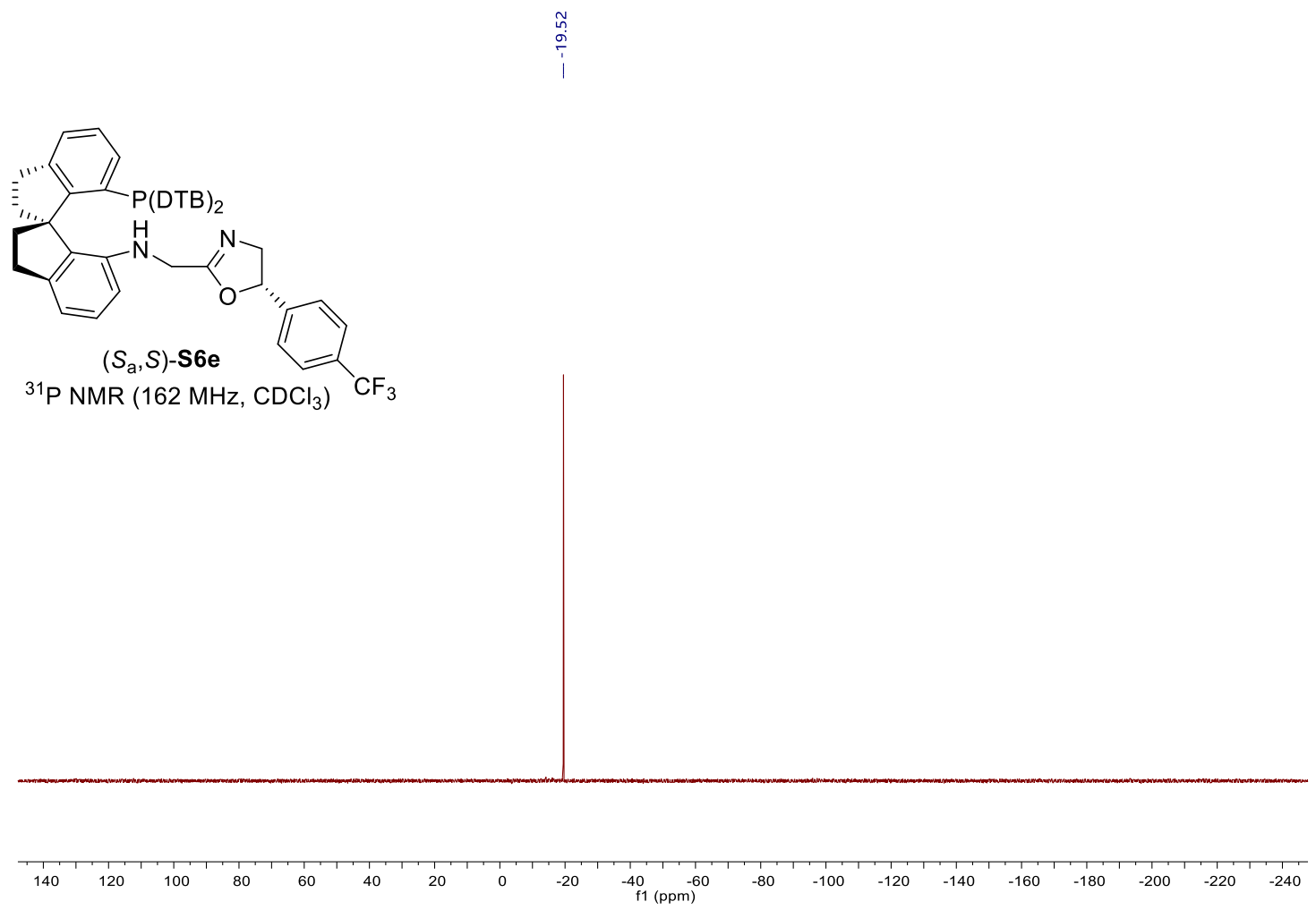

\section{Ligand $\left(S_{\mathrm{a}}, S\right)$-S6f}

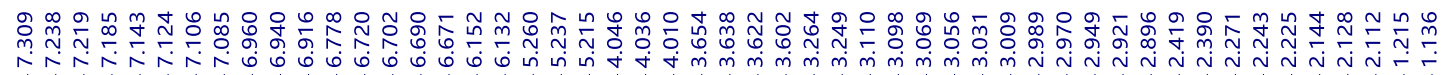<smiles>COc1cc(C)cc([C@H]2CN=C(CNc3cccc4c3[C@@]35CC[C@H]3CC[C@H]4C5)O2)c1</smiles>

${ }^{1} \mathrm{H} \mathrm{NMR}\left(400 \mathrm{MHz}, \mathrm{CDCl}_{3}\right)$
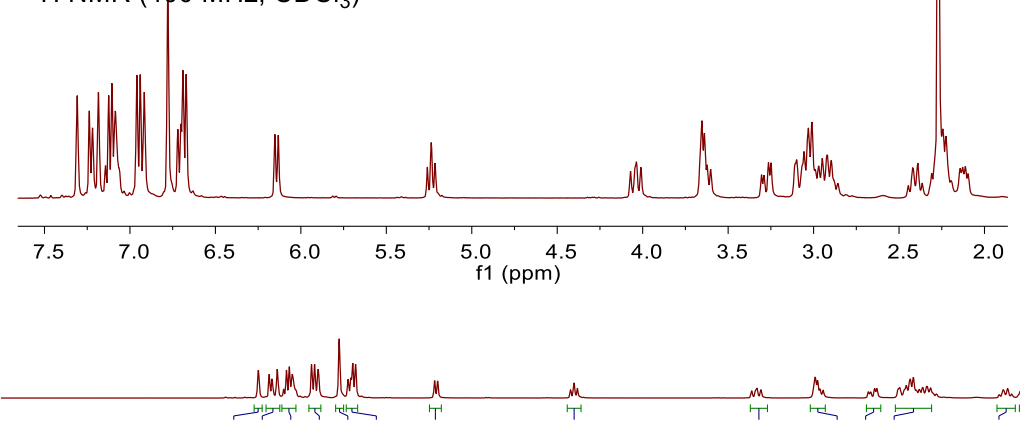

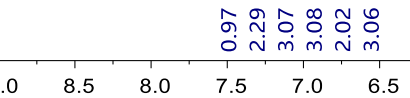
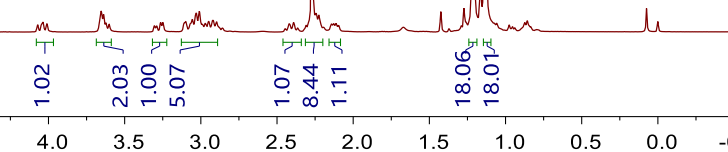

$\begin{array}{llllll}6.0 & 5.5 & 5.0 & 4.5 \\ & & & f(p p m)\end{array}$ 


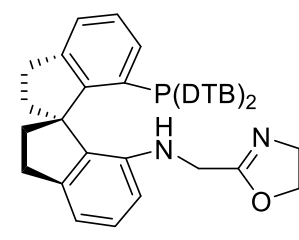

$\left(S_{a}, S\right)-\mathbf{S 6 f}$<smiles>Cc1cccc(C)c1</smiles>

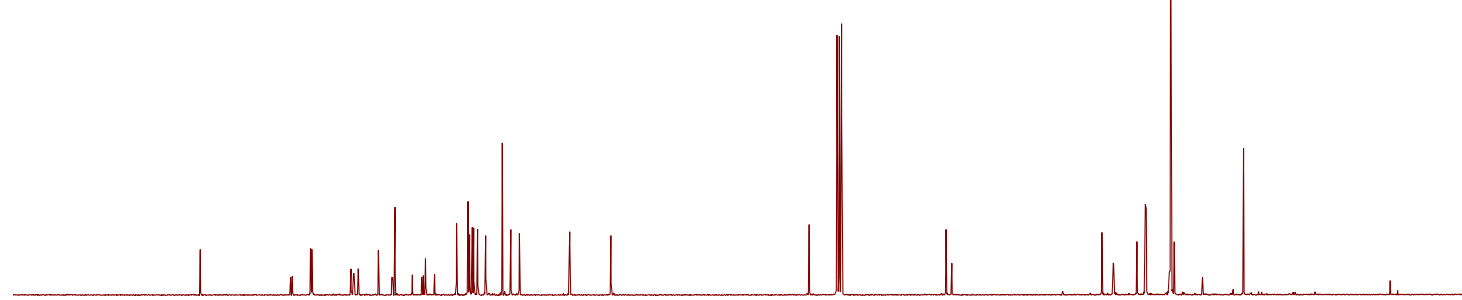

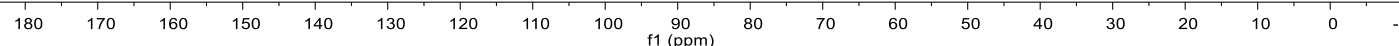

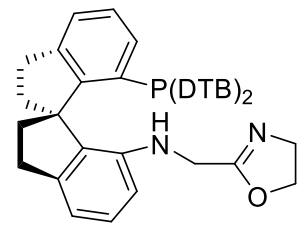

$\left(S_{\mathrm{a}}, S\right)-\mathbf{S 6 f}$<smiles>[N+]=[N+]=C1C=CC=C1</smiles>

$\mathrm{Me}$

${ }^{31} \mathrm{P}$ NMR $\left(162 \mathrm{MHz}, \mathrm{CDCl}_{3}\right)$

$\begin{array}{llllllllllllllllllllllllll}140 & 120 & 100 & 80 & 60 & 40 & 20 & 0 & -20 & -40 & -60 & -80 & -100 & -120 & -140 & -160 & -180 & -200 & -220 & -240\end{array}$ 
3-Phenoxydihydrofuran-2-one (4a)

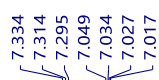

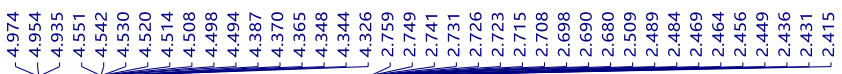<smiles>O=C1OCCC1Oc1ccccc1</smiles>

$4 a$

${ }^{1} \mathrm{H}$ NMR $\left(400 \mathrm{MHz}, \mathrm{CDCl}_{3}\right)$

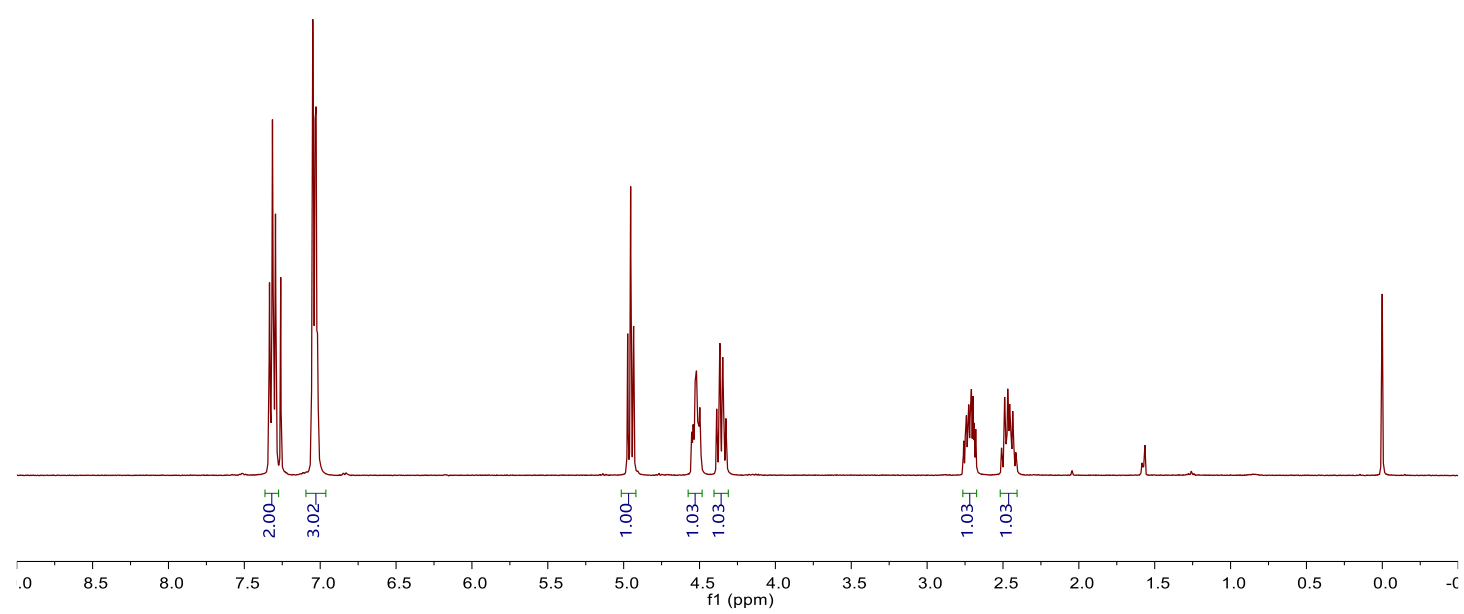

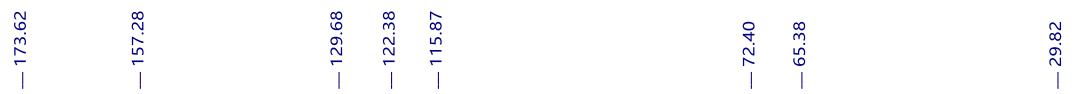

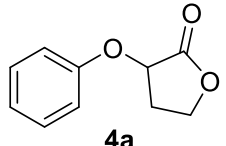

4a

${ }^{13} \mathrm{C}$ NMR $\left(101 \mathrm{MHz}, \mathrm{CDCl}_{3}\right)$

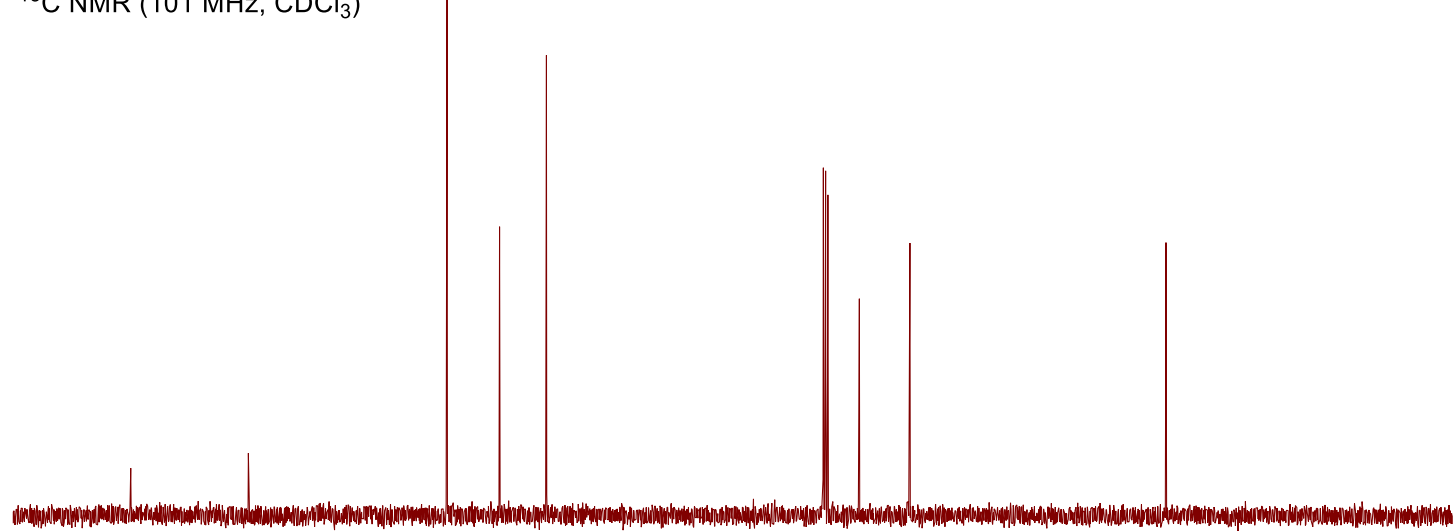

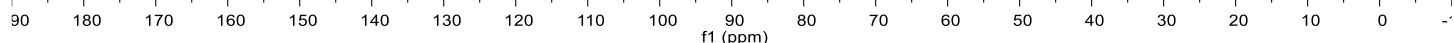


3-(p-Tolyloxy)dihydrofuran-2-one (4b)

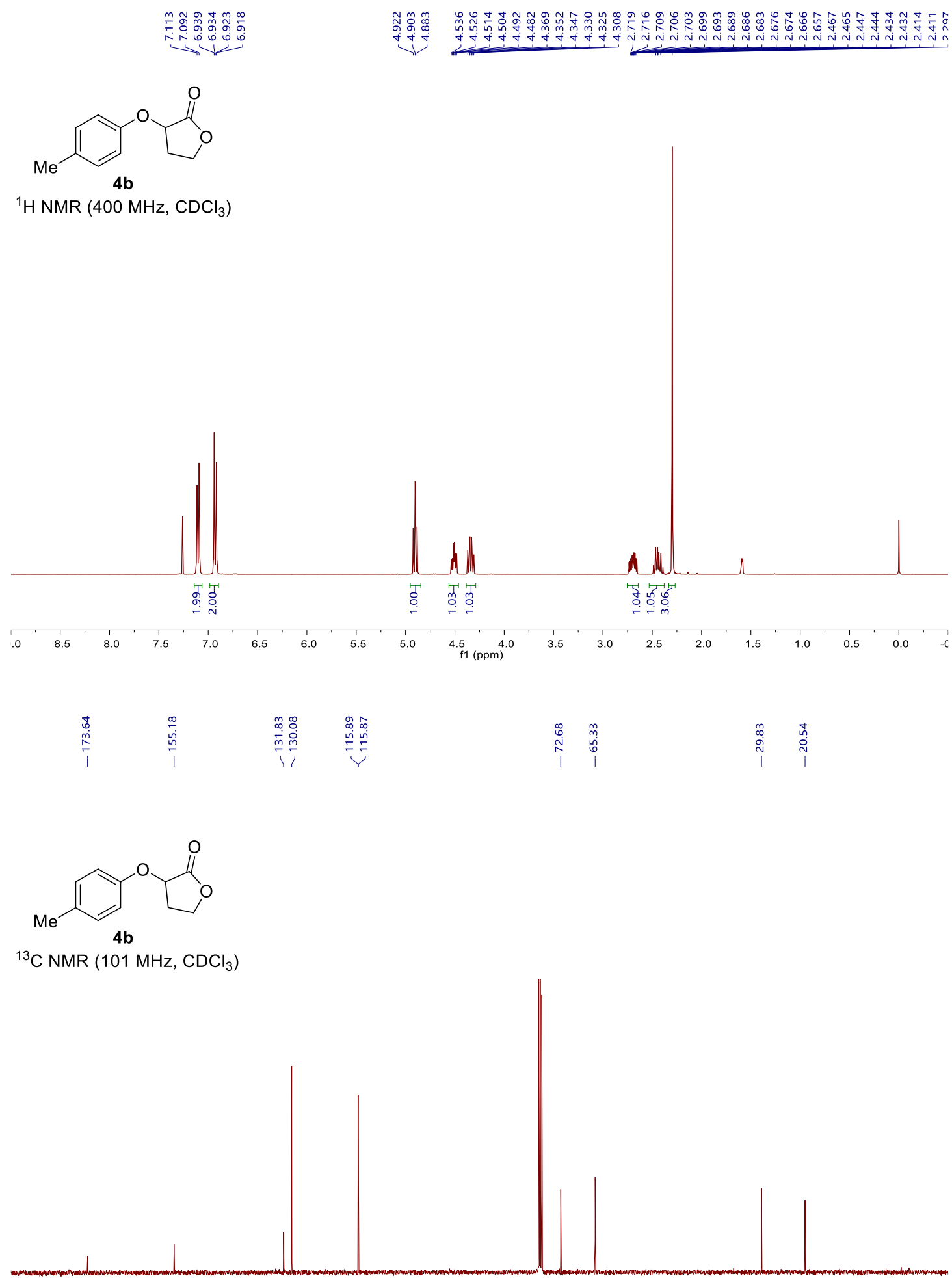

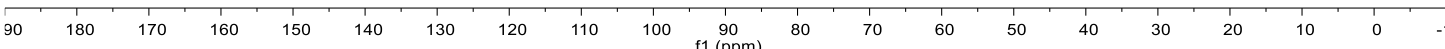


3-(4-Chlorophenoxy)dihydrofuran-2-one (4c)

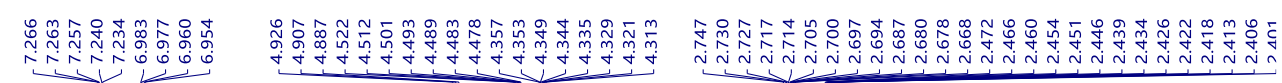<smiles>O=C1OCCC1Oc1ccc(Cl)cc1</smiles>

${ }^{1} \mathrm{H} \mathrm{NMR}\left(400 \mathrm{MHz}, \mathrm{CDCl}_{3}\right)$

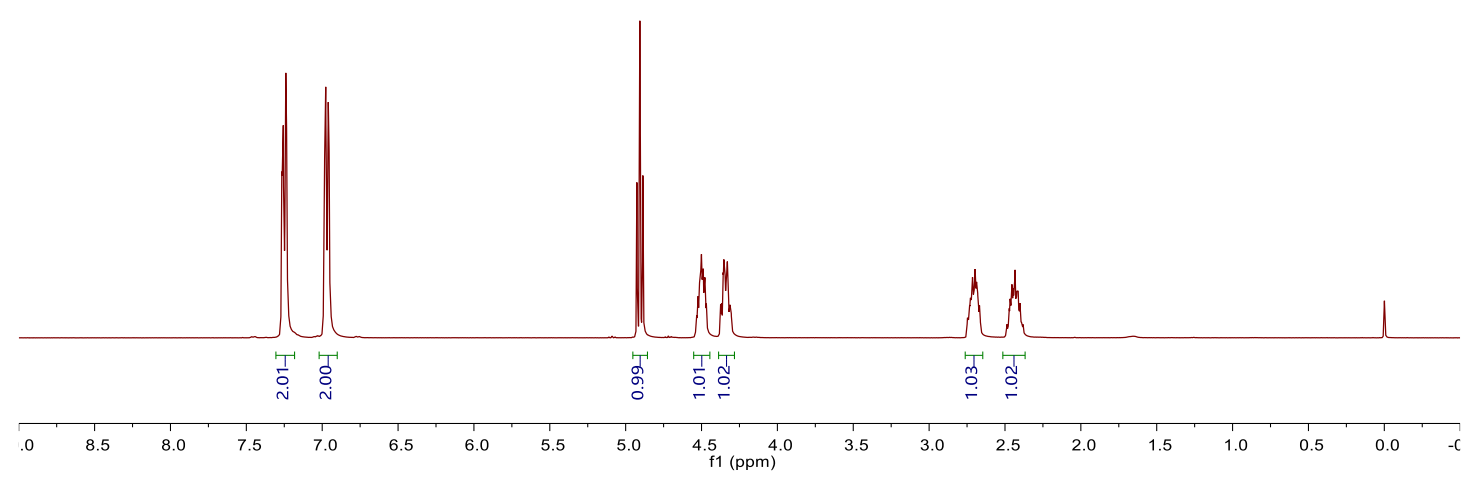

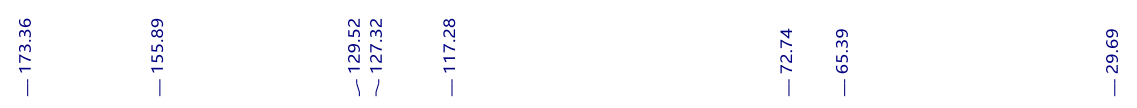

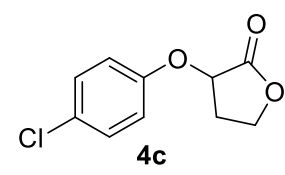

${ }^{13} \mathrm{C} \mathrm{NMR}\left(101 \mathrm{MHz}, \mathrm{CDCl}_{3}\right)$

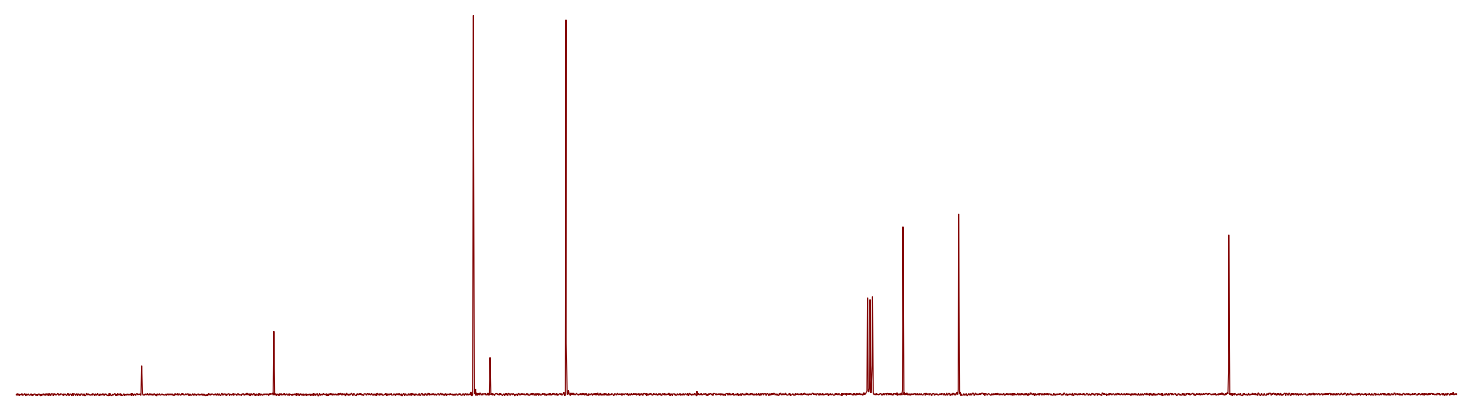

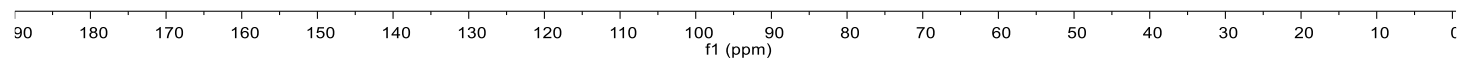


3-(4-(tert-Butyl)phenoxy)dihydrofuran-2-one (4d)

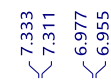

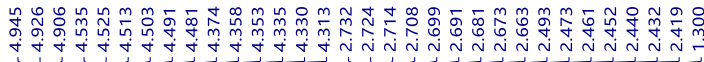

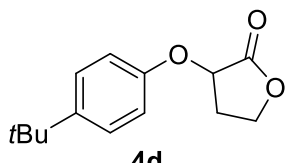

${ }^{1} \mathrm{H}$ NMR $\left(400 \mathrm{MHz}, \mathrm{CDCl}_{3}\right)$

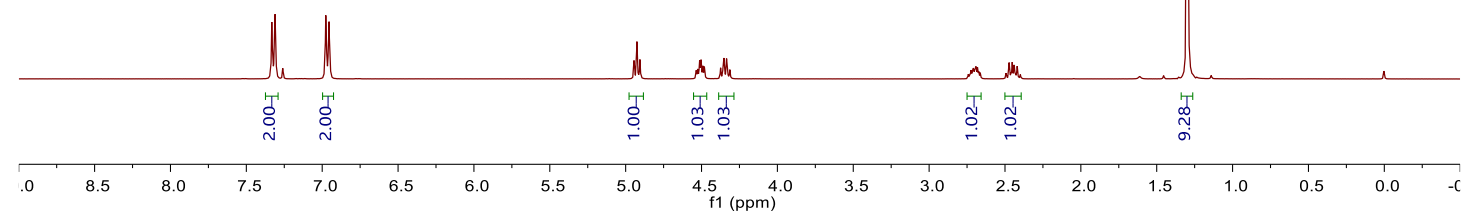

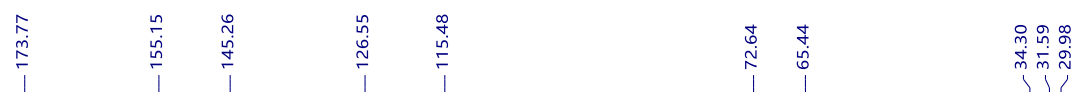

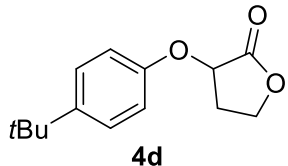

${ }^{13} \mathrm{C}$ NMR $\left(101 \mathrm{MHz}, \mathrm{CDCl}_{3}\right)$

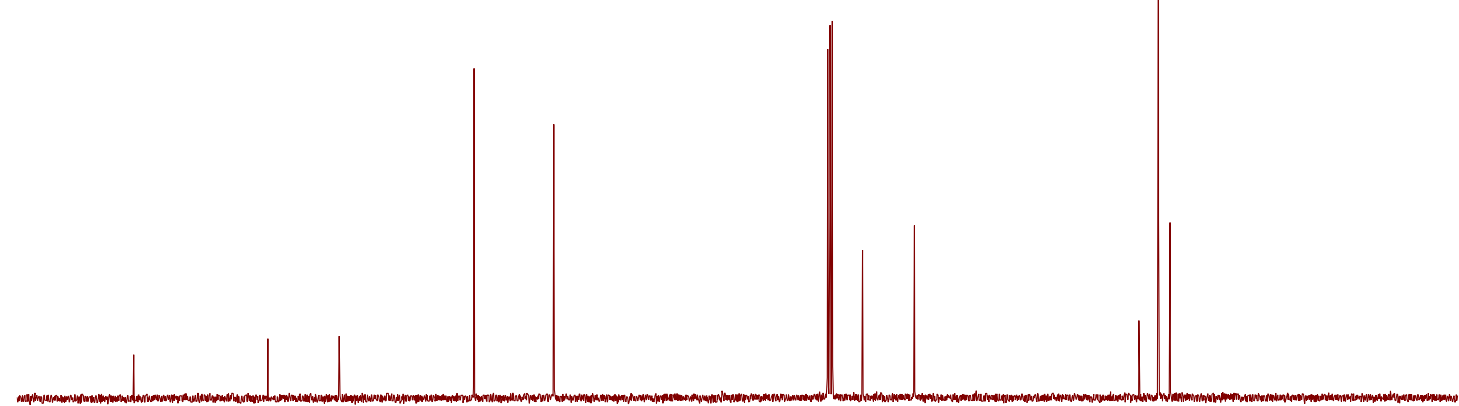

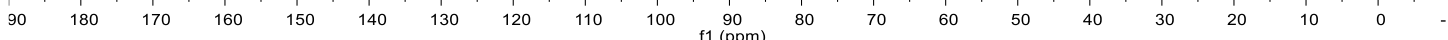




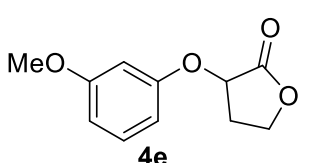

${ }^{1} \mathrm{H}$ NMR (400 MHz, $\mathrm{CDCl}_{3}$ )

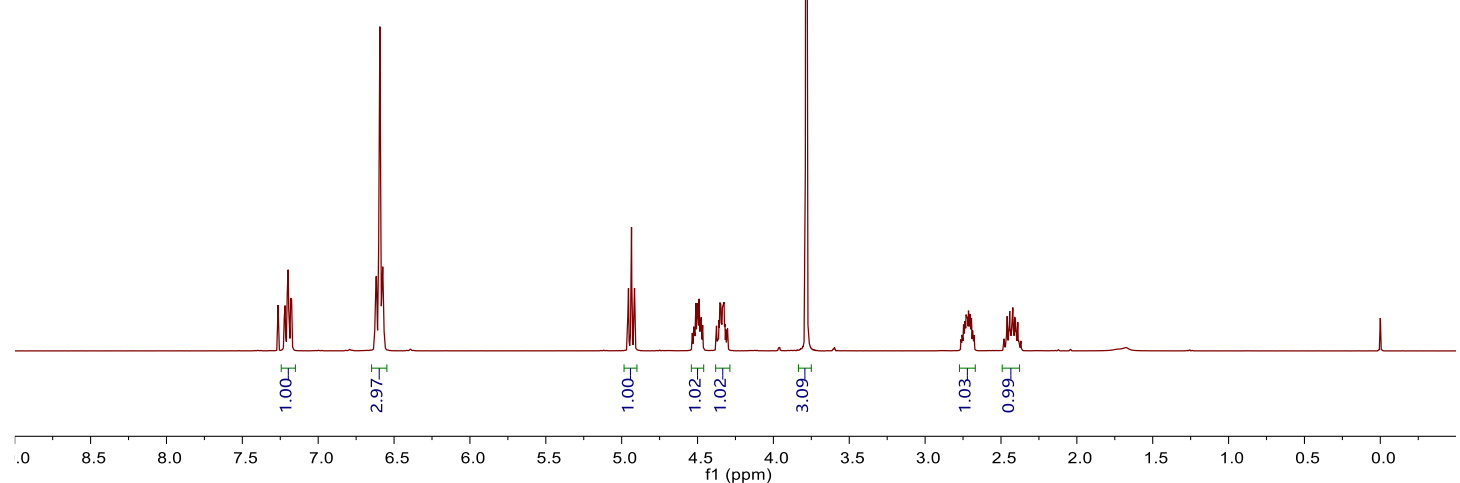

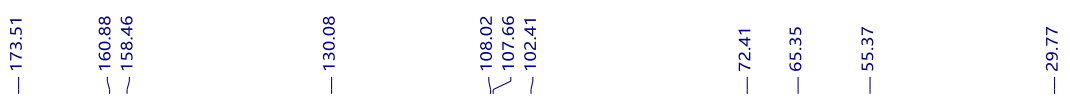

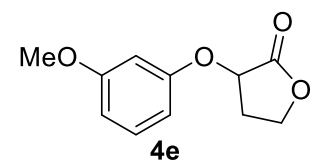

${ }^{13} \mathrm{C}$ NMR $\left(101 \mathrm{MHz}, \mathrm{CDCl}_{3}\right)$
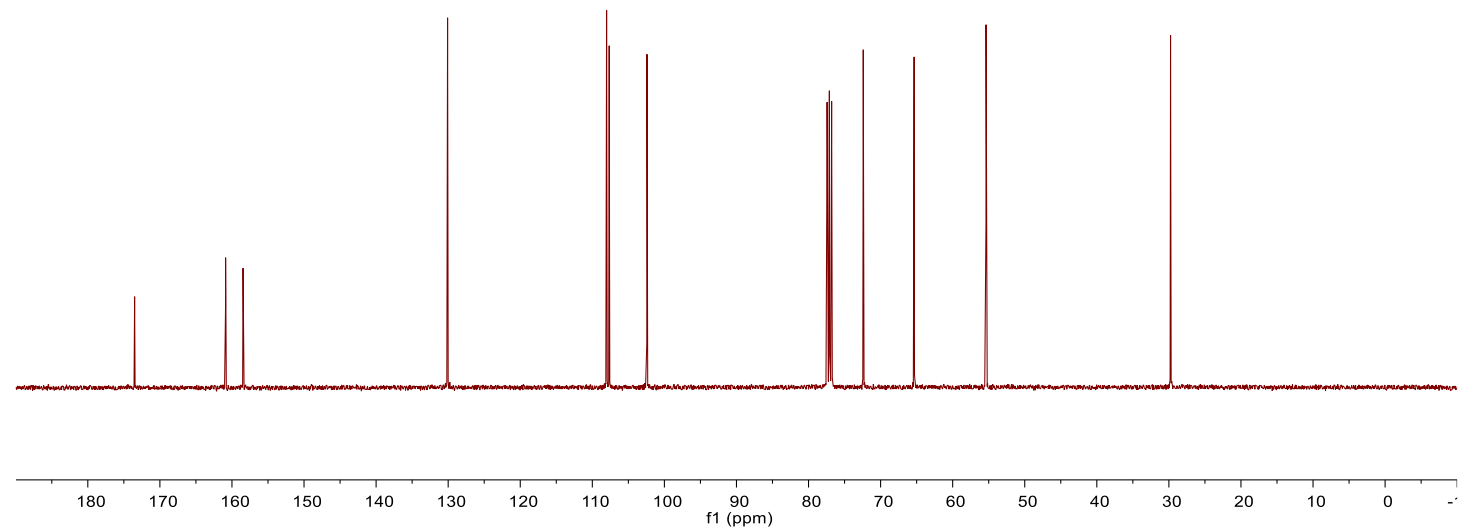


\section{3-(3-Chlorophenoxy)dihydrofuran-2-one (4f)}

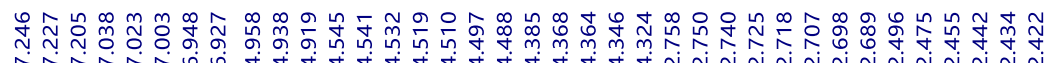

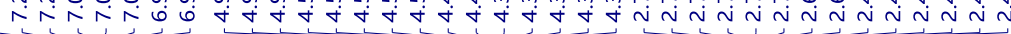

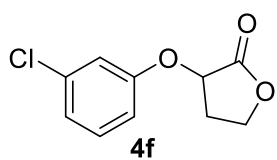

${ }^{1} \mathrm{H} \mathrm{NMR}\left(400 \mathrm{MHz}, \mathrm{CDCl}_{3}\right)$
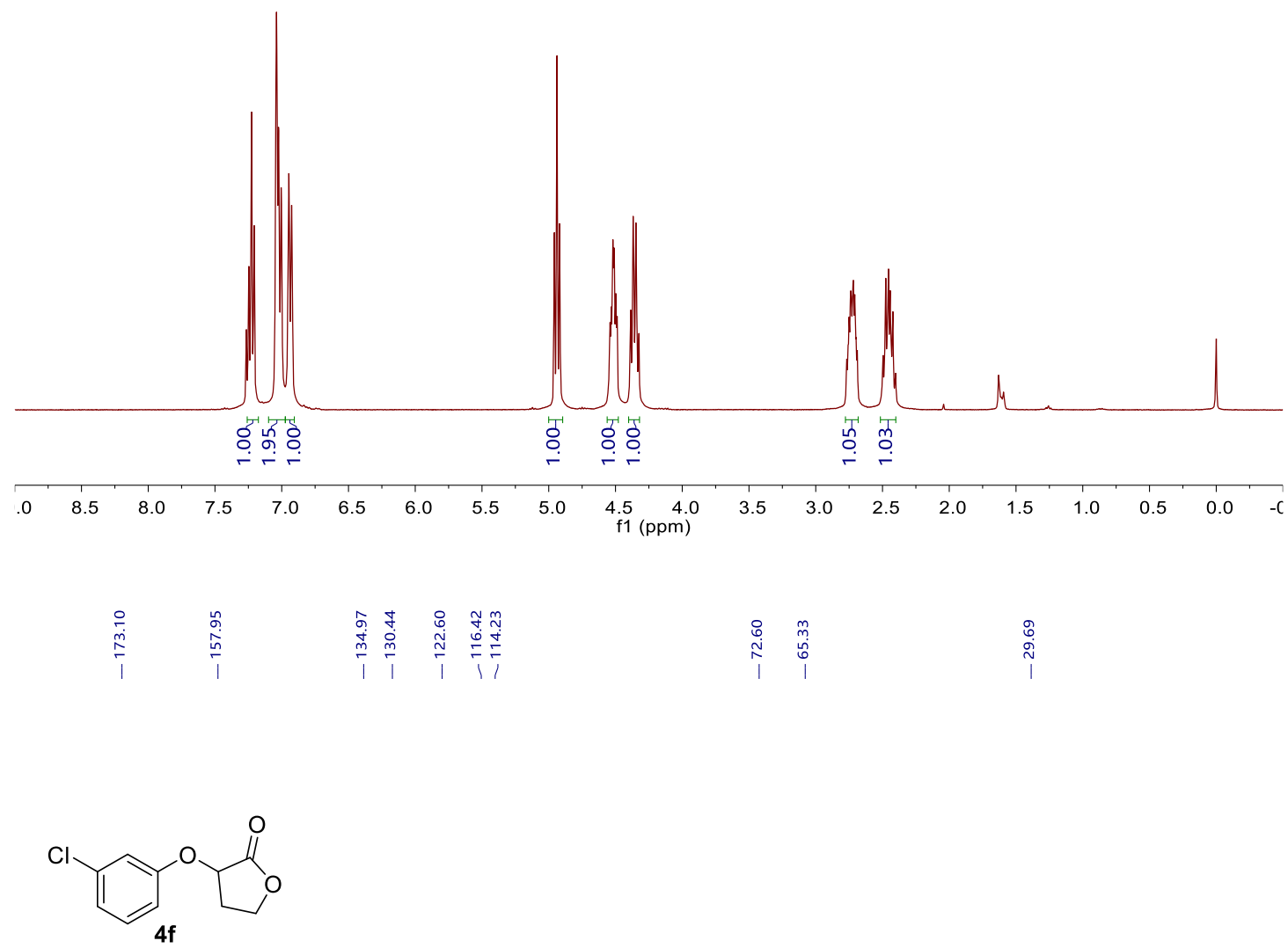

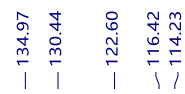

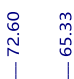

ํํํ

${ }^{13} \mathrm{C}$ NMR $\left(101 \mathrm{MHz}, \mathrm{CDCl}_{3}\right)$

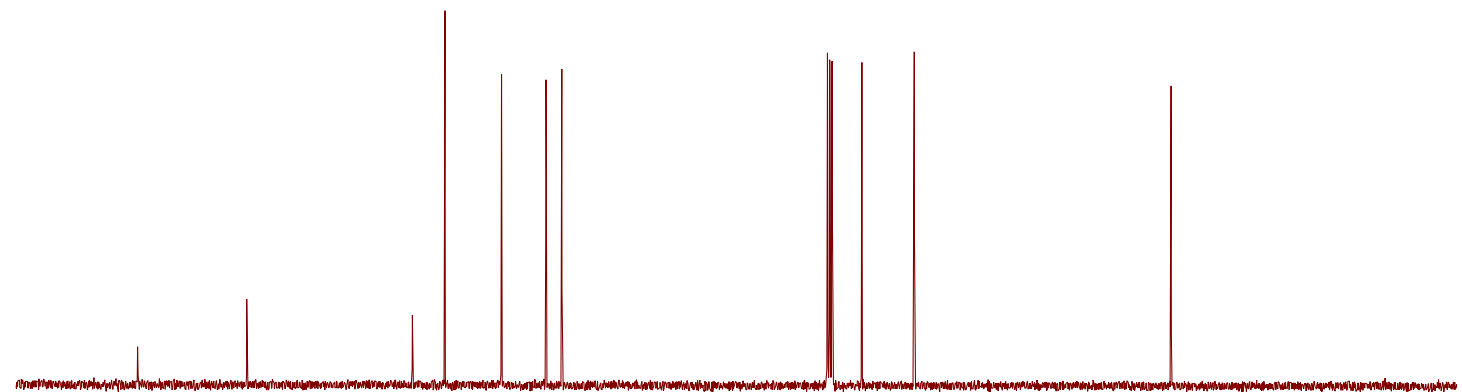

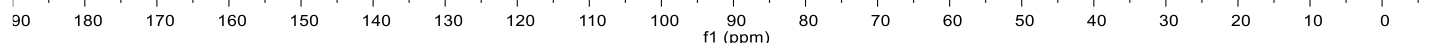




\section{3-(3-Bromophenoxy)dihydrofuran-2-one (4g)}

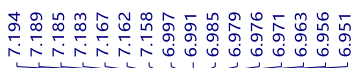

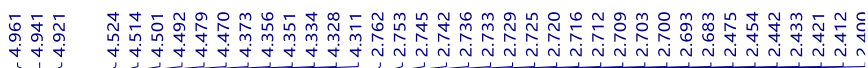<smiles>O=C1OCCC1Oc1cccc(Br)c1</smiles>

$4 \mathrm{~g}$

${ }^{1} \mathrm{H}$ NMR $\left(400 \mathrm{MHz}, \mathrm{CDCl}_{3}\right)$

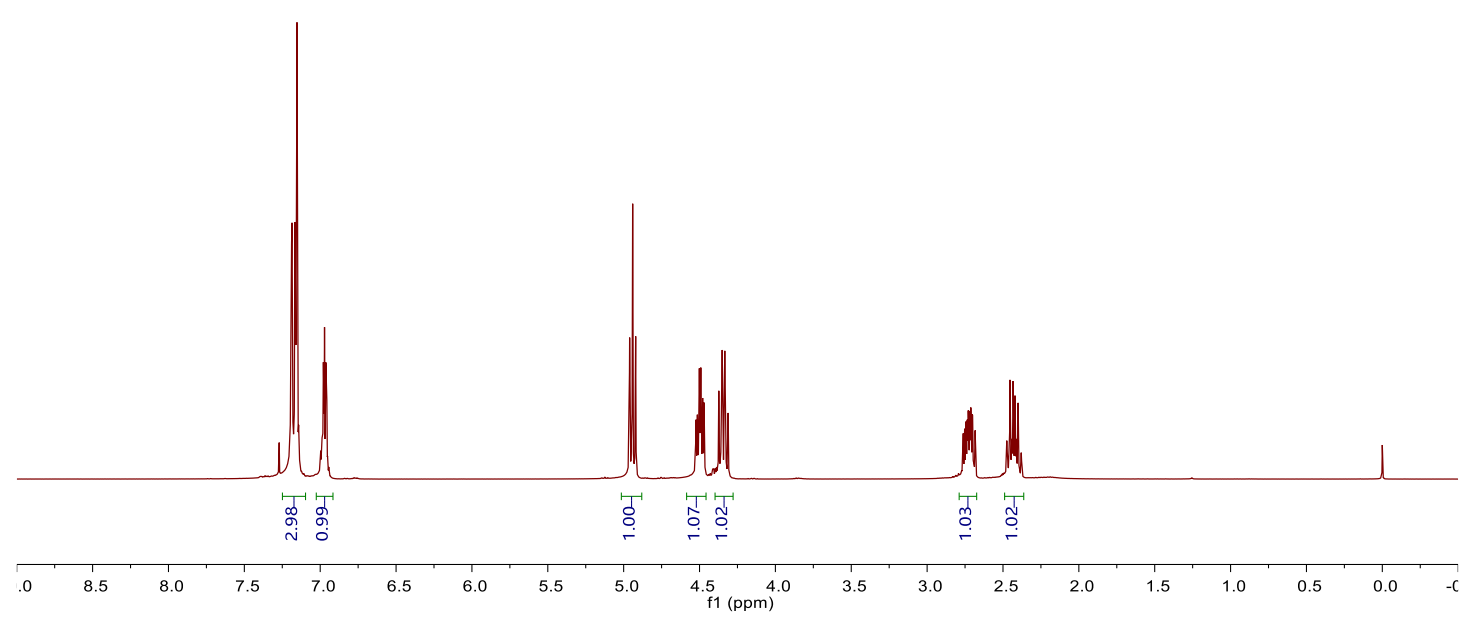

$\begin{array}{ll}\stackrel{0}{n} & \stackrel{0}{n} \\ & \frac{n}{n}\end{array}$

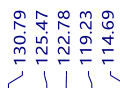

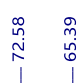

ํํำ

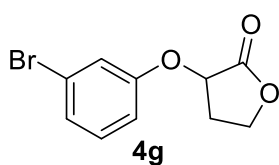

${ }^{13} \mathrm{C}$ NMR $\left(101 \mathrm{MHz}, \mathrm{CDCl}_{3}\right)$

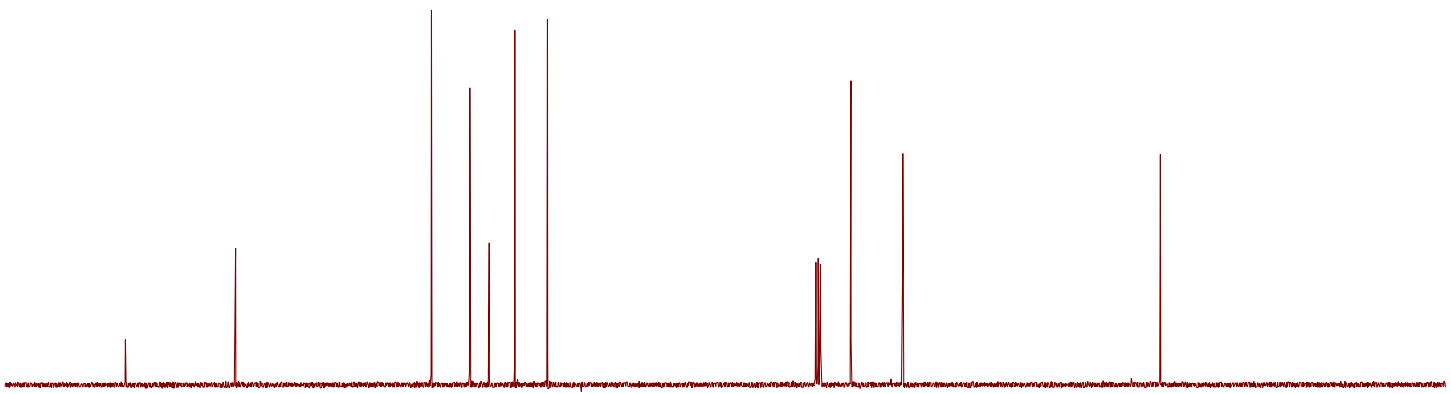

\begin{tabular}{lllllllllllllllllllllll}
\hline & 180 & 170 & 160 & 150 & 140 & 130 & 120 & 110 & 100 & 90 & 80 & 70 & 60 & 50 & 40 & 30 & 20 & 10 & 0 & -
\end{tabular} 


\section{3-(2-Methylphenoxy)oxolan-2-one (4h)}

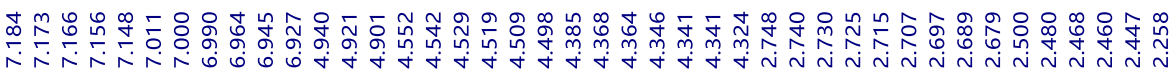<smiles>Cc1ccccc1OC1CCOC1=O</smiles>

${ }^{1} \mathrm{H} \mathrm{NMR}\left(400 \mathrm{MHz}, \mathrm{CDCl}_{3}\right)$

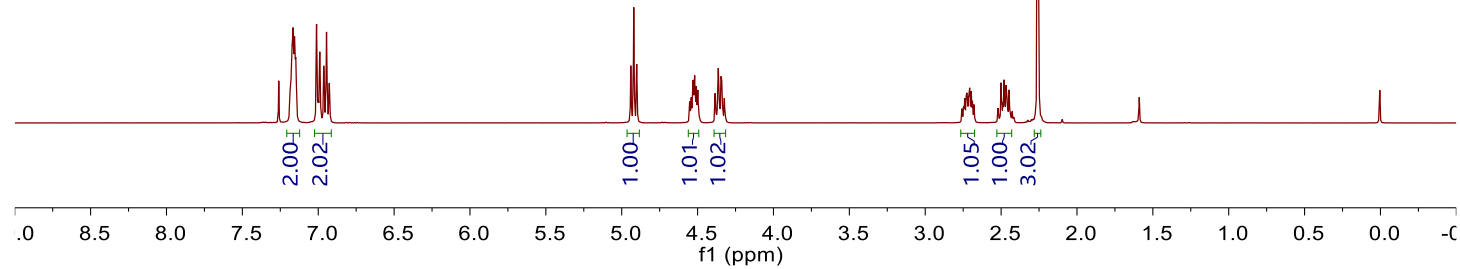

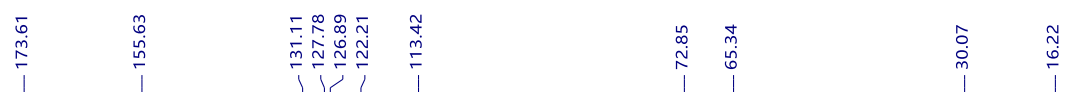<smiles>Cc1ccccc1OC1CCOC1=O</smiles>

4h

${ }^{13} \mathrm{C}$ NMR $\left(101 \mathrm{MHz}, \mathrm{CDCl}_{3}\right)$

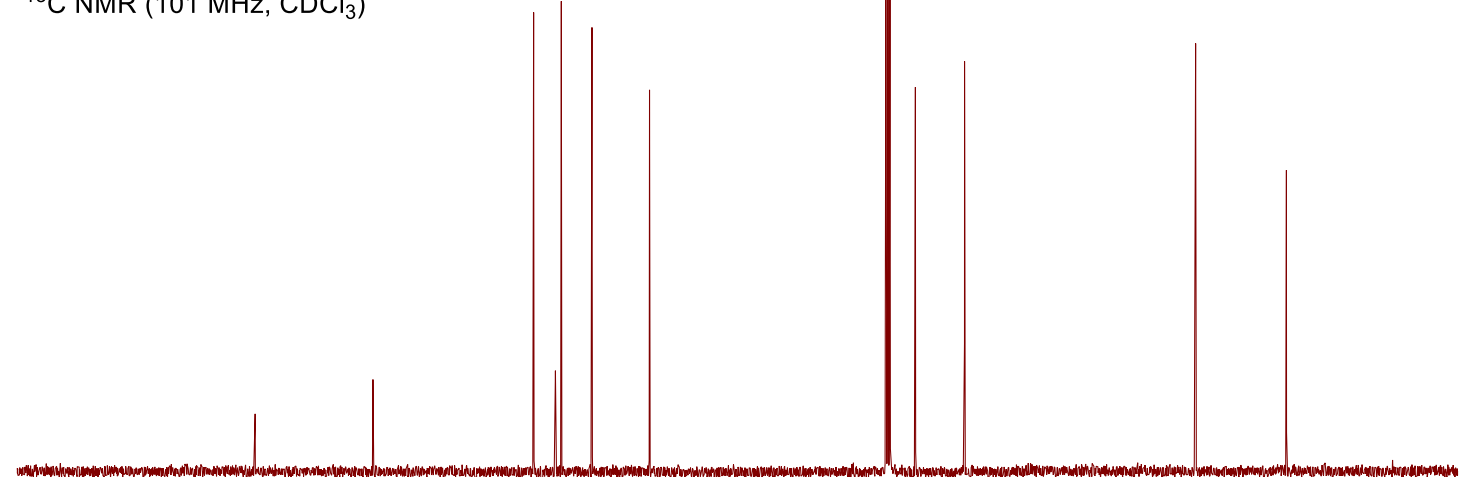

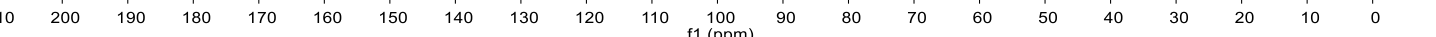




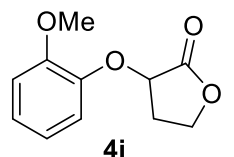

${ }^{1} \mathrm{H} \mathrm{NMR}\left(400 \mathrm{MHz}, \mathrm{CDCl}_{3}\right)$

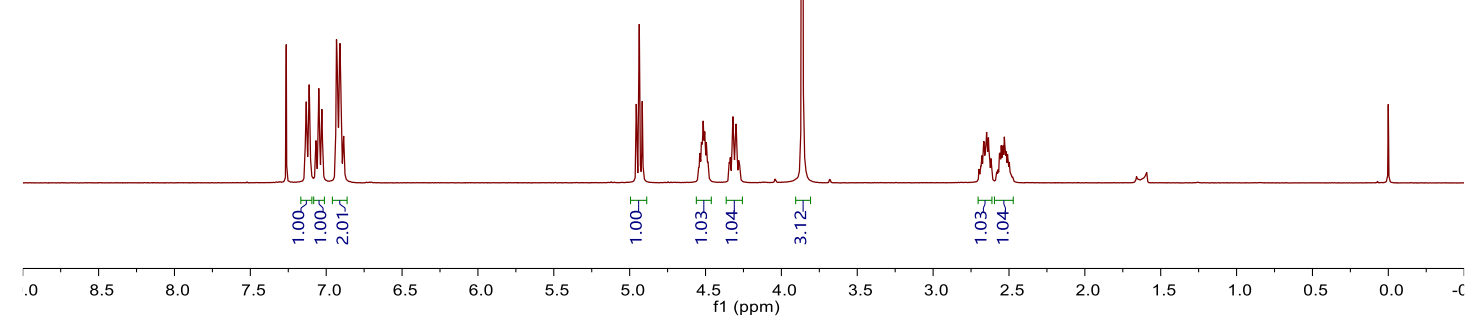

\begin{tabular}{|c|c|c|c|c|}
\hline 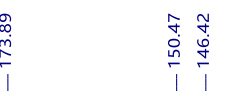 & 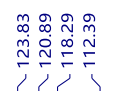 & $\underset{\substack{\bar{j} \\
\dot{j}}}{1}$ & & \\
\hline
\end{tabular}

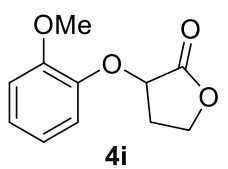

${ }^{13} \mathrm{C}$ NMR $\left(101 \mathrm{MHz}, \mathrm{CDCl}_{3}\right)$

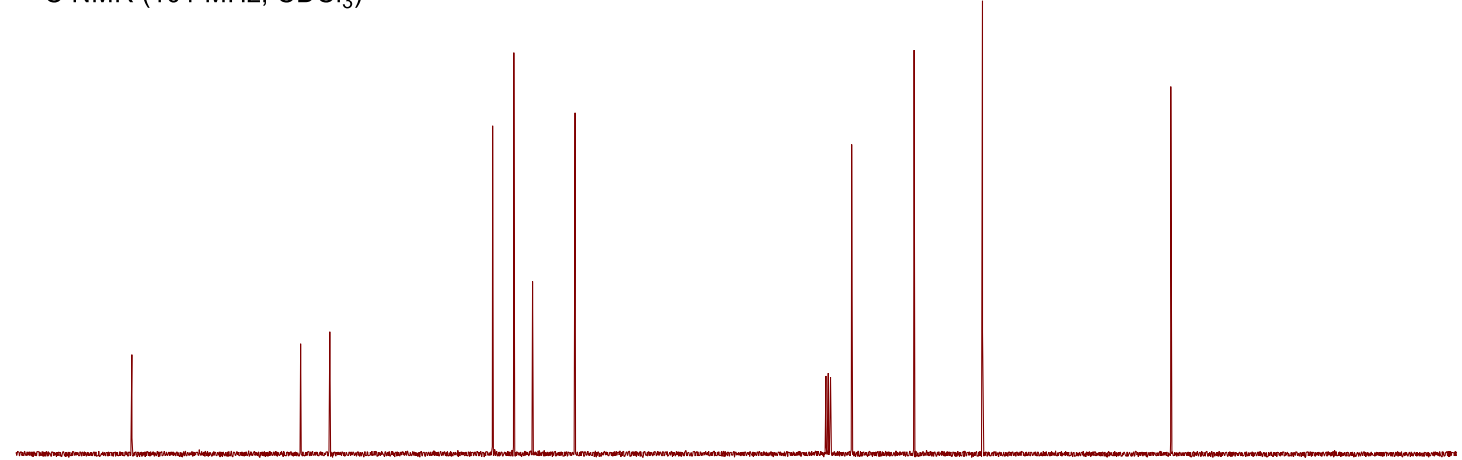

$\begin{array}{llllllllllllllllllll}1 & 180 & 170 & 160 & 150 & 140 & 130 & 120 & 110 & 100 & 90 & 90 & 70 & 60 & 50 & 40 & 30 & 20 & 10 & 0\end{array}$ 


\section{3-(2-Chlorophenoxy)dihydrofuran-2-one (4j)}<smiles>O=C1OCCC1Oc1ccccc1Cl</smiles>

${ }^{1} \mathrm{H}$ NMR $(400 \mathrm{MHz}, \mathrm{CDCl})$

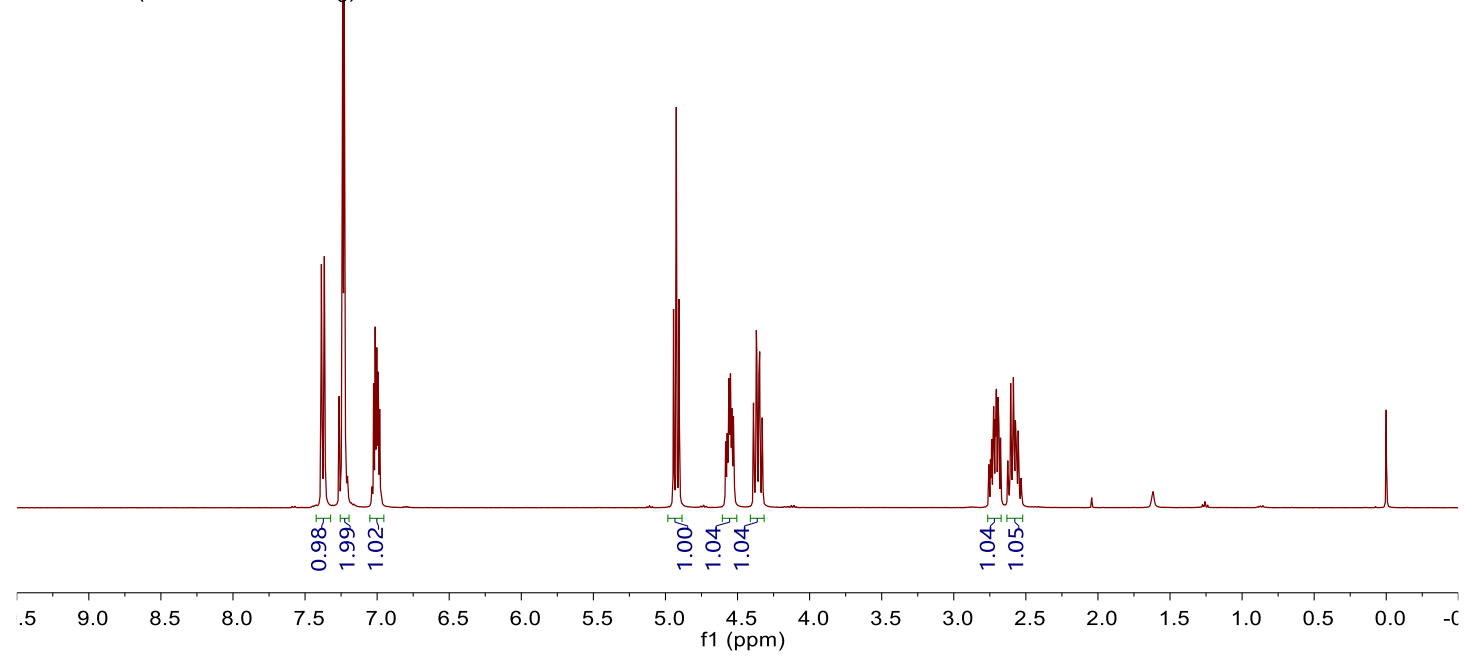

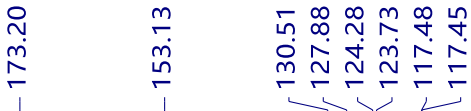

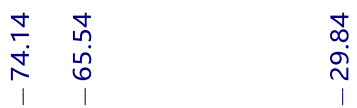<smiles>O=C1OCCC1Oc1ccccc1Cl</smiles>

${ }^{13} \mathrm{C}$ NMR $\left(101 \mathrm{MHz}, \mathrm{CDCl}_{3}\right)$

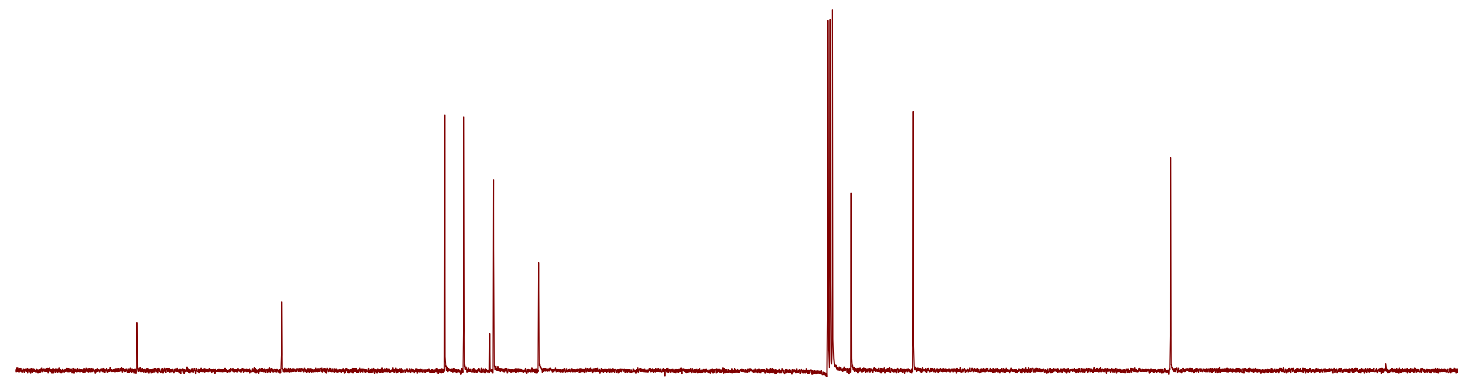

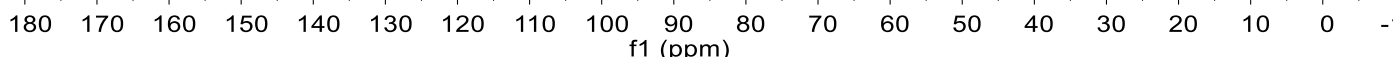




\section{3-(2-Fluorophenoxy)dihydrofuran-2-one (4k)}

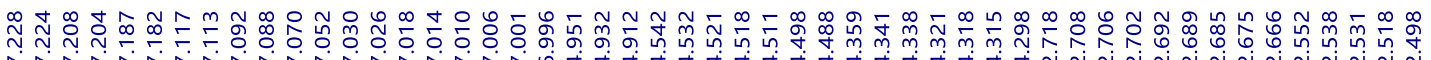

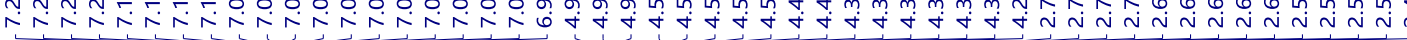<smiles>O=C1OCCC1Oc1ccccc1F</smiles>

${ }^{1} \mathrm{H} \mathrm{NMR}\left(400 \mathrm{MHz}, \mathrm{CDCl}_{3}\right)$

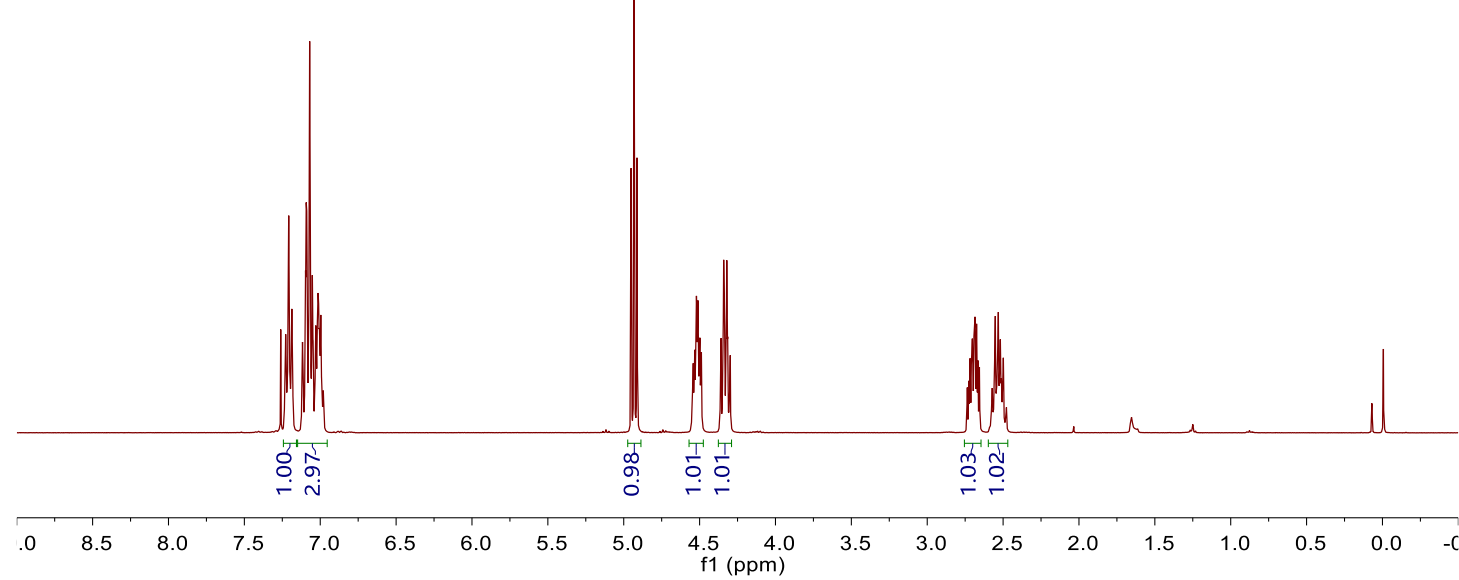

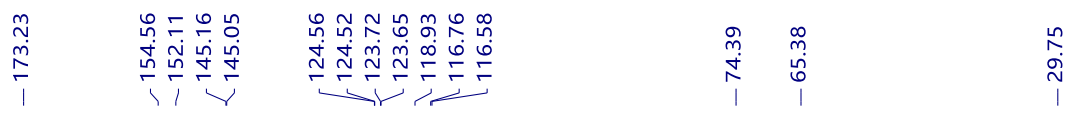

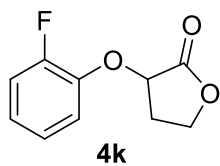

${ }^{13} \mathrm{C}$ NMR $\left(101 \mathrm{MHz}, \mathrm{CDCl}_{3}\right)$

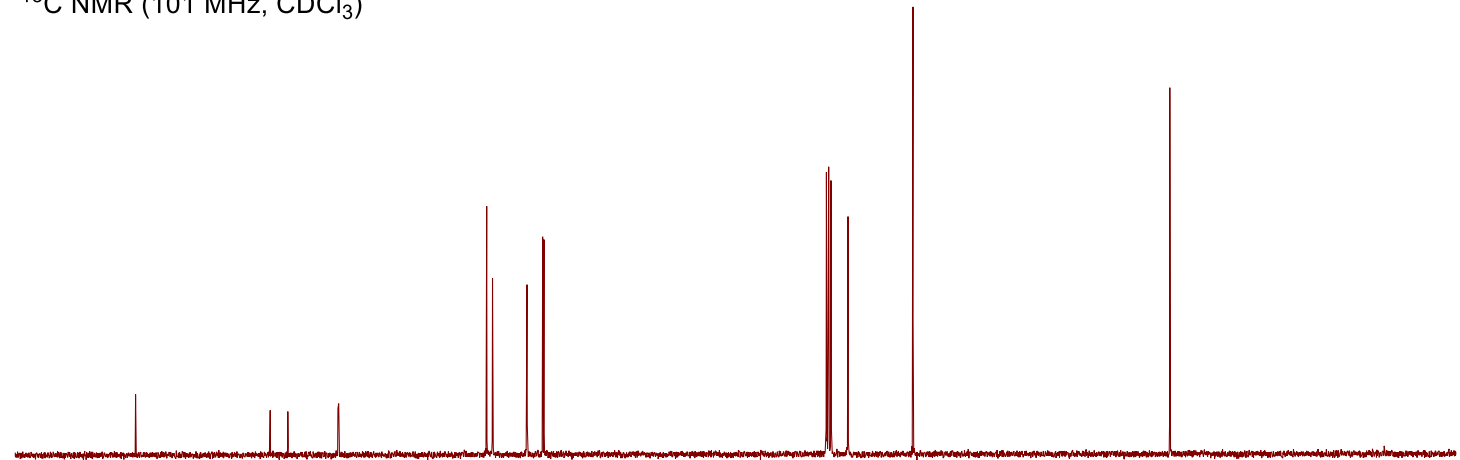

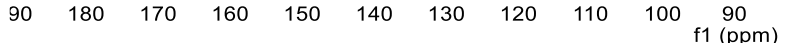


3-(2,5-Dimethylphenoxy)dihydrofuran-2-one (41)

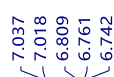

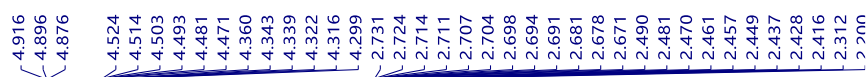<smiles>Cc1ccc([N+](=O)[O-])c(OC2CCOC2=O)c1</smiles>

${ }^{1} \mathrm{H} \mathrm{NMR}\left(400 \mathrm{MHz}, \mathrm{CDCl}_{3}\right)$

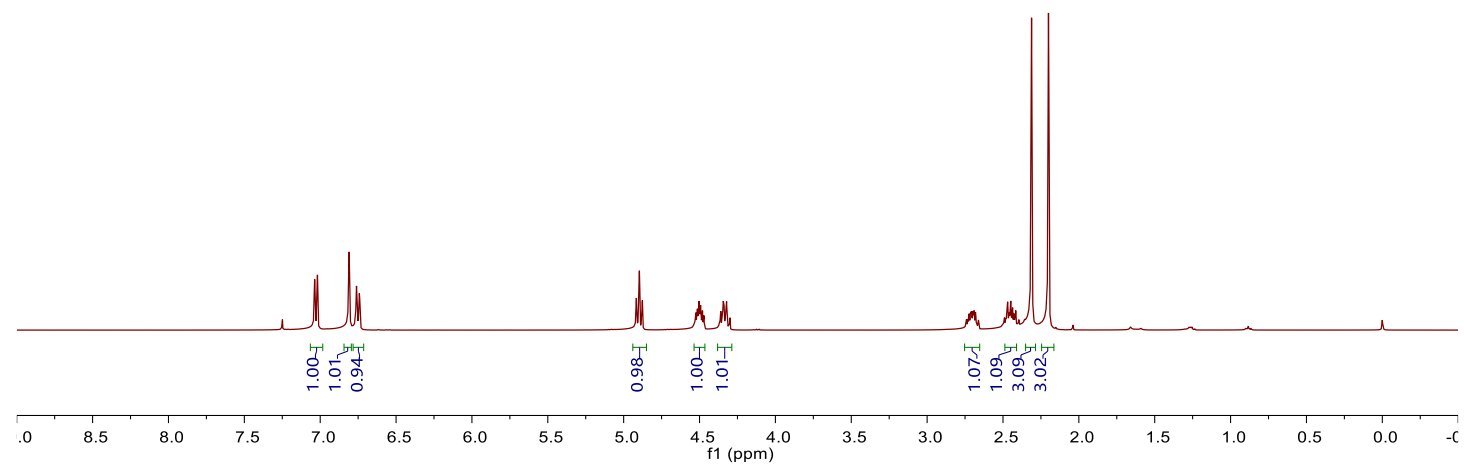

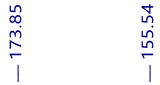

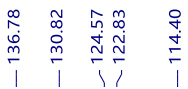

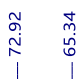

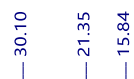

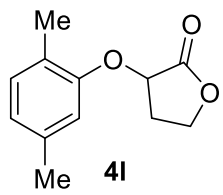

${ }^{13} \mathrm{H}$ NMR (101 MHz, $\mathrm{CDCl}_{3}$ )

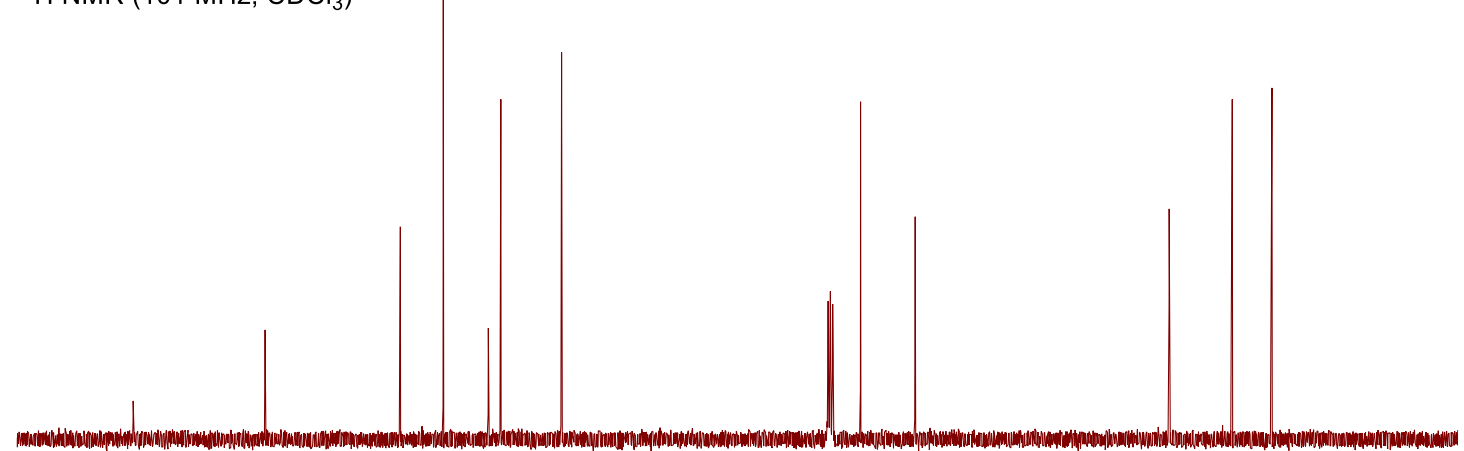

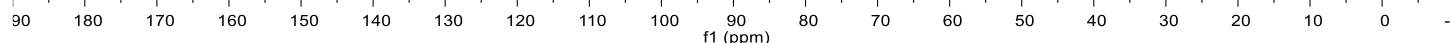




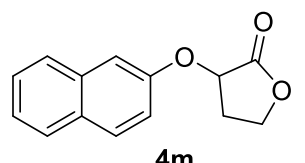

${ }^{1} \mathrm{H}$ NMR $\left(400 \mathrm{MHz}, \mathrm{CDCl}_{3}\right)$

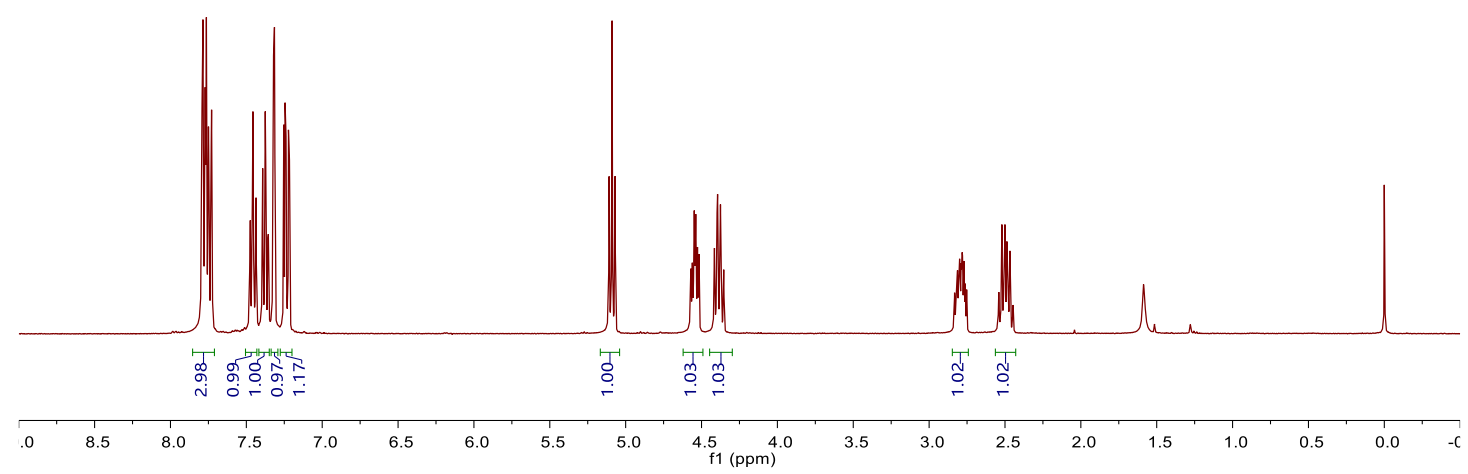

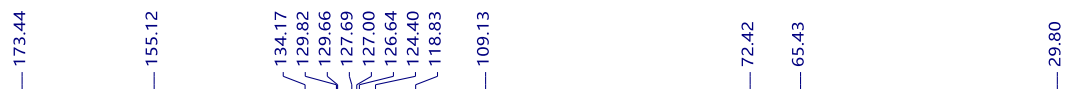

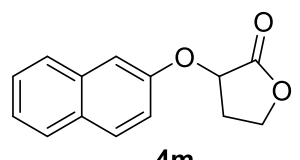

$4 \mathrm{~m}$

${ }^{13} \mathrm{C}$ NMR $\left(101 \mathrm{MHz}, \mathrm{CDCl}_{3}\right)$

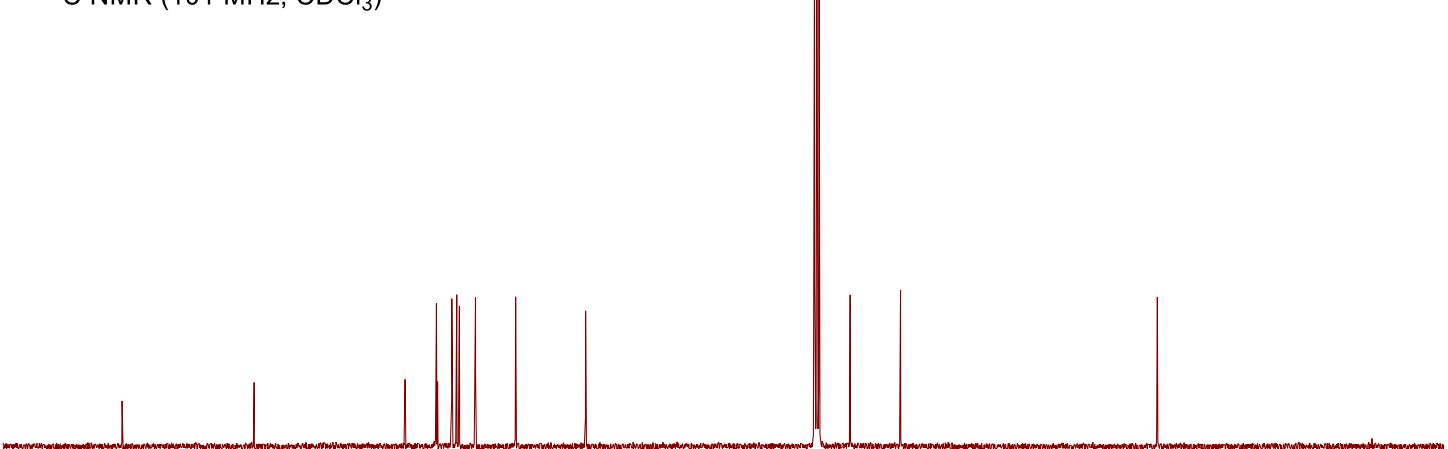

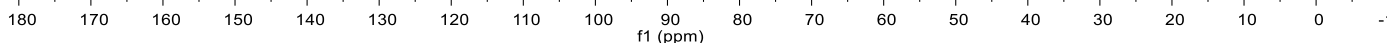


3-Phenoxytetrahydro-2H-pyran-2-one (7a)

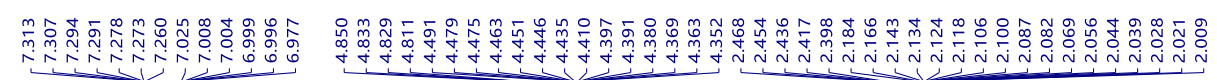

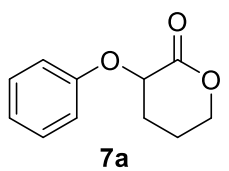

${ }^{1} \mathrm{H} \mathrm{NMR}\left(400 \mathrm{MHz}, \mathrm{CDCl}_{3}\right)$

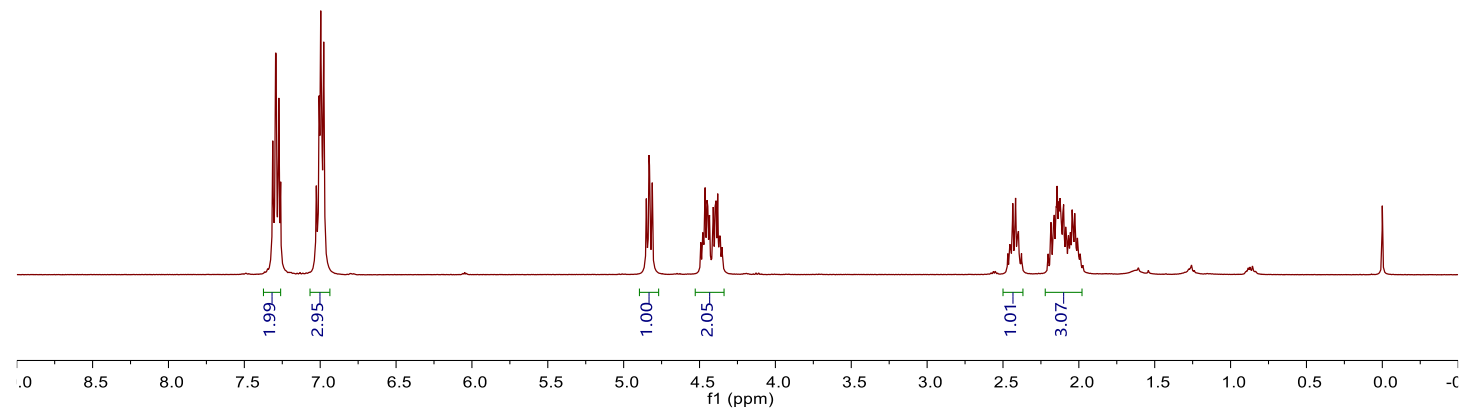

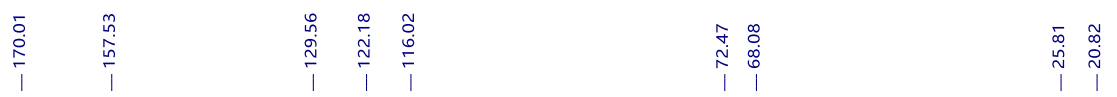

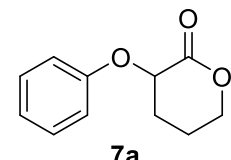

${ }^{13} \mathrm{C} \mathrm{NMR}\left(101 \mathrm{MHz}, \mathrm{CDCl}_{3}\right)$

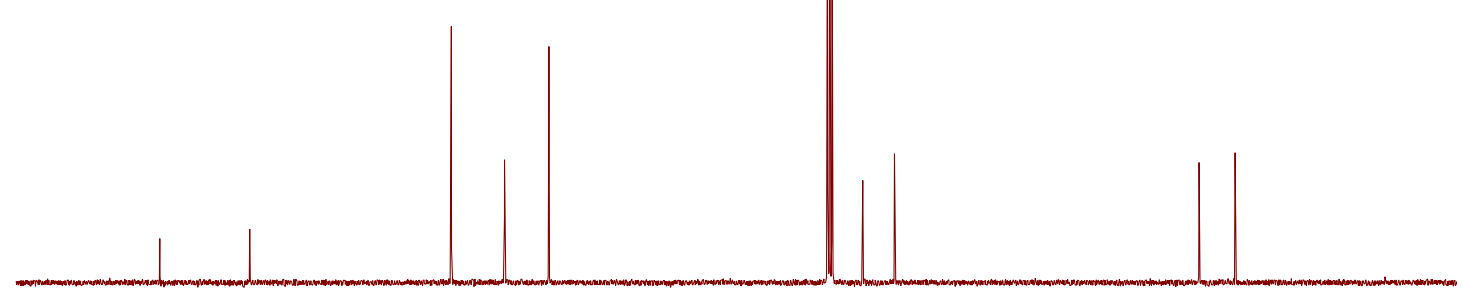

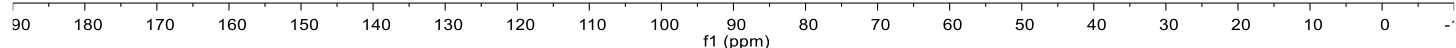




\section{3-(p-Tolyloxy)tetrahydro-2H-pyran-2-one (7b)}

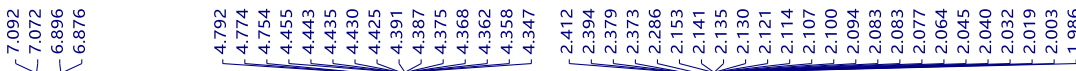

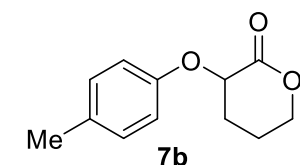

${ }^{1} \mathrm{H}$ NMR $\left(400 \mathrm{MHz}, \mathrm{CDCl}_{3}\right)$

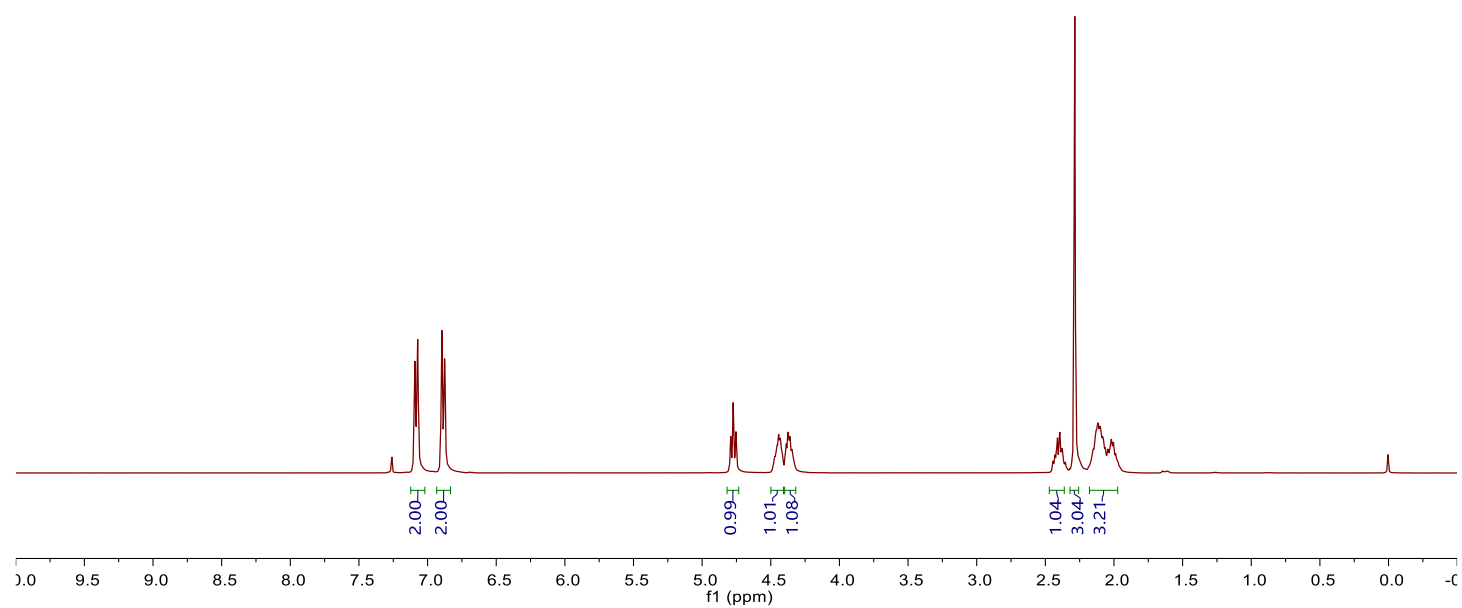

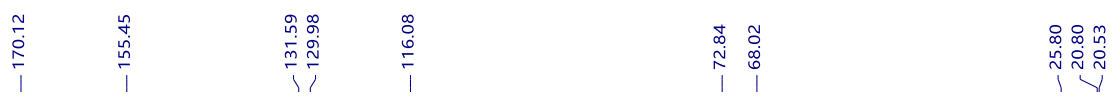

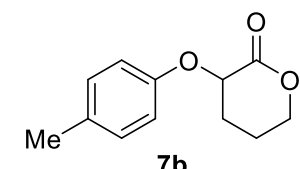

${ }^{13} \mathrm{C}$ NMR $\left(101 \mathrm{MHz}, \mathrm{CDCl}_{3}\right)$

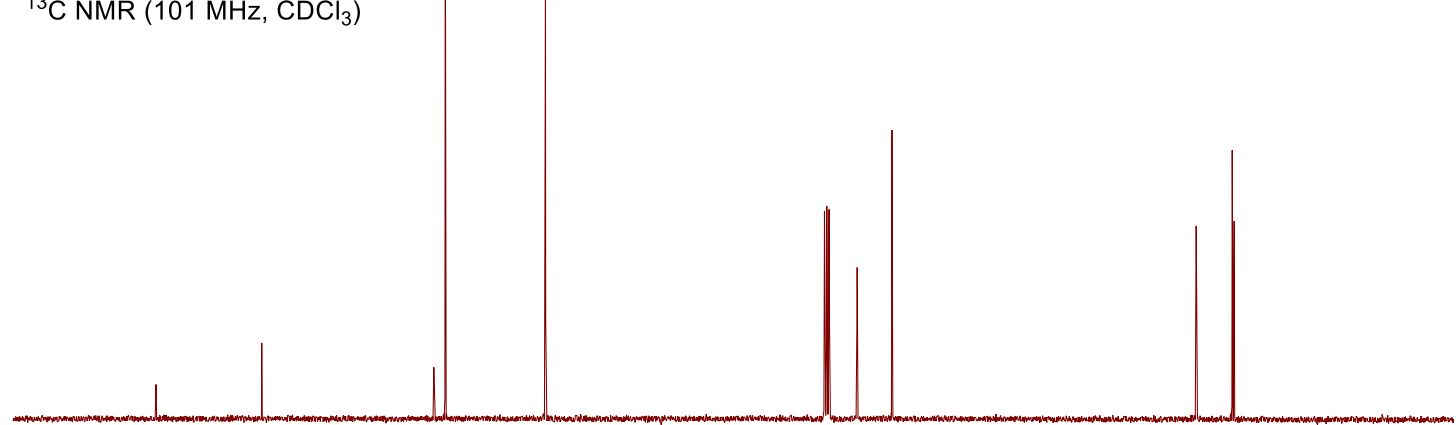

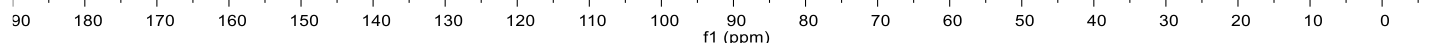


3-(m-Tolyloxy)tetrahydro-2H-pyran-2-one (7c)

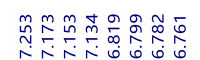

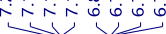

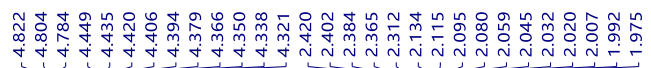

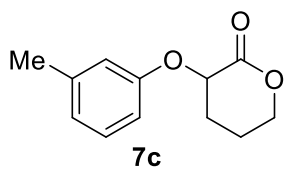

${ }^{1} \mathrm{H}$ NMR $\left(400 \mathrm{MHz}, \mathrm{CDCl}_{3}\right)$

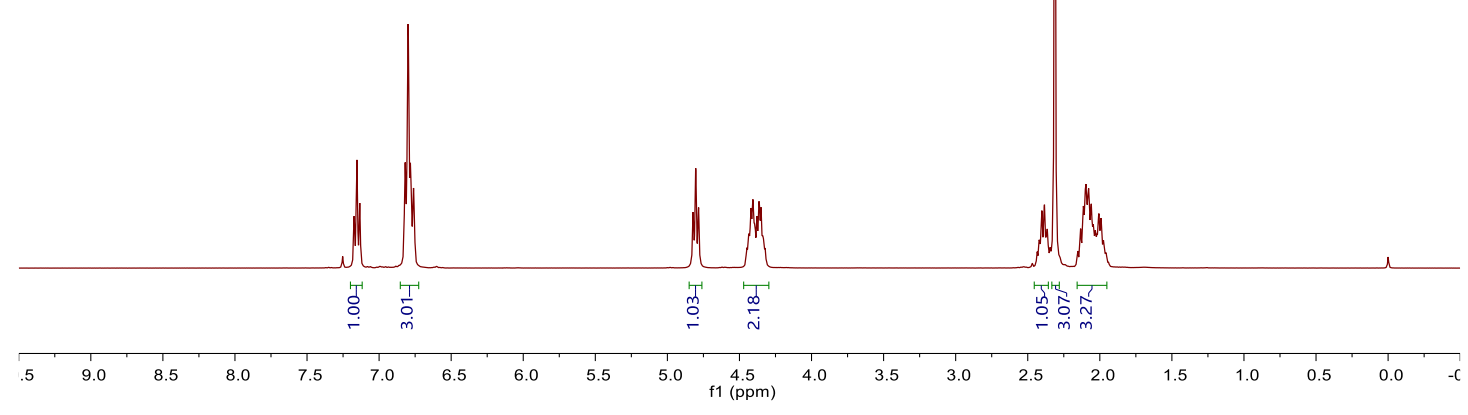

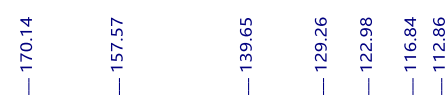

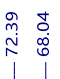

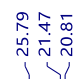<smiles>COc1cccc(OC2CCCOC2=O)c1</smiles>

${ }^{13} \mathrm{C}$ NMR $\left(101 \mathrm{MHz}, \mathrm{CDCl}_{3}\right)$

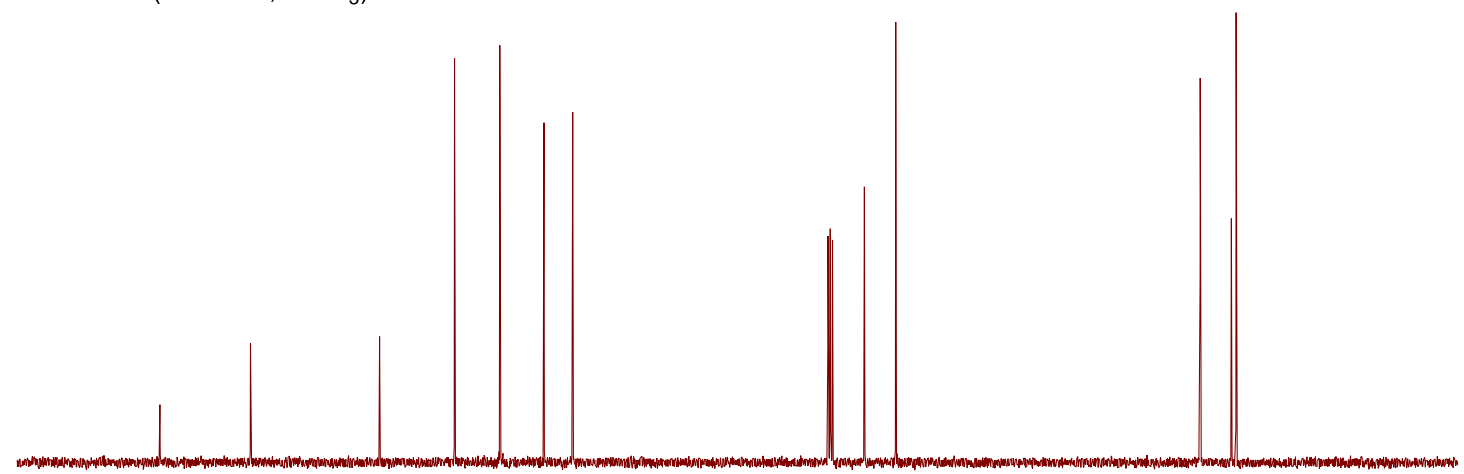

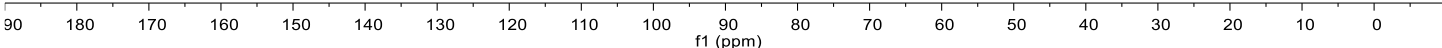


3-(o-Tolyloxy)tetrahydro-2H-pyran-2-one (7d)

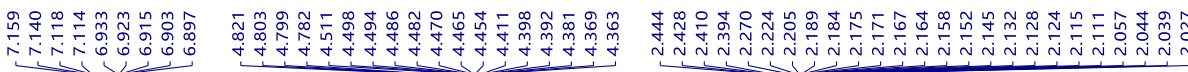<smiles>Cc1ccccc1OC1CCCOC1=O</smiles>

${ }^{1} \mathrm{H} \mathrm{NMR}\left(400 \mathrm{MHz}, \mathrm{CDCl}_{3}\right)$

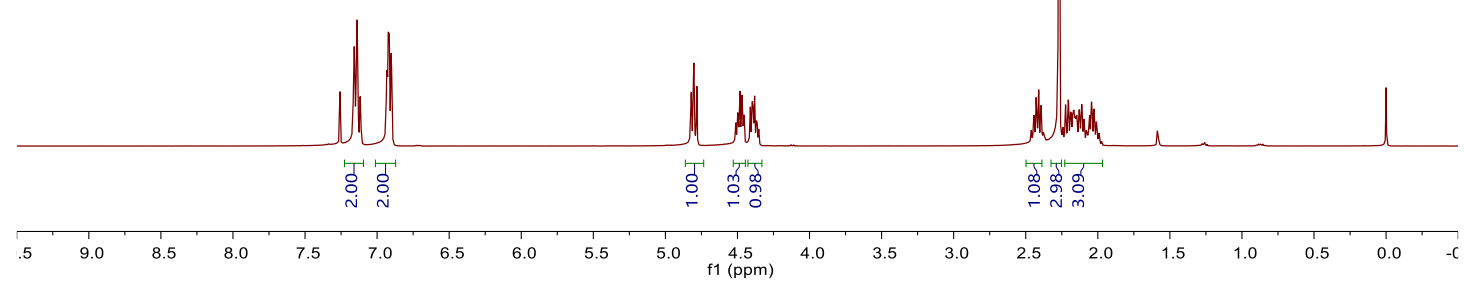

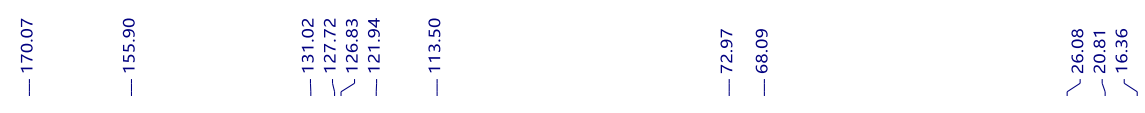

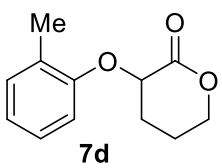

${ }^{13} \mathrm{CNMR}\left(101 \mathrm{MHz}, \mathrm{CDCl}_{3}\right)$

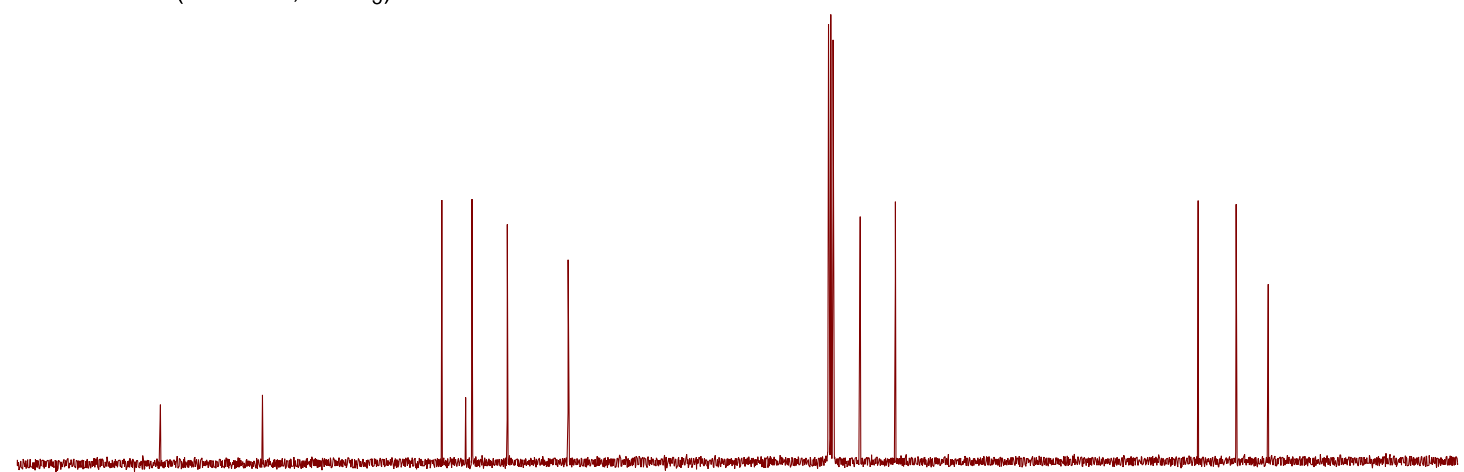

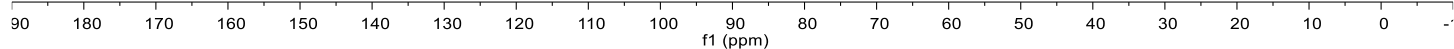




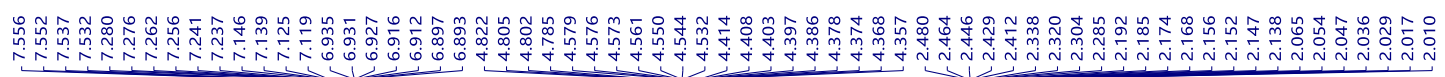<smiles>O=C1OCCCC1Oc1ccccc1Br</smiles>

${ }^{1} \mathrm{H} \mathrm{NMR}\left(400 \mathrm{MHz}, \mathrm{CDCl}_{3}\right)$
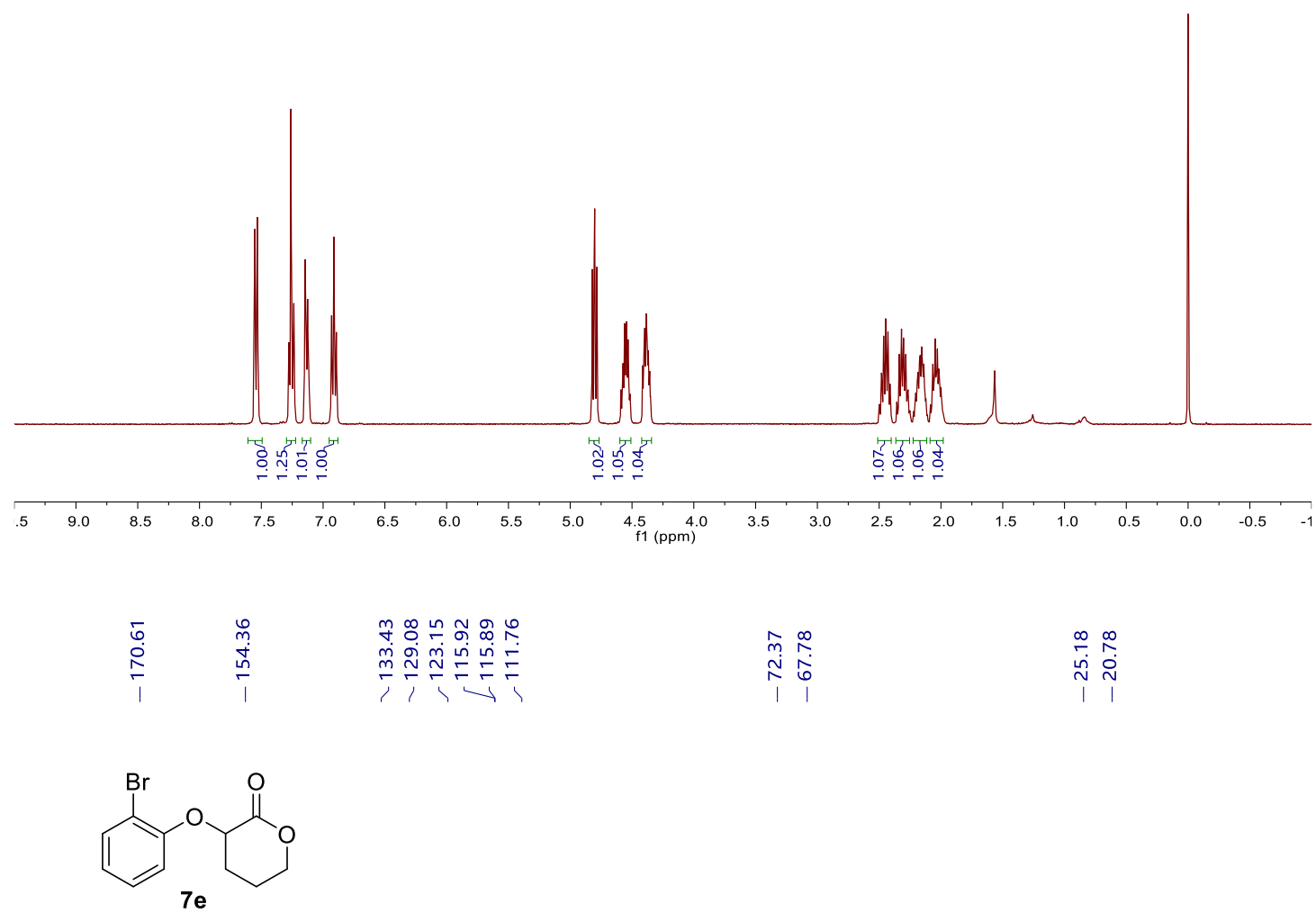

${ }^{13} \mathrm{C}$ NMR $(101 \mathrm{MHz}, \mathrm{DMSO})$

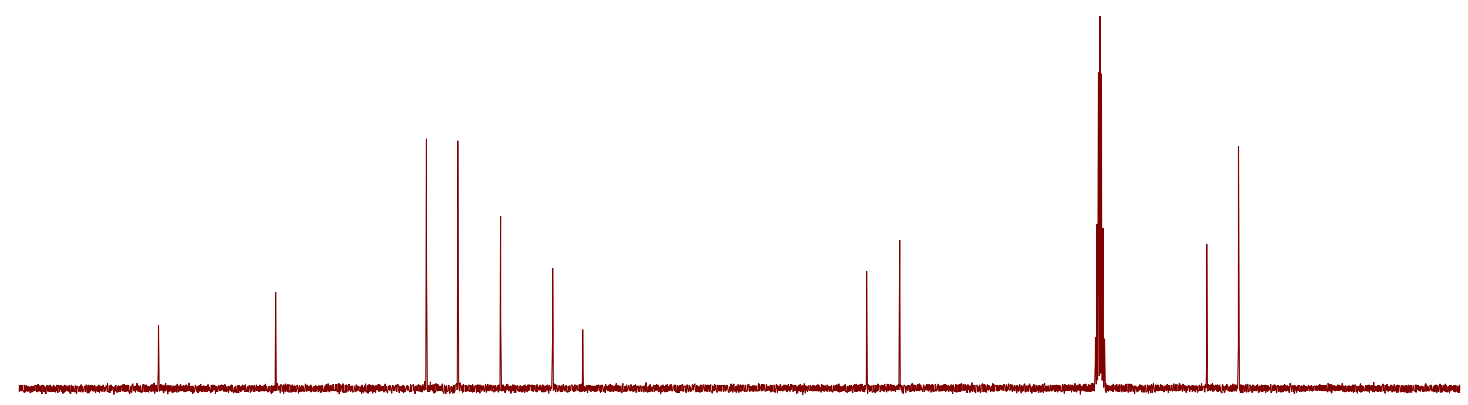

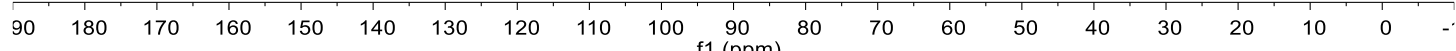


3-(2-Chlorophenoxy)tetrahydro-2H-pyran-2-one (7f)

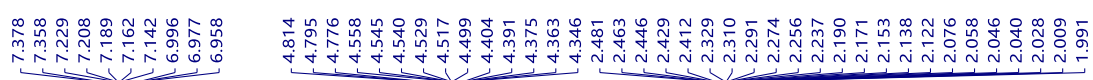

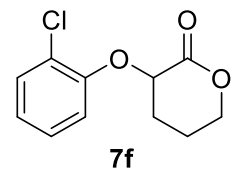

${ }^{1} \mathrm{H}$ NMR $\left(400 \mathrm{MHz}, \mathrm{CDCl}_{3}\right)$

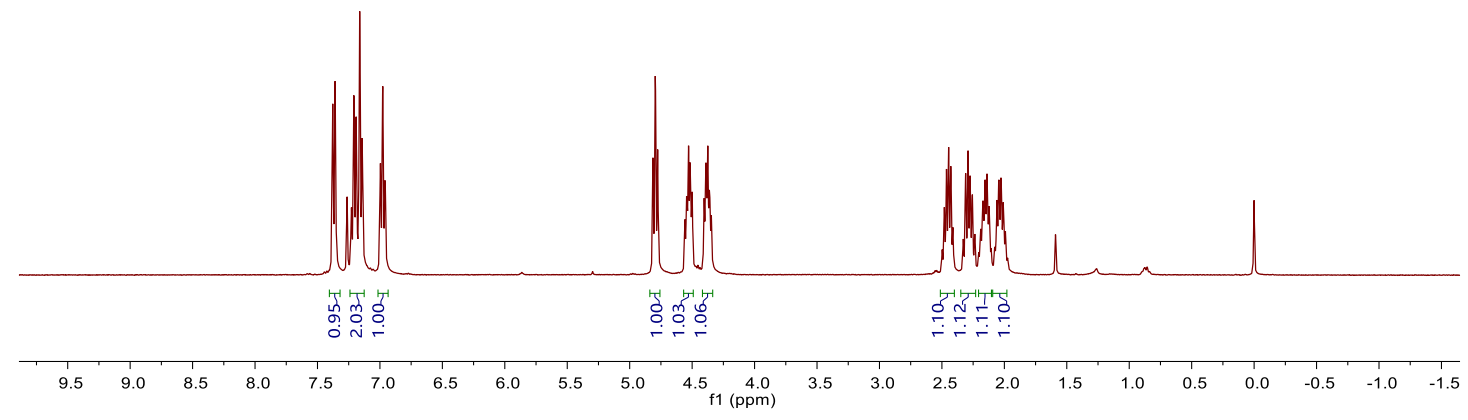

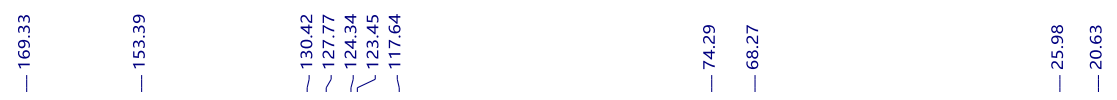<smiles>O=C1OCCCC1Oc1ccccc1Cl</smiles>

${ }^{13} \mathrm{C}$ NMR $\left(101 \mathrm{MHz}, \mathrm{CDCl}_{3}\right)$

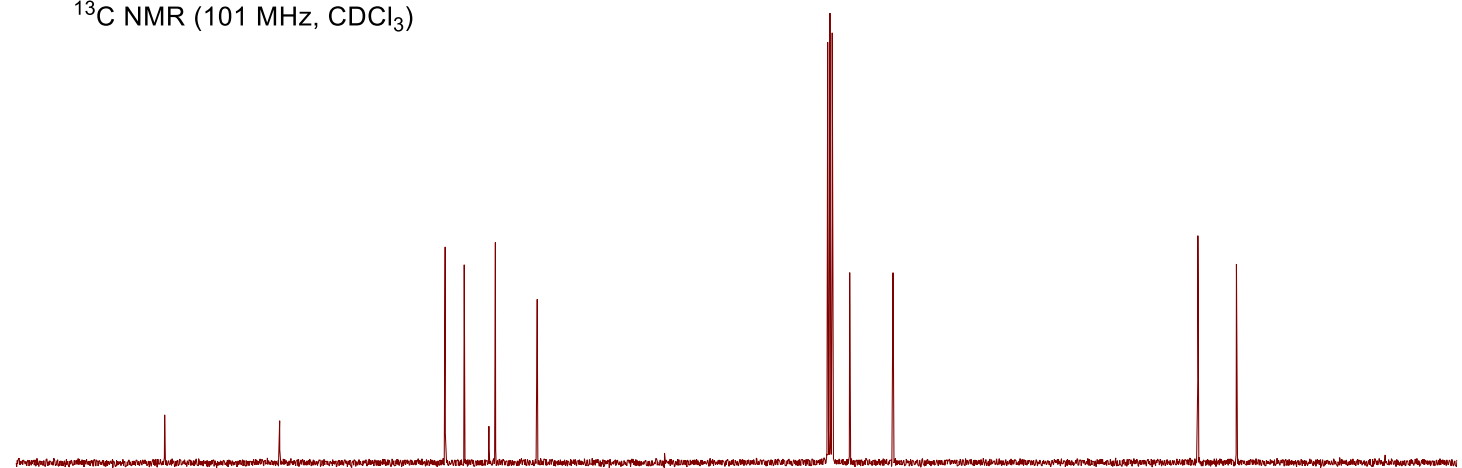

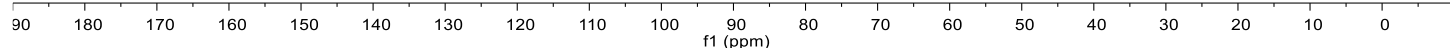


3-(2,4-Dimethylphenoxy)tetrahydro-2H-pyran-2-one (7g)

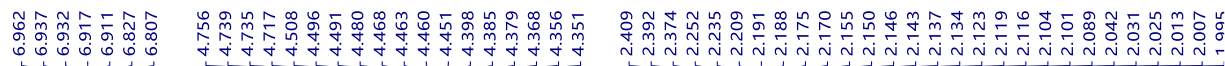<smiles>Cc1ccc(OC2CCCOC2=O)c(C)c1</smiles>

${ }^{1} \mathrm{H} \mathrm{NMR}\left(400 \mathrm{MHz}, \mathrm{CDCl}_{3}\right)$

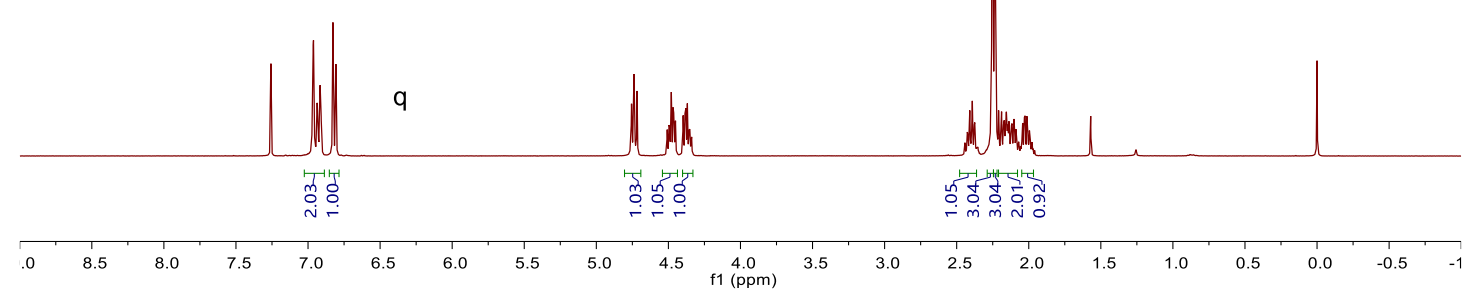

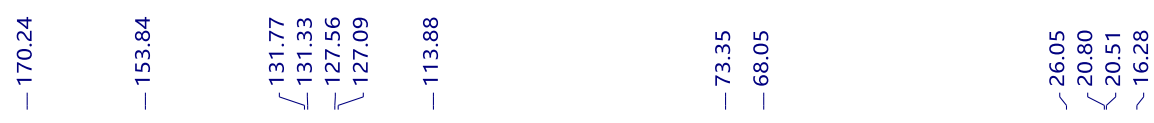

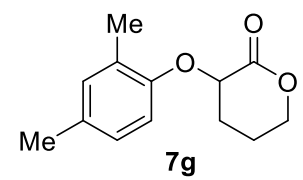

${ }^{13} \mathrm{C}$ NMR $\left(101 \mathrm{MHz}, \mathrm{CDCl}_{3}\right)$

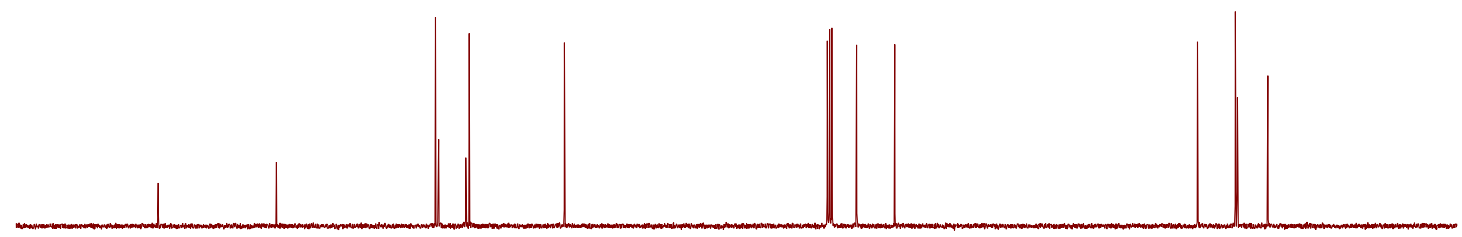

$\begin{array}{lllllllllllllllllllllllll}90 & 180 & 170 & 160 & 150 & 140 & 130 & 120 & 110 & 100 & 90 & 80 & 70 & 60 & 50 & 40 & 30 & 20 & 10 & 0 & -\end{array}$ 
(+)-2-Phenoxybutane-1,4-diol ((+)-5a)

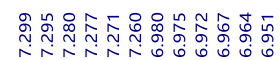

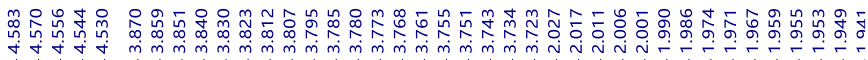

$\longrightarrow$

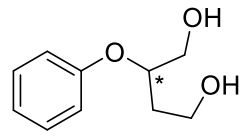

$5 a$

${ }^{1} \mathrm{H}$ NMR $\left(400 \mathrm{MHz}, \mathrm{CDCl}_{3}\right)$

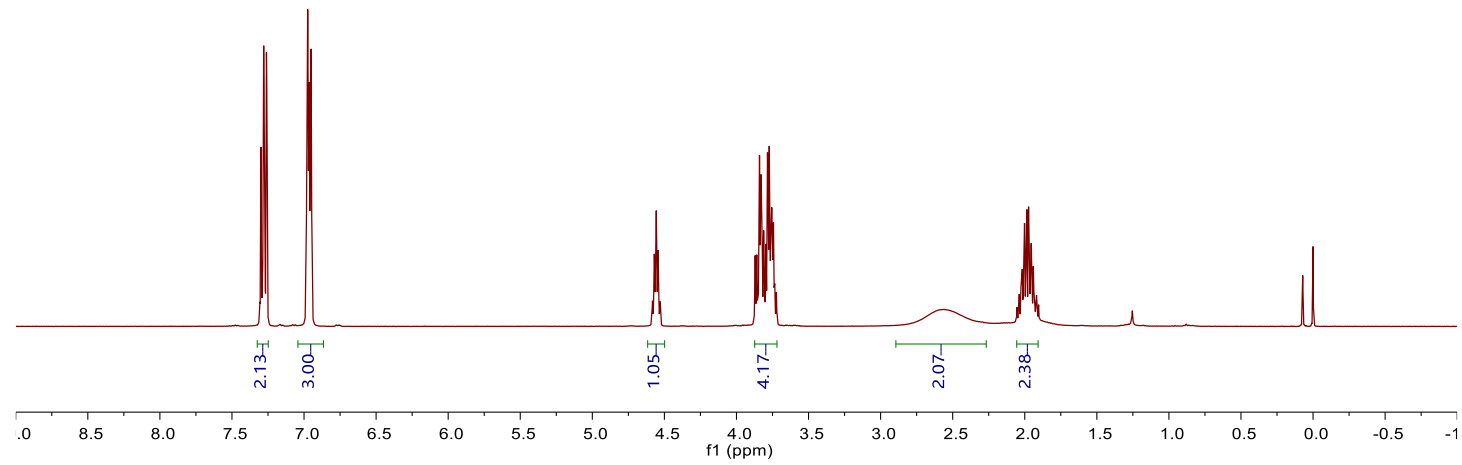

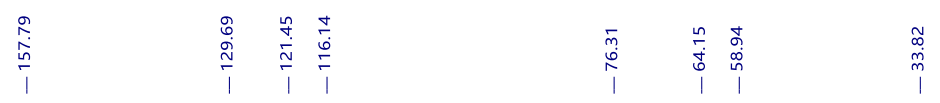

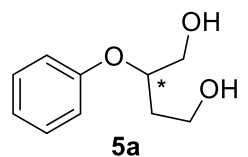

$5 a$

${ }^{13} \mathrm{C}$ NMR (101 MHz, $\mathrm{CDCl}_{3}$ )

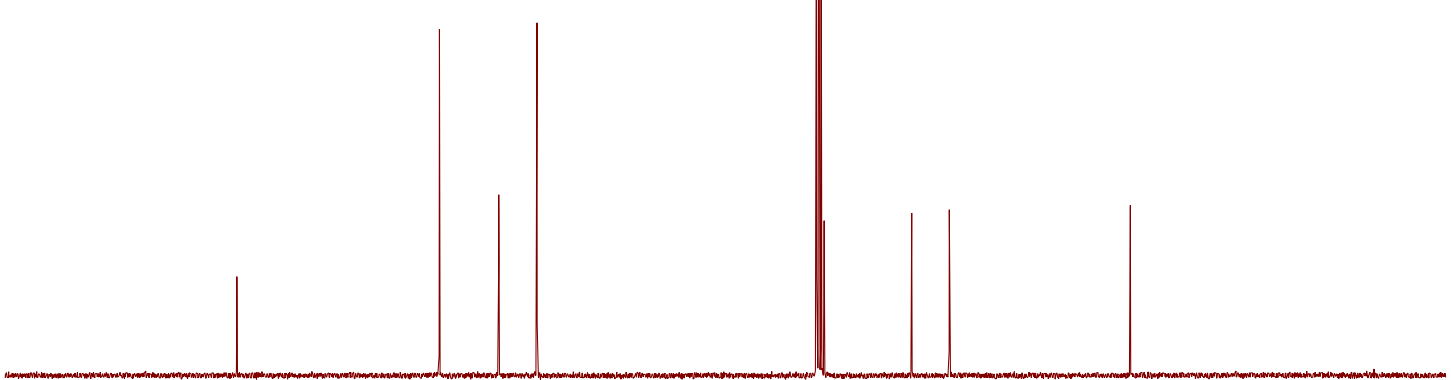

180

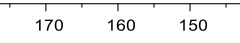

130

20

90
$\mathrm{f} 1(\mathrm{ppm})$ 
(+)2-(p-Tolyloxy)butane-1,4-diol ((+)-5b)

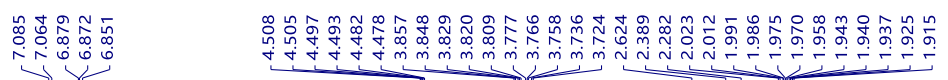

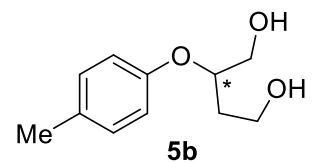

${ }^{1} \mathrm{H} \mathrm{NMR}\left(400 \mathrm{MHz}, \mathrm{CDCl}_{3}\right)$

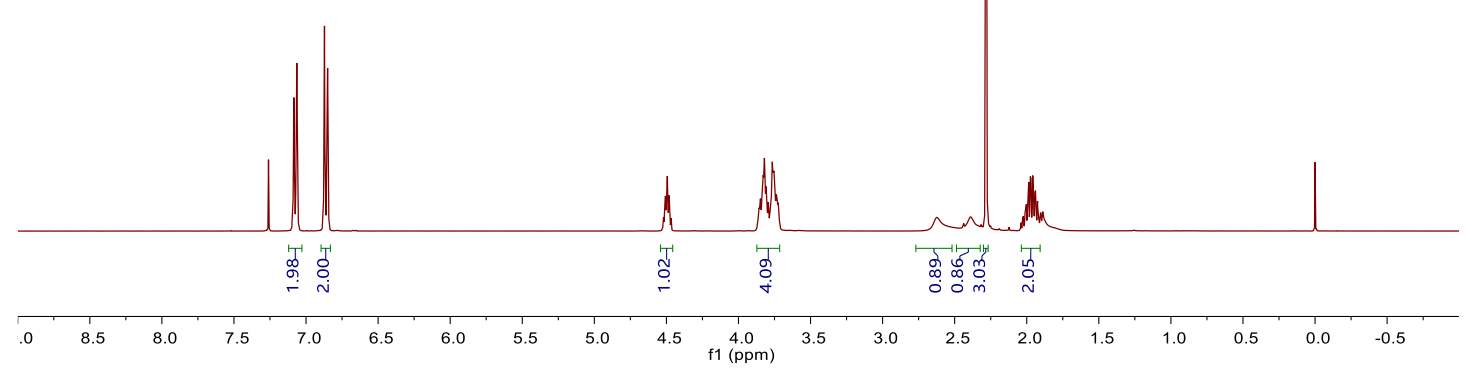

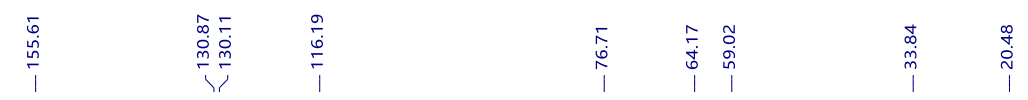

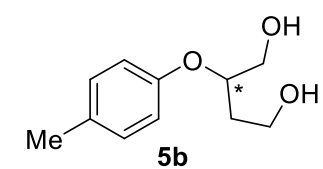

${ }^{13} \mathrm{C}$ NMR $\left(101 \mathrm{MHz}, \mathrm{CDCl}_{3}\right)$

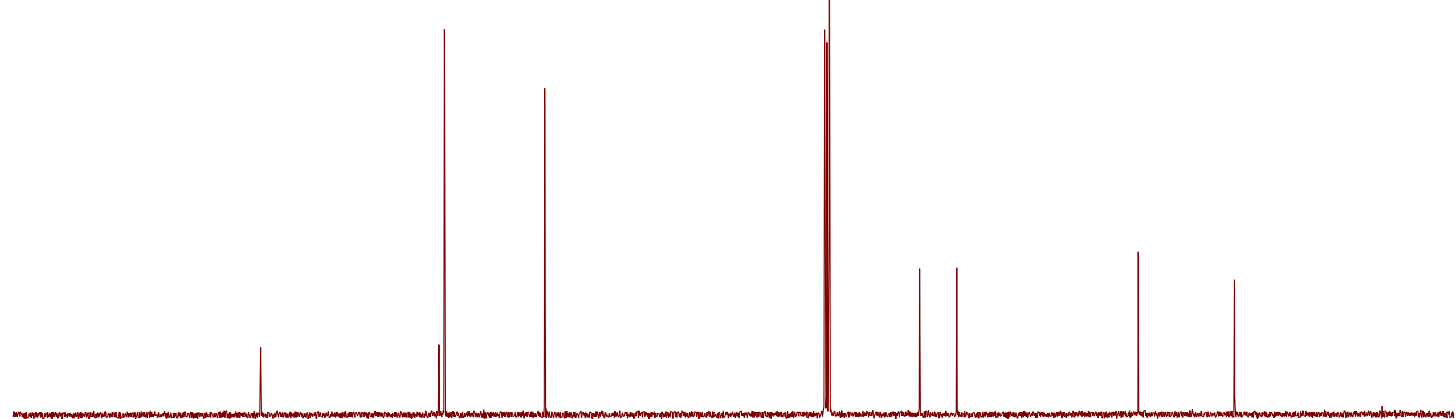

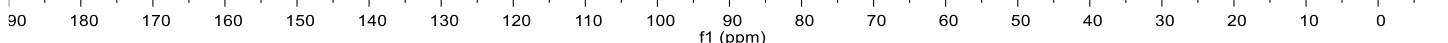


(+)-2-(4-Chlorophenoxy)butane-1,4-diol ((+)-5c)

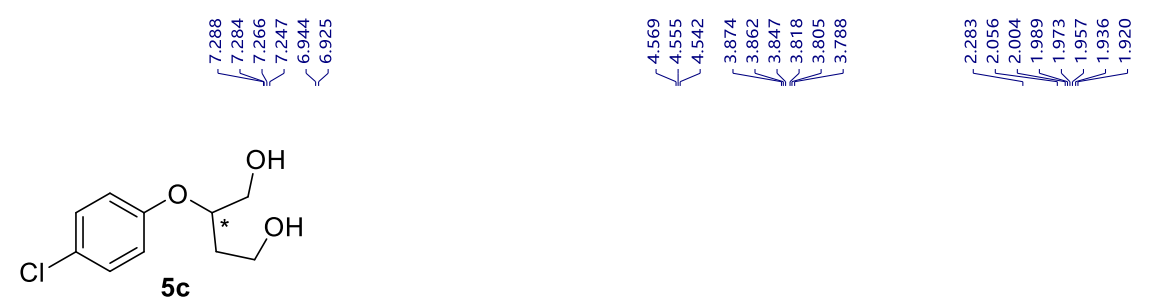

${ }^{1} \mathrm{H} \mathrm{NMR}\left(400 \mathrm{MHz}, \mathrm{CDCl}_{3}\right)$

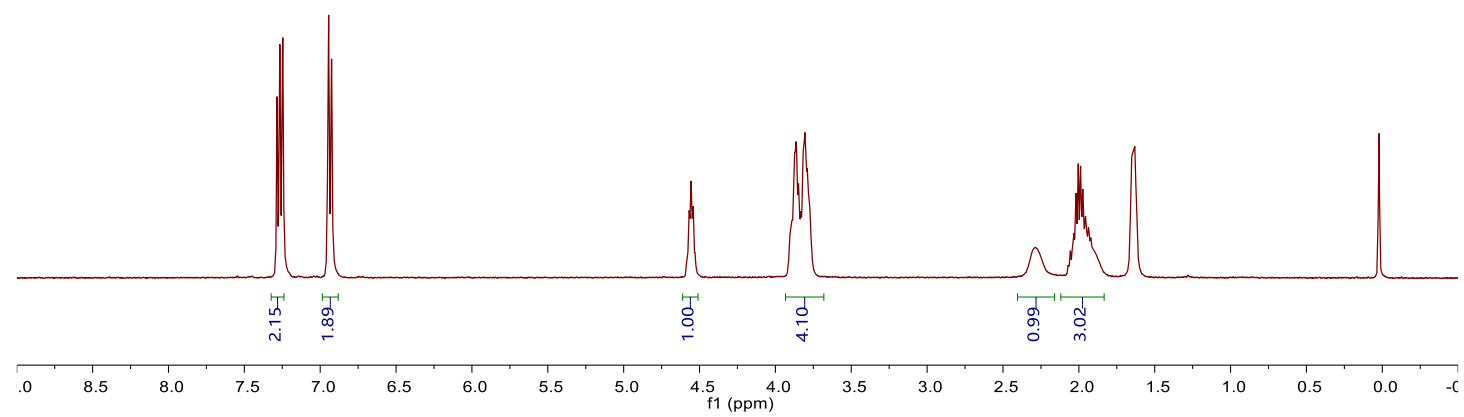

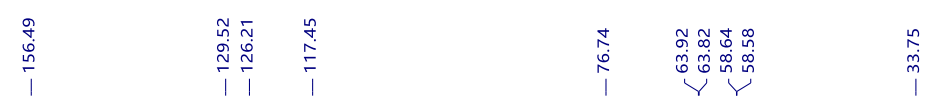

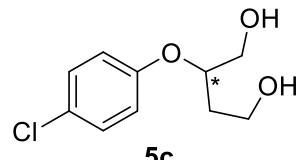

${ }^{13} \mathrm{C}$ NMR $\left(101 \mathrm{MHz}, \mathrm{CDCl}_{3}\right)$

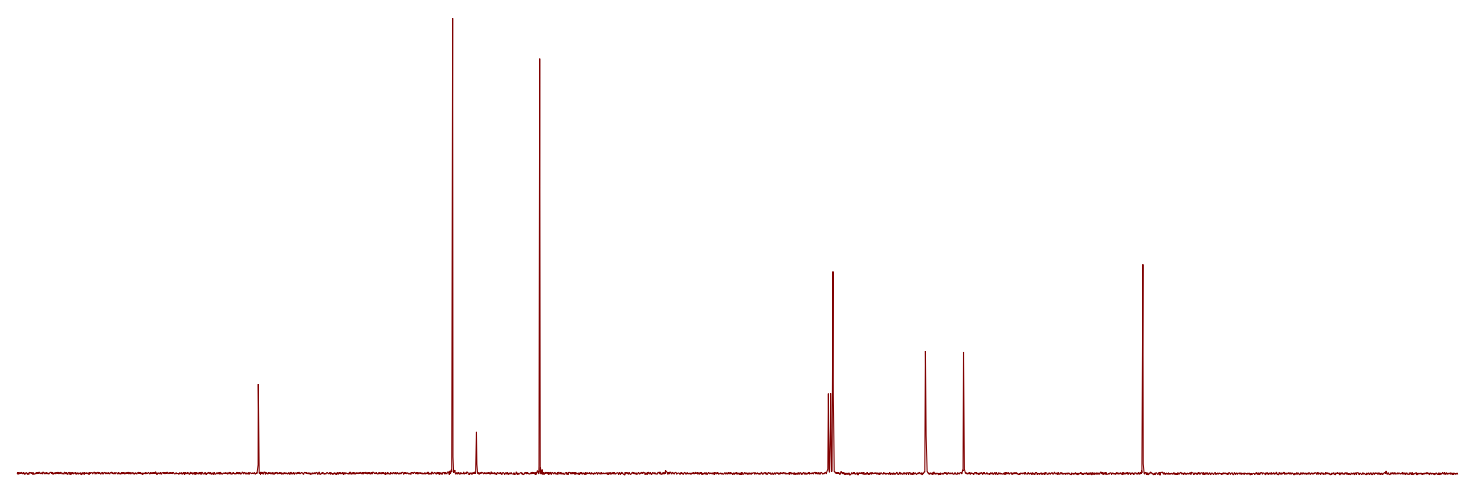

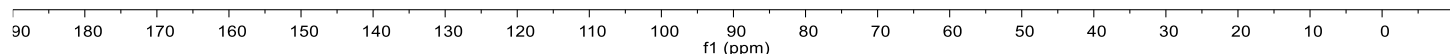


(+)-2-(4-(tert-Butyl)phenoxy)butane-1,4-diol ((+)-5d)
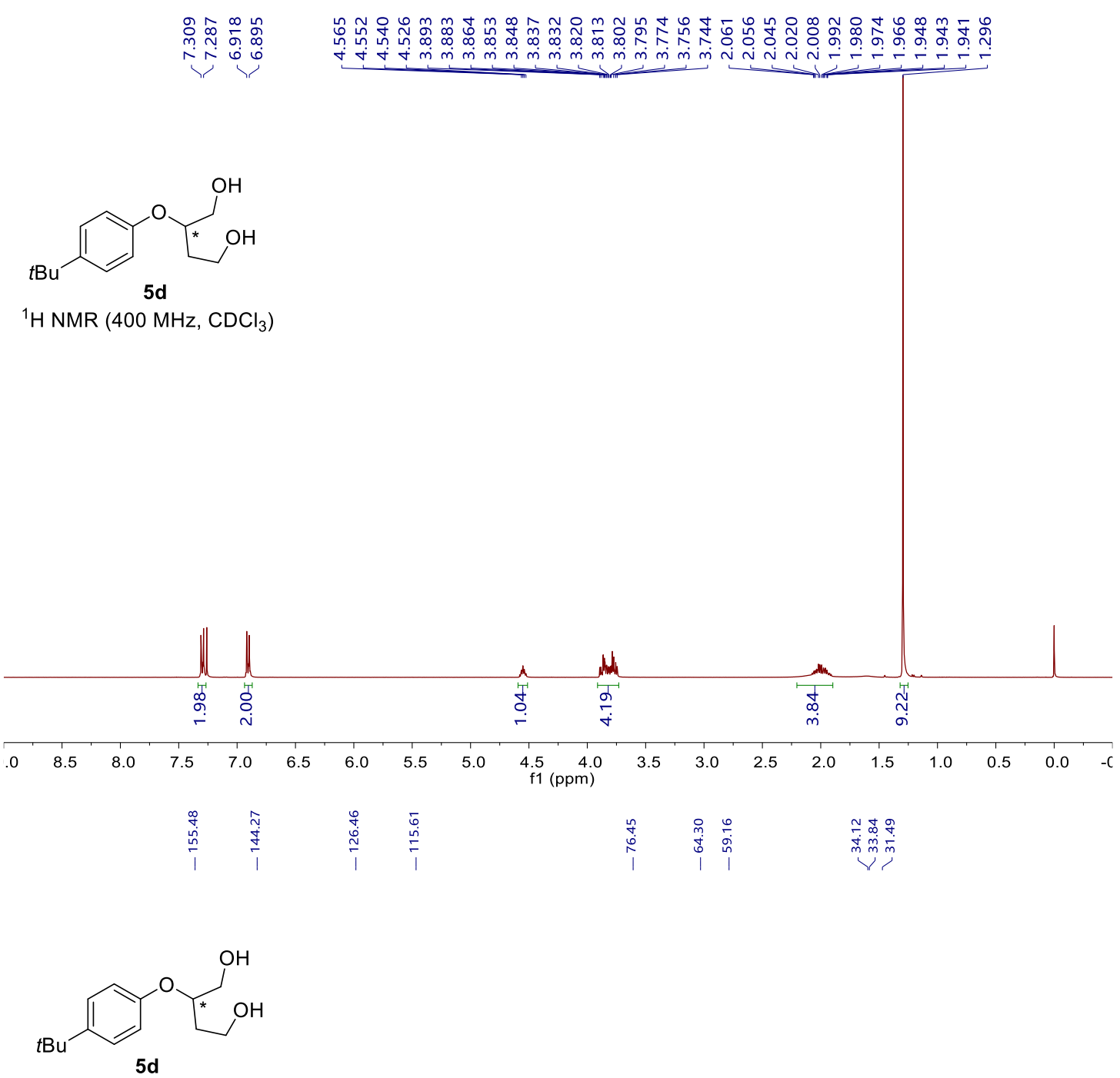

${ }^{13} \mathrm{C} \mathrm{NMR}\left(101 \mathrm{MHz}, \mathrm{CDCl}_{3}\right)$

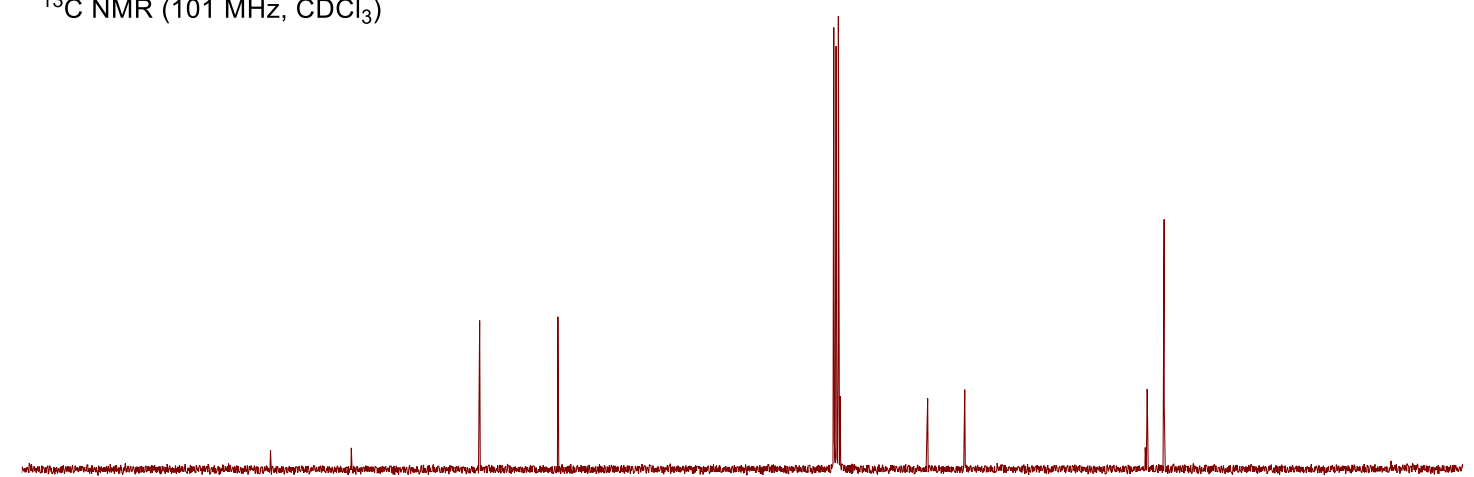

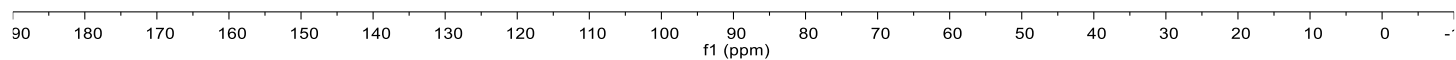


(+)-2-(3-Methoxyphenoxy)butane-1,4-diol ((+)-5e)

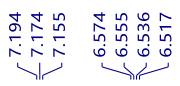

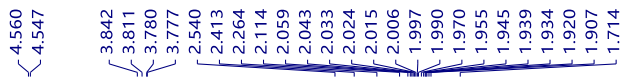

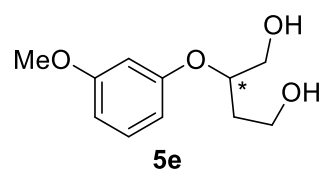

${ }^{1} \mathrm{H} \mathrm{NMR}\left(400 \mathrm{MHz}, \mathrm{CDCl}_{3}\right)$

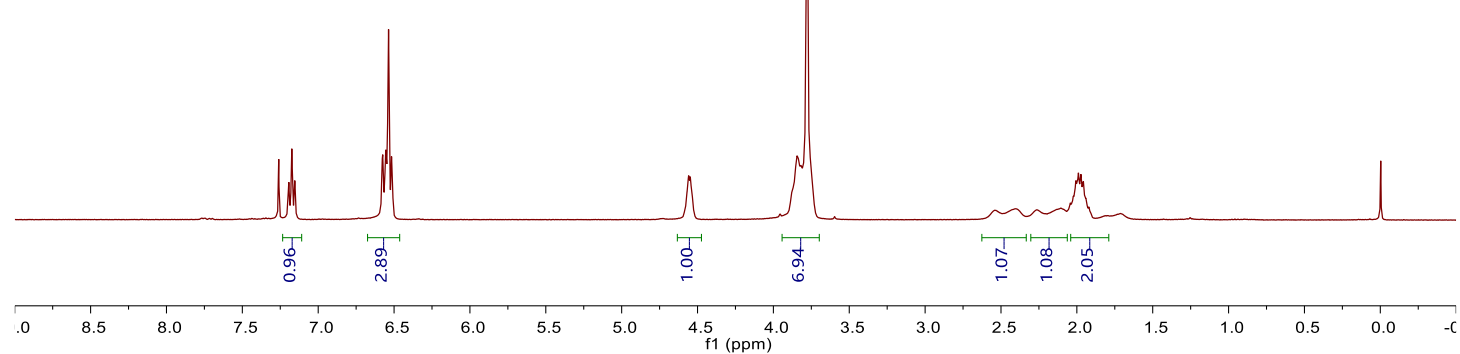

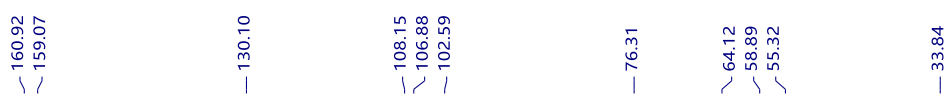

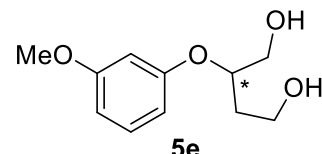

${ }^{13} \mathrm{C}$ NMR $\left(101 \mathrm{MHz}, \mathrm{CDCl}_{3}\right)$

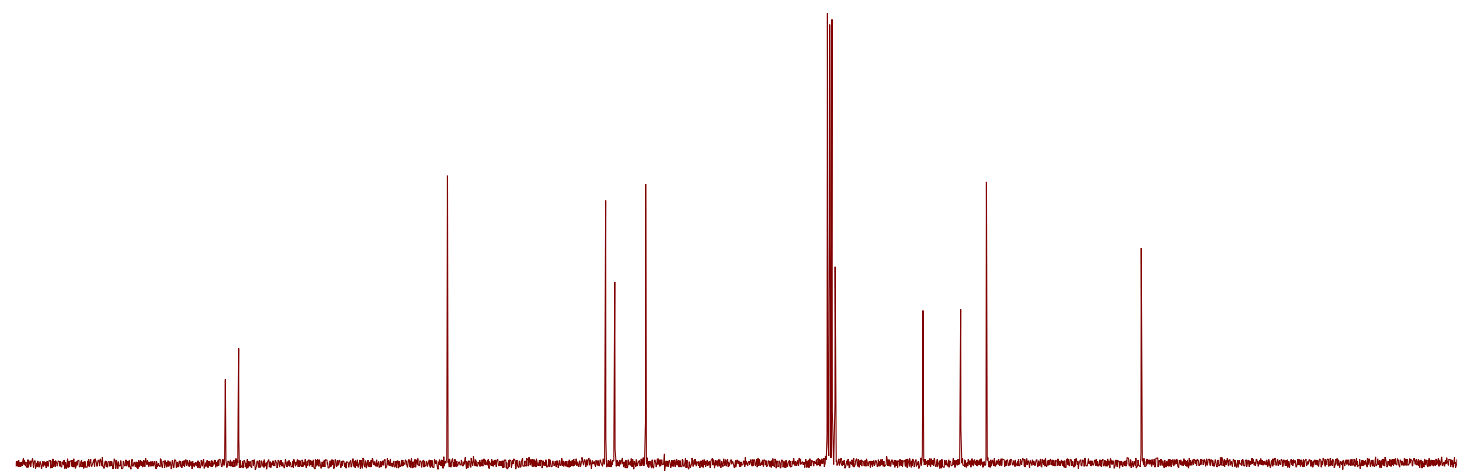

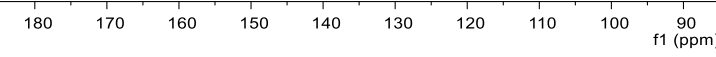


(+)-2-(3-Chlorophenoxy)butane-1,4-diol ((+)-5f)

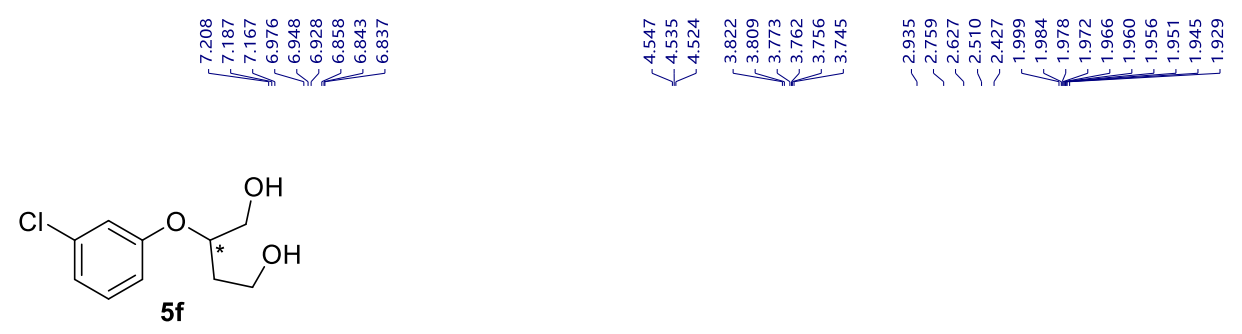

${ }^{1} \mathrm{H}$ NMR $\left(400 \mathrm{MHz}, \mathrm{CDCl}_{3}\right)$

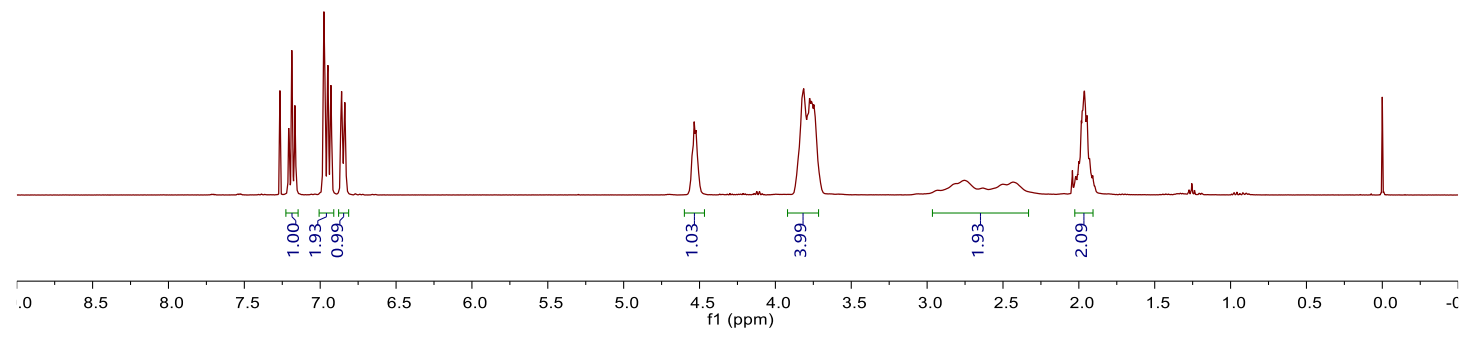

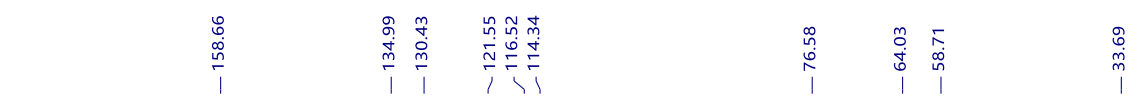

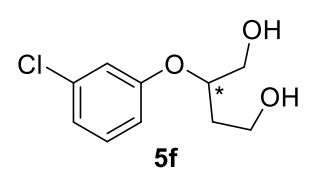

${ }^{13} \mathrm{C}$ NMR $\left(101 \mathrm{MHz}, \mathrm{CDCl}_{3}\right)$

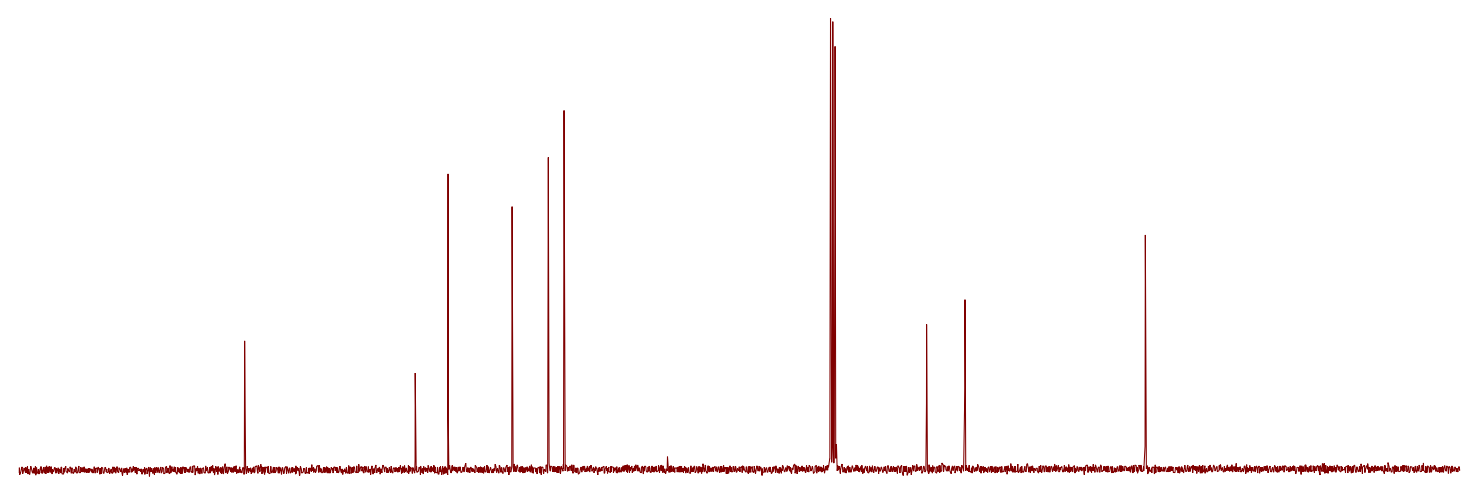

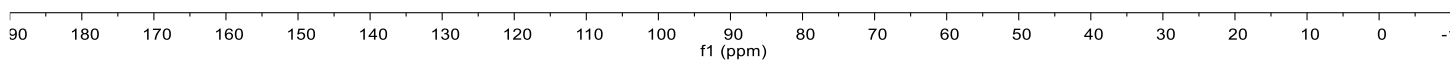


(+)-2-(3-Bromophenoxy)butane-1,4-diol ((+)-5g)

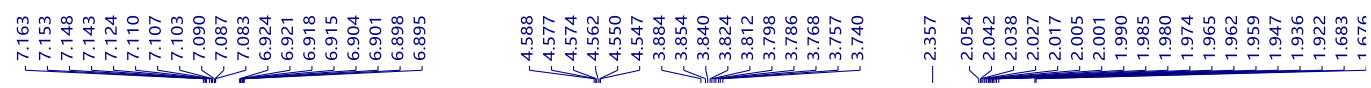<smiles>OCCC(CO)Oc1cccc(Br)c1</smiles>

${ }^{1} \mathrm{H} \mathrm{NMR}\left(400 \mathrm{MHz}, \mathrm{CDCl}_{3}\right)$

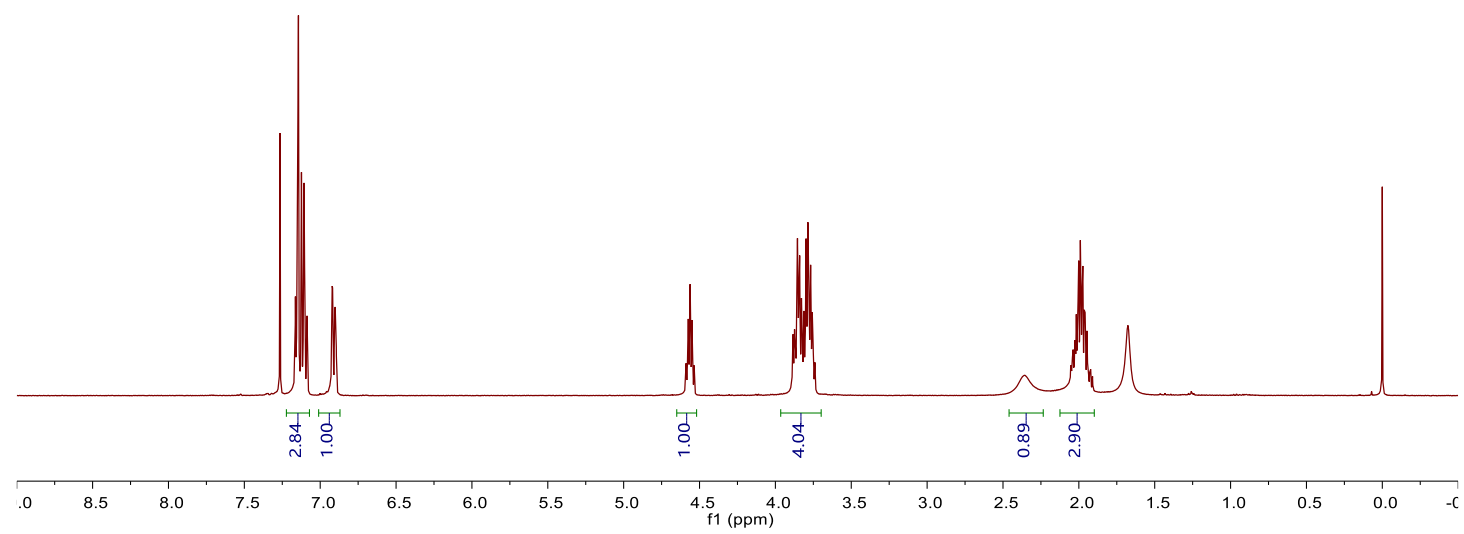

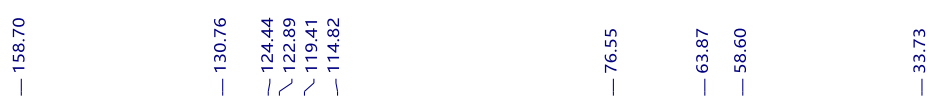

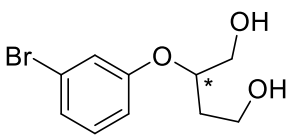

$5 \mathrm{~g}$

${ }^{13} \mathrm{C}$ NMR $\left(101 \mathrm{MHz}, \mathrm{CDCl}_{3}\right)$

f1 (ppm) 
(+)-2-(o-Tolyloxy)butane-1,4-diol ((+)-5h)

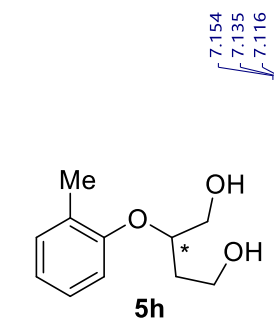

${ }^{1} \mathrm{H}$ NMR $\left(400 \mathrm{MHz}, \mathrm{CDCl}_{3}\right)$

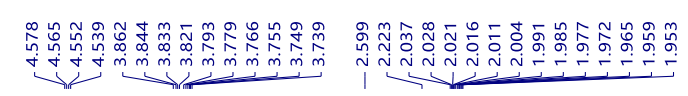

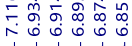

1 $\mathrm{HNMR}\left(400 \mathrm{MHz}, \mathrm{CDCl}_{3}\right)$

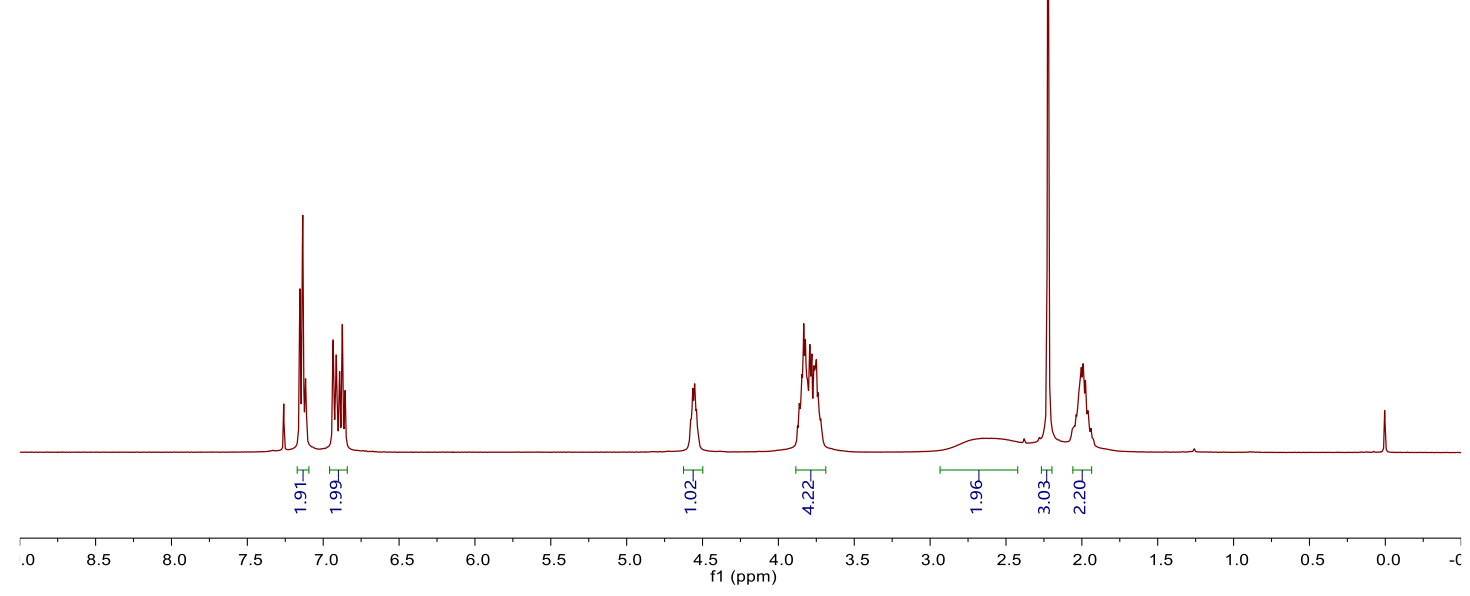

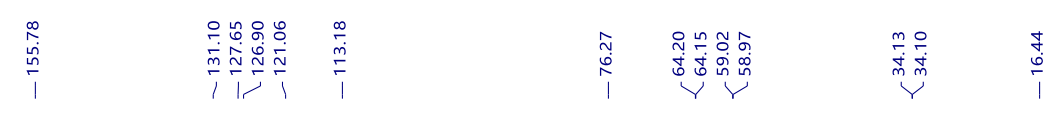

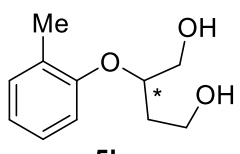

$5 \mathrm{~h}$

${ }^{13} \mathrm{C}$ NMR $\left(101 \mathrm{MHz}, \mathrm{CDCl}_{3}\right)$

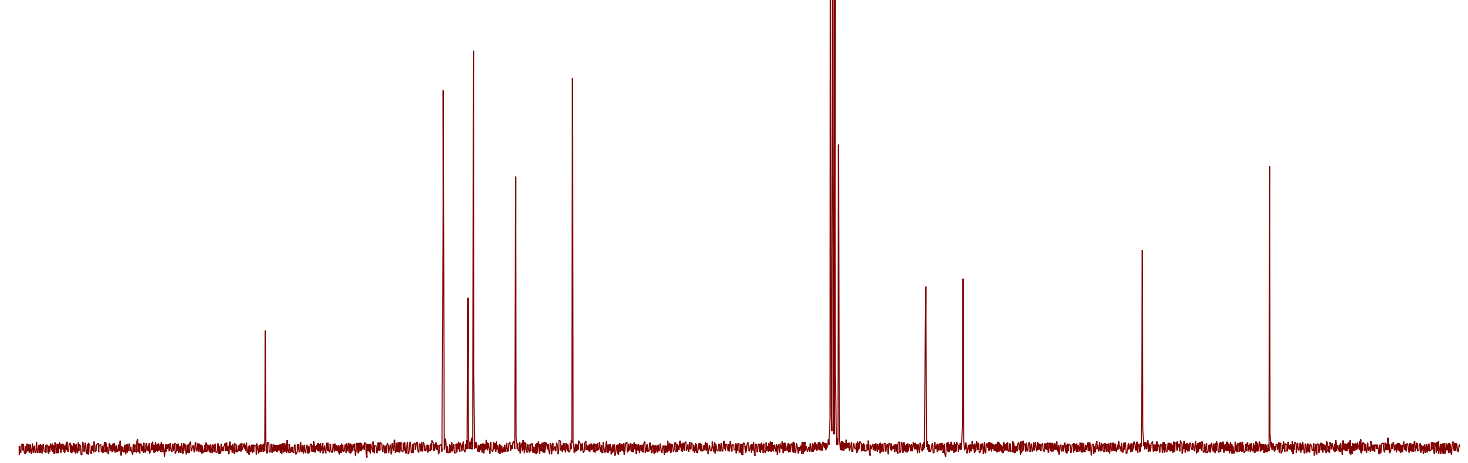

$\begin{array}{llllllllll}180 & 170 & 160 & 150 & 140 & 130 & 120 & 110 & 100 & \begin{array}{c}\mid \\ \mathrm{f} 1\end{array}(\mathrm{ppm}) \\ & & & & & & & & & \end{array}$ 
(+)-2-(2-Methoxyphenoxy)butane-1,4-diol ((+)-5i)

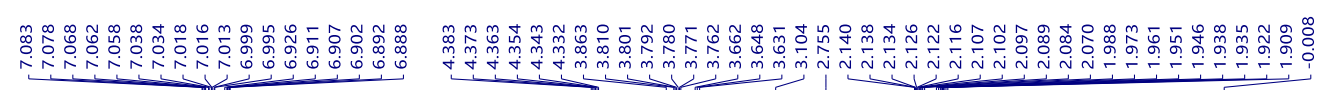

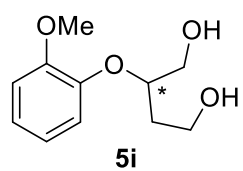

${ }^{1} \mathrm{H}$ NMR $\left(400 \mathrm{MHz}, \mathrm{CDCl}_{3}\right)$

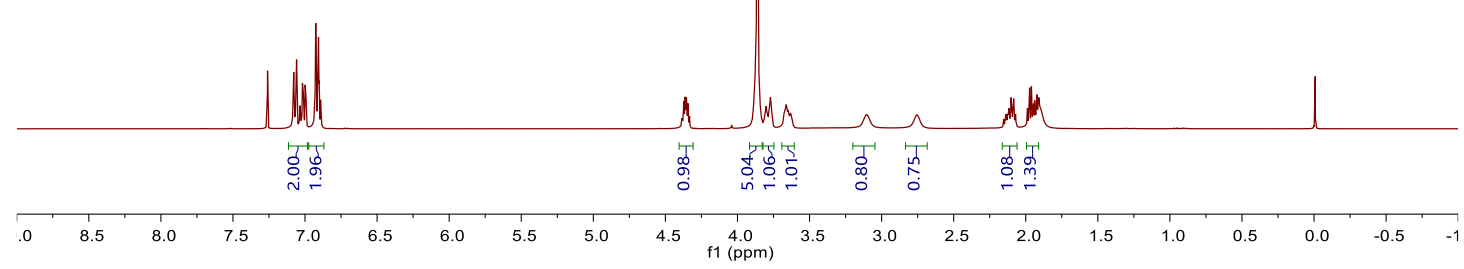

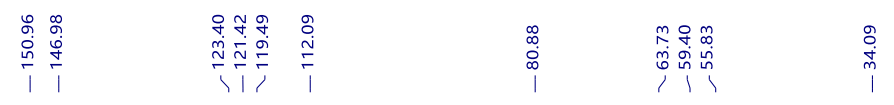

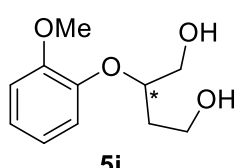

$5 \mathbf{i}$

${ }^{13} \mathrm{C}$ NMR $\left(101 \mathrm{MHz}, \mathrm{CDCl}_{3}\right)$

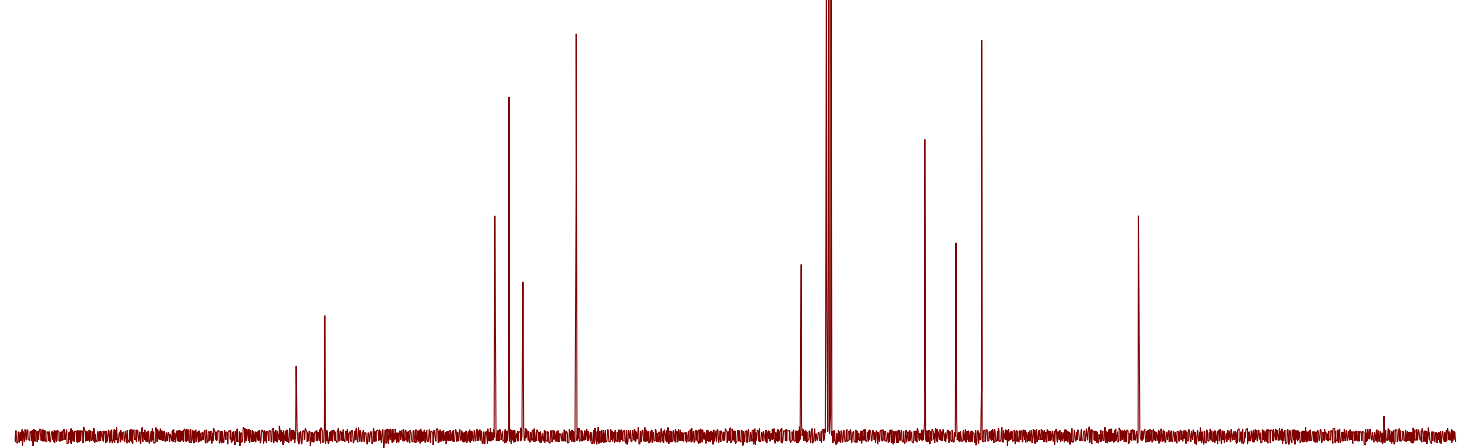

\begin{tabular}{lllllllllllllllllllllllll}
\hline 90 & 180 & 170 & 160 & 150 & 140 & 130 & 120 & 110 & 100 & 90 & 80 & 70 & 60 & 50 & 40 & 30 & 20 & 10 & 0 & -
\end{tabular} 
<smiles>OCCC(CO)Oc1ccccc1Cl</smiles>
$5 \mathrm{j}$

${ }^{1} \mathrm{H}$ NMR $\left(400 \mathrm{MHz}, \mathrm{CDCl}_{3}\right)$

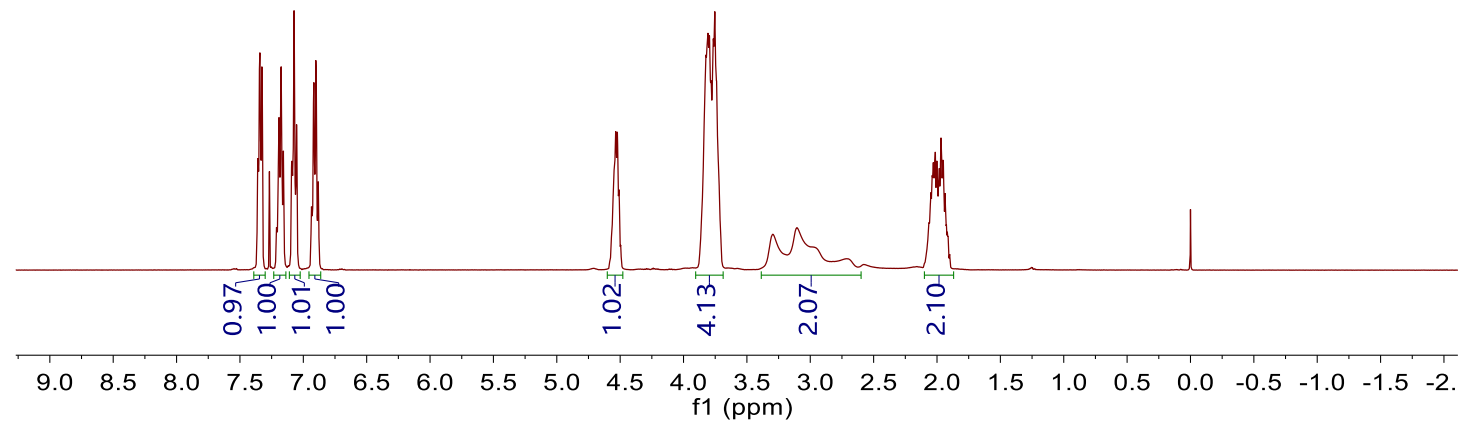

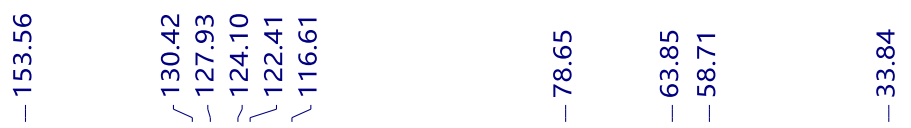<smiles>OCC[C+](CCO)Oc1ccccc1Cl</smiles>

$5 j$

${ }^{13} \mathrm{C}$ NMR $\left(101 \mathrm{MHz}, \mathrm{CDCl}_{3}\right)$

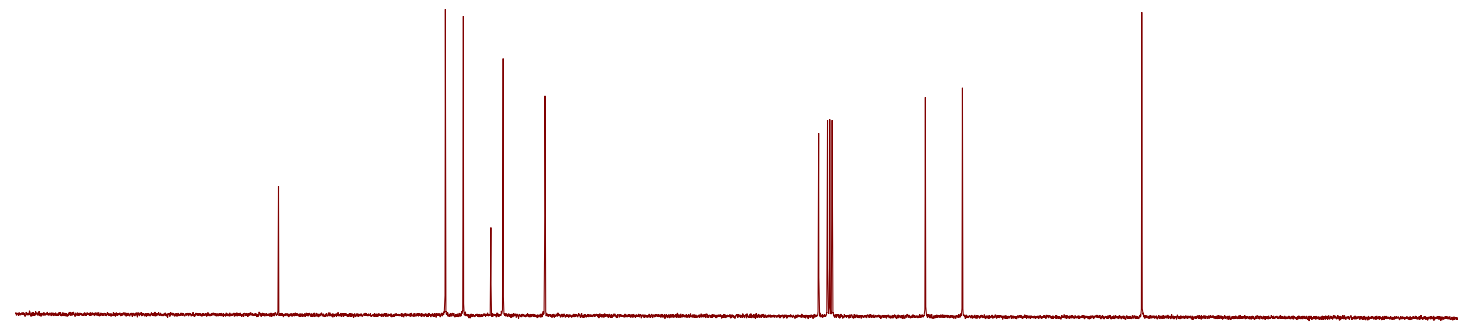

$\begin{array}{lllllllllllllllllll}180 & 170 & 160 & 150 & 140 & 130 & 120 & 110 & 100 \underset{\mathrm{f} 1}{9(\mathrm{ppm})} \mathbf{8 0} & 80 & 70 & 60 & 50 & 40 & 30 & 20 & 10 & 0 & -\end{array}$ 
(+)-2-(2-Fluorophenoxy)butane-1,4-diol ((+)-5k)

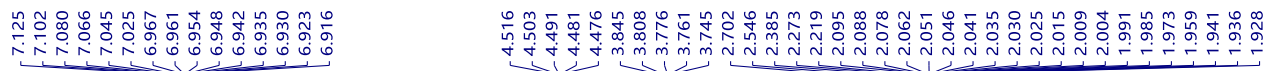

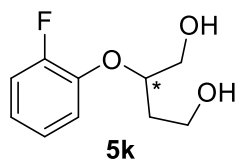

${ }^{1} \mathrm{H}$ NMR $\left(400 \mathrm{MHz}, \mathrm{CDCl}_{3}\right)$

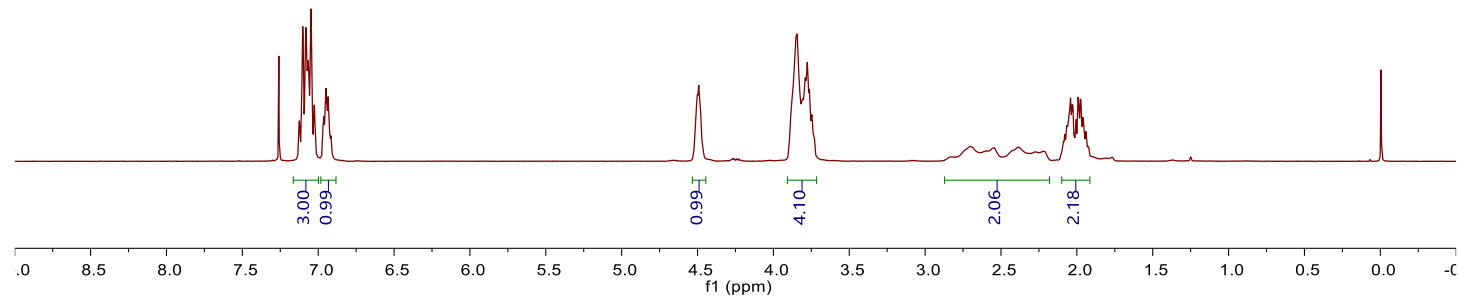

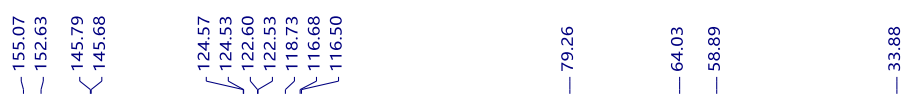

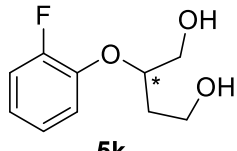

$5 \mathbf{k}$

${ }^{13} \mathrm{C}$ NMR $\left(101 \mathrm{MHz}, \mathrm{CDCl}_{3}\right)$

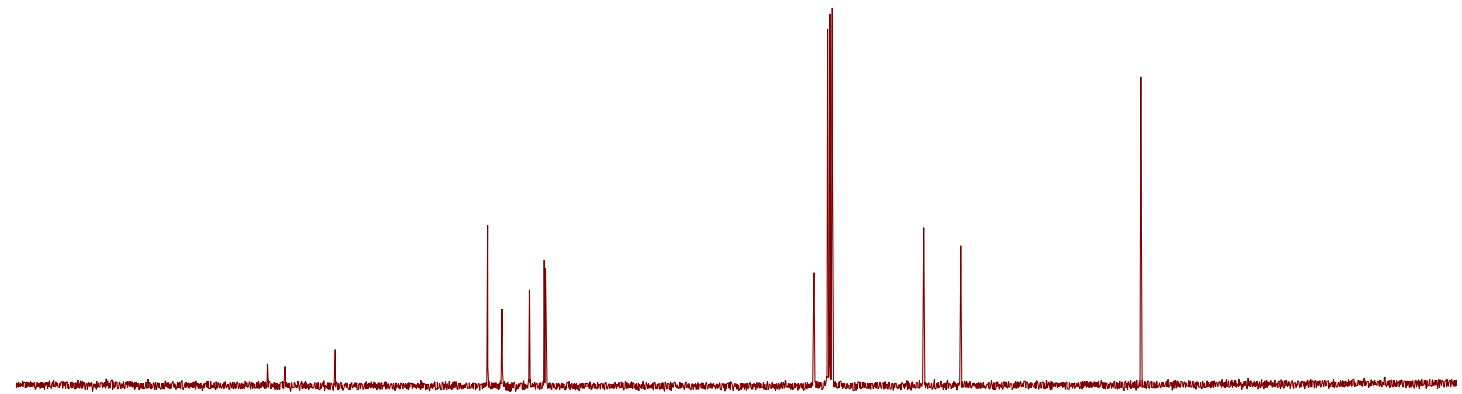

\begin{tabular}{lllllllllllllllllllll}
\hline 90 & 180 & 170 & 160 & 150 & 140 & 130 & 120 & 110 & 100 & $\begin{array}{c}90 \\
\mathrm{f} 1(\mathrm{ppm})\end{array}$ & 80 & 70 & 60 & 50 & 40 & 30 & 20 & 10 & 0 & -
\end{tabular} 
(R)-2-(2,5-Dimethylphenoxy)butane-1,4-diol ((R)-5l)
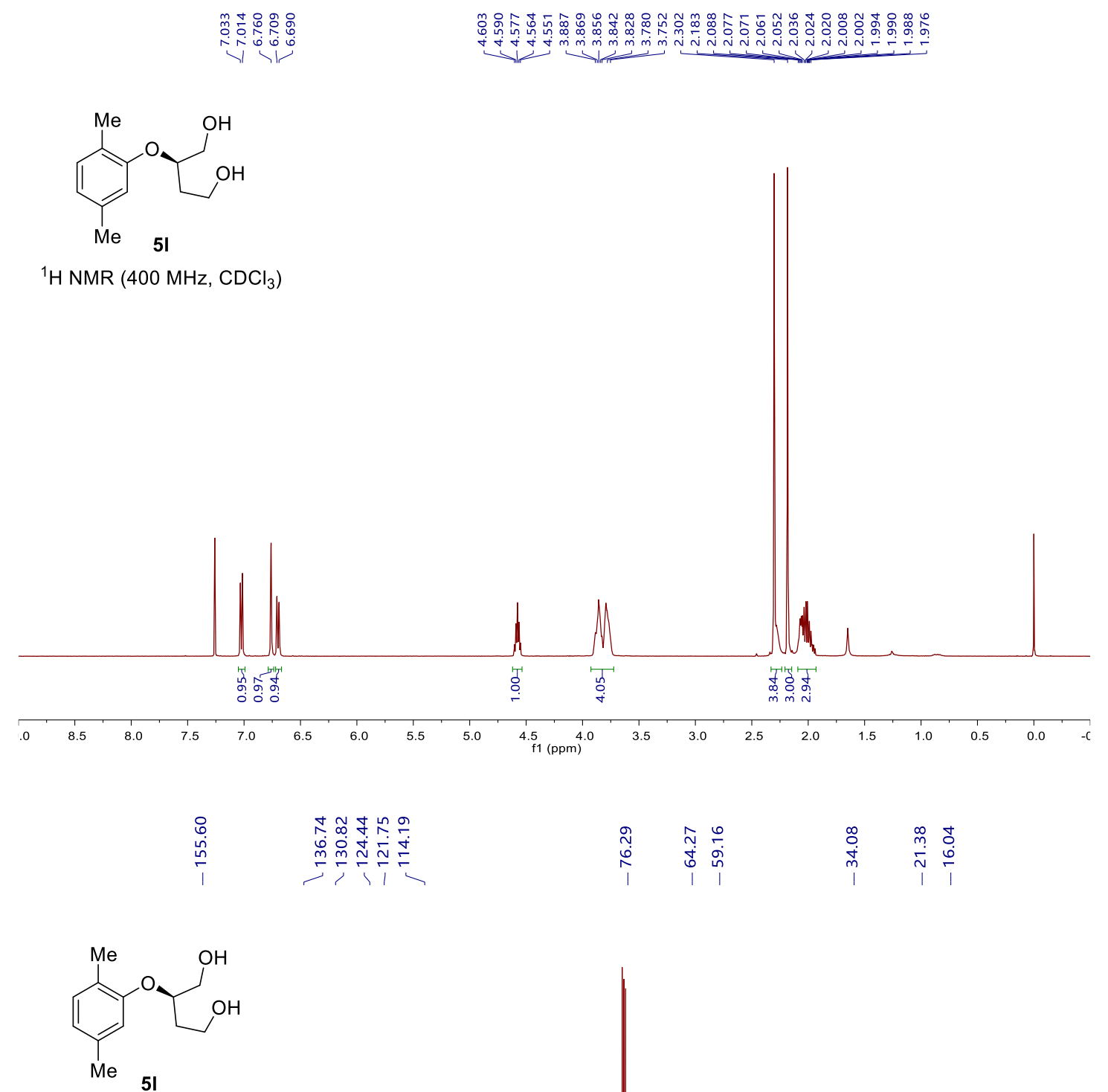

${ }^{13} \mathrm{C} \mathrm{NMR}\left(101 \mathrm{MHz}, \mathrm{CDCl}_{3}\right)$

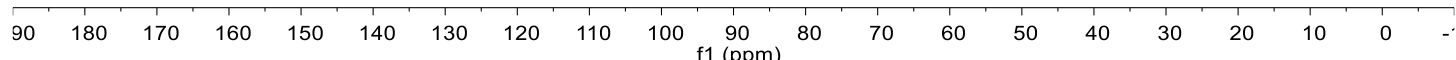


(+)-2-(Naphthalen-2-yloxy)butane-1,4-diol ((+)-5m)

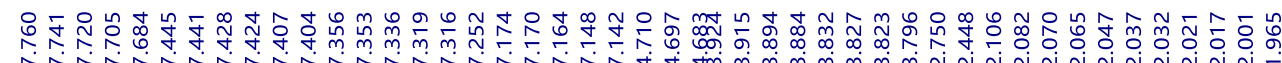

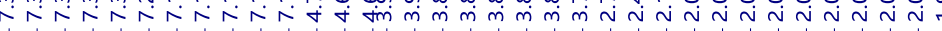

(N)

$5 \mathrm{~m}$

${ }^{1} \mathrm{H}$ NMR $\left(400 \mathrm{MHz}, \mathrm{CDCl}_{3}\right)$

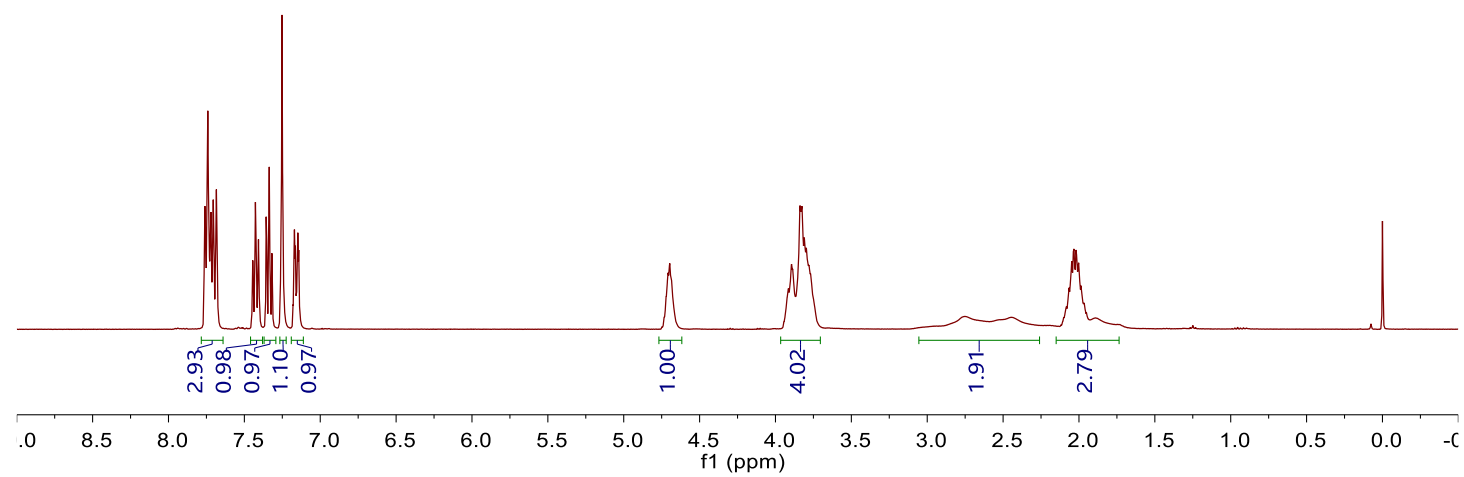

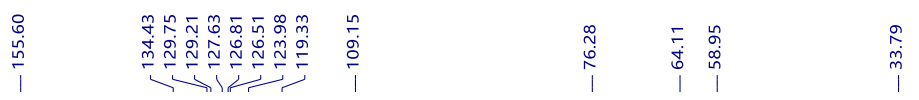

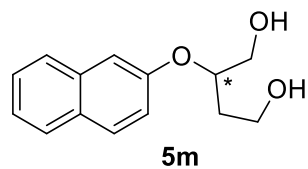

${ }^{13} \mathrm{C}$ NMR $\left(101 \mathrm{MHz}, \mathrm{CDCl}_{3}\right)$

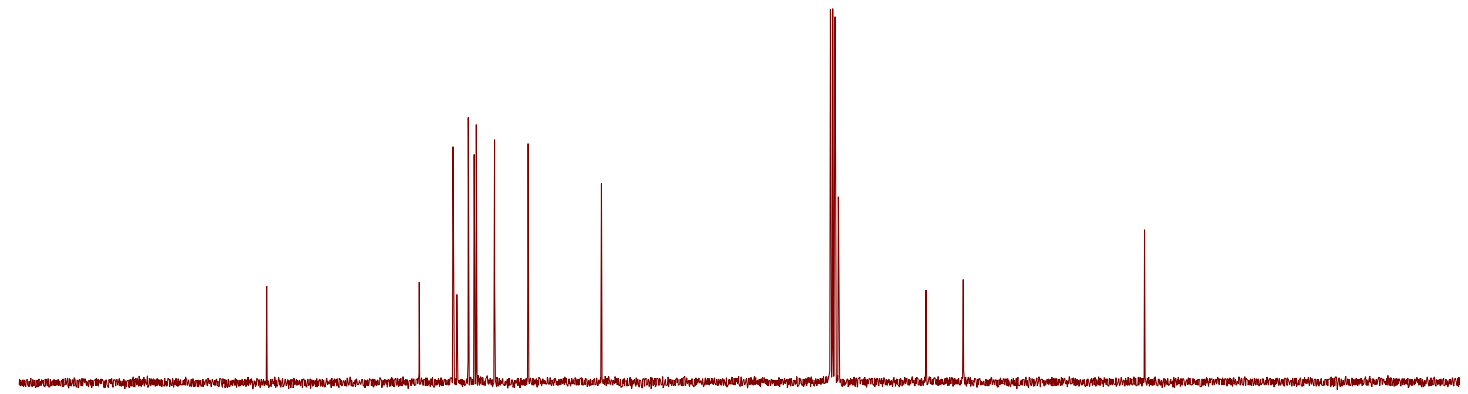

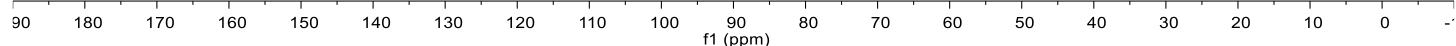


(+)-2-Phenoxypentane-1,5-diol ((+)-8a)

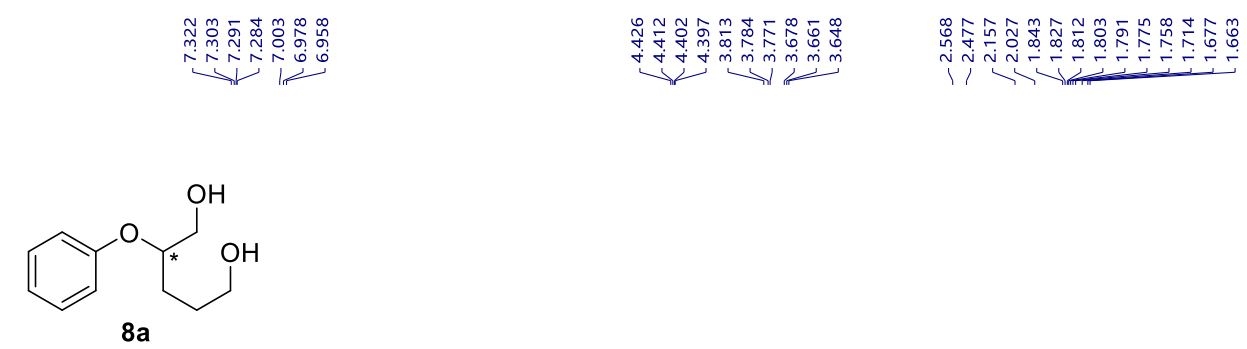

${ }^{1} \mathrm{H} \mathrm{NMR}\left(400 \mathrm{MHz}, \mathrm{CDCl}_{3}\right)$

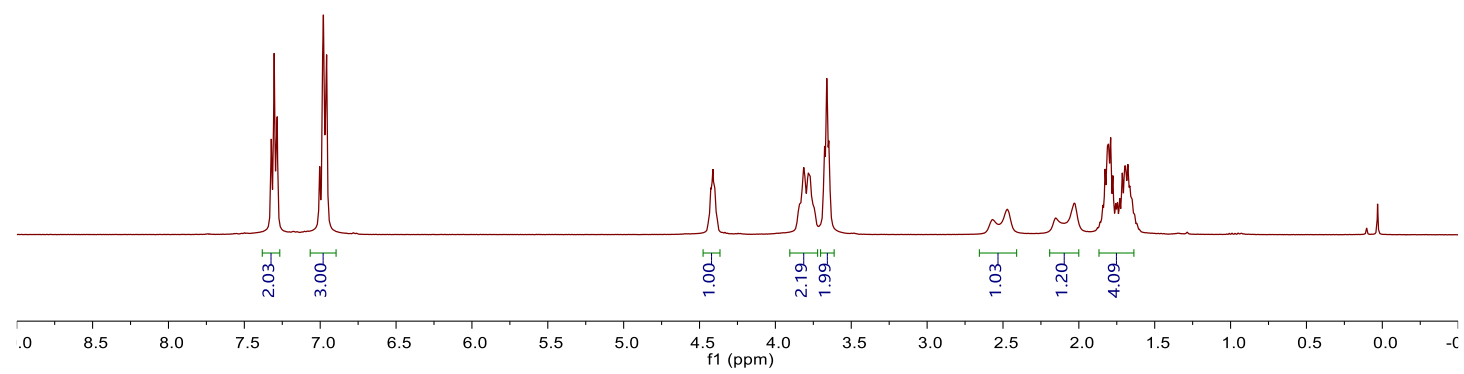

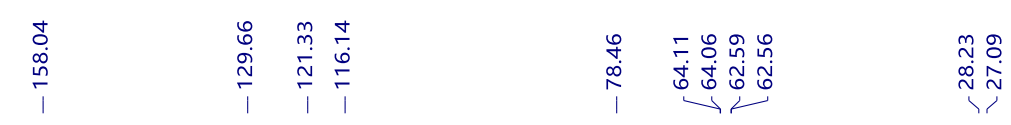

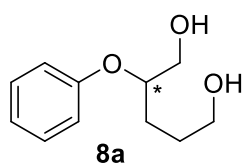

${ }^{13} \mathrm{C}$ NMR $\left(101 \mathrm{MHz}, \mathrm{CDCl}_{3}\right)$

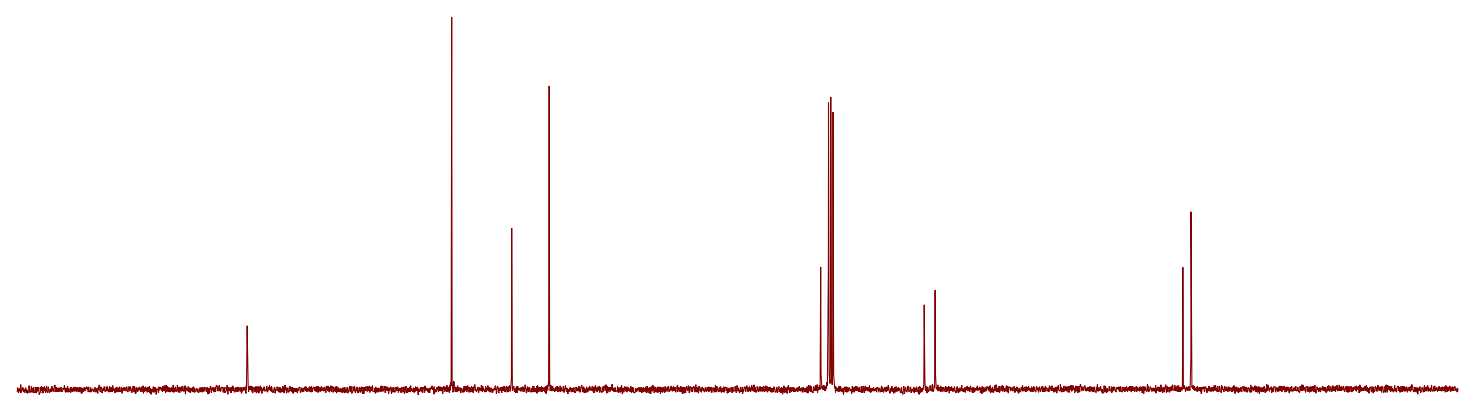

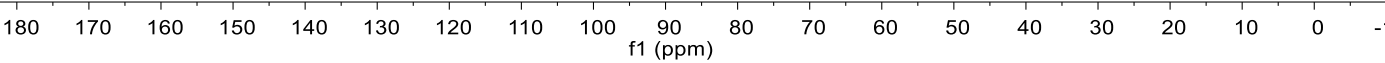


(+)-2-(p-Tolyloxy)pentane-1,5-diol ((+)-8b)

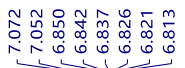

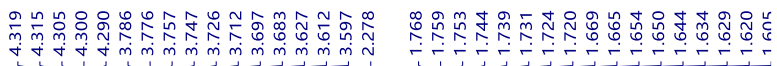

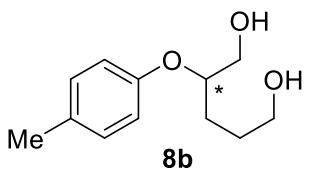

${ }^{1} \mathrm{H} \mathrm{NMR}\left(400 \mathrm{MHz}, \mathrm{CDCl}_{3}\right)$

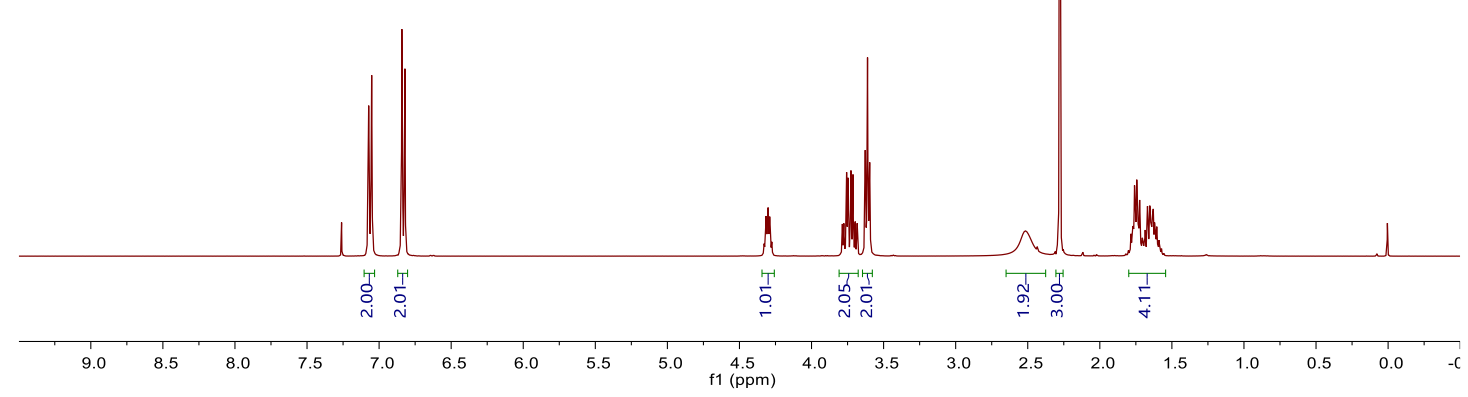

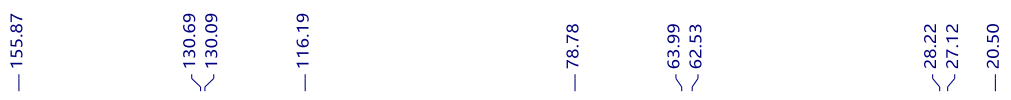

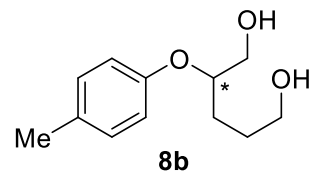

${ }^{13} \mathrm{C}$ NMR $\left(101 \mathrm{MHz}, \mathrm{CDCl}_{3}\right)$

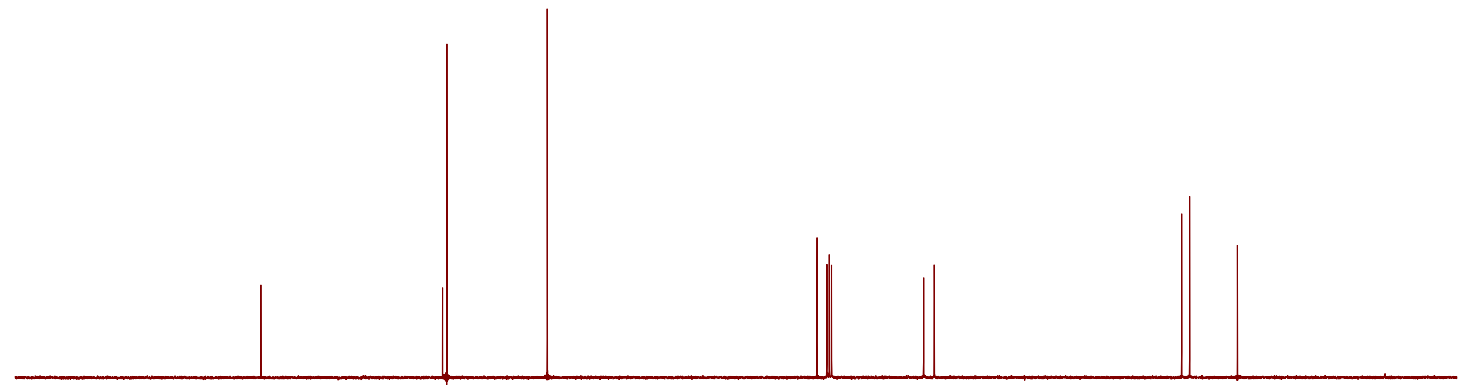

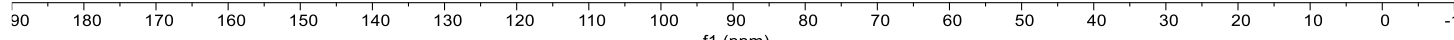


(+)-2-(m-Tolyloxy)pentane-1,5-diol ((+)-8c)

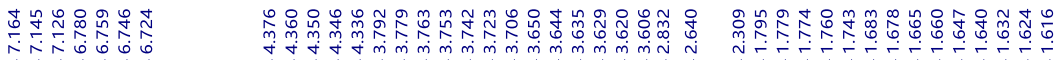

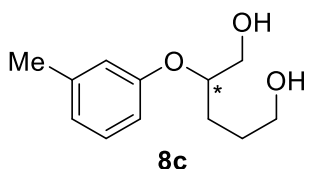

${ }^{1} \mathrm{H}$ NMR $\left(400 \mathrm{MHz}, \mathrm{CDCl}_{3}\right)$

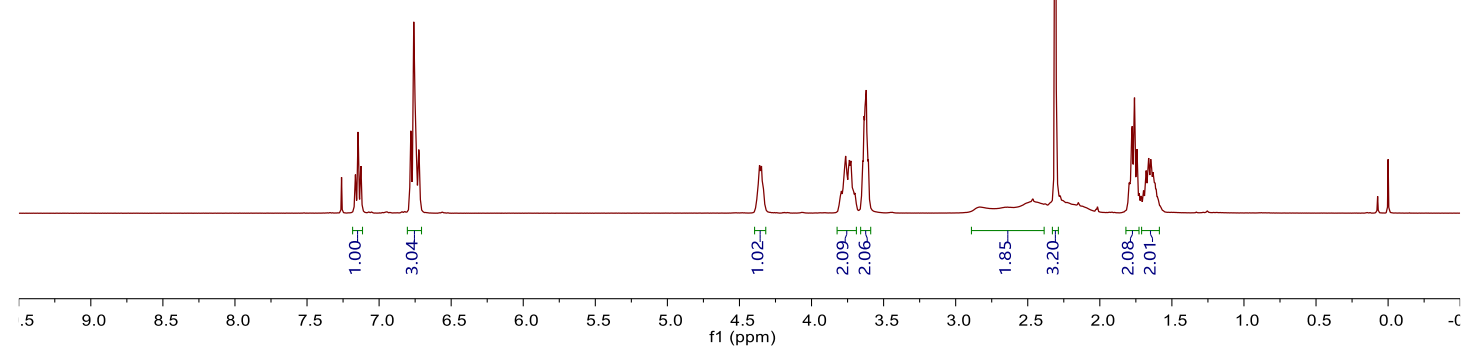

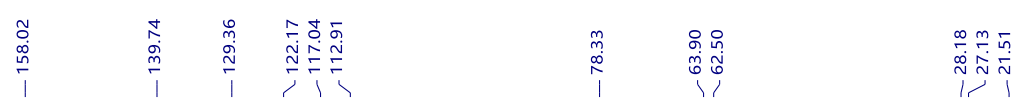

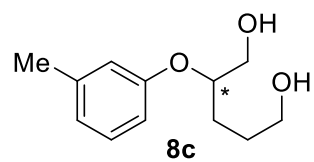

${ }^{13} \mathrm{C}$ NMR $\left(101 \mathrm{MHz}, \mathrm{CDCl}_{3}\right)$

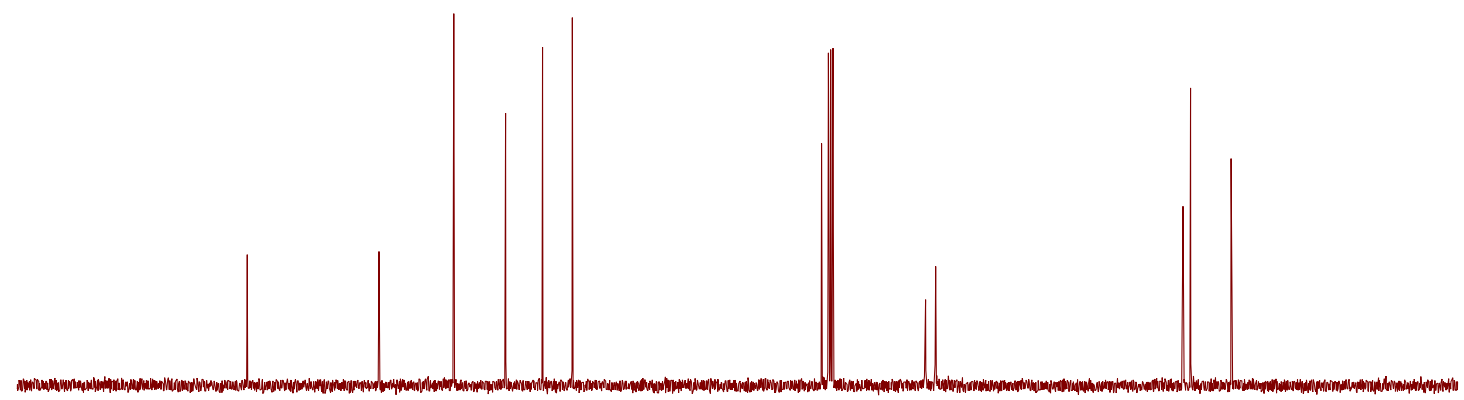

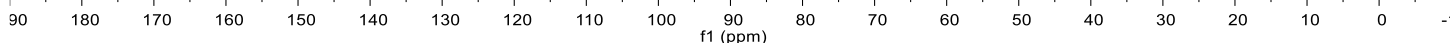


(+)-2-(o-Tolyloxy)pentane-1,5-diol ((+)-8d)

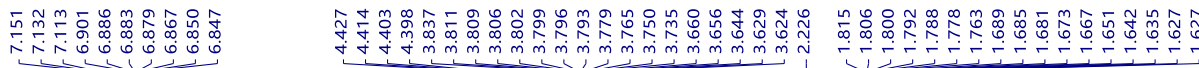

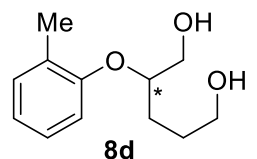

${ }^{1} \mathrm{H}$ NMR $\left(400 \mathrm{MHz}, \mathrm{CDCl}_{3}\right)$

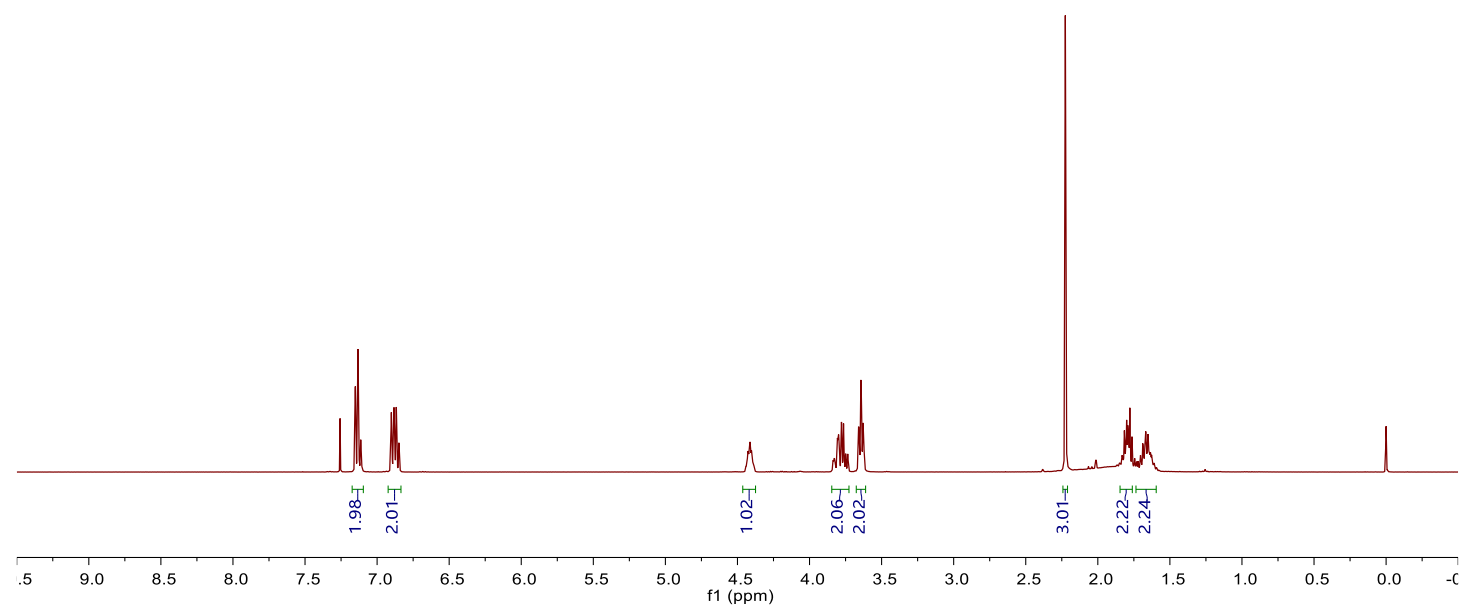

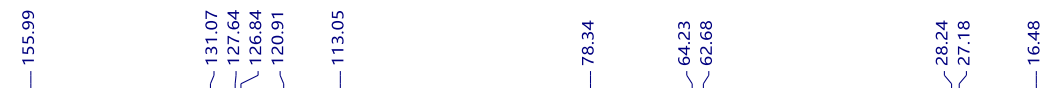

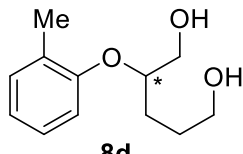

${ }^{13} \mathrm{C} \mathrm{NMR}\left(101 \mathrm{MHz}, \mathrm{CDCl}_{3}\right)$

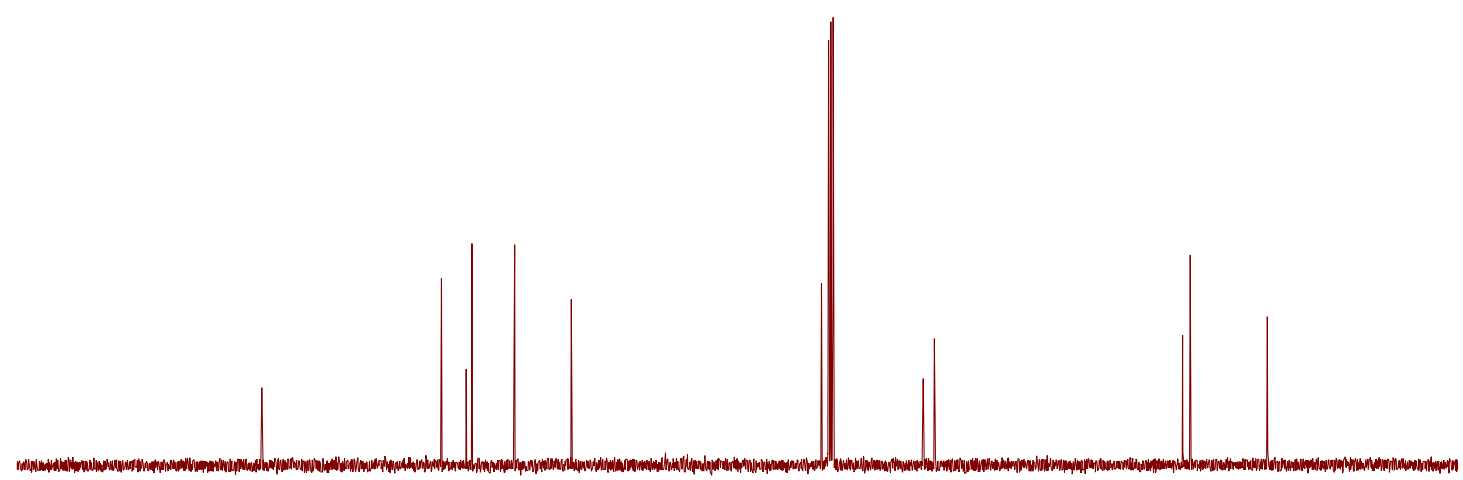

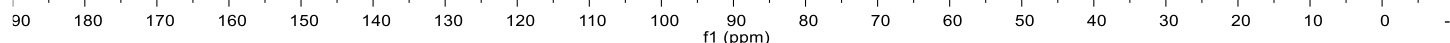


(+)-2-(2-Bromophenoxy)pentane-1,5-diol ((+)-8e)

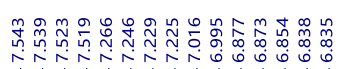

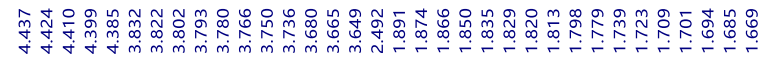

$\underbrace{\mathrm{Br}}_{8 e^{\mathrm{OH}}}$

${ }^{1} \mathrm{H}$ NMR $\left(400 \mathrm{MHz}, \mathrm{CDCl}_{3}\right)$

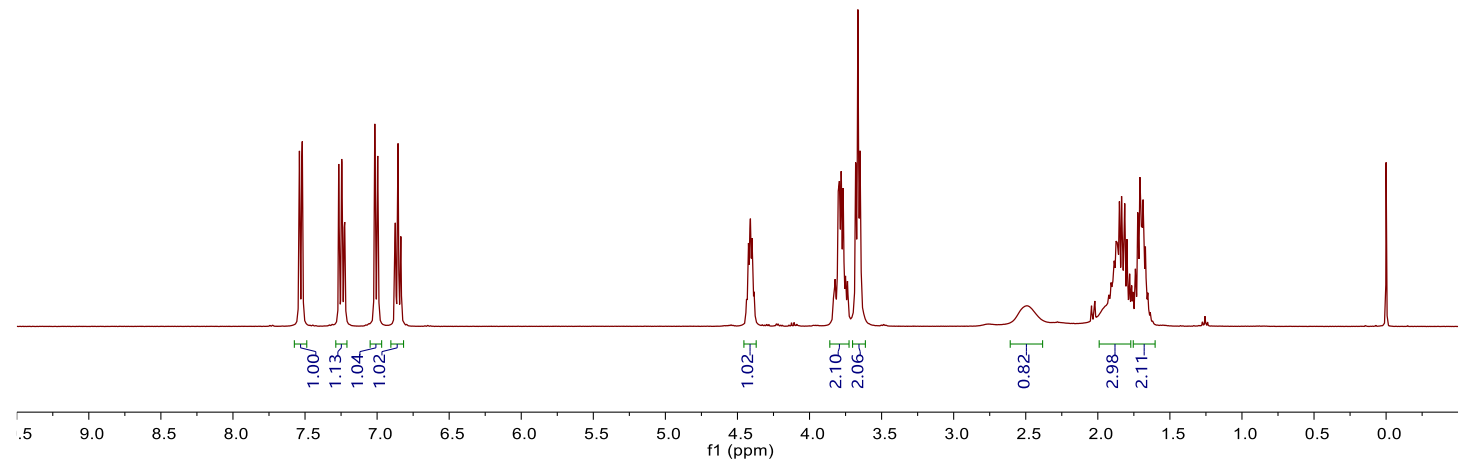

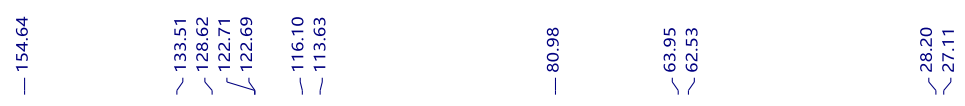<smiles>OCCC[C@@H](CO)Oc1ccccc1Br</smiles>

${ }^{13} \mathrm{C}$ NMR $\left(101 \mathrm{MHz}, \mathrm{CDCl}_{3}\right)$

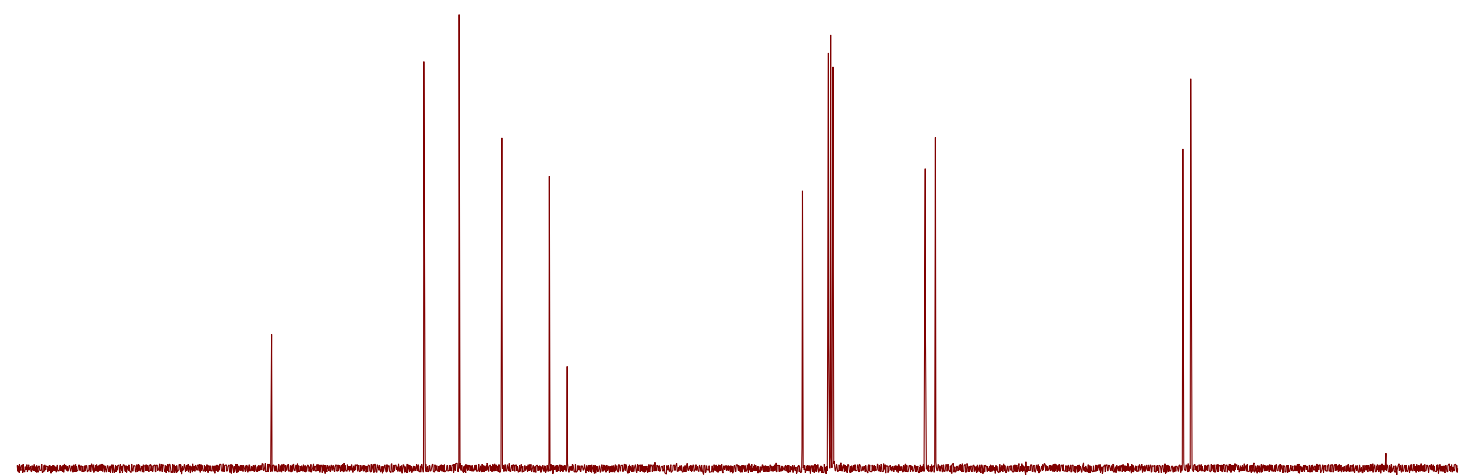

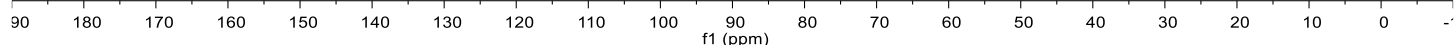




\section{(+)-2-(2-Chlorophenoxy)pentane-1,5-diol ((+)-8f)}

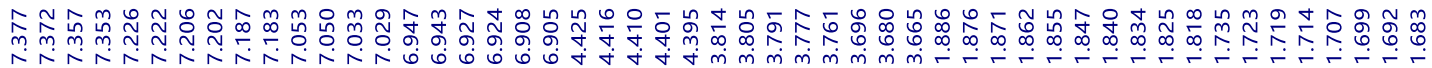<smiles>OCCCC(CO)Oc1ccccc1Cl</smiles>

${ }^{1} \mathrm{H} \mathrm{NMR}\left(400 \mathrm{MHz}, \mathrm{CDCl}_{3}\right)$

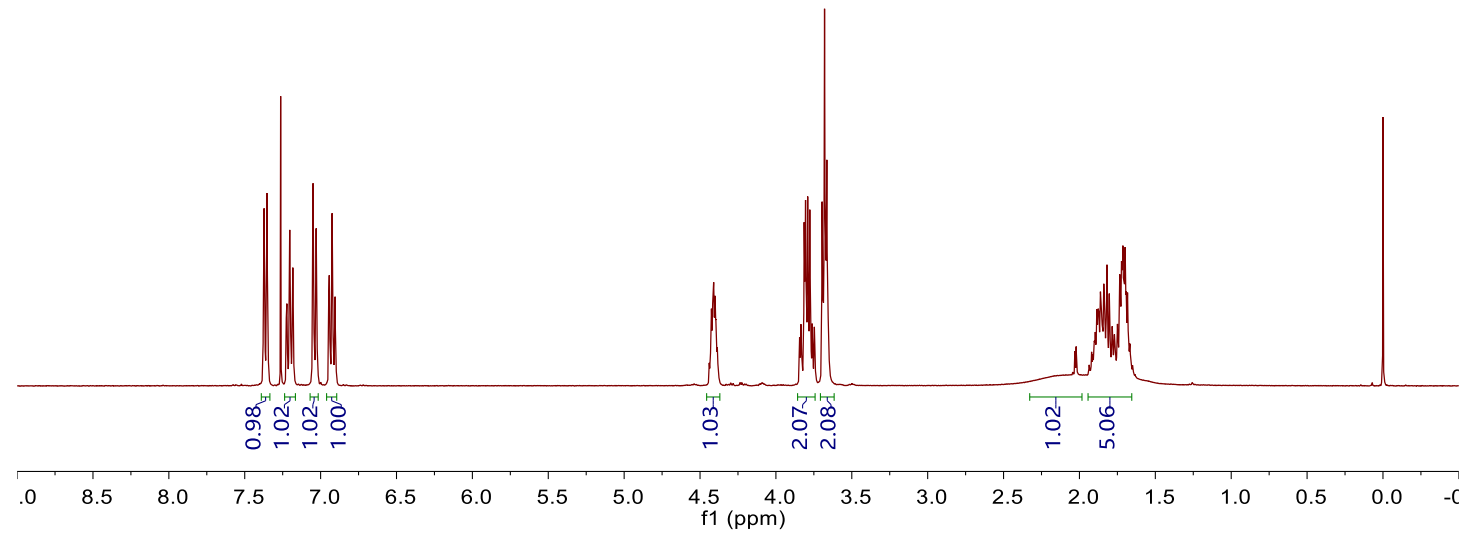

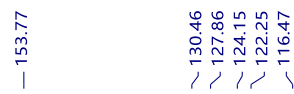

$\underset{\substack{1 \\ 0}}{1} \quad \stackrel{\substack{0 \\ 0}}{\substack{0 \\ 0}}$<smiles>OCCCC(CO)Oc1ccccc1Cl</smiles>

${ }^{13} \mathrm{C}$ NMR $\left(101 \mathrm{MHz}, \mathrm{CDCl}_{3}\right)$

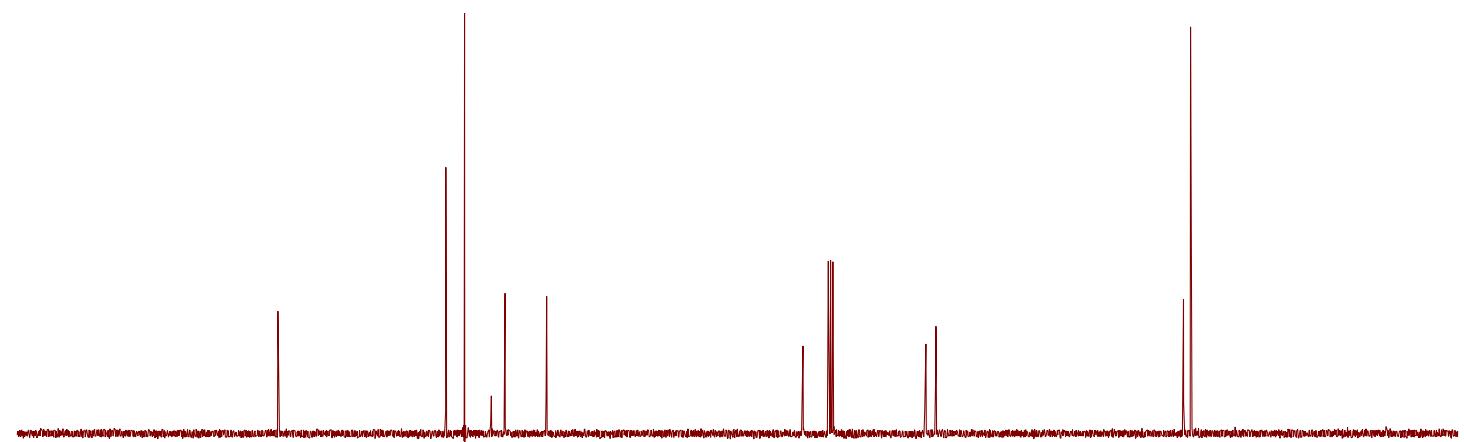

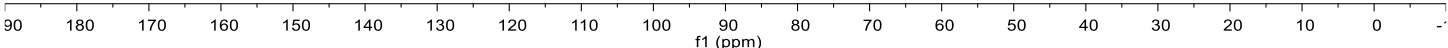


(+)-2-(2,4-Dimethylphenoxy)pentane-1,5-diol ((+)-8g)

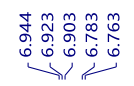

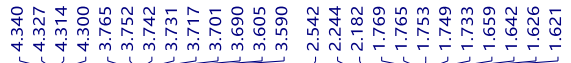

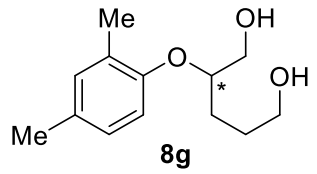

${ }^{1} \mathrm{H}$ NMR $\left(400 \mathrm{MHz}, \mathrm{CDCl}_{3}\right)$

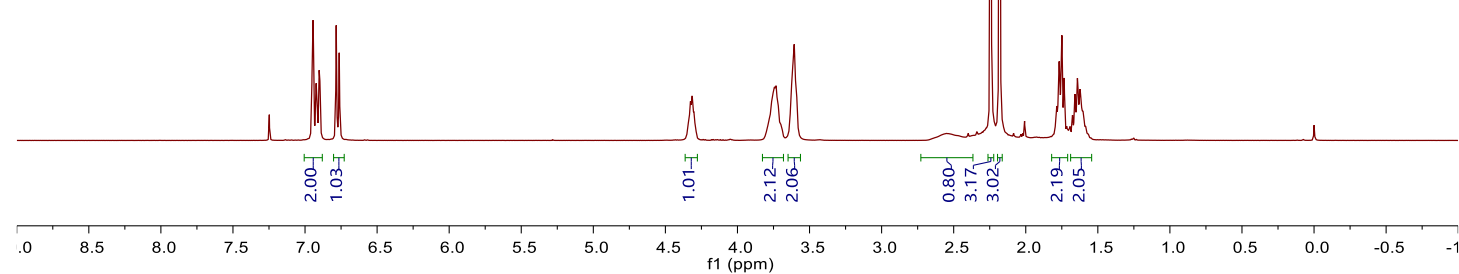

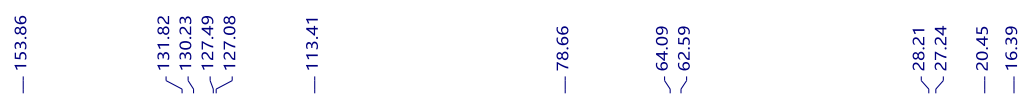<smiles>Cc1ccc(OC(=CCCO)CO)c(C)c1</smiles>

${ }^{13} \mathrm{C}$ NMR $\left(101 \mathrm{MHz}, \mathrm{CDCl}_{3}\right)$

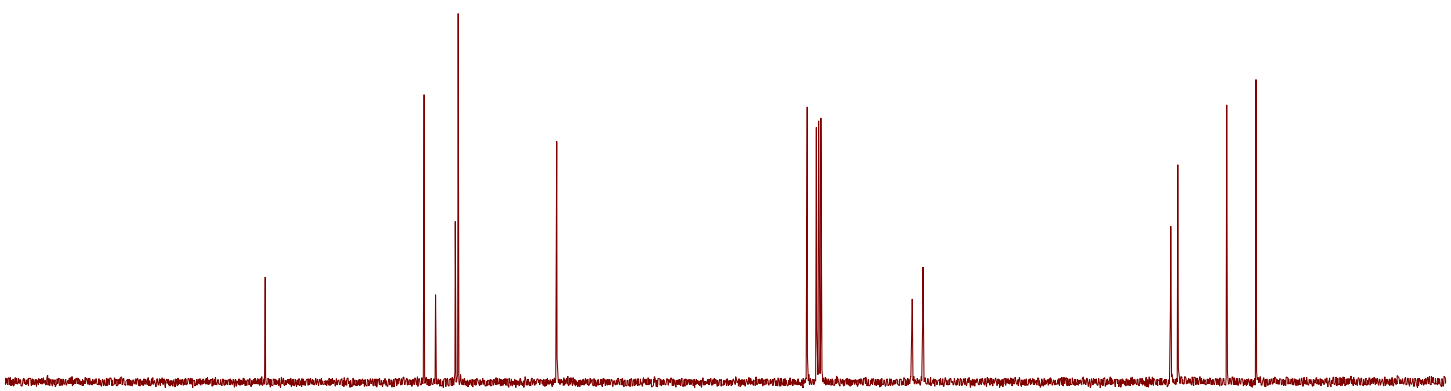

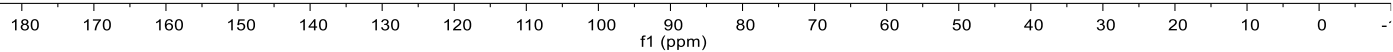




\section{(-)-3-Phenoxytetrahydrofuran ((-)-6a) $)^{8}$}

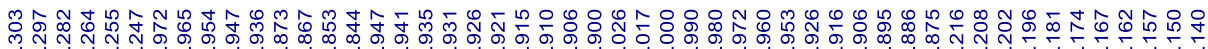

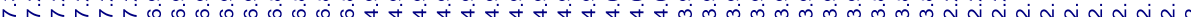<smiles>c1ccc(OC2CCOC2)cc1</smiles>

$6 \mathbf{a}$

${ }^{1} \mathrm{H}$ NMR $\left(400 \mathrm{MHz}, \mathrm{CDCl}_{3}\right)$

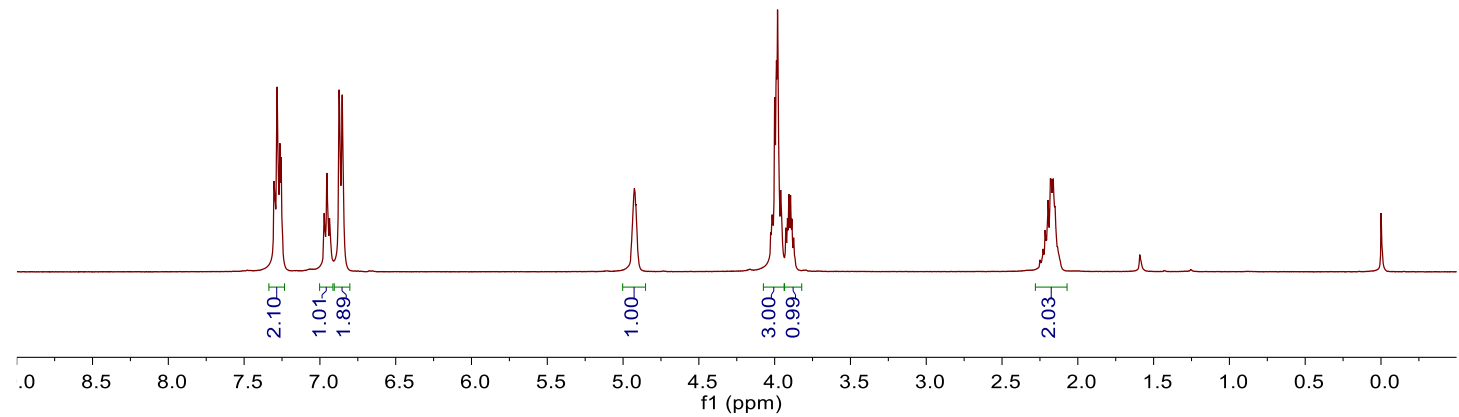

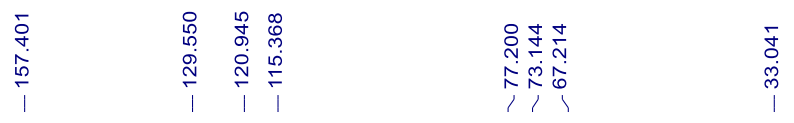

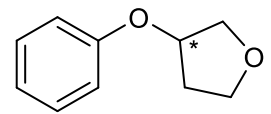

$6 a$

${ }^{13} \mathrm{C}$ NMR $\left(101 \mathrm{MHz}, \mathrm{CDCl}_{3}\right)$

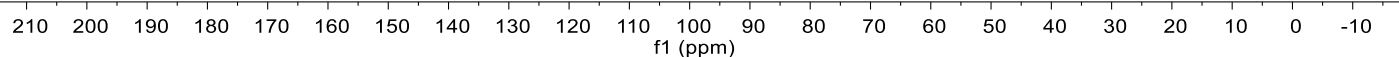


(-)-3-(p-Tolyloxy)tetrahydrofuran ((-)-6b)

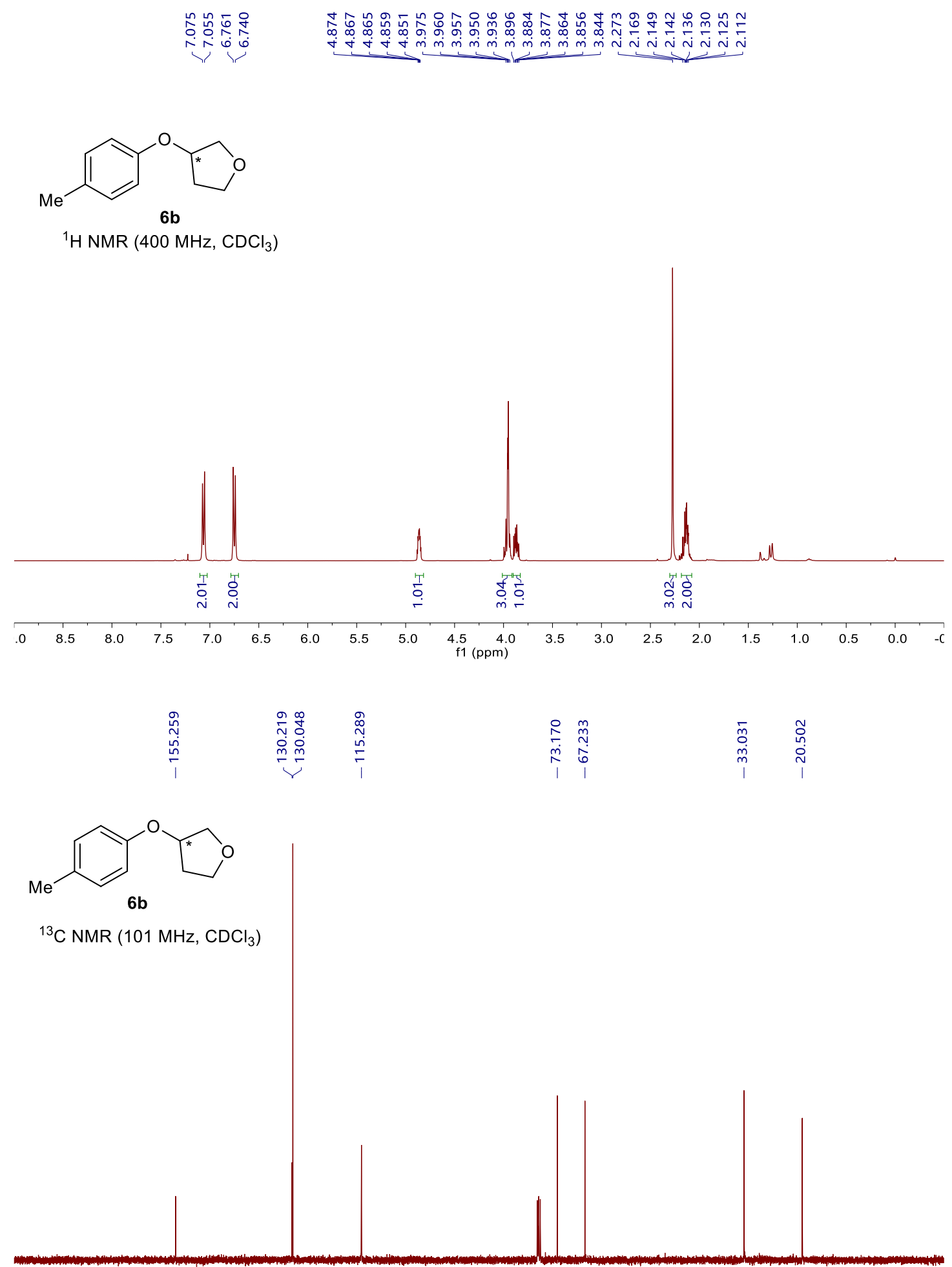

${ }^{13} \mathrm{C} \mathrm{NMR}\left(101 \mathrm{MHz}, \mathrm{CDCl}_{3}\right)$

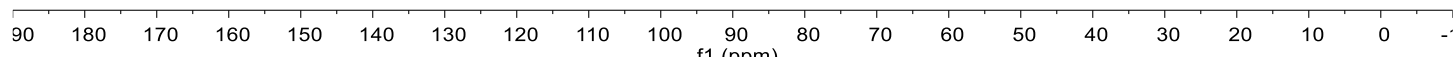


<smiles>Clc1ccc(OC2CCOC2)cc1</smiles>

${ }^{1} \mathrm{H}$ NMR $\left(400 \mathrm{MHz}, \mathrm{CDCl}_{3}\right)$
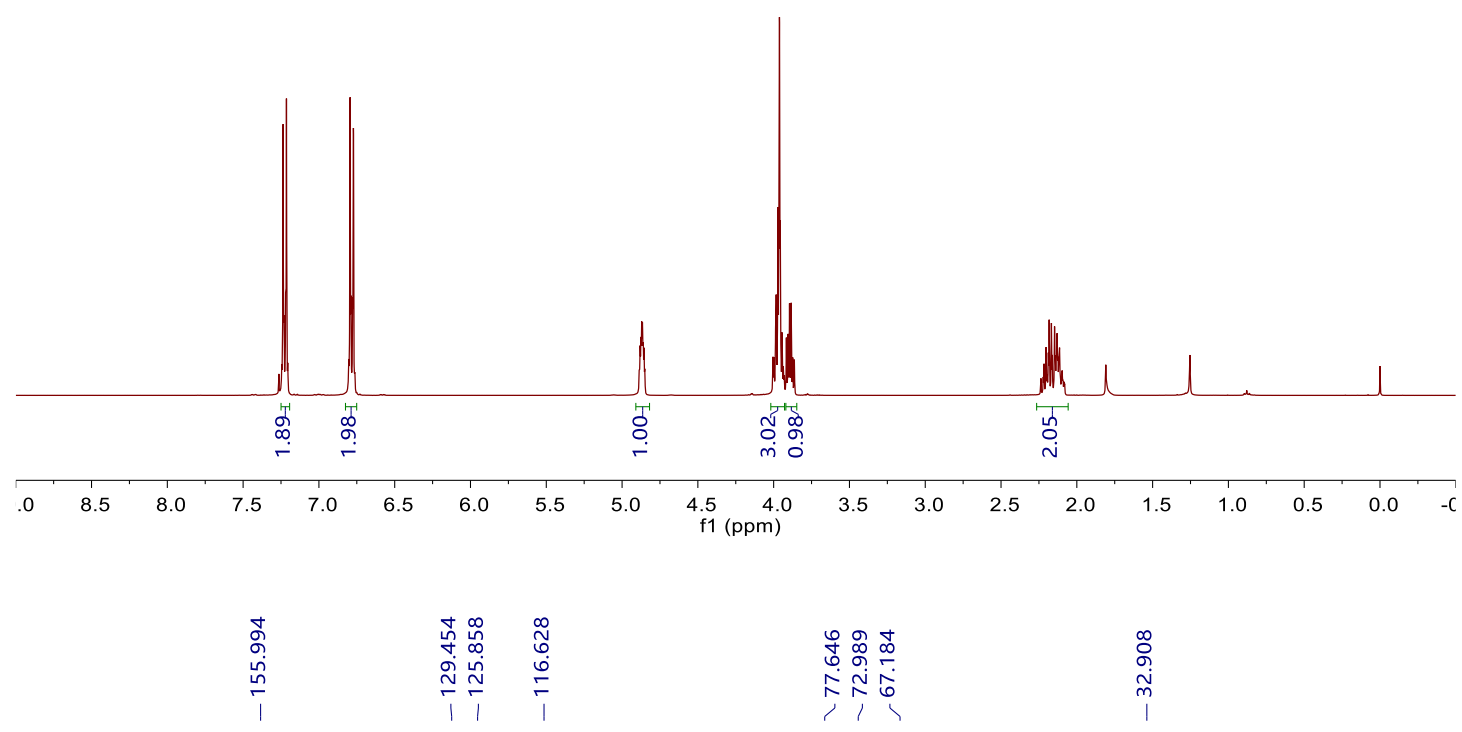<smiles>Clc1ccc(OC2CCOC2)cc1</smiles>

6c

${ }^{13} \mathrm{C}$ NMR $\left(101 \mathrm{MHz}, \mathrm{CDCl}_{3}\right)$

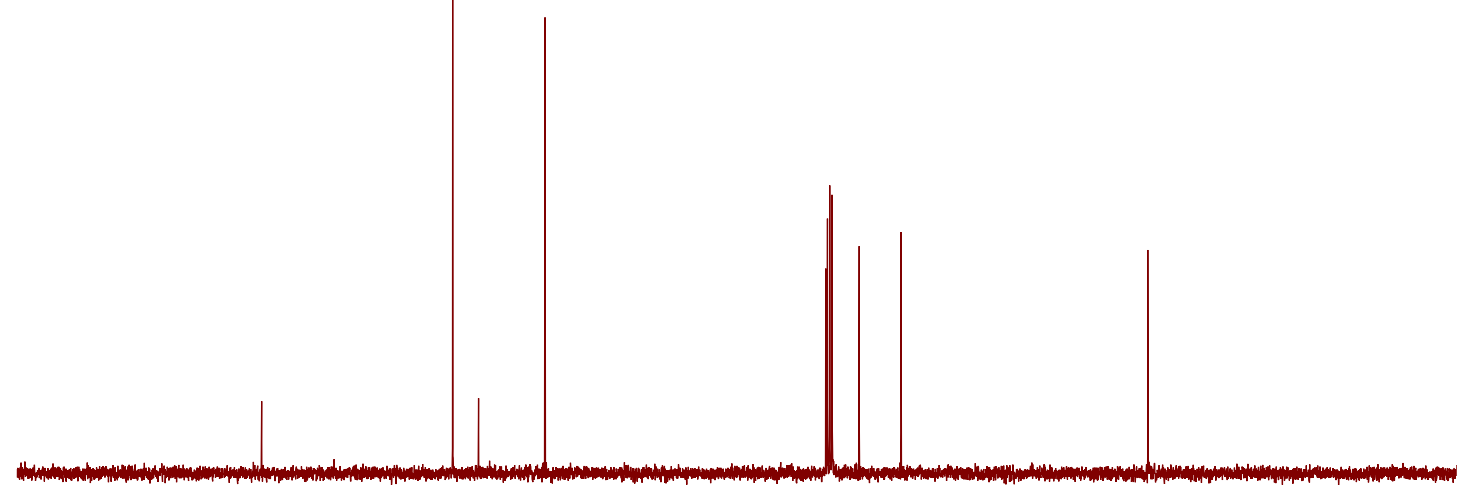

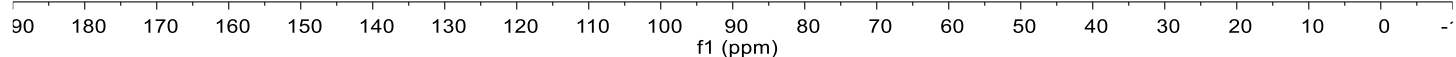



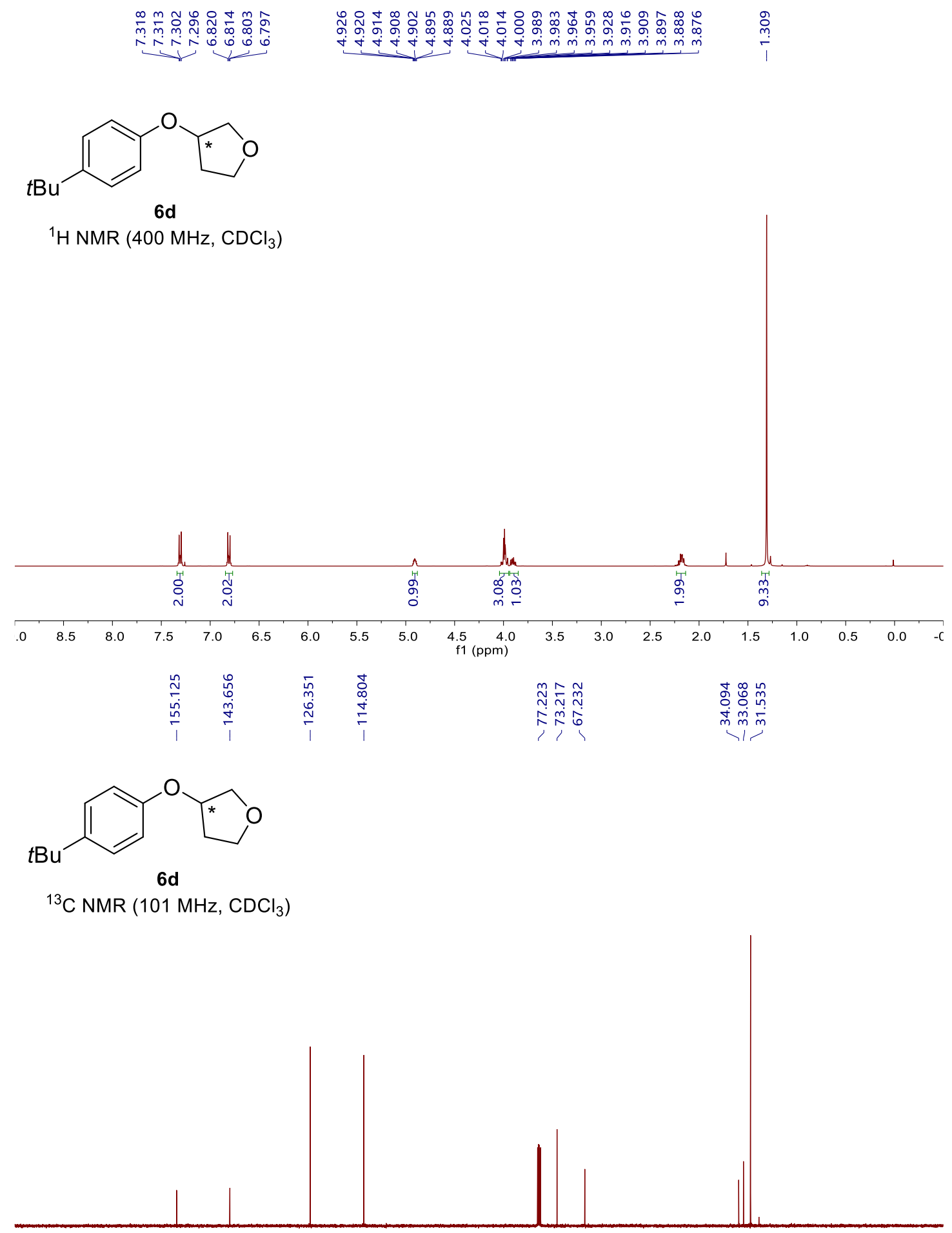

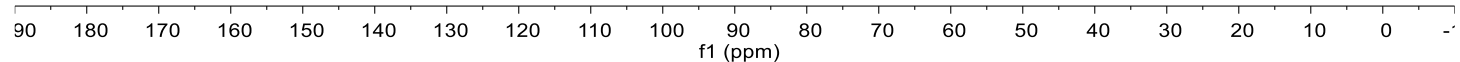



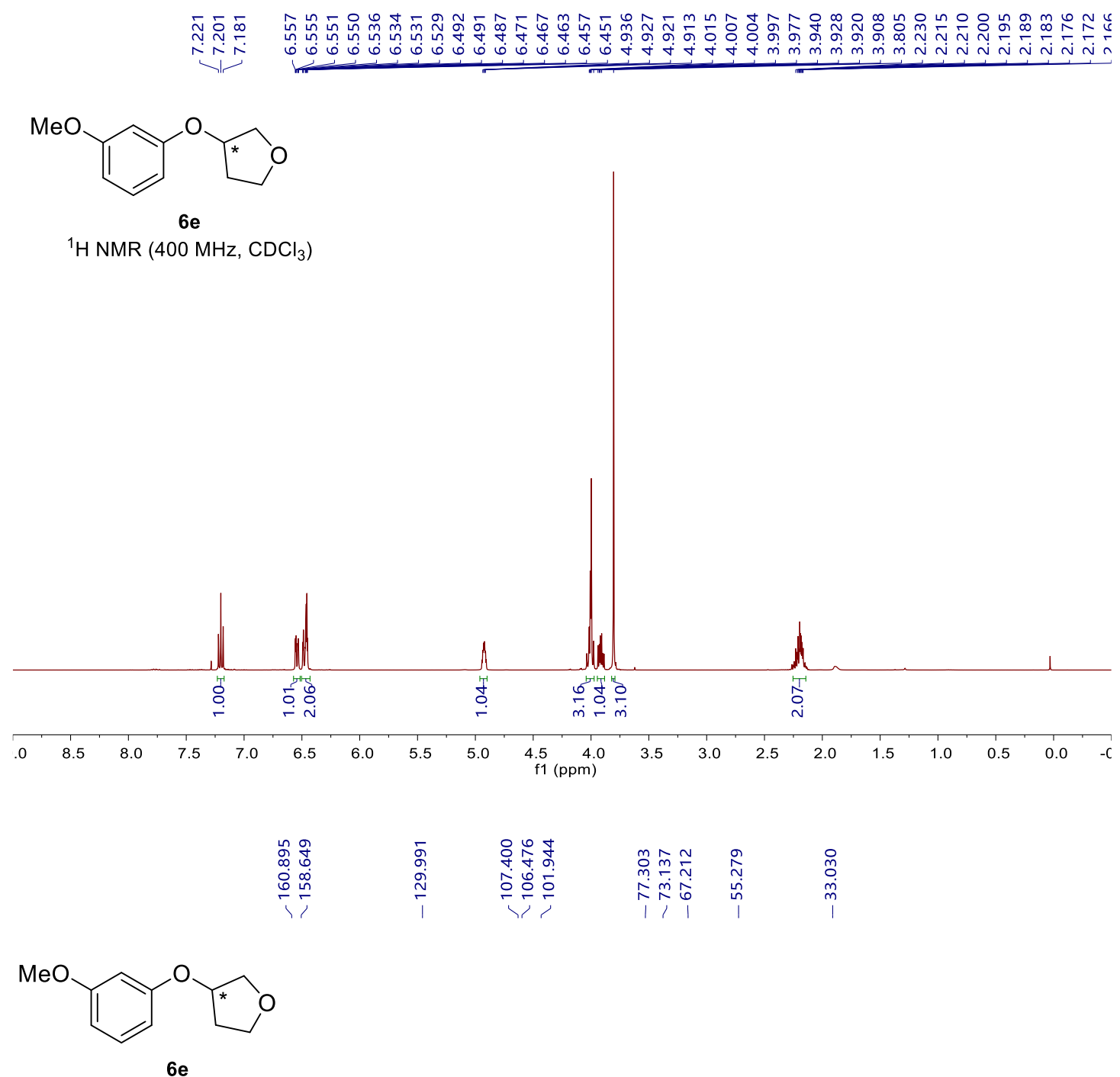

${ }^{13} \mathrm{C} \mathrm{NMR}\left(101 \mathrm{MHz}, \mathrm{CDCl}_{3}\right)$

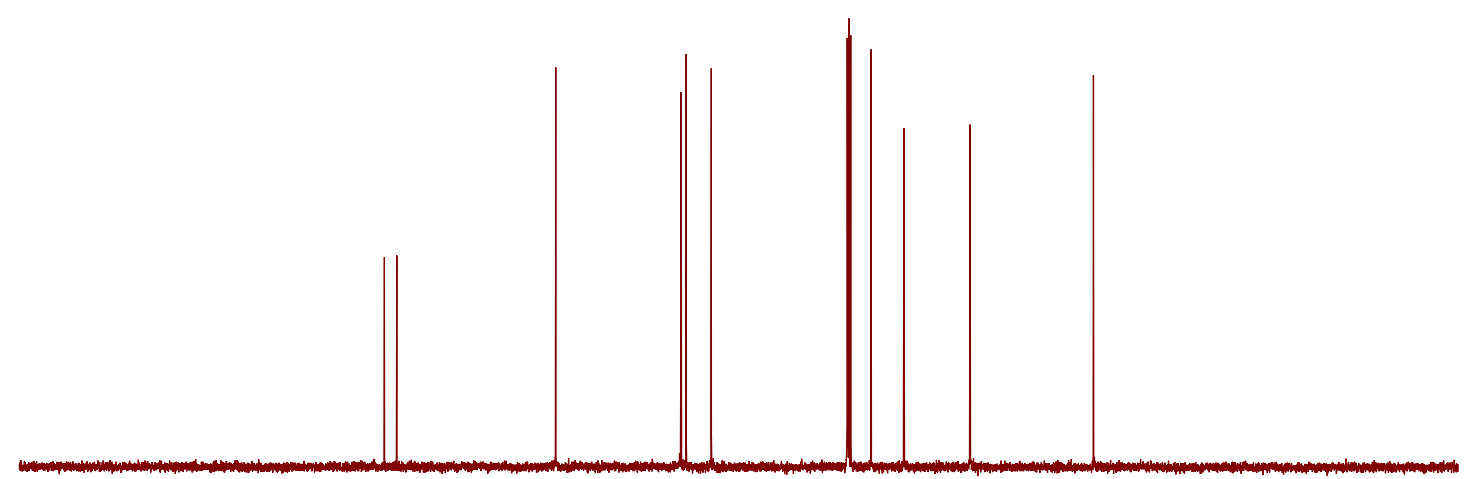

$\begin{array}{lllllllllllllllllllllllllllllll}220 & 210 & 200 & 190 & 180 & 170 & 160 & 150 & 140 & 130 & 120 & 110 & \begin{array}{c}100 \\ \mathrm{f} 1(\mathrm{ppm})\end{array} & 80 & 70 & 60 & 50 & 40 & 30 & 20 & 10 & 0 & -10 & -20 & -3 \mathrm{C}\end{array}$ 
(-)-3-(3-Chlorophenoxy)tetrahydrofuran ((-)-6f)

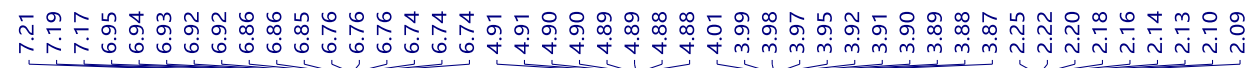

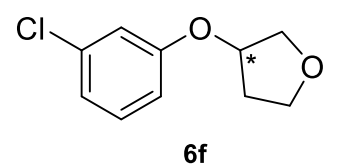

${ }^{1} \mathrm{H}$ NMR $\left(400 \mathrm{MHz}, \mathrm{CDCl}_{3}\right)$

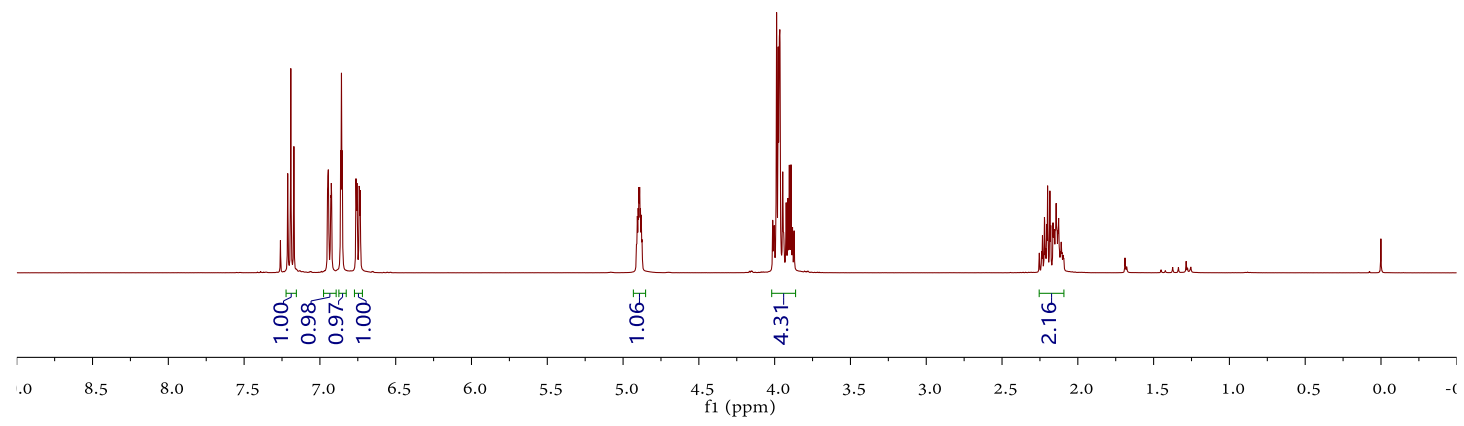

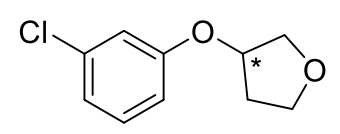

$6 f$

${ }^{13} \mathrm{C} \mathrm{NMR}\left(101 \mathrm{MHz}, \mathrm{CDCl}_{3}\right)$

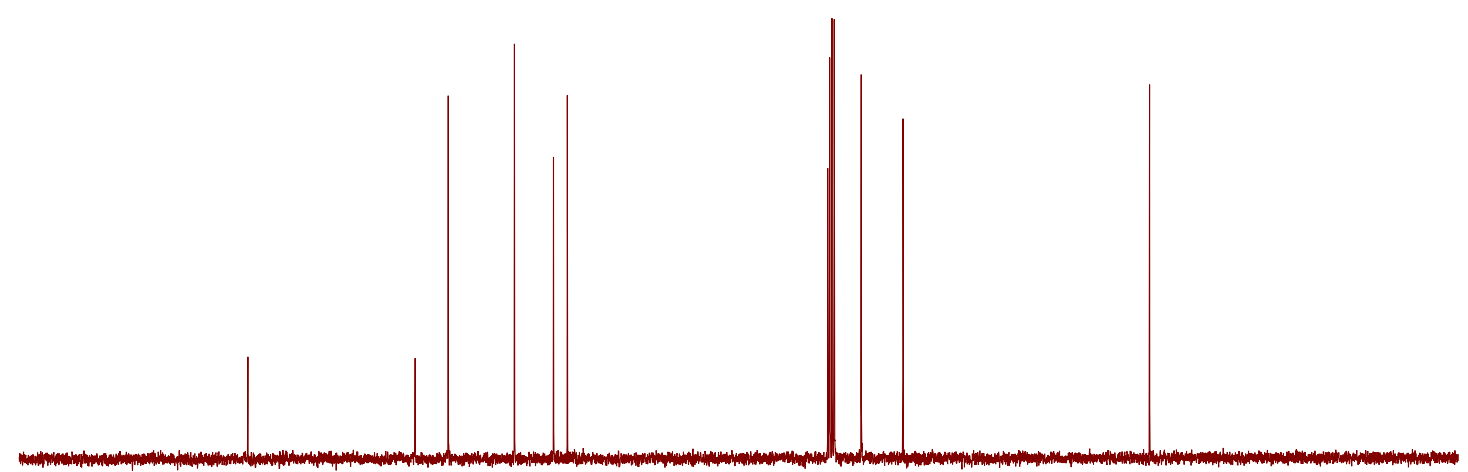

$\begin{array}{lllllllllllllllllllllll}90 & 180 & 170 & 160 & 150 & 140 & 130 & 120 & 110 & 100 & \underset{\mathrm{f} 1}{90}(\mathrm{ppm}) & 80 & 70 & 60 & 50 & 40 & 30 & 20 & 10 & 0 & -\end{array}$ 


\section{(-)-3-(3-Bromophenoxy)tetrahydrofuran ((-)-6g)}

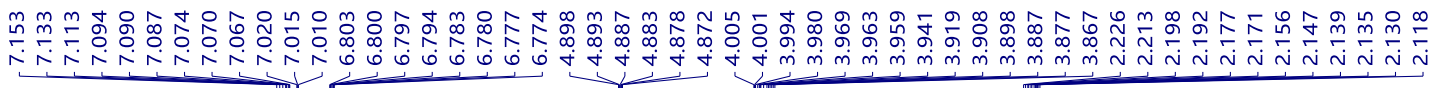<smiles>Brc1cccc(OC2CCOC2)c1</smiles>

$6 \mathrm{~g}$

${ }^{1} \mathrm{H}$ NMR $\left(400 \mathrm{MHz}, \mathrm{CDCl}_{3}\right)$
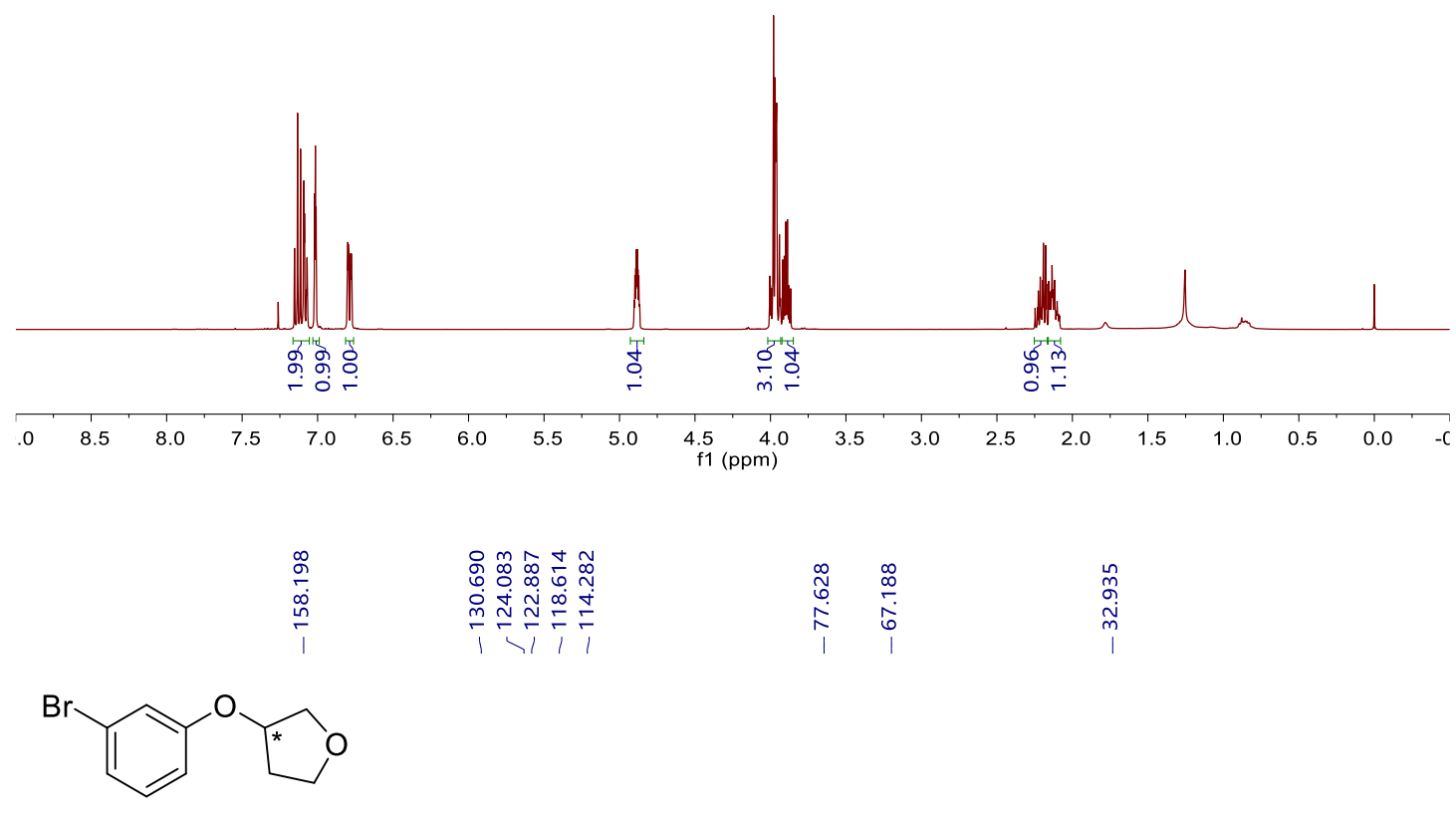

$6 \mathrm{~g}$

${ }^{13} \mathrm{C}$ NMR $\left(101 \mathrm{MHz}, \mathrm{CDCl}_{3}\right)$

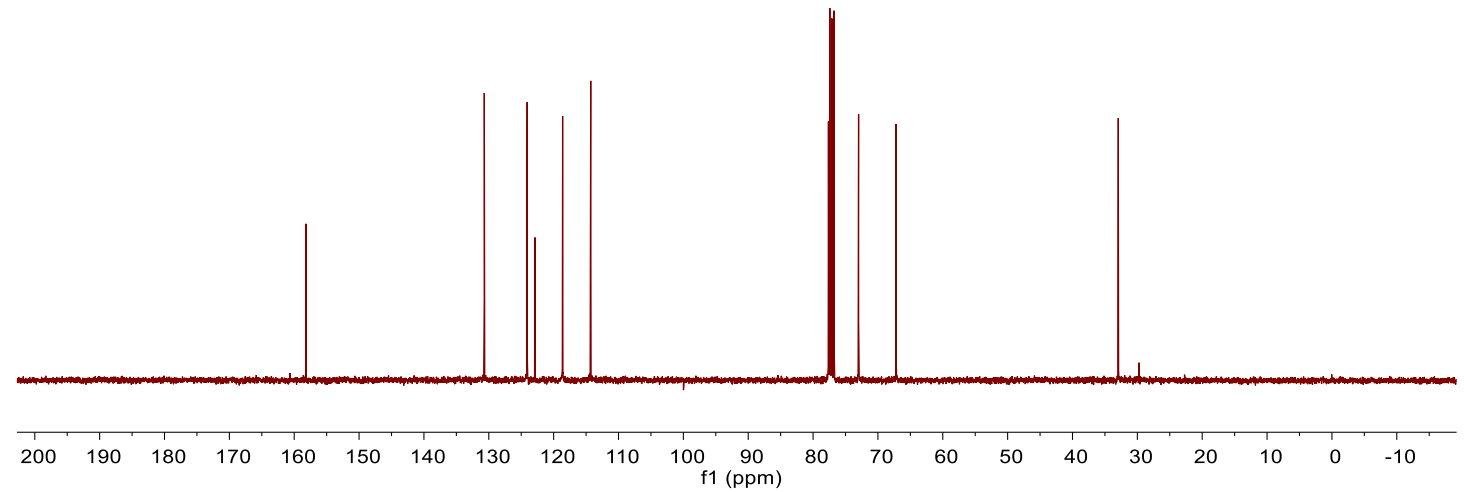


<smiles>COc1ccccc1OC1CCOC1</smiles>

$6 \mathrm{~h}$

${ }^{1} \mathrm{H} \mathrm{NMR}\left(400 \mathrm{MHz}, \mathrm{CDCl}_{3}\right)$

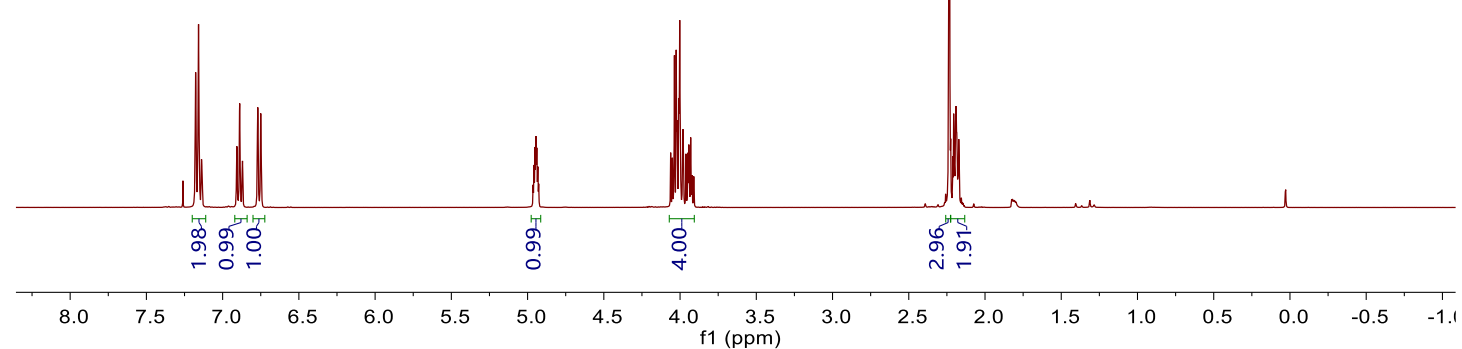

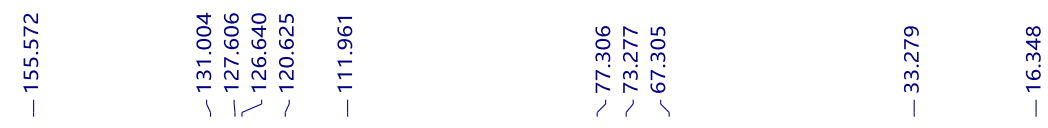<smiles>Cc1ccccc1OC1CCOC1</smiles>

$6 \mathrm{~h}$

${ }^{13} \mathrm{C}$ NMR $\left(101 \mathrm{MHz}, \mathrm{CDCl}_{3}\right)$

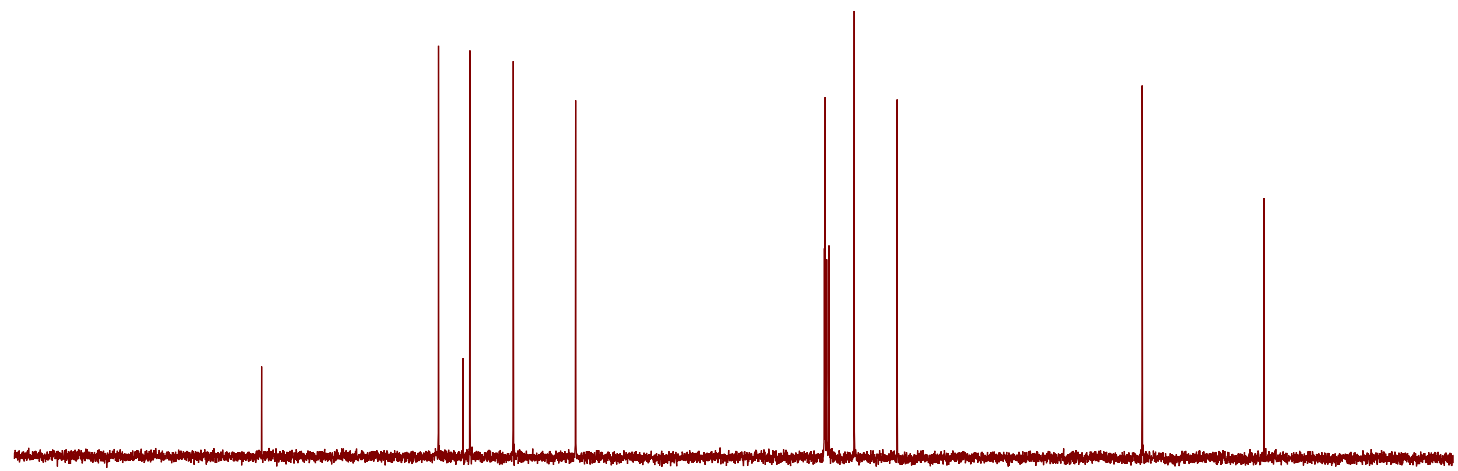

90

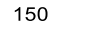

$40 \quad 130$

120

$\begin{array}{llc}110 & 100 & 90 \\ & f 1 & (p p m)\end{array}$

$\begin{array}{lll}70 & 60 \quad 50\end{array}$

$40 \quad 30 \quad 20$

$10 \quad 0$ 


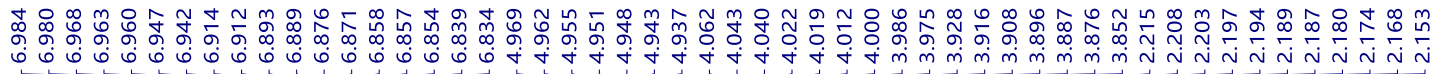<smiles>COc1ccccc1OC1CCOC1</smiles>

$6 i$

${ }^{1} \mathrm{H}$ NMR $\left(400 \mathrm{MHz}, \mathrm{CDCl}_{3}\right)$

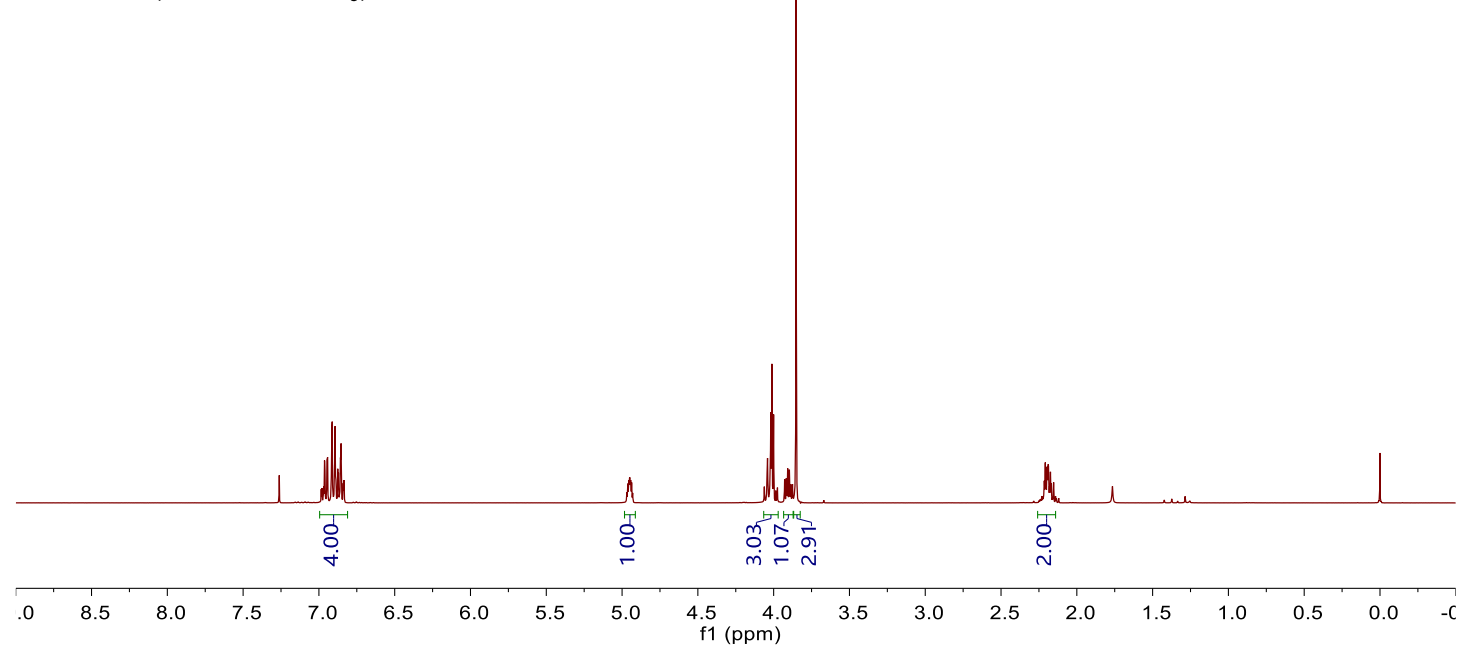

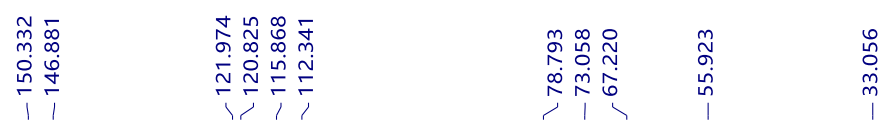<smiles>COc1ccccc1OC1CCOC1</smiles>

6i

${ }^{13} \mathrm{C}$ NMR $\left(101 \mathrm{MHz}, \mathrm{CDCl}_{3}\right)$

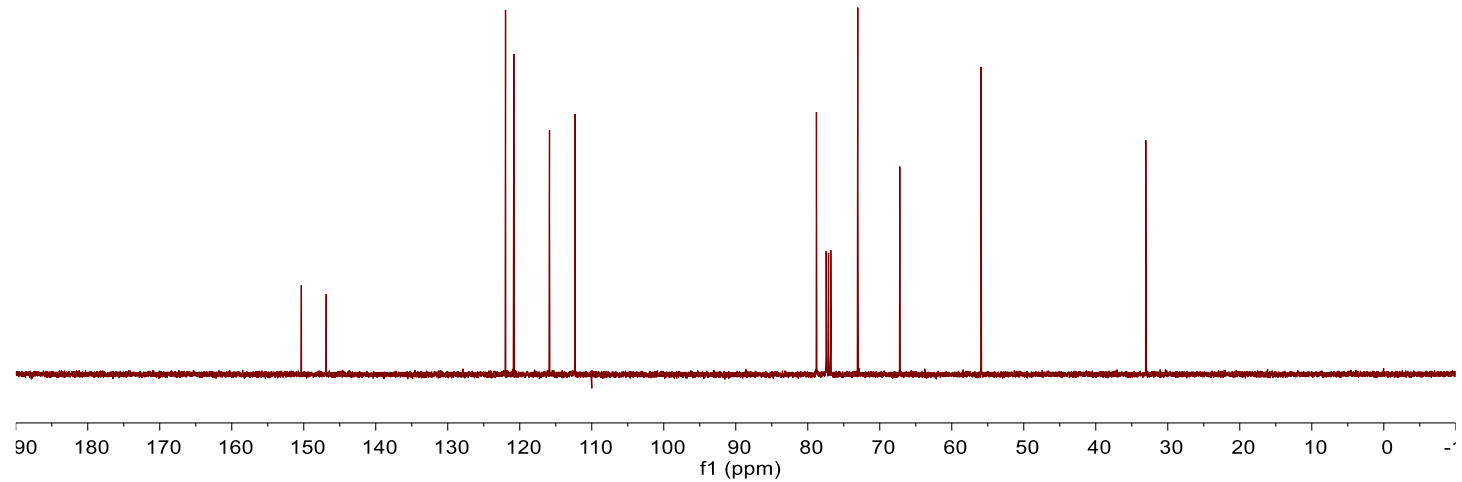


<smiles>Clc1ccccc1OC1CCOC1</smiles>

6j

${ }^{1} \mathrm{H} \mathrm{NMR}\left(400 \mathrm{MHz}, \mathrm{CDCl}_{3}\right)$

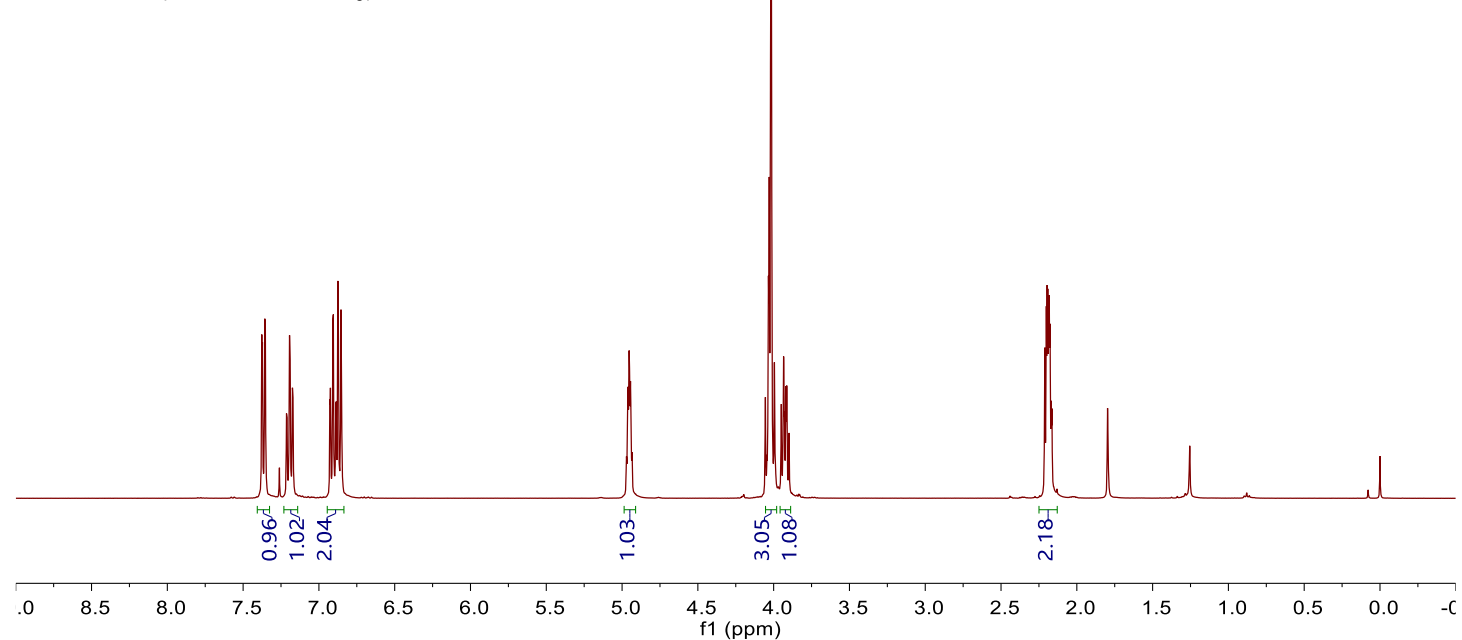

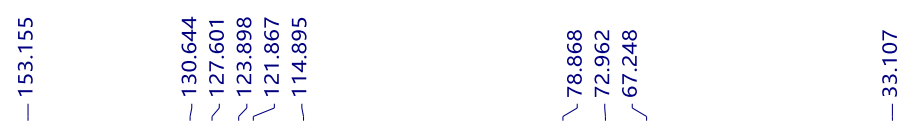<smiles>Clc1ccccc1OC1CCOC1</smiles>

6j

${ }^{13} \mathrm{C}$ NMR $\left(101 \mathrm{MHz}, \mathrm{CDCl}_{3}\right)$

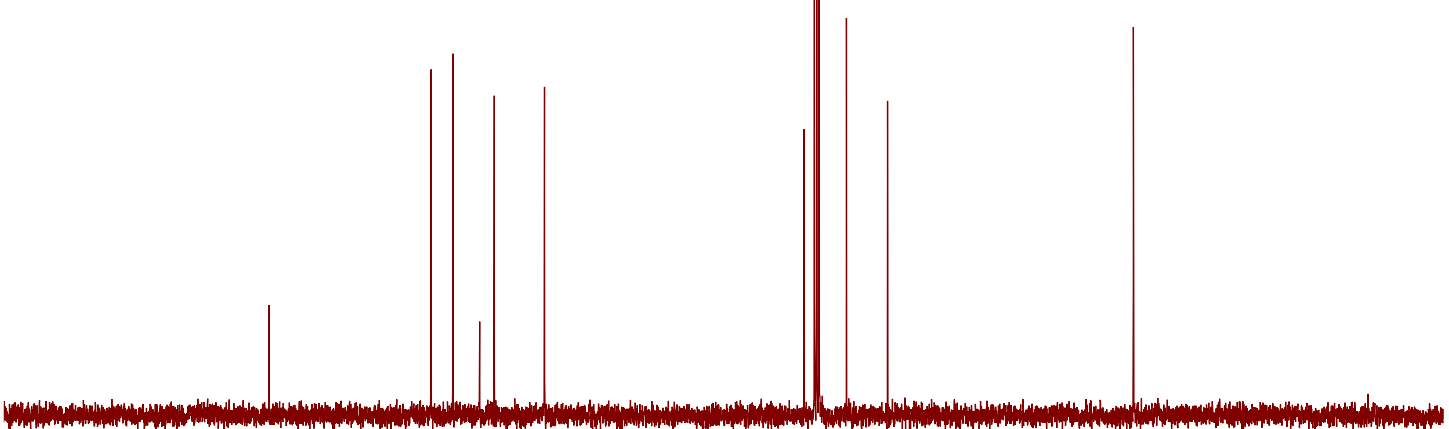

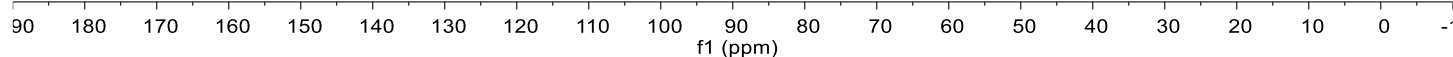




\section{(-)-3-(2-Fluorophenoxy)tetrahydrofuran ((-)-6k)}

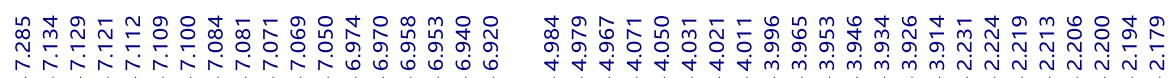<smiles>Fc1ccccc1OC1CCOC1</smiles>

$6 \mathrm{k}$

${ }^{1} \mathrm{H}$ NMR $\left(400 \mathrm{MHz}, \mathrm{CDCl}_{3}\right)$

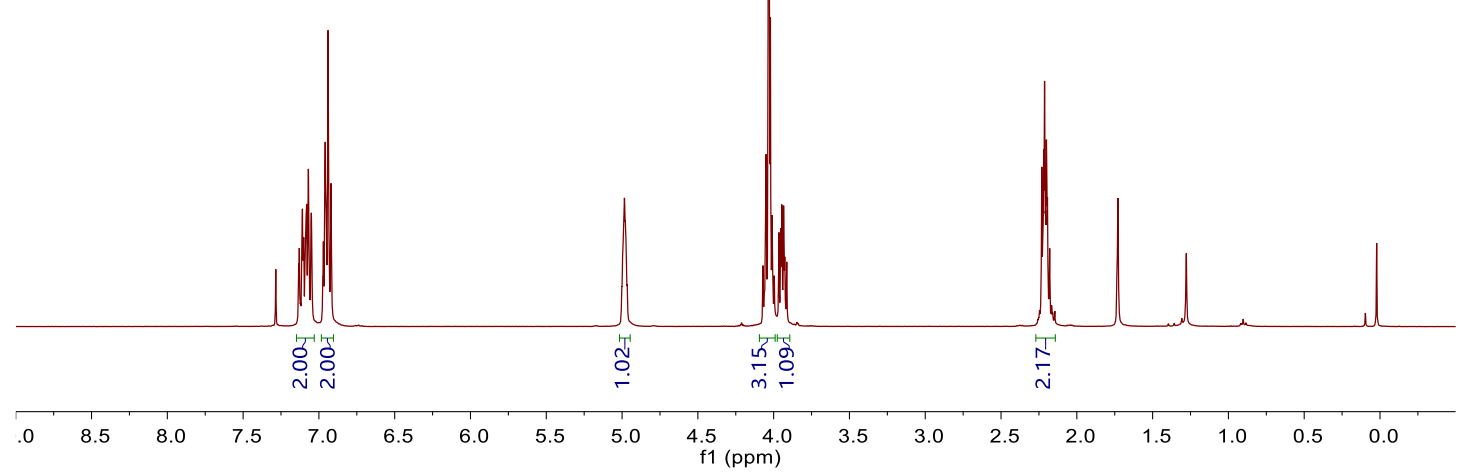

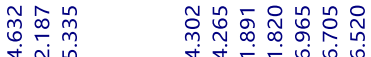

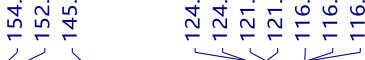

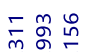

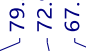

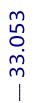<smiles>Fc1ccccc1OC1CCOC1</smiles>

6k

${ }^{13} \mathrm{C} \mathrm{NMR}\left(101 \mathrm{MHz}, \mathrm{CDCl}_{3}\right)$

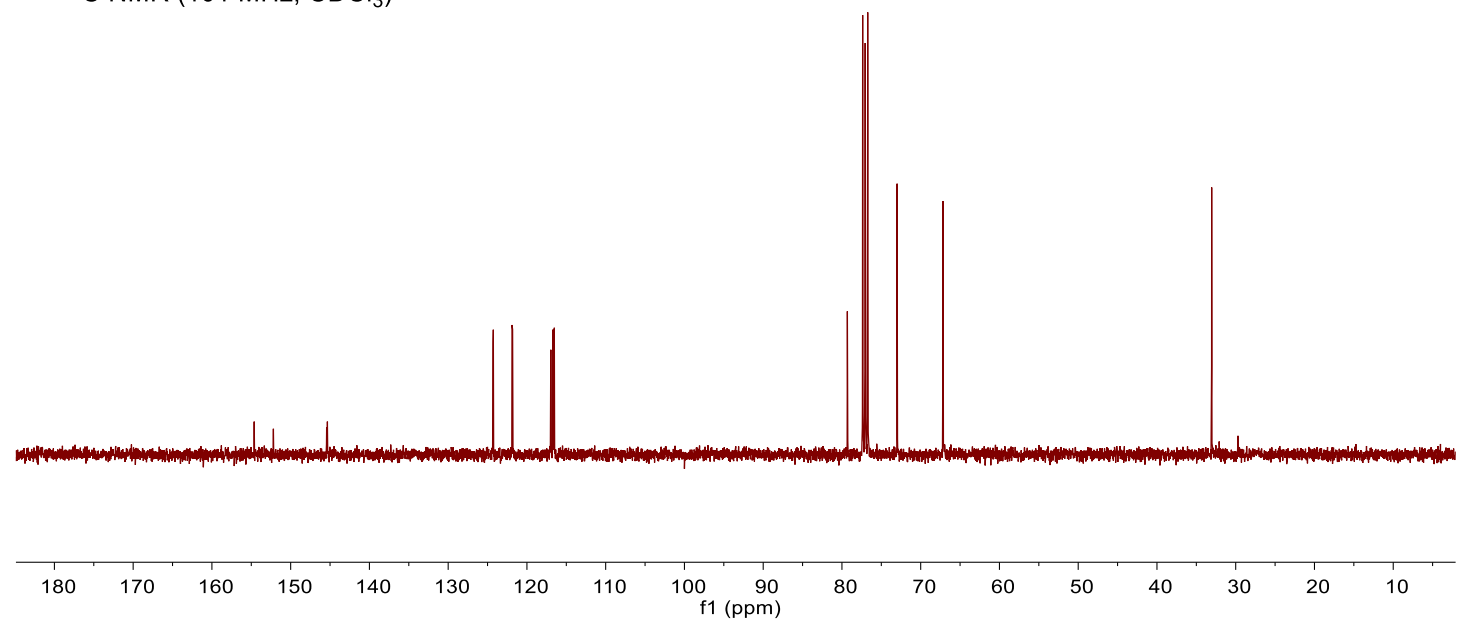


(R)-3-(2,5-Dimethylphenoxy)tetrahydrofuran ((R)-6l)

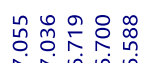

ก

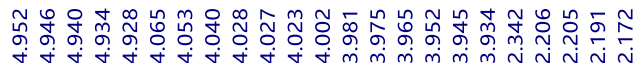

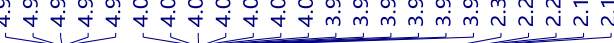<smiles>Cc1ccc(C)c(OC2CCCC2)c1</smiles>

${ }^{1} \mathrm{H} \mathrm{NMR}\left(400 \mathrm{MHz}, \mathrm{CDCl}_{3}\right)$

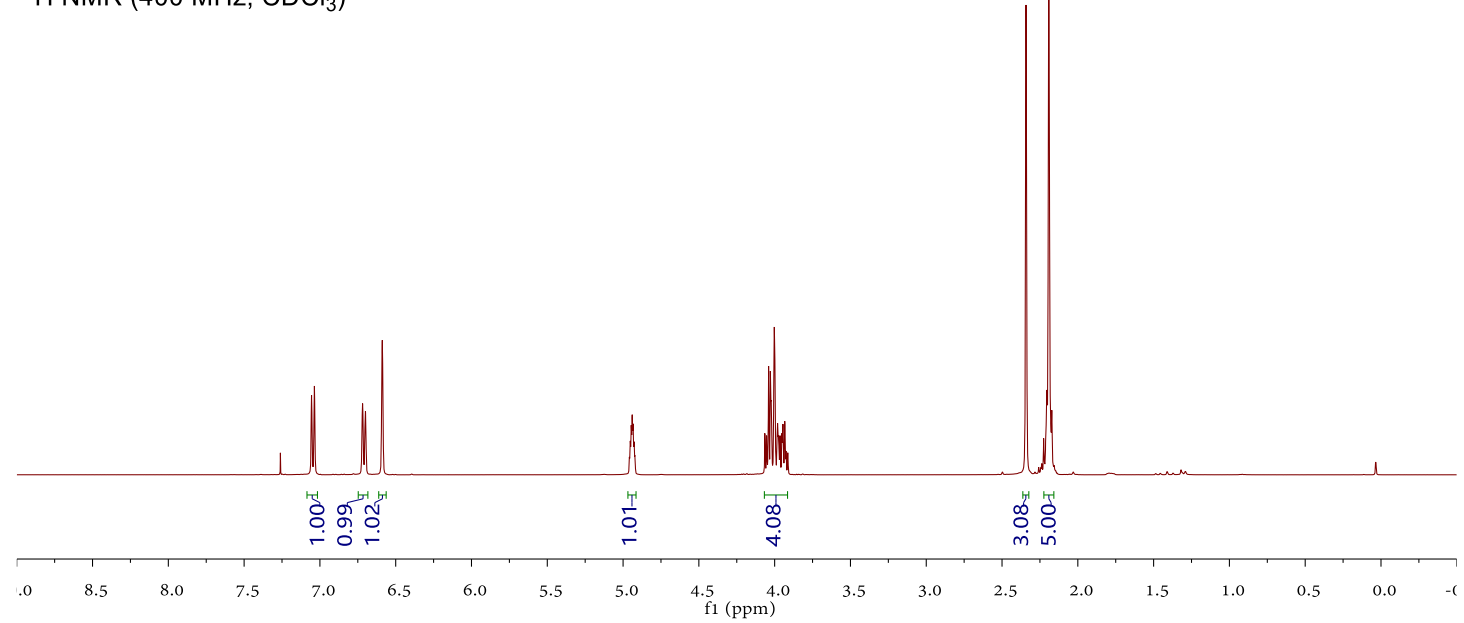

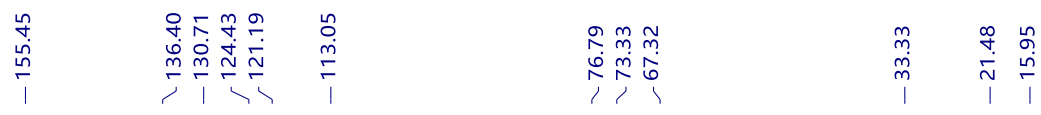

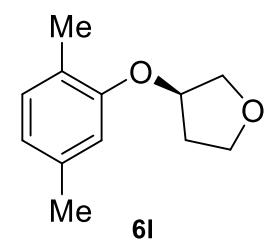

${ }^{13} \mathrm{C}$ NMR $\left(101 \mathrm{MHz}, \mathrm{CDCl}_{3}\right)$

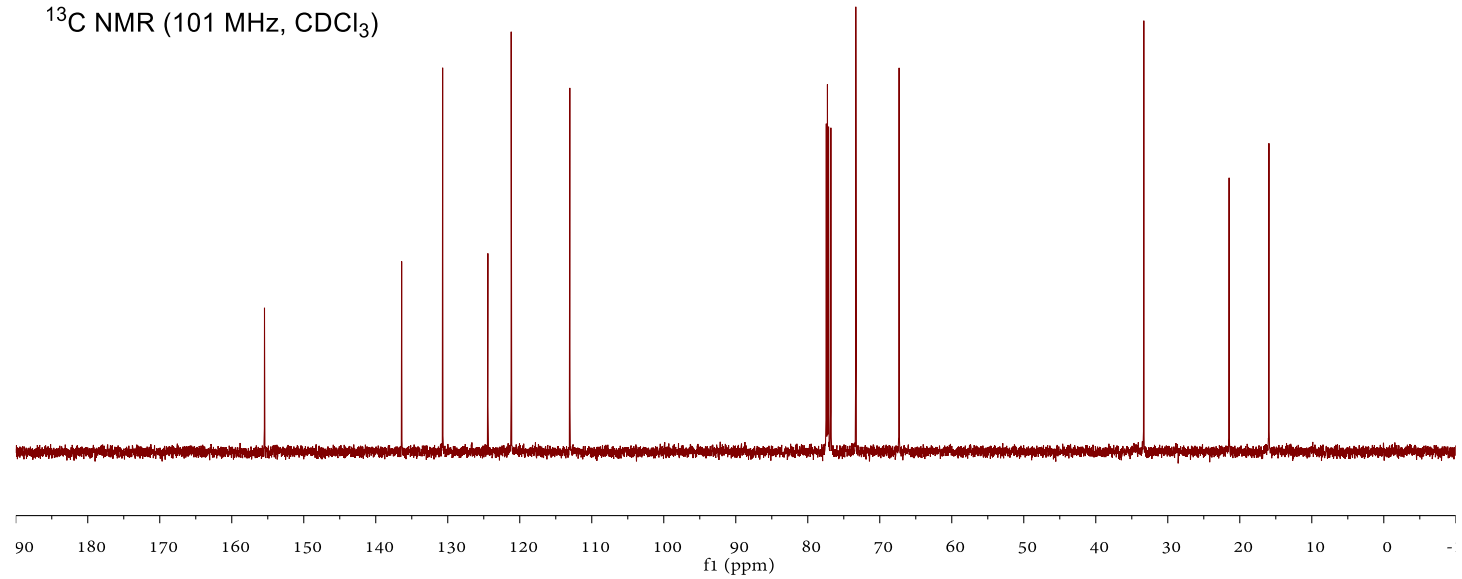




\section{(-)-3-(Naphthalen-2-yloxy)tetrahydrofuran ((-)-6m)}
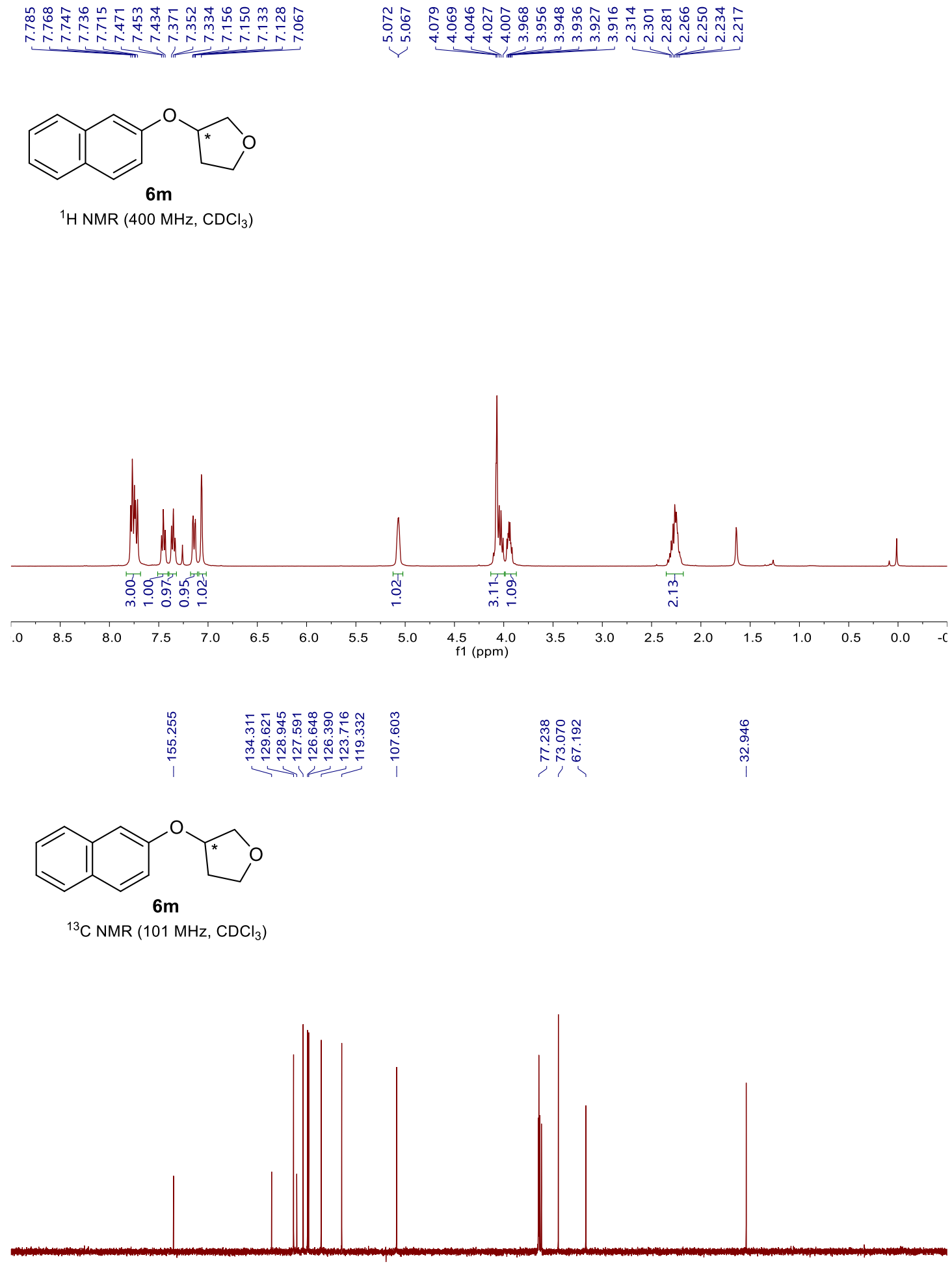

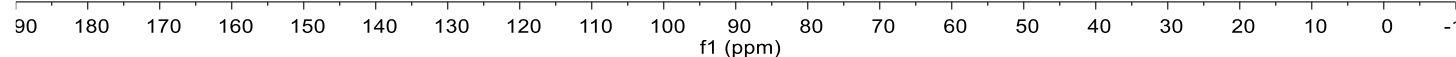




\section{Lactone 10}

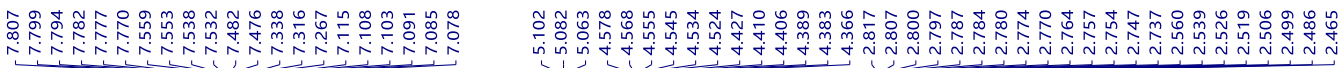<smiles>O=C(c1ccc(O[C@@H]2CCOC2=O)cc1)c1cc(Br)ccc1Cl</smiles>

\section{Lactone 10}

${ }^{1} \mathrm{H}$ NMR $\left(400 \mathrm{MHz}, \mathrm{CDCl}_{3}\right)$

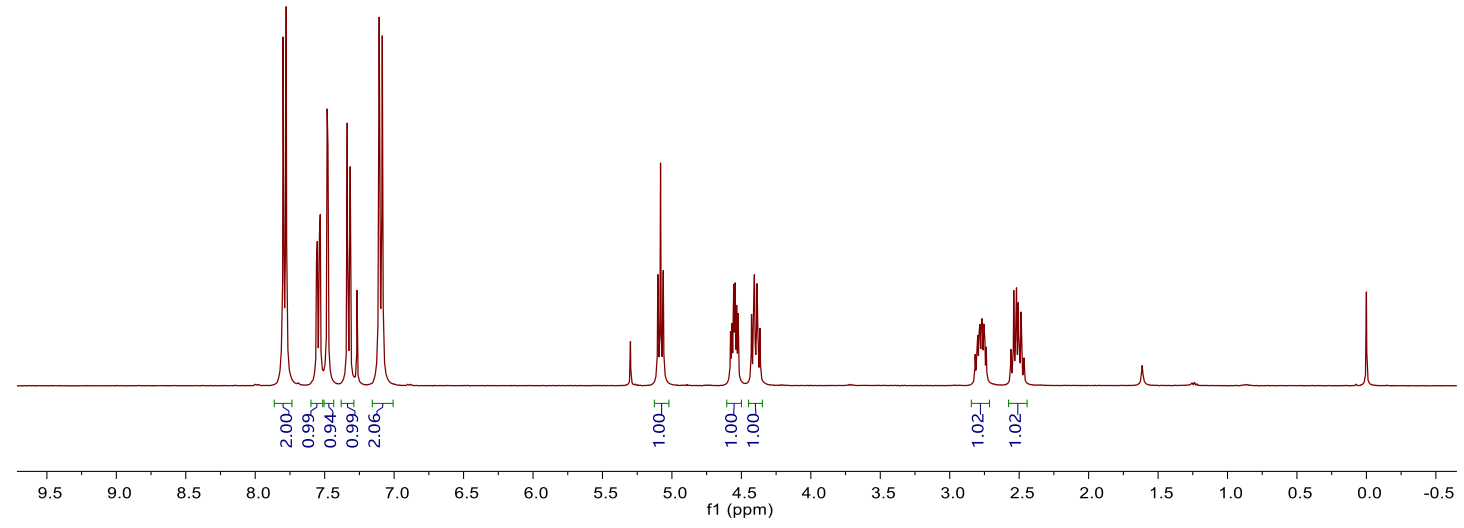

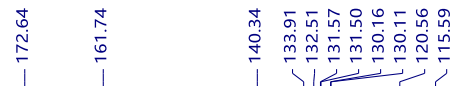

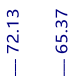<smiles>O=C(c1ccc(O[C@@H]2CCOC2=O)cc1)c1cc(Br)ccc1Cl</smiles>

${ }^{13} \mathrm{C}$ NMR (101 MHz, $\mathrm{CDCl}_{3}$ )

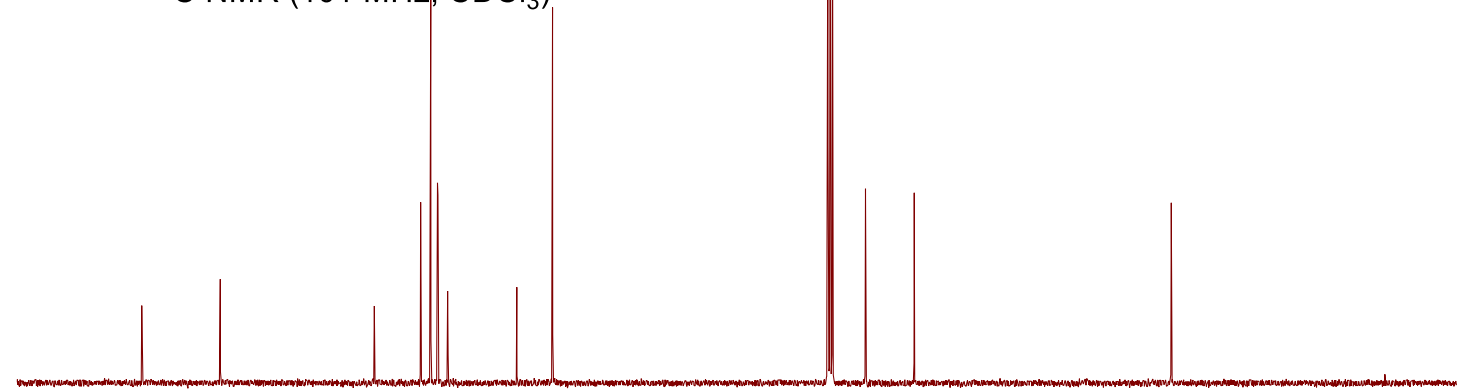

90
$\mathrm{f} 1(\mathrm{ppm})$ 


\section{Compound 11}
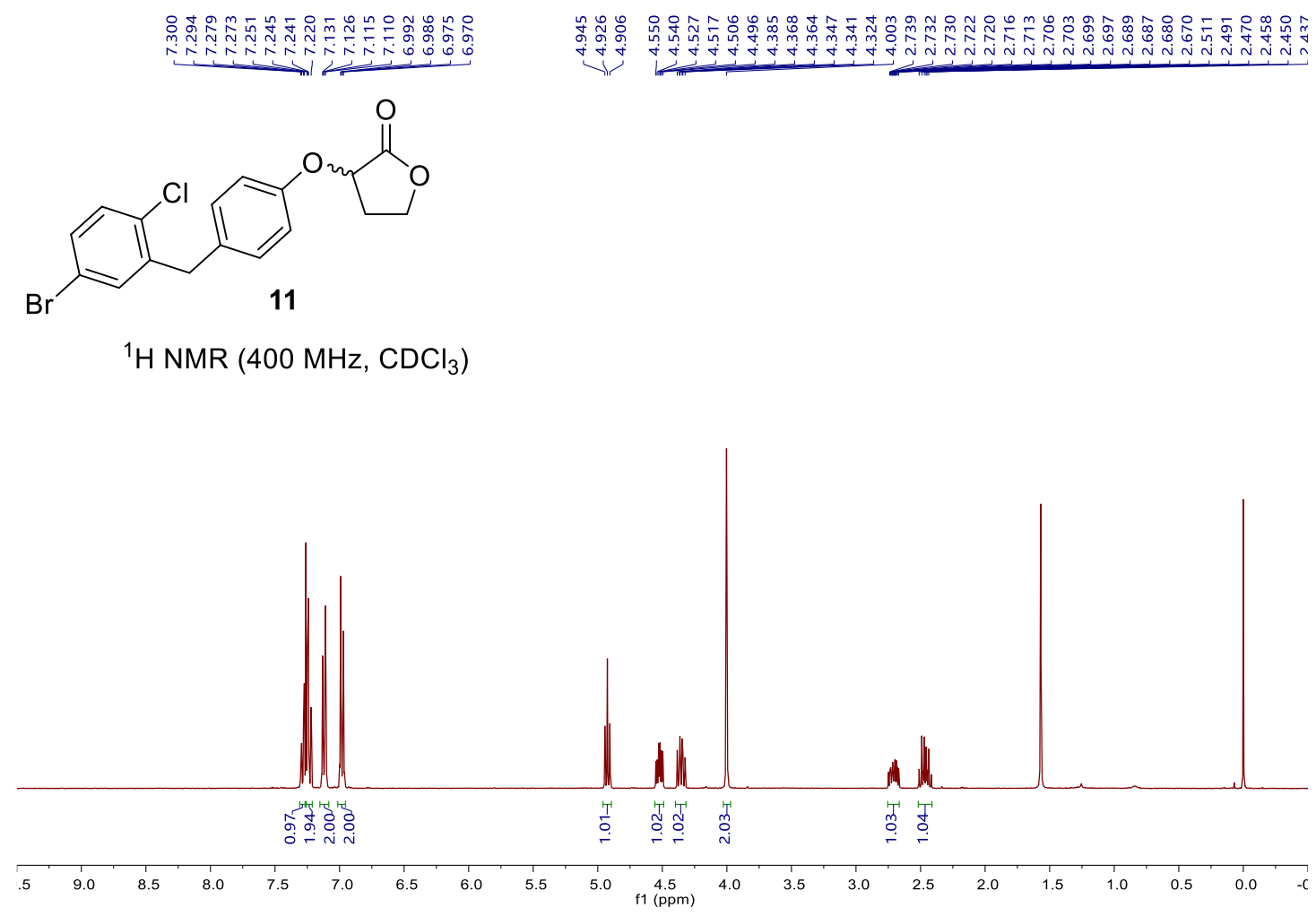

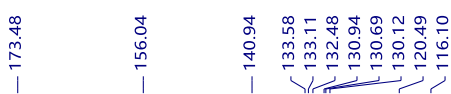

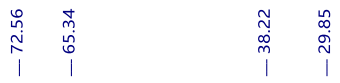

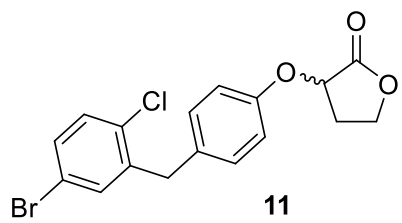

${ }^{13} \mathrm{C}$ NMR $\left(101 \mathrm{MHz}, \mathrm{CDCl}_{3}\right)$

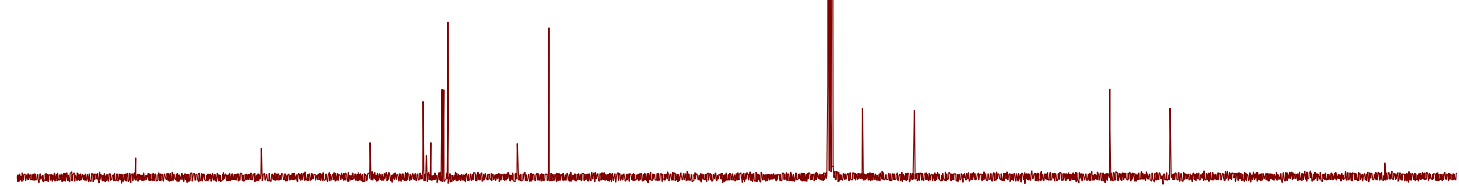

$\begin{array}{lllllllllllllllllllllllll}1 & 180 & 170 & 160 & 150 & 140 & 130 & 120 & 110 & 100 & 90 & 80 & 70 & 60 & 50 & 40 & 30 & 20 & 10 & 0 & -\end{array}$ 


\section{Compound (S)-12}

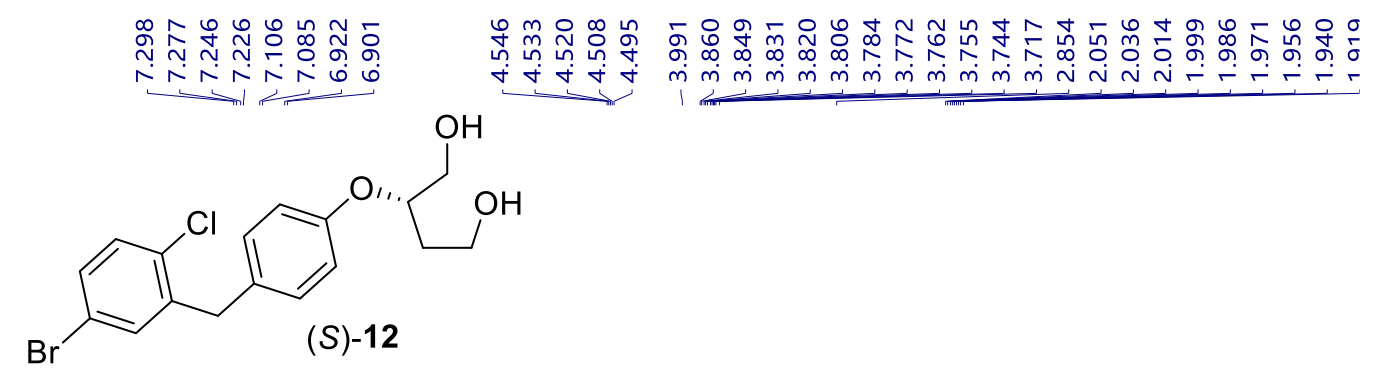

${ }^{1} \mathrm{H}$ NMR $\left(400 \mathrm{MHz}, \mathrm{CDCl}_{3}\right)$
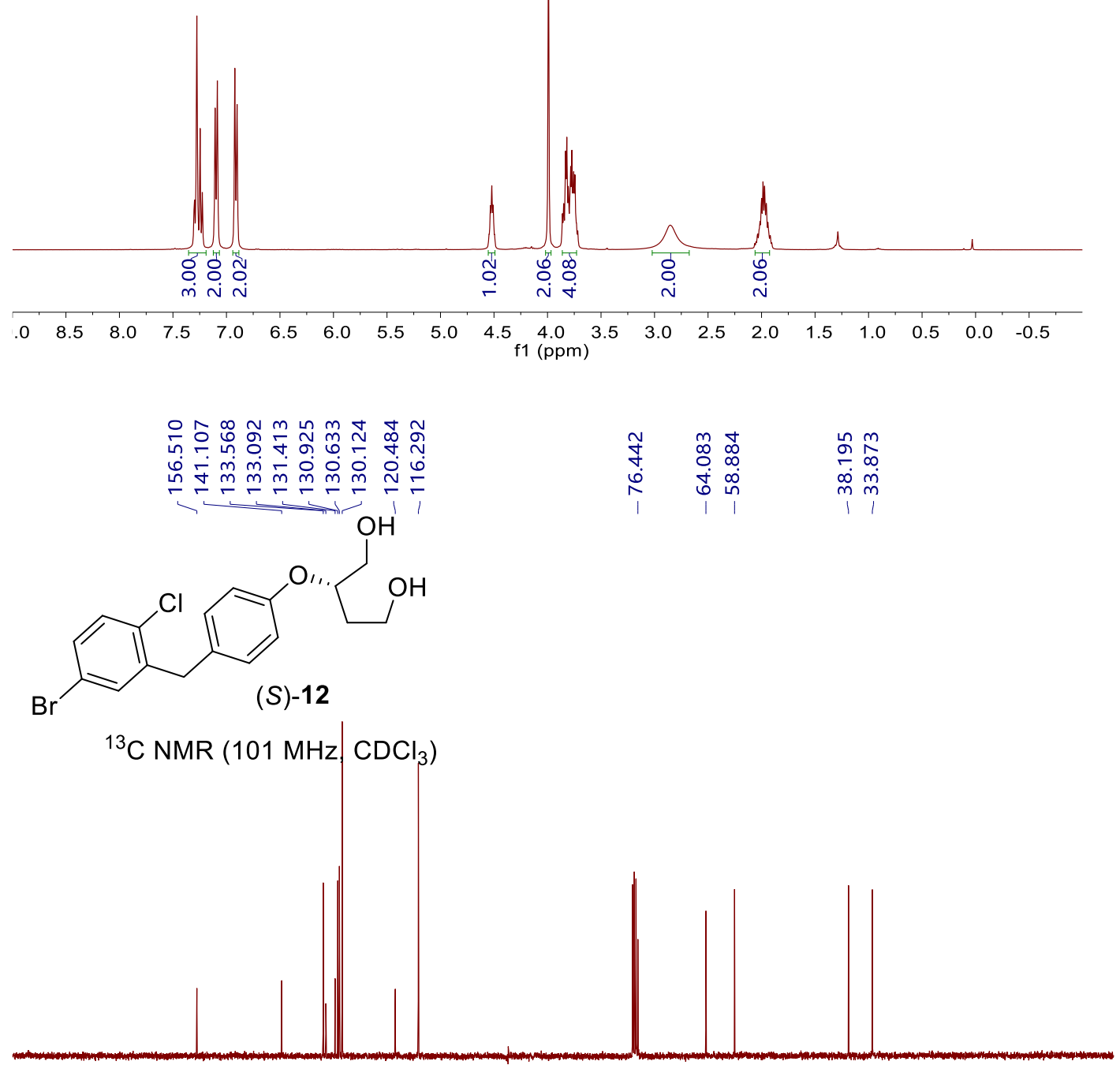

$\begin{array}{lllllllllllllllllll}90 & 180 & 170 & 160 & 150 & 140 & 130 & 120 & 110 & 100 \underset{\mathrm{f} 1}{\underset{(\mathrm{ppm})}{90}} \mathbf{8 0} & 70 & 60 & 50 & 40 & 30 & 20 & 10 & 0 & \end{array}$ 


\section{Compound (S)-13}
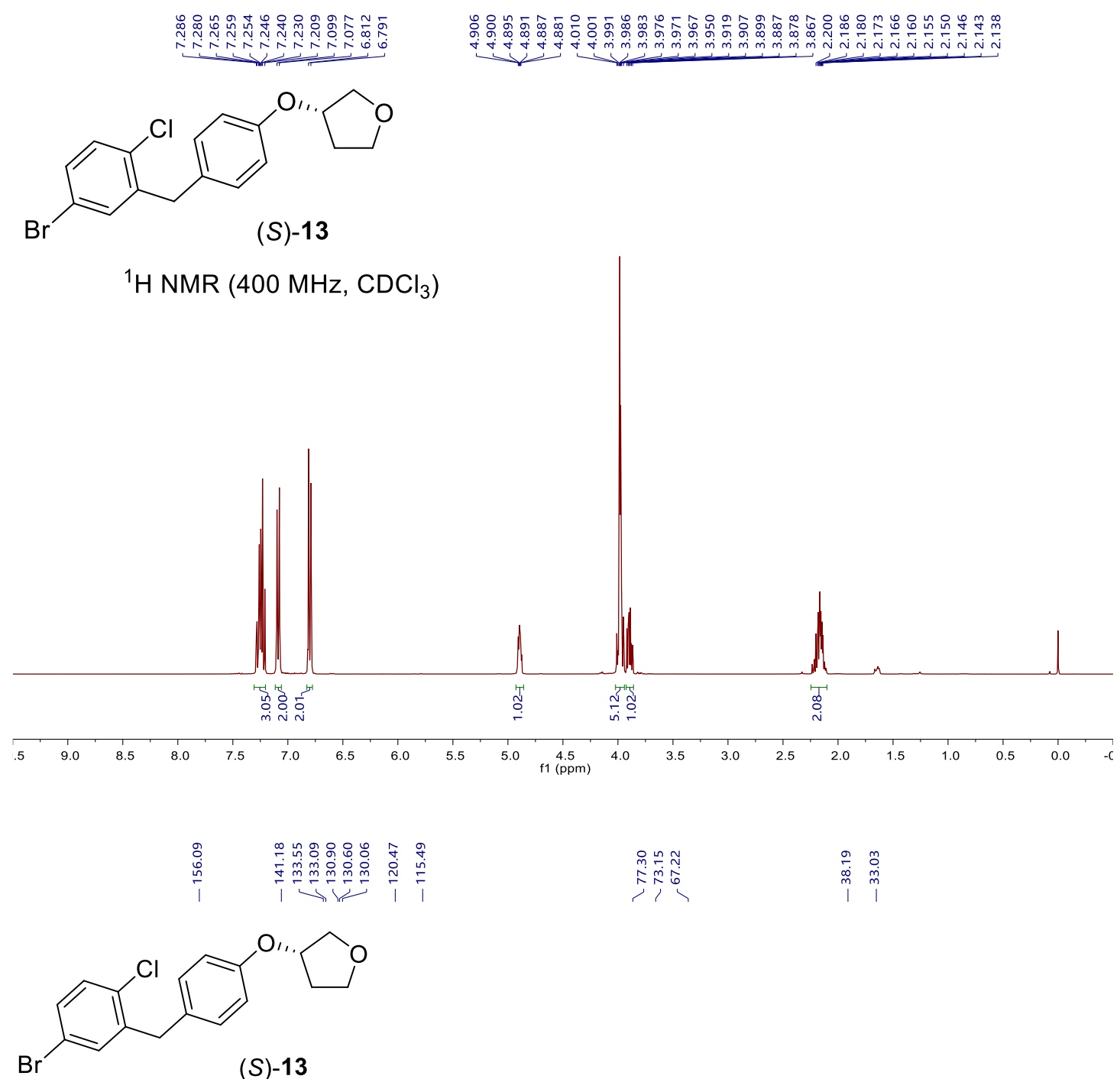

(S)-13

${ }^{13} \mathrm{C}$ NMR $\left(101 \mathrm{MHz}, \mathrm{CDCl}_{3}\right)$

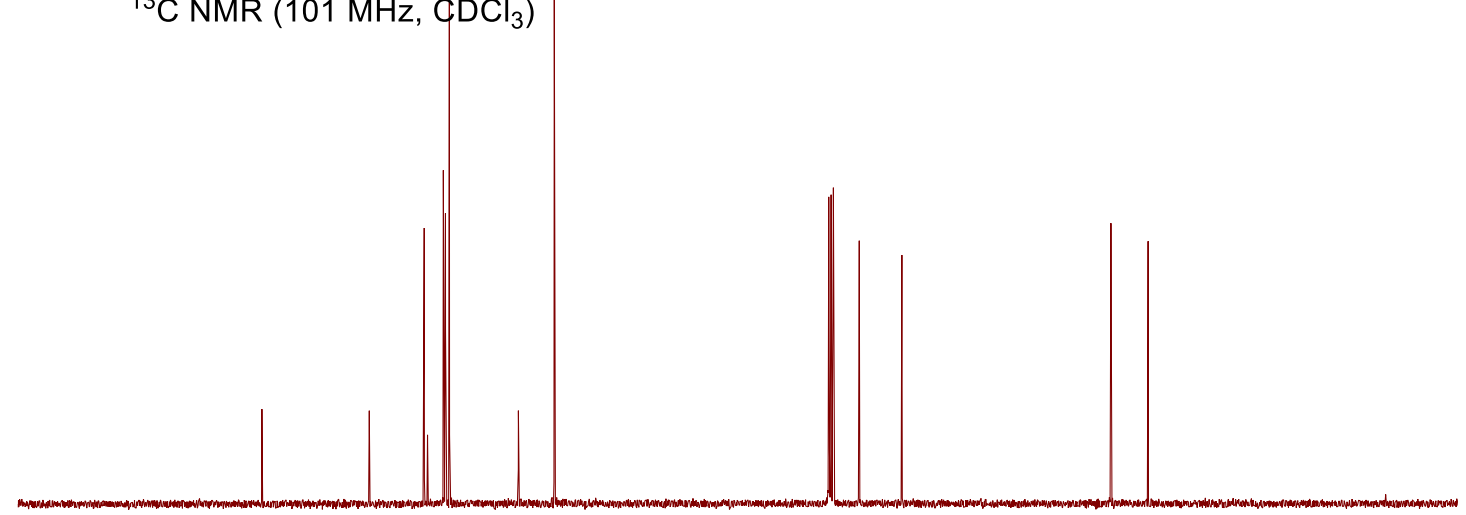

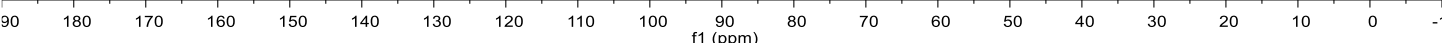


(S)-1-Methyl-3-phenoxypyrrolidine ((S)-15)

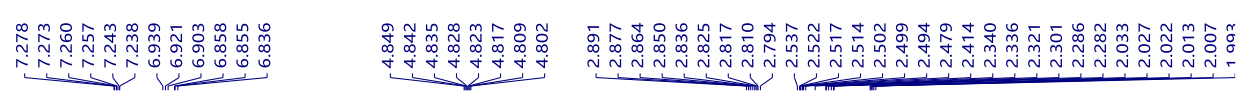

$\overbrace{}^{\mathrm{N}} \mathrm{N-Me}$

$(S)-15$

${ }^{1} \mathrm{H}$ NMR $\left(400 \mathrm{MHz}, \mathrm{CDCl}_{3}\right)$

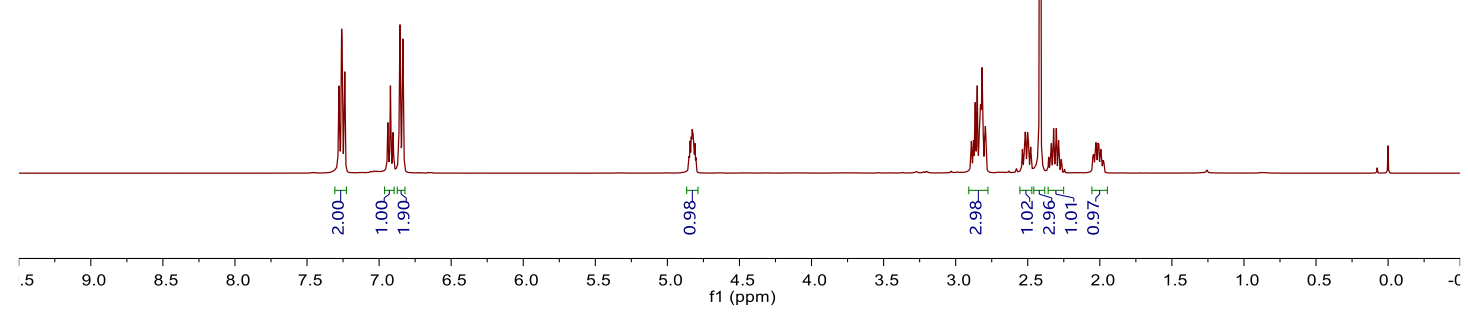

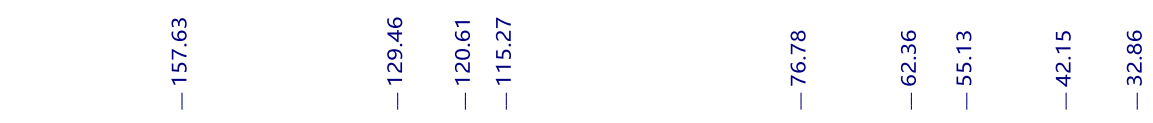<smiles>CN1CC[C@H](Oc2ccccc2)C1</smiles>

(S)-15

${ }^{13} \mathrm{C}$ NMR $\left(101 \mathrm{MHz}, \mathrm{CDCl}_{3}\right)$

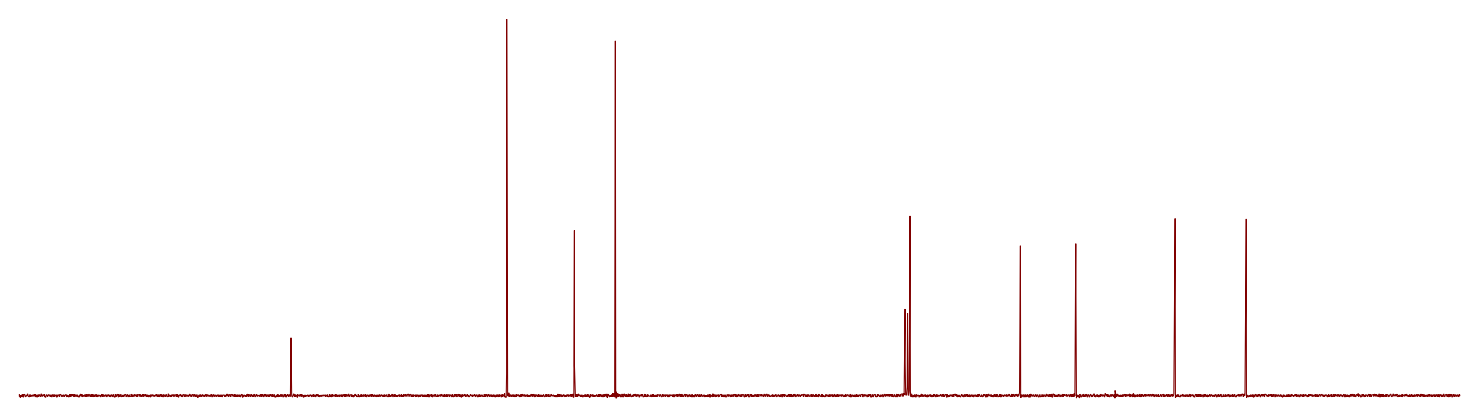

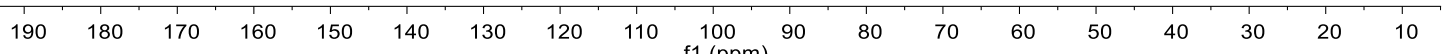




\section{(I) HPLC Charts for Hydrogenation Products}

\section{(R)-2-(Dibenzylamino)-1-(3,5-dimethylphenyl)ethanol (S2a)}
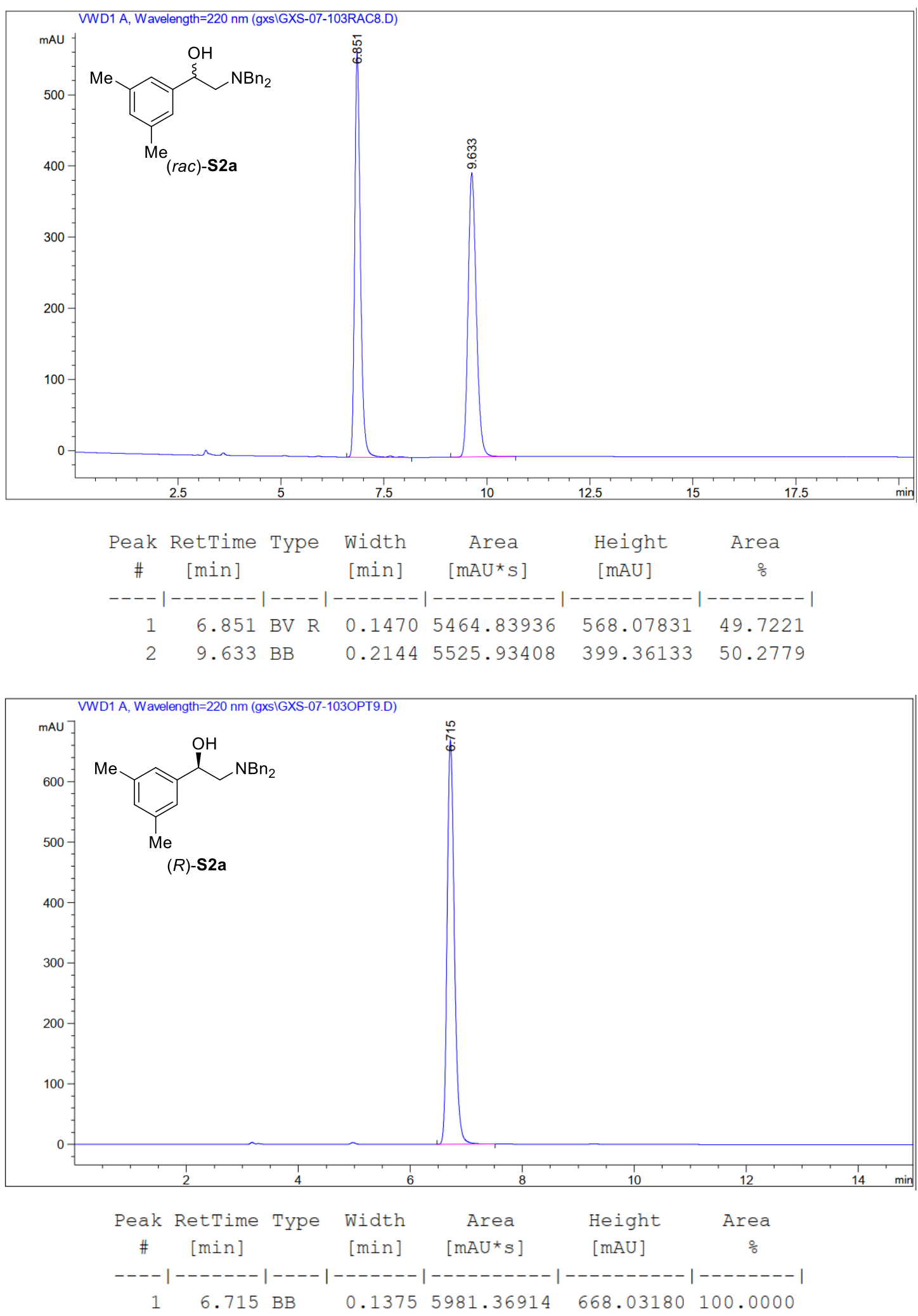


\section{(R)-2-(Dibenzylamino)-1-(4-methoxyphenyl)ethanol (S2b)}
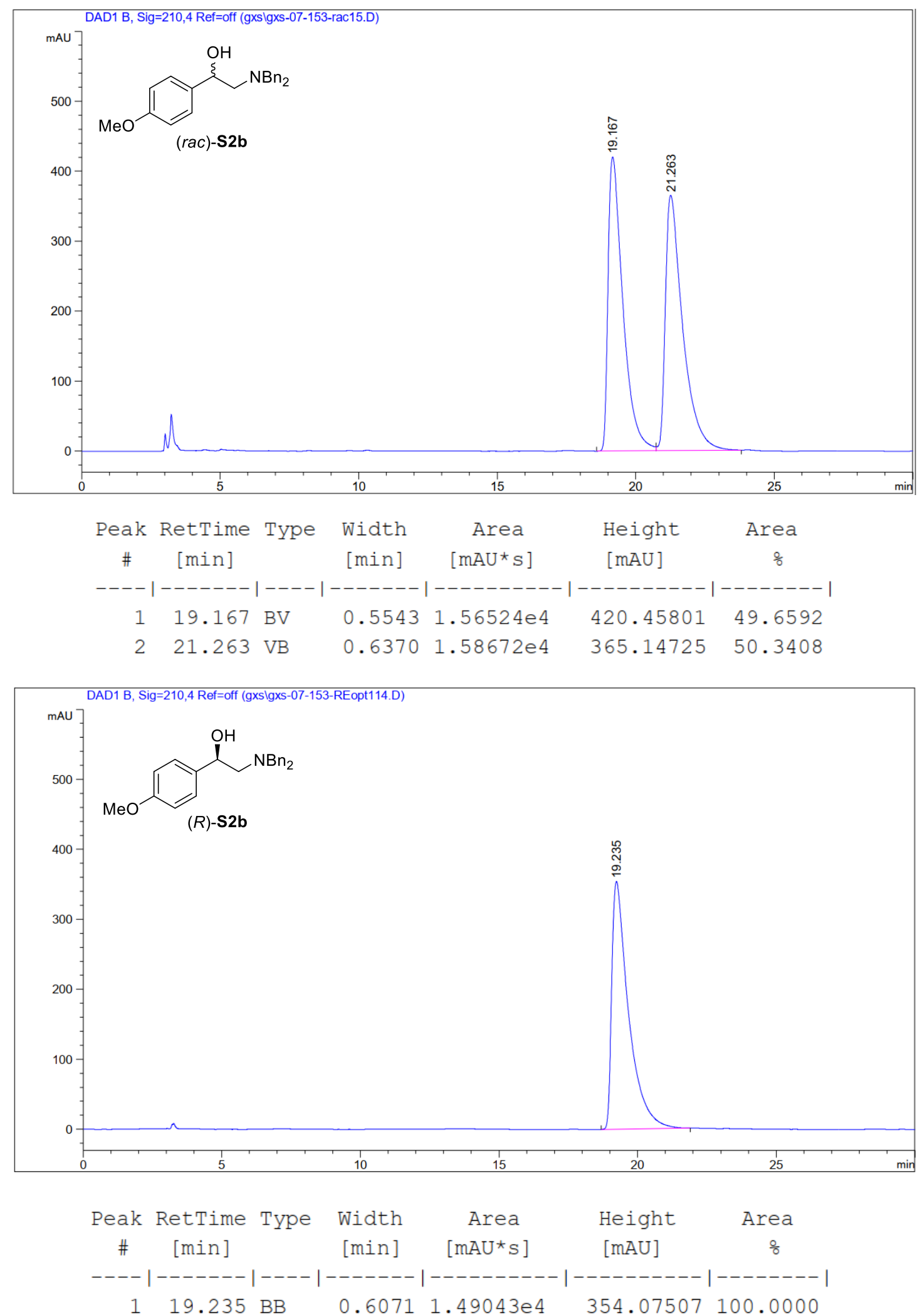
(R)-2-(Dibenzylamino)-1-(4-(trifluoromethyl)phenyl)ethanol (S2c)
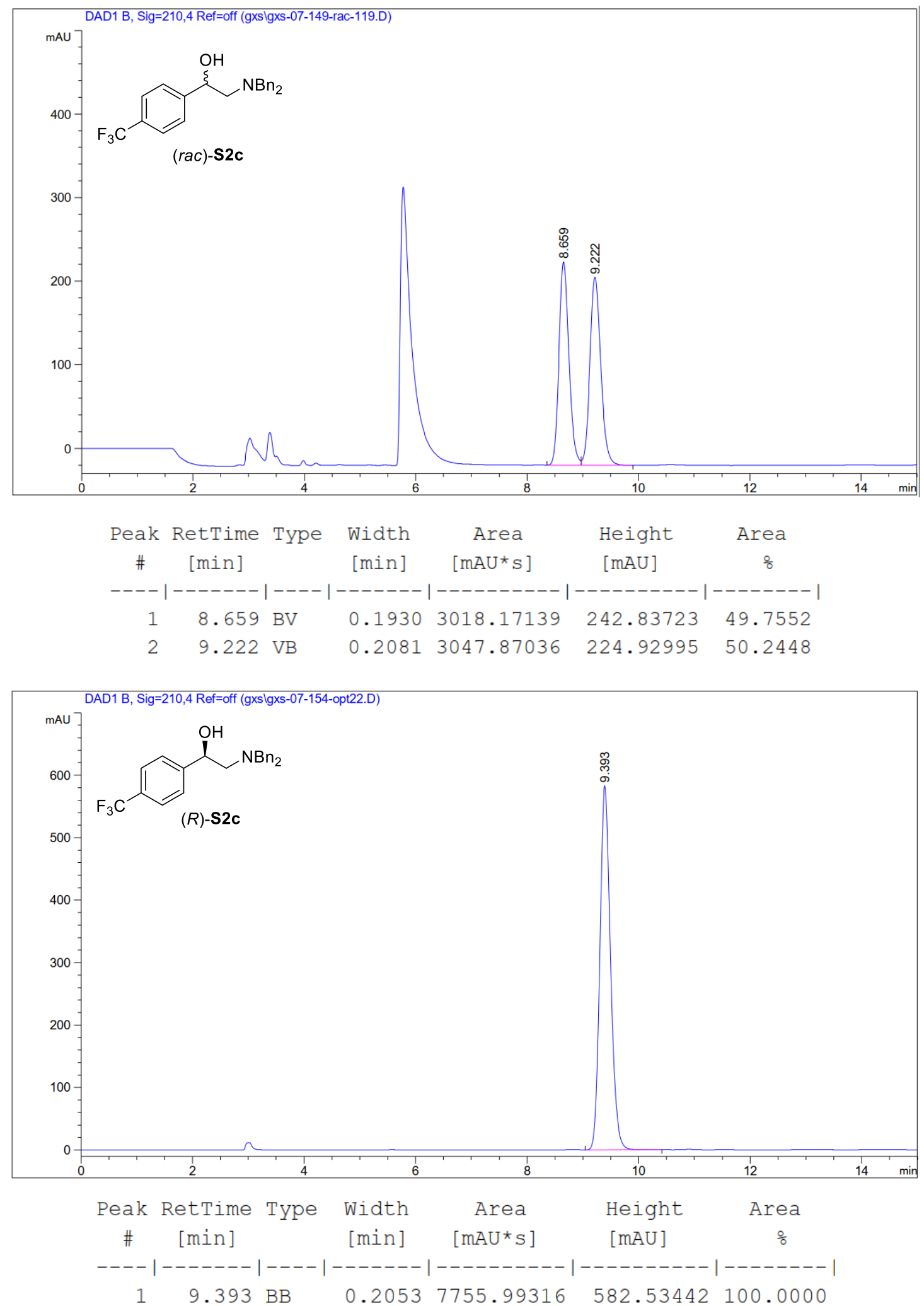


\section{(+)-2-Phenoxybutane-1,4-diol ((+)-5a)}
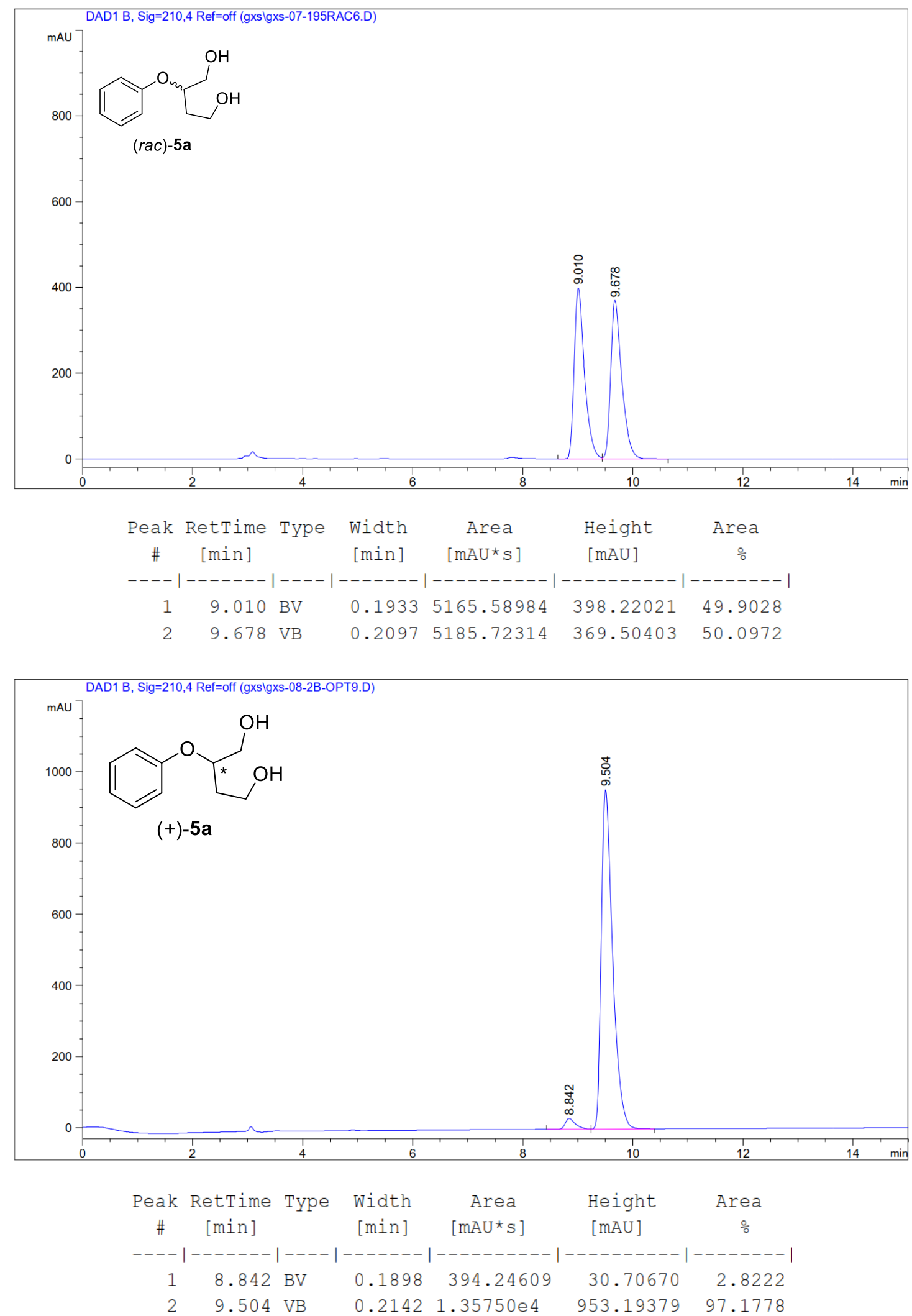


\section{$(+) 2-(p-T o l y l o x y) b u t a n e-1,4-d i o l((+)-5 b)$}
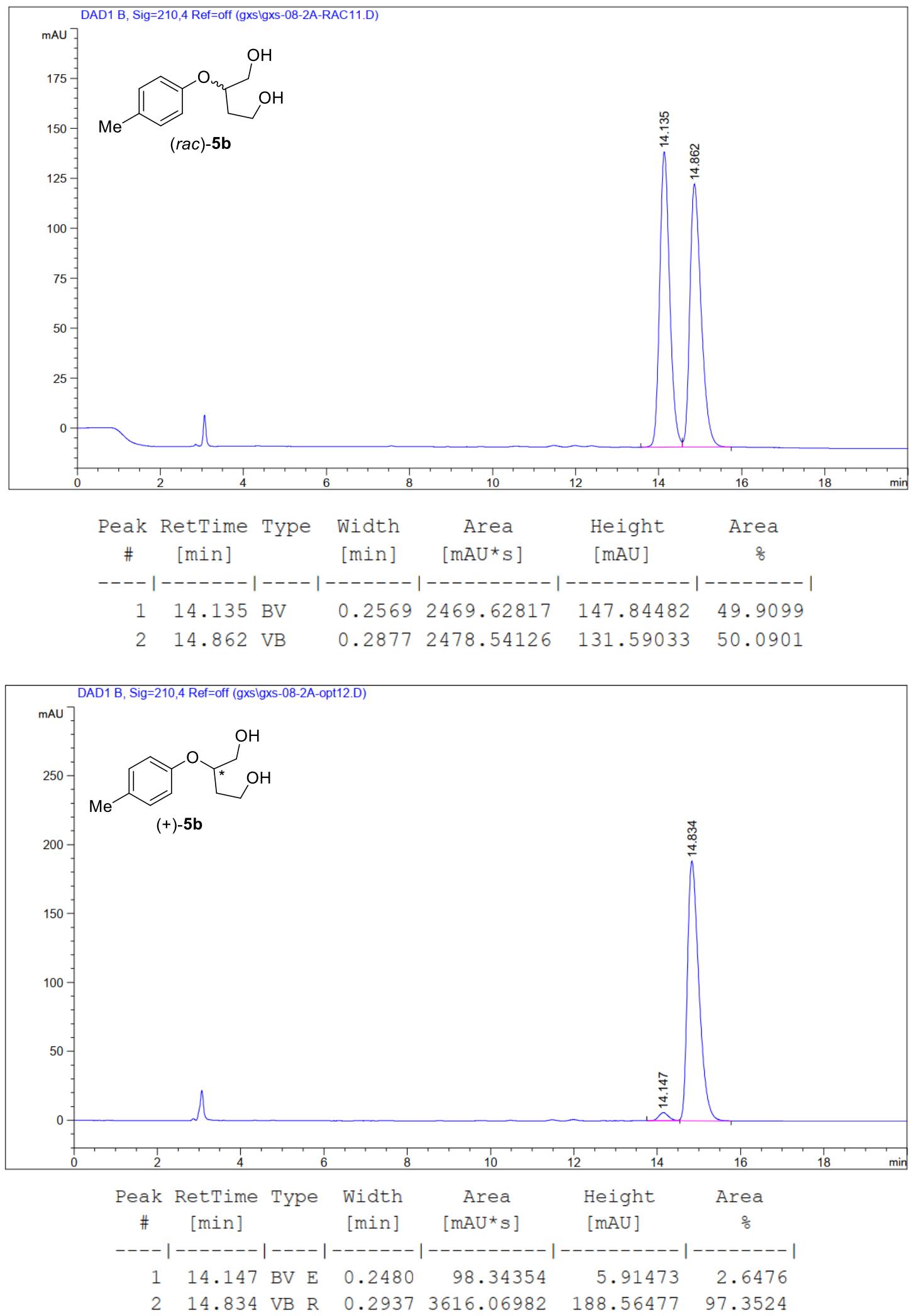
(+)-2-(4-Chlorophenoxy)butane-1,4-diol ((+)-5c)
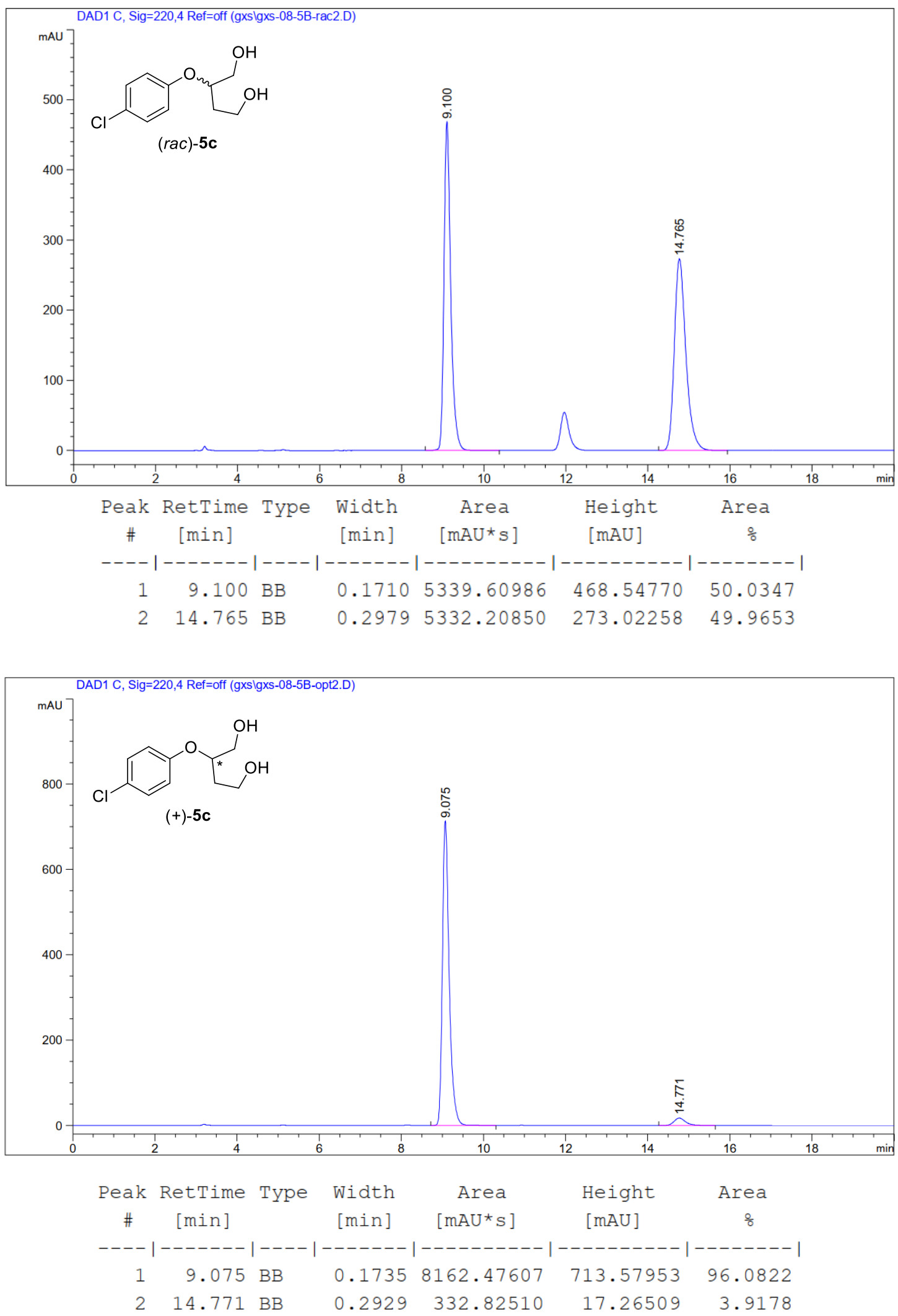


\section{(+)-2-(4-(tert-Butyl)phenoxy)butane-1,4-diol ((+)-5d)}
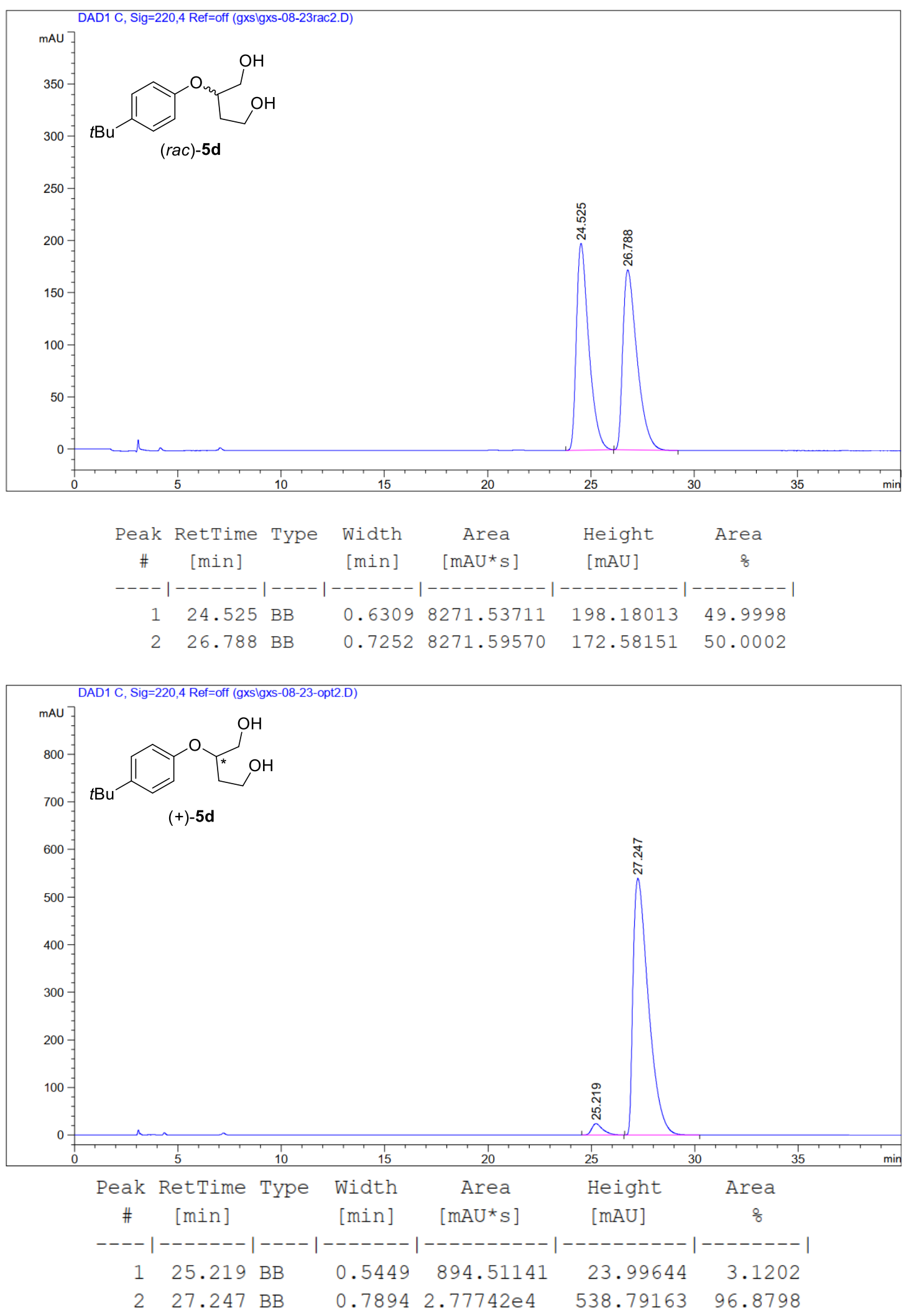


\section{(+)-2-(3-Methoxyphenoxy)butane-1,4-diol ((+)-5e)}
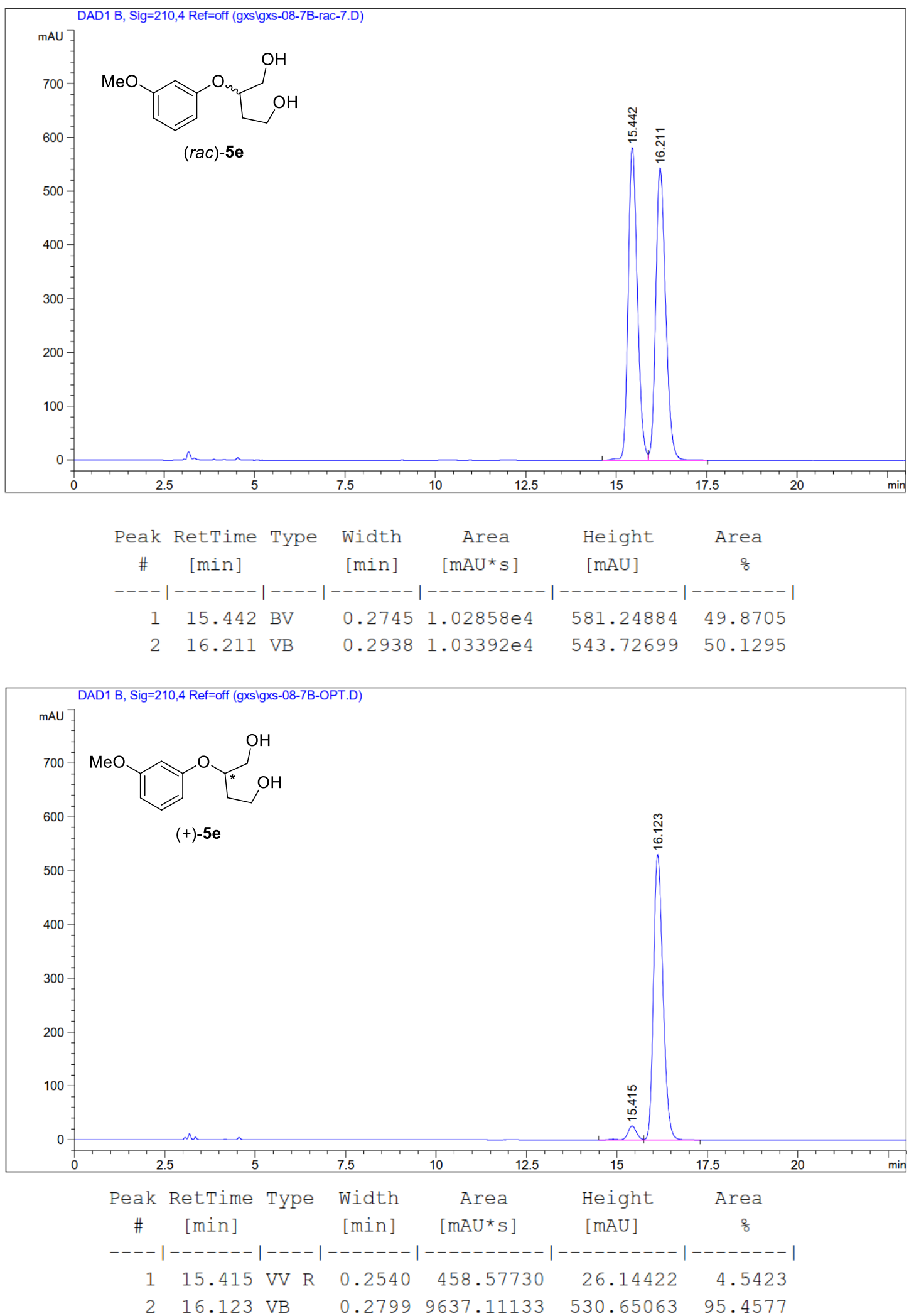
(+)-2-(3-Chlorophenoxy)butane-1,4-diol ((+)-5f)
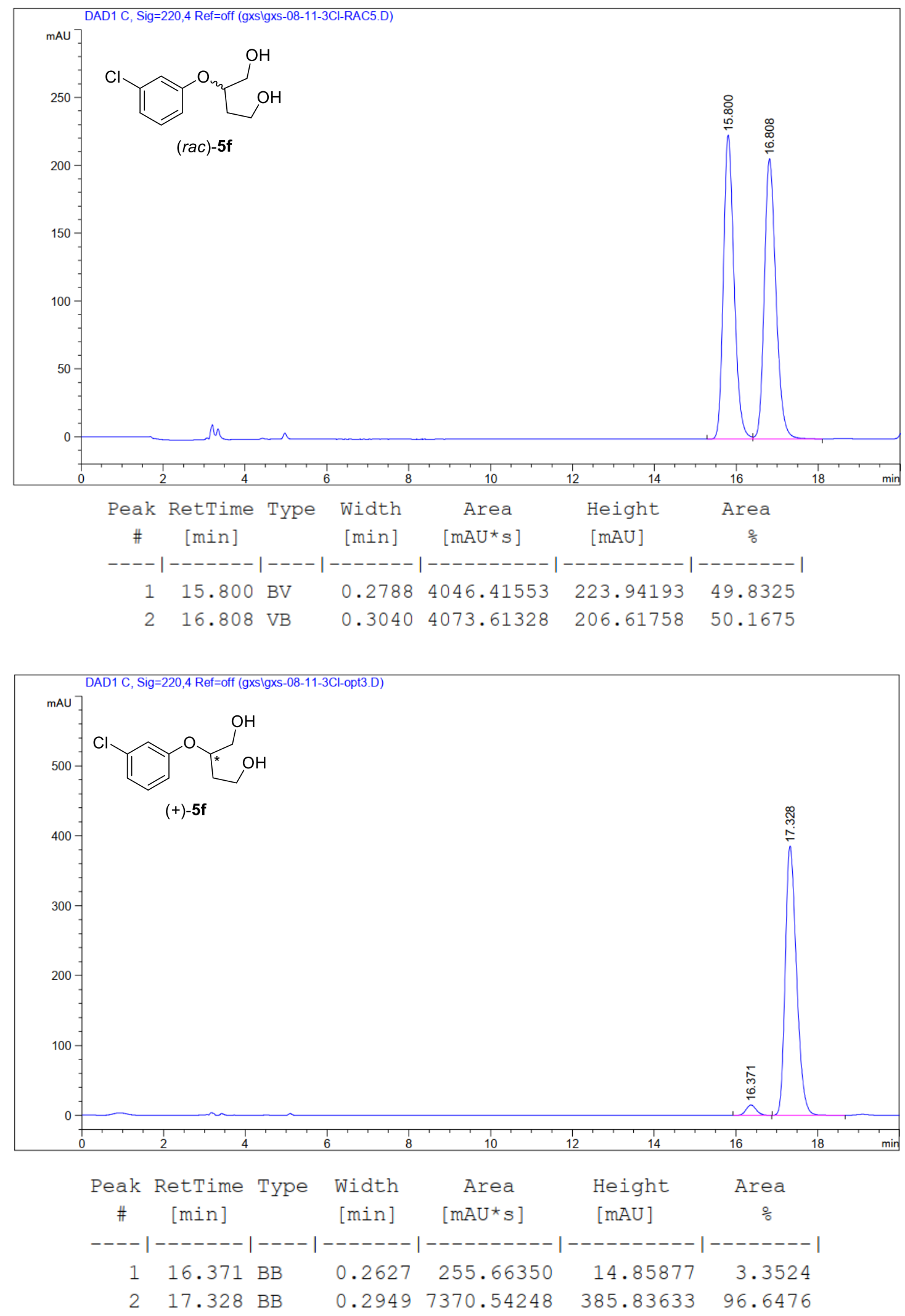
(+)-2-(3-Bromophenoxy)butane-1,4-diol ((+)-5g)
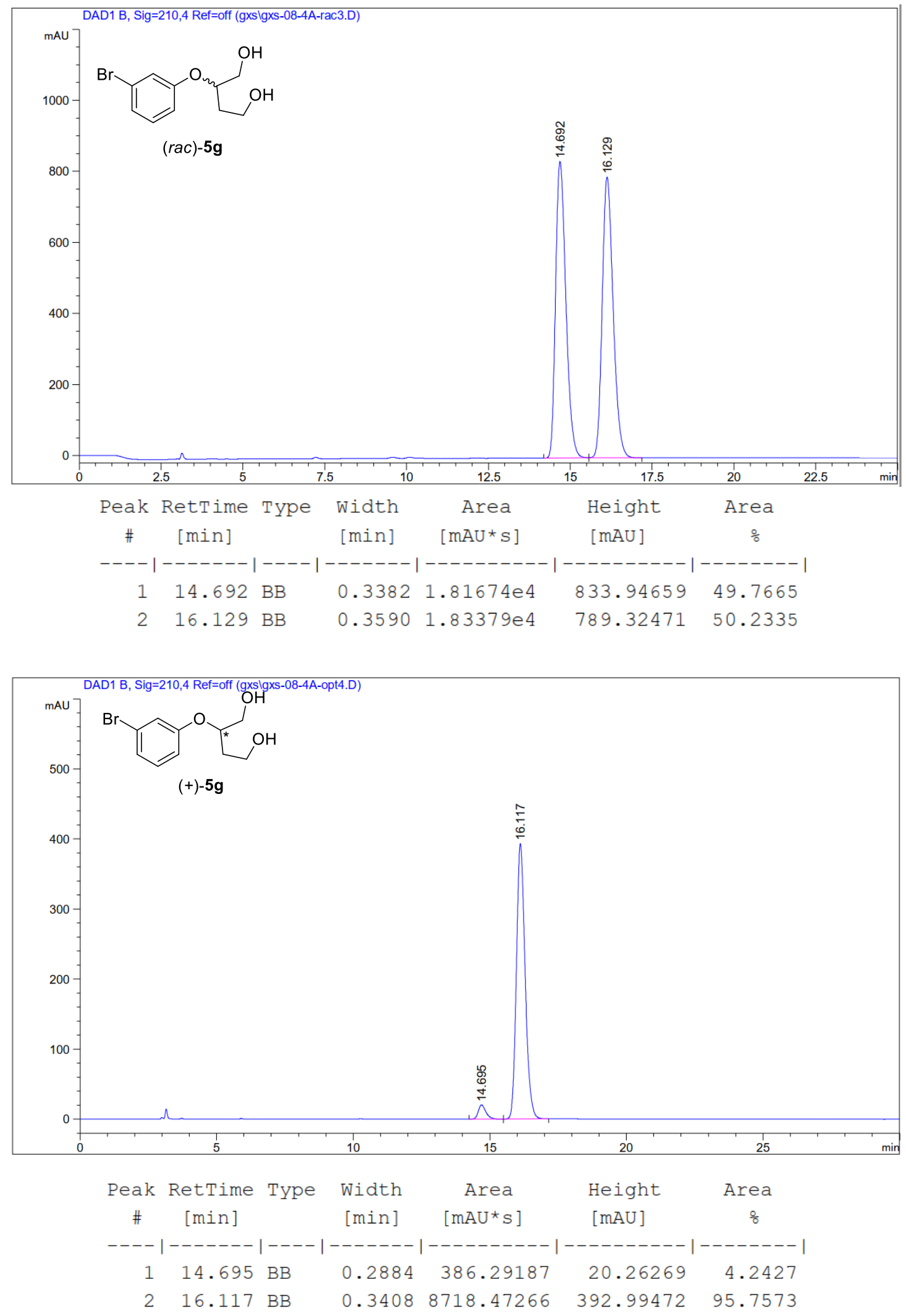
(+)-2-(o-Tolyloxy)butane-1,4-diol ((+)-5h)
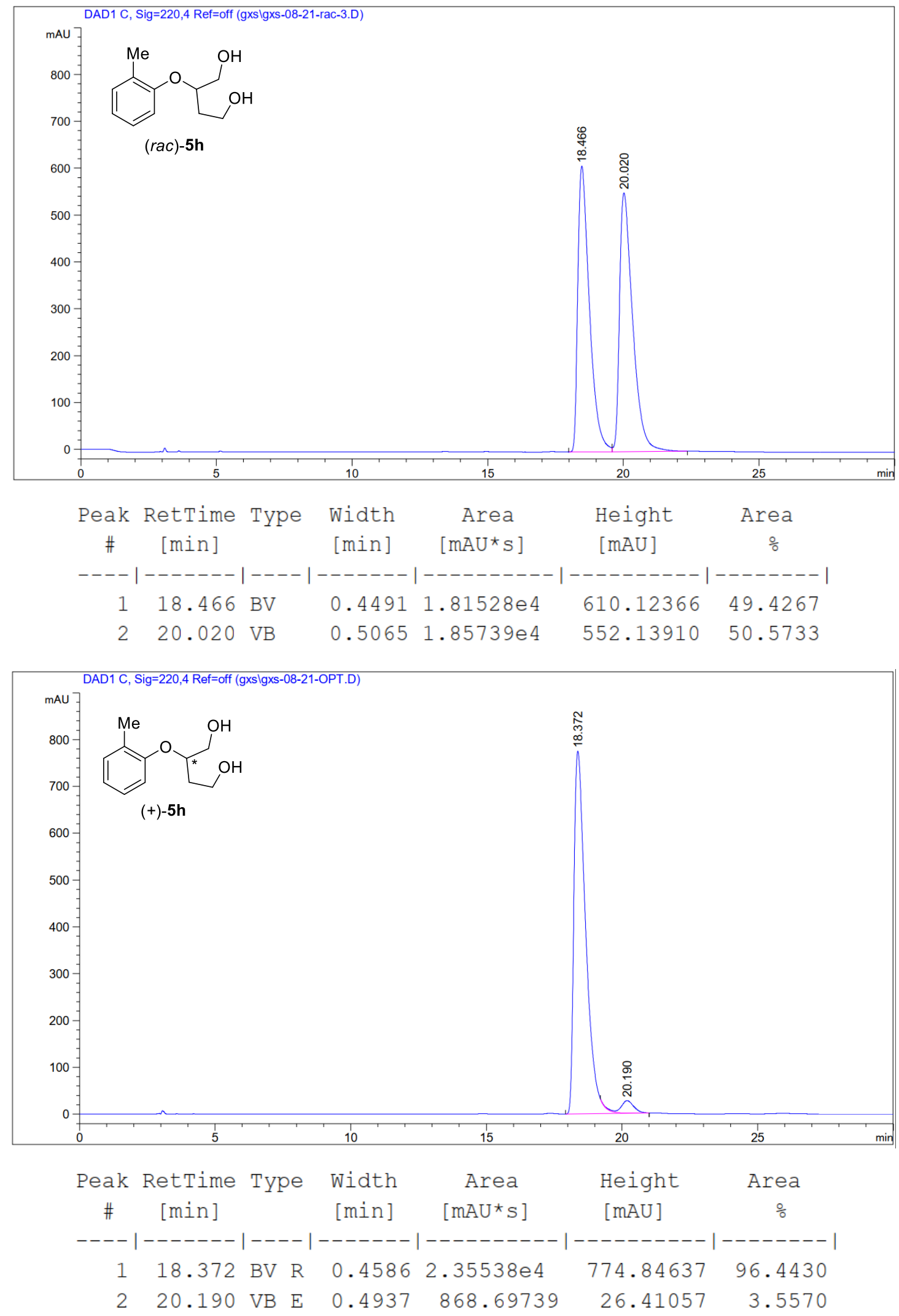


\section{(+)-2-(2-Methoxyphenoxy)butane-1,4-diol ((+)-5i)}
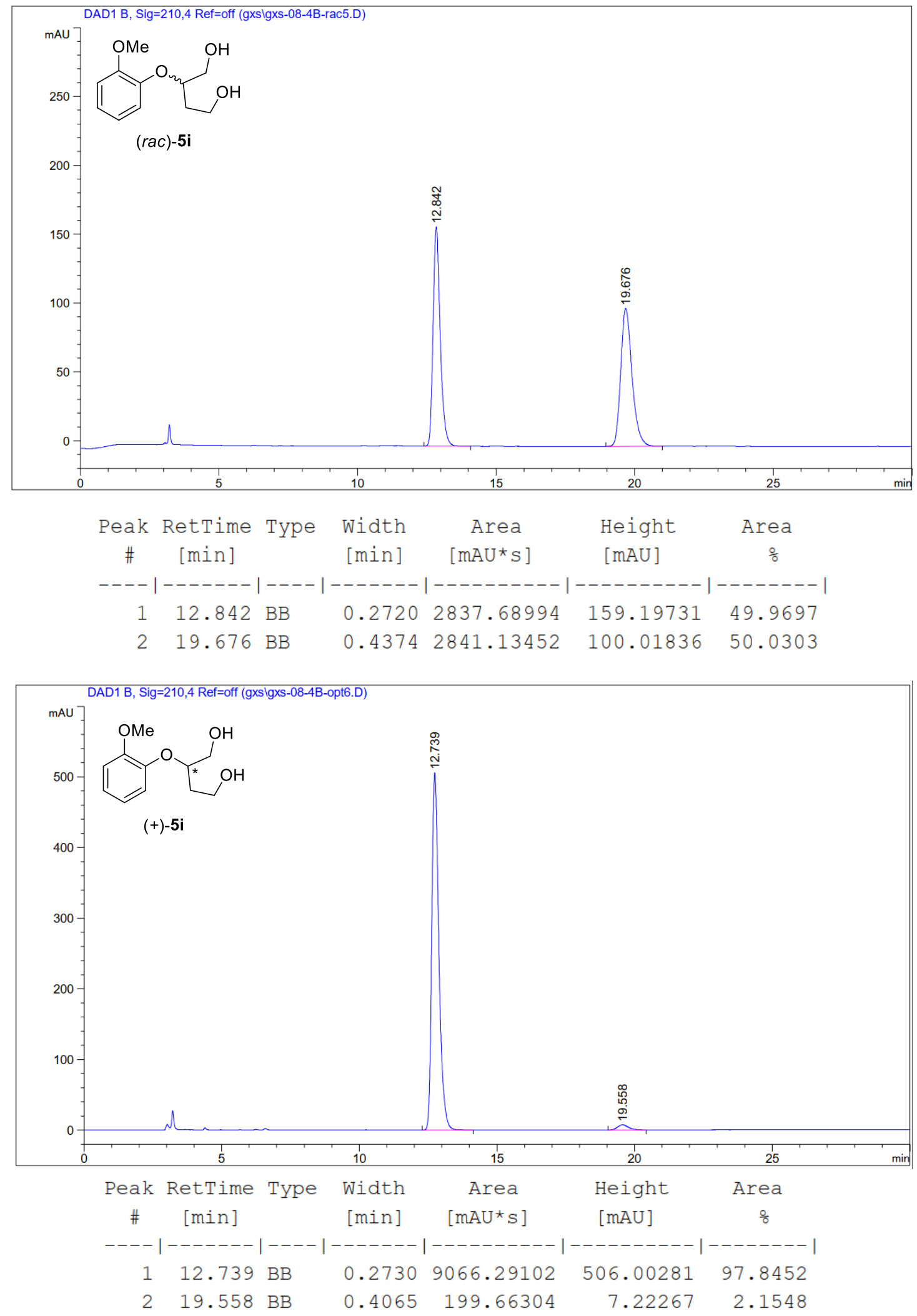
(+)-2-(2-Chlorophenoxy)butane-1,4-diol ((+)-5j)
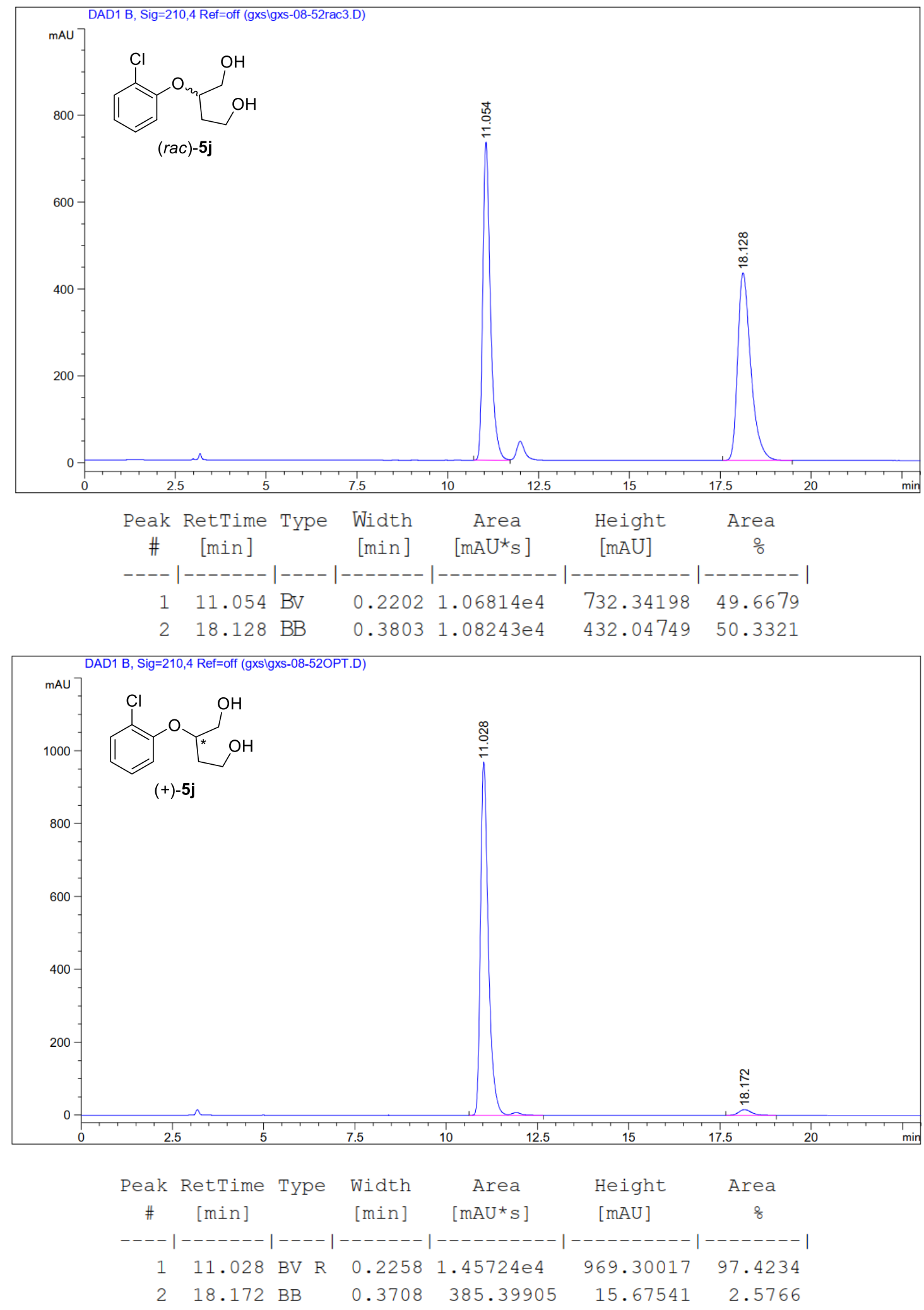
(+)-2-(2-Fluorophenoxy)butane-1,4-diol ((+)-5k)
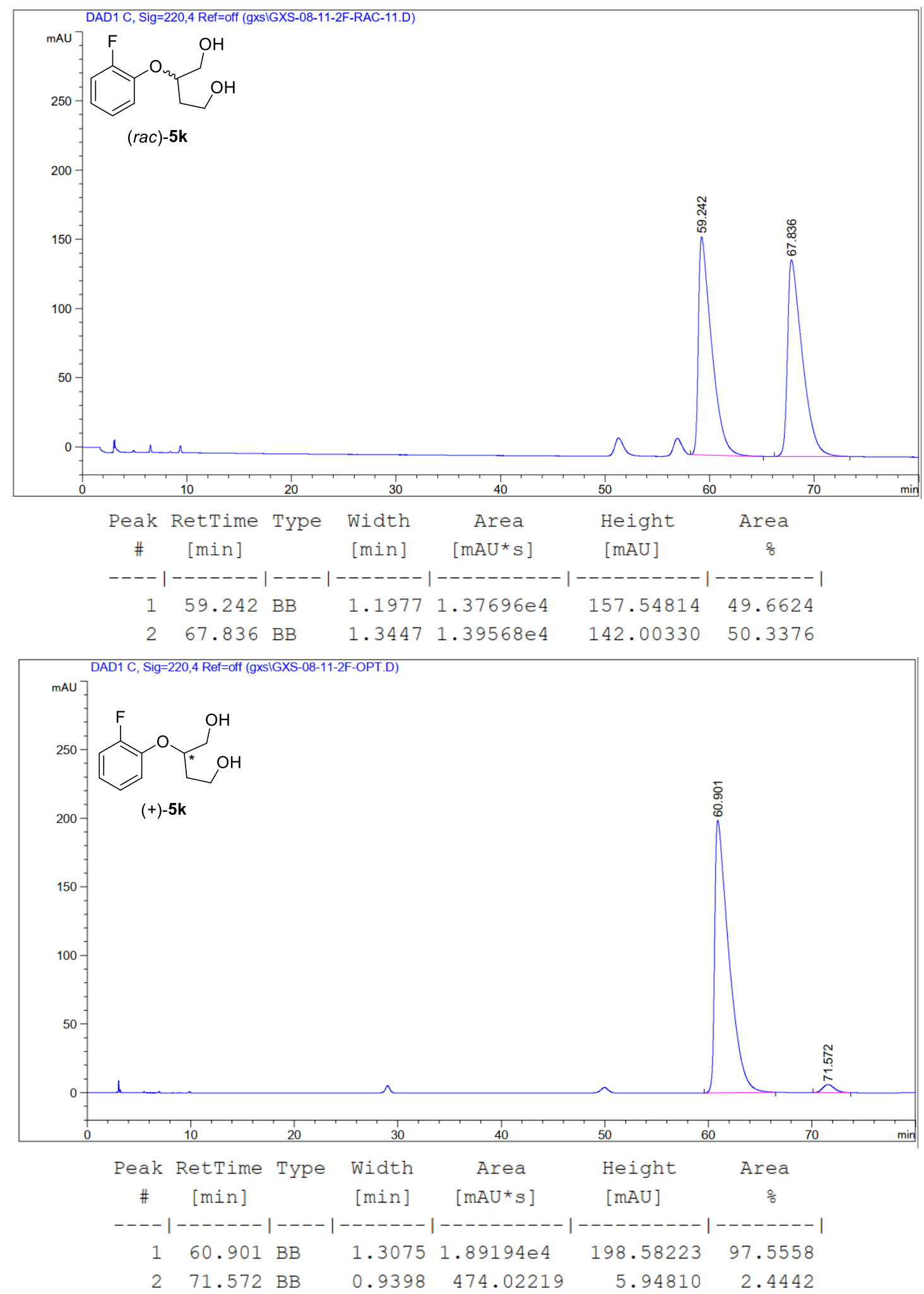


\section{(R)-2-(2,5-Dimethylphenoxy)butane-1,4-diol (5I)}
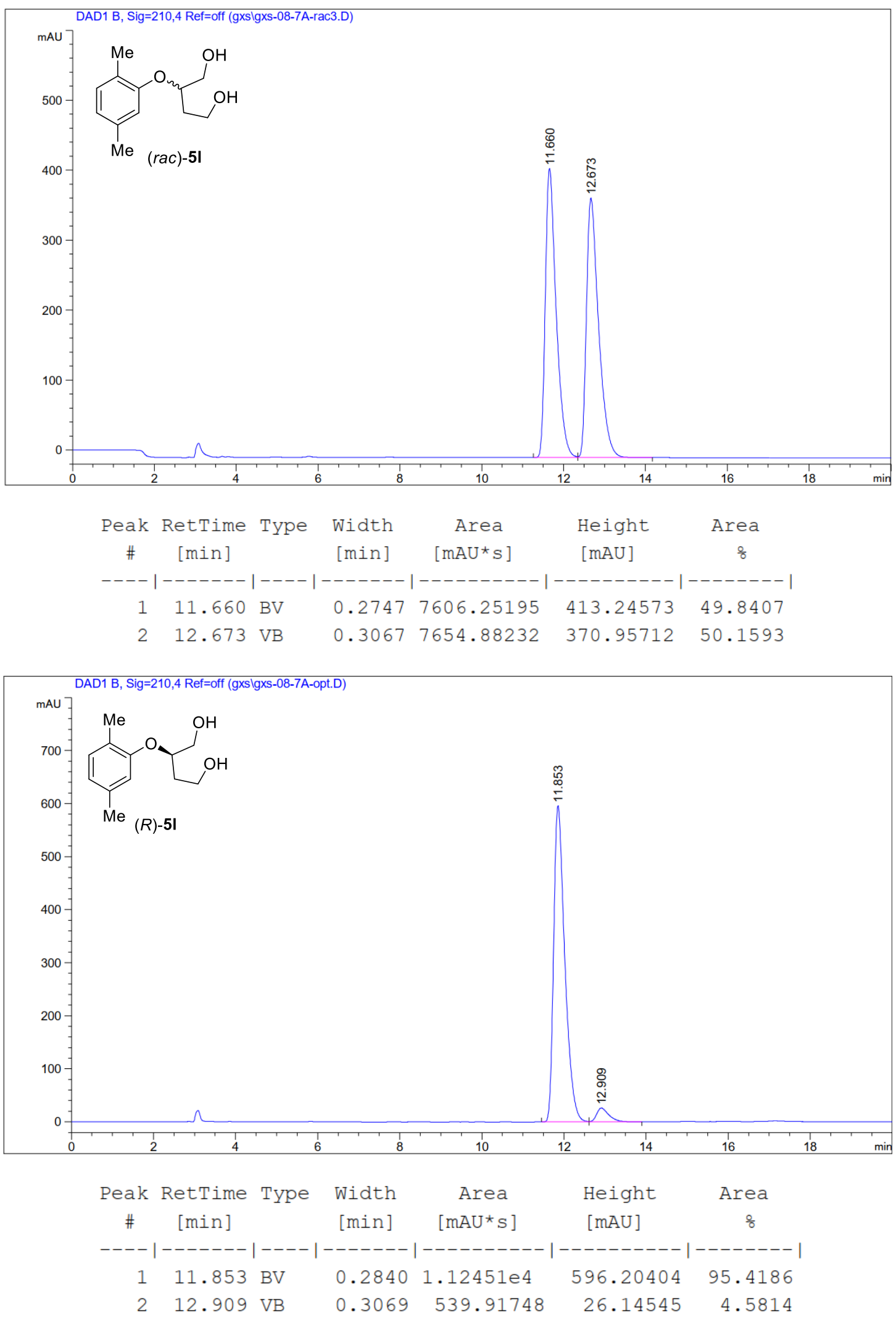
(+)-2-(Naphthalen-2-yloxy)butane-1,4-diol ((+)-5m)
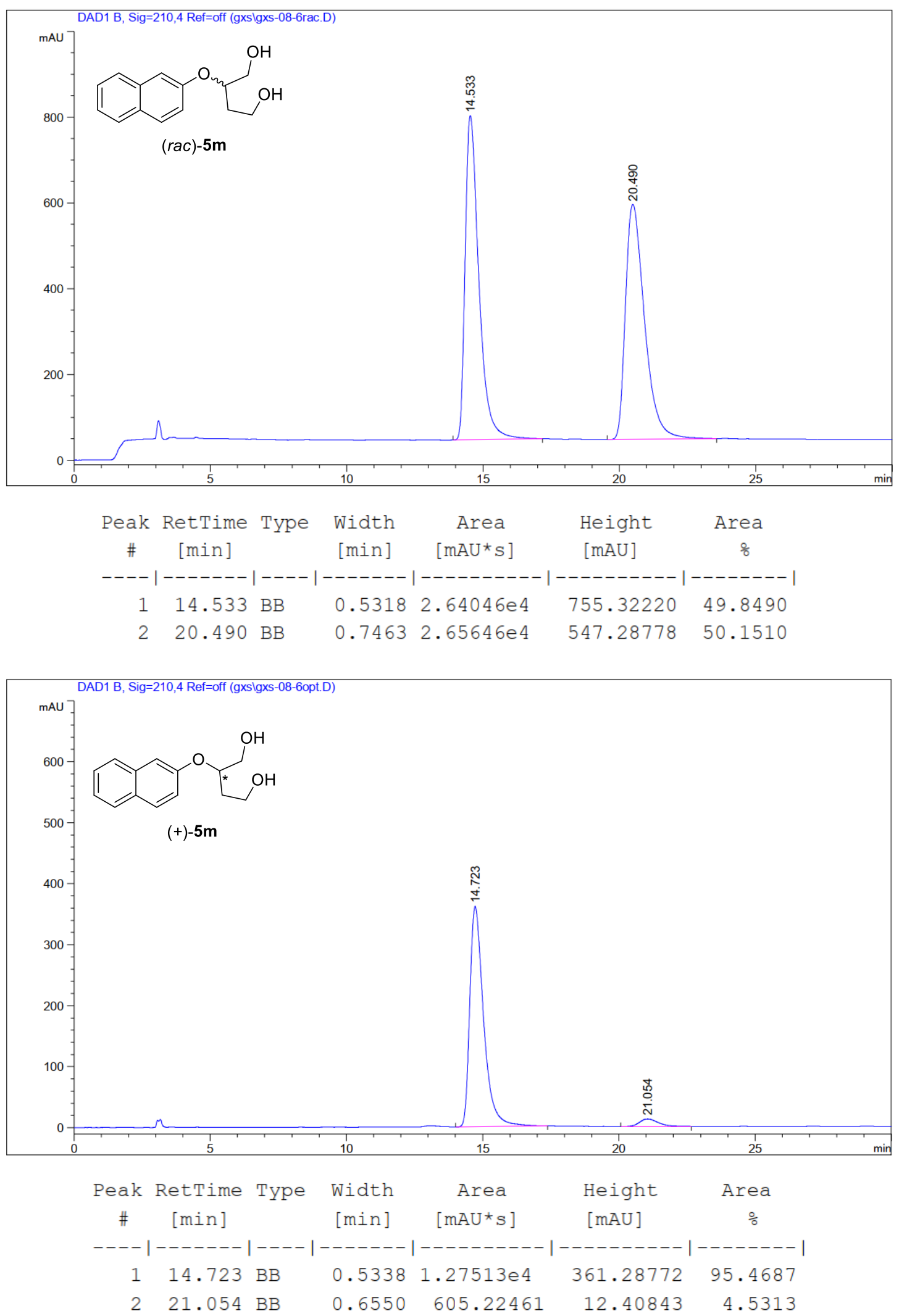
(+)-2-Phenoxypentane-1,5-diol ((+)-8a)
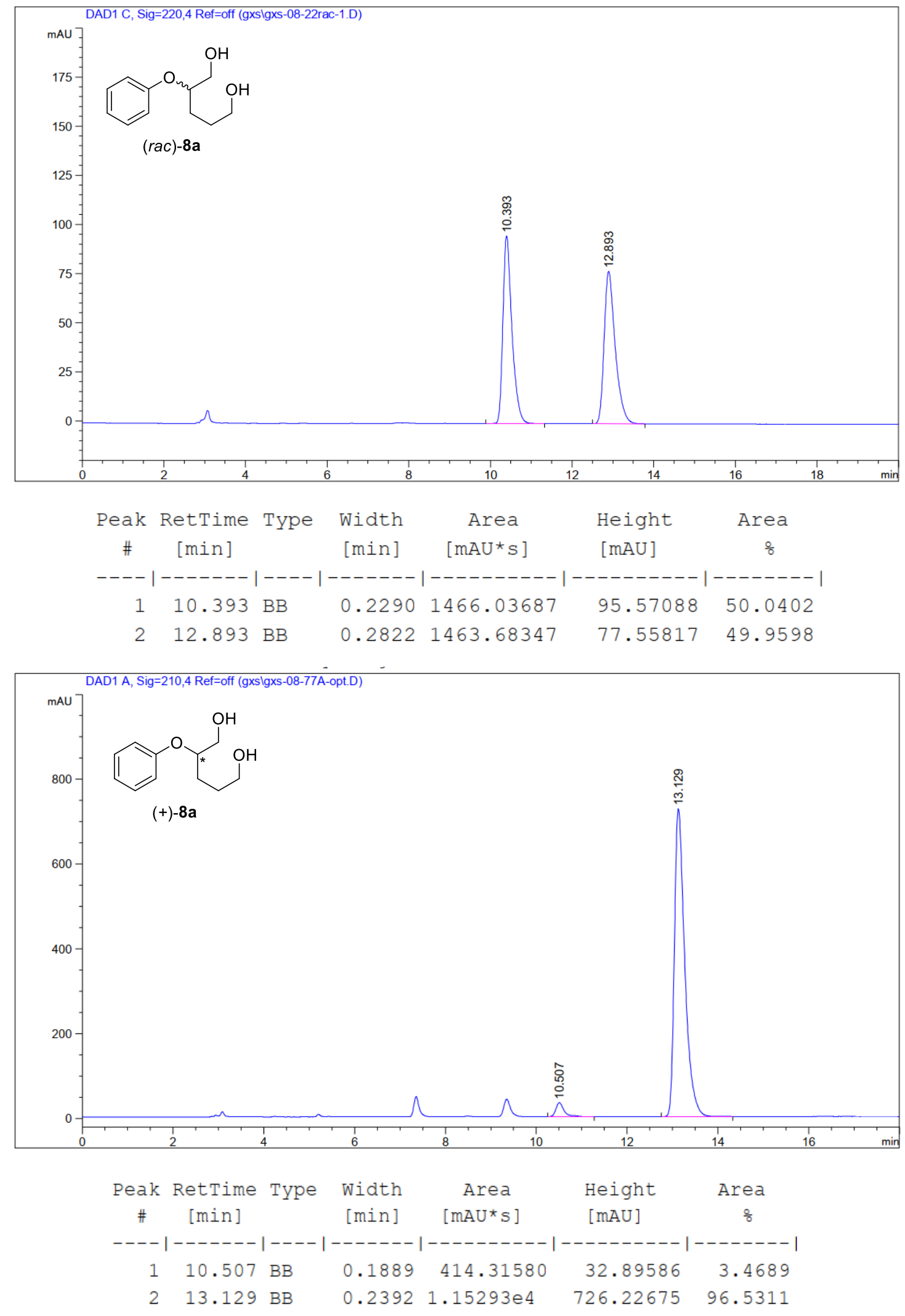
$(+)-2-(p-T o l y l o x y) p e n t a n e-1,5-d i o l ~((+)-8 b)$
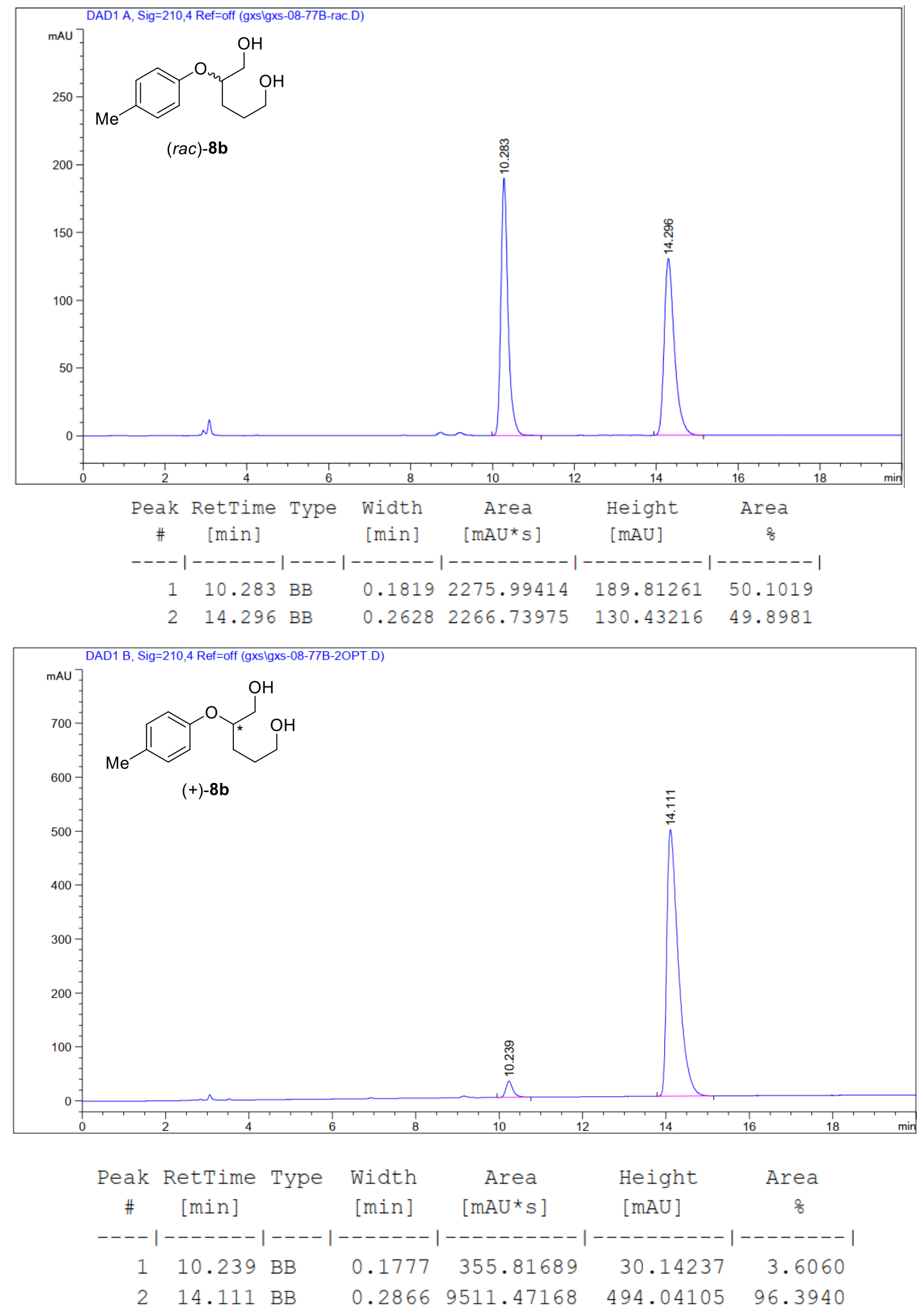
(+)-2-(m-Tolyloxy)pentane-1,5-diol ((+)-8c)
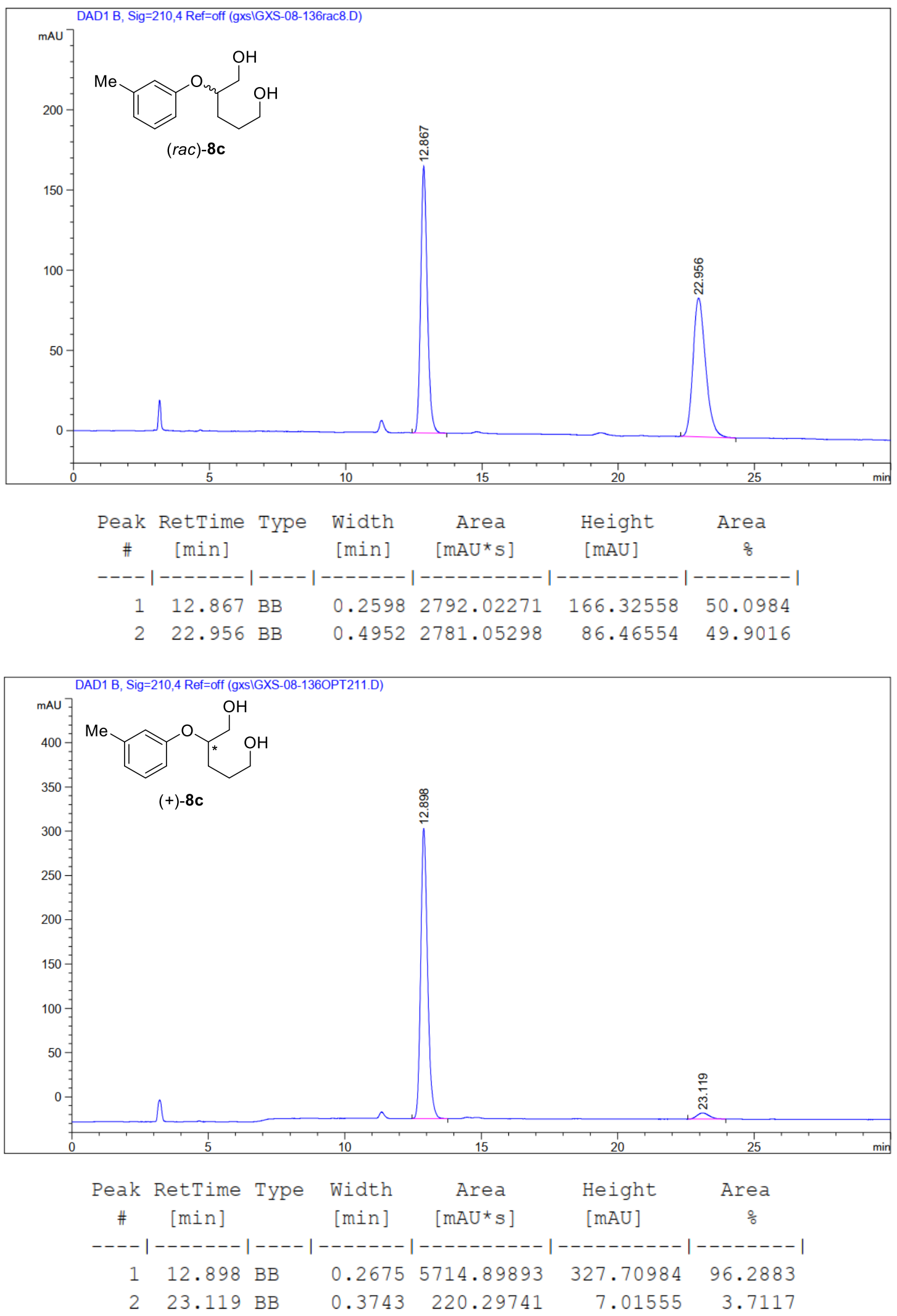
(+)-2-(o-Tolyloxy)pentane-1,5-diol ((+)-8d)
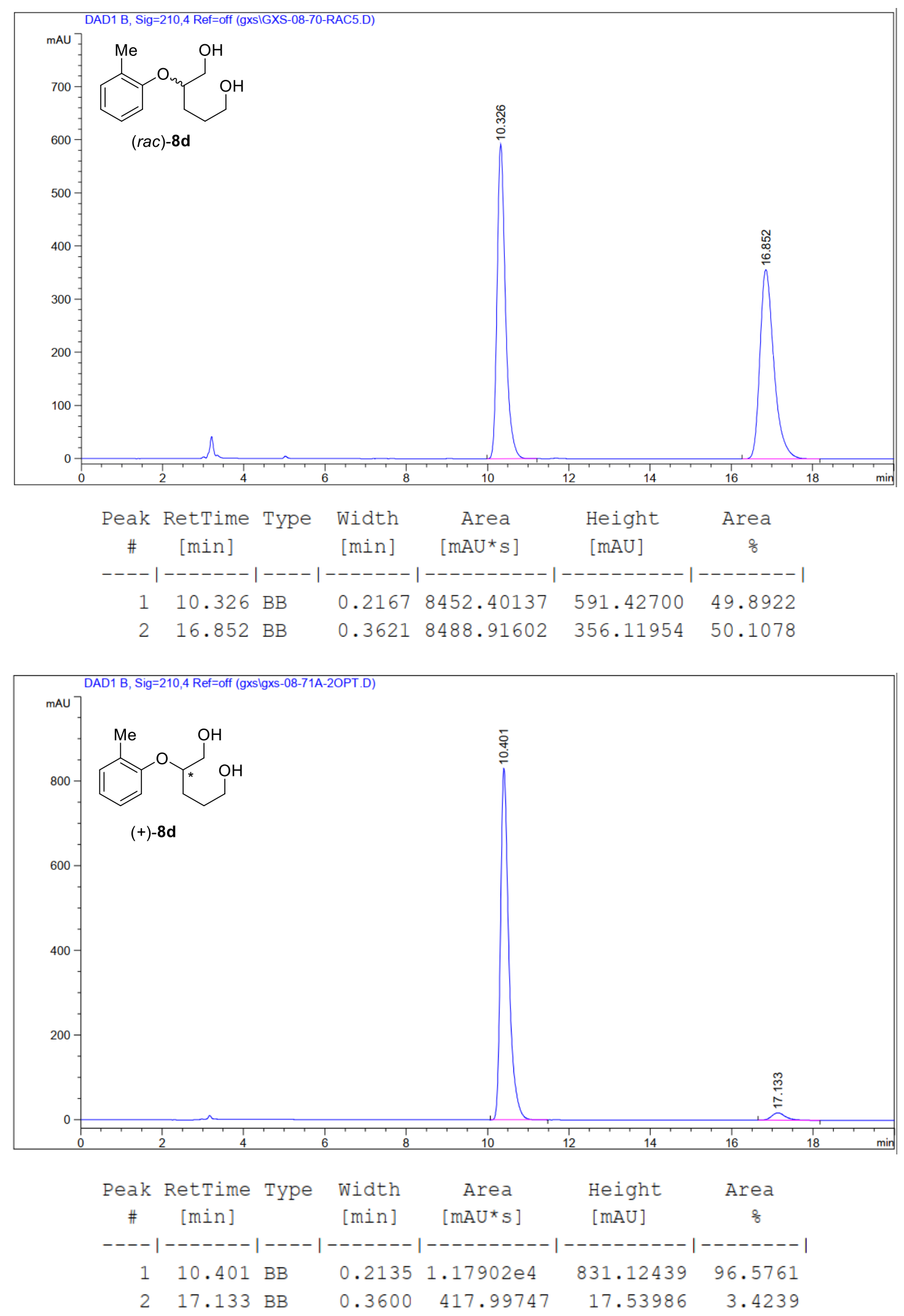
(+)-2-(2-Bromophenoxy)pentane-1,5-diol ((+)-8e)
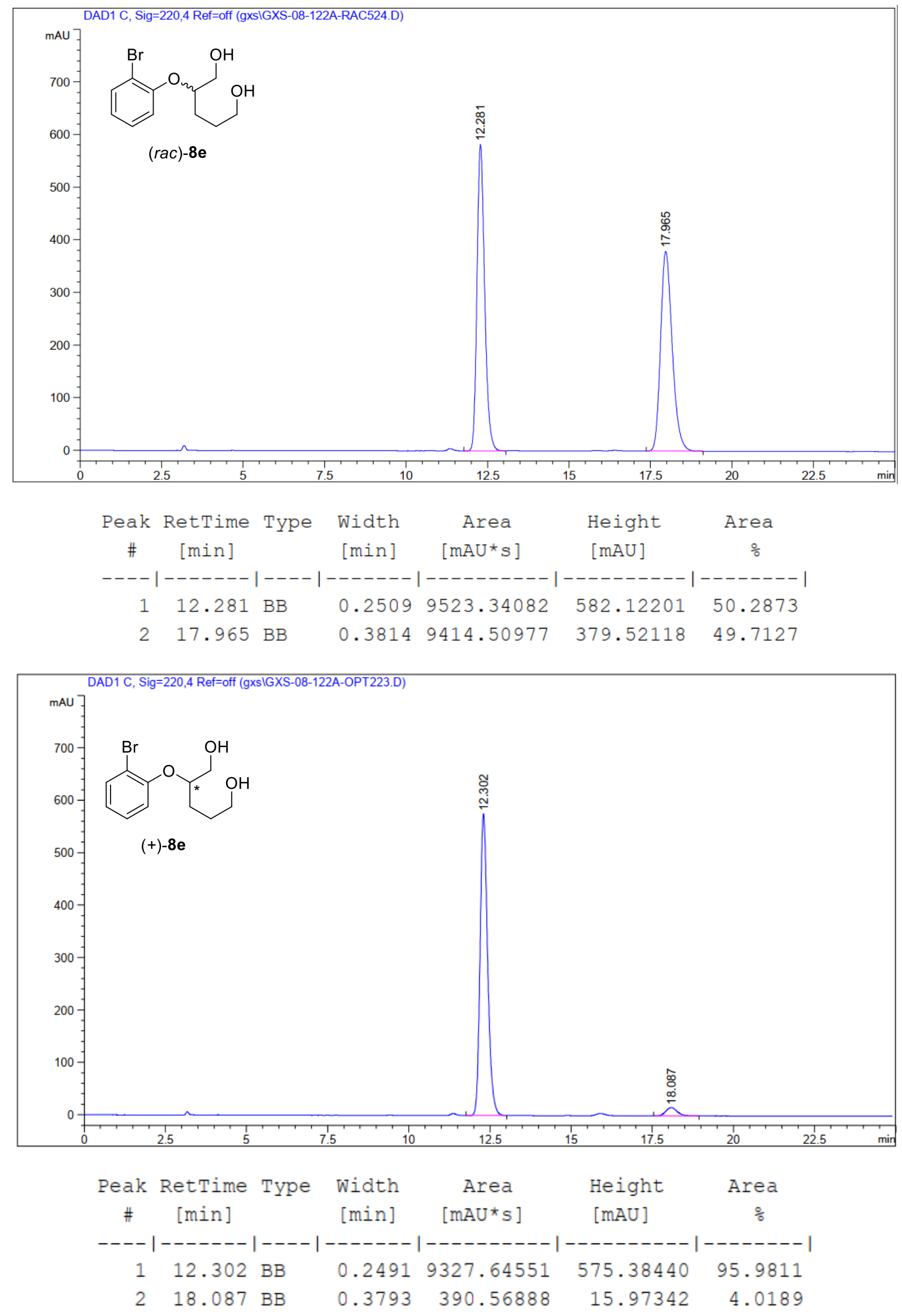
(+)-2-(2-Chlorophenoxy)pentane-1,5-diol ((+)-8f)
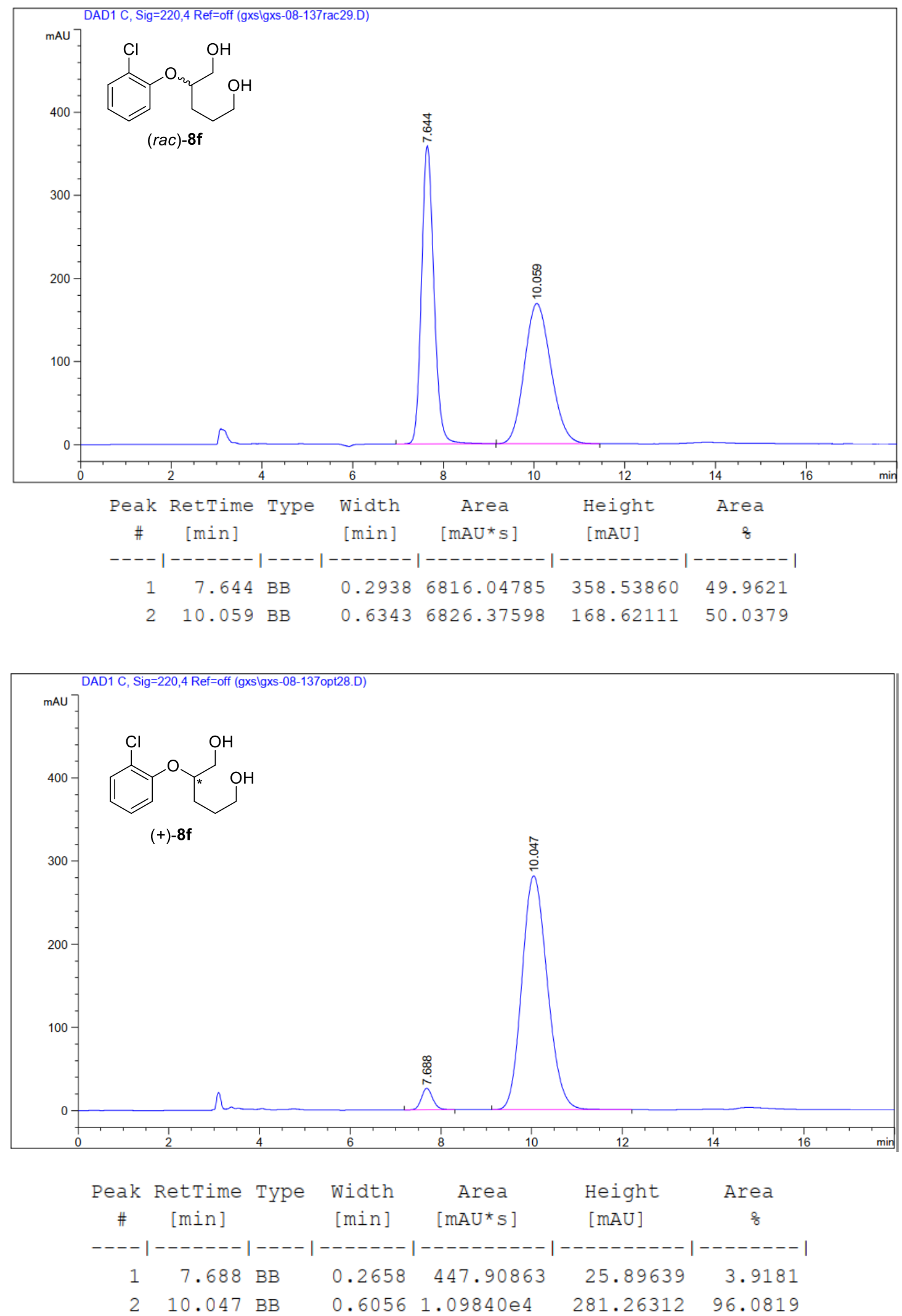
(+)-2-(2,4-Dimethylphenoxy)pentane-1,5-diol ((+)-8g)
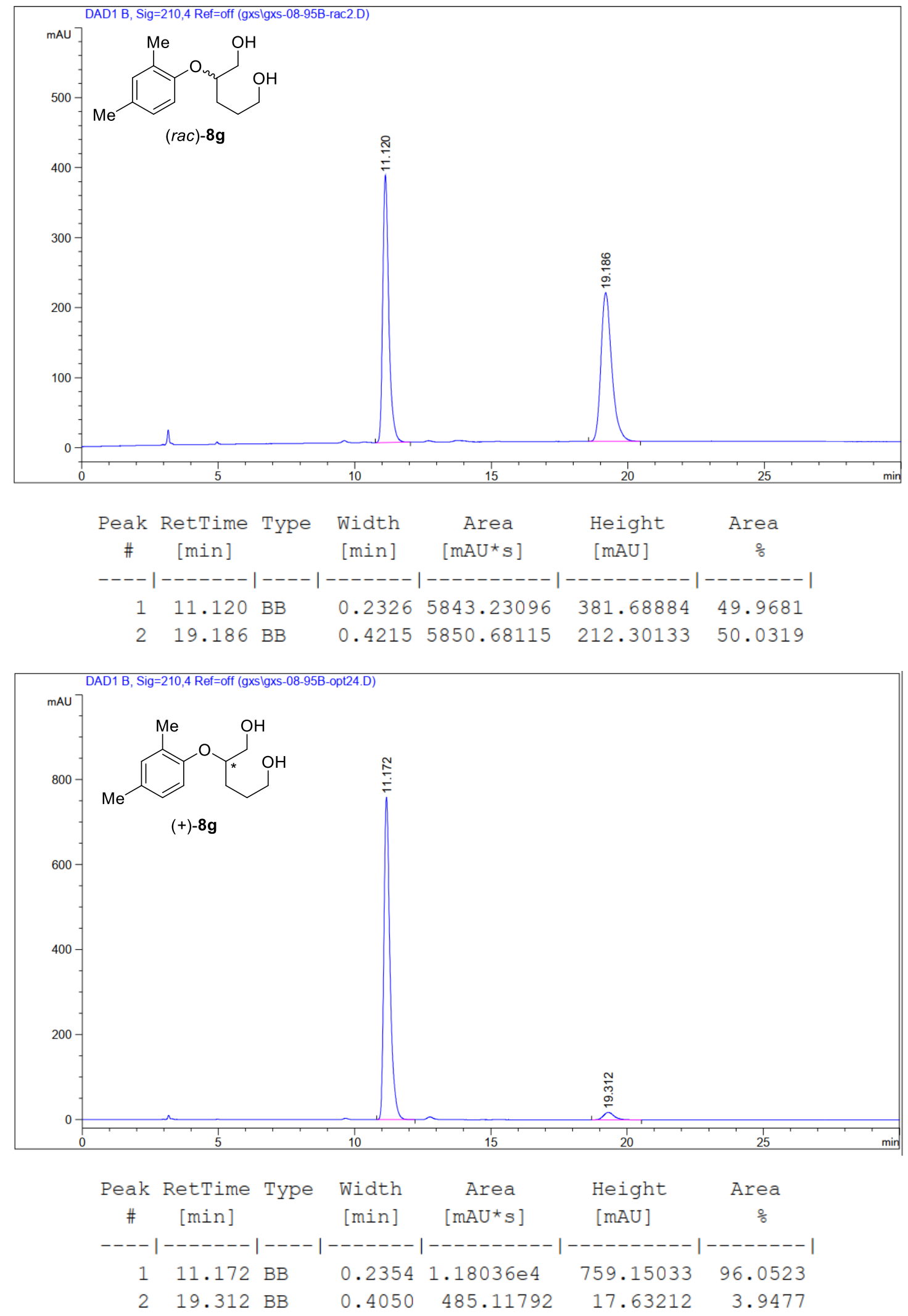
$(+)-3-P h e n o x y t e t r a h y d r o f u r a n((+)-6 a)$ (Note: derived from $(+)-5 a)$
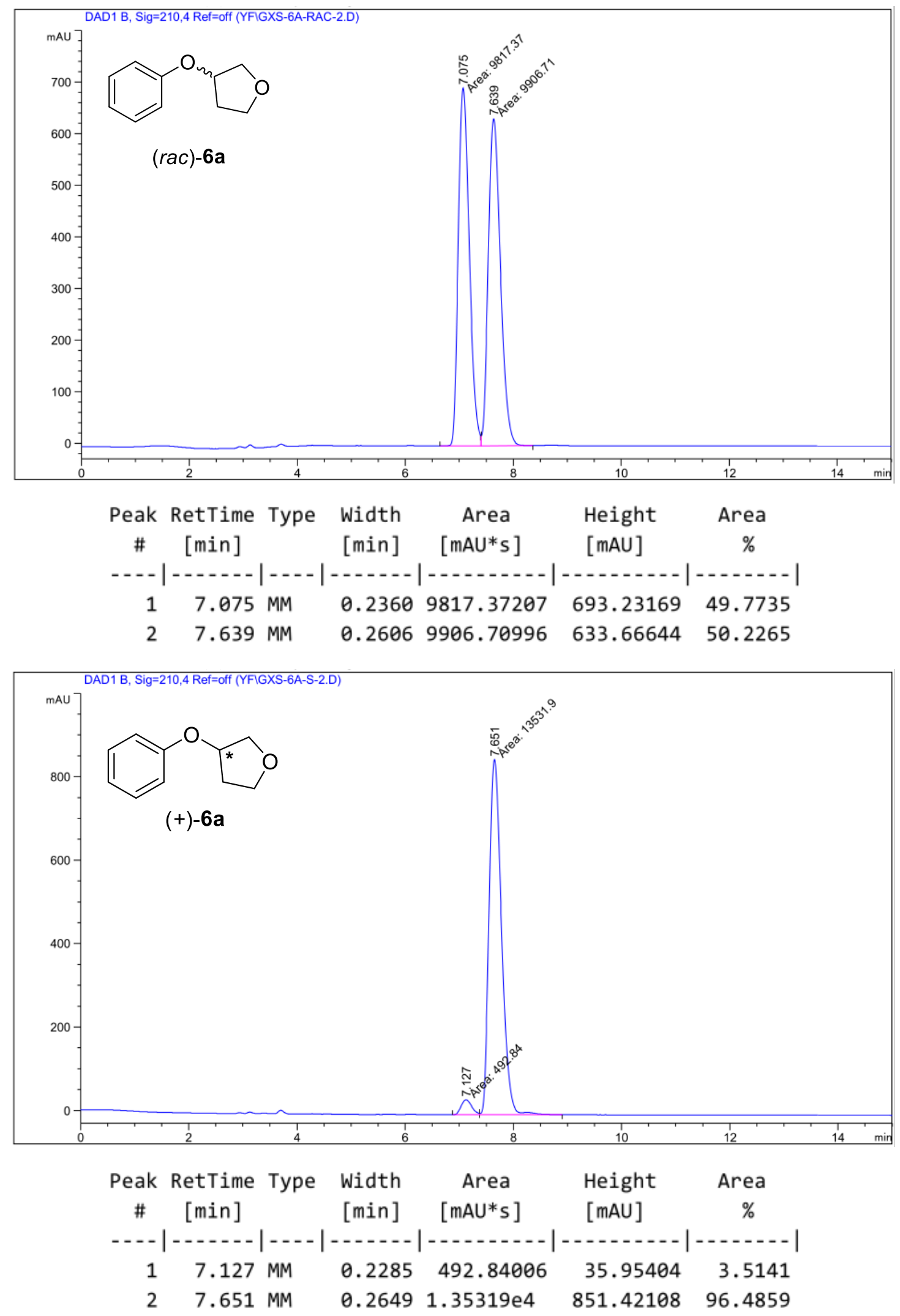
(-)-3-(p-Tolyloxy)tetrahydrofuran ((-)-6b)
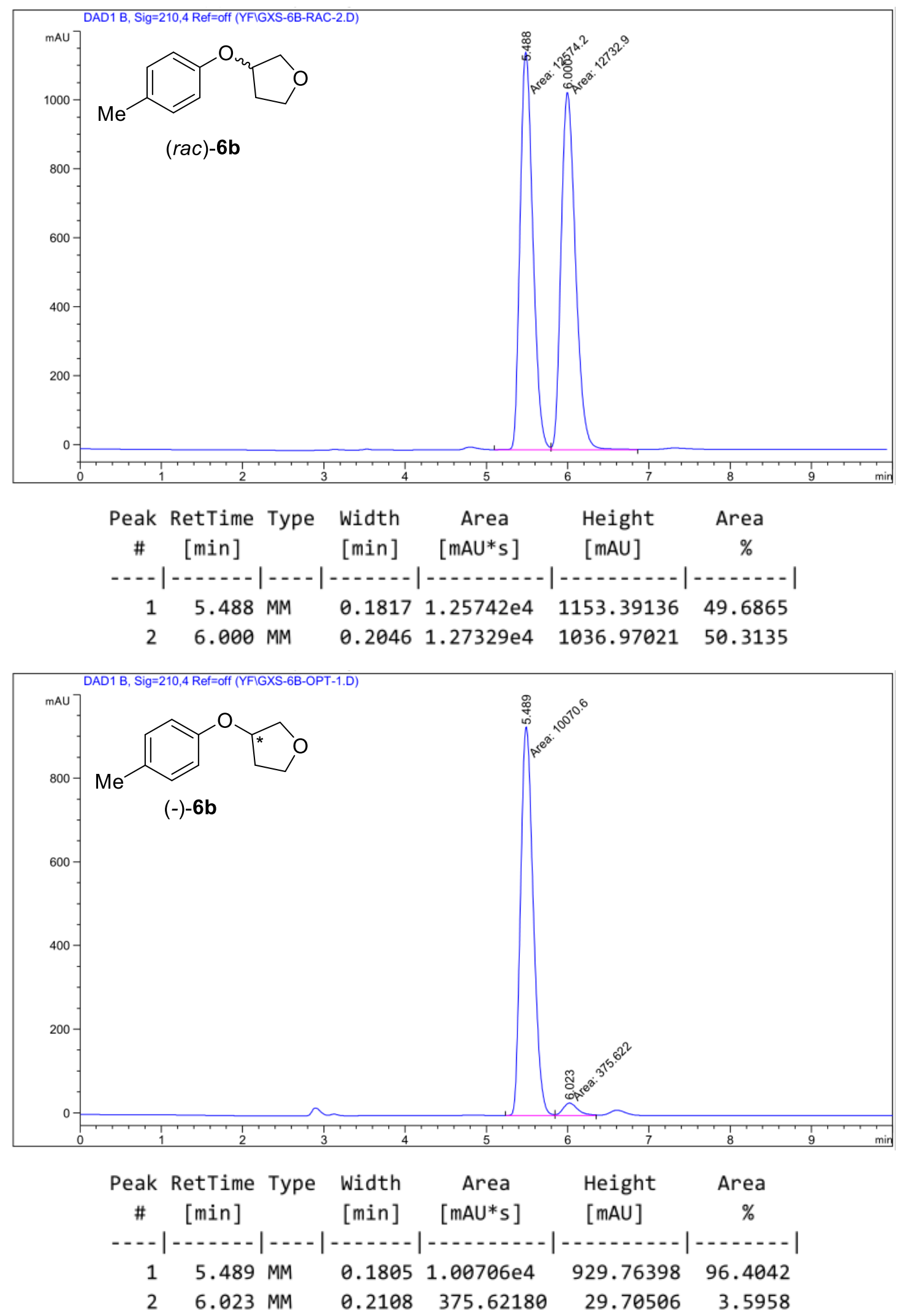
(-)-3-(o-Tolyloxy)tetrahydrofuran ((-)-6h)
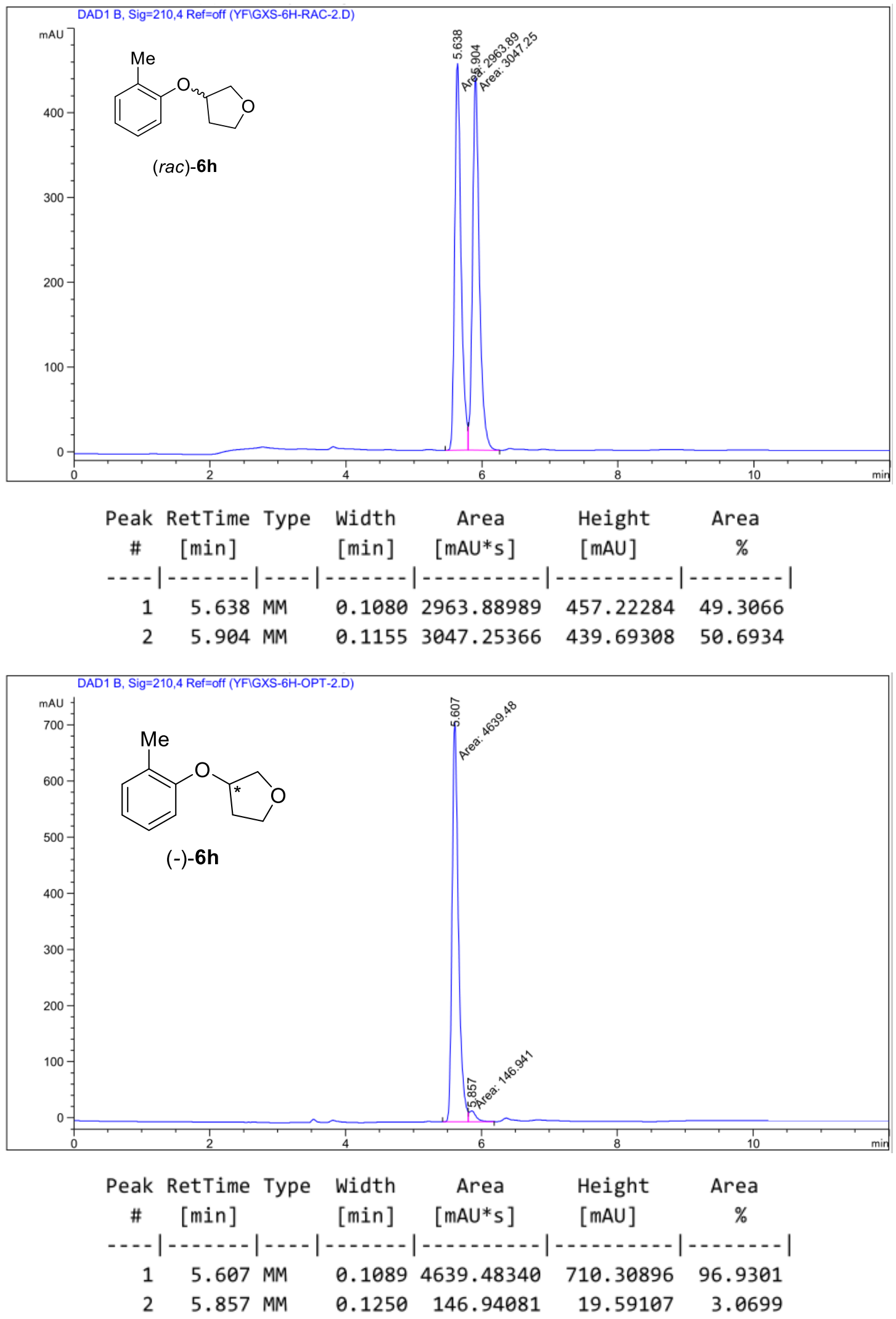
(-)-3-(2-Fluorophenoxy)tetrahydrofuran ((-)-6k)
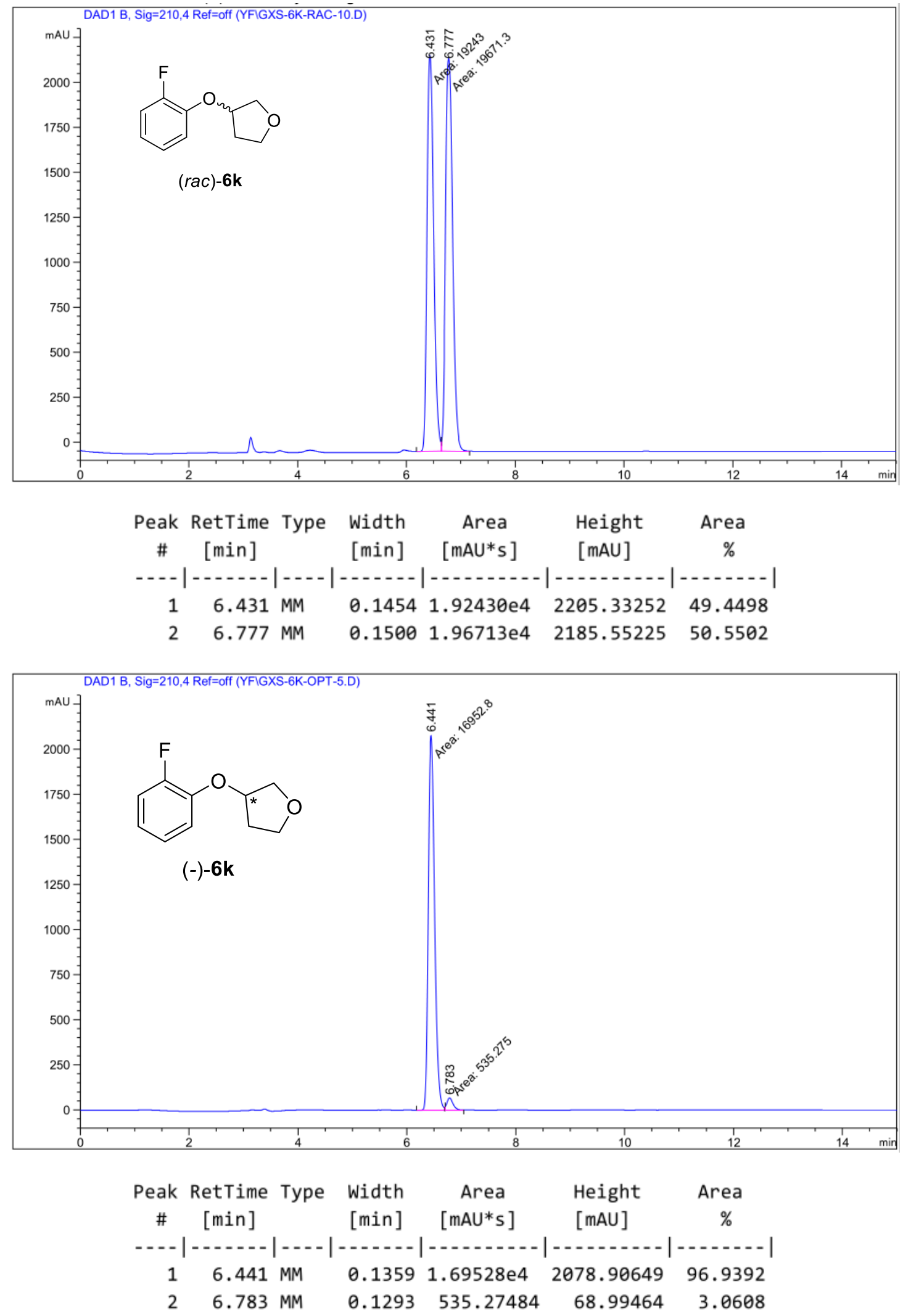
(R)-3-(2,5-Dimethylphenoxy)tetrahydrofuran ((R)-6l)
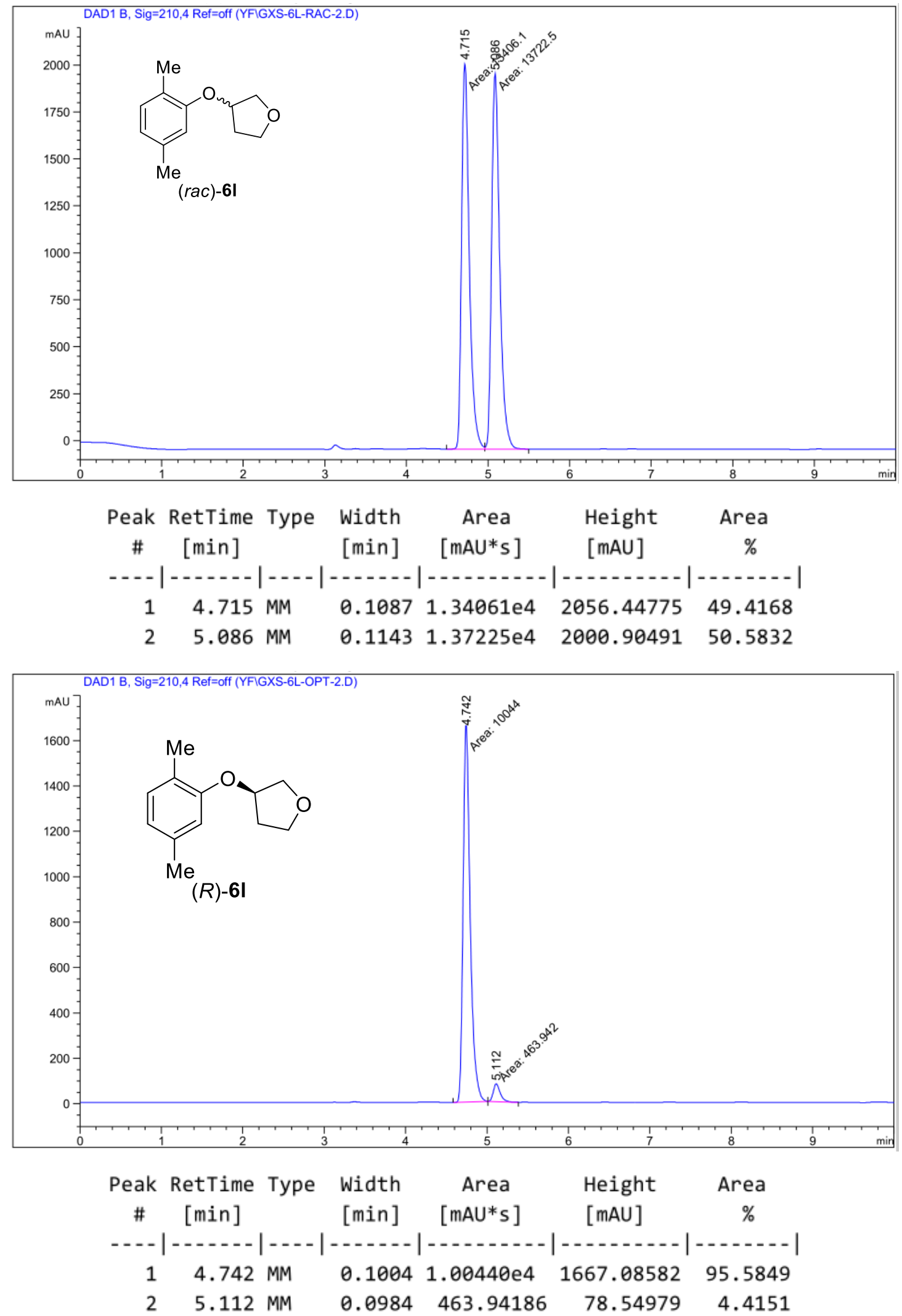
(-)-3-(Naphthalen-2-yloxy)tetrahydrofuran ((-)-6m)
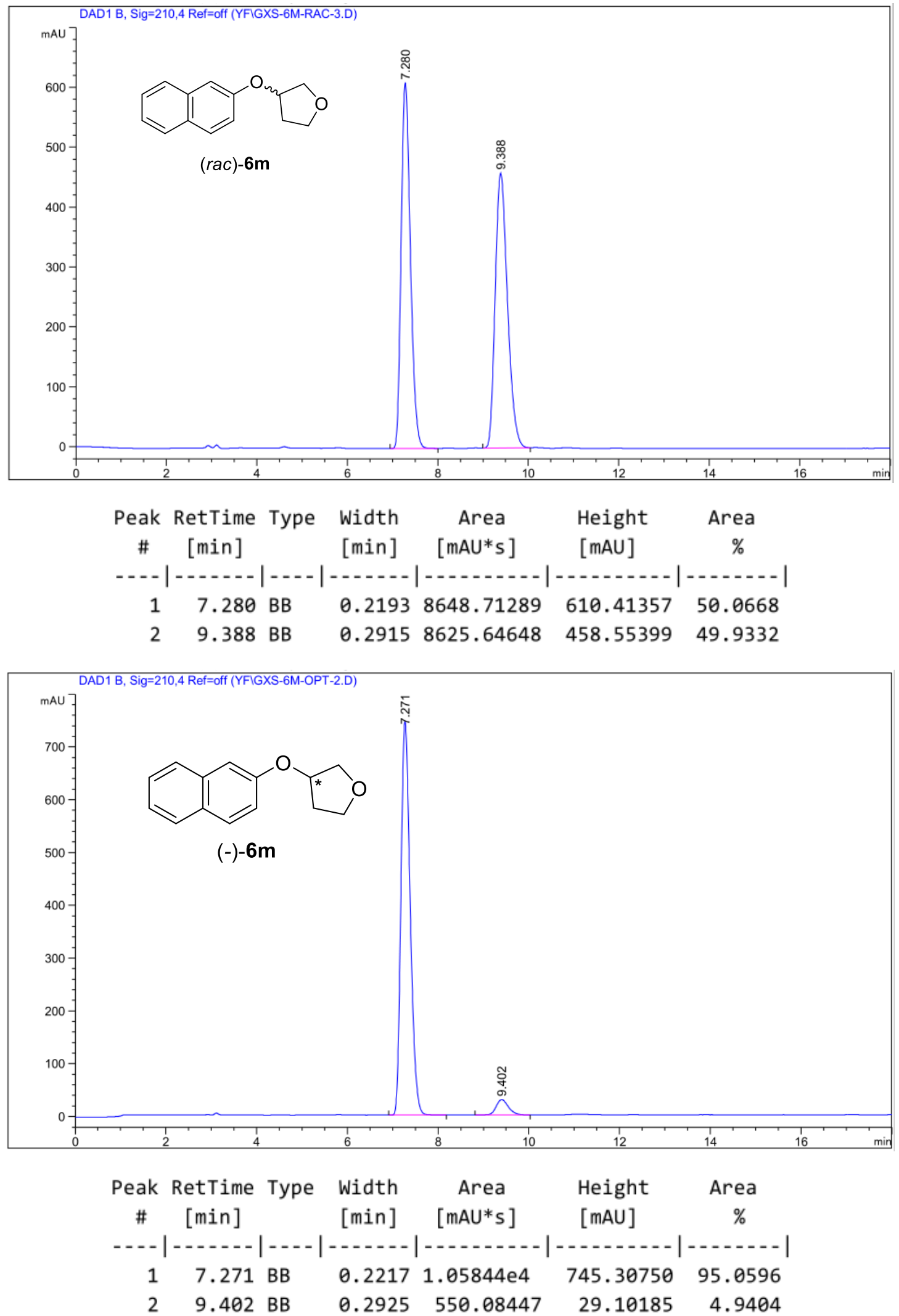


\section{(S)-2-(4-(5-bromo-2-chlorobenzyl)phenoxy)butane-1,4-diol ((S)-12)}
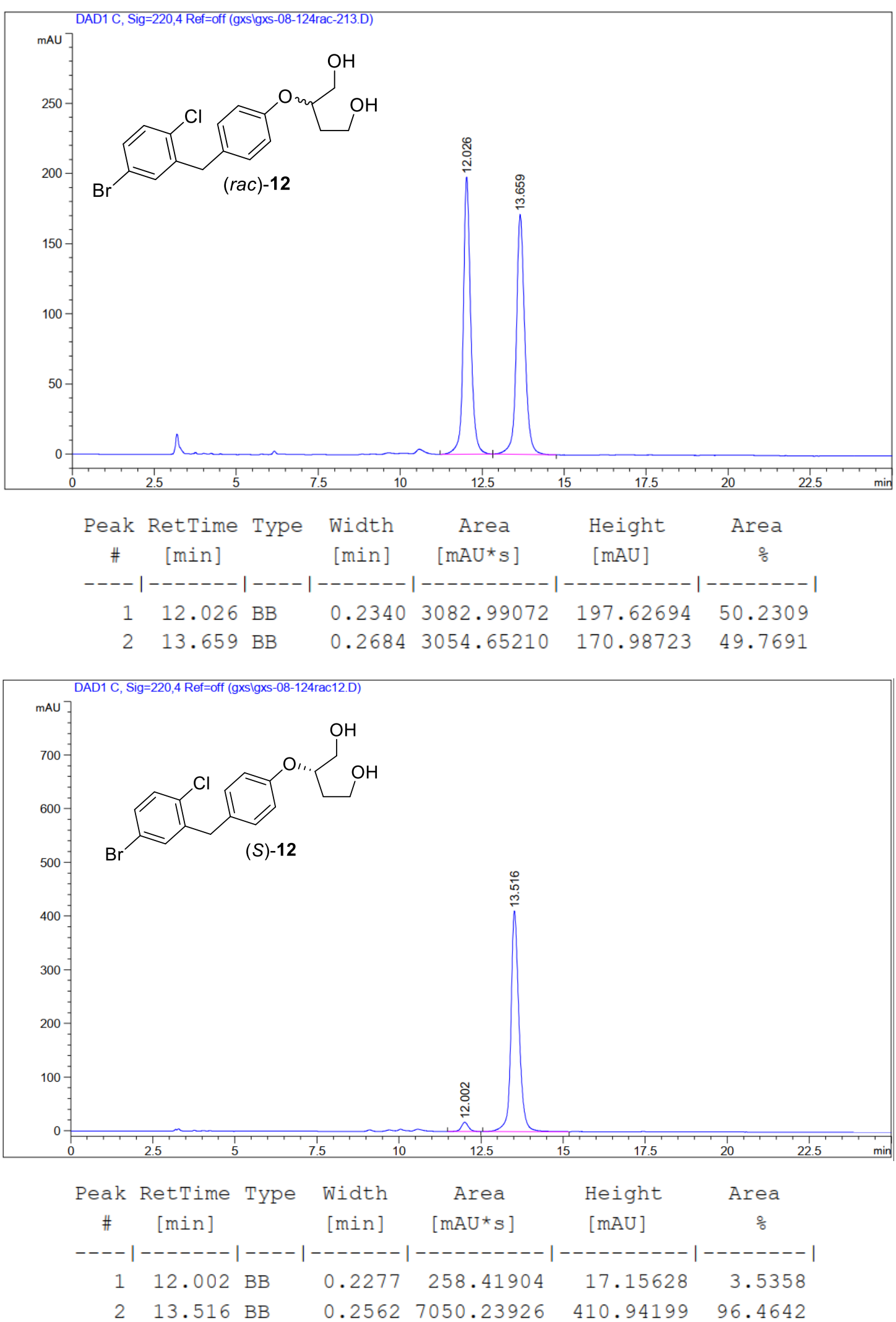


\section{Compound (S)-13}
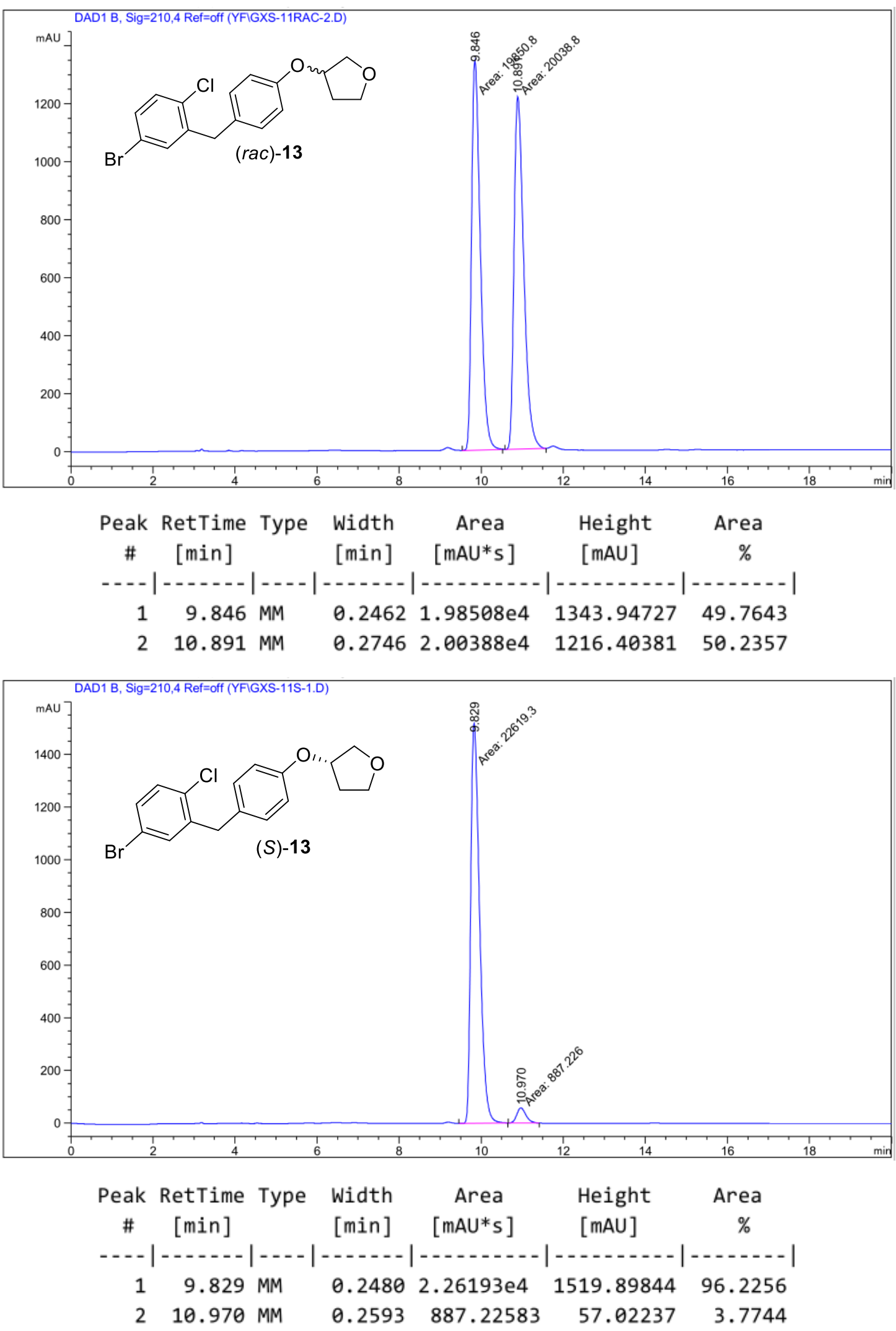
(S)-1-Methyl-3-phenoxypyrrolidine ((S)-15)
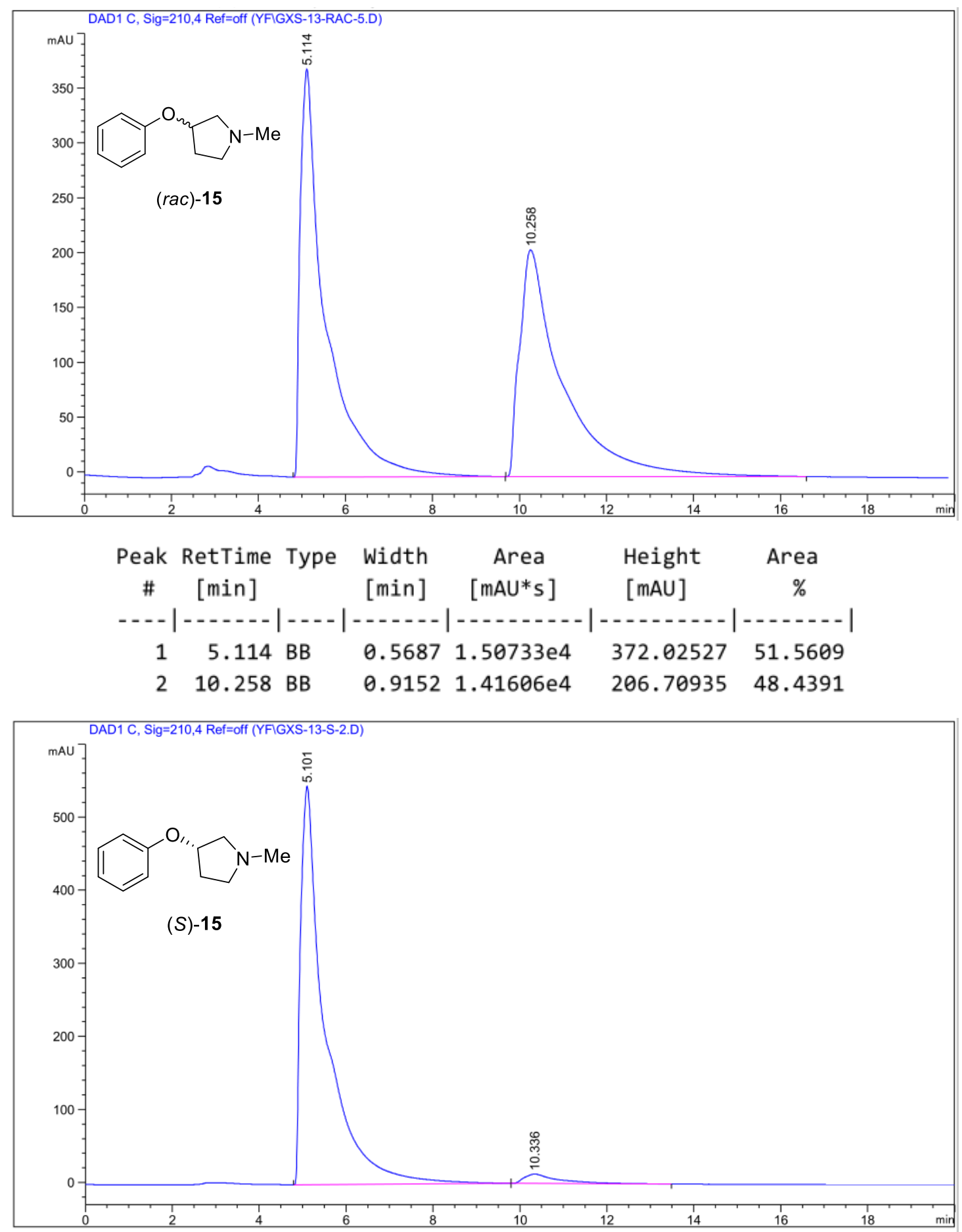

Peak RetTime Type Width Area Height Area

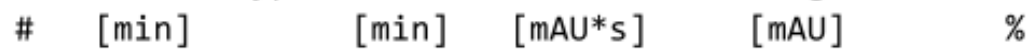

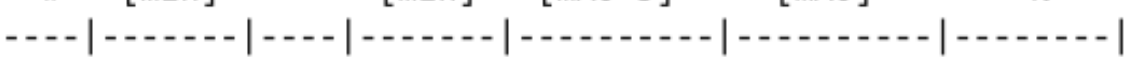

$\begin{array}{lllllll}1 & 5.101 & \text { BB } & 0.5563 & 2.16107 \mathrm{e} 4 & 545.39209 & 96.8039\end{array}$

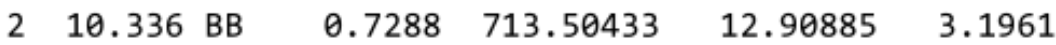

